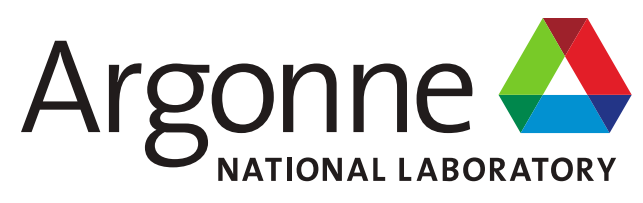

\title{
Irradiation Demonstration Element Design Parameters for MURR LEU U-Mo Fuel Conversion
}

Nuclear Science \& Engineering Division 


\title{
About Argonne National Laboratory
}

Argonne is a U.S. Department of Energy laboratory managed by UChicago Argonne, LLC under contract DE-AC02-06CH11357. The Laboratory's main facility is outside Chicago, at 9700 South Cass Avenue, Argonne, Illinois 60439. For information about Argonne and its pioneering science and technology programs, see www.anl.gov.

\section{DOCUMENT AVAILABILITY}

Online Access: U.S. Department of Energy (DOE) reports produced after 1991 and a growing number of pre-1991 documents are available free at OSTI.GOV (http://www.osti.gov/), a service of the U.S. Dept. of Energy's Office of Scientific and Technical Information.

\author{
Reports not in digital format may be purchased by the public from the \\ National Technical Information Service (NTIS): \\ U.S. Department of Commerce \\ National Technical Information Service \\ 5301 Shawnee Rd \\ Alexandria, VA 22312 \\ www.ntis.gov \\ Phone: (800) 553-NTIS (6847) or (703) \\ 605-6000 Fax: (703) 605-6900 \\ Email: orders@ntis.gov
}

\section{Reports not in digital format are available to DOE and DOE contractors from the Office of Scientific and Technical Information (OSTI):}

U.S. Department of Energy

Office of Scientific and Technical Information

P.O. Box 62

Oak Ridge, TN 37831-0062

www.osti.gov

Phone: (865) 576-8401

Fax: (865) 576-5728

Email: reports@osti.gov

Disclaimer

\section{Disclaimer}

This report was prepared as an account of work sponsored by an agency of the United States Government. Neither the United States Government nor any agency thereof, nor UChicago Argonne, LLC, nor any of their employees or officers, makes any warranty, express or implied, or assumes any legal liability or responsibility for the accuracy, completeness, or usefulness of any information, apparatus, product, or process disclosed, or represents that its use would not infringe privately owned rights. Reference herein to any specific commercial product, process, or service by trade name, trademark, manufacturer, or otherwise, does not necessarily constitute or imply its endorsement, recommendation, or favoring by the United States Government or any agency thereof. The views and opinions of document authors expressed herein do not necessarily state or reflect those of the United States Government or any agency thereof, Argonne National Laboratory, or UChicago Argonne, LLC. 
ANL/RTR/TM-18/4 Rev. 1

\section{Irradiation Demonstration Element Design Parameters for MURR LEU U-Mo Fuel Conversion}

J. Stillman'1 , E. Wilson ${ }^{1}$, D. S. Yoon ${ }^{1}$, L. Foyto' ${ }^{2}$, K. Kutikkad², J. C. McKibben², and N. Peters²

${ }^{1}$ Nuclear Science \& Engineering Division, Argonne National Laboratory

${ }^{2}$ University of Missouri-Columbia Research Reactor

Nuclear Science \& Engineering Division, Argonne National Laboratory

March 2020 
(This page left intentionally blank) 


\section{DEFINITIONS OF TERMS AND ACRONYMS}

\begin{tabular}{|c|c|}
\hline Term & Definition \\
\hline Best estimate & $\begin{array}{l}\text { Parameter that is determined with the best available methods and models } \\
\text { without including uncertainty. }\end{array}$ \\
\hline Bounding & $\begin{array}{l}\text { A condition that has been technically determined to not be exceeded under } \\
\text { given conditions, such as, for example, normal operating conditions }\end{array}$ \\
\hline Conservative & $\begin{array}{l}\text { Method, or resulting parameter, that is not best estimate and includes } \\
\text { uncertainty or margin whether discretionary or due to conservative } \\
\text { assumptions }\end{array}$ \\
\hline $\begin{array}{l}\text { Conservative } \\
\text { assumption }\end{array}$ & $\begin{array}{l}\text { Parameter or other consideration technically determined to be } \\
\text { conservative as a consequence of intentional addition of margin or the use } \\
\text { bounding data or other consideration. }\end{array}$ \\
\hline $\begin{array}{l}\text { Conservative } \\
\text { band of normal } \\
\text { operating } \\
\text { conditions }\end{array}$ & $\begin{array}{l}\text { Conservative conditions that are reasonably expected to occur up to and } \\
\text { including at the reactor licensed power, and including calculation and } \\
\text { measurement uncertainties }\end{array}$ \\
\hline DDE & Design demonstration element tested as an experiment in a test reactor \\
\hline $\begin{array}{l}\text { Design } \\
\text { parameter }\end{array}$ & Parameter used in design, qualification tests, and licensing \\
\hline Fission density & The number of nuclear fission events of all isotopes per volume of fuel \\
\hline $\begin{array}{l}\text { Fuel } \\
\text { qualification }\end{array}$ & $\begin{array}{l}\text { The process of designing, conducting, and evaluating experiments to assure } \\
\text { that the fuel is capable of performing without failure during reactor } \\
\text { operations up to reported performance limits. Fuel qualification also } \\
\text { includes measurements and reporting of fuel properties that can be used in } \\
\text { performance and safety modeling. }\end{array}$ \\
\hline $\begin{array}{l}\text { Irradiation } \\
\text { conditions }\end{array}$ & $\begin{array}{l}\text { Conditions occurring in a reactor during operation at power and that, in } \\
\text { the context of fuel design and qualification, refer to conditions experienced } \\
\text { in reactor driver fuel or fuel testing. }\end{array}$ \\
\hline HEU & Highly-enriched uranium with $\geq 20$ weight $\%$ enrichment \\
\hline LCO & Limiting Condition of Operation \\
\hline LEU & Low-enriched uranium with $<20$ weight $\%$ enrichment \\
\hline Licensed power & $\begin{array}{l}\text { The power approved by the regulatory authority up to which the reactor } \\
\text { can operate, including any amendments or changes that modify a safety } \\
\text { basis. For a reactor without a defined licensed duration this term is taken } \\
\text { to be the equivalent of approved operating power. }\end{array}$ \\
\hline LSSS & Limiting Safety System Setting \\
\hline LTA & Lead test assembly \\
\hline Margin & $\begin{array}{l}\text { A parameter where the value is determined other than on a best estimate } \\
\text { basis, and to be conservative is increased (or decreased) on a discretionary } \\
\text { basis or due to conservative assumptions }\end{array}$ \\
\hline $\begin{array}{l}\text { Maximum local } \\
\text { parameter }\end{array}$ & $\begin{array}{l}\text { Bounding value among considered operational states, and for a size scale } \\
\text { relevant to the safety basis under which a reactor can operate. For } \\
\text { example, maximum local fission density is calculated as an input to } \\
\text { qualification tests, and then used in the licensing basis as a limit during fuel } \\
\text { management. Also commonly referred to as a peak local parameter. }\end{array}$ \\
\hline M3 & NNSA Office of Material Management and Minimization \\
\hline
\end{tabular}




\begin{tabular}{|c|c|}
\hline Term & Definition \\
\hline MURR & University of Missouri Research Reactor \\
\hline NNSA & US National Nuclear Security Administration \\
\hline Nominal & Value of a parameter under normal operating conditions \\
\hline $\begin{array}{l}\text { Normal } \\
\text { operating } \\
\text { conditions }\end{array}$ & $\begin{array}{l}\text { Conditions that are reasonably expected to occur up to and including at the } \\
\text { reactor licensed power. These do not include calculation or measurement } \\
\text { uncertainties. }\end{array}$ \\
\hline NRC & US Nuclear Regulatory Commission \\
\hline Power density & Heat generated by fission reactions deposited in a fuel volume \\
\hline Prototypic & Conditions that are considered representative of normal operations. \\
\hline PSAR & $\begin{array}{l}\text { Preliminary SAR submitted to, and reviewed by, the regulator of the } \\
\text { facility. A PSAR is distinct from the SAR since the SAR is submitted to } \\
\text { modify a license. }\end{array}$ \\
\hline $\mathrm{R} \& \mathrm{D}$ & Research and development \\
\hline Safety basis & $\begin{array}{l}\text { A SAR, referenced supporting information, and other regulatory materials } \\
\text { that provide the basis for safe operation of a reactor facility }\end{array}$ \\
\hline Safety limit & A regulator approved limit ensuring safe reactor operation \\
\hline SAR & $\begin{array}{l}\text { Safety Analysis Report submitted to, and approved by, the regulator of the } \\
\text { facility }\end{array}$ \\
\hline Steady-state & Conditions that can be persistent and are not transient \\
\hline TS & $\begin{array}{l}\text { Technical specification on reactor operations that are approved by the } \\
\text { regulator. Technical specifications include licensed power, LCO, LSSS, and } \\
\text { safety limit requirements. }\end{array}$ \\
\hline Target test value & $\begin{array}{l}\text { The goal value of a design parameter to be achieved during testing, such as } \\
\text { during an irradiation experiment }\end{array}$ \\
\hline UMo & Uranium molybdenum alloy fuel \\
\hline U-10Mo & $\begin{array}{l}\text { Uranium - } 10 \mathrm{wt} \% \text { molybdenum alloy fuel being developed as a monolithic } \\
\text { metallic alloy fuel }\end{array}$ \\
\hline USHPRR & US high-performance research reactor \\
\hline
\end{tabular}




\section{PREFACE}

This report contains the results of reactor design and performance calculations for conversion of the University of Missouri Research Reactor (MURR ${ }^{\circledR}$ ) from the use of highly-enriched uranium (HEU) fuel to the use of low-enriched uranium (LEU) fuel. The analyses were performed by staff members of the U.S. Department of Energy National Nuclear Security Administration (NNSA) Office of Material Management and Minimization (M3) Reactor Conversion Program at the Argonne National Laboratory and the MURR Facility.

In the framework of non-proliferation policies, the international community presently aims to minimize the amount of nuclear material available that could be used for nuclear weapons. In this geopolitical context most research and test reactors, both domestic and international, have completed or are in the process of conversion to the use of LEU fuel. At this time worldwide 100 reactors have been converted to LEU fuel, or are verified to have permanently shutdown and no longer use HEU. Among remaining HEU reactors worldwide, a number of high performance reactors that refuel frequently remain for which new higher-density fuel is required. A new type of LEU fuel based on an alloy of uranium and molybdenum has been developed by the NNSA U.S. High Performance Research Reactor (USHPRR) program across multiple US national laboratories. This U$10 \mathrm{Mo}$ fuel is expected to allow the conversion of U.S. domestic high performance research reactors like MURR.

As an overview to the fuel testing of the USHPRR program, 142 small and large-size UMo monolithic fuel plates have been irradiated with during the R\&D phase of the program. Over half of these plates were fabricated with the down-selected fuel material system with roll-bonded zirconium interlayers between the cladding and U-10Mo fuel core. All plates from the down-selected system showed good irradiation performance when tested in the design parameter envelope required by the USHPRR. In future testing, many MURR-specific plates are planned to be irradiated during the qualification phase of the program. In addition, a full prototypic MURR element as a Design Demonstration Element (DDE) experiment is to be irradiated in a test reactor. Including irradiation of MURR's prototypic element, fuel qualification plans are to irradiate approximately at least a half-dozen full-size USHPRR U-10Mo elements for MURR, MITR, NBSR and ATR (which, in typical 2-pump operational mode, will achieve a fission density and power density similar to the maximum values for MURR) prior to conversion of MURR to the new LEU U-10Mo fuel.

The original version of this report documented the best estimate nominal steady-state irradiation conditions to be used in the design of the MURR DDE experiment. The maximum calculated irradiation parameters in MURR cores fueled with LEU U-10Mo for key sets of plates were included. Also included were best estimate data for all fuel plates in the MURR element under prototypic conditions.

The motivation for Revision 1 of this report is to include additional information for the design of the MURR DDE to obtain conditions when the experiment is irradiated in the test reactor that are regime appropriate for normal operations in MURR. Specifically, this additional information includes a range for the end-channel thicknesses, the nominal inter-element spacing, and nominal thermal-hydraulic conditions for prototypic operations at MURR.

The data presented herein serve as design parameters for irradiation qualification tests and, upon successful completion of fuel qualification, allows the program to bound MURR and other USHPRR irradiation conditions, and so allow a licensing basis for a conversion Safety Analysis Report (SAR)

Irradiation Demonstration Element Design Parameters for MURR LEU U-Mo Fuel Conversion 
specific to MURR. Within a program working on fuel fabrication at a commercial scale and fuel irradiation qualification, the source of the design parameters is fuel element design and safety and operational analyses that have been completed. This work provides the technical basis for what the MURR reactor recently submitted to the U.S. Nuclear Regulatory Commission (NRC) as a Preliminary SAR for LEU conversion. A final SAR and the ability to convert is awaiting successful completion of fuel fabrication deployment and fuel irradiation qualification efforts.

In addition to this report giving parameters from operational and safety analyses, for fabrication there are related documents for fuel element specifications and drawings. The design of the fuel element incorporates manufacturing tolerance requirements. These documents have also been completed consistent with the design and safety basis that has been submitted to the NRC in the Preliminary SAR for MURR conversion to LEU U-10Mo fuel. Thus, the sum of the technical work completed to date provides a comprehensive basis for fabricating and irradiating fuel elements consistent with what is planned to be later required to license and operate MURR. 


\section{Table of Contents}

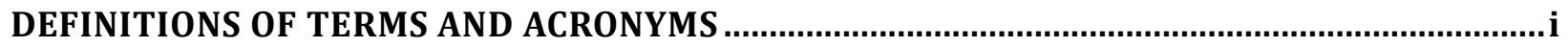

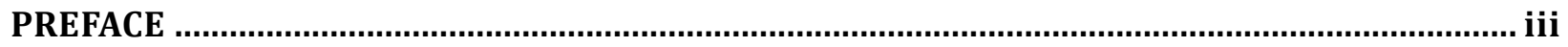

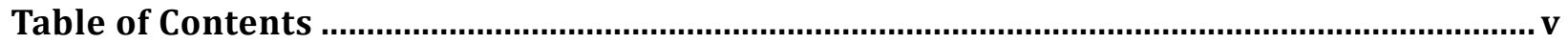

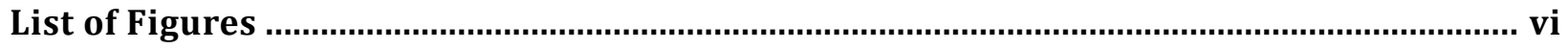

List of Tables

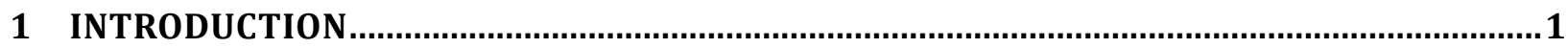

2 FUEL ELEMENT GEOMETRY

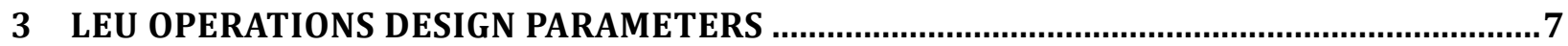

3.1 Scope of Steady-State Core Conditions Evaluated ……........................................................................ 8

3.2 Axial and Azimuthal Discretization of the Results ...........................................................................10

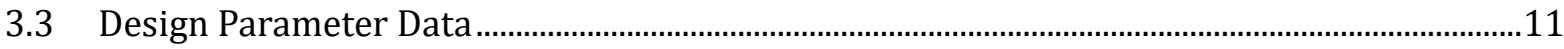

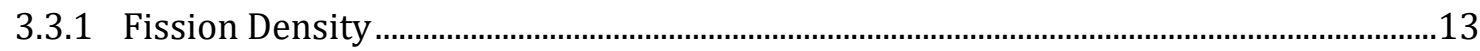

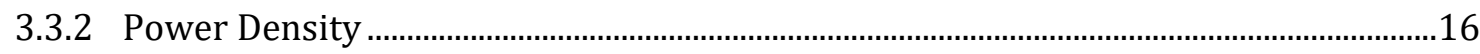

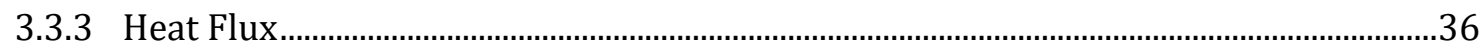

3.3.4 Steady-State Temperatures for LEU Fuel Plates ..............................................................43

4 SUMMARY OF MURR LEU IRRADIATION PARAMETERS .................................................. 48

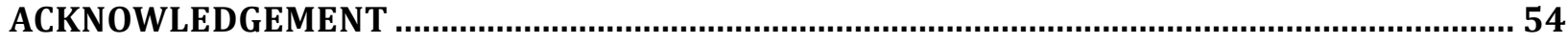

REFERENCES

APPENDIX A: Plate Specific Fission Density Data in MURR LEU Element .......................... 57

APPENDIX B: Plate Specific Power Density Data in MURR LEU Element ............................ 81 


\section{List of Figures}

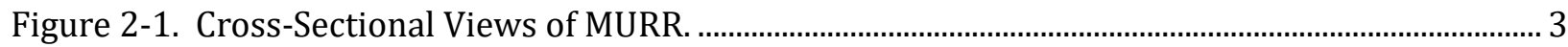

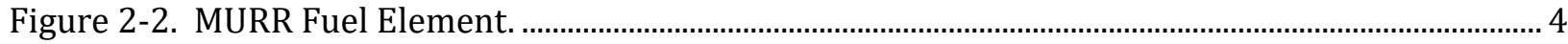

Figure 2-3. Cross-Sectional View of MURR LEU Fuel Element................................................................ 5

Figure 2-4. Cross-Sectional View of MURR LEU Element Plates 1-5 and 19-23...................................... 6

Figure 3-1. Fission Density by Plate in Discharged LEU Fuel.........................................................................15

Figure 3-2. Axial Profile of Discharge Fission Density in LEU Fuel Plates. ..................................................16

Figure 3-3. Power Density Profile $(\mathrm{kW} / \mathrm{cm} 3)$ for Plate with Maximum Power Density of All SteadyState Cores: Fresh LEU Element in Core 5B1, Position X7, No Xe, Plate 1, Empty FT. .............................18

Figure 3-4. Evolution of Power Density Profile $(\mathrm{kW} / \mathrm{cm} 3)$ for LEU Fuel Plate 1, Element Burnup from 0 to $77 \mathrm{MWd}$ : Core 7A, no Xe, FT loaded with typical samples. 24

Figure 3-5. Evolution of Power Density Profile $(\mathrm{kW} / \mathrm{cm} 3)$ for LEU Fuel Plate 1, Element Burnup from 96 to $170 \mathrm{MWd}$ : Core 7A, no Xe, FT loaded with typical samples. 25

Figure 3-6. Evolution of Heat Flux Profile (W/cm2) for LEU Fuel Plate 22, Element Burnup from 0 to 77 MWd: Core 7A, no Xe, FT loaded with typical samples. 41

Figure 3-7. Evolution of Heat Flux Profile (W/cm2) for LEU Fuel Plate 22, Element Burnup from 96 to $170 \mathrm{MWd}$ : Core 7A, no Xe, FT loaded with typical samples.

Figure 3-8. Axial Fuel Temperature Profile by Plate for EOL LEU Element in Core 7A..........................47

Figure 4-1. Evolution of Heat Flux Profile (W/cm2) for LEU Fuel Plate 22, Element 49 


\section{List of Tables}

Table 2-1. Nominal LEU Fuel Element and Plate Dimensions and Loadings. ............................................ 6

Table 3-1. Nomenclature for LEU Core Case Names: $c f b$ (e.g. 5B1) …………............................................... 8

Table 3-2. Summary of Power Distribution Evaluations for LEU Cores....................................................... 9

Table 3-3. Beginning-of-Cycle Burnups for Reference LEU Mixed Burnup Core $(c=7,8)$......................10

Table 3-4. Summary of Local Power Parameters Evaluated for LEU Cores.................................................12

Table 3-5. Maximum Power Density for Various Cores....................................................................................13

Table 3-6. Plate Discharge Fission Density for LEU Fuel..........................................................................14

Table 3-7. Discharge Fission Density (x1020 fissions $/ \mathrm{cm}^{3}$ ) in LEU Fuel Plates......................................15

Table 3-8. Power Density Data $\left(\mathrm{kW} / \mathrm{cm}^{3}\right)$ for Plate with Maximum Power Density of All Steady-State Cores: Fresh LEU Element in Core 5B1, Position X7, No Xe, Plate 1, Empty FT...........................................17

Table 3-9. Power Density Data $(\mathrm{kW} / \mathrm{cm} 3)$ in Fuel Plate 1 in $0 \mathrm{MWd}$ Element in Reference Mixed Burnup Core: Core 7A, Position X5, No Xe, FT loaded with typical samples..............................................20

Table 3-10. Power Density Data $(\mathrm{kW} / \mathrm{cm} 3)$ in Fuel Plate 1 in $77 \mathrm{MWd}$ Element in Reference Mixed Burnup Core: Core 7A, Position X3, No Xe, FT loaded with typical samples..............................................21

Table 3-11. Power Density Data (kW/cm3) in Fuel Plate 1 in 96 MWd Element in Reference Mixed Burnup Core: Core 7A, Position X2, No Xe, FT loaded with typical samples..............................................22

Table 3-12. Power Density Data (kW/cm3) in Fuel Plate 1 in 170 MWd Burnup Element in Reference Mixed Burnup Core: Core 7A, Position X4, No Xe, FT loaded with typical samples. ................................23

Table 3-13. Power Density Data $(\mathrm{kW} / \mathrm{cm} 3)$ in Fuel Plate 1 in $3 \mathrm{MWd}$ Burnup Element at Eq. Xe Conditions in Reference Mixed Burnup Core: Core 8A, Position X5, FT loaded with typical samples.

Table 3-14. Power Density Data $(\mathrm{kW} / \mathrm{cm} 3)$ in Fuel Plate 1 in 80 MWd Burnup Element at Eq. Xe Conditions in Reference Mixed Burnup Core: Core 8A, Position X3, FT loaded with typical samples.

Table 3-15. Power Density Data (kW/cm3) in Fuel Plate 1 in 99 MWd Burnup Element at Eq. Xe Conditions in Reference Mixed Burnup Core: Core 8A, Position X2, FT loaded with typical samples.

Table 3-16. Power Density Data (kW/cm3) in Fuel Plate 1 in 173 MWd Burnup Element at Eq. Xe Conditions in Reference Mixed Burnup Core: Core 8A, Position X4, FT loaded with typical samples.

Table 3-17. Power Density Data $\left(\mathrm{kW} / \mathrm{cm}^{3}\right)$ in Fuel Plate 22 in 0 MWd Burnup Element at No Xe Conditions in Reference Mixed Burnup Core: Core 7A, Position X1, FT loaded with typical samples.

Table 3-18. Power Density Data $\left(\mathrm{kW} / \mathrm{cm}^{3}\right)$ in Fuel Plate 22 in $3 \mathrm{MWd}$ Burnup Element at Eq. Xe Conditions in Reference Mixed Burnup Core: Core 8A, Positions X1, FT loaded with typical samples.

Table 3-19. Power Density Data $\left(\mathrm{kW} / \mathrm{cm}^{3}\right)$ in Fuel Plate 22 in $80 \mathrm{MWd}$ Burnup Element at Eq. Xe Conditions in Reference Mixed Burnup Core: Core 8A, Positions X7, FT loaded with typical samples. 
Table 3-20. Power Density Data $\left(\mathrm{kW} / \mathrm{cm}^{3}\right)$ in Fuel Plate 22 in 99 MWd Burnup Element at Eq. Xe Conditions in Reference Mixed Burnup Core: Core 8A, Positions X6, FT loaded with typical samples.

Table 3-21. Power Density Data $\left(\mathrm{kW} / \mathrm{cm}^{3}\right)$ in Fuel Plate 22 in $173 \mathrm{MWd}$ Burnup Element at Eq. Xe Conditions in Reference Mixed Burnup Core: Core 8A, Positions X8, FT loaded with typical samples.

Table 3-22. Heat Flux Data $\left(\mathrm{W} / \mathrm{cm}^{2}\right)$ for Fuel Plate 22 in 0 MWd Burnup Element in Reference Mixed Burnup Core: Core 7A, Position X1, No Xe, FT loaded with typical samples. 37

Table 3-23. Heat Flux Data (W/ $\mathrm{cm}^{2}$ ) for Fuel Plate 22 in 77 MWd Burnup Element in Reference Mixed Burnup Core: Core 7A, Position X7, No Xe, FT loaded with typical samples. 38

Table 3-24. Heat Flux Data (W/ $\mathrm{cm}^{2}$ ) for Fuel Plate 22 in 96 MWd Burnup Element in Reference Mixed Burnup Core: Core 7A, Position X6, No Xe, FT loaded with typical samples. 39

Table 3-25. Heat Flux Data (W/ $\left.\mathrm{cm}^{2}\right)$ in Fuel Plate 22 in 170 MWd Burnup Element in Reference Mixed Burnup Core: Core 7A, Position X8, No Xe, FT loaded with typical samples..............................................40

Table 3-26. Nominal MURR Core Operating Parameters with LEU Fuel.....................................................43

Table 3-27. Thermal Conductivity Values for LEU Fuel Plate Materials (W/m-C).................................44

Table 3-28. Predicted Oxide Layer Thickness for LEU Fuel Plates............................................................. 45

Table 3-29. Fuel Conditions for Prototypic MURR Core with LEU Fuel. ......................................................46

Table 4-1. End Channel and Inter-Element Spacing Dimensions for MURR DDE...................................49

Table 4-2. Nominal Thermal-Hydraulic Conditions for Prototypic MURR Element. ..............................50

Table 4-3. Summary of MUR DDE Target Test Values for Plates 1 Through 8. .........................................51

Table 4-4. Summary of MURR DDE Target Test Values for Plates 9 Through 16.....................................52

Table 4-5. Summary of MURR DDE Target Test Values for Plates 17 Through 23 and the Element

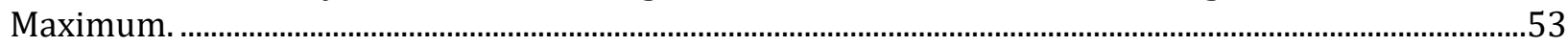

Table A- 1. Fission Density Data (fissions $/ \mathrm{cm}^{3}$ ) in Fuel Plate 1 in Element Discharged from Reference Mixed Burnup Core: FT loaded with typical samples.

Table A-2. Fission Density Data (fissions $/ \mathrm{cm}^{3}$ ) in Fuel Plate 2 in Element Discharged from Reference Mixed Burnup Core: FT loaded with typical samples. .59

Table A-3. Fission Density Data (fissions $/ \mathrm{cm}^{3}$ ) in Fuel Plate 3 in Element Discharged from Reference Mixed Burnup Core: FT loaded with typical samples. 60

Table A 4. Fission Density Data (fissions $/ \mathrm{cm}^{3}$ ) in Fuel Plate 4 in Element Discharged from Reference Mixed Burnup Core: FT loaded with typical samples. . .61

Table A-5. Fission Density Data (fissions $/ \mathrm{cm}^{3}$ ) in Fuel Plate 5 in Element Discharged from Reference Mixed Burnup Core: FT loaded with typical samples.

Table A-6. Fission Density Data (fissions $/ \mathrm{cm}^{3}$ ) in Fuel Plate 6 in Element Discharged from Reference Mixed Burnup Core: FT loaded with typical samples. ................................................................................63

Table A-7. Fission Density Data (fissions/ $\mathrm{cm}^{3}$ ) in Fuel Plate 7 in Element Discharged from Reference

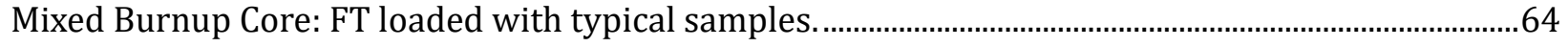

Table A-8. Fission Density Data (fissions $/ \mathrm{cm}^{3}$ ) in Fuel Plate 8 in Element Discharged from Reference Mixed Burnup Core: FT loaded with typical samples. ...............................................................................65 
Table A-9. Fission Density Data (fissions $/ \mathrm{cm}^{3}$ ) in Fuel Plate 9 in Element Discharged from Reference Mixed Burnup Core: FT loaded with typical samples. 66

Table A-10. Fission Density Data (fissions/ $\mathrm{cm}^{3}$ ) in Fuel Plate 10 in Element Discharged from Reference Mixed Burnup Core: FT loaded with typical samples.

Table A-11. Fission Density Data (fissions $/ \mathrm{cm}^{3}$ ) in Fuel Plate 11 in Element Discharged from Reference Mixed Burnup Core: FT loaded with typical samples. 68

Table A-12. Fission Density Data (fissions $/ \mathrm{cm}^{3}$ ) in Fuel Plate 12 in Element Discharged from Reference Mixed Burnup Core: FT loaded with typical samples.

Table A-13. Fission Density Data (fissions $/ \mathrm{cm}^{3}$ ) in Fuel Plate 13 in Element Discharged from Reference Mixed Burnup Core: FT loaded with typical samples. 70

Table A-14. Fission Density Data (fissions $/ \mathrm{cm}^{3}$ ) in Fuel Plate 14 in Element Discharged from Reference Mixed Burnup Core: FT loaded with typical samples. .71

Table A 15. Fission Density Data (fissions/ $\mathrm{cm}^{3}$ ) in Fuel Plate 15 in Element Discharged from Reference Mixed Burnup Core: FT loaded with typical samples. 72

Table A-16. Fission Density Data (fissions $/ \mathrm{cm}^{3}$ ) in Fuel Plate 16 in Element Discharged from Reference Mixed Burnup Core: FT loaded with typical samples. 73

Table A-17. Fission Density Data (fissions $/ \mathrm{cm}^{3}$ ) in Fuel Plate 17 in Element Discharged from Reference Mixed Burnup Core: FT loaded with typical samples. 74

Table A-18. Fission Density Data (fissions $/ \mathrm{cm}^{3}$ ) in Fuel Plate 18 in Element Discharged from Reference Mixed Burnup Core: FT loaded with typical samples. 75

Table A-19. Fission Density Data (fissions/ $\mathrm{cm}^{3}$ ) in Fuel Plate 19 in Element Discharged from Reference Mixed Burnup Core: FT loaded with typical samples. 76

Table A-20. Fission Density Data (fissions $/ \mathrm{cm}^{3}$ ) in Fuel Plate 20 in Element Discharged from Reference Mixed Burnup Core: FT loaded with typical samples. 77

Table A-21. Fission Density Data (fissions $/ \mathrm{cm}^{3}$ ) in Fuel Plate 21 in Element Discharged from Reference Mixed Burnup Core: FT loaded with typical samples. 78

Table A-22. Fission Density Data (fissions $/ \mathrm{cm}^{3}$ ) in Fuel Plate 22 in Element Discharged from Reference Mixed Burnup Core: FT loaded with typical samples. 79

Table A-23. Fission Density Data (fissions $/ \mathrm{cm}^{3}$ ) in Fuel Plate 23 in Element Discharged from Reference Mixed Burnup Core: FT loaded with typical samples. .80

Table B-1. Power Density Data $\left(\mathrm{kW} / \mathrm{cm}^{3}\right)$ in Fuel Plate 1 in Prototypic Core. ..........................................82

Table B-2. Power Density Data $\left(\mathrm{kW} / \mathrm{cm}^{3}\right)$ in Fuel Plate 2 in Prototypic Core. .........................................87

Table B- 3. Power Density Data $\left(\mathrm{kW} / \mathrm{cm}^{3}\right)$ in Fuel Plate 3 in Prototypic Core. ......................................... 92

Table B- 4. Power Density Data $\left(\mathrm{kW} / \mathrm{cm}^{3}\right)$ in Fuel Plate 4 in Prototypic Core. ........................................97

Table B-5. Power Density Data $\left(\mathrm{kW} / \mathrm{cm}^{3}\right)$ in Fuel Plate 5 in Prototypic Core. ......................................... 102

Table B-6. Power Density Data $\left(\mathrm{kW} / \mathrm{cm}^{3}\right)$ in Fuel Plate 6 in Prototypic Core. ....................................... 107

Table B-7. Power Density Data $\left(\mathrm{kW} / \mathrm{cm}^{3}\right)$ in Fuel Plate 7 in Prototypic Core. ....................................... 112

Table B-8. Power Density Data $\left(\mathrm{kW} / \mathrm{cm}^{3}\right)$ in Fuel Plate 8 in Prototypic Core. ...................................... 117

Table B-9. Power Density Data $\left(\mathrm{kW} / \mathrm{cm}^{3}\right)$ in Fuel Plate 9 in Prototypic Core. ...................................... 122

Table B-10. Power Density Data $\left(\mathrm{kW} / \mathrm{cm}^{3}\right)$ in Fuel Plate 10 in Prototypic Core...................................127 
Table B-11. Power Density Data $\left(\mathrm{kW} / \mathrm{cm}^{3}\right)$ in Fuel Plate 11 in Prototypic Core.................................... 132

Table B-12. Power Density Data $\left(\mathrm{kW} / \mathrm{cm}^{3}\right)$ in Fuel Plate 12 in Prototypic Core...................................137

Table B-13. Power Density Data $\left(\mathrm{kW} / \mathrm{cm}^{3}\right)$ in Fuel Plate 13 in Prototypic Core................................... 142

Table B-14. Power Density Data $\left(\mathrm{kW} / \mathrm{cm}^{3}\right)$ in Fuel Plate 14 in Prototypic Core....................................147

Table B-15. Power Density Data $\left(\mathrm{kW} / \mathrm{cm}^{3}\right)$ in Fuel Plate 15 in Prototypic Core.................................... 152

Table B-16. Power Density Data $\left(\mathrm{kW} / \mathrm{cm}^{3}\right)$ in Fuel Plate 16 in Prototypic Core................................... 157

Table B-17. Power Density Data $\left(\mathrm{kW} / \mathrm{cm}^{3}\right)$ in Fuel Plate 17 in Prototypic Core..................................... 162

Table B-18. Power Density Data $\left(\mathrm{kW} / \mathrm{cm}^{3}\right)$ in Fuel Plate 18 in Prototypic Core...................................167

Table B-19. Power Density Data $\left(\mathrm{kW} / \mathrm{cm}^{3}\right)$ in Fuel Plate 19 in Prototypic Core.................................. 172

Table B-20. Power Density Data $\left(\mathrm{kW} / \mathrm{cm}^{3}\right)$ in Fuel Plate 20 in Prototypic Core..................................177

Table B-21. Power Density Data $\left(\mathrm{kW} / \mathrm{cm}^{3}\right)$ in Fuel Plate 21 in Prototypic Core...................................182

Table B-22. Power Density Data $\left(\mathrm{kW} / \mathrm{cm}^{3}\right)$ in Fuel Plate 22 in Prototypic Core...................................187

Table B-23. Power Density Data $\left(\mathrm{kW} / \mathrm{cm}^{3}\right)$ in Fuel Plate 23 in Prototypic Core.................................... 192 


\section{INTRODUCTION}

The University of Missouri is working in conjunction with the National Nuclear Security Administration (NNSA) Material Management and Minimization (M3) Reactor Conversion Program at Argonne National Laboratory to perform fuel element design and fuel cycle performance analyses [1], and steady-state thermal-hydraulic safety analyses [2] to support conversion of the University of Missouri Research Reactor (MURR®) from highly-enriched uranium (HEU) to low-enriched uranium (LEU) fuel. The conversion objectives are to develop a fuel element design that will ensure safe reactor operations, acceptable shutdown and safety margins, as well as maintain the existing experimental performance of the facility.

An LEU fuel element was designed by staff members at Argonne and MURR as a part of the Reactor Conversion Pillar in the M3 U.S. High Performance Research Reactor (USHPRR) LEU conversion program. A set of assumptions to reliably manufacture the fuel plates were provided by the Fuel Fabrication and Fuel Qualification USHPRR Pillar areas in the M3 Reactor Conversion Program. Consequently, the dimensions of the element were developed to meet the conversion goals and provide the best fuel cycle performance, while adhering to this set of manufacturing assumptions. Reference 1 describes the fuel design process.

Irradiation experiment conceptual design parameters for the LEU fuel element under nominal steadystate operating conditions in MURR were previously reported in Reference 3. Data for key fuel plates under conditions that are expected to yield the maximum local fission density, fission rate density, heat flux, and fuel and cladding temperatures. Core states that are not prototypic for MURR while operating at power were included in identifying the maximum irradiation parameters. The information in this report updates and replaces the data reported in Reference 3.

The original version of this report documented the best estimate nominal steady-state irradiation conditions of a key set of plates containing maximum local irradiation conditions found in prototypic MURR cores. The irradiation conditions corresponded to the planned normal equilibrium operation of the reactor after conversion, i.e., for a core fueled with LEU U-10Mo and after the transition phase.

The motivation for Revision 1 of this report is to include additional information for the design of the MURR DDE to obtain conditions when the experiment is irradiated in the test reactor that are regime appropriate for normal operations in MURR. Specifically, this additional information includes a range for the end-channel thicknesses, the nominal inter-element spacing, and nominal thermal-hydraulic conditions for prototypic operations at MURR.

The data presented in this report serve as design parameters for irradiation qualification tests. These irradiation design parameters are intended for designing the MURR DDE to bound normal equilibrium operations in MURR. The irradiation conditions during the transition to normal equilibrium operations will not be bounded by the MURR DDE, but by other dedicated experiments (e.g., MP, FSP, ET-1).

Upon successful completion of fuel qualification, the data allow the program to bound MURR and other USHPRR irradiation conditions, and so allow a licensing basis for a conversion Safety Analysis Report (SAR) specific to MURR. Within a program working on fuel fabrication at a commercial scale and fuel irradiation qualification, the source of the design parameters is fuel element design and safety analysis that has been completed. This work provides the technical basis for what the MURR reactor recently submitted to the NRC as a Preliminary SAR (PSAR) for LEU conversion. [4] A final 
SAR, and the ability to convert is awaiting successful completion of fuel fabrication deployment and fuel irradiation qualification efforts.

The set of design parameters that have been identified by the USHPRR program for irradiation experiments, based on known or potential impacts on fuel performance, are: 1) fuel element/plate geometry, 2) local fission density, and 3) local power density. [5] Furthermore, temperatures achieved in the experiment should be regime appropriate. The MURR DDE irradiation experiment of a full prototypic MURR element should be designed so that target test values match as closely as possible the set of design parameters at prototypic conditions that are given in this report.

The proposed LEU fuel element has an overall design and exterior dimensions that are similar to those of the current HEU fuel elements. There are 23 fuel plates in the LEU design. The overall thickness of each plate is 44 mil, except for the exterior plate that is furthest from the center flux trap (plate 23), which is 49 mil thick. The proposed LEU fuel plates have U-10Mo monolithic fuel foils with a $235 \mathrm{U}$ enrichment of $19.75 \%$ varying from 9 mil to 20 mil thick, and clad with aluminum AA6061. A thin interlayer of zirconium exists between the fuel core and the aluminum cladding as a diffusion barrier. The thinnest nominal combined zirconium and aluminum cladding thickness on each side of the fuel plates is 12 mil.

The LEU U-10Mo monolithic fuel is not yet qualified as driver fuel in research reactors, but is under intense development under the auspices of the M3 Fuel Fabrication and Fuel Qualification Pillars. To assist in the design of mini- and full-size plates for the irradiation experiments, as well as the planning and execution of the experimental campaign, calculated design parameters have been compiled and are presented in this report.

A number of core states that could exist during steady-state operations of MURR were examined in Reference 1 . These core states included variations in the burnup for the mixture of fuel elements loaded in the core, the core xenon buildup state, center flux trap contents, and control blade state. In this report, best estimate design parameters are presented for the LEU elements under the conditions that are expected in normal steady-state operations in MURR. Data for the highest plate cumulative fission density, power density (heat flux though redundant is also presented), and fuel and cladding temperatures under prototypic conditions are presented. 


\section{FUEL ELEMENT GEOMETRY}

The MURR core has a fixed geometry consisting of eight fuel elements, each having identical physical dimensions. The fuel elements are placed vertically around an annulus between two cylindrical aluminum reactor pressure vessels. Cross-sectional views of the MURR reactor core and a close-up view of the core are shown in Figure 2-1.

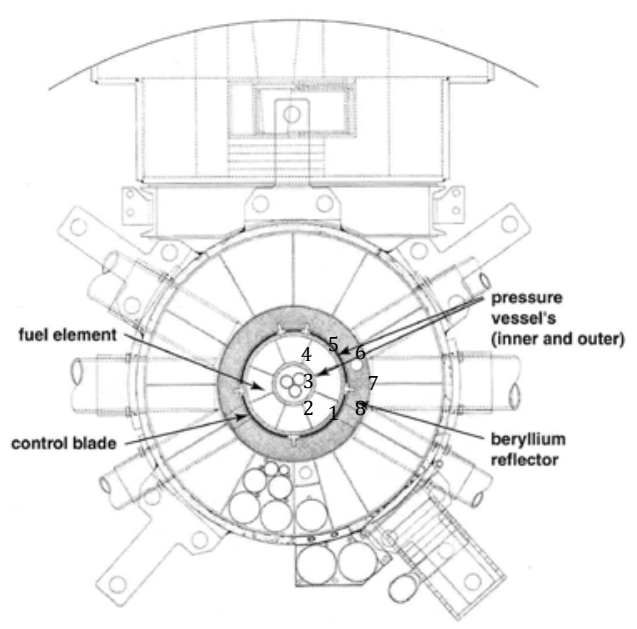

MURR Reactor

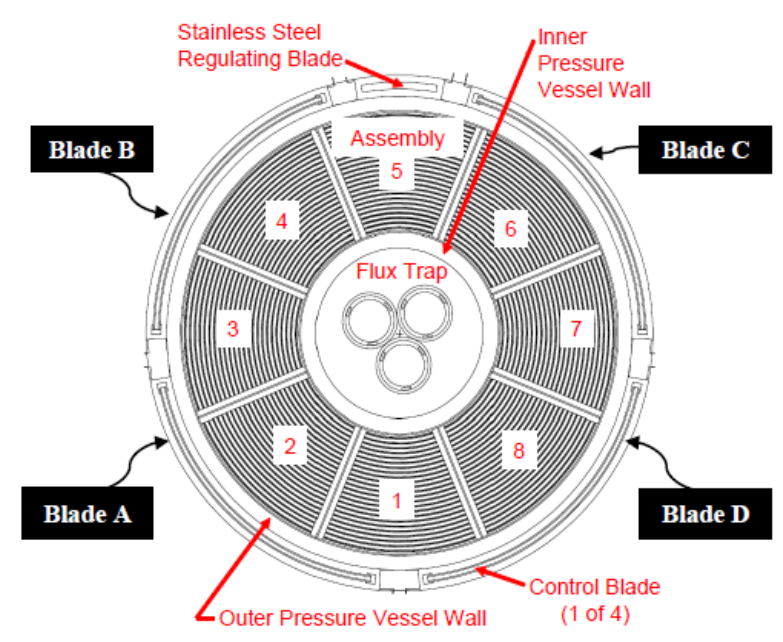

MURR Core

Figure 2-1. Cross-Sectional Views of MURR.

Figure 2-2 provides an illustration of the MURR fuel element. The curved fuel plates are swaged into two aluminum AA6061 side plates that are 0.150 inches (150 mil) thick, 3.16 inches wide, and 31.75 inches long. The plates fit into grooves cut into the side plate. A comb is attached over the fuel plates at their top and bottom to provide additional structural support and help maintain fuel plate spacing. The side plates are attached to the top and bottom end fittings. Rollers on the inside and outside edges of the end fittings facilitate insertion of the elements into the annular pressure vessel of the MURR. They also ensure that the minimum outer channel clearances are maintained. The overall length of the element is 32.5 inches.

The current HEU fuel element used in MURR has 24 curved plates that form a 45-degree arc. The HEU fuel plates are 50 mil thick ( 0.050 inches). The fuel meat is 20 mil thick in each plate and consists of $\mathrm{UAl}_{\mathrm{x}}$ aluminide dispersion fuel containing uranium with a ${ }^{235} \mathrm{U}$ enrichment of approximately $93 \%$. The HEU plates are nominally clad with 15 mil of aluminum AA6061. The fuel plates are 25.5 inches long, with an active fuel meat length of 24 inches. The fuel plate and fuel meat width varies by plate.

In order to meet the reactivity requirements of the MURR operating cycle with LEU fuel, it is necessary to have a much higher uranium density than the currently qualified $\mathrm{UAl}_{\mathrm{x}}$ or silicide dispersion fuels. [6] U-10Mo monolithic foils that can provide a uranium density of $15.3 \mathrm{gU} / \mathrm{cm}^{3}$ have been selected for the LEU fuel, pending successful fuel qualification. A fuel element design with U10Mo LEU fuel developed in Reference 1 was found to safely meet the MURR operating cycle requirements and maintain existing reactor performance.

The LEU fuel element design has 23 fuel plates. A cross-sectional drawing of the fuel plates in the element is shown in Figure 2-3. Just as for the HEU element, each LEU fuel plate is 25.5 inches long, 
with a 24.0 inch fueled length. Because of the difference in the number of fuel plates, the LEU fuel plate and fuel foil widths are different than the HEU element. The unfueled edge on each side of the fuel plates is 145 mil, of which nominally 69 mil extends into a 75 mil deep groove cut into the element side plates. The other portions of the element construction (e.g., side plate length, width, and thickness; and end fittings) will be identical to the HEU fuel element.

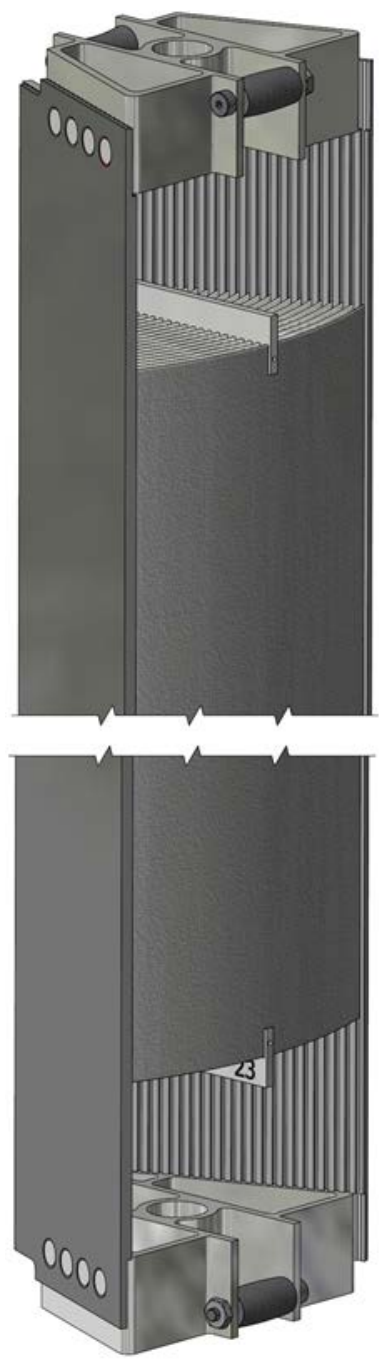

Figure 2-2. MURR Fuel Element. 


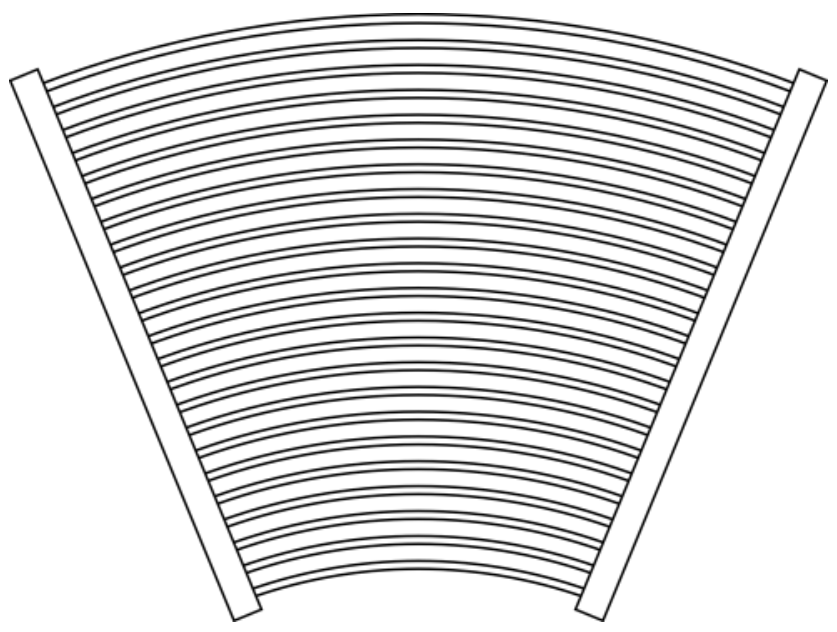

Figure 2-3. Cross-Sectional View of MURR LEU Fuel Element.

A complete description of the LEU element fuel design and associated tolerances is provided in References 4 and 7. Table 2-1 provides a summary of LEU fuel element dimensions and design parameters. The LEU element uses graded foil thicknesses to flatten the radial heat flux profile. This can be seen in Figure 2-4, which shows a cross-sectional view of the MURR LEU element plates 1-5 and 19-23 derived from the MCNP neutronics model. The different thicknesses of the fuel cores in the plates can be seen in the figure. Note that plates 6-18, which are not shown in Figure 2.4, have the same fuel foil and cladding thicknesses as plates 4-5 and 19-22. Full-thickness fuel foils with a fuel core thickness of 20 mil are loaded in plates 4-22. The thinnest fuel foil is loaded in plate 1, with a fuel core thickness of 9 mil. The cladding thickness also varies by plate, with the thinnest nominal cladding being 12 mil.

The cladding thicknesses on plates 1 and 23 are thicker (17.5 and 16 mil, respectively) because the outer plates are more susceptible to being scratched during handling. The fuel cladding consists of aluminum AA6061 and a thin (1 mil) zirconium layer at the fuel-cladding interface as an interaction barrier. The Zr interlayer is not shown in Figure 2-4, as it is combined with the AA6061 cladding in neutronics modeling. Plates 1 through 22 are designed to have a nominal thickness of 44 mil. Making the plates thinner than the HEU fuel element, as well having one less plate, gives a higher water-tofuel ratio in the LEU element and increases the reactivity. Plate 23 is the widest fuel plate and is designed to have a nominal thickness of 49 mil, which gives greater resistance against bending forces in the MURR coolant flow field. 
Table 2-1. Nominal LEU Fuel Element and Plate Dimensions and Loadings.

\begin{tabular}{|c|c|c|c|c|}
\hline $\begin{array}{l}\text { Channel } \\
\text { or Plate }\end{array}$ & $\begin{array}{l}\text { Meat } \\
\text { thickness } \\
\left(\text { mil) }{ }^{1}\right.\end{array}$ & $\begin{array}{l}\text { AA6061 Cladding } \\
\text { +Zr Interlayer } \\
\text { thickness (mil) }\end{array}$ & $\begin{array}{l}\text { Plate thickness } \\
\text { (mil) }\end{array}$ & $\begin{array}{c}\text { Coolant } \\
\text { Channel } \\
\text { thickness (mil) }\end{array}$ \\
\hline 1 & 9 & 17.5 & 44 & $80.5^{2}$ \\
\hline 2 & 12 & 16 & 44 & 93 \\
\hline 3 & 16 & 14 & 44 & 93 \\
\hline 4 & 20 & 12 & 44 & 93 \\
\hline 5 & 20 & 12 & 44 & 93 \\
\hline 6 & 20 & 12 & 44 & 92 \\
\hline 7 & 20 & 12 & 44 & 92 \\
\hline 8 & 20 & 12 & 44 & 92 \\
\hline 9 & 20 & 12 & 44 & 92 \\
\hline 10 & 20 & 12 & 44 & 92 \\
\hline 11 & 20 & 12 & 44 & 92 \\
\hline 12 & 20 & 12 & 44 & 92 \\
\hline 13 & 20 & 12 & 44 & 92 \\
\hline 14 & 20 & 12 & 44 & 92 \\
\hline 15 & 20 & 12 & 44 & 92 \\
\hline 16 & 20 & 12 & 44 & 92 \\
\hline 17 & 20 & 12 & 44 & 92 \\
\hline 18 & 20 & 12 & 44 & 92 \\
\hline 19 & 20 & 12 & 44 & 92 \\
\hline 20 & 20 & 12 & 44 & 93 \\
\hline 21 & 20 & 12 & 44 & 93 \\
\hline 22 & 20 & 12 & 44 & 93 \\
\hline 23 & 17 & 16 & 49 & 93 \\
\hline 24 & & & & $80.5^{3}$ \\
\hline
\end{tabular}

$1 \pm 1$ mil for all plates

2 Plate 1 to outer edge of inner roller

3 Plate 23 to outer edge of outer roller

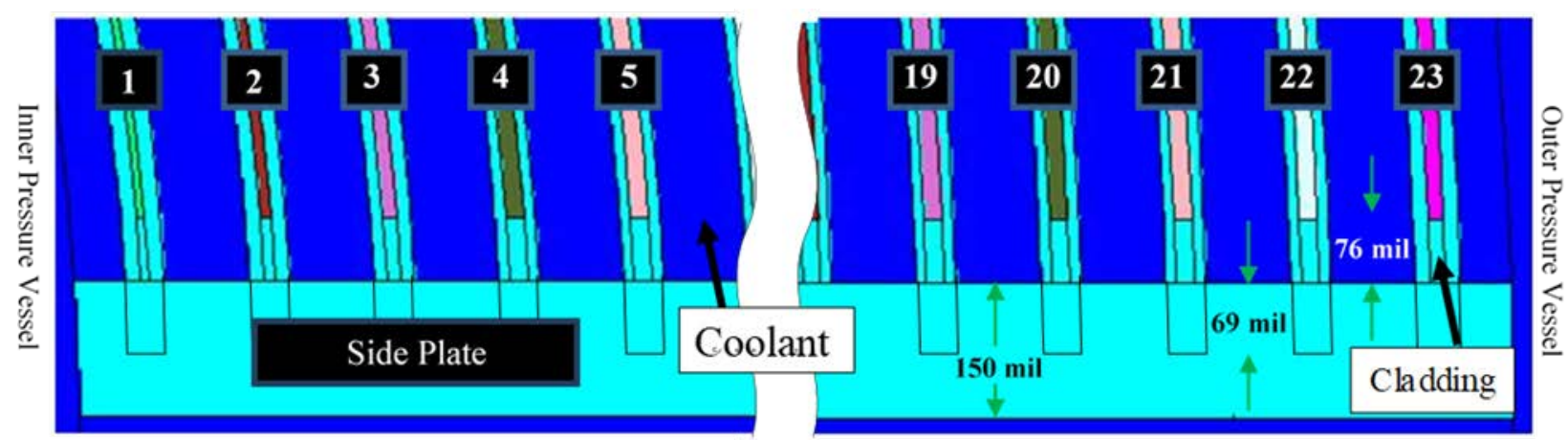

Figure 2-4. Cross-Sectional View of MURR LEU Element Plates 1-5 and 19-23. 


\section{LEU OPERATIONS DESIGN PARAMETERS}

The MURR is currently licensed for a maximum steady-state power level of $10 \mathrm{MW}$ thermal with HEU fuel. This power level provides neutron flux levels in the center flux trap and irradiation positions in the graphite reflector region that enable MURR to fulfill its mission of providing experimental and irradiation services to a variety of users.

The goal of the analysis for conversion to LEU fuel is to develop a fuel element design that will continue to ensure safe reactor operations, as well as maintaining existing performance. The LEU fuel element was designed according to a set of manufacturing assumptions that were provided by the Fuel Fabrication and Fuel Qualification Pillars of the Reduced Enrichment for Research and Test (RERTR) program to reliably manufacture the U-10Mo monolithic fuel plates. It has also been shown in Reference 1 that a power uprate to $12 \mathrm{MW}$ will be needed for the LEU fueled core to continue to meet the facility's mission. A detailed thermal-hydraulics analysis was performed to establish the steady-state safety basis of the proposed LEU fuel and power uprate $[2,4]$.

Clarification of the power normalization employed in the analysis is in order to ensure that there is a proper understanding of the basis of the results presented in previous work and in this report. The calculated heat flux profiles presented in Reference 1 are based on f7 tallies in MCNP, which conservatively assumes that all heat resulting from fission is deposited at the site of the fission event (i.e., in the fuel core of the plate). The gamma heating in the beryllium reflector and other ex-core components which contributes to the total reactor plant power was neglected in the power normalization. Thus, the data presented in Reference 1 are normalized to a core (i.e., fuel plates and associated coolant) power level of $12 \mathrm{MW}$. Note that for the steady-state thermal-hydraulic safety basis analysis performed in References 2 and 4, gamma heating in the ex-core components was taken into account in the power normalization so as to not be overly conservative in predicting the margins to safety.

For the data presented here, gamma heating in the beryllium and graphite reflectors and other excore components of MURR has been accounted for in the power normalization to provide more representative values for the design parameters for irradiation experiments. Analysts at MURR have calculated that for the LEU-fueled core the fraction of the plant power generated in ex-core structures is $3.6 \%$ of the total power [8]. Consequently, for the results presented here, the plate power density and heat flux profiles have been normalized to a core power level of $11.57 \mathrm{MW}$, or $96.4 \%$ of a total plant power of $12 \mathrm{MW}$. There is, however, no adjustment to the plate heat flux or power density to account for fission heat deposited directly in the coolant (in other words, the same assumptions as made for the f7 tallies), which remains a conservative assumption.

Calculated results for the fission density were also presented in Reference 1 . These data were derived from REBUS-DIF3D depletion modeling, which was based on a power normalization to a total plant power of $12 \mathrm{MW}$. In other words, heat generation in the ex-core components (reflectors, pool, pressure vessels, flux trap) is included in the power normalization in the REBUS-DIF3D calculations. Consequently, the fission rate and fission density calculated by the REBUS-DIF3D model are properly normalized for the LEU-fueled MURR operating at the anticipated uprated power of $12 \mathrm{MW}$. As such, no renormalization of the fission density results presented in Reference 1 is needed. 


\subsection{Scope of Steady-State Core Conditions Evaluated}

Power peaking in MURR is dependent upon the mixture of burnup states among the elements in the core, upon the core xenon buildup state, upon critical control blade compositions and positions, and upon the experiment/sample loadings, particularly in the flux trap. Twenty-four cores loaded with the proposed LEU fuel design that cover the range of expected variations in these conditions were examined. In the results presented on the following pages, cases are labeled with a nomenclature $c f b$, where $c$ indicates the fuel burnup and xenon condition, $f$ indicates the flux trap loading, and $b$ indicates the control blade conditions. The nomenclature of the core labels is defined in Table 3-1. The derivation of each of the components of the core state is described below. A summary of the MCNP cases for these twenty-four cores is provided in Table 3-2.

Table 3-1. Nomenclature for LEU Core Case Names: $c f b$ (e.g. 5B1)

\begin{tabular}{|l|l|}
\hline$c=5$ & All fresh LEU elements, BOC (no Xe) \\
\hline$c=6$ & All fresh LEU elements, day 2 (eq. Xe) \\
\hline$c=7$ & Reference mixed burnup LEU core, BOC (no Xe) \\
\hline$c=8$ & Reference mixed burnup LEU core, day 2 (eq. Xe) \\
\hline
\end{tabular}

\begin{tabular}{|l|l|}
\hline$f=\mathrm{A}$ & Flux trap holder with typical samples loaded in center flux trap \\
\hline$f=\mathrm{B}$ & No flux trap holder or samples loaded in center flux trap -water only \\
\hline
\end{tabular}

\begin{tabular}{|l|l|}
\hline$b=n u l l$ & All blades fresh and banked. \\
\hline$b=1$ & $\begin{array}{l}\text { Blades C and D at 8 years prior use, blades A and B fresh. Blades C and D } \\
\text { positioned 1 inch higher than A and B, per Technical Specification (TS) } \\
\text { limit. }\end{array}$ \\
\hline$b=2$ & $\begin{array}{l}\text { Blades A and D at 8 years prior use, blades B and C fresh. Blades A and D } \\
\text { positioned 1 inch higher than B and C, per TS limit. }\end{array}$ \\
\hline
\end{tabular}

Core burnup state. The MURR operates continuously with the exception of a weekly scheduled shutdown. Over the past 41 years of operation, the MURR has averaged approximately 6.3 days/week at full power. The weekly shutdown provides an opportunity to access samples in the center flux trap, to perform surveillance tests and maintenance, and to replace all eight fuel elements in the core. Replacing all fuel elements provides the chance to remix or shuffle the elements that will be used in the core and a xenon free core for restart. The active fuel cycle typically consists of 32 fuel elements, corresponding to sixteen pairs of elements. A core loading will always consist of four different pairs of elements, with the two elements of each pair loaded opposite of each other in the core (e.g., positions 1 and 5 in Figure 2.1 are paired together).

A fuel cycle simulation of the complex MURR fuel shuffling sequence was performed with REBUSDIF3D to obtain a "pseudo-equilibrium" reference mixed burnup core [1] that is prototypic for MURR operations. Elements are loaded in the core about 19 times before discharge from the fuel cycle. This is the same number of times the HEU fuel is typically loaded in the MURR before discharge from the fuel cycle. While the core residence time is the same for both the LEU and HEU fuel, the discharge burnup of the LEU elements is $20 \%$ greater (about $180 \mathrm{MWd}$ ) due to the higher core operating power level. An MCNP model of a chosen reference mixed-burnup core that is prototypic for MURR operations was used to evaluate experimental performance, calculate reactivity parameters, and calculate the detailed power distributions needed for the steady-state thermal-hydraulics analyses. The fuel compositions for the MCNP analyses were derived from the REBUS-DIF3D results. 
Table 3-2. Summary of Power Distribution Evaluations for LEU Cores.

\begin{tabular}{|c|c|c|c|c|c|c|c|c|c|c|c|c|c|}
\hline \multicolumn{4}{|c|}{$\begin{array}{l}\text { Core state that may bound power } \\
\text { peaking }\end{array}$} & \multicolumn{4}{|c|}{$\begin{array}{l}\text { Element Burnup } \\
\text { (MWd) } \\
\text { at Beginning of Cycle }\end{array}$} & \multirow[b]{2}{*}{$\begin{array}{l}\text { Fresh } \\
\text { Blades }\end{array}$} & \multirow[b]{2}{*}{$\begin{array}{l}\text { Depleted } \\
\text { Blades }\end{array}$} & \multicolumn{3}{|c|}{$\begin{array}{l}\text { Critical Blade Position } \\
\text { (Inches withdrawn) }\end{array}$} & \multirow[b]{2}{*}{$\begin{array}{c}\text { MCNP } \\
\text { k-eff }\end{array}$} \\
\hline Case & $\begin{array}{l}\text { Burnup } \\
\text { State }\end{array}$ & $\begin{array}{c}\text { Xe } \\
\text { State }\end{array}$ & $\begin{array}{l}\text { Flux } \\
\text { Trap }\end{array}$ & $\begin{array}{l}\mathrm{X} 1 \\
\mathrm{X} 5\end{array}$ & $\begin{array}{l}\mathrm{X} 2 \\
\mathrm{X} 6\end{array}$ & $\begin{array}{l}\mathrm{X} 3 \\
\mathrm{X} 7\end{array}$ & $\begin{array}{l}\mathrm{X} 4 \\
\mathrm{X} 8\end{array}$ & & & $\begin{array}{l}\text { Fresh } \\
\text { Blades }\end{array}$ & $\begin{array}{l}\text { Depleted } \\
\text { Blades }\end{array}$ & $\begin{array}{c}\text { Reg } \\
\text { Blade }\end{array}$ & \\
\hline $5 \mathrm{~A}$ & Fresh & No Xe & Samples & 0 & 0 & 0 & 0 & $A, B, C, D$ & & 13.259 & & 10 & 1.00016 \\
\hline $5 \mathrm{~A} 1$ & Fresh & No Xe & Samples & 0 & 0 & 0 & 0 & A, B & C, D & 10.656 & 11.656 & 10 & 0.99993 \\
\hline $5 \mathrm{~A} 2$ & Fresh & No Xe & Samples & 0 & 0 & 0 & 0 & B, C & A, D & 10.814 & 11.814 & 10 & 0.99999 \\
\hline $6 \mathrm{~A}$ & Fresh & Eq. Xe & Samples & 0 & 0 & 0 & 0 & $A, B, C, D$ & & 17.193 & & 15 & 0.99985 \\
\hline $6 \mathrm{~A} 1$ & Fresh & Eq. Xe & Samples & 0 & 0 & 0 & 0 & A, B & C, D & 14.912 & 15.912 & 15 & 1.00001 \\
\hline $6 \mathrm{~A} 2$ & Fresh & Eq. Xe & Samples & 0 & 0 & 0 & 0 & $\mathrm{~B}, \mathrm{C}$ & A, D & 15.027 & 16.027 & 15 & 0.99990 \\
\hline $7 \mathrm{~A}$ & Mixed BU & No Xe & Samples & 0 & 96 & 77 & 170 & $A, B, C, D$ & & 17.567 & & 10 & 1.00008 \\
\hline 7A1 & Mixed BU & No Xe & Samples & 0 & 96 & 77 & 170 & A, B & C, D & 15.209 & 16.209 & 10 & 0.99975 \\
\hline $7 \mathrm{~A} 2$ & Mixed BU & No Xe & Samples & 0 & 96 & 77 & 170 & $\mathrm{~B}, \mathrm{C}$ & A, D & 15.351 & 16.351 & 10 & 1.00014 \\
\hline $8 \mathrm{~A}$ & Mixed BU & Eq. Xe & Samples & 0 & 96 & 77 & 170 & $A, B, C, D$ & & 24.314 & & 15 & 1.00001 \\
\hline $8 \mathrm{~A} 1$ & Mixed BU & Eq. Xe & Samples & 0 & 96 & 77 & 170 & A, B & C, D & 22.765 & 23.765 & 15 & 1.00004 \\
\hline $8 \mathrm{~A} 2$ & Mixed BU & Eq. Xe & Samples & 0 & 96 & 77 & 170 & $\mathrm{~B}, \mathrm{C}$ & A, D & 22.698 & 23.698 & 15 & 0.99986 \\
\hline $5 B$ & Fresh & No Xe & Empty & 0 & 0 & 0 & 0 & $A, B, C, D$ & & 13.650 & & 10 & 0.99998 \\
\hline $5 \mathrm{~B} 1$ & Fresh & No Xe & Empty & 0 & 0 & 0 & 0 & A, B & C, D & 11.080 & 12.080 & 10 & 1.00003 \\
\hline $5 \mathrm{~B} 2$ & Fresh & No Xe & Empty & 0 & 0 & 0 & 0 & $\mathrm{~B}, \mathrm{C}$ & A, D & 11.258 & 12.258 & 10 & 1.00009 \\
\hline $6 \mathrm{~B}$ & Fresh & Eq. Xe & Empty & 0 & 0 & 0 & 0 & $A, B, C, D$ & & 17.891 & & 15 & 0.99997 \\
\hline $6 \mathrm{~B} 1$ & Fresh & Eq. Xe & Empty & 0 & 0 & 0 & 0 & A, B & C, D & 15.580 & 16.580 & 15 & 0.99988 \\
\hline $6 \mathrm{~B} 2$ & Fresh & Eq. Xe & Empty & 0 & 0 & 0 & 0 & $\mathrm{~B}, \mathrm{C}$ & A, D & 15.741 & 16.741 & 15 & 1.00017 \\
\hline $7 \mathrm{~B}$ & Mixed BU & No Xe & Empty & 0 & $\overline{96}$ & 77 & 170 & $A, B, C, D$ & & 18.193 & & 10 & 1.00013 \\
\hline 7B1 & Mixed BU & No Xe & Empty & 0 & 96 & 77 & 170 & A, B & C, D & 15.933 & 16.933 & 10 & 1.00012 \\
\hline $7 \mathrm{~B} 2$ & Mixed BU & No Xe & Empty & 0 & 96 & 77 & 170 & $\mathrm{~B}, \mathrm{C}$ & A, D & 16.030 & 17.030 & 10 & 1.00003 \\
\hline $8 \mathrm{~B}$ & Mixed BU & Eq. Xe & Empty & 0 & 96 & 77 & 170 & $A, B, C, D$ & & 26.000 & & 15 & 0.99917 \\
\hline 8B1 & Mixed BU & Eq. Xe & Empty & 0 & 96 & 77 & 170 & A, B & C, D & 25.621 & 26.000 & 15 & 0.99998 \\
\hline $8 \mathrm{~B} 2$ & Mixed BU & Eq. Xe & Empty & 0 & 96 & 77 & 170 & $\mathrm{~B}, \mathrm{C}$ & A, D & 25.312 & 26.000 & 15 & 0.99986 \\
\hline
\end{tabular}

"Samples" indicates a typical loading of samples in all three flux trap tubes.

"Empty" indicates neither samples nor tubes in the flux trap (i.e., "empty island" configuration).

Full blade withdrawal is 26 inches. 
Table 3-3 below summarizes the anticipated burnup ranges of LEU elements in the eight core positions of MURR, as well as the burnups of the elements at the beginning-of-cycle (BOC) for the selected reference mixed-burnup core. Note that cores with eight fresh elements were also evaluated, but these will only be loaded for startup testing of the converted core.

Flux trap conditions. Two center flux trap conditions were considered. The prototypic case, indicated by $\mathrm{f}=\mathrm{A}$ in the case name label, has the flux trap holder loaded with typical samples for MURR operations. A non-prototypic "empty flux trap" case, in which the flux trap holder is not inserted and only pool water is in the center flux trap, is indicated by $f=\mathrm{B}$. In all cases, the irradiation positions in the graphite reflector were modeled with typical samples for those locations.

Table 3-3. Beginning-of-Cycle Burnups for Reference LEU Mixed Burnup Core $(c=7,8)$.

\begin{tabular}{|c|c|c|}
\hline Core Positions & $\begin{array}{c}\text { Typical B0C Burnup Range } \\
\text { (MWd) }\end{array}$ & $\begin{array}{c}\text { Reference Core BOC } \\
\text { Burnup (MWd) }\end{array}$ \\
\hline X1 and X5 & 0 to $50(20$ avg.) & 0 \\
\hline X3 and X7 & 40 to $90(64$ avg.) & 77 \\
\hline X2 and X6 & 85 to $135(108$ avg.) & 96 \\
\hline X4 and X8 & 125 to $170(153$ avg.) & 170 \\
\hline
\end{tabular}

1 Burnup values from fuel cycle simulation in REBUS-DIF3D

Control blade conditions. Reactivity control of MURR is accomplished by control blades that move vertically in an annular gap between the outer reactor pressure vessel and a beryllium reflector (see Figure 2-1). The blades control the reactivity by absorbing neutrons and reducing neutron reflection from the beryllium reflector back into the core. There are four shim control blades constructed of BORAL $^{\circledR}$, and one stainless steel regulating blade.

Within the first two days of the one week cycle, the control blades move from a typical position of 17 inches withdrawn at BOC to 23 inches withdrawn in response to the reactivity effects of xenon. The change in the control blade position affects the axial shape of the neutron flux and power peaking. Furthermore, while the control blades are typically "banked" at the same axial position, the MURR TS allow for the blade tips to differ in height relative to one another by up to one inch (from the highest to the lowest control blade). This blade height mismatch can cause a tilting of the neutron flux shape across the core. Lastly, the BORAL ${ }^{\circledR}$ material depletes significantly with blade use in the region near the blade tip. To accommodate this effect, the MURR control blades are shuffled in a multi-year scheme analogous to fuel shuffling. Blades are inserted for a two year cycle and typically average about 4 years of use before being retired, but can be used for as long as 10 years. Thus, in addition to a case with fresh control blades banked at the same height, the effects of control blade depletion and mismatch of the control blade heights relative to one another were evaluated. The two alternate control blade pattern cases identified in Table 3-1 were selected to maximize the impact on the power distribution in the core. These cases were evaluated for safety analyses and are considered extreme cases within the conservative band of operating conditions for MURR. The case with fresh blades that are banked is considered more prototypic for MURR operations.

\subsection{Axial and Azimuthal Discretization of the Results}

Detailed power distribution tallies were compiled using MCNP. In these tallies, the fuel core in each plate was divided into 24 one-inch axial segments. While the power distribution varies most significantly in the axial and radial (plate-by-plate) dimensions of the fuel element, the power density also varies along the width of the fuel core, and can peak sharply at the edge of the fueled section of the plate due to an increase in the local water-to-fuel ratio near the edge of the fuel core. Lateral 
conduction of the heat generated by fission to the unfueled portion of the fuel plate is not modeled in the one-dimensional steady-state thermal-hydraulics analysis employed for MURR. Rather, an appropriately-sized finite "hot-stripe" width was selected for the power tally segment width that yields conservative estimates of the fuel temperature and the heat conduction into the coolant channel for flow instability and critical heat flux analyses. Three-dimensional computational fluid dynamics (CFD) analyses were performed to determine the proper discretization of the fuel plates in the azimuthal dimension $[9,10]$. It was concluded from that analysis that the MCNP power distributions should be tallied by azimuthally subdividing the fuel core of each fuel plate into nine tally stripes; three stripes of $0.5 \mathrm{~cm}(5 \mathrm{~mm})$ each along both edges of the fuel core, and three stripes of equal angle in the middle portion of the fuel core to cover the balance of the fuel width. The resulting tally mesh size used for the MURR safety basis and for calculating the power density irradiation parameters is $0.5 \times 2.54 \mathrm{~cm}$. For the fission density irradiation parameter, the mesh size is $0.5 \times 5.08 \mathrm{~cm}$.

\subsection{Design Parameter Data}

MURR can operate under a range of conditions while safely fulfilling its mission. As discussed above, these conditions include the burnup of the fuel elements used in the weekly operating cycle, the experiment loading, and the prior history and the relative positioning of the control blades used for reactivity control. Each of these can have an impact on safety margins and local irradiation parameters such as power density, fission density, or heat flux. Other conditions that may vary during operation include the coolant flow rate through the core, the coolant pressure, and the coolant inlet temperature to the core. However, while coolant conditions will have an impact on safety margins, they will have little or no effect on local irradiation parameters. Additionally, variations in the coolant conditions from normal operating conditions are usually short in duration (minutes or hours) compared to variations in fuel, experiment, or control blade utilization (weeks).

Steady-state neutronics analysis under nominal operating conditions for the set of twenty-four cores identified in Section 3.1 were performed to predict key local heating rates in Reference 1. For completeness, the results for all cases are summarized in Table 3-4. Note that the power density and heat flux data in Table 3-4 have been renormalized to a core power level of $11.57 \mathrm{MW}$, as discussed in Section 3.0, above. Among these cases, only the two cases that are shaded in blue (Core 7A and 8A) are considered prototypic for MURR operations.

Local irradiation conditions expected during nominal steady-state operations are of interest as design parameters for the purpose of planning and evaluating irradiation experiments. A key design parameter which has been observed to affect fuel thermo-physical properties and the blister threshold temperature in U-10Mo monolithic fuel is the cumulative fission density in the fuel. The discharge fission density of the fuel in the MURR fuel element under prototypic conditions will vary by plate, as well as axial and azimuthal position in the plate. Furthermore, each of the USHPRR reactors is expected to discharge fuel over a wide range of fission densities. Post-irradiation examination of the irradiated fuel will include inspection for fuel integrity, as well as measurements and modeling of irradiation-induced swelling, thermal conductivity and other properties that are affected by fuel burnup, and blister threshold measurements for the irradiated fuel. A detailed examination of the fission density data for the discharged MURR fuel element under prototypic conditions is provided in Section 3.3.1 and Appendix A.

Another design parameter being experimentally tested to determine if it impacts fuel performance is the power density in the fuel during operation. The exact geometry and cooling conditions of the MURR fuel plate design, which can affect the heat flux and fuel temperature, may not be reproduced 
in fuel irradiation experiments, especially in plate-level tests. However, irradiation experiments can be designed to reproduce the prototypic power density/fission rate in the fuel plates. Table 3-4 shows that the maximum local power density occurs in Core 5B1, which is a core loaded with all fresh LEU elements at BOC, an empty flux trap, and the control blades in a skewed position (refer to Table 3-1 above for a description of the control blade pattern). Table 3-5 compares the calculated maximum power density by element for this core, as well as the greatest power density for fuel elements in the cores labeled 7A and 8A. The greatest power density values for Core 7A (no Xe conditions) and Core 8A (equilibrium Xe conditions) are 9\% and 19\% lower, respectively, compared with Core 5B1. However, Cores 7A and 8A will be prototypic of MURR operations following conversion, as they are modeled with a mixture of fresh and previously burned LEU elements and have experiments loaded in the center flux trap. A detailed examination of the power density data for MURR fuel elements under prototypic conditions is provided in Section 3.3.2 and Appendix B.

Table 3-4. Summary of Local Power Parameters Evaluated for LEU Cores.

\begin{tabular}{|c|c|c|c|c|}
\hline \multirow[b]{2}{*}{ Core } & \multicolumn{2}{|c|}{$\begin{array}{c}\text { Local Power Density } \\
\left(\mathrm{kW} / \mathrm{cm}^{3}\right)\end{array}$} & \multicolumn{2}{|c|}{$\begin{array}{c}\text { Local Heat Flux } \\
\left(\mathrm{W} / \mathrm{cm}^{2}\right)\end{array}$} \\
\hline & Maximum & Location 1 & Maximum & Location 1 \\
\hline $5 \mathrm{~A}$ & 14.374 & X3 P01 S1 A17 & 214.2 & X8 P23 S9 A17 \\
\hline $5 \mathrm{~A} 1$ & 14.719 & X7 P01 S9 A16 & 224.9 & X7 P23 S1 A17 \\
\hline $5 \mathrm{~A} 2$ & 14.601 & X2 P01 S9 A16 & 221.2 & X1 P23 S9 A17 \\
\hline $6 \mathrm{~A}$ & 13.772 & X4 P01 S9 A14 & 204.3 & X8 P23 S9 A15 \\
\hline $6 \mathrm{~A} 1$ & 13.680 & X8 P01 S1 A15 & 214.2 & X6 P23 S9 A15 \\
\hline $6 \mathrm{~A} 2$ & 13.575 & X2 P01 S9 A14 & 208.6 & X8 P23 S9 A15 \\
\hline $7 \mathrm{~A}$ & 14.400 & X5 P01 S1 A15 & 203.2 & X1 P23 S1 A16 \\
\hline 7A1 & 14.191 & X5 P01 S9 A14 & 219.9 & X1 P23 S1 A15 \\
\hline 7A2 & 14.075 & X1 P01 S9 A15 & 210.2 & X1 P23 S9 A17 \\
\hline $8 \mathrm{~A}$ & 12.780 & X5 P01 S1 A12 & 186.1 & X1 P23 S1 A14 \\
\hline 8A1 & 12.709 & X1 P01 S1 A12 & 194.6 & X1 P23 S1 A14 \\
\hline $8 \mathrm{~A} 2$ & 12.583 & X5 P01 S1 A14 & 189.6 & X1 P23 S1 A11 \\
\hline $5 B$ & 15.415 & X2 P01 S9 A17 & 212.4 & X8 P23 S9 A17 \\
\hline $5 B 1$ & 15.826 & X7 P01 S1 A16 & 225.6 & X6 P23 S9 A16 \\
\hline $5 B 2$ & 15.439 & X1 P01 S1 A16 & 221.6 & X8 P23 S9 A17 \\
\hline $6 \mathrm{~B}$ & 14.617 & X1 P01 S1 A15 & 202.6 & X6 P23 S9 A15 \\
\hline $6 \mathrm{~B} 1$ & 14.187 & X7 P01 S9 A14 & 209.4 & X7 P23 S1 A13 \\
\hline $6 \mathrm{~B} 2$ & 14.171 & X8 P01 S9 A15 & 205.8 & X8 P23 S9 A14 \\
\hline $7 \mathrm{~B}$ & 14.647 & X5 P01 S1 A14 & 201.4 & X1 P23 S1 A15 \\
\hline 7B1 & 14.762 & X5 P01 S1 A15 & 218.2 & X1 P23 S1 A15 \\
\hline 7B2 & 14.757 & X1 P01 S1 A15 & 209.2 & X1 P23 S1 A14 \\
\hline $8 \mathrm{~B}$ & 13.039 & X1 P01 S1 A13 & 183.8 & X1 P23 S1 A13 \\
\hline $8 B 1$ & 12.943 & X1 P01 S1 A10 & 184.4 & X1 P23 S1 A13 \\
\hline $8 B 2$ & 12.815 & X5 P01 S9 A12 & 184.7 & X1 P23 S1 A10 \\
\hline
\end{tabular}

$1 \mathrm{Xi}$ is element number, as shown in Figure 2.1. Pkk is plate, numbered 1-23 moving from inner pressure vessel to outer pressure vessel. $\mathrm{Sj}$ is azimuthal stripe in plate, numbered 1-9 moving clockwise, looking from above. Azz is axial level, numbered 1-24 from top of the fuel core in the fuel plate. 
Table 3-5. Maximum Power Density for Various Cores.

\begin{tabular}{|c|c|c|c|c|c|c|}
\hline \multirow[b]{2}{*}{$\begin{array}{c}\text { Core } \\
\text { Position }\end{array}$} & \multicolumn{3}{|c|}{ Fuel Element Burnup (MWd) } & \multicolumn{3}{|c|}{ Maximum Power Density $\left(\mathrm{kW} / \mathrm{cm}^{3}\right)$} \\
\hline & $\begin{array}{c}5 \mathrm{~B} 1 \\
\text { (No Xe } \\
\text { conditions) }\end{array}$ & $\begin{array}{c}7 \mathrm{~A} \\
\text { (No Xe } \\
\text { conditions) }\end{array}$ & $\begin{array}{c}\text { 8A } \\
\text { (Eq. Xe } \\
\text { conditions) }\end{array}$ & $\begin{array}{c}5 \mathrm{~B} 1 \\
\text { (No Xe } \\
\text { conditions) }\end{array}$ & $\begin{array}{c}\text { 7A } \\
\text { (No Xe } \\
\text { conditions) }\end{array}$ & $\begin{array}{c}\text { 8A } \\
\text { (Eq. Xe } \\
\text { conditions) }\end{array}$ \\
\hline 1 & 0 & 0 & 3 & 15.1 & 13.9 & 12.5 \\
\hline 2 & 0 & 96 & 99 & 14.6 & 11.5 & 10.2 \\
\hline 3 & 0 & 77 & 80 & 14.3 & 12.0 & 10.5 \\
\hline 4 & 0 & 170 & 173 & 14.4 & 9.6 & 8.2 \\
\hline 5 & 0 & 0 & 3 & 15.0 & 14.4 & 12.8 \\
\hline 6 & 0 & 96 & 99 & 15.6 & 11.5 & 10.2 \\
\hline 7 & 0 & 77 & 80 & 15.8 & 11.8 & 10.6 \\
\hline 8 & 0 & 170 & 174 & 15.3 & 9.5 & 8.5 \\
\hline \multicolumn{4}{|c|}{ Location of Maximum $^{1}$} & X5 P01 S1 A15 & X5 P01 S1 A12 & X7 P01 S1 A16 \\
\hline
\end{tabular}

${ }^{1}$ Nomenclature for location of maximum value is described in footnote of Table 3-4.

It should also be noted that the specified geometry of the LEU fuel element design is also considered part of the set of design parameters for irradiation experiment design. The nominal LEU fuel element and plate dimensions are summarized in Table 2-1.

The MURR DDE irradiation experiment of a full prototypic MURR element should be designed so that target test values match as closely as possible the set of design parameters which are given in this report at prototypic conditions.

Best estimate heat flux and temperature data for a prototypic case at nominal operating conditions will also be examined in this report. Plate-level data for the MURR fuel element heat flux are presented in Section 3.3.3. Plate-level data for the fuel and cladding temperature are presented in Section 3.3.4. Heat flux is not a design parameter since it is determined by the combination of the specified geometry and power density. It is the geometry and power density, as well as fission density, that are of interest for irradiation experiments for fuel qualification to determine whether there is an impact on fuel performance. However, heat flux is often reported in the safety basis analysis, and so is listed here as a helpful tie to both values reported in the MURR PSAR [4], as well as to aid in checking experiment design.

Heat flux also affects the fuel and cladding temperatures reached during operation. It should be noted that corrosion of the cladding is not being tested in the fuel qualification program since the AA6061 aluminum alloy cladding is not being changed by conversion. Moreover, the coolant water chemistry in a test reactor is not the same as MURR, and so any measured data are not applicable to MURR. The predicted LEU corrosion thicknesses used in safety basis analyses have been based on experimentally measured and correlated values in the MURR reactor coolant chemistry.

\subsubsection{Fission Density}

Table 3-6 provides the best estimate cumulative fission density averaged for each plate, and the maximum local fission density for each plate with axial and azimuthal factors applied, for an LEU element discharged from a reference "pseudo-equilibrium" fuel cycle simulation that is prototypic for the fuel management in MURR. The total burnup for this element calculated in the REBUS-DIF3D fuel cycle simulation was $180.1 \mathrm{MWd}$. The fuel was not subdivided azimuthally in the REBUS-DIF3D 
model to avoid having an excessively large number of depletion zones in the model. Rather, the azimuthal power peaking factor for each plate from the MCNP power distribution analysis was applied to the axially-dependent fission density calculated from the REBUS-DIF3D analysis to obtain the maximum local burnup values which occur along the edge of the fuel foil. It should be noted that the depletion modeling in REBUS-DIF3D divided the fuel in each plate into 12 axial depletion zones of 2 inches each in length. Consequently, the mesh size for the fission density data presented in this section is $0.5 \times 5.08 \mathrm{~cm}$.

The best estimate maximum local burnup for the LEU fuel in MURR is $3.37 \times 10^{21}$ fissions $/ \mathrm{cm}^{3}$ and occurs in plate 1 . This includes fission in all fissionable isotopes, not solely $235 \mathrm{U}$. Figure 3-1 provides a plot of the radial profile (plate-by-plate) of the fission density in a discharged LEU fuel element. Both plate average and maximum local burnup values with all peaking factors applied are presented. Table 3-7 compares the average and maximum burnup values by axial segment for the 3 most highlyburned plates in the discharged element. The axial burnup profiles for these plates are plotted in Figure 3-2.

Table 3-6. Plate Discharge Fission Density for LEU Fuel.

\begin{tabular}{|c|c|c|c|c|c|}
\hline \multirow[b]{2}{*}{ Plate } & \multirow{2}{*}{$\begin{array}{l}\text { Plate Average } \\
\text { Fission Density } \\
\left(\mathrm{x} 10^{20} \text { fiss } / \mathrm{cm}^{3}\right)\end{array}$} & \multirow[b]{2}{*}{$\begin{array}{c}\text { Axial Burnup } \\
\text { Peaking Factor }\end{array}$} & \multirow[b]{2}{*}{$\begin{array}{c}\text { Azimuthal } \\
\text { Peaking factor }\end{array}$} & \multicolumn{2}{|c|}{ Maximum Local Burnup } \\
\hline & & & & $\begin{array}{l}\text { Fission Density } \\
\left(\mathrm{x} 10^{20} \text { fiss } / \mathrm{cm}^{3}\right)\end{array}$ & $\begin{array}{l}\text { Atom \% } \\
\text { Burnup }\end{array}$ \\
\hline 1 & 26.56 & 1.223 & 1.038 & 33.72 & $43.5 \%$ \\
\hline 2 & 20.60 & 1.245 & 1.087 & 27.86 & $35.9 \%$ \\
\hline 3 & 15.68 & 1.254 & 1.144 & 22.50 & $29.0 \%$ \\
\hline 4 & 12.25 & 1.262 & 1.196 & 18.49 & $23.9 \%$ \\
\hline 5 & 10.36 & 1.264 & 1.234 & 16.17 & $20.9 \%$ \\
\hline 6 & 9.34 & 1.263 & 1.251 & 14.76 & $19.0 \%$ \\
\hline 7 & 8.58 & 1.263 & 1.250 & 13.54 & $17.5 \%$ \\
\hline 8 & 8.07 & 1.263 & 1.251 & 12.75 & $16.4 \%$ \\
\hline 9 & 7.72 & 1.263 & 1.247 & 12.16 & $15.7 \%$ \\
\hline 10 & 7.46 & 1.264 & 1.241 & 11.71 & $15.1 \%$ \\
\hline 11 & 7.28 & 1.265 & 1.240 & 11.43 & $14.7 \%$ \\
\hline 12 & 7.15 & 1.266 & 1.243 & 11.26 & $14.5 \%$ \\
\hline 13 & 7.18 & 1.267 & 1.245 & 11.33 & $14.6 \%$ \\
\hline 14 & 7.13 & 1.269 & 1.255 & 11.35 & $14.6 \%$ \\
\hline 15 & 7.11 & 1.271 & 1.264 & 11.42 & $14.7 \%$ \\
\hline 16 & 7.15 & 1.274 & 1.273 & 11.60 & $15.0 \%$ \\
\hline 17 & 7.28 & 1.278 & 1.288 & 11.98 & $15.5 \%$ \\
\hline 18 & 7.52 & 1.282 & 1.302 & 12.55 & $16.2 \%$ \\
\hline 19 & 8.05 & 1.287 & 1.307 & 13.53 & $17.5 \%$ \\
\hline 20 & 8.84 & 1.293 & 1.297 & 14.83 & $19.1 \%$ \\
\hline 21 & 10.34 & 1.297 & 1.268 & 17.01 & $22.0 \%$ \\
\hline 22 & 13.22 & 1.300 & 1.202 & 20.66 & $26.7 \%$ \\
\hline 23 & 18.01 & 1.289 & 1.115 & 25.89 & $33.4 \%$ \\
\hline
\end{tabular}




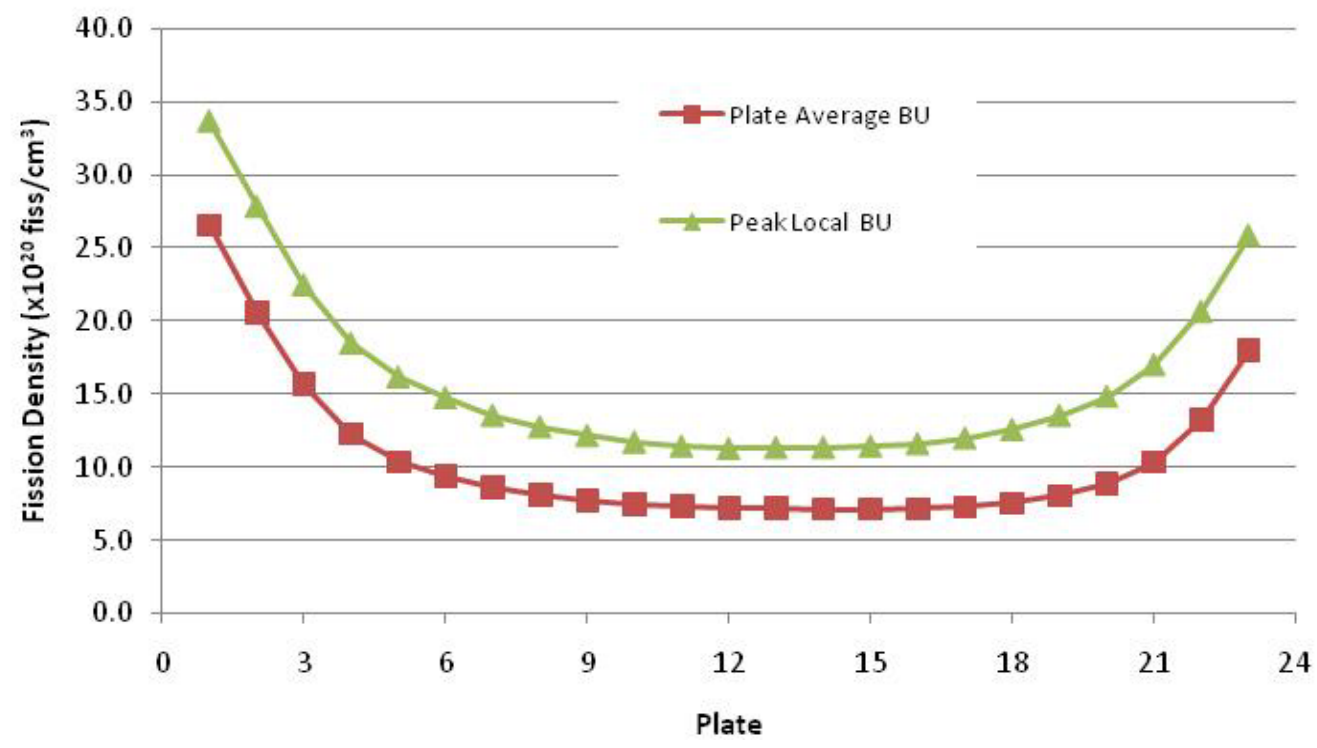

Figure 3-1. Fission Density by Plate in Discharged LEU Fuel.

Table 3-7. Discharge Fission Density $\left(\mathrm{x10}^{20}\right.$ fissions $\left./ \mathrm{cm}^{3}\right)$ in LEU Fuel Plates.

\begin{tabular}{|c|c|c|c|c|c|c|}
\hline \multirow{2}{*}{$\begin{array}{c}\text { Distance from } \\
\text { top of fuel } \\
\text { (inches) }\end{array}$} & \multicolumn{2}{|c|}{ Plate 1 } & \multicolumn{2}{c|}{ Plate 2 } & \multicolumn{2}{c|}{ Plate 23 } \\
\cline { 2 - 7 } & Average & Maximum & $\begin{array}{c}\text { Averag } \\
\text { e }\end{array}$ & Maximum & $\begin{array}{c}\text { Averag } \\
\text { e }\end{array}$ & Maximum \\
\hline 1.0 & 18.1 & 18.8 & 13.4 & 14.6 & 6.0 & 6.6 \\
\hline 3.0 & 21.0 & 21.9 & 15.8 & 17.1 & 10.6 & 11.8 \\
\hline 5.0 & 25.2 & 26.1 & 19.2 & 20.9 & 16.0 & 17.8 \\
\hline 7.0 & 28.7 & 29.8 & 22.3 & 24.2 & 19.4 & 21.7 \\
\hline 9.0 & 31.2 & 32.4 & 24.4 & 26.5 & 21.6 & 24.1 \\
\hline 11.0 & 32.5 & 33.7 & 25.6 & 27.8 & 22.9 & 25.5 \\
\hline 13.0 & 32.5 & 33.7 & 25.7 & $\mathbf{2 7 . 9}$ & 23.2 & $\mathbf{2 5 . 9}$ \\
\hline 15.0 & 31.1 & 32.3 & 24.5 & 26.6 & 22.7 & 25.3 \\
\hline 17.0 & 28.7 & 29.8 & 22.6 & 24.5 & 21.4 & 23.9 \\
\hline 19.0 & 25.9 & 26.9 & 20.2 & 22.0 & 19.5 & 21.7 \\
\hline 21.0 & 22.8 & 23.6 & 17.5 & 19.0 & 17.1 & 19.1 \\
\hline 23.0 & 21.2 & 22.0 & 16.1 & 17.5 & 15.9 & 17.7 \\
\hline
\end{tabular}



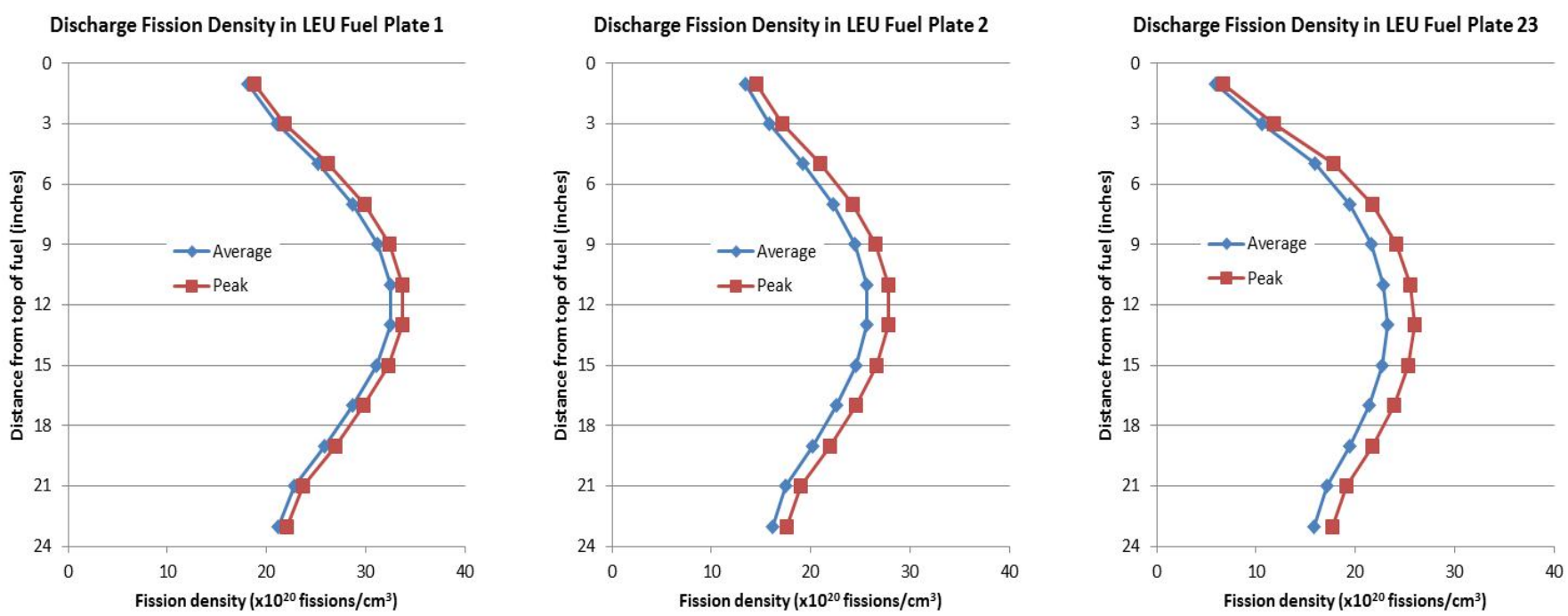

Figure 3-2. Axial Profile of Discharge Fission Density in LEU Fuel Plates.

Detailed axial and azimuthal fission density profiles for all 23 fuel plates in a discharged LEU fuel element under prototypic operating conditions are provided in Appendix A. As discussed above, the fission density data were not calculated with any azimuthal detail in the REBUS-DIF3D analysis. Instead, the local fission density values for the fuel plates were calculated by integrating the detailed time-dependent two-dimensional power density profiles calculated by MCNP over the life of the element and normalizing the results to match the maximum fission density for each plate listed in Table 3-6. A power generation of 201.4 MeV (or 3.23E-11 J) per fission event was used in the analysis. The mesh size for the fission density data provided in Appendix A is $0.5 \times 2.54 \mathrm{~cm}$.

\subsubsection{Power Density}

Table 3-8 provides the best estimate two-dimensional power density profile for the plate that has the maximum power density of all the steady-state cores examined. This occurs in plate 1 of a fresh fuel element in core position 7 of Core 5B1. A contour plot of the power density for this plate is shown in Figure 3-3. 
Table 3-8. Power Density Data $\left(\mathrm{kW} / \mathrm{cm}^{3}\right)$ for Plate with Maximum Power Density of All Steady-State Cores: Fresh LEU Element in Core 5B1, Position X7, No Xe, Plate 1, Empty FT.

\section{Fuel core width 1.690 inches Fuel core thickness 9 mil}

\begin{tabular}{|c|c|c|c|c|c|c|c|c|c|}
\hline $\begin{array}{c}\text { Axial position } \\
\text { from top of } \\
\text { fuel (inches) }\end{array}$ & $\begin{array}{c}0.000 \\
\text { to } \\
0.197 \\
\text { inches }\end{array}$ & $\begin{array}{c}0.197 \\
\text { to } \\
\text { inches }\end{array}$ & $\begin{array}{c}0.394 \\
\text { to } \\
\text { inches } \\
\text { inches }\end{array}$ & $\begin{array}{c}0.591 \\
\text { to } \\
\text { inches }\end{array}$ & $\begin{array}{c}0.760 \\
\text { to } \\
0.930 \\
\text { inches }\end{array}$ & $\begin{array}{c}0.930 \\
\text { to } \\
1.099 \\
\text { inches }\end{array}$ & $\begin{array}{c}1.099 \\
\text { to } \\
1.296 \\
\text { inches }\end{array}$ & $\begin{array}{c}1.296 \\
\text { to } \\
1.493 \\
\text { inches }\end{array}$ & $\begin{array}{c}1.493 \\
\text { to } \\
\text { inches }\end{array}$ \\
\hline 0.5 & 3.78 & 3.79 & 3.66 & 3.66 & 3.58 & 3.79 & 3.74 & 3.92 & 3.77 \\
\hline 1.5 & 4.10 & 4.02 & 3.71 & 3.61 & 3.81 & 3.97 & 3.83 & 3.81 & 3.93 \\
\hline 2.5 & 4.57 & 4.69 & 4.51 & 4.56 & 4.23 & 4.58 & 4.55 & 4.51 & 4.41 \\
\hline 3.5 & 5.72 & 5.36 & 5.33 & 5.20 & 5.28 & 5.46 & 5.47 & 5.27 & 5.53 \\
\hline 4.5 & 6.43 & 6.27 & 6.37 & 6.02 & 5.85 & 6.26 & 6.21 & 6.43 & 6.59 \\
\hline 5.5 & 7.44 & 7.20 & 6.90 & 7.32 & 6.65 & 7.18 & 7.18 & 7.17 & 7.50 \\
\hline 6.5 & 8.35 & 8.23 & 8.41 & 8.33 & 8.21 & 8.18 & 8.26 & 8.06 & 8.17 \\
\hline 7.5 & 9.22 & 9.30 & 9.15 & 9.13 & 8.59 & 8.97 & 9.06 & 9.34 & 9.86 \\
\hline 8.5 & 10.50 & 10.18 & 9.93 & 10.05 & 9.53 & 10.01 & 10.05 & 9.88 & 10.47 \\
\hline 9.5 & 11.58 & 11.27 & 10.88 & 11.31 & 10.77 & 11.10 & 11.06 & 11.27 & 11.47 \\
\hline 10.5 & 12.68 & 12.07 & 12.01 & 12.10 & 11.41 & 12.37 & 12.25 & 12.05 & 12.49 \\
\hline 11.5 & 13.11 & 13.06 & 12.86 & 12.86 & 12.68 & 13.31 & 12.87 & 12.97 & 13.32 \\
\hline 12.5 & 14.46 & 13.87 & 13.60 & 13.62 & 12.97 & 13.87 & 13.94 & 13.92 & 14.01 \\
\hline 13.5 & 14.77 & 14.68 & 14.38 & 14.31 & 13.59 & 14.34 & 14.26 & 14.18 & 14.86 \\
\hline 14.5 & 15.19 & 15.06 & 14.78 & 14.62 & 14.08 & 14.75 & 14.40 & 14.53 & 14.92 \\
\hline 15.5 & 15.83 & 14.92 & 14.63 & 14.76 & 13.99 & 14.64 & 14.68 & 14.67 & 15.53 \\
\hline 16.5 & 15.48 & 15.25 & 15.00 & 14.60 & 13.53 & 14.73 & 14.79 & 15.16 & 15.27 \\
\hline 17.5 & 15.63 & 14.78 & 14.37 & 14.27 & 13.66 & 14.14 & 14.42 & 14.24 & 15.17 \\
\hline 18.5 & 14.97 & 14.03 & 13.92 & 14.07 & 13.01 & 14.18 & 13.65 & 13.98 & 14.75 \\
\hline 19.5 & 13.82 & 13.09 & 13.05 & 12.96 & 12.32 & 13.43 & 13.29 & 13.59 & 13.95 \\
\hline 20.5 & 12.16 & 12.10 & 11.89 & 12.00 & 11.36 & 12.11 & 11.93 & 12.14 & 12.49 \\
\hline 21.5 & 11.31 & 10.80 & 10.79 & 10.66 & 10.03 & 10.63 & 10.94 & 11.08 & 11.56 \\
\hline 22.5 & 10.19 & 9.53 & 9.67 & 9.88 & 9.28 & 9.81 & 9.48 & 9.90 & 10.27 \\
\hline 23.5 & 10.27 & 9.99 & 9.85 & 9.49 & 9.23 & 9.63 & 9.79 & 10.12 & 10.42 \\
\hline Ax. Avg. & 10.90 & 10.56 & 10.40 & 10.39 & 9.90 & 10.48 & 10.42 & 10.51 & 10.86 \\
\hline & & & & & & & & & \\
\hline
\end{tabular}

POWER DENSITY VALUES NORMALIZED TO A CORE POWER OF 11.57 MW AND ASSUME ALL FISSION ENERGY (EXCLUDING GAMMA-HEATING OF EX-CORE COMPONENTS) IS DEPOSITED IN THE FUEL. 


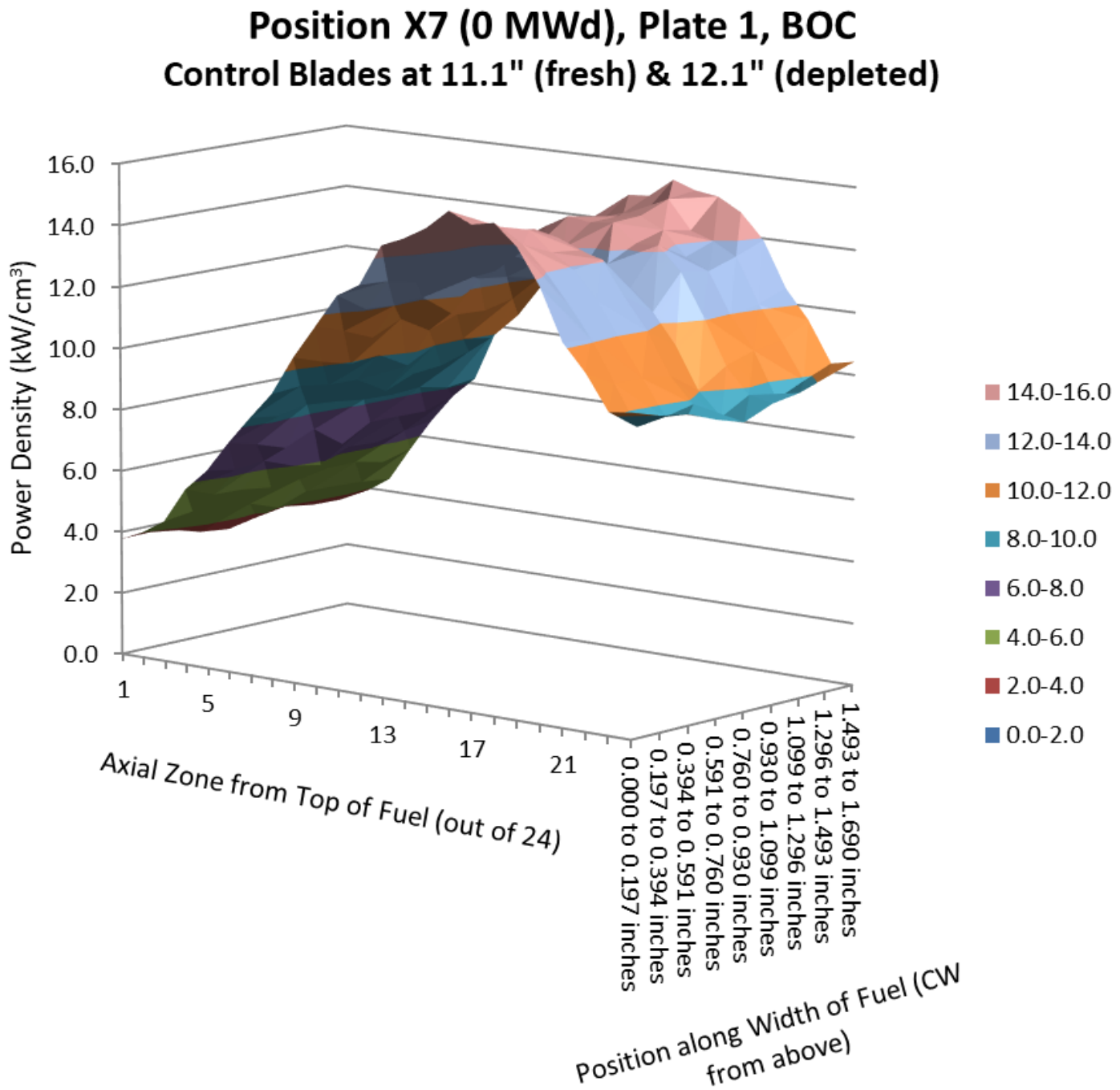

Figure 3-3. Power Density Profile $(\mathrm{kW} / \mathrm{cm} 3)$ for Plate with Maximum Power Density of All Steady-State Cores: Fresh LEU Element in Core 5B1, Position X7, No Xe, Plate 1, Empty FT.

POWER DENSITY VALUES NORMALIZED TO A CORE POWER OF 11.57 MW AND ASSUME ALL FISSION ENERGY (EXCLUDING GAMMA-HEATING OF EX-CORE COMPONENTS) IS DEPOSITED IN THE FUEL. 
While this plate has the maximum power density among all the plates in all steady-state cores examined, Core 5B1 is not prototypic for MURR operations. Tables 3-9 to 3-12 provide the power density profiles for plate 1 in elements at four different core positions at BOC in Core 7A. This core has a mixture of fuel elements at burnups that are expected to be typical for the MURR prototypic "pseudo-equilibrium" operating cycle, as well as experiments in the flux trap and irradiation materials in the graphite reflector that are typical for MURR operations. The burnups of the fuel elements in this reference core were derived from the extensive fuel cycle simulation that was representative of typical fuel management in MURR. It should be noted that the burnup indicated in these tables is the average burnup for the entire element, not the plate burnup. It should also be noted that the data presented in these tables are under xenon-free conditions, even for those plates that have experienced prior burnup.

Each profile in Tables 3-9 to 3-12 is for a fuel plate 1 (the plate adjacent to the center flux trap) in an element that has reached a different burnup in its expected lifetime under steady-state operating conditions. Since Core 7A has a mixture of fuel elements at burnups that are expected to be typical for MURR at the beginning of weekly operations under a "pseudo-equilibrium" fuel management schedule, the data in Tables 3-9 to 3-12 are representative of the power density in a MURR fuel plate with the thinnest fuel core thickness over its anticipated lifetime. Likewise, Figures 3-4 and 3-5 are for illustrative purposes and show the power density profile contours for the plate at each point in its burnup history, and illustrate the significant reduction and flattening of the power density in the plate that occurs during the fuel element's use.

The core axial and radial power distribution is influenced by the critical control blade insertion height in MURR. The insertion of the control blades at the xenon-free state that exists at the beginning of the weekly MURR operating cycle causes axial power peaking towards the bottom of the core. The fuel irradiation experiments that are being planned by the Fuel Qualification Pillar will not be conducted in MURR and may not be able to achieve this same axial power shape. Since the equilibrium xenon concentration is reached after about 48 hours of operation in a typical 6.3-day cycle in MURR, the power density data at equilibrium xenon conditions are representative of the fuel operating conditions for most of its residence in the MURR core. As can be seen in Table 3-2, the control blades are about 6 to 7 inches more fully withdrawn at equilibrium xenon, largely due to the reactivity poisoning effect of the xenon. This results in a power distribution that is less peaked axially, and also less radially peaked towards plate 1 .

Tables 3-13 to 3-16 provide best estimate power density data for fuel plate 1 in a typical MURR LEU element's lifetime at different burnup states and at equilibrium xenon conditions. The element burnup values are about 3 MWd greater than those presented in Tables 3-9 to 3-12, respectively, corresponding to the increased element burnup two days after the start of the weekly cycle. The maximum power density in MURR plate 1 under equilibrium xenon irradiation conditions that are prototypic for the weekly operating cycle in MURR is $12.8 \mathrm{~kW} / \mathrm{cm}^{3}$. This is $11 \%$ lower than the maximum power density in the same plate under xenon-free conditions in MURR, and 19\% lower than the maximum power density for a fresh plate 1 for all steady-state cases examined.

The MURR fuel plates are loaded with fuel foils of differing thicknesses. The thinnest fuel foil thickness among all the foils used in the MURR LEU fuel element design is loaded in plate 1, which has a fuel core thickness of 9 mil. Loading this thin fuel foil in the high importance region near the center flux trap results in high power density (as discussed above) and higher fission density compared to other plates in the discharged element (as discussed in Section 3.3.1). This design feature also reduces the plate heat flux and fuel temperature compared to other plates.

\section{Irradiation Demonstration Element Design Parameters for MURR LEU U-Mo Fuel Conversion}


Table 3-9. Power Density Data (kW/cm3) in Fuel Plate 1 in 0 MWd Element in Reference Mixed Burnup Core: Core 7A, Position X5, No Xe, FT loaded with typical samples.

\section{Fuel core width 1.690 inches Fuel core thickness 9 Mil}

\begin{tabular}{|c|c|c|c|c|c|c|c|c|c|}
\hline $\begin{array}{l}\text { Axial position } \\
\text { from top of } \\
\text { fuel (inches) }\end{array}$ & $\begin{array}{c}0.000 \\
\text { to } \\
0.197 \\
\text { inches }\end{array}$ & $\begin{array}{c}0.197 \\
\text { to } \\
0.394 \\
\text { inches }\end{array}$ & $\begin{array}{c}0.394 \\
\text { to } \\
0.591 \\
\text { inches }\end{array}$ & $\begin{array}{c}0.591 \\
\text { to } \\
0.760 \\
\text { inches }\end{array}$ & $\begin{array}{c}0.760 \\
\text { to } \\
0.930 \\
\text { inches }\end{array}$ & $\begin{array}{c}0.930 \\
\text { to } \\
1.099 \\
\text { inches }\end{array}$ & $\begin{array}{c}1.099 \\
\text { to } \\
1.296 \\
\text { inches }\end{array}$ & $\begin{array}{c}1.296 \\
\text { to } \\
1.493 \\
\text { inches }\end{array}$ & $\begin{array}{c}1.493 \\
\text { to } \\
1.690 \\
\text { inches }\end{array}$ \\
\hline 0.5 & 4.20 & 3.67 & 3.71 & 3.47 & 3.76 & 3.66 & 3.54 & 3.54 & 3.61 \\
\hline 1.5 & 4.03 & 3.78 & 3.80 & 3.73 & 3.53 & 3.80 & 3.54 & 3.75 & 3.74 \\
\hline 2.5 & 4.66 & 4.63 & 4.40 & 4.43 & 3.98 & 4.52 & 4.19 & 4.47 & 4.68 \\
\hline 3.5 & 5.50 & 5.45 & 5.39 & 5.41 & 5.04 & 5.15 & 5.52 & 5.37 & 5.43 \\
\hline 4.5 & 6.67 & 6.10 & 6.36 & 6.53 & 6.07 & 6.42 & 6.39 & 6.30 & 6.55 \\
\hline 5.5 & 7.38 & 7.26 & 7.08 & 7.14 & 6.70 & 7.35 & 7.11 & 7.27 & 7.41 \\
\hline 6.5 & 8.25 & 8.14 & 7.85 & 7.76 & 7.49 & 7.98 & 7.90 & 8.08 & 8.15 \\
\hline 7.5 & 9.17 & 8.59 & 8.92 & 8.66 & 8.12 & 8.71 & 8.33 & 8.65 & 8.99 \\
\hline 8.5 & 10.13 & 9.69 & 9.61 & 9.65 & 9.47 & 9.93 & 9.42 & 9.19 & 9.94 \\
\hline 9.5 & 11.32 & 10.66 & 10.42 & 10.62 & 10.03 & 10.74 & 10.84 & 10.71 & 10.93 \\
\hline 10.5 & 12.18 & 11.97 & 11.54 & 11.73 & 11.00 & 11.71 & 11.79 & 11.86 & 11.98 \\
\hline 11.5 & 12.84 & 12.56 & 12.50 & 12.35 & 11.70 & 13.06 & 12.61 & 12.77 & 13.08 \\
\hline 12.5 & 13.73 & 13.72 & 13.09 & 13.45 & 12.53 & 12.86 & 12.87 & 13.24 & 13.43 \\
\hline 13.5 & 14.32 & 13.64 & 13.22 & 13.27 & 12.92 & 13.28 & 13.83 & 13.62 & 13.94 \\
\hline 14.5 & 14.40 & 13.67 & 13.22 & 13.34 & 13.15 & 13.53 & 13.59 & 13.74 & 13.86 \\
\hline 15.5 & 13.69 & 13.42 & 13.27 & 12.90 & 12.64 & 13.77 & 13.14 & 13.63 & 13.70 \\
\hline 16.5 & 13.33 & 12.68 & 12.50 & 12.87 & 12.33 & 12.65 & 12.60 & 12.96 & 13.04 \\
\hline 17.5 & 12.81 & 12.60 & 12.12 & 12.17 & 11.30 & 11.79 & 11.55 & 11.86 & 12.25 \\
\hline 18.5 & 11.98 & 11.43 & 11.37 & 11.44 & 11.02 & 10.99 & 11.19 & 11.75 & 11.66 \\
\hline 19.5 & 11.13 & 10.68 & 10.73 & 11.05 & 10.43 & 10.23 & 10.35 & 10.46 & 10.81 \\
\hline 20.5 & 10.60 & 10.41 & 10.06 & 10.20 & 9.44 & 9.80 & 9.74 & 10.48 & 10.13 \\
\hline 21.5 & 9.90 & 9.55 & 9.12 & 9.17 & 9.06 & 9.21 & 9.37 & 9.43 & 9.47 \\
\hline 22.5 & 8.99 & 8.67 & 8.84 & 8.47 & 8.05 & 8.14 & 7.96 & 8.37 & 8.58 \\
\hline 23.5 & 9.11 & 8.91 & 8.89 & 8.85 & 8.35 & 8.92 & 8.95 & 8.90 & 9.07 \\
\hline Ax. Avg. & 10.02 & 9.66 & 9.50 & 9.53 & 9.09 & 9.51 & 9.43 & 9.60 & 9.77 \\
\hline
\end{tabular}

POWER DENSITY VALUES NORMALIZED TO A CORE POWER OF 11.57 MW AND ASSUME ALL FISSION ENERGY (EXCLUDING GAMMA-HEATING OF EX-CORE COMPONENTS) IS DEPOSITED IN THE FUEL. 
Table 3-10. Power Density Data (kW/cm3) in Fuel Plate 1 in 77 MWd Element in Reference Mixed Burnup Core: Core 7A, Position X3, No Xe, FT loaded with typical samples.

\section{Fuel core width 1.690 inches Fuel core thickness 9 mil}

\begin{tabular}{|c|c|c|c|c|c|c|c|c|c|}
\hline $\begin{array}{c}\text { Axial position } \\
\text { from top of } \\
\text { fuel (inches) }\end{array}$ & $\begin{array}{c}0.000 \\
\text { to } \\
0.197 \\
\text { inches }\end{array}$ & $\begin{array}{c}0.197 \\
\text { to } \\
\text { inches }\end{array}$ & $\begin{array}{c}0.394 \\
\text { to } \\
\text { inches }\end{array}$ & $\begin{array}{c}0.591 \\
\text { to } \\
\text { inches } \\
\text { inches }\end{array}$ & $\begin{array}{c}0.760 \\
\text { to } \\
0.930 \\
\text { inches }\end{array}$ & $\begin{array}{c}0.930 \\
\text { to } \\
1.099 \\
\text { inches }\end{array}$ & $\begin{array}{c}1.099 \\
\text { to } \\
1.296 \\
\text { inches }\end{array}$ & $\begin{array}{c}1.296 \\
\text { to } \\
1.493 \\
\text { inches }\end{array}$ & $\begin{array}{c}1.493 \\
\text { to } \\
\text { inches }\end{array}$ \\
\hline 0.5 & 3.63 & 3.50 & 3.52 & 3.41 & 3.43 & 3.37 & 3.35 & 3.60 & 3.65 \\
\hline 1.5 & 3.83 & 3.61 & 3.47 & 3.43 & 3.51 & 3.50 & 3.52 & 3.56 & 3.66 \\
\hline 2.5 & 4.10 & 3.93 & 3.90 & 4.08 & 3.91 & 3.91 & 3.92 & 3.80 & 4.03 \\
\hline 3.5 & 4.88 & 4.86 & 4.65 & 4.76 & 4.34 & 4.56 & 4.71 & 4.59 & 4.80 \\
\hline 4.5 & 5.52 & 5.51 & 5.48 & 5.33 & 4.90 & 5.25 & 5.43 & 5.40 & 5.35 \\
\hline 5.5 & 6.14 & 6.35 & 6.15 & 6.09 & 5.89 & 5.95 & 5.95 & 5.95 & 6.30 \\
\hline 6.5 & 6.86 & 6.58 & 6.61 & 6.66 & 6.34 & 6.85 & 6.66 & 6.72 & 6.96 \\
\hline 7.5 & 7.94 & 7.53 & 7.33 & 7.56 & 7.39 & 7.66 & 7.37 & 7.50 & 7.59 \\
\hline 8.5 & 8.39 & 8.27 & 8.36 & 8.02 & 7.86 & 8.03 & 7.90 & 8.20 & 8.28 \\
\hline 9.5 & 9.29 & 8.98 & 8.93 & 8.94 & 8.47 & 9.07 & 8.99 & 9.11 & 9.29 \\
\hline 10.5 & 10.09 & 9.78 & 9.58 & 9.89 & 8.96 & 9.44 & 9.55 & 9.49 & 9.87 \\
\hline 11.5 & 10.81 & 10.68 & 10.31 & 10.37 & 9.68 & 10.27 & 10.20 & 10.54 & 10.57 \\
\hline 12.5 & 11.44 & 11.14 & 11.02 & 11.01 & 10.47 & 10.90 & 10.80 & 10.90 & 10.91 \\
\hline 13.5 & 11.45 & 11.44 & 10.96 & 11.21 & 10.53 & 11.06 & 11.27 & 11.32 & 11.72 \\
\hline 14.5 & $\mathbf{1 1 . 9 9}$ & 11.65 & 11.21 & 11.24 & 10.95 & 11.40 & 11.39 & 11.50 & 11.83 \\
\hline 15.5 & 11.65 & 11.20 & 11.01 & 11.17 & 10.48 & 11.01 & 11.17 & 11.38 & 11.60 \\
\hline 16.5 & 11.46 & 11.15 & 11.13 & 10.84 & 10.59 & 10.78 & 10.71 & 10.85 & 11.43 \\
\hline 17.5 & 11.24 & 10.99 & 10.48 & 10.37 & 9.81 & 10.61 & 10.16 & 10.75 & 10.41 \\
\hline 18.5 & 10.48 & 10.29 & 10.02 & 10.06 & 9.43 & 10.24 & 9.72 & 9.90 & 10.16 \\
\hline 19.5 & 10.12 & 9.66 & 9.67 & 9.48 & 9.18 & 9.56 & 9.57 & 9.29 & 9.54 \\
\hline 20.5 & 9.50 & 9.21 & 8.86 & 9.30 & 8.57 & 8.96 & 9.08 & 8.92 & 9.06 \\
\hline 21.5 & 8.38 & 8.35 & 8.17 & 7.85 & 7.86 & 8.17 & 8.16 & 8.07 & 8.12 \\
\hline 22.5 & 7.79 & 7.86 & 7.50 & 7.30 & 6.97 & 7.14 & 7.36 & 7.76 & 7.90 \\
\hline 23.5 & 8.49 & 8.06 & 7.76 & 7.86 & 7.30 & 7.92 & 7.86 & 7.82 & 8.34 \\
\hline Ax. Avg. & 8.56 & 8.35 & 8.17 & 8.17 & 7.79 & 8.15 & 8.12 & 8.21 & 8.39 \\
\hline & & & & & & & & & \\
\hline & & & & & & & & &
\end{tabular}

POWER DENSITY VALUES NORMALIZED TO A CORE POWER OF 11.57 MW AND ASSUME ALL FISSION ENERGY (EXCLUDING GAMMA-HEATING OF EX-CORE COMPONENTS) IS DEPOSITED IN THE FUEL. 
Table 3-11. Power Density Data (kW/cm3) in Fuel Plate 1 in 96 MWd Element in Reference Mixed Burnup Core: Core 7A, Position X2, No Xe, FT loaded with typical samples.

\section{Fuel core width 1.690 inches Fuel core thickness 99 mil}

\begin{tabular}{|c|c|c|c|c|c|c|c|c|c|}
\hline $\begin{array}{c}\text { Axial position } \\
\text { from top of } \\
\text { fuel (inches) }\end{array}$ & $\begin{array}{c}0.000 \\
\text { to } \\
0.197 \\
\text { inches }\end{array}$ & $\begin{array}{c}0.197 \\
\text { to } \\
\text { inches }\end{array}$ & $\begin{array}{c}0.394 \\
\text { to } \\
\text { inches }\end{array}$ & $\begin{array}{c}0.591 \\
\text { to } \\
\text { inches }\end{array}$ & $\begin{array}{c}0.760 \\
\text { to } \\
\text { inc.930 } \\
\text { inches }\end{array}$ & $\begin{array}{c}0.930 \\
\text { to } \\
1.099 \\
\text { inches }\end{array}$ & $\begin{array}{c}1.099 \\
\text { to } \\
1.296 \\
\text { inches }\end{array}$ & $\begin{array}{c}1.296 \\
\text { to } \\
1.493 \\
\text { inches }\end{array}$ & $\begin{array}{c}1.493 \\
\text { to } \\
\text { inches }\end{array}$ \\
\hline 0.5 & 3.49 & 3.36 & 3.25 & 3.10 & 3.06 & 3.20 & 3.40 & 3.38 & 3.47 \\
\hline 1.5 & 3.58 & 3.41 & 3.33 & 3.72 & 3.27 & 3.54 & 3.32 & 3.41 & 3.56 \\
\hline 2.5 & 4.10 & 3.93 & 3.93 & 3.83 & 3.82 & 3.91 & 3.93 & 3.84 & 4.14 \\
\hline 3.5 & 4.62 & 4.84 & 4.53 & 4.71 & 4.44 & 4.77 & 4.69 & 4.65 & 4.73 \\
\hline 4.5 & 5.29 & 5.13 & 5.14 & 5.21 & 4.83 & 5.19 & 5.02 & 5.19 & 5.38 \\
\hline 5.5 & 6.34 & 6.19 & 5.84 & 5.82 & 5.69 & 5.94 & 5.88 & 5.94 & 6.21 \\
\hline 6.5 & 6.62 & 6.31 & 6.39 & 6.52 & 6.07 & 6.49 & 6.36 & 6.44 & 6.69 \\
\hline 7.5 & 7.56 & 7.68 & 7.28 & 7.26 & 6.93 & 7.46 & 7.35 & 7.48 & 7.24 \\
\hline 8.5 & 8.33 & 8.21 & 8.13 & 8.04 & 7.54 & 7.90 & 8.18 & 8.38 & 8.06 \\
\hline 9.5 & 9.22 & 8.85 & 8.75 & 8.93 & 8.59 & 8.97 & 8.71 & 8.90 & 9.27 \\
\hline 10.5 & 9.58 & 9.47 & 9.28 & 9.39 & 8.97 & 9.24 & 9.24 & 9.47 & 9.56 \\
\hline 11.5 & 10.28 & 9.86 & 10.04 & 9.95 & 9.60 & 10.10 & 10.03 & 10.19 & 10.41 \\
\hline 12.5 & 10.64 & 10.68 & 10.27 & 10.26 & 9.97 & 10.21 & 10.58 & 10.36 & 10.61 \\
\hline 13.5 & 11.16 & 10.72 & 10.45 & 10.86 & 10.18 & 10.88 & 10.65 & 10.70 & 11.20 \\
\hline 14.5 & 11.53 & 11.02 & 10.89 & 10.61 & 10.55 & 10.86 & 11.27 & 11.15 & 11.41 \\
\hline 15.5 & 10.99 & 10.67 & 10.80 & 10.64 & 10.42 & 10.81 & 10.73 & 11.18 & 11.16 \\
\hline 16.5 & 11.04 & 10.72 & 10.63 & 10.70 & 10.47 & 11.05 & 10.27 & 10.97 & 11.38 \\
\hline 17.5 & 10.52 & 10.34 & 10.38 & 10.11 & 9.99 & 10.46 & 10.15 & 10.48 & 10.83 \\
\hline 18.5 & 10.50 & 10.03 & 9.93 & 10.09 & 9.78 & 10.18 & 9.93 & 10.05 & 10.29 \\
\hline 19.5 & 9.55 & 9.59 & 9.57 & 9.27 & 9.21 & 9.24 & 9.58 & 9.72 & 9.69 \\
\hline 20.5 & 9.42 & 8.87 & 8.77 & 8.72 & 8.21 & 8.92 & 8.61 & 9.10 & 9.44 \\
\hline 21.5 & 8.72 & 8.28 & 7.72 & 7.86 & 7.56 & 8.02 & 8.10 & 7.97 & 8.32 \\
\hline 22.5 & 7.80 & 7.26 & 7.27 & 7.34 & 7.01 & 7.60 & 7.43 & 7.39 & 7.63 \\
\hline 23.5 & 8.20 & 8.16 & 7.88 & 7.53 & 7.46 & 7.48 & 7.71 & 7.50 & 8.00 \\
\hline Ax. Avg. & 8.30 & 8.06 & 7.94 & 7.94 & 7.65 & 8.02 & 7.96 & 8.07 & 8.28 \\
\hline & & & & & & & & & \\
\hline & & & & & & & & &
\end{tabular}

POWER DENSITY VALUES NORMALIZED TO A CORE POWER OF 11.57 MW AND ASSUME ALL FISSION ENERGY (EXCLUDING GAMMA-HEATING OF EX-CORE COMPONENTS) IS DEPOSITED IN THE FUEL. 
Table 3-12. Power Density Data $(\mathrm{kW} / \mathrm{cm} 3)$ in Fuel Plate 1 in 170 MWd Burnup Element in Reference Mixed Burnup Core: Core 7A, Position X4, No Xe, FT loaded with typical samples.

\section{Fuel core width 1.690 inches Fuel core thickness 99 mil}

\begin{tabular}{|c|c|c|c|c|c|c|c|c|c|}
\hline $\begin{array}{l}\text { Axial position } \\
\text { from top of } \\
\text { fuel (inches) }\end{array}$ & $\begin{array}{c}0.000 \\
\text { to } \\
0.197 \\
\text { inches }\end{array}$ & $\begin{array}{c}0.197 \\
\text { to } \\
0.394 \\
\text { inches }\end{array}$ & $\begin{array}{c}0.394 \\
\text { to } \\
0.591 \\
\text { inches }\end{array}$ & $\begin{array}{c}0.591 \\
\text { to } \\
0.760 \\
\text { inches }\end{array}$ & $\begin{array}{c}0.760 \\
\text { to } \\
0.930 \\
\text { inches }\end{array}$ & $\begin{array}{c}0.930 \\
\text { to } \\
1.099 \\
\text { inches }\end{array}$ & $\begin{array}{c}1.099 \\
\text { to } \\
1.296 \\
\text { inches }\end{array}$ & $\begin{array}{c}1.296 \\
\text { to } \\
1.493 \\
\text { inches }\end{array}$ & $\begin{array}{c}1.493 \\
\text { to } \\
1.690 \\
\text { inches }\end{array}$ \\
\hline 0.5 & 3.09 & 3.07 & 3.06 & 3.18 & 2.81 & 3.05 & 2.99 & 3.13 & 3.12 \\
\hline 1.5 & 3.10 & 3.09 & 3.13 & 3.06 & 2.98 & 3.21 & 3.11 & 3.29 & 3.32 \\
\hline 2.5 & 3.56 & 3.48 & 3.56 & 3.53 & 3.33 & 3.48 & 3.55 & 3.60 & 3.62 \\
\hline 3.5 & 4.17 & 4.25 & 4.12 & 4.20 & 4.14 & 4.26 & 4.04 & 4.15 & 4.11 \\
\hline 4.5 & 4.64 & 4.48 & 4.46 & 4.45 & 4.50 & 4.48 & 4.45 & 4.51 & 4.72 \\
\hline 5.5 & 5.21 & 5.11 & 5.10 & 4.97 & 5.01 & 5.27 & 5.24 & 5.15 & 5.53 \\
\hline 6.5 & 5.49 & 5.58 & 5.63 & 5.64 & 5.14 & 5.52 & 5.57 & 5.62 & 5.55 \\
\hline 7.5 & 6.19 & 6.03 & 5.87 & 6.36 & 6.03 & 6.31 & 6.26 & 6.36 & 6.38 \\
\hline 8.5 & 6.72 & 6.41 & 6.33 & 6.47 & 6.30 & 6.41 & 6.68 & 6.78 & 6.72 \\
\hline 9.5 & 7.23 & 7.22 & 6.87 & 7.18 & 6.72 & 6.85 & 7.15 & 7.26 & 7.33 \\
\hline 10.5 & 7.63 & 7.47 & 7.46 & 7.62 & 7.44 & 7.50 & 7.53 & 8.00 & 7.88 \\
\hline 11.5 & 8.40 & 8.31 & 8.27 & 8.21 & 7.78 & 8.32 & 8.17 & 8.54 & 8.85 \\
\hline 12.5 & 9.02 & 8.79 & 8.44 & 8.85 & 8.41 & 8.74 & 8.82 & 9.04 & 9.15 \\
\hline 13.5 & 9.41 & 9.17 & 8.93 & 9.13 & 8.47 & 9.06 & 8.97 & 9.23 & 9.16 \\
\hline 14.5 & 9.51 & 9.35 & 9.37 & 9.32 & 8.75 & 9.32 & 9.14 & 9.31 & 9.60 \\
\hline 15.5 & 9.14 & 9.09 & 9.23 & 9.25 & 8.61 & 8.74 & 9.15 & 9.14 & 9.17 \\
\hline 16.5 & 9.25 & 8.85 & 8.86 & 9.04 & 8.75 & 8.92 & 8.99 & 9.49 & 9.32 \\
\hline 17.5 & 8.85 & 8.62 & 8.38 & 8.71 & 8.31 & 8.89 & 8.85 & 8.60 & 8.73 \\
\hline 18.5 & 8.56 & 8.64 & 8.43 & 8.50 & 8.07 & 8.40 & 8.74 & 8.30 & 9.03 \\
\hline 19.5 & 8.00 & 7.83 & 7.95 & 7.78 & 7.56 & 7.83 & 7.84 & 8.14 & 8.11 \\
\hline 20.5 & 7.76 & 7.59 & 7.49 & 7.69 & 7.22 & 7.46 & 7.50 & 7.80 & 7.85 \\
\hline 21.5 & 7.26 & 7.02 & 6.93 & 7.27 & 6.90 & 7.19 & 7.20 & 6.99 & 7.33 \\
\hline 22.5 & 6.76 & 6.77 & 6.74 & 6.64 & 6.28 & 6.46 & 6.42 & 6.90 & 6.80 \\
\hline 23.5 & 7.37 & 6.86 & 6.73 & 6.94 & 6.49 & 7.03 & 7.02 & 6.85 & 7.21 \\
\hline Ax. Avg. & 6.93 & 6.79 & 6.72 & 6.83 & 6.50 & 6.78 & 6.81 & 6.93 & 7.02 \\
\hline
\end{tabular}

POWER DENSITY VALUES NORMALIZED TO A CORE POWER OF 11.57 MW AND ASSUME ALL FISSION ENERGY (EXCLUDING GAMMA-HEATING OF EX-CORE COMPONENTS) IS DEPOSITED IN THE FUEL. 


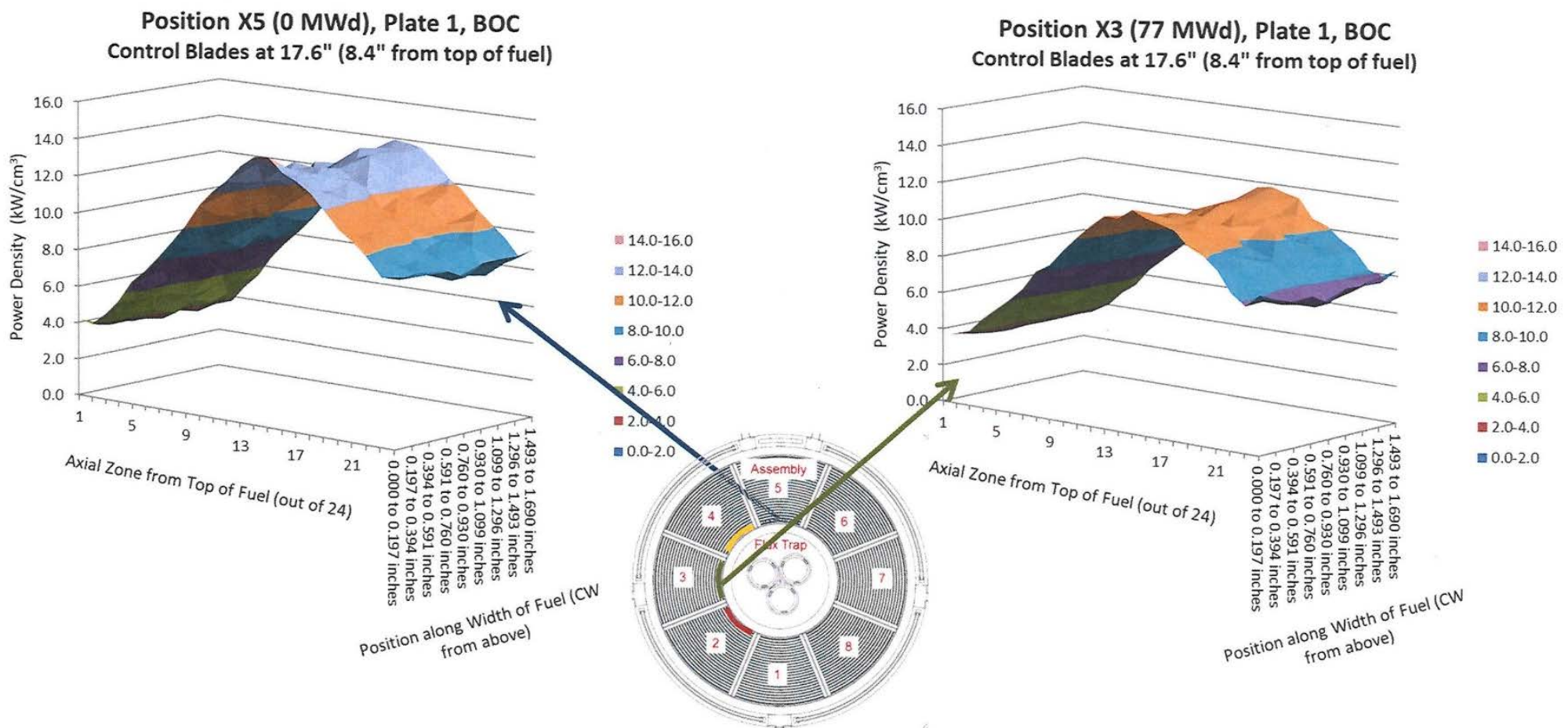

Position X5, 0 MWd, No Xe, Plate 1, FT loaded with typical samples

Position X3, 77 MWd, No Xe, Plate 1, FT loaded with typical samples

Figure 3-4. Evolution of Power Density Profile (kW/cm3) for LEU Fuel Plate 1, Element Burnup from 0 to 77 MWd: Core 7A, no Xe, FT loaded with typical samples.

POWER DENSITY VALUES NORMALIZED TO A CORE POWER OF 11.57 MW AND ASSUME ALL FISSION ENERGY (EXCLUDING GAMMAHEATING OF EX-CORE COMPONENTS) IS DEPOSITED IN THE FUEL. 


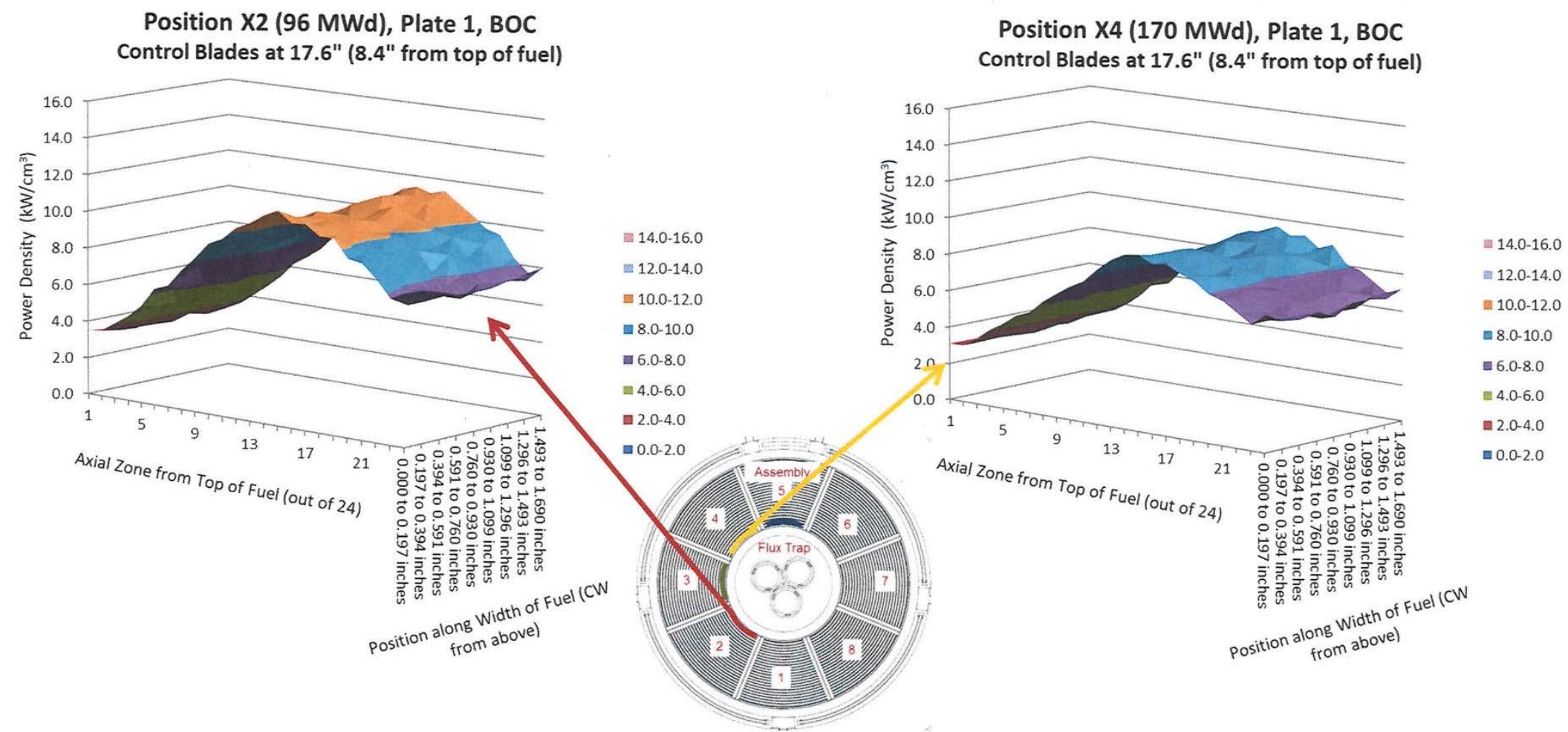

Position X2, 96 MWd, No Xe, Plate 1, FT loaded with typical samples

Position X4, 170 MWd, No Xe, Plate 1, FT loaded with typical samples

Figure 3-5. Evolution of Power Density Profile $(\mathrm{kW} / \mathrm{cm} 3)$ for LEU Fuel Plate 1, Element Burnup from 96 to 170 MWd: Core 7A, no Xe, FT loaded with typical samples.

POWER DENSITY VALUES NORMALIZED TO A CORE POWER OF 11.57 MW AND ASSUME ALL FISSION ENERGY (EXCLUDING GAMMAHEATING OF EX-CORE COMPONENTS) IS DEPOSITED IN THE FUEL. 
Table 3-13. Power Density Data $(\mathrm{kW} / \mathrm{cm} 3)$ in Fuel Plate 1 in 3 MWd Burnup Element at Eq. Xe Conditions in Reference Mixed Burnup Core: Core 8A, Position X5, FT loaded with typical samples.

\section{Fuel core width 1.690 inches Fuel core thickness 9 mil}

\begin{tabular}{|c|c|c|c|c|c|c|c|c|c|}
\hline $\begin{array}{c}\text { Axial position } \\
\text { from top of } \\
\text { fuel (inches) }\end{array}$ & $\begin{array}{c}0.000 \\
\text { to } \\
0.197 \\
\text { inches }\end{array}$ & $\begin{array}{c}0.197 \\
\text { to } \\
0.394 \\
\text { inches }\end{array}$ & $\begin{array}{c}0.394 \\
\text { to } \\
0.591 \\
\text { inches }\end{array}$ & $\begin{array}{c}0.591 \\
\text { to } \\
0.760 \\
\text { inches }\end{array}$ & $\begin{array}{c}0.760 \\
\text { to } \\
0.930 \\
\text { inches }\end{array}$ & $\begin{array}{c}0.930 \\
\text { to } \\
1.099 \\
\text { inches }\end{array}$ & $\begin{array}{c}1.099 \\
\text { to } \\
1.296 \\
\text { inches }\end{array}$ & $\begin{array}{c}1.296 \\
\text { to } \\
1.493 \\
\text { inches }\end{array}$ & $\begin{array}{c}1.493 \\
\text { to } \\
1.690 \\
\text { inches }\end{array}$ \\
\hline 0.5 & 5.72 & 5.41 & 5.43 & 5.70 & 4.94 & 5.37 & 5.35 & 5.33 & 5.59 \\
\hline 1.5 & 5.78 & 5.49 & 5.48 & 5.46 & 5.19 & 5.57 & 5.32 & 5.52 & 5.52 \\
\hline 2.5 & 6.27 & 6.24 & 6.47 & 6.25 & 6.15 & 6.34 & 6.16 & 6.38 & 6.52 \\
\hline 3.5 & 7.45 & 7.11 & 7.13 & 6.88 & 6.98 & 7.16 & 7.16 & 7.45 & 7.56 \\
\hline 4.5 & 8.53 & 8.16 & 7.86 & 8.05 & 7.75 & 7.96 & 8.34 & 8.35 & 8.86 \\
\hline 5.5 & 9.28 & 9.02 & 9.06 & 8.68 & 8.24 & 8.60 & 8.72 & 9.02 & 9.62 \\
\hline 6.5 & 10.34 & 9.44 & 9.57 & 9.52 & 9.08 & 9.49 & 9.55 & 9.91 & 9.75 \\
\hline 7.5 & 10.62 & 10.23 & 9.99 & 9.98 & 9.48 & 9.89 & 9.97 & 9.98 & 10.09 \\
\hline 8.5 & 11.20 & 10.69 & 10.39 & 10.53 & 9.99 & 10.51 & 10.42 & 10.41 & 10.16 \\
\hline 9.5 & 11.73 & 11.28 & 11.23 & 11.14 & 10.46 & 11.16 & 11.14 & 11.13 & 11.54 \\
\hline 10.5 & 12.19 & 11.32 & 11.31 & 11.54 & 11.08 & 11.40 & 11.27 & 11.77 & 11.79 \\
\hline 11.5 & 12.78 & 12.19 & 11.66 & 11.59 & 11.23 & 11.75 & 11.55 & 11.98 & 12.39 \\
\hline 12.5 & 12.47 & 12.04 & 11.85 & 12.14 & 11.30 & 11.56 & 11.60 & 11.72 & 12.06 \\
\hline 13.5 & 12.47 & 12.04 & 11.61 & 11.67 & 11.17 & 11.93 & 11.86 & 12.12 & 12.25 \\
\hline 14.5 & 12.27 & 11.82 & 11.61 & 11.38 & 10.65 & 11.37 & 11.49 & 11.84 & 12.07 \\
\hline 15.5 & 11.35 & 11.14 & 10.81 & 11.24 & 10.29 & 10.92 & 10.65 & 11.13 & 11.65 \\
\hline 16.5 & 10.59 & 10.28 & 10.22 & 10.10 & 9.87 & 10.13 & 10.00 & 10.35 & 10.94 \\
\hline 17.5 & 10.10 & 9.81 & 9.44 & 9.94 & 9.01 & 9.50 & 9.38 & 9.53 & 9.60 \\
\hline 18.5 & 9.20 & 8.78 & 8.94 & 8.65 & 8.53 & 8.76 & 8.81 & 8.80 & 8.83 \\
\hline 19.5 & 8.79 & 8.52 & 8.38 & 8.27 & 7.51 & 8.32 & 8.14 & 8.12 & 8.31 \\
\hline 20.5 & 7.96 & 8.04 & 7.88 & 7.72 & 7.34 & 7.39 & 7.67 & 7.68 & 7.51 \\
\hline 21.5 & 7.31 & 7.06 & 6.95 & 6.99 & 6.63 & 7.13 & 6.61 & 6.74 & 7.13 \\
\hline 22.5 & 6.86 & 6.43 & 6.23 & 6.64 & 5.84 & 6.24 & 6.42 & 6.67 & 6.58 \\
\hline 23.5 & 7.19 & 6.75 & 6.71 & 7.06 & 6.32 & 6.56 & 6.46 & 6.58 & 7.00 \\
\hline Ax. Avg. & 9.52 & 9.14 & 9.01 & 9.05 & 8.55 & 8.96 & 8.92 & 9.11 & 9.31 \\
\hline
\end{tabular}

POWER DENSITY VALUES NORMALIZED TO A CORE POWER OF 11.57 MW AND ASSUME ALL FISSION ENERGY (EXCLUDING GAMMA-HEATING OF EX-CORE COMPONENTS) IS DEPOSITED IN THE FUEL. 
Table 3-14. Power Density Data (kW/cm3) in Fuel Plate 1 in 80 MWd Burnup Element at Eq. Xe Conditions in Reference Mixed Burnup Core: Core 8A, Position X3, FT loaded with typical samples.

\section{Fuel core width 1.690 inches Fuel core thickness 9 mil}

\begin{tabular}{|c|c|c|c|c|c|c|c|c|c|}
\hline $\begin{array}{l}\text { Axial position } \\
\text { from top of } \\
\text { fuel (inches) }\end{array}$ & $\begin{array}{c}0.000 \\
\text { to } \\
0.197 \\
\text { inches }\end{array}$ & $\begin{array}{c}0.197 \\
\text { to } \\
0.394 \\
\text { inches }\end{array}$ & $\begin{array}{c}0.394 \\
\text { to } \\
0.591 \\
\text { inches }\end{array}$ & $\begin{array}{c}0.591 \\
\text { to } \\
0.760 \\
\text { inches }\end{array}$ & $\begin{array}{c}0.760 \\
\text { to } \\
0.930 \\
\text { inches }\end{array}$ & $\begin{array}{c}0.930 \\
\text { to } \\
1.099 \\
\text { inches }\end{array}$ & $\begin{array}{c}1.099 \\
\text { to } \\
1.296 \\
\text { inches }\end{array}$ & $\begin{array}{c}1.296 \\
\text { to } \\
1.493 \\
\text { inches }\end{array}$ & $\begin{array}{c}1.493 \\
\text { to } \\
1.690 \\
\text { inches }\end{array}$ \\
\hline 0.5 & 5.43 & 5.21 & 5.04 & 5.14 & 4.88 & 5.32 & 5.21 & 5.15 & 5.42 \\
\hline 1.5 & 5.64 & 5.29 & 5.03 & 5.22 & 4.93 & 5.14 & 4.98 & 5.11 & 5.17 \\
\hline 2.5 & 5.91 & 5.82 & 5.73 & 5.74 & 5.54 & 5.76 & 5.52 & 5.61 & 5.97 \\
\hline 3.5 & 6.70 & 6.34 & 6.49 & 6.51 & 6.15 & 6.62 & 6.49 & 6.48 & 6.73 \\
\hline 4.5 & 7.34 & 7.13 & 7.06 & 7.35 & 6.99 & 7.21 & 6.88 & 7.12 & 7.35 \\
\hline 5.5 & 8.13 & 7.88 & 7.90 & 8.04 & 7.42 & 7.94 & 7.73 & 7.90 & 8.02 \\
\hline 6.5 & 8.76 & 8.35 & 8.11 & 8.03 & 7.63 & 8.17 & 8.01 & 8.25 & 8.35 \\
\hline 7.5 & 9.22 & 8.57 & 8.62 & 8.66 & 8.22 & 8.49 & 8.56 & 8.62 & 8.76 \\
\hline 8.5 & 9.35 & 8.89 & 8.97 & 8.71 & 8.56 & 8.78 & 8.67 & 9.10 & 9.02 \\
\hline 9.5 & 9.88 & 9.43 & 9.15 & 9.09 & 8.73 & 9.14 & 9.40 & 9.21 & 9.57 \\
\hline 10.5 & 9.97 & 9.86 & 9.41 & 9.61 & 9.10 & 9.69 & 9.47 & 9.43 & 9.91 \\
\hline 11.5 & 10.34 & 10.08 & 9.71 & 10.17 & 9.25 & 9.81 & 9.79 & 9.93 & 10.04 \\
\hline 12.5 & 10.51 & 10.06 & 10.01 & 10.19 & 9.26 & 10.20 & 9.99 & 9.73 & 10.38 \\
\hline 13.5 & 10.30 & 9.81 & 9.86 & 9.90 & 9.67 & 10.18 & 9.82 & 10.12 & 10.48 \\
\hline 14.5 & 10.25 & 9.88 & 9.75 & 9.55 & 9.34 & 9.81 & 9.80 & 9.94 & 9.93 \\
\hline 15.5 & 9.60 & 9.46 & 9.46 & 9.52 & 8.81 & 9.40 & 9.14 & 9.45 & 9.81 \\
\hline 16.5 & 9.36 & 9.12 & 8.88 & 8.97 & 8.73 & 8.81 & 8.67 & 8.71 & 9.01 \\
\hline 17.5 & 8.70 & 8.50 & 8.44 & 8.49 & 8.11 & 8.30 & 8.50 & 8.35 & 8.65 \\
\hline 18.5 & 8.38 & 8.10 & 7.88 & 7.83 & 7.36 & 7.94 & 7.79 & 7.81 & 8.38 \\
\hline 19.5 & 8.04 & 7.34 & 7.30 & 7.54 & 7.05 & 7.14 & 7.43 & 7.26 & 7.72 \\
\hline 20.5 & 7.20 & 6.98 & 6.66 & 6.98 & 6.64 & 6.60 & 6.78 & 7.00 & 6.94 \\
\hline 21.5 & 6.59 & 6.44 & 6.23 & 6.20 & 5.83 & 6.34 & 6.08 & 6.16 & 6.21 \\
\hline 22.5 & 6.03 & 5.72 & 5.69 & 5.70 & 5.73 & 5.78 & 5.88 & 5.58 & 5.81 \\
\hline 23.5 & 6.22 & 5.97 & 6.03 & 5.99 & 5.70 & 5.89 & 6.02 & 5.87 & 6.16 \\
\hline Ax. Avg. & 8.25 & 7.93 & 7.81 & 7.88 & 7.49 & 7.85 & 7.77 & 7.83 & 8.07 \\
\hline
\end{tabular}

POWER DENSITY VALUES NORMALIZED TO A CORE POWER OF 11.57 MW AND ASSUME ALL FISSION ENERGY (EXCLUDING GAMMA-HEATING OF EX-CORE COMPONENTS) IS DEPOSITED IN THE FUEL. 
Table 3-15. Power Density Data (kW/cm3) in Fuel Plate 1 in 99 MWd Burnup Element at Eq. Xe Conditions in Reference Mixed Burnup Core: Core 8A, Position X2, FT loaded with typical samples.

\section{Fuel core width 1.690 inches Fuel core thickness 9 mil}

\begin{tabular}{|c|c|c|c|c|c|c|c|c|c|}
\hline $\begin{array}{l}\text { Axial position } \\
\text { from top of } \\
\text { fuel (inches) }\end{array}$ & $\begin{array}{c}0.000 \\
\text { to } \\
0.197 \\
\text { inches }\end{array}$ & $\begin{array}{c}0.197 \\
\text { to } \\
0.394 \\
\text { inches }\end{array}$ & $\begin{array}{c}0.394 \\
\text { to } \\
0.591 \\
\text { inches }\end{array}$ & $\begin{array}{c}0.591 \\
\text { to } \\
0.760 \\
\text { inches }\end{array}$ & $\begin{array}{c}0.760 \\
\text { to } \\
0.930 \\
\text { inches }\end{array}$ & $\begin{array}{c}0.930 \\
\text { to } \\
1.099 \\
\text { inches }\end{array}$ & $\begin{array}{c}1.099 \\
\text { to } \\
1.296 \\
\text { inches }\end{array}$ & $\begin{array}{c}1.296 \\
\text { to } \\
1.493 \\
\text { inches }\end{array}$ & $\begin{array}{c}1.493 \\
\text { to } \\
1.690 \\
\text { inches }\end{array}$ \\
\hline 0.5 & 5.10 & 5.06 & 5.02 & 5.23 & 4.77 & 4.83 & 4.96 & 4.98 & 5.08 \\
\hline 1.5 & 5.40 & 5.13 & 5.24 & 4.99 & 4.99 & 5.13 & 4.87 & 5.05 & 5.36 \\
\hline 2.5 & 6.11 & 5.69 & 5.62 & 5.74 & 5.67 & 5.76 & 5.47 & 5.49 & 5.89 \\
\hline 3.5 & 6.74 & 6.66 & 6.70 & 6.75 & 6.20 & 6.33 & 6.56 & 6.58 & 6.73 \\
\hline 4.5 & 7.33 & 7.13 & 6.97 & 6.77 & 6.56 & 6.86 & 7.08 & 6.96 & 7.36 \\
\hline 5.5 & 7.83 & 7.51 & 7.62 & 7.72 & 7.56 & 7.58 & 7.69 & 7.79 & 7.91 \\
\hline 6.5 & 8.36 & 8.00 & 7.83 & 7.94 & 7.56 & 8.40 & 8.09 & 8.30 & 8.56 \\
\hline 7.5 & 9.03 & 8.71 & 8.55 & 8.71 & 7.96 & 8.37 & 8.46 & 8.83 & 8.90 \\
\hline 8.5 & 9.01 & 8.85 & 8.99 & 8.87 & 8.53 & 8.85 & 8.61 & 8.78 & 8.86 \\
\hline 9.5 & 9.65 & 9.19 & 9.24 & 9.23 & 9.00 & 9.41 & 9.14 & 9.26 & 9.41 \\
\hline 10.5 & 9.47 & 9.45 & 9.23 & 9.46 & 9.07 & 9.55 & 9.31 & 9.34 & 9.81 \\
\hline 11.5 & 9.77 & 9.69 & 9.53 & 9.45 & 9.16 & 9.18 & 9.47 & 9.57 & 9.73 \\
\hline 12.5 & 10.01 & 9.43 & 9.54 & 9.38 & 9.15 & 9.59 & 9.77 & 9.78 & 10.17 \\
\hline 13.5 & 9.79 & 9.29 & 9.34 & 9.56 & 9.11 & 9.82 & 9.60 & 9.56 & 9.95 \\
\hline 14.5 & 9.56 & 9.48 & 9.49 & 9.53 & 9.07 & 9.52 & 9.45 & 9.72 & 9.72 \\
\hline 15.5 & 9.26 & 9.33 & 9.08 & 8.81 & 8.54 & 9.30 & 9.00 & 9.41 & 9.34 \\
\hline 16.5 & 9.14 & 8.66 & 8.88 & 8.79 & 8.27 & 8.79 & 8.81 & 9.00 & 9.03 \\
\hline 17.5 & 8.53 & 8.29 & 8.14 & 8.20 & 8.03 & 8.36 & 8.25 & 8.28 & 8.42 \\
\hline 18.5 & 8.02 & 8.15 & 7.90 & 8.03 & 7.40 & 7.69 & 7.85 & 7.94 & 8.18 \\
\hline 19.5 & 7.47 & 7.20 & 7.07 & 7.39 & 7.05 & 7.29 & 7.08 & 7.48 & 7.67 \\
\hline 20.5 & 6.88 & 6.84 & 6.39 & 6.76 & 6.35 & 6.84 & 6.67 & 6.88 & 6.98 \\
\hline 21.5 & 6.26 & 6.24 & 6.01 & 6.17 & 5.80 & 6.03 & 5.96 & 6.17 & 6.40 \\
\hline 22.5 & 5.96 & 5.78 & 5.68 & 5.55 & 5.35 & 5.61 & 5.74 & 5.54 & 6.07 \\
\hline 23.5 & 6.01 & 5.86 & 5.64 & 5.75 & 5.38 & 5.70 & 5.76 & 5.91 & 5.98 \\
\hline Ax. Avg. & 7.95 & 7.74 & 7.65 & 7.70 & 7.36 & 7.70 & 7.65 & 7.78 & 7.98 \\
\hline
\end{tabular}

POWER DENSITY VALUES NORMALIZED TO A CORE POWER OF 11.57 MW AND ASSUME ALL FISSION ENERGY (EXCLUDING GAMMA-HEATING OF EX-CORE COMPONENTS) IS DEPOSITED IN THE FUEL. 
Table 3-16. Power Density Data (kW/cm3) in Fuel Plate 1 in 173 MWd Burnup Element at Eq. Xe Conditions in Reference Mixed Burnup Core: Core 8A, Position X4, FT loaded with typical samples.

\section{Fuel core width 1.690 inches Fuel core thickness 9 mil}

\begin{tabular}{|c|c|c|c|c|c|c|c|c|c|}
\hline $\begin{array}{l}\text { Axial position } \\
\text { from top of } \\
\text { fuel (inches) }\end{array}$ & $\begin{array}{c}0.000 \\
\text { to } \\
0.197 \\
\text { inches }\end{array}$ & $\begin{array}{c}0.197 \\
\text { to } \\
0.394 \\
\text { inches }\end{array}$ & $\begin{array}{c}0.394 \\
\text { to } \\
0.591 \\
\text { inches }\end{array}$ & $\begin{array}{c}0.591 \\
\text { to } \\
0.760 \\
\text { inches }\end{array}$ & $\begin{array}{c}0.760 \\
\text { to } \\
0.930 \\
\text { inches }\end{array}$ & $\begin{array}{c}0.930 \\
\text { to } \\
1.099 \\
\text { inches }\end{array}$ & $\begin{array}{c}1.099 \\
\text { to } \\
1.296 \\
\text { inches }\end{array}$ & $\begin{array}{c}1.296 \\
\text { to } \\
1.493 \\
\text { inches }\end{array}$ & $\begin{array}{c}1.493 \\
\text { to } \\
1.690 \\
\text { inches }\end{array}$ \\
\hline 0.5 & 4.65 & 4.38 & 4.52 & 4.43 & 4.28 & 4.78 & 4.32 & 4.50 & 4.78 \\
\hline 1.5 & 4.71 & 4.67 & 4.67 & 4.43 & 4.15 & 4.52 & 4.62 & 4.74 & 4.77 \\
\hline 2.5 & 4.97 & 4.95 & 4.78 & 4.84 & 4.58 & 4.87 & 4.93 & 4.99 & 4.99 \\
\hline 3.5 & 5.84 & 5.39 & 5.58 & 5.61 & 5.18 & 5.50 & 5.60 & 5.91 & 5.97 \\
\hline 4.5 & 6.05 & 5.90 & 6.10 & 5.92 & 5.74 & 6.28 & 6.18 & 6.11 & 6.18 \\
\hline 5.5 & 6.86 & 6.71 & 6.62 & 6.70 & 6.13 & 6.64 & 6.66 & 6.54 & 6.76 \\
\hline 6.5 & 7.01 & 6.66 & 6.77 & 6.84 & 6.50 & 6.94 & 6.75 & 6.64 & 7.02 \\
\hline 7.5 & 7.20 & 6.95 & 6.97 & 7.29 & 6.66 & 7.02 & 6.83 & 7.11 & 7.16 \\
\hline 8.5 & 7.34 & 7.24 & 6.74 & 7.08 & 7.01 & 7.32 & 7.01 & 7.32 & 7.29 \\
\hline 9.5 & 7.81 & 7.71 & 7.61 & 7.45 & 7.10 & 7.54 & 7.37 & 7.69 & 7.65 \\
\hline 10.5 & 7.68 & 7.64 & 7.66 & 7.66 & 7.34 & 7.50 & 7.64 & 7.48 & 7.67 \\
\hline 11.5 & 7.97 & 7.69 & 8.05 & 7.82 & 7.30 & 8.11 & 7.59 & 7.86 & 8.11 \\
\hline 12.5 & 8.06 & 8.33 & 7.83 & 8.11 & 7.67 & 7.99 & 8.09 & 8.04 & 8.03 \\
\hline 13.5 & 8.18 & 7.86 & 7.89 & 7.89 & 7.62 & 7.94 & 8.12 & 8.19 & 8.09 \\
\hline 14.5 & 8.14 & 8.16 & 7.92 & 8.21 & 7.70 & 8.08 & 7.72 & 8.04 & 8.21 \\
\hline 15.5 & 7.51 & 7.62 & 7.25 & 7.54 & 7.25 & 7.75 & 7.39 & 7.51 & 7.61 \\
\hline 16.5 & 7.52 & 7.29 & 7.30 & 7.45 & 7.22 & 7.64 & 7.42 & 7.37 & 7.69 \\
\hline 17.5 & 6.75 & 6.74 & 6.78 & 6.93 & 6.46 & 6.90 & 6.91 & 6.94 & 7.26 \\
\hline 18.5 & 7.12 & 6.62 & 6.66 & 6.49 & 6.35 & 6.90 & 6.67 & 6.84 & 7.04 \\
\hline 19.5 & 6.26 & 6.16 & 5.94 & 5.96 & 5.84 & 6.28 & 6.37 & 6.49 & 6.37 \\
\hline 20.5 & 6.21 & 6.27 & 6.02 & 5.88 & 5.75 & 5.75 & 5.91 & 6.09 & 6.14 \\
\hline 21.5 & 5.42 & 5.43 & 5.48 & 5.32 & 5.16 & 5.29 & 5.40 & 5.43 & 5.67 \\
\hline 22.5 & 5.01 & 4.88 & 4.78 & 5.01 & 4.66 & 4.99 & 5.03 & 5.12 & 5.14 \\
\hline 23.5 & 5.38 & 5.31 & 5.39 & 5.12 & 5.16 & 5.11 & 5.16 & 5.15 & 5.39 \\
\hline Ax. Avg. & 6.65 & 6.52 & 6.48 & 6.50 & 6.20 & 6.57 & 6.49 & 6.59 & 6.70 \\
\hline
\end{tabular}

POWER DENSITY VALUES NORMALIZED TO A CORE POWER OF 11.57 MW AND ASSUME ALL FISSION ENERGY (EXCLUDING GAMMA-HEATING OF EX-CORE COMPONENTS) IS DEPOSITED IN THE FUEL. 
Plates 4-22 contain full-thickness fuel foils, with a fuel core thickness of 20 mil. Among all plates in the MURR fuel element, plate 22 has been found to have the maximum steady-state fuel temperature (see Section 3.3.4). Best estimate power density data for plate 22 were collected and are summarized in Tables 3-17 to 3-21. Just as for the data collected for plate 1, the data are representative of the power density profile in plate 22 over it's lifetime from beginning of life (BOL) to near discharge. Table 3-17 provides the power density data in a fresh plate 22 in Core 7A, which is a core configuration that is typical for weekly MURR operations under xenon-free conditions. Tables 3-18 to 3-21 provide the power density for this plate at other points in the lifetime of the fuel element under equilibrium xenon conditions. The burnup indicated in these tables is the average burnup for the entire element, not the plate burnup.

Best estimate power density data for all plates in a MURR fuel element under prototypic steady-state conditions are provided in Appendix B. The data are representative over the lifetime of these plates in the MURR "pseudo-equilibrium" fuel cycle from BOL to near discharge. 
Table 3-17. Power Density Data $\left(\mathrm{kW} / \mathrm{cm}^{3}\right)$ in Fuel Plate 22 in 0 MWd Burnup Element at No Xe Conditions in Reference Mixed Burnup Core: Core 7A, Position X1, FT loaded with typical samples.

\section{Fuel core width 3.938 inches Fuel core thickness 20 mil}

\begin{tabular}{|c|c|c|c|c|c|c|c|c|c|}
\hline $\begin{array}{l}\text { Axial position } \\
\text { from top of } \\
\text { fuel (inches) }\end{array}$ & $\begin{array}{c}0.000 \\
\text { to } \\
0.197 \\
\text { inches }\end{array}$ & $\begin{array}{c}0.197 \\
\text { to } \\
0.394 \\
\text { inches }\end{array}$ & $\begin{array}{c}0.394 \\
\text { to } \\
0.591 \\
\text { inches }\end{array}$ & $\begin{array}{c}0.591 \\
\text { to } \\
1.510 \\
\text { inches }\end{array}$ & $\begin{array}{c}1.510 \\
\text { to } \\
2.428 \\
\text { inches }\end{array}$ & $\begin{array}{c}2.428 \\
\text { to } \\
3.347 \\
\text { inches }\end{array}$ & $\begin{array}{c}3.347 \\
\text { to } \\
3.544 \\
\text { inches }\end{array}$ & $\begin{array}{c}3.544 \\
\text { to } \\
3.741 \\
\text { inches }\end{array}$ & $\begin{array}{c}3.741 \\
\text { to } \\
3.938 \\
\text { inches }\end{array}$ \\
\hline 0.5 & 0.78 & 0.75 & 0.66 & 0.75 & 0.90 & 0.78 & 0.66 & 0.73 & 0.81 \\
\hline 1.5 & 0.77 & 0.65 & 0.68 & 0.75 & 0.89 & 0.74 & 0.72 & 0.70 & 0.75 \\
\hline 2.5 & 0.89 & 0.91 & 0.86 & 0.94 & 1.03 & 0.89 & 0.80 & 0.78 & 0.89 \\
\hline 3.5 & 1.09 & 1.02 & 1.01 & 1.06 & 1.20 & 1.05 & 0.95 & 0.96 & 1.04 \\
\hline 4.5 & 1.29 & 1.11 & 1.16 & 1.24 & 1.41 & 1.23 & 1.18 & 1.08 & 1.24 \\
\hline 5.5 & 1.42 & 1.39 & 1.37 & 1.42 & 1.72 & 1.44 & 1.36 & 1.36 & 1.46 \\
\hline 6.5 & 1.71 & 1.56 & 1.55 & 1.79 & 2.03 & 1.75 & 1.66 & 1.67 & 1.91 \\
\hline 7.5 & 2.27 & 2.14 & 2.13 & 2.26 & 2.56 & 2.23 & 2.14 & 2.15 & 2.25 \\
\hline 8.5 & 3.83 & 3.44 & 3.47 & 3.28 & 3.21 & 3.23 & 3.24 & 3.38 & 3.86 \\
\hline 9.5 & 5.35 & 4.70 & 4.31 & 4.17 & 3.93 & 4.17 & 4.48 & 4.56 & 5.21 \\
\hline 10.5 & 6.02 & 5.17 & 5.03 & 4.70 & 4.47 & 4.81 & 5.00 & 5.19 & 5.84 \\
\hline 11.5 & 6.64 & 5.64 & 5.48 & 5.08 & 4.92 & 5.14 & 5.64 & 5.73 & 6.58 \\
\hline 12.5 & 6.73 & 6.02 & 5.74 & 5.51 & 5.08 & 5.45 & 5.74 & 5.98 & 6.74 \\
\hline 13.5 & 7.32 & 6.29 & 6.18 & 5.66 & 5.39 & 5.59 & 5.83 & 5.97 & 7.13 \\
\hline 14.5 & 7.32 & 6.44 & 6.00 & 5.76 & 5.48 & 5.67 & 6.03 & 6.38 & 7.40 \\
\hline 15.5 & 7.23 & 6.23 & 5.87 & 5.73 & 5.49 & 5.75 & 6.09 & 5.95 & 7.18 \\
\hline 16.5 & 7.23 & 6.21 & 6.00 & 5.73 & 5.39 & 5.55 & 5.68 & 6.17 & 7.02 \\
\hline 17.5 & 7.05 & 6.06 & 5.80 & 5.46 & 5.25 & 5.56 & 5.59 & 6.01 & 6.83 \\
\hline 18.5 & 6.59 & 5.78 & 5.58 & 5.24 & 4.94 & 5.22 & 5.36 & 5.86 & 6.51 \\
\hline 19.5 & 6.13 & 5.28 & 5.22 & 4.94 & 4.79 & 4.95 & 5.33 & 5.27 & 6.28 \\
\hline 20.5 & 5.83 & 4.95 & 4.90 & 4.51 & 4.48 & 4.58 & 4.71 & 4.95 & 5.81 \\
\hline 21.5 & 5.27 & 4.50 & 4.29 & 4.20 & 3.98 & 4.14 & 4.22 & 4.39 & 5.07 \\
\hline 22.5 & 4.63 & 3.99 & 4.00 & 3.72 & 3.57 & 3.65 & 3.92 & 4.06 & 4.69 \\
\hline 23.5 & 5.54 & 4.52 & 4.30 & 4.01 & 3.92 & 3.94 & 4.12 & 4.36 & 4.95 \\
\hline Ax. Avg. & 4.54 & 3.95 & 3.82 & 3.66 & 3.58 & 3.65 & 3.77 & 3.90 & 4.48 \\
\hline
\end{tabular}

POWER DENSITY VALUES NORMALIZED TO A CORE POWER OF 11.57 MW AND ASSUME ALL FISSION ENERGY (EXCLUDING GAMMA-HEATING OF EX-CORE COMPONENTS) IS DEPOSITED IN THE FUEL. 
Table 3-18. Power Density Data $\left(\mathrm{kW} / \mathrm{cm}^{3}\right)$ in Fuel Plate 22 in $3 \mathrm{MWd}$ Burnup Element at Eq. Xe Conditions in Reference Mixed Burnup Core: Core 8A, Positions X1, FT loaded with typical samples.

\section{Fuel core width 3.938 inches Fuel core thickness 20 mil}

\begin{tabular}{|c|c|c|c|c|c|c|c|c|c|}
\hline $\begin{array}{l}\text { Axial position } \\
\text { from top of } \\
\text { fuel (inches) }\end{array}$ & $\begin{array}{c}0.000 \\
\text { to } \\
0.197 \\
\text { inches }\end{array}$ & $\begin{array}{c}0.197 \\
\text { to } \\
0.394 \\
\text { inches }\end{array}$ & $\begin{array}{c}0.394 \\
\text { to } \\
0.591 \\
\text { inches }\end{array}$ & $\begin{array}{c}0.591 \\
\text { to } \\
1.510 \\
\text { inches }\end{array}$ & $\begin{array}{c}1.510 \\
\text { to } \\
2.428 \\
\text { inches }\end{array}$ & $\begin{array}{c}2.428 \\
\text { to } \\
3.347 \\
\text { inches }\end{array}$ & $\begin{array}{c}3.347 \\
\text { to } \\
3.544 \\
\text { inches }\end{array}$ & $\begin{array}{c}3.544 \\
\text { to } \\
3.741 \\
\text { inches }\end{array}$ & $\begin{array}{c}3.741 \\
\text { to } \\
3.938 \\
\text { inches }\end{array}$ \\
\hline 0.5 & 1.66 & 1.40 & 1.34 & 1.42 & 1.68 & 1.41 & 1.22 & 1.39 & 1.55 \\
\hline 1.5 & 2.08 & 1.79 & 1.74 & 1.77 & 1.88 & 1.79 & 1.64 & 1.74 & 2.13 \\
\hline 2.5 & 3.12 & 2.82 & 2.58 & 2.51 & 2.51 & 2.53 & 2.65 & 2.73 & 3.07 \\
\hline 3.5 & 3.93 & 3.33 & 3.15 & 3.06 & 2.98 & 3.12 & 3.15 & 3.42 & 3.78 \\
\hline 4.5 & 4.42 & 3.87 & 3.75 & 3.54 & 3.39 & 3.49 & 3.60 & 3.89 & 4.27 \\
\hline 5.5 & 5.09 & 4.19 & 4.01 & 3.92 & 3.75 & 3.89 & 4.15 & 4.20 & 4.77 \\
\hline 6.5 & 5.62 & 4.67 & 4.38 & 4.21 & 4.08 & 4.19 & 4.49 & 4.54 & 5.53 \\
\hline 7.5 & 5.89 & 4.90 & 4.73 & 4.56 & 4.32 & 4.53 & 4.73 & 5.01 & 5.71 \\
\hline 8.5 & 6.06 & 5.32 & 5.04 & 4.71 & 4.51 & 4.80 & 4.84 & 5.23 & 5.95 \\
\hline 9.5 & 6.20 & 5.55 & 5.12 & 4.89 & 4.67 & 4.98 & 5.20 & 5.26 & 6.10 \\
\hline 10.5 & 6.32 & 5.60 & 5.48 & 4.98 & 4.77 & 5.08 & 5.23 & 5.73 & 6.33 \\
\hline 11.5 & 6.65 & 5.76 & 5.42 & 5.10 & 4.84 & 5.03 & 5.30 & 5.59 & 6.39 \\
\hline 12.5 & 6.36 & 5.51 & 5.41 & 5.04 & 4.79 & 5.05 & 5.15 & 5.62 & 6.35 \\
\hline 13.5 & 6.42 & 5.52 & 5.36 & 5.11 & 4.90 & 5.11 & 5.17 & 5.44 & 6.36 \\
\hline 14.5 & 6.21 & 5.36 & 5.32 & 4.95 & 4.74 & 4.91 & 5.19 & 5.37 & 6.17 \\
\hline 15.5 & 6.19 & 5.23 & 4.89 & 4.83 & 4.64 & 4.78 & 4.87 & 5.35 & 5.88 \\
\hline 16.5 & 6.03 & 5.13 & 4.93 & 4.66 & 4.45 & 4.55 & 4.76 & 5.06 & 5.82 \\
\hline 17.5 & 5.44 & 5.01 & 4.66 & 4.45 & 4.21 & 4.37 & 4.53 & 4.76 & 5.38 \\
\hline 18.5 & 5.34 & 4.60 & 4.32 & 4.20 & 3.93 & 4.12 & 4.33 & 4.56 & 5.31 \\
\hline 19.5 & 4.93 & 4.30 & 4.08 & 3.94 & 3.78 & 3.89 & 4.02 & 4.32 & 4.92 \\
\hline 20.5 & 4.43 & 3.77 & 3.59 & 3.51 & 3.37 & 3.47 & 3.71 & 4.05 & 4.30 \\
\hline 21.5 & 4.13 & 3.50 & 3.39 & 3.21 & 3.03 & 3.16 & 3.22 & 3.55 & 3.97 \\
\hline 22.5 & 3.63 & 3.13 & 2.99 & 2.87 & 2.70 & 2.72 & 2.97 & 3.08 & 3.66 \\
\hline 23.5 & 3.89 & 3.35 & 3.24 & 3.02 & 2.87 & 3.07 & 3.28 & 3.44 & 3.91 \\
\hline Ax. Avg. & 5.00 & 4.32 & 4.12 & 3.94 & 3.78 & 3.92 & 4.06 & 4.31 & 4.90 \\
\hline
\end{tabular}

POWER DENSITY VALUES NORMALIZED TO A CORE POWER OF 11.57 MW AND ASSUME ALL FISSION ENERGY (EXCLUDING GAMMA-HEATING OF EX-CORE COMPONENTS) IS DEPOSITED IN THE FUEL. 
Table 3-19. Power Density Data $\left(\mathrm{kW} / \mathrm{cm}^{3}\right)$ in Fuel Plate 22 in 80 MWd Burnup Element at Eq. Xe Conditions in Reference Mixed Burnup Core: Core 8A, Positions X7, FT loaded with typical samples.

\section{Fuel core width 3.938 inches Fuel core thickness 20 mil}

\begin{tabular}{|c|c|c|c|c|c|c|c|c|c|}
\hline $\begin{array}{l}\text { Axial position } \\
\text { from top of } \\
\text { fuel (inches) }\end{array}$ & $\begin{array}{c}0.000 \\
\text { to } \\
0.197 \\
\text { inches }\end{array}$ & $\begin{array}{c}0.197 \\
\text { to } \\
0.394 \\
\text { inches }\end{array}$ & $\begin{array}{c}0.394 \\
\text { to } \\
0.591 \\
\text { inches }\end{array}$ & $\begin{array}{c}0.591 \\
\text { to } \\
1.510 \\
\text { inches }\end{array}$ & $\begin{array}{c}1.510 \\
\text { to } \\
2.428 \\
\text { inches }\end{array}$ & $\begin{array}{c}2.428 \\
\text { to } \\
3.347 \\
\text { inches }\end{array}$ & $\begin{array}{c}3.347 \\
\text { to } \\
3.544 \\
\text { inches }\end{array}$ & $\begin{array}{c}3.544 \\
\text { to } \\
3.741 \\
\text { inches }\end{array}$ & $\begin{array}{c}3.741 \\
\text { to } \\
3.938 \\
\text { inches }\end{array}$ \\
\hline 0.5 & 1.39 & 1.34 & 1.21 & 1.28 & 1.47 & 1.65 & 1.49 & 1.46 & 1.56 \\
\hline 1.5 & 1.97 & 1.72 & 1.85 & 1.72 & 1.80 & 1.95 & 1.83 & 1.81 & 2.11 \\
\hline 2.5 & 3.02 & 2.69 & 2.66 & 2.54 & 2.48 & 2.58 & 2.61 & 2.70 & 3.13 \\
\hline 3.5 & 3.81 & 3.48 & 3.23 & 3.16 & 3.10 & 3.13 & 3.16 & 3.36 & 3.77 \\
\hline 4.5 & 4.31 & 3.94 & 3.53 & 3.56 & 3.41 & 3.55 & 3.39 & 3.76 & 4.20 \\
\hline 5.5 & 4.90 & 4.36 & 4.23 & 4.04 & 3.82 & 3.87 & 4.03 & 4.18 & 4.77 \\
\hline 6.5 & 5.36 & 4.61 & 4.21 & 4.31 & 4.03 & 4.12 & 4.28 & 4.50 & 5.22 \\
\hline 7.5 & 5.65 & 5.05 & 4.66 & 4.55 & 4.29 & 4.39 & 4.46 & 4.59 & 5.59 \\
\hline 8.5 & 5.71 & 5.08 & 4.97 & 4.74 & 4.54 & 4.68 & 4.71 & 5.04 & 5.71 \\
\hline 9.5 & 5.98 & 5.17 & 5.10 & 4.93 & 4.68 & 4.86 & 4.88 & 5.18 & 5.93 \\
\hline 10.5 & 6.12 & 5.63 & 5.21 & 5.06 & 4.82 & 4.83 & 4.91 & 5.24 & 5.90 \\
\hline 11.5 & 6.25 & 5.59 & 5.44 & 5.16 & 4.85 & 4.86 & 5.00 & 5.19 & 6.08 \\
\hline 12.5 & 6.15 & 5.37 & 5.39 & 5.10 & 4.76 & 4.77 & 5.14 & 5.29 & 5.93 \\
\hline 13.5 & 6.13 & 5.68 & 5.18 & 5.02 & 4.66 & 4.74 & 5.04 & 5.16 & 6.09 \\
\hline 14.5 & 6.13 & 5.26 & 5.18 & 4.89 & 4.56 & 4.67 & 4.91 & 5.19 & 5.97 \\
\hline 15.5 & 5.98 & 5.19 & 4.72 & 4.70 & 4.40 & 4.39 & 4.60 & 4.87 & 5.60 \\
\hline 16.5 & 5.64 & 4.88 & 4.55 & 4.53 & 4.21 & 4.30 & 4.56 & 4.74 & 5.24 \\
\hline 17.5 & 5.30 & 4.70 & 4.46 & 4.21 & 3.88 & 4.05 & 4.21 & 4.36 & 5.21 \\
\hline 18.5 & 5.11 & 4.28 & 4.15 & 3.99 & 3.75 & 3.79 & 4.02 & 4.29 & 4.81 \\
\hline 19.5 & 4.43 & 4.16 & 3.91 & 3.66 & 3.47 & 3.54 & 3.71 & 3.96 & 4.59 \\
\hline 20.5 & 4.13 & 3.60 & 3.67 & 3.44 & 3.17 & 3.21 & 3.41 & 3.62 & 4.14 \\
\hline 21.5 & 3.56 & 3.30 & 3.07 & 2.99 & 2.85 & 2.98 & 3.02 & 3.23 & 3.72 \\
\hline 22.5 & 3.28 & 2.98 & 2.94 & 2.64 & 2.58 & 2.63 & 2.78 & 2.83 & 3.46 \\
\hline 23.5 & 3.68 & 3.11 & 3.05 & 2.93 & 2.82 & 2.88 & 2.99 & 3.12 & 3.59 \\
\hline Ax. Avg. & 4.75 & 4.22 & 4.02 & 3.88 & 3.68 & 3.77 & 3.88 & 4.07 & 4.68 \\
\hline
\end{tabular}

POWER DENSITY VALUES NORMALIZED TO A CORE POWER OF 11.57 MW AND ASSUME ALL FISSION ENERGY (EXCLUDING GAMMA-HEATING OF EX-CORE COMPONENTS) IS DEPOSITED IN THE FUEL. 
Table 3-20. Power Density Data $\left(\mathrm{kW} / \mathrm{cm}^{3}\right)$ in Fuel Plate 22 in 99 MWd Burnup Element at Eq. Xe Conditions in Reference Mixed Burnup Core: Core 8A, Positions X6, FT loaded with typical samples.

\section{Fuel core width 3.938 inches Fuel core thickness 20 mil}

\begin{tabular}{|c|c|c|c|c|c|c|c|c|c|}
\hline $\begin{array}{l}\text { Axial position } \\
\text { from top of } \\
\text { fuel (inches) }\end{array}$ & $\begin{array}{c}0.000 \\
\text { to } \\
0.197 \\
\text { inches }\end{array}$ & $\begin{array}{c}0.197 \\
\text { to } \\
0.394 \\
\text { inches }\end{array}$ & $\begin{array}{c}0.394 \\
\text { to } \\
0.591 \\
\text { inches }\end{array}$ & $\begin{array}{c}0.591 \\
\text { to } \\
1.510 \\
\text { inches }\end{array}$ & $\begin{array}{c}1.510 \\
\text { to } \\
2.428 \\
\text { inches }\end{array}$ & $\begin{array}{c}2.428 \\
\text { to } \\
3.347 \\
\text { inches }\end{array}$ & $\begin{array}{c}3.347 \\
\text { to } \\
3.544 \\
\text { inches }\end{array}$ & $\begin{array}{c}3.544 \\
\text { to } \\
3.741 \\
\text { inches }\end{array}$ & $\begin{array}{c}3.741 \\
\text { to } \\
3.938 \\
\text { inches }\end{array}$ \\
\hline 0.5 & 1.57 & 1.35 & 1.24 & 1.18 & 1.12 & 1.17 & 1.29 & 1.27 & 1.49 \\
\hline 1.5 & 1.96 & 1.89 & 1.80 & 1.61 & 1.55 & 1.62 & 1.61 & 1.74 & 1.95 \\
\hline 2.5 & 3.07 & 2.44 & 2.55 & 2.44 & 2.38 & 2.48 & 2.60 & 2.67 & 3.07 \\
\hline 3.5 & 3.53 & 3.11 & 3.09 & 3.01 & 3.00 & 3.12 & 3.26 & 3.37 & 3.75 \\
\hline 4.5 & 4.03 & 3.71 & 3.36 & 3.47 & 3.43 & 3.54 & 3.63 & 3.81 & 4.20 \\
\hline 5.5 & 4.38 & 3.90 & 3.89 & 3.80 & 3.75 & 3.88 & 4.02 & 4.39 & 4.99 \\
\hline 6.5 & 4.80 & 4.37 & 4.28 & 4.08 & 4.18 & 4.26 & 4.40 & 4.57 & 5.32 \\
\hline 7.5 & 5.01 & 4.56 & 4.39 & 4.44 & 4.42 & 4.48 & 4.61 & 4.91 & 5.51 \\
\hline 8.5 & 5.33 & 4.88 & 4.65 & 4.65 & 4.52 & 4.84 & 4.94 & 5.00 & 5.76 \\
\hline 9.5 & 5.31 & 4.90 & 4.89 & 4.79 & 4.81 & 4.99 & 4.95 & 5.33 & 5.97 \\
\hline 10.5 & 5.35 & 4.92 & 4.78 & 4.83 & 4.81 & 4.94 & 5.32 & 5.15 & 5.88 \\
\hline 11.5 & 5.73 & 5.16 & 4.99 & 4.99 & 4.82 & 5.07 & 5.11 & 5.47 & 6.15 \\
\hline 12.5 & 5.69 & 5.14 & 4.99 & 4.96 & 4.93 & 5.10 & 5.41 & 5.32 & 6.17 \\
\hline 13.5 & 5.79 & 5.11 & 4.86 & 4.88 & 4.89 & 4.96 & 5.15 & 5.43 & 6.09 \\
\hline 14.5 & 5.61 & 5.05 & 4.82 & 4.91 & 4.80 & 4.96 & 5.19 & 5.44 & 6.21 \\
\hline 15.5 & 5.49 & 5.07 & 4.56 & 4.77 & 4.57 & 4.79 & 4.98 & 5.06 & 5.86 \\
\hline 16.5 & 5.18 & 4.82 & 4.60 & 4.52 & 4.53 & 4.67 & 4.69 & 5.00 & 5.70 \\
\hline 17.5 & 5.06 & 4.50 & 4.44 & 4.33 & 4.27 & 4.30 & 4.39 & 4.51 & 5.24 \\
\hline 18.5 & 4.75 & 4.19 & 4.13 & 4.07 & 4.00 & 4.13 & 4.07 & 4.25 & 4.84 \\
\hline 19.5 & 4.33 & 3.94 & 3.70 & 3.74 & 3.77 & 3.69 & 3.79 & 3.94 & 4.54 \\
\hline 20.5 & 4.05 & 3.59 & 3.47 & 3.43 & 3.31 & 3.42 & 3.37 & 3.70 & 4.05 \\
\hline 21.5 & 3.55 & 3.09 & 3.07 & 3.01 & 2.94 & 3.07 & 3.05 & 3.35 & 3.65 \\
\hline 22.5 & 3.18 & 2.82 & 2.75 & 2.71 & 2.63 & 2.73 & 2.71 & 2.84 & 3.48 \\
\hline 23.5 & 3.57 & 2.98 & 2.87 & 2.87 & 2.81 & 2.86 & 2.87 & 3.07 & 3.64 \\
\hline Ax. Avg. & 4.43 & 3.98 & 3.84 & 3.81 & 3.76 & 3.88 & 3.97 & 4.15 & 4.73 \\
\hline
\end{tabular}

POWER DENSITY VALUES NORMALIZED TO A CORE POWER OF 11.57 MW AND ASSUME ALL FISSION ENERGY (EXCLUDING GAMMA-HEATING OF EX-CORE COMPONENTS) IS DEPOSITED IN THE FUEL. 
Table 3-21. Power Density Data $\left(\mathrm{kW} / \mathrm{cm}^{3}\right)$ in Fuel Plate 22 in $173 \mathrm{MWd}$ Burnup Element at Eq. Xe Conditions in Reference Mixed Burnup Core: Core 8A, Positions X8, FT loaded with typical samples.

\section{Fuel core width 3.938 inches $\quad$ Fuel core thickness 20 mil}

\begin{tabular}{|c|c|c|c|c|c|c|c|c|c|}
\hline $\begin{array}{c}\text { Axial position } \\
\text { from top of } \\
\text { fuel (inches) }\end{array}$ & $\begin{array}{c}0.000 \\
\text { to } \\
0.197 \\
\text { inches }\end{array}$ & $\begin{array}{c}0.197 \\
\text { to } \\
\text { inches }\end{array}$ & $\begin{array}{c}0.394 \\
\text { to } \\
0.591 \\
\text { inches }\end{array}$ & $\begin{array}{c}0.591 \\
\text { to } \\
1.510 \\
\text { inches }\end{array}$ & $\begin{array}{c}1.510 \\
\text { to } \\
2.428 \\
\text { inches }\end{array}$ & $\begin{array}{c}2.428 \\
\text { to } \\
3.347 \\
\text { inches }\end{array}$ & $\begin{array}{c}3.347 \\
\text { to } \\
3.544 \\
\text { inches }\end{array}$ & $\begin{array}{c}3.544 \\
\text { to } \\
3.741 \\
\text { inches }\end{array}$ & $\begin{array}{c}3.741 \\
\text { to } \\
\text { inches }\end{array}$ \\
\hline 0.5 & 1.51 & 1.32 & 1.25 & 1.19 & 1.13 & 1.17 & 1.24 & 1.30 & 1.41 \\
\hline 1.5 & 2.05 & 1.70 & 1.78 & 1.71 & 1.61 & 1.60 & 1.70 & 1.70 & 2.04 \\
\hline 2.5 & 2.99 & 2.67 & 2.57 & 2.53 & 2.44 & 2.55 & 2.63 & 2.81 & 3.06 \\
\hline 3.5 & 3.80 & 3.30 & 3.20 & 3.12 & 3.07 & 3.13 & 3.17 & 3.37 & 3.76 \\
\hline 4.5 & 4.13 & 3.67 & 3.65 & 3.47 & 3.40 & 3.49 & 3.59 & 3.69 & 4.27 \\
\hline 5.5 & 4.73 & 4.10 & 3.96 & 3.92 & 3.86 & 3.92 & 3.91 & 4.22 & 4.67 \\
\hline 6.5 & 4.80 & 4.39 & 4.12 & 4.18 & 4.03 & 4.14 & 4.36 & 4.44 & 4.87 \\
\hline 7.5 & 5.38 & 4.68 & 4.52 & 4.35 & 4.33 & 4.45 & 4.38 & 4.69 & 5.18 \\
\hline 8.5 & 5.35 & 4.79 & 4.59 & 4.50 & 4.52 & 4.50 & 4.78 & 5.10 & 5.51 \\
\hline 9.5 & 5.59 & 4.95 & 4.86 & 4.68 & 4.71 & 4.75 & 4.87 & 5.10 & 5.81 \\
\hline 10.5 & 5.66 & 5.11 & 4.89 & 4.87 & 4.70 & 4.84 & 4.72 & 5.03 & 5.61 \\
\hline 11.5 & 5.50 & 5.06 & 4.81 & 4.81 & 4.72 & 4.93 & 5.00 & 5.14 & 5.83 \\
\hline 12.5 & 5.63 & 4.90 & 4.81 & 4.80 & 4.74 & 4.85 & 5.02 & 5.06 & 5.65 \\
\hline 13.5 & 5.43 & 4.92 & 4.65 & 4.69 & 4.64 & 4.83 & 4.91 & 5.08 & 5.71 \\
\hline 14.5 & 5.32 & 4.71 & 4.56 & 4.60 & 4.49 & 4.83 & 4.99 & 5.17 & 5.68 \\
\hline 15.5 & 5.28 & 4.66 & 4.46 & 4.60 & 4.47 & 4.62 & 4.72 & 4.90 & 5.39 \\
\hline 16.5 & 5.13 & 4.46 & 4.46 & 4.41 & 4.31 & 4.54 & 4.46 & 4.74 & 5.32 \\
\hline 17.5 & 5.00 & 4.40 & 4.24 & 4.12 & 4.15 & 4.29 & 4.47 & 4.48 & 5.06 \\
\hline 18.5 & 4.63 & 4.23 & 4.09 & 4.02 & 3.98 & 3.94 & 3.96 & 4.36 & 4.84 \\
\hline 19.5 & 4.39 & 3.94 & 3.77 & 3.63 & 3.62 & 3.66 & 3.79 & 3.94 & 4.41 \\
\hline 20.5 & 3.76 & 3.70 & 3.44 & 3.41 & 3.33 & 3.46 & 3.40 & 3.54 & 3.98 \\
\hline 21.5 & 3.57 & 3.22 & 3.12 & 2.99 & 3.00 & 3.04 & 3.23 & 3.24 & 3.49 \\
\hline 22.5 & 3.26 & 2.93 & 2.67 & 2.67 & 2.68 & 2.79 & 2.80 & 2.86 & 3.32 \\
\hline 23.5 & 3.65 & 3.18 & 3.04 & 2.90 & 2.79 & 2.97 & 3.02 & 3.04 & 3.56 \\
\hline Ax. Avg. & 4.44 & 3.96 & 3.81 & 3.76 & 3.70 & 3.80 & 3.88 & 4.04 & 4.52 \\
\hline & & & & & & & & & \\
\hline
\end{tabular}

POWER DENSITY VALUES NORMALIZED TO A CORE POWER OF 11.57 MW AND ASSUME ALL FISSION ENERGY (EXCLUDING GAMMA-HEATING OF EX-CORE COMPONENTS) IS DEPOSITED IN THE FUEL. 


\subsubsection{Heat Flux}

The local plate surface heat flux is not a design parameter for irradiation experiments because it can be derived from the specified fuel plate geometry and power density, both of which are design parameters. Nonetheless, heat flux is often reported in the safety basis analysis, and so is provided in this report to aid in checking the MURR DDE experiment design.

Although the local plate surface heat flux is affected by coolant flow conditions on each side of the fuel plate, a simple estimate of the heat flux can be calculated by assuming that all heat deposited in the fuel core from fission, neutron capture, and gamma heating is conducted evenly from the two radial surfaces of the fuel plate. The estimate can be further simplified by neglecting lateral heat conduction that will be higher near the edge of the fuel core (heat conducting along the cladding towards the side plate). Thus, the heat flux can be simply estimated by multiplying the power density by the fuel core thickness and dividing by two. The local heat flux calculated in this way is not a target test value.

The heat flux profile data provided in Tables 3-22 to 3-25, and the corresponding heat flux contour plots in Figures 3-6 and 3-7, are for illustrative purposes to show the evolution of the heat flux profile in plate 22 of a MURR LEU fuel element over its anticipated lifetime. Plate 22 was selected for illustration because it is the plate with the maximum fuel temperature in a prototypic core (see Section 3.3.4). The heat flux data were extracted from the MCNP calculation for Core 7A for elements at four different burnup state points. This core has a mixture of fuel elements at burnups that are expected to be typical for the MURR prototypic "pseudo-equilibrium" operating cycle, as well as experiments in the flux trap and irradiation materials in the graphite reflector that are typical for MURR operations. It should be noted that the burnup indicated in these tables and figures is the average burnup for the entire element, not the plate burnup. It should also be noted that the data presented in these tables are for a core under xenon-free conditions.

The data show that the maximum local heat flux in plate 22 decreases by about $10 \%$ over its lifetime. It is observed that maximum local heat flux increases about 3\% from an element burnup of 77 to 96 MWd (compare Tables 3-22 and 3-25). This is because the element is repositioned in the neutronics analysis from core position 7 to core position 6, which is typical for the MURR fuel management. As can be inferred from the control blade positions indicated in Figure 2-1, the axial power profile of a fuel element in core position 6 is more strongly influenced by the axial positioning of the control blades, resulting in a larger axial power peaking factor. Once the element is repositioned to core position 8 near the end of its life, the maximum local heat flux reduces significantly. 
Table 3-22. Heat Flux Data $\left(\mathrm{W} / \mathrm{cm}^{2}\right)$ for Fuel Plate 22 in 0 MWd Burnup Element in Reference Mixed Burnup Core: Core 7A, Position X1, No Xe, FT loaded with typical samples.

\section{Fuel core width 3.938 inches Fuel core thickness 20 mil}

\begin{tabular}{|c|c|c|c|c|c|c|c|c|c|}
\hline $\begin{array}{l}\text { Axial position } \\
\text { from top of } \\
\text { fuel (inches) }\end{array}$ & $\begin{array}{c}0.000 \\
\text { to } \\
0.197 \\
\text { inches }\end{array}$ & $\begin{array}{c}0.197 \\
\text { to } \\
0.394 \\
\text { inches }\end{array}$ & $\begin{array}{c}0.394 \\
\text { to } \\
0.591 \\
\text { inches }\end{array}$ & $\begin{array}{c}0.591 \\
\text { to } \\
1.510 \\
\text { inches }\end{array}$ & $\begin{array}{c}1.510 \\
\text { to } \\
2.428 \\
\text { inches }\end{array}$ & $\begin{array}{c}2.428 \\
\text { to } \\
3.347 \\
\text { inches }\end{array}$ & $\begin{array}{c}3.347 \\
\text { to } \\
3.544 \\
\text { inches }\end{array}$ & $\begin{array}{c}3.544 \\
\text { to } \\
3.741 \\
\text { inches }\end{array}$ & $\begin{array}{c}3.741 \\
\text { to } \\
3.938 \\
\text { inches }\end{array}$ \\
\hline 0.5 & 19.72 & 19.06 & 16.66 & 18.98 & 22.92 & 19.92 & 16.64 & 18.46 & 20.66 \\
\hline 1.5 & 19.47 & 16.61 & 17.31 & 19.04 & 22.51 & 18.77 & 18.17 & 17.70 & 19.08 \\
\hline 2.5 & 22.59 & 23.08 & 21.86 & 23.85 & 26.27 & 22.49 & 20.29 & 19.80 & 22.66 \\
\hline 3.5 & 27.68 & 25.88 & 25.66 & 27.00 & 30.50 & 26.70 & 24.10 & 24.43 & 26.31 \\
\hline 4.5 & 32.66 & 28.15 & 29.50 & 31.42 & 35.93 & 31.35 & 30.07 & 27.54 & 31.48 \\
\hline 5.5 & 35.98 & 35.22 & 34.88 & 36.13 & 43.64 & 36.62 & 34.47 & 34.47 & 37.00 \\
\hline 6.5 & 43.51 & 39.75 & 39.47 & 45.48 & 51.57 & 44.56 & 42.18 & 42.54 & 48.64 \\
\hline 7 & 57.57 & 54.36 & 54.01 & 57.40 & 65.05 & 56.64 & 54.36 & 54.71 & 57.22 \\
\hline 8.5 & 97.22 & 87.50 & 88.09 & 83.27 & 81.58 & 82.01 & 82.17 & 85.81 & 97.98 \\
\hline 9.5 & 136.01 & 119.44 & 109.52 & 105.82 & 99.72 & 105.82 & 113.67 & 115.74 & 132.30 \\
\hline 10.5 & 152.82 & 131.40 & 127.68 & 119.51 & 113.56 & 122.11 & 127.06 & 131.89 & 148.24 \\
\hline 11.5 & 168.72 & 143.32 & 139.27 & 129.00 & 124.95 & 130.49 & 143.19 & 145.48 & 167.10 \\
\hline 12.5 & 170.84 & 152.80 & 145.70 & 140.02 & 128.94 & 138.46 & 145.70 & 151.95 & 171.12 \\
\hline 13.5 & 185.99 & 159.82 & 156.87 & 143.86 & 136.91 & 142.08 & 148.14 & 151.54 & 181.11 \\
\hline 14.5 & 186.05 & 163.64 & 152.36 & 146.19 & 139.12 & 143.94 & 153.11 & 161.99 & 187.86 \\
\hline 15.5 & 183.61 & 158.19 & 149.22 & 145.48 & 139.50 & 145.93 & 154.60 & 151.16 & 182.26 \\
\hline 16.5 & 183.64 & 157.83 & 152.37 & 145.44 & 137.03 & 141.02 & 144.26 & 156.65 & 178.33 \\
\hline 17.5 & 179.19 & 153.86 & 147.38 & 138.75 & 133.42 & 141.34 & 142.06 & 152.71 & 173.58 \\
\hline 18.5 & 167.47 & 146.70 & 141.79 & 133.18 & 125.53 & 132.50 & 136.19 & 148.89 & 165.42 \\
\hline 19.5 & 155.62 & 134.11 & 132.68 & 125.43 & 121.67 & 125.82 & 135.27 & 133.85 & 159.50 \\
\hline 20.5 & 147.96 & 125.64 & 124.44 & 114.48 & 113.88 & 116.40 & 119.52 & 125.64 & 147.48 \\
\hline 21.5 & 133.86 & 114.38 & 108.86 & 106.59 & 101.07 & 105.07 & 107.13 & 111.46 & 128.88 \\
\hline 22.5 & 117.60 & 101.31 & 101.50 & 94.43 & 90.65 & 92.78 & 99.57 & 103.06 & 119.15 \\
\hline 23.5 & 140.62 & 114.84 & 109.24 & 101.85 & 99.63 & 100.05 & 104.60 & 110.83 & 125.83 \\
\hline Ax. Avg. & 115.22 & 100.31 & 96.96 & 93.04 & 91.03 & 92.66 & 95.72 & 99.06 & 113.69 \\
\hline
\end{tabular}

HEAT FLUX VALUES NORMALIZED TO A CORE POWER OF 11.57 MW AND ASSUME ALL FISSION ENERGY (EXCLUDING GAMMA-HEATING OF EX-CORE COMPONENTS) IS DEPOSITED IN THE FUEL. HEAT FLUX VALUES ALSO ASSUME THAT THE HEAT DEPOSITED IN THE FUEL CONDUCTS OUT EVENLY FROM THE TWO SURFACES OF THE FUEL PLATE WITHOUT ANY LATERAL OR AXIAL CONDUCTION IN THE CLADDING. 
Table 3-23. Heat Flux Data (W/ $\left.\mathrm{cm}^{2}\right)$ for Fuel Plate 22 in 77 MWd Burnup Element in Reference Mixed Burnup Core: Core 7A, Position X7, No Xe, FT loaded with typical samples.

\section{Fuel core width 3.938 inches Fuel core thickness 20 mil}

\begin{tabular}{|c|c|c|c|c|c|c|c|c|c|}
\hline $\begin{array}{l}\text { Axial position } \\
\text { from top of } \\
\text { fuel (inches) }\end{array}$ & $\begin{array}{c}0.000 \\
\text { to } \\
0.197 \\
\text { inches }\end{array}$ & $\begin{array}{c}0.197 \\
\text { to } \\
0.394 \\
\text { inches }\end{array}$ & $\begin{array}{c}0.394 \\
\text { to } \\
0.591 \\
\text { inches }\end{array}$ & $\begin{array}{c}0.591 \\
\text { to } \\
1.510 \\
\text { inches }\end{array}$ & $\begin{array}{c}1.510 \\
\text { to } \\
2.428 \\
\text { inches }\end{array}$ & $\begin{array}{c}2.428 \\
\text { to } \\
3.347 \\
\text { inches }\end{array}$ & $\begin{array}{c}3.347 \\
\text { to } \\
3.544 \\
\text { inches }\end{array}$ & $\begin{array}{c}3.544 \\
\text { to } \\
3.741 \\
\text { inches }\end{array}$ & $\begin{array}{c}3.741 \\
\text { to } \\
3.938 \\
\text { inches }\end{array}$ \\
\hline 0.5 & 19.14 & 16.97 & 18.23 & 18.05 & 21.81 & 23.98 & 21.00 & 18.75 & 21.44 \\
\hline 1.5 & 18.76 & 16.92 & 16.23 & 16.11 & 20.42 & 22.35 & 20.90 & 18.95 & 19.84 \\
\hline 2.5 & 22.03 & 18.50 & 19.74 & 19.85 & 25.68 & 26.59 & 21.61 & 24.04 & 25.37 \\
\hline 3.5 & 24.70 & 23.38 & 21.65 & 23.03 & 28.68 & 31.59 & 27.50 & 26.04 & 26.64 \\
\hline 4.5 & 27.74 & 27.16 & 25.55 & 27.48 & 34.46 & 38.43 & 32.33 & 32.65 & 32.13 \\
\hline 5.5 & 38.09 & 30.29 & 31.80 & 32.30 & 39.83 & 44.34 & 38.09 & 37.45 & 38.05 \\
\hline 6.5 & 44.15 & 40.27 & 37.19 & 37.90 & 47.64 & 52.24 & 43.40 & 40.45 & 44.42 \\
\hline 7.5 & 56.13 & 52.56 & 50.20 & 51.69 & 59.82 & 63.74 & 57.98 & 55.38 & 62.64 \\
\hline 5 & 93.61 & 84.57 & 79.06 & 80.13 & 79.14 & 82.43 & 80.38 & 83.25 & 94.92 \\
\hline 9.5 & 127.58 & 114.03 & 106.88 & 107.31 & 100.69 & 101.87 & 105.49 & 110.51 & 122.88 \\
\hline 10.5 & 144.76 & 130.73 & 122.93 & 119.45 & 112.14 & 115.37 & 118.13 & 124.49 & 138.40 \\
\hline 11.5 & 155.85 & 142.12 & 135.46 & 130.09 & 124.60 & 123.43 & 126.30 & 134.67 & 155.46 \\
\hline 12.5 & 169.43 & 151.29 & 141.60 & 136.28 & 127.55 & 129.87 & 130.82 & 138.46 & 159.06 \\
\hline 13.5 & 172.72 & 151.67 & 142.47 & 137.59 & 130.21 & 131.74 & 136.06 & 150.84 & 169.24 \\
\hline 14.5 & 170.12 & 155.67 & 145.70 & 142.47 & 129.28 & 132.93 & 137.84 & 141.63 & 167.74 \\
\hline 15.5 & 170.76 & 149.80 & 144.49 & 138.90 & 130.38 & 133.17 & 142.67 & 145.89 & 162.66 \\
\hline 16.5 & 172.89 & 149.51 & 141.81 & 134.66 & 127.37 & 130.94 & 134.24 & 149.10 & 169.18 \\
\hline 17.5 & 169.22 & 144.53 & 139.19 & 131.99 & 122.38 & 126.38 & 133.32 & 141.86 & 164.02 \\
\hline 18.5 & 155.57 & 138.71 & 136.92 & 128.23 & 116.48 & 122.36 & 125.04 & 132.83 & 151.61 \\
\hline 19.5 & 153.30 & 132.02 & 122.83 & 121.38 & 112.19 & 112.68 & 120.29 & 128.88 & 144.71 \\
\hline 20.5 & 137.04 & 117.22 & 114.66 & 111.54 & 103.08 & 105.76 & 109.65 & 119.67 & 132.92 \\
\hline 21.5 & 127.71 & 108.70 & 101.07 & 98.63 & 95.17 & 98.33 & 100.66 & 108.19 & 124.05 \\
\hline 22.5 & 111.82 & 100.28 & 93.90 & 90.40 & 85.96 & 88.09 & 92.61 & 100.55 & 113.39 \\
\hline 23.5 & 122.21 & 106.71 & 101.34 & 101.84 & 93.94 & 96.98 & 100.43 & 102.76 & 127.18 \\
\hline Ax. Avg. & 108.59 & 96.03 & 91.29 & 89.02 & 86.20 & 89.02 & 89.84 & 94.48 & 107.04 \\
\hline
\end{tabular}

HEAT FLUX VALUES NORMALIZED TO A CORE POWER OF 11.57 MW AND ASSUME ALL FISSION ENERGY (EXCLUDING GAMMA-HEATING OF EX-CORE COMPONENTS) IS DEPOSITED IN THE FUEL. HEAT FLUX VALUES ALSO ASSUME THAT THE HEAT DEPOSITED IN THE FUEL CONDUCTS OUT EVENLY FROM THE TWO SURFACES OF THE FUEL PLATE WITHOUT ANY LATERAL OR AXIAL CONDUCTION IN THE CLADDING. 
Table 3-24. Heat Flux Data (W/ $\left.\mathrm{cm}^{2}\right)$ for Fuel Plate 22 in 96 MWd Burnup Element in Reference Mixed Burnup Core: Core 7A, Position X6, No Xe, FT loaded with typical samples.

\section{Fuel core width 3.938 inches Fuel core thickness 20 mil}

\begin{tabular}{|c|c|c|c|c|c|c|c|c|c|}
\hline $\begin{array}{l}\text { Axial position } \\
\text { from top of } \\
\text { fuel (inches) }\end{array}$ & $\begin{array}{c}0.000 \\
\text { to } \\
0.197 \\
\text { inches }\end{array}$ & $\begin{array}{c}0.197 \\
\text { to } \\
0.394 \\
\text { inches }\end{array}$ & $\begin{array}{c}0.394 \\
\text { to } \\
0.591 \\
\text { inches }\end{array}$ & $\begin{array}{c}0.591 \\
\text { to } \\
1.510 \\
\text { inches }\end{array}$ & $\begin{array}{c}1.510 \\
\text { to } \\
2.428 \\
\text { inches }\end{array}$ & $\begin{array}{c}2.428 \\
\text { to } \\
3.347 \\
\text { inches }\end{array}$ & $\begin{array}{c}3.347 \\
\text { to } \\
3.544 \\
\text { inches }\end{array}$ & $\begin{array}{c}3.544 \\
\text { to } \\
3.741 \\
\text { inches }\end{array}$ & $\begin{array}{c}3.741 \\
\text { to } \\
3.938 \\
\text { inches }\end{array}$ \\
\hline 0.5 & 25.19 & 19.60 & 19.41 & 16.92 & 16.32 & 14.82 & 16.35 & 17.74 & 19.28 \\
\hline 1.5 & 25.66 & 20.52 & 19.16 & 16.32 & 15.32 & 15.49 & 17.94 & 17.56 & 18.90 \\
\hline 2.5 & 26.32 & 23.64 & 19.08 & 19.39 & 18.47 & 18.30 & 18.84 & 18.63 & 21.14 \\
\hline 3.5 & 31.13 & 27.28 & 25.61 & 22.49 & 21.07 & 20.96 & 21.72 & 23.18 & 28.13 \\
\hline 4.5 & 37.18 & 32.12 & 29.13 & 25.74 & 24.92 & 24.84 & 24.52 & 24.68 & 29.53 \\
\hline 5.5 & 41.24 & 35.16 & 35.16 & 31.00 & 29.05 & 29.27 & 29.46 & 33.91 & 34.88 \\
\hline 6.5 & 48.69 & 44.62 & 44.47 & 37.19 & 34.20 & 35.21 & 35.51 & 36.00 & 40.18 \\
\hline 7.5 & 58.78 & 54.81 & 50.85 & 48.16 & 46.05 & 47.52 & 46.79 & 48.74 & 57.45 \\
\hline 8.5 & 92.14 & 82.62 & 77.50 & 77.66 & 75.06 & 76.48 & 79.00 & 82.77 & 89.93 \\
\hline 9.5 & 122.75 & 108.55 & 113.25 & 103.86 & 100.97 & 104.92 & 103.32 & 109.83 & 131.29 \\
\hline 10.5 & 136.39 & 118.64 & 120.35 & 121.45 & 115.58 & 120.59 & 124.76 & 131.98 & 147.65 \\
\hline 11.5 & 144.62 & 137.22 & 129.42 & 128.62 & 126.38 & 131.53 & 134.84 & 139.73 & 154.14 \\
\hline 12.5 & 151.24 & 143.32 & 137.35 & 137.07 & 132.49 & 136.38 & 141.24 & 149.43 & 160.40 \\
\hline 13.5 & 165.72 & 146.30 & 140.40 & 138.39 & 137.24 & 144.43 & 145.44 & 150.62 & 167.74 \\
\hline 14.5 & 164.77 & 146.85 & 144.65 & 142.44 & 141.12 & 146.26 & 149.64 & 154.93 & 170.20 \\
\hline 15.5 & 166.69 & 146.08 & 144.32 & 140.67 & 141.25 & 144.32 & 148.56 & 152.80 & 176.49 \\
\hline 16.5 & 159.84 & 144.14 & 138.67 & 139.10 & 138.24 & 145.01 & 145.44 & 150.77 & 170.06 \\
\hline 17.5 & 156.03 & 141.92 & 136.61 & 137.73 & 133.82 & 138.43 & 136.33 & 142.90 & 166.50 \\
\hline 18.5 & 156.09 & 138.55 & 128.64 & 129.58 & 127.57 & 132.93 & 136.94 & 142.83 & 153.94 \\
\hline 19.5 & 148.11 & 130.31 & 120.11 & 120.98 & 119.74 & 121.10 & 124.59 & 130.31 & 146.87 \\
\hline 20.5 & 130.28 & 114.77 & 115.57 & 113.39 & 108.68 & 112.35 & 119.82 & 120.63 & 135.33 \\
\hline 21.5 & 120.86 & 108.56 & 105.74 & 102.30 & 99.69 & 101.47 & 103.45 & 107.20 & 123.47 \\
\hline 22.5 & 109.66 & 96.45 & 95.98 & 91.63 & 88.80 & 93.43 & 96.64 & 97.49 & 112.77 \\
\hline 23.5 & 119.98 & 99.88 & 103.20 & 98.48 & 95.56 & 98.98 & 98.07 & 101.29 & 119.68 \\
\hline Ax. Avg. & 105.79 & 94.28 & 91.44 & 89.16 & 86.97 & 89.80 & 91.63 & 95.28 & 107.34 \\
\hline
\end{tabular}

HEAT FLUX VALUES NORMALIZED TO A CORE POWER OF 11.57 MW AND ASSUME ALL FISSION ENERGY (EXCLUDING GAMMA-HEATING OF EX-CORE COMPONENTS) IS DEPOSITED IN THE FUEL. HEAT FLUX VALUES ALSO ASSUME THAT THE HEAT DEPOSITED IN THE FUEL CONDUCTS OUT EVENLY FROM THE TWO SURFACES OF THE FUEL PLATE WITHOUT ANY LATERAL OR AXIAL CONDUCTION IN THE CLADDING. 
Table 3-25. Heat Flux Data $\left(\mathrm{W} / \mathrm{cm}^{2}\right)$ in Fuel Plate 22 in 170 MWd Burnup Element in Reference Mixed Burnup Core: Core 7A, Position X8, No Xe, FT loaded with typical samples.

\section{Fuel core width 3.938 inches Fuel core thickness 20 mil}

\begin{tabular}{|c|c|c|c|c|c|c|c|c|c|}
\hline $\begin{array}{l}\text { Axial position } \\
\text { from top of } \\
\text { fuel (inches) }\end{array}$ & $\begin{array}{c}0.000 \\
\text { to } \\
0.197 \\
\text { inches }\end{array}$ & $\begin{array}{c}0.197 \\
\text { to } \\
0.394 \\
\text { inches }\end{array}$ & $\begin{array}{c}0.394 \\
\text { to } \\
0.591 \\
\text { inches }\end{array}$ & $\begin{array}{c}0.591 \\
\text { to } \\
1.510 \\
\text { inches }\end{array}$ & $\begin{array}{c}1.510 \\
\text { to } \\
2.428 \\
\text { inches }\end{array}$ & $\begin{array}{c}2.428 \\
\text { to } \\
3.347 \\
\text { inches }\end{array}$ & $\begin{array}{c}3.347 \\
\text { to } \\
3.544 \\
\text { inches }\end{array}$ & $\begin{array}{c}3.544 \\
\text { to } \\
3.741 \\
\text { inches }\end{array}$ & $\begin{array}{l}3.741 \\
\text { to } \\
3.938 \\
\text { inches }\end{array}$ \\
\hline 0.5 & 20.81 & 17.58 & 16.72 & 15.19 & 14.59 & 14.70 & 15.80 & 18.70 & 17.91 \\
\hline 1.5 & 18.67 & 16.01 & 17.27 & 15.72 & 14.54 & 15.09 & 14.52 & 14.35 & 18.15 \\
\hline 2.5 & 21.63 & 18.64 & 17.07 & 17.30 & 16.98 & 17.05 & 18.86 & 21.33 & 21.08 \\
\hline 3.5 & 25.96 & 23.61 & 21.75 & 21.49 & 20.41 & 20.98 & 21.36 & 21.88 & 23.46 \\
\hline 4.5 & 28.19 & 25.62 & 24.89 & 24.02 & 22.28 & 23.44 & 24.92 & 26.18 & 29.52 \\
\hline 5.5 & 34.98 & 31.02 & 29.59 & 28.34 & 26.47 & 28.98 & 30.37 & 29.88 & 35.44 \\
\hline 6.5 & 41.27 & 36.97 & 33.86 & 32.20 & 31.18 & 31.64 & 32.20 & 35.35 & 38.89 \\
\hline 7.5 & 55.31 & 47.39 & 45.83 & 44.82 & 43.90 & 45.32 & 43.16 & 48.59 & 54.57 \\
\hline 8.5 & 86.38 & 81.33 & 74.92 & 73.42 & 72.13 & 73.57 & 75.98 & 76.51 & 89.70 \\
\hline 9.5 & 121.88 & 106.02 & 101.85 & 98.19 & 96.97 & 100.12 & 101.24 & 106.83 & 116.90 \\
\hline 10.5 & 136.98 & 121.00 & 119.13 & 113.76 & 110.96 & 114.58 & 120.18 & 120.76 & 132.20 \\
\hline 11.5 & 146.05 & 125.62 & 123.48 & 124.61 & 120.95 & 123.98 & 123.35 & 130.41 & 148.83 \\
\hline 12.5 & 151.82 & 133.96 & 128.97 & 128.84 & 125.82 & 129.50 & 130.68 & 139.35 & 149.07 \\
\hline 13.5 & 158.60 & 138.86 & 132.69 & 133.65 & 131.59 & 135.43 & 138.58 & 144.89 & 157.91 \\
\hline 14.5 & 155.97 & 145.40 & 138.59 & 132.89 & 133.03 & 138.73 & 139.42 & 148.88 & 163.89 \\
\hline 15.5 & 57.22 & 141.46 & 139.65 & 136.99 & 135.46 & 136.02 & 140.90 & 141.32 & 162.52 \\
\hline 16.5 & 153.83 & 139.12 & 133.16 & 134.55 & 133.99 & 136.63 & 145.64 & 146.61 & 165.20 \\
\hline 17.5 & 153.81 & 134.67 & 130.79 & 129.72 & 127.98 & 132.53 & 140.83 & 138.95 & 157.29 \\
\hline 18.5 & 148.38 & 128.93 & 122.80 & 127.37 & 123.84 & 129.72 & 134.41 & 140.55 & 157.38 \\
\hline 19.5 & 137.21 & 123.47 & 120.16 & 119.06 & 118.57 & 120.78 & 124.09 & 130.10 & 144.32 \\
\hline 20.5 & 134.12 & 117.23 & 113.35 & 111.64 & 109.12 & 111.75 & 111.98 & 121.80 & 132.98 \\
\hline 21.5 & 121.12 & 102.81 & 103.54 & 102.30 & 97.33 & 100.23 & 109.23 & 110.37 & 122.36 \\
\hline 22.5 & 108.99 & 98.82 & 94.44 & 90.52 & 89.12 & 91.73 & 92.85 & 93.41 & 111.61 \\
\hline 23.5 & 116.71 & 105.92 & 100.83 & 99.81 & 96.36 & 99.92 & 100.53 & 107.75 & 120.98 \\
\hline Ax. Avg. & 101.46 & 90.04 & 86.88 & 85.65 & 83.89 & 86.35 & 88.81 & 92.24 & 103.04 \\
\hline
\end{tabular}

HEAT FLUX VALUES NORMALIZED TO A CORE POWER OF 11.57 MW AND ASSUME ALL FISSION ENERGY (EXCLUDING GAMMA-HEATING OF EX-CORE COMPONENTS) IS DEPOSITED IN THE FUEL. HEAT FLUX VALUES ALSO ASSUME THAT THE HEAT DEPOSITED IN THE FUEL CONDUCTS OUT EVENLY FROM THE TWO SURFACES OF THE FUEL PLATE WITHOUT ANY LATERAL OR AXIAL CONDUCTION IN THE CLADDING. 


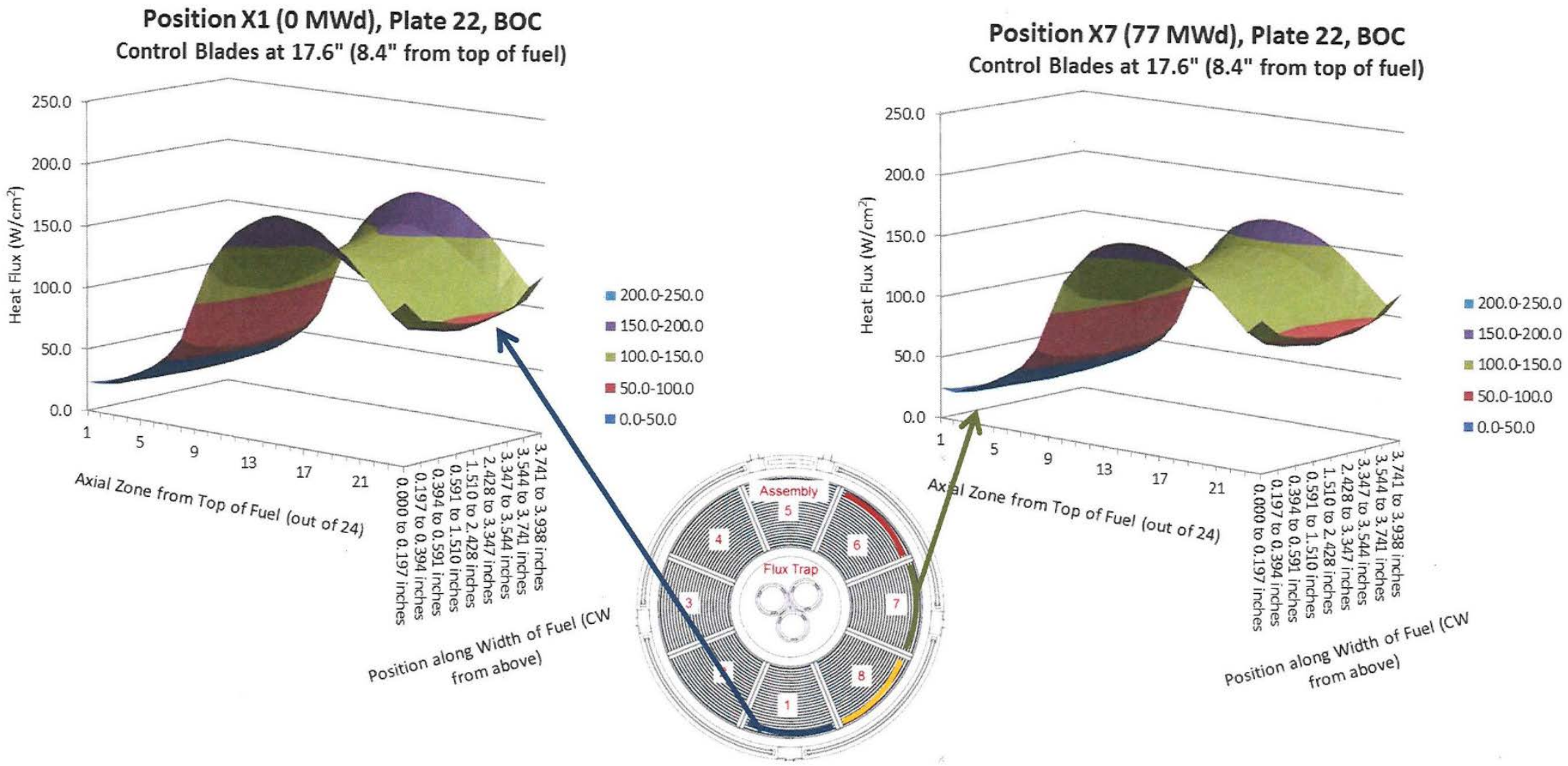

Position X1, 0 MWd, No Xe, Plate 22

Position X7, 77 MWd, No Xe, Plate 22

Figure 3-6. Evolution of Heat Flux Profile (W/cm2) for LEU Fuel Plate 22, Element Burnup from 0 to 77 MWd: Core 7A, no Xe, FT loaded with typical samples.

HEAT FLUX VALUES NORMALIZED TO A CORE POWER OF 11.57 MW AND ASSUME ALL FISSION ENERGY (EXCLUDING GAMMAHEATING OF EX-CORE COMPONENTS) IS DEPOSITED IN THE FUEL. HEAT FLUX VALUES ALSO ASSUME THAT THE HEAT DEPOSITED IN THE FUEL CONDUCTS OUT EVENLY FROM THE TWO SURFACES OF THE FUEL PLATE WITHOUT ANY LATERAL OR AXIAL CONDUCTION IN THE CLADDING. 


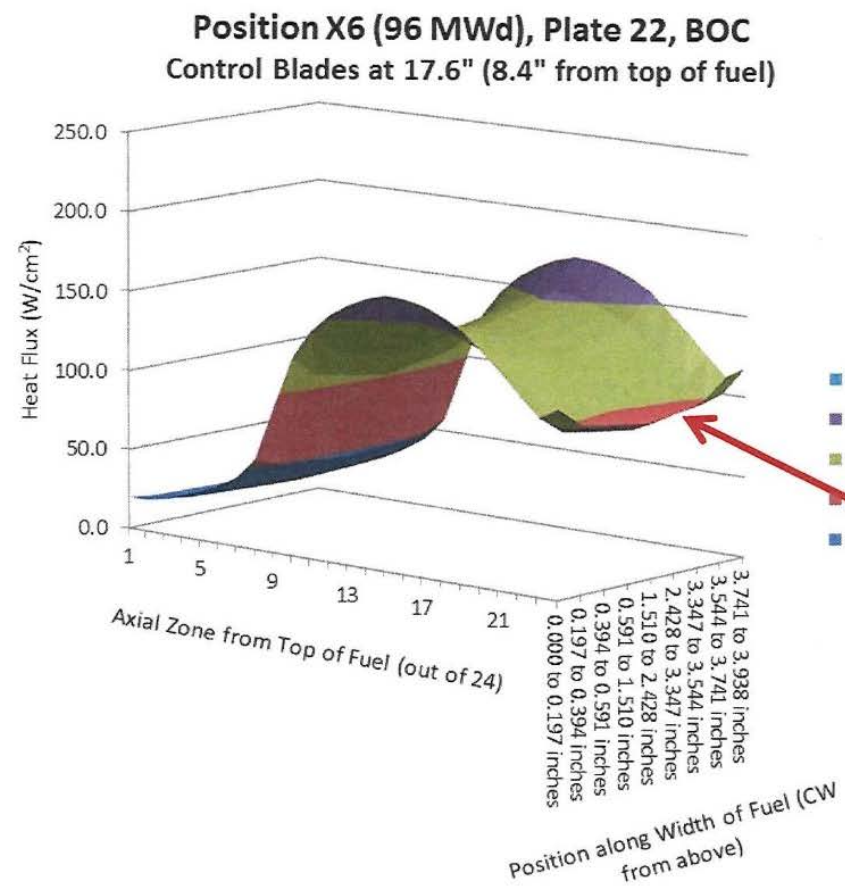

=150.0-250.0 $=150.0-200.0$
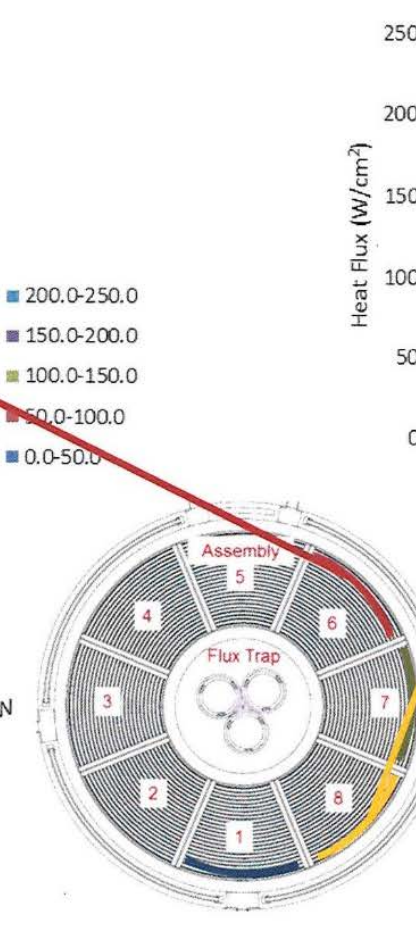

Position X6, 96 MWd, No Xe, Plate 22
Position X8 (170 MWd), Plate 22, BOC

Control Blades at 17.6" (8.4" from top of fuel)

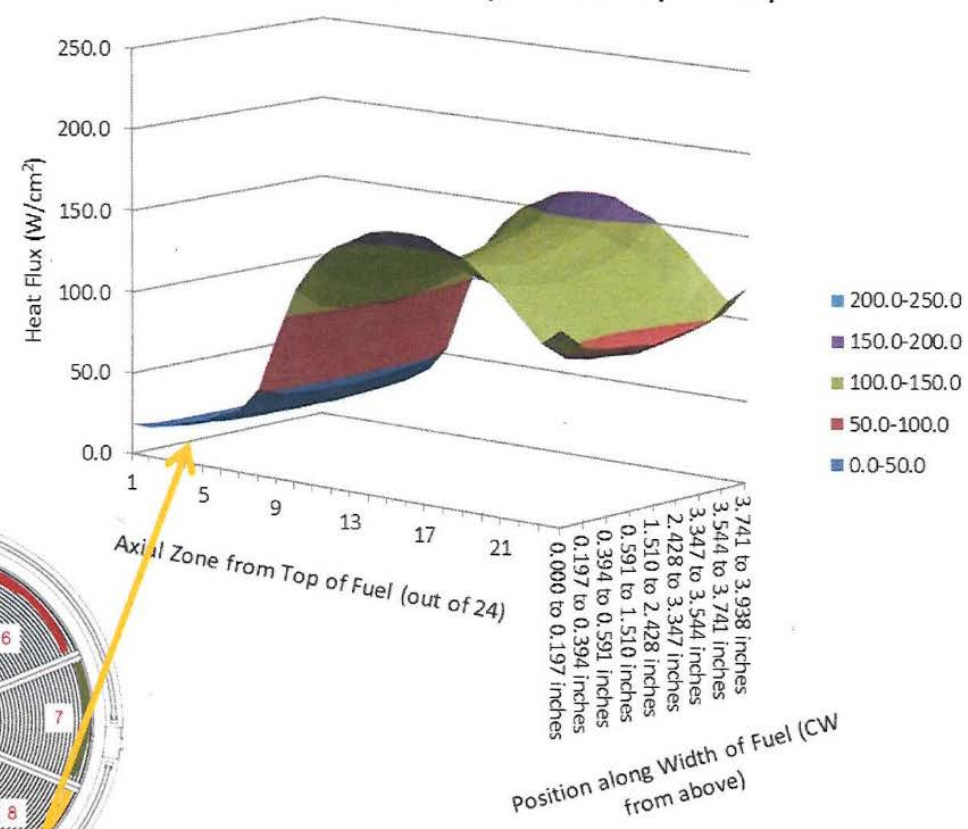

Position X8, 170 MWd, No Xe, Plate 22

Figure 3-7. Evolution of Heat Flux Profile (W/cm2) for LEU Fuel Plate 22, Element Burnup from 96 to 170 MWd: Core 7A, no Xe, FT loaded with typical samples.

HEAT FLUX VALUES NORMALIZED TO A CORE POWER OF 11.57 MW AND ASSUME ALL FISSION ENERGY (EXCLUDING GAMMA-HEATING OF EX-CORE COMPONENTS) IS DEPOSITED IN THE FUEL. HEAT FLUX VALUES ALSO ASSUME THAT THE HEAT DEPOSITED IN THE FUEL CONDUCTS OUT EVENLY FROM THE TWO SURFACES OF THE FUEL PLATE WITHOUT ANY LATERAL OR AXIAL CONDUCTION IN THE CLADDING. 


\subsubsection{Steady-State Temperatures for LEU Fuel Plates}

This section presents calculated temperature data for MURR fuel plates under prototypic conditions. Temperatures were calculated using version 4.3 of the PLTEMP/ANL code [11]. All eight elements in the MURR core are modeled. In the one-dimensional analysis, temperatures were evaluated for the hottest azimuthal stripe (highest power density) of each fuel plate in the core, which is located along the edge of the fuel foil near the element side plate. As discussed in Section 3.2, since lateral heat conduction is neglected in the analysis, an appropriately-sized stripe width was determined from a detailed three-dimensional CFD analysis $[9,10]$.

Table 3-26 summarizes the LEU core operating parameters assumed in the analysis. The total core power of $11.57 \mathrm{MW}$ represents the portion of the total reactor power of $12 \mathrm{MW}$ following conversion that is deposited in the fuel elements and primary coolant. The balance of the reactor power $(0.43$ MW) is deposited in the reflectors and ex-core components and is removed by the pool coolant system. The nominal coolant inlet temperature for MURR is $120 \mathrm{oF}$, but varies throughout the year in a normal operating band from $118{ }^{\circ} \mathrm{F}$ to $125{ }^{\circ} \mathrm{F}$. An inlet coolant temperature of $122{ }^{\circ} \mathrm{F}$, which is slightly above the middle of this operating band, was selected as a prototypic value for the temperature analysis. It should be noted that the pressurizer pressure assumed in this analysis is at the upper range of the normal operating band for MURR, but this will have little effect on the predicted temperatures.

Table 3-26. Nominal MURR Core Operating Parameters with LEU Fuel.

\begin{tabular}{|l|c|}
\hline Total Reactor Power & $12 \mathrm{MW}$ \\
\hline $\begin{array}{l}\text { Fraction of Power Deposited in Primary Coolant } \\
\text { System }\end{array}$ & $96.4 \%$ \\
\hline Total Core Power & $11.57 \mathrm{MW}$ \\
\hline Coolant Inlet Temperature & $122^{\circ} \mathrm{F}\left(50^{\circ} \mathrm{C}\right)$ \\
\hline Total Core Coolant Flow Rate & $3800 \mathrm{gpm}$ \\
\hline Pressurizer Pressure & 84 psia \\
\hline
\end{tabular}

The construction of the LEU fuel plates is described in Section 2, and consists of a U-10Mo fuel core, a thin (1 mil nominal) layer of zirconium, and aluminum AA6061 cladding. The zirconium acts as an interaction barrier between the fuel core and aluminum. Dimensions for each fuel plate in the element are given in Table 2-1. While the fuel element is in use, a thin layer of oxide gradually forms on the outer surface of the fuel plate cladding and thickens with hours of operation. The oxide layer growth rate and thermal treatment are described later in this section.

The thermal conductivity values of the LEU fuel plate materials assumed in the temperature calculations are summarized in Table 3-27. The U-10Mo thermal conductivity values are the same as used in the steady-state thermal-hydraulics analysis reported in Reference 2. The U-10Mo thermal conductivity decreases with fuel burnup. Two values are provided in Table 3-27, one for the fresh fuel and another for the fuel when it is near EOL. These values are consistent with experimentally measured values. For the discharged fuel, the thermal conductivity is conservatively based on a fuel burnup of $40 \times 10^{20}$ fissions $/ \mathrm{cm}^{3}$. This is well above the predicted maximum LEU fuel burnup of $33.7 \times 10^{20}$ fissions $/ \mathrm{cm}^{3}$ reported for MURR in Section 3.3.1. Between these two extremes, linear interpolation based on fuel element burnup is used in the PLTEMP/ANL model. 
It should be noted that the MURR LEU accident analysis reported in References 4 and 12 was based on more recent measurements of the thermal conductivity of irradiated U-10Mo, and future measurements of prototypic material are expected as part of ongoing fuel qualification work by the Fuel Qualification Pillar. The values for the U-10Mo thermal conductivity assumed in this analysis are lower than the best-estimate temperature- and burnup-dependent fit of the thermal conductivity reported in Reference 12 . Therefore, the results reported here are conservative relative to results that would be obtained using the more recent data.

Table 3-27. Thermal Conductivity Values for LEU Fuel Plate Materials (W/m-C).

\begin{tabular}{|l|c|c|}
\hline Material & $\begin{array}{c}\text { Fresh Element } \\
\text { (0 MWd) }\end{array}$ & $\begin{array}{c}\text { Discharged Element } \\
\text { (180 MWd) }\end{array}$ \\
\hline Fuel core (U-10Mo) & 16.2 & 11.3 \\
\hline Zirconium layer & 19.6 & 19.6 \\
\hline Aluminum AA6061 & 166.1 & 166.1 \\
\hline Oxide layer & 2.25 & 2.25 \\
\hline
\end{tabular}

Values for the thermal conductivity of the zirconium layer and the aluminum AA6061 were derived from the same temperature dependent correlations used in the MURR accident analysis and reported in Section 2 of Reference 12. The thermal conductivity values for the zirconium and AA6061 used in the PLTEMP/ANL analysis were derived at $125^{\circ} \mathrm{C}$, which is representative for the temperatures of these materials under prototypic conditions. The more conservative values that were used in Reference 2 (150.0 and $19.0 \mathrm{~W} / \mathrm{m}-\mathrm{C}$ for the AA6061 and zirconium, respectively) result in a maximum fuel temperature that is only a few-tenths of a degree warmer. A representative value for thermal conductivity of the oxide layer, which was identified in Reference 2 and used in the accident analysis reported in Reference 12, was used in the present analysis.

When steady-state thermal-hydraulic safety calculations are performed, hot channel factors are applied to account for factors such as the statistical variations in manufacturing of the fuel element and the uncertainty of the method used for the neutronics calculation, which would affect the calculated heat flux. This ensures that the thermal-hydraulic safety margins are maintained under even the most extreme conditions. However, the temperatures calculated here under prototypic operating conditions serve as a nominal basis of comparison during design of the MURR DDE experiment. As such, hot channel factors related to the fuel manufacturing and calculational uncertainty were not included in the temperature analysis performed here.

However, the impact of the coolant channel restriction that may occur with depletion of the fuel under normal operating conditions, as well as the increased thermal resistance across the cladding due to the growth of an oxide layer on the surface of the fuel plates were included in the temperature analysis. This methodology was presented as a part of prior design parameters activities [3], and is summarized briefly here.

The thicknesses of the coolant channels between the LEU fuel plates will become narrower over the life of the fuel element as a result of three phenomena. First, as the fuel undergoes fission, swelling of the monolithic U-10Mo fuel core from the production of gaseous and solid fission products will occur. The second phenomenon is the effect of irradiation-enhanced creep of the fuel core which will cause localized bulging of the fuel plate near the edges, which are constrained by the cladding at the edges next to the side plates. Third, oxide growth from the aluminum-water reaction will constrict the coolant channel. The amount of oxide growth on the fuel plate depends on the temperature at the 
water-plate surface, operating time, water $\mathrm{pH}$ level, and any protective coating placed on the outside surface of the fuel cladding.

The fuel swelling and fuel creep effects were assessed from experimental data reported in References 13 and 14. Based on correlations in the references, the maximum local increase in the fuel core thickness over the life of the LEU fuel from these two phenomena is predicted to be 4 mil. This value is currently under investigation.

An aluminum-water reaction on the surface of the aluminum cladding forms an oxide layer that increases with operating time and temperature. This causes both a restriction of the coolant channel, as well as increases the thermal resistance of the cladding, raising the $\Delta T$ from the plate surface to the fuel. A correlation of the oxide growth rate developed by Griess [15] was modified to take into account the specific operating conditions of the MURR. The "modified Griess correlation" parameters were empirically adjusted based on experimental measurements of the oxide layer thickness formed on the convex surface of plate 24 of several discharged MURR HEU fuel elements and steady-state analysis of the HEU fuel plate surface temperature calculated by PLTEMP/ANL.

Table 3-28 summarizes the oxide layer thickness predicted by the modified Griess correlation for LEU fuel plates at four different element burnups. The correlation is formulated based on hours of operation and plate surface temperature, which changes over the lifetime of the element. The oxide layer thickness was calculated based on the predicted fuel plate surface temperature on the concave surface of LEU fuel plate 23, which is the maximum for all fuel plates over the majority of the LEU element lifetime. The oxide thickness values reported in Table 3-28 were conservatively applied as a thermal resistance layer to all plates in the fuel element.

Table 3-28. Predicted Oxide Layer Thickness for LEU Fuel Plates.

\begin{tabular}{|c|c|c|}
\hline LEU Element Burnup (MWd) & Hours of Operation & $\begin{array}{c}\text { Oxide Layer Thickness } \\
\text { (mil) }\end{array}$ \\
\hline 0 & 0 & 0 \\
\hline 77 & 1358 & 0.55 \\
\hline 96 & 1662 & 0.63 \\
\hline $180^{2}$ & 2880 & 0.93 \\
\hline
\end{tabular}

${ }^{1}$ Calculated from modified Griess correlation [3] on concave surface of LEU fuel plate 23 at prototypic operating conditions.

${ }^{2}$ LEU EOL element discharge burnup.

While the predicted oxide layer thickness at the LEU discharge burnup is less than 1 mil, it was conservatively assumed for the purpose of estimating the coolant channel restriction that the oxide layer on the surface of the fuel plates grows to 2 mil at end-of-life (EOL). Thus, the total maximum channel restriction due to the combined effects of fuel swelling, irradiation-enhanced creep, and oxide growth for the LEU fuel in MURR was assumed to be 8 mil for channels that are heated by two fuel plates ( 2 mil for each fuel plate from fuel swelling and creep, plus 2 mil for each plate from oxide), and 4 mil for channels heated by only one plate. This effect was included in the PLTEMP/ANL model, with the assumption that the channel restriction increases linearly with burnup over the life of the fuel from 0 to $180 \mathrm{MWd}$.

Table 3-29 provides a summary of the maximum fuel and cladding surface temperatures for each fuel element under prototypic operating conditions in MURR for Cores 7A (no xenon) and 8A (equilibrium xenon). The plate surface is the interface between the coolant and oxide layer, if this layer exists, or 
the outer cladding surface for the case of fresh fuel. The temperatures were calculated by PLTEMP/ANL assuming the prototypic operating conditions listed in Table 3-28 and without any additional uncertainties. As described above, the effects of fuel burnup/ on the U-10Mo thermal conductivity, the thickness of the coolant channels, and the oxide as a thermal resistance layer were modeled in PLTEMP/ANL. Temperatures were calculated for the hottest azimuthal stripe of the LEU fuel plates. As discussed in Section 3.2, the hot stripe is one of the $0.5 \mathrm{~cm}$ azimuthal stripes along the edge of the fuel core that is adjacent to the unfueled portion of the plate that is swaged into the side plate. The local temperatures presented in this report are for a mesh size that is $0.5 \times 2.54 \mathrm{~cm}$.

Table 3-29. Fuel Conditions for Prototypic MURR Core with LEU Fuel.

\begin{tabular}{|c|c|c|c|c|c|c|c|}
\hline \multirow[b]{3}{*}{$\begin{array}{c}\text { Core } \\
\text { Position }\end{array}$} & \multirow{3}{*}{$\begin{array}{l}\text { Element } \\
\text { Burnup } \\
\text { at BOC } \\
\text { (MWd) }\end{array}$} & \multicolumn{3}{|c|}{$\begin{array}{c}\text { Core 7A: No Xe (BOC) } \\
\text { Control blades at 8.4" from top of } \\
\text { fuel }\end{array}$} & \multicolumn{3}{|c|}{$\begin{array}{c}\text { Core 8A: Eq. Xe (Day } 2 \text { of week) } \\
\text { Control blades at } 1.7 \text { " from top of } \\
\text { fuel }\end{array}$} \\
\hline & & \multirow[b]{2}{*}{$\begin{array}{c}\text { Power } \\
\text { (MW) }\end{array}$} & \multicolumn{2}{|c|}{$\begin{array}{c}\text { Maximum } \\
\text { Temperature, }^{\circ} \mathrm{C} \\
\text { (Plate) }\end{array}$} & \multirow[b]{2}{*}{$\begin{array}{c}\text { Power } \\
\text { (MW) }\end{array}$} & \multicolumn{2}{|c|}{$\begin{array}{c}\text { Maximum Temperature, } \\
\text { oC (Plate) }\end{array}$} \\
\hline & & & Fuel & $\begin{array}{c}\text { Plate } \\
\text { Surface }\end{array}$ & & Fuel & $\begin{array}{c}\text { Plate } \\
\text { Surface }\end{array}$ \\
\hline $\mathrm{X} 1$ & 0 & 1.52 & $135.0(23)$ & $115.2(23)$ & 1.52 & $130.1(23)$ & $112.1(23)$ \\
\hline $\mathrm{X} 5$ & 0 & 1.52 & $130.0(23)$ & $112.0(23)$ & 1.47 & $126.0(23)$ & $108.8(23)$ \\
\hline X3 & 77 & 1.44 & $139.9(23)$ & $110.3(23)$ & 1.44 & $135.2(23)$ & $107.6(23)$ \\
\hline $\mathrm{X7}$ & 77 & 1.44 & $140.4(23)$ & $110.4(23)$ & 1.45 & $136.9(23)$ & $108.7(23)$ \\
\hline $\mathrm{X} 2$ & 96 & 1.44 & $143.7(23)$ & $111.1(23)$ & 1.46 & $135.7(23)$ & $106.8(23)$ \\
\hline $\mathrm{X} 6$ & 96 & 1.44 & $145.0(23)$ & $112.0(23)$ & 1.44 & $138.3(23)$ & $108.5(23)$ \\
\hline $\mathrm{X} 4$ & 170 & 1.38 & $147.5(22)$ & $107.9(23)$ & 1.38 & $139.6(22)$ & $104.6(23)$ \\
\hline $\mathrm{X} 8$ & 171 & 1.39 & $149.0(22)$ & $108.7(22)$ & 1.41 & $140.1(22)$ & $104.9(22)$ \\
\hline
\end{tabular}

Under xenon-free conditions, the power fraction for the EOL element in the X8 position is about $10 \%$ lower than the fresh element in the adjacent position X1, but the maximum fuel temperature in the element at EOL is about $14{ }^{\circ} \mathrm{C}$ greater than that in the fresh element. This is because the decreased thermal conductivity of the U-10Mo fuel at EOL, the constriction of the coolant channels in the depleted elements from oxide formation and fuel swelling, and the increased thermal resistance due to oxide growth on the cladding surface result in higher maximum fuel temperatures in depleted elements than in the fresh fuel elements. It is also observed that for more than half of the lifetime of the fuel element the maximum fuel temperature occurs in plate 23. As the element approaches its end-of-life, the maximum fuel temperature occurs in fuel plate 22 . This is due to fuel depletion effects, which reduces the heat flux in plate 23 relative to plate 22 . Furthermore, there is an 8-mil restriction of the coolant channels on both sides of plate 22 due to irradiation and oxide-growth effects; the coolant channel on the outboard side of plate 23 is constricted only 4 mil at EOL. The maximum plate surface temperature at the clad-water interface occurs in the fresh fuel element because of the higher heat flux relative to burned elements. Under equilibrium xenon conditions, the control blades are nearly fully withdrawn, which decreases the axial power peaking. Consequently, the maximum fuel temperature is lower in all elements at equilibrium xenon conditions. The maximum fuel temperature still occurs in the EOL element in position X8.

Figure 3-8 plots the maximum fuel temperature by axial position for all plates in the EOL element under xenon-free conditions in the prototypic Core 7A. The axial power distributions in the 
outermost plates are strongly affected by the control blade position. It can be seen that there is a corresponding effect on the maximum fuel temperature profile.

Lastly, the maximum local temperatures reported here are based on conditions for the design of irradiation experiments that are relevant to nominal steady-state conditions in MURR. Temperatures due to conditions that occur during off-nominal or transitory conditions, such as found in an analysis of limiting safety system setting (LSSS) or transient and accident scenarios, are not reported here but have been calculated elsewhere and compared to a fuel temperature safety limit. $[4,11]$

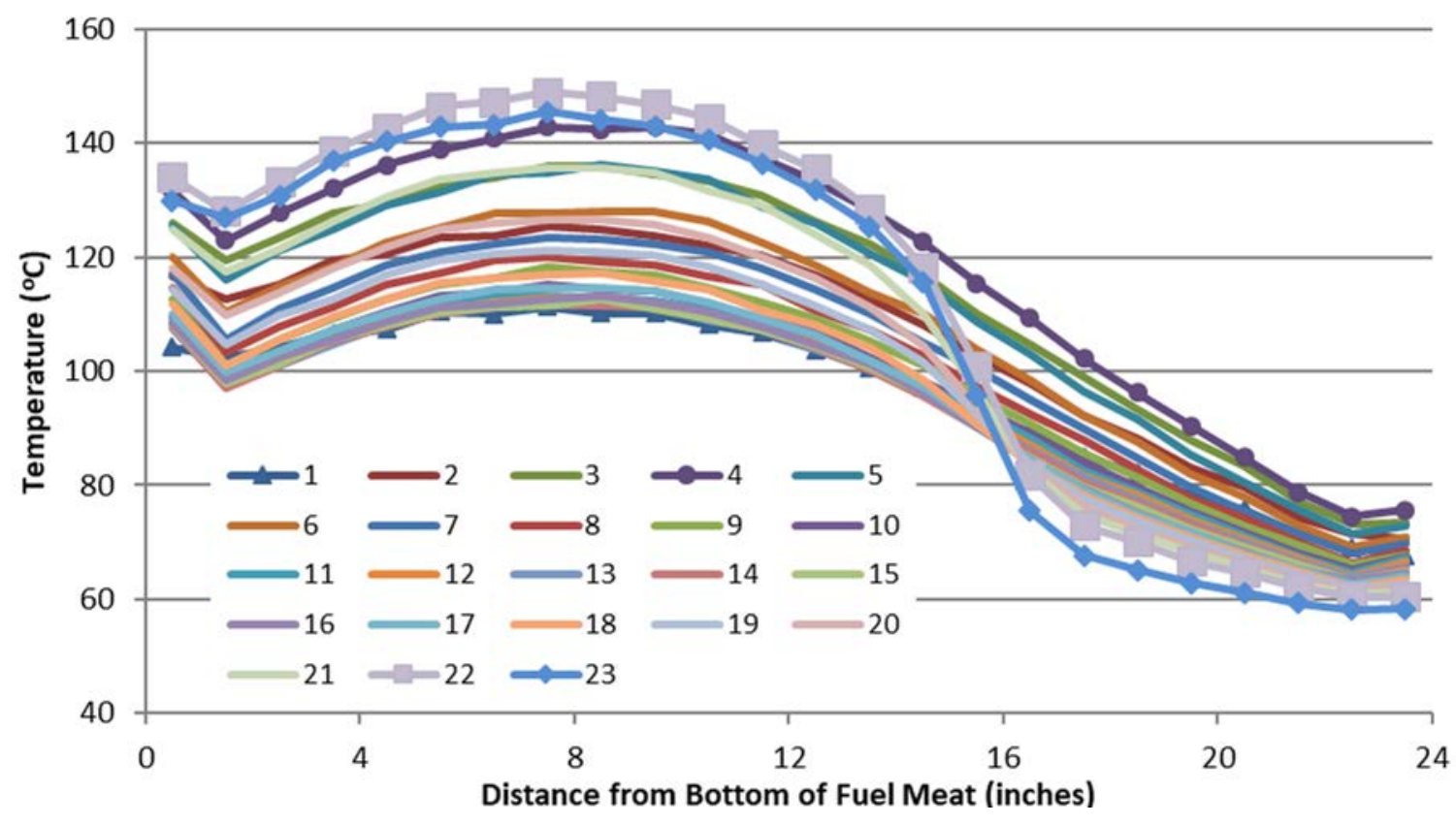

Figure 3-8. Axial Fuel Temperature Profile by Plate for EOL LEU Element in Core 7A. 


\section{SUMMARY OF MURR LEU IRRADIATION PARAMETERS}

Irradiation experiment conceptual design parameters for the LEU fuel element under nominal steady-state operating conditions in MURR were previously reported in Reference 3. Data for key fuel plates under conditions that are expected to yield the maximum fission rate density and cumulative fission density were provided, but core states that are not prototypic for MURR were included in identifying the maximum irradiation parameters in Reference 3. Thus, the information in this report updates and replaces the maximum fission rate density of $5.09 \times 10^{14}$ fissions $/ \mathrm{cm}^{3}-\mathrm{s}$ reported in Reference 3 as an irradiation experiment design parameter. The maximum cumulative fission density of $3.4 \times 10^{21}$ fissions $/ \mathrm{cm}^{3}$ for plate 1 is the same as previously reported, but platespecific data for all plates under prototypic conditions are provided here to supplement the previous data.

The preceding sections provide plate performance data for a variety of core states that could exist during steady-state operations of the MURR. The plates presented were selected from LEU elements under the conditions that are expected to yield the highest plate fission density and power density. Data for heat flux, and fuel and cladding temperatures are also provided. Data for prototypic core configurations are discussed in detail. Limited data for non-prototypic core configurations are also provided.

It should be noted that this data represents, within the local plate shapes specific to MURR, maximum local performance parameters expected for the MURR LEU fuel based on nominal steady-state conditions without engineering hot channel uncertainty factors. Safety analyses have accounted for such uncertainties, including manufacturing tolerances and other uncertainties in reactor geometry as described References 2, 4, and 11.

The MURR DDE is intended to test LEU fuel element performance under prototypic conditions to observe how the element behaves under conditions comparable to what will be experienced in MURR after conversion during planned nominal equilibrium operation with the reactor core fully loaded with LEU fuel elements. Fuel qualification plans are to irradiate approximately at least a half-dozen full-size USHPRR U-10Mo elements for MURR, MITR, NBSR, and ATR (which, in typical 2-pump operational mode, will achieve a fission density and power density similar to the prototypic maximum values for MURR) prior to conversion of MURR to the new LEU U-10Mo fuel. Other experiments being conducted by the Fuel Qualification Pillar will be conducted at a plate level and will be bounding and beyond the prototypic values to augment data demonstrating adequate fuel performance.

The purpose of this report is to collect and present best estimate design parameter data for fission density and power density, along with auxiliary information, under nominal conditions in MURR. The MURR DDE experiment will be placed in an irradiation vehicle which will support the element in an experimental location in a test reactor. The irradiation vehicle and experimental campaign of the MURR DDE should be designed so that the element operates at conditions comparable to those which are expected for typical operations in MURR for those design parameters which are expected to impact fuel performance. At this time this includes the known effects of fission density and the potential impacts of power density in the specific geometry of the MURR fuel element. Temperatures are regime appropriate and have not impacted fuel performance at the temperatures experienced by MURR fuel. 
All necessary dimensions for the preliminary MURR LEU fuel element relevant to MURR DDE can be found in Reference 7, except the end channel dimensions and inter-element spacing for the element in the MURR core. Values for these data are summarized in Table 4-1. A schematic view of MURR DDE is presented in Figure 4-1 to illustrate the dimensions presented in Table 4-1. The end channel dimensions are given as a range. The inter-element spacing is not considered part of the effective coolant flow area for the MURR element, although it may be important to include as part of the MURR DDE design to obtain regime appropriate conditions when the experiment is irradiated in the test reactor. In normal MURR operations, there is no coolant flow through the inter-element spacing because the way the element rests on the fuel element support spider blocks the bottom of the flow channel. The combined spacing on each side of the experiment should sum to the specified value. No required range or tolerance is specified for this value.

Table 4-1. End Channel and Inter-Element Spacing Dimensions for MURR DDE.

\begin{tabular}{|c|c|}
\hline Dimension & Value, inches \\
\hline Inner End Channel & $0.082-0.108$ \\
\hline Outer End Channel & $0.083-0.109$ \\
\hline $\begin{array}{c}\text { Inter-Element Spacing } \\
\text { (both sides combined) }\end{array}$ & 0.040 \\
\hline
\end{tabular}

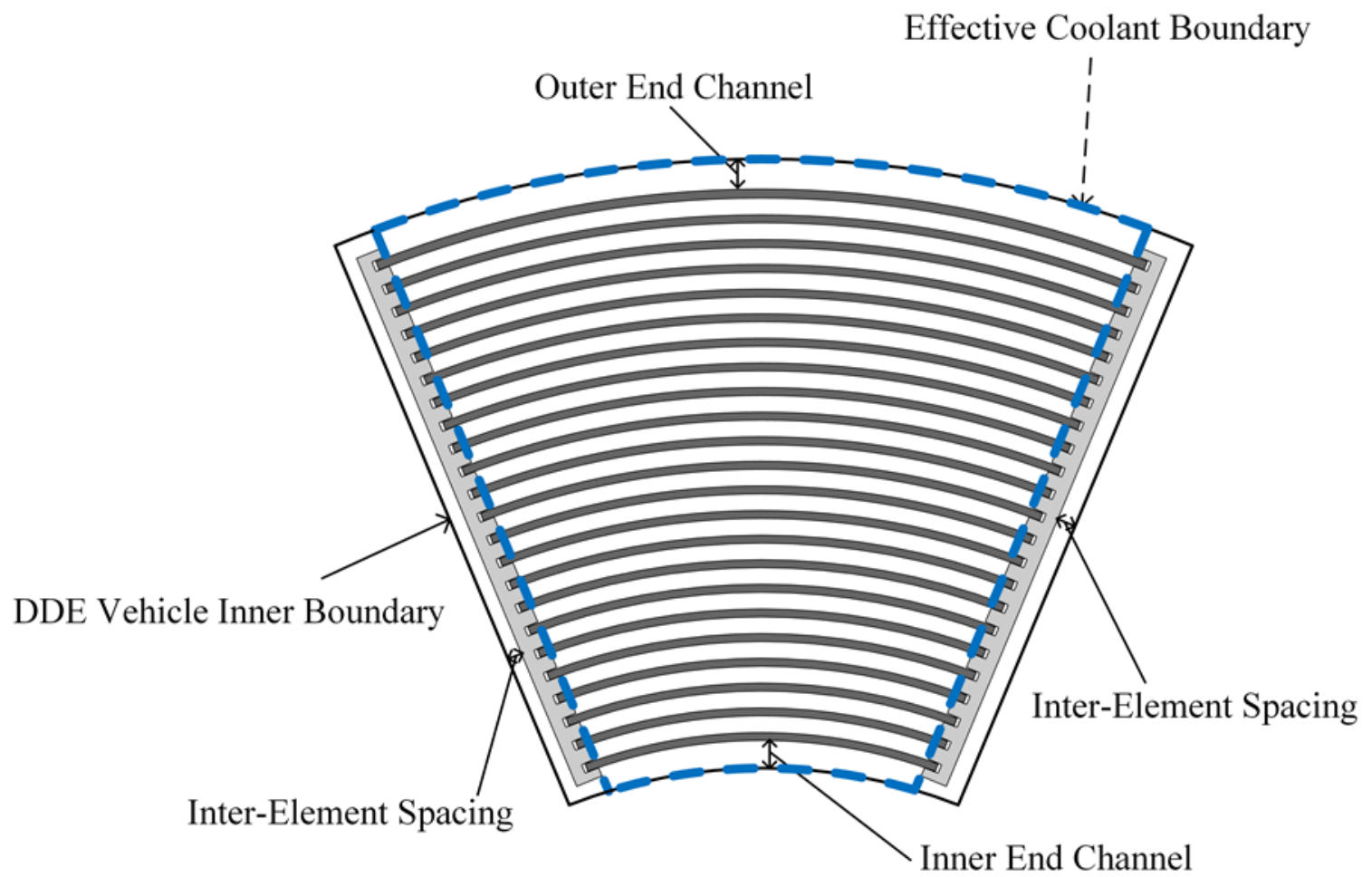

Figure 4-1. Evolution of Heat Flux Profile $(\mathrm{W} / \mathrm{cm} 2)$ for LEU Fuel Plate 22, Element

Thermal-hydraulic conditions for prototypic operations in MURR are summarized in Table 4-2. These include target test values, which the irradiation experiment needs to approximately reproduce in order to ensure regime appropriate conditions in the experiment, and reference conditions which provide additional information about prototypic conditions in MURR. 
Table 4-2. Nominal Thermal-Hydraulic Conditions for Prototypic MURR Element.

\begin{tabular}{|c|c|c|}
\hline & Condition & Value \\
\hline $\begin{array}{l}\text { Target Test } \\
\text { Value }\end{array}$ & Coolant Inlet Flow Rate & $0.02957 \mathrm{~m}^{3} / \mathrm{s}(468.8 \mathrm{gpm})$ \\
\hline \multirow{6}{*}{$\begin{array}{l}\text { Reference } \\
\text { Conditions }\end{array}$} & Coolant Inlet Temperature & $48.9^{\circ} \mathrm{C}\left(120^{\circ} \mathrm{F}\right)$ \\
\hline & $\begin{array}{l}\text { Total Coolant Flow Area } \\
\text { (cross-sectional, assumes } \\
\text { average end channels from } \\
\text { Table 4.1, but not } \\
\text { inter-element spacing) }\end{array}$ & $4.33 \mathrm{E}-3 \mathrm{~m}^{2}\left(4.66 \mathrm{E}-2 \mathrm{ft}^{2}\right)$ \\
\hline & $\begin{array}{l}\text { Inter-Element Spacing Area } \\
\text { (both sides combined } \\
\text { covering } 0.040^{\prime \prime} \text { ) }\end{array}$ & $8.155 \mathrm{E}-5 \mathrm{~m}^{2}\left(8.778 \mathrm{E}-4 \mathrm{ft}^{2}\right)$ \\
\hline & $\begin{array}{c}\text { Channel-Averaged Inlet Flow } \\
\text { Velocity }\end{array}$ & $6.82 \mathrm{~m} / \mathrm{s}(22.38 \mathrm{ft} / \mathrm{s})$ \\
\hline & $\begin{array}{l}\text { Maximum Cladding Surface } \\
\text { Temperature }\end{array}$ & $115^{\circ} \mathrm{C}\left(239^{\circ} \mathrm{F}\right)$ \\
\hline & $\begin{array}{l}\text { Maximum Fuel Centerline } \\
\text { Temperature }\end{array}$ & $149^{\circ} \mathrm{C}\left(300^{\circ} \mathrm{F}\right)$ \\
\hline
\end{tabular}

Regarding the irradiation design parameters, the set of target test values for the MURR DDE, as well as the maxima of selected auxiliary data, are summarized for all plates in the LEU element in Tables 4-3 to 4-5.

While the set of irradiation parameters presented here provide data on select plates of interest to fuel irradiation experiments, it should be noted that further data may be useful among the wide variety of individual plate irradiation conditions that would be present in MURR LEU cores. Thus, further cooperative work should continue between the Reactor Conversion, including MURR, and the Fuel Fabrication and Fuel Qualification Pillars to ensure that appropriate data is available to plan irradiations which include maximum local conditions, as well as anticipated gradients from withinplate distributions of irradiation parameters relevant to fuel performance. 
Table 4-3. Summary of MUR DDE Target Test Values for Plates 1 Through 8.

\begin{tabular}{|c|c|c|c|c|c|c|c|c|}
\hline & Plate 1 & Plate 2 & Plate 3 & Plate 4 & Plate 5 & Plate 6 & Plate 7 & Plate 8 \\
\hline \multicolumn{9}{|l|}{ Design Parameter } \\
\hline $\begin{array}{l}\text { Local EOL Fission Density (total } \\
\text { fissions } / \mathrm{cm}^{3} \text { from all isotopes) }\end{array}$ & $3.37 \mathrm{E}+21$ & $2.79 \mathrm{E}+21$ & $2.25 \mathrm{E}+21$ & $1.85 \mathrm{E}+21$ & $1.62 \mathrm{E}+21$ & $1.48 \mathrm{E}+21$ & $1.35 \mathrm{E}+21$ & $1.27 \mathrm{E}+21$ \\
\hline \multicolumn{9}{|l|}{$\begin{array}{l}\text { Maximum Local Power Density } \\
\left(\mathrm{kW} / \mathrm{cm}^{3}\right)\end{array}$} \\
\hline Fresh Element, No Xe & 14.40 & 10.61 & 8.34 & 6.70 & 5.89 & 5.31 & 4.95 & 4.75 \\
\hline Fresh Element, Eq. Xe & 12.78 & 9.39 & 7.26 & 5.96 & 5.25 & 4.63 & 4.34 & 4.05 \\
\hline EOL Element, Eq. Xe & 8.55 & 7.48 & 6.17 & 5.20 & 4.65 & 4.28 & 3.95 & 3.87 \\
\hline \multicolumn{9}{|l|}{ Auxiliary Data } \\
\hline \multicolumn{9}{|l|}{ Maximum Local Heat Flux $\left(W / \mathrm{cm}^{2}\right)$} \\
\hline Fresh Element, No Xe & 164.6 & 161.7 & 169.5 & 170.1 & 149.6 & 134.8 & 125.8 & 120.8 \\
\hline Fresh Element, Eq. Xe & 146.1 & 143.2 & 147.4 & 151.4 & 133.2 & 117.7 & 110.1 & 102.9 \\
\hline EOL Element, Eq. Xe & 97.7 & 114.0 & 125.3 & 132.1 & 118.0 & 108.8 & 100.3 & 98.3 \\
\hline \multicolumn{9}{|l|}{ Maximum Fuel Temperature $\left({ }^{\circ} \mathrm{C}\right)$} \\
\hline Fresh Element, No Xe & 113.0 & 117.3 & 121.8 & 124.5 & 116.3 & 110.6 & 106.8 & 103.6 \\
\hline Fresh Element, Eq. Xe & 106.4 & 109.7 & 113.5 & 116.1 & 108.9 & 104.0 & 99.5 & 97.6 \\
\hline EOL Element, No Xe & 109.1 & 122.8 & 133.1 & 140.7 & 133.3 & 125.5 & 121.0 & 117.6 \\
\hline EOL Element, Eq. Xe & 102.5 & 114.7 & 123.9 & 130.8 & 123.1 & 116.8 & 112.0 & 109.3 \\
\hline \multicolumn{9}{|c|}{ Maximum Plate Surface Temperature $\left({ }^{\circ} \mathrm{C}\right)$} \\
\hline Fresh Element, No Xe & 101.1 & 103.4 & 105.3 & 105.8 & 100.2 & 96.1 & 93.1 & 90.6 \\
\hline Fresh Element, Eq. Xe & 96.0 & 97.5 & 99.1 & 99.8 & 94.7 & 91.3 & 88.0 & 86.4 \\
\hline EOL Element, No Xe & 89.4 & 96.4 & 101.1 & 103.3 & 99.5 & 95.7 & 92.8 & 91.0 \\
\hline EOL Element, Eq. Xe & 84.9 & 91.6 & 95.7 & 97.7 & 93.8 & 90.2 & 87.8 & 85.9 \\
\hline
\end{tabular}


Table 4-4. Summary of MURR DDE Target Test Values for Plates 9 Through 16.

\begin{tabular}{|c|c|c|c|c|c|c|c|c|}
\hline & Plate 9 & Plate 10 & Plate 11 & Plate 12 & Plate 13 & Plate 14 & Plate 15 & Plate 16 \\
\hline \multicolumn{9}{|l|}{ Design Parameter } \\
\hline $\begin{array}{l}\text { Local EOL Fission Density (total } \\
\text { fissions } / \mathrm{cm}^{3} \text { from all isotopes) }\end{array}$ & $1.22 \mathrm{E}+21$ & $1.17 \mathrm{E}+21$ & $1.14 \mathrm{E}+21$ & $1.13 E+21$ & $1.13 \mathrm{E}+21$ & $1.13 \mathrm{E}+21$ & $1.14 \mathrm{E}+21$ & $1.16 \mathrm{E}+21$ \\
\hline \multicolumn{9}{|l|}{$\begin{array}{l}\text { Maximum Local Power Density } \\
\left(\mathrm{kW} / \mathrm{cm}^{3}\right)\end{array}$} \\
\hline Fresh Element, No Xe & 4.52 & 4.35 & 4.26 & 4.23 & 4.14 & 4.16 & 4.21 & 4.27 \\
\hline Fresh Element, Eq. Xe & 3.93 & 3.84 & 3.73 & 3.63 & 3.68 & 3.54 & 3.61 & 3.78 \\
\hline EOL Element, Eq. Xe & 3.68 & 3.54 & 3.42 & 3.43 & 3.36 & 3.41 & 3.50 & 3.56 \\
\hline \multicolumn{9}{|l|}{ Auxiliary Data } \\
\hline \multicolumn{9}{|l|}{ Maximum Local Heat Flux $\left(W / \mathrm{cm}^{2}\right)$} \\
\hline Fresh Element, No Xe & 114.7 & 110.5 & 108.3 & 107.4 & 105.1 & 105.7 & 106.8 & 108.6 \\
\hline Fresh Element, Eq. Xe & 99.8 & 97.6 & 94.8 & 92.2 & 93.6 & 90.0 & 91.8 & 96.0 \\
\hline EOL Element, Eq. Xe & 93.4 & 89.9 & 86.7 & 87.2 & 85.2 & 86.7 & 88.9 & 90.3 \\
\hline \multicolumn{9}{|l|}{ Maximum Fuel Temperature $\left({ }^{\circ} \mathrm{C}\right)$} \\
\hline Fresh Element, No Xe & 101.4 & 100.3 & 98.7 & 98.5 & 97.9 & 97.4 & 97.4 & 98.2 \\
\hline Fresh Element, Eq. Xe & 95.0 & 94.1 & 92.9 & 92.4 & 91.9 & 92.4 & 92.7 & 93.1 \\
\hline EOL Element, No Xe & 116.0 & 113.0 & 111.6 & 110.9 & 109.7 & 109.6 & 110.5 & 111.0 \\
\hline EOL Element, Eq. Xe & 106.9 & 105.1 & 103.7 & 103.6 & 103.0 & 103.1 & 103.7 & 104.9 \\
\hline \multicolumn{9}{|c|}{ Maximum Plate Surface Temperature $\left({ }^{\circ} \mathrm{C}\right)$} \\
\hline Fresh Element, No Xe & 89.2 & 88.3 & 87.1 & 86.9 & 86.4 & 86.2 & 86.0 & 86.6 \\
\hline Fresh Element, Eq. Xe & 84.4 & 83.8 & 82.8 & 82.5 & 82.1 & 82.3 & 82.7 & 82.8 \\
\hline EOL Element, No Xe & 89.7 & 87.9 & 87.2 & 86.6 & 86.1 & 85.8 & 86.0 & 86.2 \\
\hline EOL Element, Eq. Xe & 84.7 & 83.5 & 82.6 & 82.6 & 82.2 & 82.0 & 82.4 & 83.3 \\
\hline
\end{tabular}


Table 4-5. Summary of MURR DDE Target Test Values for Plates 17 Through 23 and the Element Maximum.

\begin{tabular}{|c|c|c|c|c|c|c|c|c|}
\hline & Plate 17 & Plate 18 & Plate 19 & Plate 20 & Plate 21 & Plate 22 & Plate 23 & Element \\
\hline \multicolumn{9}{|l|}{ Design Parameter } \\
\hline $\begin{array}{l}\text { Local EOL Fission Density (total } \\
\text { fissions } / \mathrm{cm}^{3} \text { from all isotopes) }\end{array}$ & $1.20 \mathrm{E}+21$ & $1.26 \mathrm{E}+21$ & $1.35 \mathrm{E}+21$ & $1.48 \mathrm{E}+21$ & $1.70 \mathrm{E}+21$ & $2.07 \mathrm{E}+21$ & $2.59 \mathrm{E}+21$ & $3.37 \mathrm{E}+21$ \\
\hline \multicolumn{9}{|l|}{$\begin{array}{l}\text { Maximum Local Power Density } \\
\left(\mathrm{kW} / \mathrm{cm}^{3}\right)\end{array}$} \\
\hline Fresh Element, No Xe & 4.30 & 4.54 & 4.87 & 5.25 & 6.01 & 7.40 & 9.69 & 14.40 \\
\hline Fresh Element, Eq. Xe & 3.79 & 4.07 & 4.26 & 4.74 & 5.27 & 6.65 & 8.88 & 12.78 \\
\hline EOL Element, Eq. Xe & 3.58 & 3.83 & 4.02 & 4.38 & 4.84 & 5.83 & 6.96 & 8.50 \\
\hline \multicolumn{9}{|l|}{ Auxiliary Data } \\
\hline \multicolumn{9}{|l|}{ Maximum Local Heat Flux $\left(W / \mathrm{cm}^{2}\right)$} \\
\hline Fresh Element, No Xe & 109.1 & 115.4 & 123.8 & 133.2 & 152.7 & 187.9 & 209.3 & 209.3 \\
\hline Fresh Element, Eq. Xe & 96.1 & 103.4 & 108.1 & 120.4 & 133.9 & 169.0 & 191.8 & 191.8 \\
\hline EOL Element, Eq. Xe & 90.9 & 97.3 & 102.2 & 111.2 & 122.9 & 148.2 & 150.2 & 150.2 \\
\hline \multicolumn{9}{|l|}{ Maximum Fuel Temperature $\left({ }^{\circ} \mathrm{C}\right)$} \\
\hline Fresh Element, No Xe & 99.1 & 101.0 & 103.6 & 108.3 & 115.6 & 127.3 & 131.9 & 131.9 \\
\hline Fresh Element, Eq. Xe & 94.4 & 96.7 & 99.0 & 104.4 & 111.0 & 123.0 & 127.1 & 127.1 \\
\hline EOL Element, No Xe & 112.5 & 114.8 & 118.9 & 124.1 & 132.9 & 145.8 & 142.7 & 145.8 \\
\hline EOL Element, Eq. Xe & 106.7 & 108.5 & 112.2 & 117.9 & 125.7 & 137.2 & 134.7 & 137.2 \\
\hline \multicolumn{9}{|c|}{ Maximum Plate Surface Temperature $\left({ }^{\circ} \mathrm{C}\right)$} \\
\hline Fresh Element, No Xe & 87.2 & 88.8 & 90.5 & 94.0 & 99.1 & 107.4 & 111.5 & 111.5 \\
\hline Fresh Element, Eq. Xe & 83.9 & 85.5 & 87.4 & 91.2 & 96.1 & 104.4 & 108.5 & 108.5 \\
\hline EOL Element, No Xe & 87.5 & 88.7 & 91.0 & 93.9 & 98.6 & 105.2 & 104.6 & 105.2 \\
\hline EOL Element, Eq. Xe & 84.4 & 85.5 & 87.7 & 91.1 & 95.7 & 101.6 & 101.4 & 101.6 \\
\hline
\end{tabular}




\section{ACKNOWLEDGEMENT}

This work was sponsored by the U.S. Department of Energy, Office of Material Management and Minimization in the U.S. National Nuclear Security Administration Office of Defense Nuclear Nonproliferation under Contract DE-AC02-06CH11357. 


\section{REFERENCES}

1. Stillman, J. A., et al, Technical Basis in Support of the Conversion of the University of Missouri Research Reactor (MURR) Core from Highly-Enriched to Low-Enriched Uranium - Core Neutron Physics, ANL/RERTR/TM-12-30, Argonne National Laboratory, September 2012.

2. Feldman, E. E., et al., Technical Basis in Support of the Conversion of the University of Missouri Research Reactor (MURR) Core from Highly-Enriched to Low-Enriched Uranium - Steady-State Thermal-Hydraulic Analysis, ANL/RERTR/TM-12-37, Argonne National Laboratory, 2012.

3. Stillman, J. A., et al, Irradiation Experiment Conceptual Design Parameters for MURR LEU U-Mo Fuel Conversion, Argonne National Laboratory, ANL/RERTR/TM-13-1 Rev. 1, June 2013.

4. Low-Enriched Uranium Conversion Preliminary Safety Analysis Report for the University of Missouri Research Reactor, University of Missouri Research Reactor, August 2017.

5. Glagolenko, I., Mini-plate (MP-1) Irradiation Experiment Execution Plan, Idaho National Laboratory, PLN-4891, February 11, 2016.

6. Letter Request to USNRC, "Application for Unique Purpose Exemption from Conversion from HEU Fuel, Facility License No. R-103," University of Missouri Research Reactor, September 1986; and supplemented by Letter Response to USNRC Request for Additional Information Supporting the Unique Purpose Exemption Request, February 1987.

7. University of Missouri at Columbia Test Research Training Reactor 4 MURR U-10Mo LEU Fuel Element Assembly and Details, Drawing 2852, University of Missouri Research Reactor (MURR), June 5, 2018.

8. Peters, Nickie and Kutikkad, Kiratadas, Spatial Distribution of the Effective Fission Energy Deposition Within the MURR Core: HEU vs. LEU Fuel Comparison, University of Missouri, 2012.

9. Tzanos, C. and Stillman, J., Effect of Lateral Conduction on the Azimuthal Distribution of Heat Flux in the Hot Plate of a Research Reactor, ANS Summer Meeting, June 2012.

10. Stillman, J. A., et al, Safety Analysis of the Mo-99 Production Upgrade to the University of Missouri Research Reactor (MURR) with Highly Enriched and Low-Enriched Uranium Fuel, ANL/RTR/TM-18/16, Argonne National Laboratory, October 2019.

11. Kalimullah, M., Olson, A. P., and Feldman, E. E., A User's Guide to the PLTEMP/ANL Code, ANL/RTR/TM-18/17, Version 4.3, Rev. 0, Argonne National Laboratory, November, 2018.

12. Stillman, J. A., et al, Accident Analyses for Conversion of the University of Missouri Research Reactor (MURR) from Highly-Enriched to Low-Enriched Uranium, ANL/GTRI/TM-14/5, Revision 1, Argonne National Laboratory, February 2017.

13. Kim, Y. S. and Hofman, G. L., Fission Product Induced Swelling of U-Mo Alloy Fuel, Journal of Nuclear Materials 419 (2011) 291-301.

14. Hofman, G. L, et al, Fission Induced Swelling and Creep of Uranium-Molybdenum Alloy Fuel, 13th International Topical Meeting on Research Reactor Fuel Management (RRFM09), Vienna, Austria, March 22-25, 2009. 
15. J. C. Griess, H. C. Savage, and J. L. English, Effect of Heat Flux on the Corrosion of Aluminum by Water. Part IV. Tests Relative to the Advanced Test Reactor and Correlation with Previous Results, ORNL3541, Oak Ridge National Laboratory, February 1964. 


\section{APPENDIX A: Plate Specific Fission Density Data in MURR LEU Element}


Table A- 1. Fission Density Data (fissions $/ \mathrm{cm}^{3}$ ) in Fuel Plate 1 in Element Discharged from Reference Mixed Burnup Core: FT loaded with typical samples.

\section{Fuel core width $\quad 1.690$ inches Fuel core thickness 9 mil}

\begin{tabular}{|c|c|c|c|c|c|c|c|c|c|}
\hline $\begin{array}{c}\text { Axial } \\
\text { position } \\
\text { from top of } \\
\text { fuel (inches) }\end{array}$ & $\begin{array}{c}0.000 \text { to } \\
0.197 \\
\text { inches }\end{array}$ & $\begin{array}{c}0.197 \text { to } \\
0.394 \\
\text { inches }\end{array}$ & $\begin{array}{c}0.394 \text { to } \\
0.591 \\
\text { inches }\end{array}$ & $\begin{array}{c}0.591 \text { to } \\
0.760 \\
\text { inches }\end{array}$ & $\begin{array}{c}0.760 \text { to } \\
0.929 \\
\text { inches }\end{array}$ & $\begin{array}{c}0.929 \text { to } \\
1.099 \\
\text { inches }\end{array}$ & $\begin{array}{c}1.099 \text { to } \\
1.296 \\
\text { inches }\end{array}$ & $\begin{array}{l}1.296 \text { to } \\
1.493 \\
\text { inches }\end{array}$ & $\begin{array}{c}1.493 \text { to } \\
1.690 \\
\text { inches }\end{array}$ \\
\hline 0.5 & $1.67 \mathrm{E}+21$ & $1.63 \mathrm{E}+21$ & $1.59 \mathrm{E}+21$ & $1.63 \mathrm{E}+21$ & $1.50 \mathrm{E}+21$ & $1.62 \mathrm{E}+21$ & $1.62 \mathrm{E}+21$ & $1.62 \mathrm{E}+21$ & $1.70 \mathrm{E}+21$ \\
\hline 1.5 & $1.73 E+21$ & $1.66 \mathrm{E}+21$ & $1.64 \mathrm{E}+21$ & $1.62 \mathrm{E}+21$ & $1.56 \mathrm{E}+21$ & $1.65 E+21$ & $1.62 \mathrm{E}+21$ & $1.65 \mathrm{E}+21$ & $1.70 \mathrm{E}+21$ \\
\hline 2.5 & $1.92 \mathrm{E}+21$ & $1.84 \mathrm{E}+21$ & $1.84 \mathrm{E}+21$ & $1.86 \mathrm{E}+21$ & $1.77 \mathrm{E}+21$ & $1.85 \mathrm{E}+21$ & $1.84 \mathrm{E}+21$ & $1.85 \mathrm{E}+21$ & $1.92 \mathrm{E}+21$ \\
\hline 3.5 & $2.20 \mathrm{E}+21$ & $2.14 \mathrm{E}+21$ & $2.14 \mathrm{E}+21$ & $2.13 \mathrm{E}+21$ & $2.04 \mathrm{E}+21$ & $2.13 E+21$ & $2.14 \mathrm{E}+21$ & $2.16 \mathrm{E}+21$ & $2.22 \mathrm{E}+21$ \\
\hline 4.5 & $2.41 E+21$ & $2.36 \mathrm{E}+21$ & $2.33 E+21$ & $2.31 \mathrm{E}+21$ & $2.23 E+21$ & $2.33 E+21$ & $2.34 \mathrm{E}+21$ & $2.35 \mathrm{E}+21$ & $2.43 E+21$ \\
\hline 5.5 & $2.64 \mathrm{E}+21$ & $2.56 \mathrm{E}+21$ & $2.55 E+21$ & $2.55 \mathrm{E}+21$ & $2.40 \mathrm{E}+21$ & $2.55 E+21$ & $2.52 \mathrm{E}+21$ & $2.56 \mathrm{E}+21$ & $2.65 \mathrm{E}+21$ \\
\hline 6.5 & $2.78 \mathrm{E}+21$ & $2.64 \mathrm{E}+21$ & $2.63 E+21$ & $2.64 \mathrm{E}+21$ & $2.51 \mathrm{E}+21$ & $2.67 \mathrm{E}+21$ & $2.65 E+21$ & $2.68 \mathrm{E}+21$ & $2.74 \mathrm{E}+21$ \\
\hline 7.5 & $2.89 \mathrm{E}+21$ & $2.80 \mathrm{E}+21$ & $2.77 \mathrm{E}+21$ & $2.79 \mathrm{E}+21$ & $2.62 \mathrm{E}+21$ & $2.75 \mathrm{E}+21$ & $2.77 \mathrm{E}+21$ & $2.82 \mathrm{E}+21$ & $2.87 \mathrm{E}+21$ \\
\hline 8.5 & $3.00 \mathrm{E}+21$ & $2.91 \mathrm{E}+21$ & $2.87 E+21$ & $2.86 \mathrm{E}+21$ & $2.77 E+21$ & $2.90 \mathrm{E}+21$ & $2.86 \mathrm{E}+21$ & $2.91 \mathrm{E}+21$ & $2.95 \mathrm{E}+21$ \\
\hline 9.5 & $3.17 E+21$ & $3.07 \mathrm{E}+21$ & $3.02 E+21$ & $3.02 \mathrm{E}+21$ & $2.90 \mathrm{E}+21$ & $3.05 E+21$ & $3.04 \mathrm{E}+21$ & $3.07 \mathrm{E}+21$ & $3.16 \mathrm{E}+21$ \\
\hline 10.5 & $3.24 E+21$ & $3.16 \mathrm{E}+21$ & $3.11 E+21$ & $3.12 \mathrm{E}+21$ & $3.00 \mathrm{E}+21$ & $3.14 \mathrm{E}+21$ & $3.10 \mathrm{E}+21$ & $3.14 \mathrm{E}+21$ & $3.23 E+21$ \\
\hline 11.5 & $3.34 \mathrm{E}+21$ & $3.27 \mathrm{E}+21$ & $3.20 \mathrm{E}+21$ & $3.22 \mathrm{E}+21$ & $3.05 E+21$ & $3.20 \mathrm{E}+21$ & $3.17 \mathrm{E}+21$ & $3.26 \mathrm{E}+21$ & $3.32 \mathrm{E}+21$ \\
\hline 12.5 & $3.37 E+21$ & $3.26 \mathrm{E}+21$ & $3.21 \mathrm{E}+21$ & $3.23 E+21$ & $3.05 E+21$ & $3.25 E+21$ & $3.24 \mathrm{E}+21$ & $3.25 \mathrm{E}+21$ & $3.35 \mathrm{E}+21$ \\
\hline 13.5 & $3.33 E+21$ & $3.20 \mathrm{E}+21$ & $3.17 E+21$ & $3.18 \mathrm{E}+21$ & $3.09 E+21$ & $3.23 E+21$ & $3.19 E+21$ & $3.25 \mathrm{E}+21$ & $3.33 E+21$ \\
\hline 14.5 & $3.26 \mathrm{E}+21$ & $3.19 \mathrm{E}+21$ & $3.13 E+21$ & $3.14 \mathrm{E}+21$ & $3.00 \mathrm{E}+21$ & $3.18 \mathrm{E}+21$ & $3.15 E+21$ & $3.20 \mathrm{E}+21$ & $3.25 \mathrm{E}+21$ \\
\hline 15.5 & $3.09 \mathrm{E}+21$ & $3.03 \mathrm{E}+21$ & $2.99 \mathrm{E}+21$ & $3.03 \mathrm{E}+21$ & $2.88 \mathrm{E}+21$ & $3.03 E+21$ & $2.95 \mathrm{E}+21$ & $3.03 E+21$ & $3.12 \mathrm{E}+21$ \\
\hline 16.5 & $2.99 \mathrm{E}+21$ & $2.88 \mathrm{E}+21$ & $2.83 E+21$ & $2.87 \mathrm{E}+21$ & $2.77 \mathrm{E}+21$ & $2.90 \mathrm{E}+21$ & $2.86 \mathrm{E}+21$ & $2.90 \mathrm{E}+21$ & $2.98 \mathrm{E}+21$ \\
\hline 17.5 & $2.76 \mathrm{E}+21$ & $2.70 \mathrm{E}+21$ & $2.64 \mathrm{E}+21$ & $2.68 \mathrm{E}+21$ & $2.56 \mathrm{E}+21$ & $2.68 \mathrm{E}+21$ & $2.69 \mathrm{E}+21$ & $2.69 \mathrm{E}+21$ & $2.76 \mathrm{E}+21$ \\
\hline 18.5 & $2.62 E+21$ & $2.53 \mathrm{E}+21$ & $2.50 \mathrm{E}+21$ & $2.51 \mathrm{E}+21$ & $2.38 \mathrm{E}+21$ & $2.51 \mathrm{E}+21$ & $2.51 \mathrm{E}+21$ & $2.54 \mathrm{E}+21$ & $2.64 \mathrm{E}+21$ \\
\hline 19.5 & $2.43 E+21$ & $2.35 \mathrm{E}+21$ & $2.31 \mathrm{E}+21$ & $2.34 \mathrm{E}+21$ & $2.24 \mathrm{E}+21$ & $2.35 E+21$ & $2.34 \mathrm{E}+21$ & $2.35 \mathrm{E}+21$ & $2.43 E+21$ \\
\hline 20.5 & $2.29 \mathrm{E}+21$ & $2.23 \mathrm{E}+21$ & $2.18 \mathrm{E}+21$ & $2.21 \mathrm{E}+21$ & $2.11 \mathrm{E}+21$ & $2.18 \mathrm{E}+21$ & $2.18 \mathrm{E}+21$ & $2.24 \mathrm{E}+21$ & $2.28 \mathrm{E}+21$ \\
\hline 21.5 & $2.07 \mathrm{E}+21$ & $2.03 E+21$ & $1.99 \mathrm{E}+21$ & $1.98 \mathrm{E}+21$ & $1.90 \mathrm{E}+21$ & $2.02 \mathrm{E}+21$ & $1.99 \mathrm{E}+21$ & $2.03 E+21$ & $2.08 \mathrm{E}+21$ \\
\hline 22.5 & $1.93 E+21$ & $1.87 \mathrm{E}+21$ & $1.83 E+21$ & $1.86 \mathrm{E}+21$ & $1.76 \mathrm{E}+21$ & $1.85 \mathrm{E}+21$ & $1.87 \mathrm{E}+21$ & $1.87 \mathrm{E}+21$ & $1.92 \mathrm{E}+21$ \\
\hline 23.5 & $2.02 E+21$ & $1.93 \mathrm{E}+21$ & $1.92 \mathrm{E}+21$ & $1.93 \mathrm{E}+21$ & $1.84 \mathrm{E}+21$ & $1.90 \mathrm{E}+21$ & $1.92 \mathrm{E}+21$ & $1.93 \mathrm{E}+21$ & $2.01 \mathrm{E}+21$ \\
\hline Ax. Avg. & $2.63 E+21$ & $2.55 \mathrm{E}+21$ & $2.52 E+21$ & $2.53 E+21$ & $2.41 \mathrm{E}+21$ & $2.54 \mathrm{E}+21$ & $2.52 \mathrm{E}+21$ & $2.56 \mathrm{E}+21$ & $2.63 E+21$ \\
\hline
\end{tabular}


Table A-2. Fission Density Data (fissions/ $\mathrm{cm}^{3}$ ) in Fuel Plate 2 in Element Discharged from Reference Mixed Burnup Core: FT loaded with typical samples.

Fuel core width 1.797 inches Fuel core thickness 12 mil

\begin{tabular}{|c|c|c|c|c|c|c|c|c|c|}
\hline $\begin{array}{c}\text { Axial } \\
\text { position } \\
\text { from top of } \\
\text { fuel (inches) }\end{array}$ & $\begin{array}{c}0.000 \text { to } \\
0.197 \\
\text { inches }\end{array}$ & $\begin{array}{c}0.197 \text { to } \\
0.394 \\
\text { inches }\end{array}$ & $\begin{array}{c}0.394 \text { to } \\
0.591 \\
\text { inches }\end{array}$ & $\begin{array}{c}0.591 \text { to } \\
0.796 \\
\text { inches }\end{array}$ & $\begin{array}{c}0.796 \text { to } \\
1.001 \\
\text { inches }\end{array}$ & $\begin{array}{l}1.001 \text { to } \\
1.207 \\
\text { inches }\end{array}$ & $\begin{array}{l}1.207 \text { to } \\
1.403 \\
\text { inches }\end{array}$ & $\begin{array}{l}1.403 \text { to } \\
1.600 \\
\text { inches }\end{array}$ & $\begin{array}{l}1.600 \text { to } \\
1.797 \\
\text { inches }\end{array}$ \\
\hline 0.5 & $1.40 \mathrm{E}+21$ & $1.25 \mathrm{E}+21$ & $1.22 \mathrm{E}+21$ & $1.24 \mathrm{E}+21$ & $1.19 \mathrm{E}+21$ & $1.24 \mathrm{E}+21$ & $1.25 \mathrm{E}+21$ & $1.28 \mathrm{E}+21$ & $1.38 \mathrm{E}+21$ \\
\hline 1.5 & $1.36 \mathrm{E}+21$ & $1.25 \mathrm{E}+21$ & $1.21 \mathrm{E}+21$ & $1.18 \mathrm{E}+21$ & $1.14 \mathrm{E}+21$ & $1.20 \mathrm{E}+21$ & $1.20 \mathrm{E}+21$ & $1.24 \mathrm{E}+21$ & $1.36 \mathrm{E}+21$ \\
\hline 2.5 & $1.51 \mathrm{E}+21$ & $1.42 \mathrm{E}+21$ & $1.37 \mathrm{E}+21$ & $1.38 \mathrm{E}+21$ & $1.30 \mathrm{E}+21$ & $1.38 \mathrm{E}+21$ & $1.36 \mathrm{E}+21$ & $1.43 \mathrm{E}+21$ & $1.52 \mathrm{E}+21$ \\
\hline 3.5 & $1.77 \mathrm{E}+21$ & $1.65 \mathrm{E}+21$ & $1.61 \mathrm{E}+21$ & $1.60 \mathrm{E}+21$ & $1.55 \mathrm{E}+21$ & $1.59 \mathrm{E}+21$ & $1.62 \mathrm{E}+21$ & $1.65 \mathrm{E}+21$ & $1.77 \mathrm{E}+21$ \\
\hline 4.5 & $1.96 \mathrm{E}+21$ & $1.82 \mathrm{E}+21$ & $1.77 \mathrm{E}+21$ & $1.77 \mathrm{E}+21$ & $1.70 \mathrm{E}+21$ & $1.77 \mathrm{E}+21$ & $1.78 \mathrm{E}+21$ & $1.81 \mathrm{E}+21$ & $1.93 \mathrm{E}+21$ \\
\hline 5.5 & $2.14 E+21$ & $1.99 \mathrm{E}+21$ & $1.93 E+21$ & $1.95 E+21$ & $1.86 \mathrm{E}+21$ & $1.94 E+21$ & $1.92 E+21$ & $2.00 \mathrm{E}+21$ & $2.16 \mathrm{E}+21$ \\
\hline 6.5 & $2.27 \mathrm{E}+21$ & $2.09 \mathrm{E}+21$ & $2.04 \mathrm{E}+21$ & $2.06 \mathrm{E}+21$ & $1.95 \mathrm{E}+21$ & $2.06 \mathrm{E}+21$ & $2.06 \mathrm{E}+21$ & $2.10 \mathrm{E}+21$ & $2.28 \mathrm{E}+21$ \\
\hline 7.5 & $2.40 \mathrm{E}+21$ & $2.21 \mathrm{E}+21$ & $2.15 E+21$ & $2.16 \mathrm{E}+21$ & $2.06 \mathrm{E}+21$ & $2.15 \mathrm{E}+21$ & $2.15 \mathrm{E}+21$ & $2.21 \mathrm{E}+21$ & $2.39 \mathrm{E}+21$ \\
\hline 8.5 & $2.46 \mathrm{E}+21$ & $2.32 \mathrm{E}+21$ & $2.26 \mathrm{E}+21$ & $2.25 \mathrm{E}+21$ & $2.15 E+21$ & $2.25 \mathrm{E}+21$ & $2.25 \mathrm{E}+21$ & $2.31 \mathrm{E}+21$ & $2.51 E+21$ \\
\hline 9.5 & $2.66 \mathrm{E}+21$ & $2.45 \mathrm{E}+21$ & $2.37 \mathrm{E}+21$ & $2.36 \mathrm{E}+21$ & $2.26 \mathrm{E}+21$ & $2.37 \mathrm{E}+21$ & $2.40 \mathrm{E}+21$ & $2.42 \mathrm{E}+21$ & $2.60 \mathrm{E}+21$ \\
\hline 10.5 & $2.67 E+21$ & $2.52 \mathrm{E}+21$ & $2.42 \mathrm{E}+21$ & $2.43 \mathrm{E}+21$ & $2.32 \mathrm{E}+21$ & $2.45 \mathrm{E}+21$ & $2.46 \mathrm{E}+21$ & $2.53 E+21$ & $2.73 E+21$ \\
\hline 11.5 & $2.79 E+21$ & $2.57 E+21$ & $2.51 E+21$ & $2.50 \mathrm{E}+21$ & $2.37 E+21$ & $2.52 \mathrm{E}+21$ & $2.50 \mathrm{E}+21$ & $2.56 \mathrm{E}+21$ & $2.76 \mathrm{E}+21$ \\
\hline 12.5 & $2.77 E+21$ & $2.57 \mathrm{E}+21$ & $2.51 \mathrm{E}+21$ & $2.51 \mathrm{E}+21$ & $2.41 \mathrm{E}+21$ & $2.52 \mathrm{E}+21$ & $2.52 \mathrm{E}+21$ & $2.59 \mathrm{E}+21$ & $2.77 E+21$ \\
\hline 13.5 & $2.75 E+21$ & $2.58 \mathrm{E}+21$ & $2.51 \mathrm{E}+21$ & $2.49 \mathrm{E}+21$ & $2.36 \mathrm{E}+21$ & $2.48 \mathrm{E}+21$ & $2.49 \mathrm{E}+21$ & $2.54 \mathrm{E}+21$ & $2.76 \mathrm{E}+21$ \\
\hline 14.5 & $2.71 \mathrm{E}+21$ & $2.50 \mathrm{E}+21$ & $2.44 \mathrm{E}+21$ & $2.41 \mathrm{E}+21$ & $2.32 \mathrm{E}+21$ & $2.44 \mathrm{E}+21$ & $2.44 \mathrm{E}+21$ & $2.52 \mathrm{E}+21$ & $2.70 \mathrm{E}+21$ \\
\hline 15.5 & $2.56 \mathrm{E}+21$ & $2.41 \mathrm{E}+21$ & $2.33 E+21$ & $2.34 \mathrm{E}+21$ & $2.22 \mathrm{E}+21$ & $2.33 \mathrm{E}+21$ & $2.34 \mathrm{E}+21$ & $2.40 \mathrm{E}+21$ & $2.56 \mathrm{E}+21$ \\
\hline 16.5 & $2.47 \mathrm{E}+21$ & $2.29 \mathrm{E}+21$ & $2.20 \mathrm{E}+21$ & $2.21 \mathrm{E}+21$ & $2.12 \mathrm{E}+21$ & $2.21 \mathrm{E}+21$ & $2.23 E+21$ & $2.30 \mathrm{E}+21$ & $2.48 \mathrm{E}+21$ \\
\hline 17.5 & $2.28 \mathrm{E}+21$ & $2.14 \mathrm{E}+21$ & $2.07 \mathrm{E}+21$ & $2.05 \mathrm{E}+21$ & $1.97 \mathrm{E}+21$ & $2.07 \mathrm{E}+21$ & $2.07 \mathrm{E}+21$ & $2.13 \mathrm{E}+21$ & $2.28 \mathrm{E}+21$ \\
\hline 18.5 & $2.17 \mathrm{E}+21$ & $2.02 \mathrm{E}+21$ & $1.95 \mathrm{E}+21$ & $1.96 \mathrm{E}+21$ & $1.83 \mathrm{E}+21$ & $1.94 \mathrm{E}+21$ & $1.94 \mathrm{E}+21$ & $1.99 \mathrm{E}+21$ & $2.17 \mathrm{E}+21$ \\
\hline 19.5 & $2.00 \mathrm{E}+21$ & $1.87 \mathrm{E}+21$ & $1.81 \mathrm{E}+21$ & $1.79 \mathrm{E}+21$ & $1.71 E+21$ & $1.80 \mathrm{E}+21$ & $1.82 \mathrm{E}+21$ & $1.86 \mathrm{E}+21$ & $1.97 \mathrm{E}+21$ \\
\hline 20.5 & $1.85 E+21$ & $1.74 \mathrm{E}+21$ & $1.68 \mathrm{E}+21$ & $1.66 \mathrm{E}+21$ & $1.59 E+21$ & $1.69 \mathrm{E}+21$ & $1.66 \mathrm{E}+21$ & $1.75 E+21$ & $1.86 \mathrm{E}+21$ \\
\hline 21.5 & $1.69 \mathrm{E}+21$ & $1.55 \mathrm{E}+21$ & $1.51 \mathrm{E}+21$ & $1.51 \mathrm{E}+21$ & $1.44 \mathrm{E}+21$ & $1.52 \mathrm{E}+21$ & $1.53 \mathrm{E}+21$ & $1.57 \mathrm{E}+21$ & $1.70 \mathrm{E}+21$ \\
\hline 22.5 & $1.52 \mathrm{E}+21$ & $1.41 \mathrm{E}+21$ & $1.37 \mathrm{E}+21$ & $1.38 \mathrm{E}+21$ & $1.31 \mathrm{E}+21$ & $1.40 \mathrm{E}+21$ & $1.39 \mathrm{E}+21$ & $1.44 \mathrm{E}+21$ & $1.56 \mathrm{E}+21$ \\
\hline 23.5 & $1.67 \mathrm{E}+21$ & $1.52 \mathrm{E}+21$ & $1.49 \mathrm{E}+21$ & $1.48 \mathrm{E}+21$ & $1.42 \mathrm{E}+21$ & $1.47 \mathrm{E}+21$ & $1.48 \mathrm{E}+21$ & $1.53 \mathrm{E}+21$ & $1.68 \mathrm{E}+21$ \\
\hline Ax. Avg. & $2.16 \mathrm{E}+21$ & $2.01 \mathrm{E}+21$ & $1.95 \mathrm{E}+21$ & $1.95 \mathrm{E}+21$ & $1.86 \mathrm{E}+21$ & $1.95 \mathrm{E}+21$ & $1.95 \mathrm{E}+21$ & $2.01 \mathrm{E}+21$ & $2.16 \mathrm{E}+21$ \\
\hline
\end{tabular}


Table A-3. Fission Density Data (fissions $/ \mathrm{cm}^{3}$ ) in Fuel Plate 3 in Element Discharged from Reference Mixed Burnup Core: FT loaded with typical samples.

\section{Fuel core width 1.905 inches Fuel core thickness 16 mil}

\begin{tabular}{|c|c|c|c|c|c|c|c|c|c|}
\hline $\begin{array}{c}\text { Axial } \\
\text { position } \\
\text { from top of } \\
\text { fuel (inches) }\end{array}$ & $\begin{array}{c}0.000 \text { to } \\
0.197 \\
\text { inches }\end{array}$ & $\begin{array}{c}0.197 \text { to } \\
0.394 \\
\text { inches }\end{array}$ & $\begin{array}{c}0.394 \text { to } \\
0.591 \\
\text { inches }\end{array}$ & $\begin{array}{c}0.591 \text { to } \\
0.832 \\
\text { inches }\end{array}$ & $\begin{array}{c}0.832 \text { to } \\
1.073 \\
\text { inches }\end{array}$ & $\begin{array}{l}1.073 \text { to } \\
1.314 \\
\text { inches }\end{array}$ & $\begin{array}{l}1.314 \text { to } \\
1.511 \\
\text { inches }\end{array}$ & $\begin{array}{l}1.511 \text { to } \\
1.708 \\
\text { inches }\end{array}$ & $\begin{array}{l}1.708 \text { to } \\
1.905 \\
\text { inches }\end{array}$ \\
\hline 0.5 & $1.14 \mathrm{E}+21$ & $9.85 \mathrm{E}+20$ & $9.38 \mathrm{E}+20$ & $9.26 \mathrm{E}+20$ & $8.92 \mathrm{E}+20$ & $9.44 \mathrm{E}+20$ & $9.50 \mathrm{E}+20$ & $9.74 \mathrm{E}+20$ & $1.15 \mathrm{E}+21$ \\
\hline 1.5 & $1.09 \mathrm{E}+21$ & $9.27 \mathrm{E}+20$ & $8.84 \mathrm{E}+20$ & $8.74 \mathrm{E}+20$ & $8.37 E+20$ & $8.77 E+20$ & $8.68 E+20$ & $9.34 \mathrm{E}+20$ & $1.08 \mathrm{E}+21$ \\
\hline 2.5 & $1.22 \mathrm{E}+21$ & $1.09 \mathrm{E}+21$ & $1.02 \mathrm{E}+21$ & $1.03 \mathrm{E}+21$ & $9.75 E+20$ & $1.01 \mathrm{E}+21$ & $1.03 E+21$ & $1.08 \mathrm{E}+21$ & $1.23 \mathrm{E}+21$ \\
\hline 3.5 & $1.40 \mathrm{E}+21$ & $1.24 \mathrm{E}+21$ & $1.21 \mathrm{E}+21$ & $1.18 \mathrm{E}+21$ & $1.13 E+21$ & $1.18 \mathrm{E}+21$ & $1.20 \mathrm{E}+21$ & $1.25 \mathrm{E}+21$ & $1.42 \mathrm{E}+21$ \\
\hline 4.5 & $1.57 \mathrm{E}+21$ & $1.39 \mathrm{E}+21$ & $1.34 \mathrm{E}+21$ & $1.33 \mathrm{E}+21$ & $1.27 E+21$ & $1.32 \mathrm{E}+21$ & $1.32 \mathrm{E}+21$ & $1.41 \mathrm{E}+21$ & $1.56 \mathrm{E}+21$ \\
\hline 5.5 & $1.72 \mathrm{E}+21$ & $1.53 \mathrm{E}+21$ & $1.46 \mathrm{E}+21$ & $1.45 \mathrm{E}+21$ & $1.39 \mathrm{E}+21$ & $1.45 \mathrm{E}+21$ & $1.47 \mathrm{E}+21$ & $1.54 \mathrm{E}+21$ & $1.72 \mathrm{E}+21$ \\
\hline 6.5 & $1.82 \mathrm{E}+21$ & $1.63 \mathrm{E}+21$ & $1.55 \mathrm{E}+21$ & $1.55 \mathrm{E}+21$ & $1.47 \mathrm{E}+21$ & $1.57 \mathrm{E}+21$ & $1.57 \mathrm{E}+21$ & $1.62 \mathrm{E}+21$ & $1.82 \mathrm{E}+21$ \\
\hline 7.5 & $1.94 \mathrm{E}+21$ & $1.71 \mathrm{E}+21$ & $1.65 E+21$ & $1.61 \mathrm{E}+21$ & $1.54 \mathrm{E}+21$ & $1.63 \mathrm{E}+21$ & $1.63 \mathrm{E}+21$ & $1.71 \mathrm{E}+21$ & $1.93 \mathrm{E}+21$ \\
\hline 8.5 & $2.01 \mathrm{E}+21$ & $1.79 \mathrm{E}+21$ & $1.74 \mathrm{E}+21$ & $1.71 \mathrm{E}+21$ & $1.61 \mathrm{E}+21$ & $1.70 \mathrm{E}+21$ & $1.69 \mathrm{E}+21$ & $1.77 \mathrm{E}+21$ & $1.99 \mathrm{E}+21$ \\
\hline 9.5 & $2.12 \mathrm{E}+21$ & $1.87 \mathrm{E}+21$ & $1.78 \mathrm{E}+21$ & $1.78 \mathrm{E}+21$ & $1.70 \mathrm{E}+21$ & $1.79 \mathrm{E}+21$ & $1.79 \mathrm{E}+21$ & $1.87 \mathrm{E}+21$ & $2.14 \mathrm{E}+21$ \\
\hline 10.5 & $2.18 \mathrm{E}+21$ & $1.93 \mathrm{E}+21$ & $1.85 E+21$ & $1.85 \mathrm{E}+21$ & $1.76 \mathrm{E}+21$ & $1.83 \mathrm{E}+21$ & $1.85 E+21$ & $1.93 \mathrm{E}+21$ & $2.20 \mathrm{E}+21$ \\
\hline 11.5 & $2.20 \mathrm{E}+21$ & $1.95 \mathrm{E}+21$ & $1.88 \mathrm{E}+21$ & $1.88 \mathrm{E}+21$ & $1.79 \mathrm{E}+21$ & $1.87 \mathrm{E}+21$ & $1.89 \mathrm{E}+21$ & $1.98 \mathrm{E}+21$ & $2.22 \mathrm{E}+21$ \\
\hline 12.5 & $2.25 E+21$ & $1.96 \mathrm{E}+21$ & $1.89 \mathrm{E}+21$ & $1.91 \mathrm{E}+21$ & $1.81 \mathrm{E}+21$ & $1.90 \mathrm{E}+21$ & $1.91 \mathrm{E}+21$ & $1.99 \mathrm{E}+21$ & $2.23 \mathrm{E}+21$ \\
\hline 13.5 & $2.22 \mathrm{E}+21$ & $1.96 \mathrm{E}+21$ & $1.88 \mathrm{E}+21$ & $1.85 \mathrm{E}+21$ & $1.79 \mathrm{E}+21$ & $1.87 \mathrm{E}+21$ & $1.89 \mathrm{E}+21$ & $1.95 \mathrm{E}+21$ & $2.20 \mathrm{E}+21$ \\
\hline 14.5 & $2.18 \mathrm{E}+21$ & $1.90 \mathrm{E}+21$ & $1.82 \mathrm{E}+21$ & $1.83 \mathrm{E}+21$ & $1.75 \mathrm{E}+21$ & $1.83 \mathrm{E}+21$ & $1.84 \mathrm{E}+21$ & $1.91 \mathrm{E}+21$ & $2.18 \mathrm{E}+21$ \\
\hline 15.5 & $2.08 \mathrm{E}+21$ & $1.84 \mathrm{E}+21$ & $1.76 \mathrm{E}+21$ & $1.74 \mathrm{E}+21$ & $1.66 \mathrm{E}+21$ & $1.77 \mathrm{E}+21$ & $1.78 \mathrm{E}+21$ & $1.86 \mathrm{E}+21$ & $2.09 \mathrm{E}+21$ \\
\hline 16.5 & $1.99 \mathrm{E}+21$ & $1.76 \mathrm{E}+21$ & $1.69 \mathrm{E}+21$ & $1.67 E+21$ & $1.59 \mathrm{E}+21$ & $1.67 E+21$ & $1.68 \mathrm{E}+21$ & $1.77 \mathrm{E}+21$ & $1.98 \mathrm{E}+21$ \\
\hline 17.5 & $1.84 \mathrm{E}+21$ & $1.66 \mathrm{E}+21$ & $1.58 \mathrm{E}+21$ & $1.56 \mathrm{E}+21$ & $1.46 \mathrm{E}+21$ & $1.56 \mathrm{E}+21$ & $1.58 \mathrm{E}+21$ & $1.67 \mathrm{E}+21$ & $1.84 \mathrm{E}+21$ \\
\hline 18.5 & $1.75 E+21$ & $1.53 \mathrm{E}+21$ & $1.47 \mathrm{E}+21$ & $1.46 \mathrm{E}+21$ & $1.39 \mathrm{E}+21$ & $1.47 \mathrm{E}+21$ & $1.47 \mathrm{E}+21$ & $1.54 \mathrm{E}+21$ & $1.76 \mathrm{E}+21$ \\
\hline 19.5 & $1.62 E+21$ & $1.43 \mathrm{E}+21$ & $1.38 \mathrm{E}+21$ & $1.36 \mathrm{E}+21$ & $1.31 \mathrm{E}+21$ & $1.37 \mathrm{E}+21$ & $1.36 \mathrm{E}+21$ & $1.44 \mathrm{E}+21$ & $1.59 \mathrm{E}+21$ \\
\hline 20.5 & $1.49 \mathrm{E}+21$ & $1.30 \mathrm{E}+21$ & $1.27 \mathrm{E}+21$ & $1.24 \mathrm{E}+21$ & $1.20 \mathrm{E}+21$ & $1.25 \mathrm{E}+21$ & $1.28 \mathrm{E}+21$ & $1.32 \mathrm{E}+21$ & $1.49 \mathrm{E}+21$ \\
\hline 21.5 & $1.32 \mathrm{E}+21$ & $1.17 \mathrm{E}+21$ & $1.13 E+21$ & $1.14 \mathrm{E}+21$ & $1.07 \mathrm{E}+21$ & $1.11 \mathrm{E}+21$ & $1.11 \mathrm{E}+21$ & $1.19 \mathrm{E}+21$ & $1.35 \mathrm{E}+21$ \\
\hline 22.5 & $1.21 \mathrm{E}+21$ & $1.05 \mathrm{E}+21$ & $1.02 \mathrm{E}+21$ & $9.99 \mathrm{E}+20$ & $9.55 \mathrm{E}+20$ & $1.01 \mathrm{E}+21$ & $1.02 \mathrm{E}+21$ & $1.08 \mathrm{E}+21$ & $1.23 \mathrm{E}+21$ \\
\hline 23.5 & $1.38 \mathrm{E}+21$ & $1.18 \mathrm{E}+21$ & $1.15 \mathrm{E}+21$ & $1.13 \mathrm{E}+21$ & $1.07 \mathrm{E}+21$ & $1.13 \mathrm{E}+21$ & $1.13 \mathrm{E}+21$ & $1.19 \mathrm{E}+21$ & $1.38 \mathrm{E}+21$ \\
\hline Ax. Avg. & $1.74 \mathrm{E}+21$ & $1.53 \mathrm{E}+21$ & $1.47 \mathrm{E}+21$ & $1.46 \mathrm{E}+21$ & $1.39 \mathrm{E}+21$ & $1.46 \mathrm{E}+21$ & $1.47 \mathrm{E}+21$ & $1.54 \mathrm{E}+21$ & $1.74 \mathrm{E}+21$ \\
\hline
\end{tabular}


Table A 4. Fission Density Data (fissions $/ \mathrm{cm}^{3}$ ) in Fuel Plate 4 in Element Discharged from Reference Mixed Burnup Core: FT loaded with typical samples.

\section{Fuel core width 2.012 inches Fuel core thickness 20 mil}

\begin{tabular}{|c|c|c|c|c|c|c|c|c|c|}
\hline $\begin{array}{c}\text { Axial } \\
\text { position } \\
\text { from top of } \\
\text { fuel (inches) }\end{array}$ & $\begin{array}{c}0.000 \text { to } \\
0.197 \\
\text { inches }\end{array}$ & $\begin{array}{c}0.197 \text { to } \\
0.394 \\
\text { inches }\end{array}$ & $\begin{array}{c}0.394 \text { to } \\
0.591 \\
\text { inches }\end{array}$ & $\begin{array}{c}0.591 \text { to } \\
0.868 \\
\text { inches }\end{array}$ & $\begin{array}{l}0.868 \text { to } \\
1.145 \\
\text { inches }\end{array}$ & $\begin{array}{l}1.145 \text { to } \\
1.422 \\
\text { inches }\end{array}$ & $\begin{array}{l}1.422 \text { to } \\
1.619 \\
\text { inches }\end{array}$ & $\begin{array}{l}1.619 \text { to } \\
1.815 \\
\text { inches }\end{array}$ & $\begin{array}{l}1.815 \text { to } \\
2.012 \\
\text { inches }\end{array}$ \\
\hline 0.5 & $9.30 \mathrm{E}+20$ & $7.85 \mathrm{E}+20$ & $7.39 \mathrm{E}+20$ & $7.31 \mathrm{E}+20$ & $7.05 \mathrm{E}+20$ & $7.29 \mathrm{E}+20$ & $7.50 \mathrm{E}+20$ & $7.85 \mathrm{E}+20$ & $9.48 \mathrm{E}+20$ \\
\hline 1.5 & $8.55 E+20$ & $7.24 \mathrm{E}+20$ & $6.75 E+20$ & $6.60 \mathrm{E}+20$ & $6.34 \mathrm{E}+20$ & $6.58 \mathrm{E}+20$ & $6.76 \mathrm{E}+20$ & $7.21 \mathrm{E}+20$ & $8.81 E+20$ \\
\hline 2.5 & $9.88 \mathrm{E}+20$ & $8.35 E+20$ & $7.85 E+20$ & $7.75 \mathrm{E}+20$ & $7.45 E+20$ & $7.78 \mathrm{E}+20$ & $7.81 \mathrm{E}+20$ & $8.40 \mathrm{E}+20$ & $9.95 \mathrm{E}+20$ \\
\hline 3.5 & $1.16 \mathrm{E}+21$ & $9.82 \mathrm{E}+20$ & $9.24 \mathrm{E}+20$ & $9.16 \mathrm{E}+20$ & $8.58 E+20$ & $9.05 E+20$ & $9.32 \mathrm{E}+20$ & $9.86 \mathrm{E}+20$ & $1.14 \mathrm{E}+21$ \\
\hline 4.5 & $1.28 \mathrm{E}+21$ & $1.08 \mathrm{E}+21$ & $1.03 E+21$ & $1.02 \mathrm{E}+21$ & $9.64 \mathrm{E}+20$ & $1.02 \mathrm{E}+21$ & $1.03 E+21$ & $1.08 \mathrm{E}+21$ & $1.28 \mathrm{E}+21$ \\
\hline 5.5 & $1.41 E+21$ & $1.19 \mathrm{E}+21$ & $1.13 E+21$ & $1.10 \mathrm{E}+21$ & $1.07 E+21$ & $1.11 \mathrm{E}+21$ & $1.14 \mathrm{E}+21$ & $1.21 \mathrm{E}+21$ & $1.40 \mathrm{E}+21$ \\
\hline 6.5 & $1.50 \mathrm{E}+21$ & $1.30 \mathrm{E}+21$ & $1.20 \mathrm{E}+21$ & $1.19 \mathrm{E}+21$ & $1.14 \mathrm{E}+21$ & $1.20 \mathrm{E}+21$ & $1.21 \mathrm{E}+21$ & $1.27 \mathrm{E}+21$ & $1.49 \mathrm{E}+21$ \\
\hline 7.5 & $1.59 \mathrm{E}+21$ & $1.37 \mathrm{E}+21$ & $1.30 \mathrm{E}+21$ & $1.26 \mathrm{E}+21$ & $1.19 \mathrm{E}+21$ & $1.25 \mathrm{E}+21$ & $1.27 \mathrm{E}+21$ & $1.33 \mathrm{E}+21$ & $1.59 \mathrm{E}+21$ \\
\hline 8.5 & $1.67 \mathrm{E}+21$ & $1.44 \mathrm{E}+21$ & $1.33 \mathrm{E}+21$ & $1.32 \mathrm{E}+21$ & $1.27 \mathrm{E}+21$ & $1.32 \mathrm{E}+21$ & $1.34 \mathrm{E}+21$ & $1.43 \mathrm{E}+21$ & $1.66 \mathrm{E}+21$ \\
\hline 9.5 & $1.74 \mathrm{E}+21$ & $1.49 \mathrm{E}+21$ & $1.40 \mathrm{E}+21$ & $1.37 E+21$ & $1.32 \mathrm{E}+21$ & $1.38 \mathrm{E}+21$ & $1.38 \mathrm{E}+21$ & $1.47 \mathrm{E}+21$ & $1.74 \mathrm{E}+21$ \\
\hline 10.5 & $1.80 \mathrm{E}+21$ & $1.51 \mathrm{E}+21$ & $1.45 E+21$ & $1.40 \mathrm{E}+21$ & $1.35 E+21$ & $1.40 \mathrm{E}+21$ & $1.43 E+21$ & $1.53 \mathrm{E}+21$ & $1.78 \mathrm{E}+21$ \\
\hline 11.5 & $1.83 E+21$ & $1.54 \mathrm{E}+21$ & $1.46 \mathrm{E}+21$ & $1.44 \mathrm{E}+21$ & $1.36 \mathrm{E}+21$ & $1.43 E+21$ & $1.44 \mathrm{E}+21$ & $1.54 \mathrm{E}+21$ & $1.82 \mathrm{E}+21$ \\
\hline 12.5 & $1.84 \mathrm{E}+21$ & $1.56 \mathrm{E}+21$ & $1.45 \mathrm{E}+21$ & $1.43 \mathrm{E}+21$ & $1.38 \mathrm{E}+21$ & $1.43 \mathrm{E}+21$ & $1.47 \mathrm{E}+21$ & $1.56 \mathrm{E}+21$ & $1.85 \mathrm{E}+21$ \\
\hline 13.5 & $1.83 \mathrm{E}+21$ & $1.56 \mathrm{E}+21$ & $1.45 \mathrm{E}+21$ & $1.43 \mathrm{E}+21$ & $1.37 \mathrm{E}+21$ & $1.43 \mathrm{E}+21$ & $1.47 \mathrm{E}+21$ & $1.55 \mathrm{E}+21$ & $1.81 \mathrm{E}+21$ \\
\hline 14.5 & $1.77 \mathrm{E}+21$ & $1.50 \mathrm{E}+21$ & $1.43 \mathrm{E}+21$ & $1.39 \mathrm{E}+21$ & $1.34 \mathrm{E}+21$ & $1.40 \mathrm{E}+21$ & $1.42 \mathrm{E}+21$ & $1.51 \mathrm{E}+21$ & $1.78 \mathrm{E}+21$ \\
\hline 15.5 & $1.73 E+21$ & $1.44 \mathrm{E}+21$ & $1.38 \mathrm{E}+21$ & $1.36 \mathrm{E}+21$ & $1.28 \mathrm{E}+21$ & $1.35 \mathrm{E}+21$ & $1.39 \mathrm{E}+21$ & $1.47 \mathrm{E}+21$ & $1.74 \mathrm{E}+21$ \\
\hline 16.5 & $1.63 \mathrm{E}+21$ & $1.40 \mathrm{E}+21$ & $1.31 \mathrm{E}+21$ & $1.31 \mathrm{E}+21$ & $1.25 \mathrm{E}+21$ & $1.28 \mathrm{E}+21$ & $1.31 \mathrm{E}+21$ & $1.39 \mathrm{E}+21$ & $1.61 \mathrm{E}+21$ \\
\hline 17.5 & $1.54 \mathrm{E}+21$ & $1.31 \mathrm{E}+21$ & $1.24 \mathrm{E}+21$ & $1.22 \mathrm{E}+21$ & $1.16 \mathrm{E}+21$ & $1.21 \mathrm{E}+21$ & $1.22 \mathrm{E}+21$ & $1.30 \mathrm{E}+21$ & $1.53 \mathrm{E}+21$ \\
\hline 18.5 & $1.43 \mathrm{E}+21$ & $1.21 \mathrm{E}+21$ & $1.15 \mathrm{E}+21$ & $1.15 \mathrm{E}+21$ & $1.08 \mathrm{E}+21$ & $1.14 \mathrm{E}+21$ & $1.16 \mathrm{E}+21$ & $1.23 \mathrm{E}+21$ & $1.42 \mathrm{E}+21$ \\
\hline 19.5 & $1.31 \mathrm{E}+21$ & $1.12 \mathrm{E}+21$ & $1.08 \mathrm{E}+21$ & $1.05 \mathrm{E}+21$ & $1.02 E+21$ & $1.05 E+21$ & $1.05 E+21$ & $1.12 \mathrm{E}+21$ & $1.29 \mathrm{E}+21$ \\
\hline 20.5 & $1.21 \mathrm{E}+21$ & $1.03 \mathrm{E}+21$ & $9.89 E+20$ & $9.63 E+20$ & $9.17 E+20$ & $9.44 \mathrm{E}+20$ & $9.74 \mathrm{E}+20$ & $1.02 \mathrm{E}+21$ & $1.23 E+21$ \\
\hline 21.5 & $1.09 \mathrm{E}+21$ & $9.33 \mathrm{E}+20$ & $8.69 \mathrm{E}+20$ & $8.56 \mathrm{E}+20$ & $8.21 \mathrm{E}+20$ & $8.50 \mathrm{E}+20$ & $8.78 \mathrm{E}+20$ & $9.37 \mathrm{E}+20$ & $1.10 \mathrm{E}+21$ \\
\hline 22.5 & $9.91 \mathrm{E}+20$ & $8.13 \mathrm{E}+20$ & $7.75 \mathrm{E}+20$ & $7.57 \mathrm{E}+20$ & $7.29 \mathrm{E}+20$ & $7.47 \mathrm{E}+20$ & $7.85 \mathrm{E}+20$ & $8.43 \mathrm{E}+20$ & $9.92 \mathrm{E}+20$ \\
\hline 23.5 & $1.14 \mathrm{E}+21$ & $9.46 \mathrm{E}+20$ & $8.96 \mathrm{E}+20$ & $8.94 \mathrm{E}+20$ & $8.41 \mathrm{E}+20$ & $8.82 \mathrm{E}+20$ & $8.89 \mathrm{E}+20$ & $9.62 \mathrm{E}+20$ & $1.13 \mathrm{E}+21$ \\
\hline Ax. Avg. & $1.43 \mathrm{E}+21$ & $1.21 \mathrm{E}+21$ & $1.14 \mathrm{E}+21$ & $1.12 \mathrm{E}+21$ & $1.07 \mathrm{E}+21$ & $1.12 \mathrm{E}+21$ & $1.14 \mathrm{E}+21$ & $1.21 \mathrm{E}+21$ & $1.43 \mathrm{E}+21$ \\
\hline
\end{tabular}


Table A-5. Fission Density Data (fissions $/ \mathrm{cm}^{3}$ ) in Fuel Plate 5 in Element Discharged from Reference Mixed Burnup Core: FT loaded with typical samples.

\section{Fuel core width 2.120 inches Fuel core thickness 20 mil}

\begin{tabular}{|c|c|c|c|c|c|c|c|c|c|}
\hline $\begin{array}{l}\text { Axial } \\
\text { position } \\
\text { from top of } \\
\text { fuel (inches) }\end{array}$ & $\begin{array}{c}0.000 \text { to } \\
0.197 \\
\text { inches }\end{array}$ & $\begin{array}{c}0.197 \text { to } \\
0.394 \\
\text { inches }\end{array}$ & $\begin{array}{c}0.394 \text { to } \\
0.591 \\
\text { inches }\end{array}$ & $\begin{array}{l}0.591 \text { to } \\
0.903 \\
\text { inches }\end{array}$ & $\begin{array}{c}0.903 \text { to } \\
1.216 \\
\text { inches }\end{array}$ & $\begin{array}{l}1.216 \text { to } \\
1.529 \\
\text { inches }\end{array}$ & $\begin{array}{l}1.529 \text { to } \\
1.726 \\
\text { inches }\end{array}$ & $\begin{array}{l}1.726 \text { to } \\
1.923 \\
\text { inches }\end{array}$ & $\begin{array}{l}1.923 \text { to } \\
2.120 \\
\text { inches }\end{array}$ \\
\hline 0.5 & $8.39 E+20$ & $6.75 \mathrm{E}+20$ & $6.54 \mathrm{E}+20$ & $6.31 E+20$ & $6.06 \mathrm{E}+20$ & $6.28 \mathrm{E}+20$ & $6.30 \mathrm{E}+20$ & $6.85 E+20$ & $8.35 E+20$ \\
\hline 1.5 & $53 \mathrm{E}+20$ & $6.07 \mathrm{E}+20$ & $5.65 \mathrm{E}+20$ & $5.44 \mathrm{E}+20$ & $5.29 \mathrm{E}+20$ & $5.53 \mathrm{E}+20$ & $5.81 \mathrm{E}+20$ & $6.19 \mathrm{E}+20$ & $7.59 \mathrm{E}+20$ \\
\hline 2.5 & $8.53 E+20$ & $7.09 \mathrm{E}+20$ & $6.53 \mathrm{E}+20$ & $6.43 \mathrm{E}+20$ & $6.15 E+20$ & $6.55 \mathrm{E}+20$ & $6.69 \mathrm{E}+20$ & $7.22 \mathrm{E}+20$ & $8.74 \mathrm{E}+20$ \\
\hline 3.5 & $9.88 \mathrm{E}+20$ & $8.45 E+20$ & $7.57 E+20$ & $7.59 \mathrm{E}+20$ & $7.14 \mathrm{E}+20$ & $7.58 \mathrm{E}+20$ & $7.66 \mathrm{E}+20$ & $8.31 \mathrm{E}+20$ & $1.00 \mathrm{E}+21$ \\
\hline 4.5 & $1.12 \mathrm{E}+21$ & $9.22 \mathrm{E}+20$ & $8.75 E+20$ & $8.46 \mathrm{E}+20$ & $8.12 \mathrm{E}+20$ & $8.53 E+20$ & $8.80 \mathrm{E}+20$ & $9.28 \mathrm{E}+20$ & $1.12 \mathrm{E}+21$ \\
\hline 5.5 & $1.22 \mathrm{E}+21$ & +21 & $9.59 \mathrm{E}+20$ & $2 E+20$ & $2 E+20$ & $9.31 \mathrm{E}+20$ & $9.54 \mathrm{E}+20$ & $2 E+21$ & $E+21$ \\
\hline 6.5 & $1.31 \mathrm{E}+21$ & $1.10 \mathrm{E}+21$ & $1.04 \mathrm{E}+21$ & $9.89 E+20$ & $9.50 \mathrm{E}+20$ & $1.01 \mathrm{E}+21$ & $1.04 \mathrm{E}+21$ & $1.10 \mathrm{E}+21$ & $1.31 \mathrm{E}+21$ \\
\hline 7.5 & $1.41 \mathrm{E}+21$ & $1.17 \mathrm{E}+21$ & $1.09 \mathrm{E}+21$ & $1.06 \mathrm{E}+21$ & $1.00 \mathrm{E}+21$ & $1.05 \mathrm{E}+21$ & $1.09 \mathrm{E}+21$ & $1.15 \mathrm{E}+21$ & $1.41 \mathrm{E}+21$ \\
\hline 8.5 & $1.45 E+21$ & $1.21 \mathrm{E}+21$ & $1.14 \mathrm{E}+21$ & $1.11 \mathrm{E}+21$ & $1.06 \mathrm{E}+21$ & $1.12 \mathrm{E}+21$ & $1.15 \mathrm{E}+21$ & $1.22 \mathrm{E}+21$ & $1.47 \mathrm{E}+21$ \\
\hline 9.5 & $1.53 \mathrm{E}+21$ & -21 & +21 & +21 & $1.11 \mathrm{E}+21$ & $1.17 \mathrm{E}+21$ & $E+21$ & $7 E+21$ & +21 \\
\hline 10.5 & $1.58 \mathrm{E}+21$ & $1.31 \mathrm{E}+21$ & $1.21 \mathrm{E}+21$ & $1.19 \mathrm{E}+21$ & $1.12 \mathrm{E}+21$ & $1.18 \mathrm{E}+21$ & $1.21 \mathrm{E}+21$ & $1.31 \mathrm{E}+21$ & $1.58 \mathrm{E}+21$ \\
\hline 11.5 & $1.61 \mathrm{E}+21$ & $1.33 \mathrm{E}+21$ & $1.23 \mathrm{E}+21$ & $1.20 \mathrm{E}+21$ & $1.15 \mathrm{E}+21$ & $1.20 \mathrm{E}+21$ & $1.23 \mathrm{E}+21$ & $1.31 \mathrm{E}+21$ & $1.57 \mathrm{E}+21$ \\
\hline 12.5 & $1.62 \mathrm{E}+21$ & $1.34 \mathrm{E}+21$ & $1.23 \mathrm{E}+21$ & $1.19 \mathrm{E}+21$ & $1.15 E+21$ & $1.20 \mathrm{E}+21$ & $1.24 \mathrm{E}+21$ & $1.33 \mathrm{E}+21$ & $1.60 \mathrm{E}+21$ \\
\hline 13.5 & $1.60 \mathrm{E}+21$ & $1.31 \mathrm{E}+21$ & $1.20 \mathrm{E}+21$ & $1.20 \mathrm{E}+21$ & $1.15 \mathrm{E}+21$ & $1.20 \mathrm{E}+21$ & $1.25 \mathrm{E}+21$ & $1.34 \mathrm{E}+21$ & $1.60 \mathrm{E}+21$ \\
\hline 14.5 & $1.57 \mathrm{E}+21$ & $1.29 \mathrm{E}+21$ & $1.21 \mathrm{E}+21$ & $1.16 \mathrm{E}+21$ & $1.12 \mathrm{E}+21$ & $1.17 \mathrm{E}+21$ & $1.20 \mathrm{E}+21$ & $1.29 \mathrm{E}+21$ & $1.56 \mathrm{E}+21$ \\
\hline 15.5 & $1.49 \mathrm{E}+21$ & $1.24 \mathrm{E}+21$ & $1.16 \mathrm{E}+21$ & $1.13 \mathrm{E}+21$ & $1.07 \mathrm{E}+21$ & $1.13 \mathrm{E}+21$ & $1.16 \mathrm{E}+21$ & $1.25 \mathrm{E}+21$ & $1.50 \mathrm{E}+21$ \\
\hline 16.5 & $1.43 \mathrm{E}+21$ & $1.20 \mathrm{E}+21$ & $1.12 \mathrm{E}+21$ & $1.09 \mathrm{E}+21$ & $1.05 \mathrm{E}+21$ & $1.09 \mathrm{E}+21$ & $1.12 \mathrm{E}+21$ & $1.19 \mathrm{E}+21$ & $1.43 \mathrm{E}+21$ \\
\hline 17.5 & $1.36 \mathrm{E}+21$ & $1.14 \mathrm{E}+21$ & $1.06 \mathrm{E}+21$ & $1.03 \mathrm{E}+21$ & $9.75 \mathrm{E}+20$ & $1.03 E+21$ & $1.06 \mathrm{E}+21$ & $1.13 \mathrm{E}+21$ & $1.35 \mathrm{E}+21$ \\
\hline 18.5 & $1.26 \mathrm{E}+21$ & $1.06 \mathrm{E}+21$ & $9.79 \mathrm{E}+20$ & $9.65 E+20$ & $9.20 \mathrm{E}+20$ & $9.59 \mathrm{E}+20$ & $9.99 \mathrm{E}+20$ & $1.05 \mathrm{E}+21$ & $1.24 \mathrm{E}+21$ \\
\hline 19.5 & $1.15 \mathrm{E}+21$ & $9.73 \mathrm{E}+20$ & $9.09 \mathrm{E}+20$ & $8.78 \mathrm{E}+20$ & $8.56 \mathrm{E}+20$ & $9.01 \mathrm{E}+20$ & $9.05 E+20$ & $9.55 \mathrm{E}+20$ & $1.16 \mathrm{E}+21$ \\
\hline 20.5 & $1.06 \mathrm{E}+21$ & $8.76 \mathrm{E}+20$ & $8.21 \mathrm{E}+20$ & $8.08 \mathrm{E}+20$ & $7.74 \mathrm{E}+20$ & $8.01 \mathrm{E}+20$ & $8.23 E+20$ & $8.82 \mathrm{E}+20$ & $1.06 \mathrm{E}+21$ \\
\hline 21.5 & $9.51 E+20$ & $7.88 \mathrm{E}+20$ & $7.28 \mathrm{E}+20$ & $7.17 \mathrm{E}+20$ & $6.86 \mathrm{E}+20$ & $7.12 \mathrm{E}+20$ & $7.29 \mathrm{E}+20$ & $7.92 \mathrm{E}+20$ & $9.48 \mathrm{E}+20$ \\
\hline 22.5 & $8.57 \mathrm{E}+20$ & $6.95 E+20$ & $6.51 \mathrm{E}+20$ & $6.29 E+20$ & $5.99 \mathrm{E}+20$ & $6.30 \mathrm{E}+20$ & $6.54 \mathrm{E}+20$ & $7.01 \mathrm{E}+20$ & $8.54 \mathrm{E}+20$ \\
\hline 23.5 & $1.03 \mathrm{E}+21$ & $8.29 \mathrm{E}+20$ & $7.72 \mathrm{E}+20$ & $7.72 \mathrm{E}+20$ & $7.23 \mathrm{E}+20$ & $7.59 \mathrm{E}+20$ & $7.79 \mathrm{E}+20$ & $8.33 E+20$ & $1.02 \mathrm{E}+21$ \\
\hline Ax. Avg. & $1.25 \mathrm{E}+21$ & $1.04 \mathrm{E}+21$ & $9.67 \mathrm{E}+20$ & $9.43 E+20$ & $9.02 \mathrm{E}+20$ & $9.46 \mathrm{E}+20$ & $9.70 \mathrm{E}+20$ & $1.04 \mathrm{E}+21$ & $1.25 \mathrm{E}+21$ \\
\hline
\end{tabular}


Table A-6. Fission Density Data (fissions $/ \mathrm{cm}^{3}$ ) in Fuel Plate 6 in Element Discharged from Reference Mixed Burnup Core: FT loaded with typical samples.

\section{Fuel core width 2.227 inches Fuel core thickness 20 mil}

\begin{tabular}{|c|c|c|c|c|c|c|c|c|c|}
\hline $\begin{array}{c}\text { Axial } \\
\text { position } \\
\text { from top of } \\
\text { fuel (inches) }\end{array}$ & $\begin{array}{c}0.000 \text { to } \\
0.197 \\
\text { inches }\end{array}$ & $\begin{array}{c}0.197 \text { to } \\
0.394 \\
\text { inches }\end{array}$ & $\begin{array}{c}0.394 \text { to } \\
0.591 \\
\text { inches }\end{array}$ & $\begin{array}{c}0.591 \text { to } \\
0.939 \\
\text { inches }\end{array}$ & $\begin{array}{c}0.939 \text { to } \\
1.288 \\
\text { inches }\end{array}$ & $\begin{array}{l}1.288 \text { to } \\
1.636 \\
\text { inches }\end{array}$ & $\begin{array}{l}1.636 \text { to } \\
1.833 \\
\text { inches }\end{array}$ & $\begin{array}{l}1.833 \text { to } \\
2.030 \\
\text { inches }\end{array}$ & $\begin{array}{l}2.030 \text { to } \\
2.227 \\
\text { inches }\end{array}$ \\
\hline 0.5 & $7.87 \mathrm{E}+20$ & $6.49 \mathrm{E}+20$ & $5.98 \mathrm{E}+20$ & $5.81 \mathrm{E}+20$ & $5.59 \mathrm{E}+20$ & $5.82 \mathrm{E}+20$ & $5.90 \mathrm{E}+20$ & $6.46 \mathrm{E}+20$ & $7.86 \mathrm{E}+20$ \\
\hline 1.5 & $7.07 E+20$ & $5.58 \mathrm{E}+20$ & $5.20 \mathrm{E}+20$ & $4.92 \mathrm{E}+20$ & $4.71 \mathrm{E}+20$ & $5.02 \mathrm{E}+20$ & $5.19 \mathrm{E}+20$ & $5.64 \mathrm{E}+20$ & $6.91 E+20$ \\
\hline 2.5 & $8.01 E+20$ & $6.49 \mathrm{E}+20$ & $6.00 \mathrm{E}+20$ & $5.93 E+20$ & $5.64 \mathrm{E}+20$ & $5.87 \mathrm{E}+20$ & $6.12 E+20$ & $6.43 \mathrm{E}+20$ & $7.90 \mathrm{E}+20$ \\
\hline 3.5 & $9.11 \mathrm{E}+20$ & $7.59 \mathrm{E}+20$ & $6.99 E+20$ & $6.83 E+20$ & $6.44 \mathrm{E}+20$ & $6.86 \mathrm{E}+20$ & $7.03 E+20$ & $7.63 \mathrm{E}+20$ & $9.35 \mathrm{E}+20$ \\
\hline 4.5 & $1.05 E+21$ & $8.59 \mathrm{E}+20$ & $7.97 E+20$ & $7.68 \mathrm{E}+20$ & $7.29 \mathrm{E}+20$ & $7.72 \mathrm{E}+20$ & $7.84 \mathrm{E}+20$ & $8.61 \mathrm{E}+20$ & $1.04 \mathrm{E}+21$ \\
\hline 5.5 & $1.13 \mathrm{E}+21$ & $9.44 \mathrm{E}+20$ & $8.73 \mathrm{E}+20$ & $8.49 \mathrm{E}+20$ & $8.17 \mathrm{E}+20$ & $8.49 \mathrm{E}+20$ & $8.72 \mathrm{E}+20$ & $9.29 \mathrm{E}+20$ & $1.15 \mathrm{E}+21$ \\
\hline 6.5 & $1.22 \mathrm{E}+21$ & $1.01 \mathrm{E}+21$ & $9.38 \mathrm{E}+20$ & $9.14 \mathrm{E}+20$ & $8.71 \mathrm{E}+20$ & $9.14 \mathrm{E}+20$ & $9.64 \mathrm{E}+20$ & $1.01 \mathrm{E}+21$ & $1.22 \mathrm{E}+21$ \\
\hline 7.5 & $1.29 \mathrm{E}+21$ & $1.09 \mathrm{E}+21$ & $1.01 \mathrm{E}+21$ & $9.67 E+20$ & $9.29 \mathrm{E}+20$ & $9.62 \mathrm{E}+20$ & $1.00 \mathrm{E}+21$ & $1.08 \mathrm{E}+21$ & $1.29 \mathrm{E}+21$ \\
\hline 8.5 & $1.36 \mathrm{E}+21$ & $1.11 \mathrm{E}+21$ & $1.05 E+21$ & $1.02 \mathrm{E}+21$ & $9.70 \mathrm{E}+20$ & $1.01 \mathrm{E}+21$ & $1.05 \mathrm{E}+21$ & $1.13 \mathrm{E}+21$ & $1.36 \mathrm{E}+21$ \\
\hline 9.5 & $1.42 \mathrm{E}+21$ & $1.15 \mathrm{E}+21$ & $1.08 \mathrm{E}+21$ & $1.06 \mathrm{E}+21$ & $1.00 \mathrm{E}+21$ & $1.06 \mathrm{E}+21$ & $1.09 \mathrm{E}+21$ & $1.18 \mathrm{E}+21$ & $1.42 \mathrm{E}+21$ \\
\hline 10.5 & $1.44 \mathrm{E}+21$ & $1.19 \mathrm{E}+21$ & $1.10 \mathrm{E}+21$ & $1.08 \mathrm{E}+21$ & $1.03 E+21$ & $1.09 \mathrm{E}+21$ & $1.10 \mathrm{E}+21$ & $1.19 \mathrm{E}+21$ & $1.45 \mathrm{E}+21$ \\
\hline 11.5 & $1.47 E+21$ & $1.21 \mathrm{E}+21$ & $1.13 E+21$ & $1.09 \mathrm{E}+21$ & $1.05 E+21$ & $1.09 \mathrm{E}+21$ & $1.12 \mathrm{E}+21$ & $1.21 \mathrm{E}+21$ & $1.46 \mathrm{E}+21$ \\
\hline 12.5 & $1.47 \mathrm{E}+21$ & $1.21 \mathrm{E}+21$ & $1.14 \mathrm{E}+21$ & $1.09 \mathrm{E}+21$ & $1.05 E+21$ & $1.10 \mathrm{E}+21$ & $1.12 \mathrm{E}+21$ & $1.23 \mathrm{E}+21$ & $1.48 \mathrm{E}+21$ \\
\hline 13.5 & $1.47 \mathrm{E}+21$ & $1.21 \mathrm{E}+21$ & $1.12 \mathrm{E}+21$ & $1.09 \mathrm{E}+21$ & $1.04 \mathrm{E}+21$ & $1.10 \mathrm{E}+21$ & $1.12 \mathrm{E}+21$ & $1.21 \mathrm{E}+21$ & $1.46 \mathrm{E}+21$ \\
\hline 14.5 & $1.43 \mathrm{E}+21$ & $1.20 \mathrm{E}+21$ & $1.10 \mathrm{E}+21$ & $1.06 \mathrm{E}+21$ & $1.01 \mathrm{E}+21$ & $1.08 \mathrm{E}+21$ & $1.09 \mathrm{E}+21$ & $1.17 \mathrm{E}+21$ & $1.42 \mathrm{E}+21$ \\
\hline 15.5 & $1.38 \mathrm{E}+21$ & $1.17 \mathrm{E}+21$ & $1.05 \mathrm{E}+21$ & $1.03 \mathrm{E}+21$ & $9.78 \mathrm{E}+20$ & $1.03 \mathrm{E}+21$ & $1.06 \mathrm{E}+21$ & $1.13 \mathrm{E}+21$ & $1.38 \mathrm{E}+21$ \\
\hline 16.5 & $1.32 \mathrm{E}+21$ & $1.11 \mathrm{E}+21$ & $1.03 E+21$ & $1.00 \mathrm{E}+21$ & $9.50 \mathrm{E}+20$ & $9.86 \mathrm{E}+20$ & $1.04 \mathrm{E}+21$ & $1.11 \mathrm{E}+21$ & $1.32 \mathrm{E}+21$ \\
\hline 17.5 & $1.24 \mathrm{E}+21$ & $1.04 \mathrm{E}+21$ & $9.75 \mathrm{E}+20$ & $9.45 \mathrm{E}+20$ & $9.07 \mathrm{E}+20$ & $9.38 \mathrm{E}+20$ & $9.79 \mathrm{E}+20$ & $1.04 \mathrm{E}+21$ & $1.24 \mathrm{E}+21$ \\
\hline 18.5 & $1.17 E+21$ & $9.78 \mathrm{E}+20$ & $9.08 \mathrm{E}+20$ & $8.77 \mathrm{E}+20$ & $8.38 E+20$ & $8.82 E+20$ & $9.12 E+20$ & $9.78 \mathrm{E}+20$ & $1.19 \mathrm{E}+21$ \\
\hline 19.5 & $1.07 E+21$ & $8.89 E+20$ & $8.35 E+20$ & $8.12 \mathrm{E}+20$ & $7.83 E+20$ & $8.06 \mathrm{E}+20$ & $8.27 E+20$ & $8.89 \mathrm{E}+20$ & $1.09 \mathrm{E}+21$ \\
\hline 20.5 & $9.89 E+20$ & $8.14 \mathrm{E}+20$ & $7.63 E+20$ & $7.35 \mathrm{E}+20$ & $6.98 \mathrm{E}+20$ & $7.34 \mathrm{E}+20$ & $7.63 E+20$ & $8.23 E+20$ & $9.82 \mathrm{E}+20$ \\
\hline 21.5 & $8.87 E+20$ & $7.38 \mathrm{E}+20$ & $6.65 E+20$ & $6.53 E+20$ & $6.26 \mathrm{E}+20$ & $6.49 \mathrm{E}+20$ & $6.70 \mathrm{E}+20$ & $7.41 \mathrm{E}+20$ & $8.85 E+20$ \\
\hline 22.5 & $7.97 \mathrm{E}+20$ & $6.40 \mathrm{E}+20$ & $5.89 \mathrm{E}+20$ & $5.74 \mathrm{E}+20$ & $5.43 \mathrm{E}+20$ & $5.69 \mathrm{E}+20$ & $5.83 \mathrm{E}+20$ & $6.43 \mathrm{E}+20$ & $7.92 \mathrm{E}+20$ \\
\hline 23.5 & $9.74 \mathrm{E}+20$ & $7.77 \mathrm{E}+20$ & $7.25 \mathrm{E}+20$ & $7.04 \mathrm{E}+20$ & $6.65 \mathrm{E}+20$ & $7.02 \mathrm{E}+20$ & $7.30 \mathrm{E}+20$ & $7.70 \mathrm{E}+20$ & $9.69 \mathrm{E}+20$ \\
\hline Ax. Avg. & $1.16 \mathrm{E}+21$ & $9.57 \mathrm{E}+20$ & $8.87 E+20$ & $8.60 \mathrm{E}+20$ & $8.22 \mathrm{E}+20$ & $8.62 \mathrm{E}+20$ & $8.87 E+20$ & $9.57 \mathrm{E}+20$ & $1.16 \mathrm{E}+21$ \\
\hline
\end{tabular}


Table A-7. Fission Density Data (fissions $/ \mathrm{cm}^{3}$ ) in Fuel Plate 7 in Element Discharged from Reference Mixed Burnup Core: FT loaded with typical samples.

Fuel core width 2.334 inches Fuel core thickness 20 mil

\begin{tabular}{|c|c|c|c|c|c|c|c|c|c|}
\hline $\begin{array}{c}\text { Axial } \\
\text { position } \\
\text { from top of } \\
\text { fuel (inches) }\end{array}$ & $\begin{array}{c}0.000 \text { to } \\
0.197 \\
\text { inches }\end{array}$ & $\begin{array}{c}0.197 \text { to } \\
0.394 \\
\text { inches }\end{array}$ & $\begin{array}{c}0.394 \text { to } \\
0.591 \\
\text { inches }\end{array}$ & $\begin{array}{l}0.591 \text { to } \\
0.975 \\
\text { inches }\end{array}$ & $\begin{array}{c}0.975 \text { to } \\
1.359 \\
\text { inches }\end{array}$ & $\begin{array}{l}1.359 \text { to } \\
1.743 \\
\text { inches }\end{array}$ & $\begin{array}{c}1.743 \text { to } \\
1.940 \\
\text { inches }\end{array}$ & $\begin{array}{l}1.940 \text { to } \\
2.137 \\
\text { inches }\end{array}$ & $\begin{array}{c}2.137 \text { to } \\
2.334 \\
\text { inches }\end{array}$ \\
\hline 0.5 & $7.23 E+20$ & $5.94 \mathrm{E}+20$ & $5.48 \mathrm{E}+20$ & $5.35 \mathrm{E}+20$ & $5.16 \mathrm{E}+20$ & $5.39 \mathrm{E}+20$ & $5.58 \mathrm{E}+20$ & $5.90 \mathrm{E}+20$ & $7.30 \mathrm{E}+20$ \\
\hline 1.5 & $6.26 \mathrm{E}+20$ & $5.15 \mathrm{E}+20$ & $4.72 E+20$ & $4.45 \mathrm{E}+20$ & $4.26 \mathrm{E}+20$ & $4.54 \mathrm{E}+20$ & $4.64 \mathrm{E}+20$ & $5.06 \mathrm{E}+20$ & $6.28 \mathrm{E}+20$ \\
\hline 2.5 & $7.16 \mathrm{E}+20$ & $5.92 \mathrm{E}+20$ & $5.54 \mathrm{E}+20$ & $5.26 \mathrm{E}+20$ & $5.06 \mathrm{E}+20$ & $5.27 \mathrm{E}+20$ & $5.50 \mathrm{E}+20$ & $6.10 \mathrm{E}+20$ & $7.17 \mathrm{E}+20$ \\
\hline 3.5 & $8.21 E+20$ & $6.88 \mathrm{E}+20$ & $6.40 \mathrm{E}+20$ & $6.14 \mathrm{E}+20$ & $5.85 E+20$ & $6.19 E+20$ & $6.45 E+20$ & $6.97 E+20$ & $8.38 \mathrm{E}+20$ \\
\hline 4.5 & $9.33 E+20$ & $7.79 \mathrm{E}+20$ & $7.18 \mathrm{E}+20$ & $7.00 \mathrm{E}+20$ & $6.74 \mathrm{E}+20$ & $6.91 E+20$ & $7.10 \mathrm{E}+20$ & $7.77 \mathrm{E}+20$ & $9.35 \mathrm{E}+20$ \\
\hline 5.5 & $1.02 \mathrm{E}+21$ & $8.74 \mathrm{E}+20$ & $8.15 E+20$ & $7.64 \mathrm{E}+20$ & $7.38 \mathrm{E}+20$ & $7.68 \mathrm{E}+20$ & $7.90 \mathrm{E}+20$ & $8.53 E+20$ & $1.02 \mathrm{E}+21$ \\
\hline 6.5 & $1.09 \mathrm{E}+21$ & $9.27 \mathrm{E}+20$ & $8.65 E+20$ & $8.34 \mathrm{E}+20$ & $7.85 \mathrm{E}+20$ & $8.30 \mathrm{E}+20$ & $8.45 E+20$ & $9.20 \mathrm{E}+20$ & $1.11 \mathrm{E}+21$ \\
\hline 7.5 & $1.17 \mathrm{E}+21$ & $9.82 \mathrm{E}+20$ & $9.27 \mathrm{E}+20$ & $8.76 \mathrm{E}+20$ & $8.35 E+20$ & $8.73 E+20$ & $9.22 \mathrm{E}+20$ & $9.91 E+20$ & $1.17 E+21$ \\
\hline 8.5 & $1.23 E+21$ & $1.03 \mathrm{E}+21$ & $9.45 E+20$ & $9.14 \mathrm{E}+20$ & $8.84 \mathrm{E}+20$ & $9.28 \mathrm{E}+20$ & $9.59 \mathrm{E}+20$ & $1.02 \mathrm{E}+21$ & $1.24 \mathrm{E}+21$ \\
\hline 9.5 & $1.28 \mathrm{E}+21$ & $1.04 \mathrm{E}+21$ & $9.84 \mathrm{E}+20$ & $9.52 \mathrm{E}+20$ & $9.09 E+20$ & $9.57 E+20$ & $9.96 \mathrm{E}+20$ & $1.07 \mathrm{E}+21$ & $1.28 \mathrm{E}+21$ \\
\hline 10.5 & $1.32 \mathrm{E}+21$ & $1.10 \mathrm{E}+21$ & $1.01 \mathrm{E}+21$ & $9.81 \mathrm{E}+20$ & $9.30 \mathrm{E}+20$ & $9.81 E+20$ & $1.02 \mathrm{E}+21$ & $1.09 \mathrm{E}+21$ & $1.31 \mathrm{E}+21$ \\
\hline 11.5 & $1.33 E+21$ & $1.10 \mathrm{E}+21$ & $1.02 \mathrm{E}+21$ & $9.89 E+20$ & $9.46 \mathrm{E}+20$ & $9.94 \mathrm{E}+20$ & $1.03 E+21$ & $1.10 \mathrm{E}+21$ & $1.33 \mathrm{E}+21$ \\
\hline 12.5 & $1.33 \mathrm{E}+21$ & $1.11 \mathrm{E}+21$ & $1.04 \mathrm{E}+21$ & $9.97 E+20$ & $9.44 \mathrm{E}+20$ & $9.87 E+20$ & $1.02 \mathrm{E}+21$ & $1.11 \mathrm{E}+21$ & $1.35 \mathrm{E}+21$ \\
\hline 13.5 & $1.32 \mathrm{E}+21$ & $1.10 \mathrm{E}+21$ & $1.02 \mathrm{E}+21$ & $9.81 \mathrm{E}+20$ & $9.34 \mathrm{E}+20$ & $9.82 \mathrm{E}+20$ & $1.01 \mathrm{E}+21$ & $1.10 \mathrm{E}+21$ & $1.32 \mathrm{E}+21$ \\
\hline 14.5 & $1.29 \mathrm{E}+21$ & $1.07 \mathrm{E}+21$ & $9.88 \mathrm{E}+20$ & $9.56 \mathrm{E}+20$ & $9.19 \mathrm{E}+20$ & $9.62 \mathrm{E}+20$ & $9.99 \mathrm{E}+20$ & $1.07 \mathrm{E}+21$ & $1.28 \mathrm{E}+21$ \\
\hline 15.5 & $1.26 \mathrm{E}+21$ & $1.05 \mathrm{E}+21$ & $9.61 \mathrm{E}+20$ & $9.30 \mathrm{E}+20$ & $9.00 \mathrm{E}+20$ & $9.32 \mathrm{E}+20$ & $9.65 E+20$ & $1.03 E+21$ & $1.24 \mathrm{E}+21$ \\
\hline 16.5 & $1.20 \mathrm{E}+21$ & $1.01 \mathrm{E}+21$ & $9.43 \mathrm{E}+20$ & $9.15 E+20$ & $8.70 \mathrm{E}+20$ & $8.99 \mathrm{E}+20$ & $9.31 \mathrm{E}+20$ & $1.03 \mathrm{E}+21$ & $1.20 \mathrm{E}+21$ \\
\hline 17.5 & $1.14 \mathrm{E}+21$ & $9.63 \mathrm{E}+20$ & $8.90 \mathrm{E}+20$ & $8.61 \mathrm{E}+20$ & $8.04 \mathrm{E}+20$ & $8.56 \mathrm{E}+20$ & $8.86 \mathrm{E}+20$ & $9.53 E+20$ & $1.13 E+21$ \\
\hline 18.5 & $1.06 \mathrm{E}+21$ & $8.89 E+20$ & $8.20 \mathrm{E}+20$ & $8.03 E+20$ & $7.72 E+20$ & $8.05 E+20$ & $8.46 \mathrm{E}+20$ & $8.92 E+20$ & $1.06 \mathrm{E}+21$ \\
\hline 19.5 & $9.71 E+20$ & $8.30 \mathrm{E}+20$ & $7.57 \mathrm{E}+20$ & $7.39 \mathrm{E}+20$ & $7.08 \mathrm{E}+20$ & $7.39 \mathrm{E}+20$ & $7.59 \mathrm{E}+20$ & $8.28 \mathrm{E}+20$ & $9.74 \mathrm{E}+20$ \\
\hline 20.5 & $9.01 E+20$ & $7.44 \mathrm{E}+20$ & $6.99 \mathrm{E}+20$ & $6.73 E+20$ & $6.35 E+20$ & $6.69 \mathrm{E}+20$ & $6.90 \mathrm{E}+20$ & $7.39 \mathrm{E}+20$ & $8.82 \mathrm{E}+20$ \\
\hline 21.5 & $8.05 E+20$ & $6.61 \mathrm{E}+20$ & $6.11 \mathrm{E}+20$ & $5.88 \mathrm{E}+20$ & $5.55 \mathrm{E}+20$ & $5.79 E+20$ & $6.14 \mathrm{E}+20$ & $6.61 E+20$ & $7.95 \mathrm{E}+20$ \\
\hline 22.5 & $7.18 \mathrm{E}+20$ & $5.84 \mathrm{E}+20$ & $5.27 \mathrm{E}+20$ & $5.09 \mathrm{E}+20$ & $4.90 \mathrm{E}+20$ & $5.11 \mathrm{E}+20$ & $5.32 \mathrm{E}+20$ & $5.89 \mathrm{E}+20$ & $7.01 \mathrm{E}+20$ \\
\hline 23.5 & $8.96 \mathrm{E}+20$ & $7.17 \mathrm{E}+20$ & $6.49 \mathrm{E}+20$ & $6.49 \mathrm{E}+20$ & $6.20 \mathrm{E}+20$ & $6.45 \mathrm{E}+20$ & $6.70 \mathrm{E}+20$ & $7.23 \mathrm{E}+20$ & $8.91 \mathrm{E}+20$ \\
\hline Ax. Avg. & $1.05 \mathrm{E}+21$ & $8.73 E+20$ & $8.08 \mathrm{E}+20$ & $7.80 \mathrm{E}+20$ & $7.45 \mathrm{E}+20$ & $7.80 \mathrm{E}+20$ & $8.09 E+20$ & $8.72 E+20$ & $1.05 \mathrm{E}+21$ \\
\hline
\end{tabular}


Table A-8. Fission Density Data (fissions $/ \mathrm{cm}^{3}$ ) in Fuel Plate 8 in Element Discharged from Reference Mixed Burnup Core: FT loaded with typical samples.

\section{Fuel core width 2.440 inches Fuel core thickness 20 mil}

\begin{tabular}{|c|c|c|c|c|c|c|c|c|c|}
\hline $\begin{array}{c}\text { Axial } \\
\text { position } \\
\text { from top of } \\
\text { fuel (inches) }\end{array}$ & $\begin{array}{c}0.000 \text { to } \\
0.197 \\
\text { inches }\end{array}$ & $\begin{array}{c}0.197 \text { to } \\
0.394 \\
\text { inches }\end{array}$ & $\begin{array}{c}0.394 \text { to } \\
0.591 \\
\text { inches }\end{array}$ & $\begin{array}{c}0.591 \text { to } \\
1.010 \\
\text { inches }\end{array}$ & $\begin{array}{l}1.010 \text { to } \\
1.430 \\
\text { inches }\end{array}$ & $\begin{array}{l}1.430 \text { to } \\
1.850 \\
\text { inches }\end{array}$ & $\begin{array}{l}1.850 \text { to } \\
2.047 \\
\text { inches }\end{array}$ & $\begin{array}{l}2.047 \text { to } \\
2.243 \\
\text { inches }\end{array}$ & $\begin{array}{c}2.243 \text { to } \\
2.440 \\
\text { inches }\end{array}$ \\
\hline 0.5 & $7.03 E+20$ & $5.72 \mathrm{E}+20$ & $5.21 \mathrm{E}+20$ & $5.17 \mathrm{E}+20$ & $4.95 \mathrm{E}+20$ & $5.17 \mathrm{E}+20$ & $5.22 \mathrm{E}+20$ & $5.66 \mathrm{E}+20$ & $7.01 \mathrm{E}+20$ \\
\hline 1.5 & $5.85 E+20$ & $4.83 \mathrm{E}+20$ & $4.46 \mathrm{E}+20$ & $4.25 \mathrm{E}+20$ & $4.06 \mathrm{E}+20$ & $4.20 \mathrm{E}+20$ & $4.52 \mathrm{E}+20$ & $4.81 \mathrm{E}+20$ & $5.97 E+20$ \\
\hline 2.5 & $6.83 E+20$ & $5.80 \mathrm{E}+20$ & $5.29 \mathrm{E}+20$ & $5.08 \mathrm{E}+20$ & $4.86 \mathrm{E}+20$ & $5.09 \mathrm{E}+20$ & $5.27 \mathrm{E}+20$ & $5.82 \mathrm{E}+20$ & $6.72 \mathrm{E}+20$ \\
\hline 3.5 & $7.93 E+20$ & $6.66 \mathrm{E}+20$ & $6.04 \mathrm{E}+20$ & $6.01 \mathrm{E}+20$ & $5.69 \mathrm{E}+20$ & $5.97 \mathrm{E}+20$ & $6.22 E+20$ & $6.75 \mathrm{E}+20$ & $7.93 E+20$ \\
\hline 4.5 & $8.96 \mathrm{E}+20$ & $7.46 \mathrm{E}+20$ & $6.86 \mathrm{E}+20$ & $6.64 \mathrm{E}+20$ & $6.32 E+20$ & $6.62 \mathrm{E}+20$ & $6.82 E+20$ & $7.51 \mathrm{E}+20$ & $8.87 E+20$ \\
\hline 5.5 & $9.74 E+20$ & $8.18 E+20$ & $7.67 E+20$ & $7.39 \mathrm{E}+20$ & $7.01 E+20$ & $7.33 E+20$ & $7.67 E+20$ & $8.07 E+20$ & $9.82 \mathrm{E}+20$ \\
\hline 6.5 & $1.05 E+21$ & $8.75 \mathrm{E}+20$ & $8.30 \mathrm{E}+20$ & $7.94 \mathrm{E}+20$ & $7.59 \mathrm{E}+20$ & $7.97 \mathrm{E}+20$ & $8.20 \mathrm{E}+20$ & $8.89 \mathrm{E}+20$ & $1.07 \mathrm{E}+21$ \\
\hline 7.5 & $1.13 E+21$ & $9.17 \mathrm{E}+20$ & $8.84 \mathrm{E}+20$ & $8.41 \mathrm{E}+20$ & $7.99 \mathrm{E}+20$ & $8.36 \mathrm{E}+20$ & $8.86 \mathrm{E}+20$ & $9.33 \mathrm{E}+20$ & $1.12 \mathrm{E}+21$ \\
\hline 8.5 & $1.17 \mathrm{E}+21$ & $9.61 \mathrm{E}+20$ & $8.98 \mathrm{E}+20$ & $8.85 E+20$ & $8.46 \mathrm{E}+20$ & $8.86 \mathrm{E}+20$ & $9.11 \mathrm{E}+20$ & $9.70 \mathrm{E}+20$ & $1.18 \mathrm{E}+21$ \\
\hline 9.5 & $1.23 \mathrm{E}+21$ & $9.98 \mathrm{E}+20$ & $9.44 \mathrm{E}+20$ & $9.21 E+20$ & $8.77 \mathrm{E}+20$ & $9.16 \mathrm{E}+20$ & $9.53 \mathrm{E}+20$ & $1.02 \mathrm{E}+21$ & $1.21 \mathrm{E}+21$ \\
\hline 10.5 & $1.26 \mathrm{E}+21$ & $1.03 \mathrm{E}+21$ & $9.82 E+20$ & $9.35 \mathrm{E}+20$ & $8.90 \mathrm{E}+20$ & $9.39 E+20$ & $9.68 \mathrm{E}+20$ & $1.04 \mathrm{E}+21$ & $1.24 \mathrm{E}+21$ \\
\hline 11.5 & $1.26 \mathrm{E}+21$ & $1.06 \mathrm{E}+21$ & $9.81 E+20$ & $9.53 \mathrm{E}+20$ & $9.02 E+20$ & $9.51 \mathrm{E}+20$ & $9.78 E+20$ & $1.04 \mathrm{E}+21$ & $1.25 \mathrm{E}+21$ \\
\hline 12.5 & $1.27 \mathrm{E}+21$ & $1.04 \mathrm{E}+21$ & $9.75 E+20$ & $9.55 \mathrm{E}+20$ & $9.12 \mathrm{E}+20$ & $9.55 \mathrm{E}+20$ & $9.82 \mathrm{E}+20$ & $1.06 \mathrm{E}+21$ & $1.27 E+21$ \\
\hline 13.5 & $1.26 \mathrm{E}+21$ & $1.05 \mathrm{E}+21$ & $9.70 \mathrm{E}+20$ & $9.38 \mathrm{E}+20$ & $8.94 \mathrm{E}+20$ & $9.41 \mathrm{E}+20$ & $9.81 \mathrm{E}+20$ & $1.07 \mathrm{E}+21$ & $1.25 \mathrm{E}+21$ \\
\hline 14.5 & $1.23 \mathrm{E}+21$ & $1.03 \mathrm{E}+21$ & $9.68 \mathrm{E}+20$ & $9.22 \mathrm{E}+20$ & $8.92 \mathrm{E}+20$ & $9.23 \mathrm{E}+20$ & $9.58 \mathrm{E}+20$ & $1.03 \mathrm{E}+21$ & $1.24 \mathrm{E}+21$ \\
\hline 15.5 & $1.18 \mathrm{E}+21$ & $1.02 \mathrm{E}+21$ & $9.38 \mathrm{E}+20$ & $9.02 \mathrm{E}+20$ & $8.59 \mathrm{E}+20$ & $8.98 \mathrm{E}+20$ & $9.33 \mathrm{E}+20$ & $9.94 \mathrm{E}+20$ & $1.17 \mathrm{E}+21$ \\
\hline 16.5 & $1.15 \mathrm{E}+21$ & $9.54 \mathrm{E}+20$ & $9.03 E+20$ & $8.74 \mathrm{E}+20$ & $8.19 E+20$ & $8.70 \mathrm{E}+20$ & $9.04 \mathrm{E}+20$ & $9.74 \mathrm{E}+20$ & $1.16 \mathrm{E}+21$ \\
\hline 17.5 & $1.07 \mathrm{E}+21$ & $9.18 \mathrm{E}+20$ & $8.50 \mathrm{E}+20$ & $8.30 \mathrm{E}+20$ & $7.83 \mathrm{E}+20$ & $8.21 \mathrm{E}+20$ & $8.55 \mathrm{E}+20$ & $9.12 \mathrm{E}+20$ & $1.07 \mathrm{E}+21$ \\
\hline 18.5 & $1.01 \mathrm{E}+21$ & $8.56 \mathrm{E}+20$ & $8.06 \mathrm{E}+20$ & $7.76 \mathrm{E}+20$ & $7.32 \mathrm{E}+20$ & $7.72 \mathrm{E}+20$ & $8.05 E+20$ & $8.60 \mathrm{E}+20$ & $1.01 \mathrm{E}+21$ \\
\hline 19.5 & $9.34 \mathrm{E}+20$ & $7.92 \mathrm{E}+20$ & $7.29 \mathrm{E}+20$ & $7.05 E+20$ & $6.77 E+20$ & $7.08 \mathrm{E}+20$ & $7.22 \mathrm{E}+20$ & $7.76 \mathrm{E}+20$ & $9.52 \mathrm{E}+20$ \\
\hline 20.5 & $8.51 E+20$ & $7.18 \mathrm{E}+20$ & $6.61 \mathrm{E}+20$ & $6.38 \mathrm{E}+20$ & $6.01 \mathrm{E}+20$ & $6.42 \mathrm{E}+20$ & $6.56 \mathrm{E}+20$ & $7.07 \mathrm{E}+20$ & $8.42 \mathrm{E}+20$ \\
\hline 21.5 & $7.59 \mathrm{E}+20$ & $6.33 E+20$ & $5.82 \mathrm{E}+20$ & $5.65 E+20$ & $5.39 \mathrm{E}+20$ & $5.63 \mathrm{E}+20$ & $5.82 \mathrm{E}+20$ & $6.47 \mathrm{E}+20$ & $7.53 \mathrm{E}+20$ \\
\hline 22.5 & $6.82 \mathrm{E}+20$ & $5.45 \mathrm{E}+20$ & $5.02 \mathrm{E}+20$ & $4.85 \mathrm{E}+20$ & $4.71 \mathrm{E}+20$ & $4.92 \mathrm{E}+20$ & $5.13 E+20$ & $5.61 \mathrm{E}+20$ & $6.75 \mathrm{E}+20$ \\
\hline 23.5 & $8.66 \mathrm{E}+20$ & $6.89 \mathrm{E}+20$ & $6.59 \mathrm{E}+20$ & $6.22 \mathrm{E}+20$ & $6.00 \mathrm{E}+20$ & $6.28 \mathrm{E}+20$ & $6.33 \mathrm{E}+20$ & $6.95 \mathrm{E}+20$ & $8.81 \mathrm{E}+20$ \\
\hline Ax. Avg. & $1.00 \mathrm{E}+21$ & $8.31 \mathrm{E}+20$ & $7.76 \mathrm{E}+20$ & $7.50 \mathrm{E}+20$ & $7.14 \mathrm{E}+20$ & $7.49 \mathrm{E}+20$ & $7.76 \mathrm{E}+20$ & $8.35 E+20$ & $9.99 E+20$ \\
\hline
\end{tabular}


Table A-9. Fission Density Data (fissions $/ \mathrm{cm}^{3}$ ) in Fuel Plate 9 in Element Discharged from Reference Mixed Burnup Core: FT loaded with typical samples.

Fuel core width 2.547 inches Fuel core thickness 20 mil

\begin{tabular}{|c|c|c|c|c|c|c|c|c|c|}
\hline $\begin{array}{c}\text { Axial } \\
\text { position } \\
\text { from top of } \\
\text { fuel (inches) }\end{array}$ & $\begin{array}{c}0.000 \text { to } \\
0.197 \\
\text { inches }\end{array}$ & $\begin{array}{c}0.197 \text { to } \\
0.394 \\
\text { inches }\end{array}$ & $\begin{array}{c}0.394 \text { to } \\
0.591 \\
\text { inches }\end{array}$ & $\begin{array}{c}0.591 \text { to } \\
1.046 \\
\text { inches }\end{array}$ & $\begin{array}{l}1.046 \text { to } \\
1.501 \\
\text { inches }\end{array}$ & $\begin{array}{l}1.501 \text { to } \\
1.957 \\
\text { inches }\end{array}$ & $\begin{array}{l}1.957 \text { to } \\
2.153 \\
\text { inches }\end{array}$ & $\begin{array}{c}2.153 \text { to } \\
2.350 \\
\text { inches }\end{array}$ & $\begin{array}{c}2.350 \text { to } \\
2.547 \\
\text { inches }\end{array}$ \\
\hline 0.5 & $6.60 \mathrm{E}+20$ & $5.48 \mathrm{E}+20$ & $5.03 \mathrm{E}+20$ & $4.95 \mathrm{E}+20$ & $4.72 \mathrm{E}+20$ & $4.87 \mathrm{E}+20$ & $5.10 \mathrm{E}+20$ & $5.41 \mathrm{E}+20$ & $6.65 \mathrm{E}+20$ \\
\hline 1.5 & $5.58 \mathrm{E}+20$ & $4.66 \mathrm{E}+20$ & $4.29 E+20$ & $4.09 \mathrm{E}+20$ & $3.90 \mathrm{E}+20$ & $4.09 \mathrm{E}+20$ & $4.20 \mathrm{E}+20$ & $4.56 \mathrm{E}+20$ & $5.60 \mathrm{E}+20$ \\
\hline 2.5 & $6.51 \mathrm{E}+20$ & $5.48 \mathrm{E}+20$ & $5.05 E+20$ & $4.88 \mathrm{E}+20$ & $4.68 \mathrm{E}+20$ & $4.88 \mathrm{E}+20$ & $4.99 E+20$ & $5.47 \mathrm{E}+20$ & $6.56 \mathrm{E}+20$ \\
\hline 3.5 & $7.43 E+20$ & $6.25 E+20$ & $5.86 \mathrm{E}+20$ & $5.73 E+20$ & $5.43 E+20$ & $5.71 \mathrm{E}+20$ & $6.02 E+20$ & $6.29 E+20$ & $7.62 \mathrm{E}+20$ \\
\hline 4.5 & $8.50 \mathrm{E}+20$ & $7.17 \mathrm{E}+20$ & $6.59 E+20$ & $6.41 \mathrm{E}+20$ & $6.11 E+20$ & $6.33 E+20$ & $6.49 \mathrm{E}+20$ & $7.06 \mathrm{E}+20$ & $8.33 E+20$ \\
\hline 5.5 & $9.31 E+20$ & $7.77 E+20$ & $7.35 E+20$ & $7.05 E+20$ & $6.73 E+20$ & $7.10 \mathrm{E}+20$ & $9 E+20$ & $7.81 E+20$ & $9.28 \mathrm{E}+20$ \\
\hline 6.5 & $1.01 \mathrm{E}+21$ & $8.39 \mathrm{E}+20$ & $7.89 \mathrm{E}+20$ & $7.67 \mathrm{E}+20$ & $7.31 \mathrm{E}+20$ & $7.62 \mathrm{E}+20$ & $7.95 \mathrm{E}+20$ & $8.42 \mathrm{E}+20$ & $1.00 \mathrm{E}+21$ \\
\hline 7.5 & $1.06 \mathrm{E}+21$ & $9.05 E+20$ & $8.47 E+20$ & $8.06 \mathrm{E}+20$ & $7.66 \mathrm{E}+20$ & $7.96 \mathrm{E}+20$ & $8.33 E+20$ & $9.02 \mathrm{E}+20$ & $1.07 E+21$ \\
\hline 8.5 & $1.11 \mathrm{E}+21$ & $9.36 \mathrm{E}+20$ & $8.60 \mathrm{E}+20$ & $8.40 \mathrm{E}+20$ & $8.17 E+20$ & $8.61 \mathrm{E}+20$ & $8.79 \mathrm{E}+20$ & $9.30 \mathrm{E}+20$ & $1.10 \mathrm{E}+21$ \\
\hline 9.5 & $1.15 \mathrm{E}+21$ & $9.59 \mathrm{E}+20$ & $9.04 \mathrm{E}+20$ & $8.74 \mathrm{E}+20$ & $8.40 \mathrm{E}+20$ & $8.87 \mathrm{E}+20$ & $9.11 \mathrm{E}+20$ & $9.81 \mathrm{E}+20$ & $1.15 \mathrm{E}+21$ \\
\hline 10.5 & $1.17 E+21$ & $1.00 \mathrm{E}+21$ & $9.22 E+20$ & $8.91 \mathrm{E}+20$ & $8.55 E+20$ & $8.92 \mathrm{E}+20$ & $9.22 \mathrm{E}+20$ & $9.96 \mathrm{E}+20$ & $1.18 \mathrm{E}+21$ \\
\hline 11.5 & $1.22 E+21$ & $1.01 \mathrm{E}+21$ & $9.40 \mathrm{E}+20$ & $9.05 E+20$ & $8.64 E+20$ & $9.06 \mathrm{E}+20$ & $9.47 E+20$ & $1.01 \mathrm{E}+21$ & $1.21 \mathrm{E}+21$ \\
\hline 12.5 & $1.19 \mathrm{E}+21$ & $1.01 \mathrm{E}+21$ & $9.43 \mathrm{E}+20$ & $9.16 \mathrm{E}+20$ & $8.67 E+20$ & $9.05 E+20$ & $9.35 E+20$ & $1.02 \mathrm{E}+21$ & $1.21 \mathrm{E}+21$ \\
\hline 13.5 & $1.20 \mathrm{E}+21$ & $9.98 \mathrm{E}+20$ & $9.47 \mathrm{E}+20$ & $9.00 \mathrm{E}+20$ & $8.57 E+20$ & $9.01 \mathrm{E}+20$ & $9.37 \mathrm{E}+20$ & $1.01 \mathrm{E}+21$ & $1.18 \mathrm{E}+21$ \\
\hline 14.5 & $1.17 \mathrm{E}+21$ & $9.88 \mathrm{E}+20$ & $9.29 \mathrm{E}+20$ & $8.91 \mathrm{E}+20$ & $8.55 \mathrm{E}+20$ & $8.87 \mathrm{E}+20$ & $9.16 \mathrm{E}+20$ & $9.85 \mathrm{E}+20$ & $1.18 \mathrm{E}+21$ \\
\hline 15.5 & $1.14 \mathrm{E}+21$ & $9.64 \mathrm{E}+20$ & $8.93 E+20$ & $8.70 \mathrm{E}+20$ & $8.19 E+20$ & $8.65 E+20$ & $9.00 \mathrm{E}+20$ & $9.58 \mathrm{E}+20$ & $1.14 \mathrm{E}+21$ \\
\hline 16.5 & $1.08 \mathrm{E}+21$ & $9.16 \mathrm{E}+20$ & $8.59 \mathrm{E}+20$ & $8.27 E+20$ & $7.94 \mathrm{E}+20$ & $8.31 \mathrm{E}+20$ & $8.61 \mathrm{E}+20$ & $9.20 \mathrm{E}+20$ & $1.09 \mathrm{E}+21$ \\
\hline 17.5 & $1.04 \mathrm{E}+21$ & $8.77 \mathrm{E}+20$ & $8.11 \mathrm{E}+20$ & $7.92 \mathrm{E}+20$ & $7.56 \mathrm{E}+20$ & $7.89 \mathrm{E}+20$ & $8.07 E+20$ & $8.67 \mathrm{E}+20$ & $1.02 \mathrm{E}+21$ \\
\hline 18.5 & $9.69 E+20$ & $8.17 E+20$ & $7.62 \mathrm{E}+20$ & $7.44 \mathrm{E}+20$ & $7.07 E+20$ & $7.31 \mathrm{E}+20$ & $7.76 \mathrm{E}+20$ & $8.23 E+20$ & $9.66 \mathrm{E}+20$ \\
\hline 19.5 & $8.88 E+20$ & $7.69 \mathrm{E}+20$ & $7.13 E+20$ & $6.79 E+20$ & $6.52 E+20$ & $6.78 \mathrm{E}+20$ & $7.06 \mathrm{E}+20$ & $7.64 \mathrm{E}+20$ & $8.94 E+20$ \\
\hline 20.5 & $8.07 E+20$ & $6.83 \mathrm{E}+20$ & $6.32 \mathrm{E}+20$ & $6.08 \mathrm{E}+20$ & $5.83 E+20$ & $6.13 E+20$ & $6.33 E+20$ & $6.78 \mathrm{E}+20$ & $8.07 E+20$ \\
\hline 21.5 & $7.28 \mathrm{E}+20$ & $6.08 \mathrm{E}+20$ & $5.67 \mathrm{E}+20$ & $5.38 \mathrm{E}+20$ & $5.13 E+20$ & $5.43 \mathrm{E}+20$ & $5.62 \mathrm{E}+20$ & $6.05 \mathrm{E}+20$ & $7.19 \mathrm{E}+20$ \\
\hline 22.5 & $6.46 \mathrm{E}+20$ & $5.24 \mathrm{E}+20$ & $4.75 E+20$ & $4.71 \mathrm{E}+20$ & $4.54 \mathrm{E}+20$ & $4.67 \mathrm{E}+20$ & $4.87 \mathrm{E}+20$ & $5.34 \mathrm{E}+20$ & $6.55 \mathrm{E}+20$ \\
\hline 23.5 & $8.32 \mathrm{E}+20$ & $6.80 \mathrm{E}+20$ & $6.17 \mathrm{E}+20$ & $5.91 \mathrm{E}+20$ & $5.78 \mathrm{E}+20$ & $5.99 \mathrm{E}+20$ & $6.23 \mathrm{E}+20$ & $6.58 \mathrm{E}+20$ & $8.30 \mathrm{E}+20$ \\
\hline Ax. Avg. & $9.50 \mathrm{E}+20$ & $7.99 \mathrm{E}+20$ & $7.43 E+20$ & $7.18 \mathrm{E}+20$ & $6.86 \mathrm{E}+20$ & $7.17 \mathrm{E}+20$ & $7.43 E+20$ & $7.97 \mathrm{E}+20$ & $9.50 \mathrm{E}+20$ \\
\hline
\end{tabular}


Table A-10. Fission Density Data (fissions $/ \mathrm{cm}^{3}$ ) in Fuel Plate 10 in Element Discharged from Reference Mixed Burnup Core: FT loaded with typical samples.

Fuel core width 2.654 inches $\quad$ Fuel core thickness $20 \quad$ mil

\begin{tabular}{|c|c|c|c|c|c|c|c|c|c|}
\hline $\begin{array}{c}\text { Axial } \\
\text { position } \\
\text { from top of } \\
\text { fuel (inches) }\end{array}$ & $\begin{array}{c}0.000 \text { to } \\
0.197 \\
\text { inches }\end{array}$ & $\begin{array}{c}0.197 \text { to } \\
0.394 \\
\text { inches }\end{array}$ & $\begin{array}{c}0.394 \text { to } \\
0.591 \\
\text { inches }\end{array}$ & $\begin{array}{c}0.591 \text { to } \\
1.082 \\
\text { inches }\end{array}$ & $\begin{array}{l}1.082 \text { to } \\
1.572 \\
\text { inches }\end{array}$ & $\begin{array}{l}1.572 \text { to } \\
2.063 \\
\text { inches }\end{array}$ & $\begin{array}{c}2.063 \text { to } \\
2.260 \\
\text { inches }\end{array}$ & $\begin{array}{l}2.260 \text { to } \\
2.457 \\
\text { inches }\end{array}$ & $\begin{array}{c}2.457 \text { to } \\
2.654 \\
\text { inches }\end{array}$ \\
\hline 0.5 & $6.35 \mathrm{E}+20$ & $5.35 \mathrm{E}+20$ & $4.88 \mathrm{E}+20$ & $4.72 \mathrm{E}+20$ & $4.61 \mathrm{E}+20$ & $4.76 \mathrm{E}+20$ & $4.93 E+20$ & $5.27 \mathrm{E}+20$ & $6.40 \mathrm{E}+20$ \\
\hline 1.5 & $5.36 \mathrm{E}+20$ & $4.48 \mathrm{E}+20$ & $4.15 E+20$ & $3.94 \mathrm{E}+20$ & $3.84 \mathrm{E}+20$ & $3.90 \mathrm{E}+20$ & $4.14 \mathrm{E}+20$ & $4.47 \mathrm{E}+20$ & $5.32 \mathrm{E}+20$ \\
\hline 2.5 & $6.24 \mathrm{E}+20$ & $5.23 E+20$ & $4.94 \mathrm{E}+20$ & $4.74 \mathrm{E}+20$ & $4.60 \mathrm{E}+20$ & $4.68 \mathrm{E}+20$ & $4.92 \mathrm{E}+20$ & $5.22 \mathrm{E}+20$ & $6.23 E+20$ \\
\hline 3.5 & $7.31 \mathrm{E}+20$ & $6.19 E+20$ & $5.84 \mathrm{E}+20$ & $5.54 \mathrm{E}+20$ & $5.35 E+20$ & $5.52 \mathrm{E}+20$ & $5.88 \mathrm{E}+20$ & $6.12 \mathrm{E}+20$ & $7.25 \mathrm{E}+20$ \\
\hline 4.5 & $8.16 \mathrm{E}+20$ & $6.94 \mathrm{E}+20$ & $6.42 \mathrm{E}+20$ & $6.25 E+20$ & $6.00 \mathrm{E}+20$ & $6.30 \mathrm{E}+20$ & $6.43 E+20$ & $7.00 \mathrm{E}+20$ & $8.27 E+20$ \\
\hline 5.5 & +20 & $7.54 \mathrm{E}+20$ & $7.10 \mathrm{E}+20$ & $6.84 E+20$ & $3 E+20$ & $6.86 E+20$ & $7.09 E+20$ & $7.70 \mathrm{E}+20$ & $9.07 \mathrm{E}+20$ \\
\hline 6.5 & $9.69 \mathrm{E}+20$ & $8.26 \mathrm{E}+20$ & $7.63 \mathrm{E}+20$ & $7.44 \mathrm{E}+20$ & $7.15 \mathrm{E}+20$ & $7.42 \mathrm{E}+20$ & $7.78 \mathrm{E}+20$ & $8.27 \mathrm{E}+20$ & $9.74 \mathrm{E}+20$ \\
\hline 7.5 & $1.02 \mathrm{E}+21$ & $8.90 \mathrm{E}+20$ & $8.08 \mathrm{E}+20$ & $7.83 \mathrm{E}+20$ & $7.49 \mathrm{E}+20$ & $7.82 \mathrm{E}+20$ & $8.30 \mathrm{E}+20$ & $8.76 \mathrm{E}+20$ & $1.03 E+21$ \\
\hline 8.5 & $1.06 \mathrm{E}+21$ & $9.08 \mathrm{E}+20$ & $8.52 \mathrm{E}+20$ & $8.25 E+20$ & $7.91 \mathrm{E}+20$ & $8.34 \mathrm{E}+20$ & $8.66 \mathrm{E}+20$ & $9.11 \mathrm{E}+20$ & $1.08 \mathrm{E}+21$ \\
\hline 9.5 & $1.10 \mathrm{E}+21$ & $9.42 \mathrm{E}+20$ & $8.91 \mathrm{E}+20$ & $8.48 \mathrm{E}+20$ & $8.29 \mathrm{E}+20$ & $8.60 \mathrm{E}+20$ & $8.76 \mathrm{E}+20$ & $9.41 \mathrm{E}+20$ & $1.13 \mathrm{E}+21$ \\
\hline 10.5 & $1.13 E+21$ & $9.72 \mathrm{E}+20$ & $9.04 \mathrm{E}+20$ & $8.71 \mathrm{E}+20$ & $8.27 E+20$ & $8.66 \mathrm{E}+20$ & $8.96 \mathrm{E}+20$ & $9.66 \mathrm{E}+20$ & $1.14 \mathrm{E}+21$ \\
\hline 11.5 & $1.15 E+21$ & $9.86 \mathrm{E}+20$ & $9.21 E+20$ & $8.91 E+20$ & $8.48 E+20$ & $8.74 \mathrm{E}+20$ & $9.12 E+20$ & $9.69 E+20$ & $1.16 \mathrm{E}+21$ \\
\hline 12.5 & $1.17 \mathrm{E}+21$ & $9.86 \mathrm{E}+20$ & $9.12 \mathrm{E}+20$ & $8.88 \mathrm{E}+20$ & $8.51 E+20$ & $8.79 \mathrm{E}+20$ & $9.17 \mathrm{E}+20$ & $9.80 \mathrm{E}+20$ & $1.17 E+21$ \\
\hline 13.5 & $1.15 E+21$ & $9.85 \mathrm{E}+20$ & $9.08 \mathrm{E}+20$ & $8.77 E+20$ & $8.32 \mathrm{E}+20$ & $8.78 \mathrm{E}+20$ & $9.23 E+20$ & $9.75 \mathrm{E}+20$ & $1.15 \mathrm{E}+21$ \\
\hline 14.5 & $1.13 E+21$ & $9.53 \mathrm{E}+20$ & $9.07 \mathrm{E}+20$ & $8.58 \mathrm{E}+20$ & $8.27 \mathrm{E}+20$ & $8.72 \mathrm{E}+20$ & $8.87 \mathrm{E}+20$ & $9.60 \mathrm{E}+20$ & $1.13 \mathrm{E}+21$ \\
\hline 15.5 & $1.10 \mathrm{E}+21$ & $9.39 \mathrm{E}+20$ & $8.62 \mathrm{E}+20$ & $8.34 \mathrm{E}+20$ & $8.13 E+20$ & $8.40 \mathrm{E}+20$ & $8.74 \mathrm{E}+20$ & $9.27 \mathrm{E}+20$ & $1.10 \mathrm{E}+21$ \\
\hline 16.5 & $1.04 \mathrm{E}+21$ & $8.80 \mathrm{E}+20$ & $8.32 \mathrm{E}+20$ & $8.08 \mathrm{E}+20$ & $7.66 \mathrm{E}+20$ & $8.05 E+20$ & $8.43 E+20$ & $9.01 \mathrm{E}+20$ & $1.05 \mathrm{E}+21$ \\
\hline 17.5 & $9.98 \mathrm{E}+20$ & $8.50 \mathrm{E}+20$ & $7.87 \mathrm{E}+20$ & $7.68 \mathrm{E}+20$ & $7.38 \mathrm{E}+20$ & $7.61 \mathrm{E}+20$ & $7.75 \mathrm{E}+20$ & $8.42 \mathrm{E}+20$ & $9.99 \mathrm{E}+20$ \\
\hline 18.5 & $9.25 E+20$ & $7.88 \mathrm{E}+20$ & $7.50 \mathrm{E}+20$ & $7.29 \mathrm{E}+20$ & $6.88 E+20$ & $7.14 \mathrm{E}+20$ & $7.39 \mathrm{E}+20$ & $8.09 \mathrm{E}+20$ & $9.33 E+20$ \\
\hline 19.5 & $8.63 E+20$ & $7.50 \mathrm{E}+20$ & $7.05 E+20$ & $6.70 \mathrm{E}+20$ & $6.43 E+20$ & $6.63 E+20$ & $6.92 E+20$ & $7.36 \mathrm{E}+20$ & $8.58 \mathrm{E}+20$ \\
\hline 20.5 & $7.80 \mathrm{E}+20$ & $6.59 \mathrm{E}+20$ & $6.21 E+20$ & $5.98 \mathrm{E}+20$ & $5.71 E+20$ & $6.01 \mathrm{E}+20$ & $6.15 E+20$ & $6.67 \mathrm{E}+20$ & $7.98 \mathrm{E}+20$ \\
\hline 21.5 & $7.05 E+20$ & $5.91 \mathrm{E}+20$ & $5.45 \mathrm{E}+20$ & $5.28 \mathrm{E}+20$ & $5.02 \mathrm{E}+20$ & $5.30 \mathrm{E}+20$ & $5.44 \mathrm{E}+20$ & $5.91 \mathrm{E}+20$ & $6.89 \mathrm{E}+20$ \\
\hline 22.5 & $6.31 \mathrm{E}+20$ & $5.20 \mathrm{E}+20$ & $4.76 \mathrm{E}+20$ & $4.53 \mathrm{E}+20$ & $4.33 \mathrm{E}+20$ & $4.51 \mathrm{E}+20$ & $4.73 E+20$ & $5.13 \mathrm{E}+20$ & $6.18 \mathrm{E}+20$ \\
\hline 23.5 & $8.14 \mathrm{E}+20$ & $6.63 E+20$ & $6.10 \mathrm{E}+20$ & $5.88 \mathrm{E}+20$ & $5.59 \mathrm{E}+20$ & $5.86 \mathrm{E}+20$ & $6.13 E+20$ & $6.60 \mathrm{E}+20$ & $7.97 \mathrm{E}+20$ \\
\hline Ax. Avg. & $9.15 \mathrm{E}+20$ & $7.78 \mathrm{E}+20$ & $7.24 \mathrm{E}+20$ & $6.99 \mathrm{E}+20$ & $6.70 \mathrm{E}+20$ & $6.97 E+20$ & $7.25 \mathrm{E}+20$ & $7.76 \mathrm{E}+20$ & $9.19 E+20$ \\
\hline
\end{tabular}


Table A-11. Fission Density Data (fissions $/ \mathrm{cm}^{3}$ ) in Fuel Plate 11 in Element Discharged from Reference Mixed Burnup Core: FT loaded with typical samples.

Fuel core width 2.761 inches Fuel core thickness $20 \quad$ mil

\begin{tabular}{|c|c|c|c|c|c|c|c|c|c|}
\hline $\begin{array}{c}\text { Axial } \\
\text { position } \\
\text { from top of } \\
\text { fuel (inches) }\end{array}$ & $\begin{array}{c}0.000 \text { to } \\
0.197 \\
\text { inches }\end{array}$ & $\begin{array}{c}0.197 \text { to } \\
0.394 \\
\text { inches }\end{array}$ & $\begin{array}{c}0.394 \text { to } \\
0.591 \\
\text { inches }\end{array}$ & $\begin{array}{c}0.591 \text { to } \\
1.117 \\
\text { inches }\end{array}$ & $\begin{array}{l}1.117 \text { to } \\
1.644 \\
\text { inches }\end{array}$ & $\begin{array}{l}1.644 \text { to } \\
2.170 \\
\text { inches }\end{array}$ & $\begin{array}{l}2.170 \text { to } \\
2.367 \\
\text { inches }\end{array}$ & $\begin{array}{c}2.367 \text { to } \\
2.564 \\
\text { inches }\end{array}$ & $\begin{array}{c}2.564 \text { to } \\
2.761 \\
\text { inches }\end{array}$ \\
\hline 0.5 & $6.16 \mathrm{E}+20$ & $5.20 \mathrm{E}+20$ & $4.82 \mathrm{E}+20$ & $4.67 \mathrm{E}+20$ & $4.53 \mathrm{E}+20$ & $4.69 E+20$ & $4.71 \mathrm{E}+20$ & $5.10 \mathrm{E}+20$ & $6.22 \mathrm{E}+20$ \\
\hline 1.5 & $5.26 \mathrm{E}+20$ & $4.44 \mathrm{E}+20$ & $4.14 \mathrm{E}+20$ & $3.87 \mathrm{E}+20$ & $3.73 E+20$ & $3.90 \mathrm{E}+20$ & $4.03 E+20$ & $4.27 E+20$ & $5.26 \mathrm{E}+20$ \\
\hline 2.5 & $6.15 E+20$ & $5.14 \mathrm{E}+20$ & $4.91 \mathrm{E}+20$ & $4.71 \mathrm{E}+20$ & $4.52 \mathrm{E}+20$ & $4.72 \mathrm{E}+20$ & $4.82 \mathrm{E}+20$ & $5.21 \mathrm{E}+20$ & $6.14 \mathrm{E}+20$ \\
\hline 3.5 & $7.21 \mathrm{E}+20$ & $6.11 \mathrm{E}+20$ & $5.78 \mathrm{E}+20$ & $5.47 \mathrm{E}+20$ & $5.29 \mathrm{E}+20$ & $5.42 \mathrm{E}+20$ & $5.76 \mathrm{E}+20$ & $6.16 \mathrm{E}+20$ & $7.06 \mathrm{E}+20$ \\
\hline 4.5 & $7.90 \mathrm{E}+20$ & $6.88 \mathrm{E}+20$ & $6.29 E+20$ & $6.13 E+20$ & $5.89 \mathrm{E}+20$ & $6.16 \mathrm{E}+20$ & $6.27 E+20$ & $6.96 \mathrm{E}+20$ & $8.21 E+20$ \\
\hline 5.5 & $8.88 E+20$ & $7.61 \mathrm{E}+20$ & $6.97 E+20$ & $6.80 E+20$ & $6.49 E+20$ & $6.82 E+20$ & $7.06 E+20$ & $7.54 \mathrm{E}+20$ & $8.88 E+20$ \\
\hline 6.5 & $9.37 \mathrm{E}+20$ & $8.11 \mathrm{E}+20$ & $7.63 \mathrm{E}+20$ & $7.30 \mathrm{E}+20$ & $7.05 \mathrm{E}+20$ & $7.29 \mathrm{E}+20$ & $7.71 \mathrm{E}+20$ & $8.23 \mathrm{E}+20$ & $9.62 \mathrm{E}+20$ \\
\hline 7.5 & $1.01 \mathrm{E}+21$ & $8.68 \mathrm{E}+20$ & $8.12 \mathrm{E}+20$ & $7.82 \mathrm{E}+20$ & $7.36 \mathrm{E}+20$ & $7.83 E+20$ & $8.09 \mathrm{E}+20$ & $8.80 \mathrm{E}+20$ & $1.01 \mathrm{E}+21$ \\
\hline 8.5 & $1.06 \mathrm{E}+21$ & $9.16 \mathrm{E}+20$ & $8.48 \mathrm{E}+20$ & $8.14 \mathrm{E}+20$ & $7.87 \mathrm{E}+20$ & $8.24 \mathrm{E}+20$ & $8.60 \mathrm{E}+20$ & $9.01 \mathrm{E}+20$ & $1.07 \mathrm{E}+21$ \\
\hline 9.5 & $1.09 \mathrm{E}+21$ & $9.22 \mathrm{E}+20$ & $8.78 \mathrm{E}+20$ & $8.42 \mathrm{E}+20$ & $8.08 \mathrm{E}+20$ & $8.47 \mathrm{E}+20$ & $8.71 \mathrm{E}+20$ & $9.29 \mathrm{E}+20$ & $1.10 \mathrm{E}+21$ \\
\hline 10.5 & $1.12 \mathrm{E}+21$ & $9.53 \mathrm{E}+20$ & $8.87 E+20$ & $8.60 \mathrm{E}+20$ & $8.25 E+20$ & $8.70 \mathrm{E}+20$ & $8.88 E+20$ & $9.43 E+20$ & $1.12 \mathrm{E}+21$ \\
\hline 11.5 & $1.13 E+21$ & $9.84 \mathrm{E}+20$ & $8.98 E+20$ & $8.78 E+20$ & $8.40 \mathrm{E}+20$ & $8.69 E+20$ & $9.06 E+20$ & $9.68 \mathrm{E}+20$ & $1.14 \mathrm{E}+21$ \\
\hline 12.5 & $1.13 E+21$ & $9.79 \mathrm{E}+20$ & $9.13 E+20$ & $8.75 E+20$ & $8.40 \mathrm{E}+20$ & $8.61 E+20$ & $9.07 E+20$ & $9.64 \mathrm{E}+20$ & $1.14 \mathrm{E}+21$ \\
\hline 13.5 & $1.13 \mathrm{E}+21$ & $9.69 \mathrm{E}+20$ & $9.03 E+20$ & $8.71 \mathrm{E}+20$ & $8.31 \mathrm{E}+20$ & $8.70 \mathrm{E}+20$ & $9.02 \mathrm{E}+20$ & $9.62 \mathrm{E}+20$ & $1.11 \mathrm{E}+21$ \\
\hline 14.5 & $1.10 \mathrm{E}+21$ & $9.43 E+20$ & $8.80 \mathrm{E}+20$ & $8.60 \mathrm{E}+20$ & $8.24 \mathrm{E}+20$ & $8.52 \mathrm{E}+20$ & $8.80 \mathrm{E}+20$ & $9.42 \mathrm{E}+20$ & $1.11 \mathrm{E}+21$ \\
\hline 15.5 & $1.08 \mathrm{E}+21$ & $9.22 \mathrm{E}+20$ & $8.49 \mathrm{E}+20$ & $8.21 \mathrm{E}+20$ & $8.01 \mathrm{E}+20$ & $8.22 \mathrm{E}+20$ & $8.52 \mathrm{E}+20$ & $9.13 \mathrm{E}+20$ & $1.08 \mathrm{E}+21$ \\
\hline 16.5 & $1.03 \mathrm{E}+21$ & $8.89 E+20$ & $8.23 E+20$ & $7.94 \mathrm{E}+20$ & $7.66 \mathrm{E}+20$ & $7.86 \mathrm{E}+20$ & $8.15 E+20$ & $8.88 \mathrm{E}+20$ & $1.03 E+21$ \\
\hline 17.5 & $9.80 \mathrm{E}+20$ & $8.36 \mathrm{E}+20$ & $7.80 \mathrm{E}+20$ & $7.60 \mathrm{E}+20$ & $7.20 \mathrm{E}+20$ & $7.58 \mathrm{E}+20$ & $7.81 \mathrm{E}+20$ & $8.35 \mathrm{E}+20$ & $9.81 \mathrm{E}+20$ \\
\hline 18.5 & $9.13 E+20$ & $8.00 \mathrm{E}+20$ & $7.38 \mathrm{E}+20$ & $7.24 \mathrm{E}+20$ & $6.87 E+20$ & $7.09 E+20$ & $7.49 \mathrm{E}+20$ & $7.94 \mathrm{E}+20$ & $9.22 \mathrm{E}+20$ \\
\hline 19.5 & $8.39 E+20$ & $7.23 E+20$ & $6.86 E+20$ & $6.59 \mathrm{E}+20$ & $6.24 E+20$ & $6.55 E+20$ & $6.79 E+20$ & $7.25 E+20$ & $8.49 E+20$ \\
\hline 20.5 & $7.76 \mathrm{E}+20$ & $6.55 \mathrm{E}+20$ & $6.04 \mathrm{E}+20$ & $5.94 \mathrm{E}+20$ & $5.66 \mathrm{E}+20$ & $5.93 E+20$ & $6.15 E+20$ & $6.48 \mathrm{E}+20$ & $7.74 \mathrm{E}+20$ \\
\hline 21.5 & $6.87 E+20$ & $5.78 \mathrm{E}+20$ & $5.37 \mathrm{E}+20$ & $5.18 \mathrm{E}+20$ & $4.95 E+20$ & $5.14 \mathrm{E}+20$ & $5.36 \mathrm{E}+20$ & $5.78 \mathrm{E}+20$ & $6.83 E+20$ \\
\hline 22.5 & $6.17 E+20$ & $5.07 \mathrm{E}+20$ & $4.76 \mathrm{E}+20$ & $4.51 \mathrm{E}+20$ & $4.23 \mathrm{E}+20$ & $4.54 \mathrm{E}+20$ & $4.70 \mathrm{E}+20$ & $5.13 \mathrm{E}+20$ & $6.05 \mathrm{E}+20$ \\
\hline 23.5 & $7.90 \mathrm{E}+20$ & $6.50 \mathrm{E}+20$ & $5.98 \mathrm{E}+20$ & $5.80 \mathrm{E}+20$ & $5.55 \mathrm{E}+20$ & $5.79 \mathrm{E}+20$ & $6.01 \mathrm{E}+20$ & $6.51 \mathrm{E}+20$ & $8.06 \mathrm{E}+20$ \\
\hline Ax. Avg. & $9.00 \mathrm{E}+20$ & $7.68 \mathrm{E}+20$ & $7.15 \mathrm{E}+20$ & $6.91 \mathrm{E}+20$ & $6.62 \mathrm{E}+20$ & $6.89 E+20$ & $7.15 E+20$ & $7.66 \mathrm{E}+20$ & $9.03 E+20$ \\
\hline
\end{tabular}


Table A-12. Fission Density Data (fissions $/ \mathrm{cm}^{3}$ ) in Fuel Plate 12 in Element Discharged from Reference Mixed Burnup Core: FT loaded with typical samples.

Fuel core width 2.868 inches Fuel core thickness 20 mil

\begin{tabular}{|c|c|c|c|c|c|c|c|c|c|}
\hline $\begin{array}{c}\text { Axial } \\
\text { position } \\
\text { from top of } \\
\text { fuel (inches) }\end{array}$ & $\begin{array}{c}0.000 \text { to } \\
0.197 \\
\text { inches }\end{array}$ & $\begin{array}{c}0.197 \text { to } \\
0.394 \\
\text { inches }\end{array}$ & $\begin{array}{c}0.394 \text { to } \\
0.591 \\
\text { inches }\end{array}$ & $\begin{array}{c}0.591 \text { to } \\
1.153 \\
\text { inches }\end{array}$ & $\begin{array}{l}1.153 \text { to } \\
1.715 \\
\text { inches }\end{array}$ & $\begin{array}{l}1.715 \text { to } \\
2.277 \\
\text { inches }\end{array}$ & $\begin{array}{l}2.277 \text { to } \\
2.474 \\
\text { inches }\end{array}$ & $\begin{array}{c}2.474 \text { to } \\
2.671 \\
\text { inches }\end{array}$ & $\begin{array}{c}2.671 \text { to } \\
2.868 \\
\text { inches }\end{array}$ \\
\hline 0.5 & $5.95 \mathrm{E}+20$ & $5.07 \mathrm{E}+20$ & $4.65 E+20$ & $4.56 \mathrm{E}+20$ & $4.40 \mathrm{E}+20$ & $4.54 \mathrm{E}+20$ & $4.54 \mathrm{E}+20$ & $4.88 \mathrm{E}+20$ & $5.98 \mathrm{E}+20$ \\
\hline 1.5 & $5.15 E+20$ & $4.28 \mathrm{E}+20$ & $3.97 E+20$ & $3.77 \mathrm{E}+20$ & $3.70 \mathrm{E}+20$ & $3.86 \mathrm{E}+20$ & $4.01 \mathrm{E}+20$ & $4.28 \mathrm{E}+20$ & $5.00 \mathrm{E}+20$ \\
\hline 2.5 & $6.01 E+20$ & $5.03 E+20$ & $4.74 \mathrm{E}+20$ & $4.55 E+20$ & $4.39 \mathrm{E}+20$ & $4.57 \mathrm{E}+20$ & $4.73 E+20$ & $5.03 E+20$ & $5.89 E+20$ \\
\hline 3.5 & $7.01 \mathrm{E}+20$ & $5.92 \mathrm{E}+20$ & $5.54 \mathrm{E}+20$ & $5.33 \mathrm{E}+20$ & $5.21 \mathrm{E}+20$ & $5.41 \mathrm{E}+20$ & $5.51 \mathrm{E}+20$ & $5.88 \mathrm{E}+20$ & $6.89 \mathrm{E}+20$ \\
\hline 4.5 & $7.86 \mathrm{E}+20$ & $6.63 E+20$ & $6.26 \mathrm{E}+20$ & $6.06 \mathrm{E}+20$ & $5.82 \mathrm{E}+20$ & $5.98 \mathrm{E}+20$ & $6.35 E+20$ & $6.67 \mathrm{E}+20$ & $7.82 \mathrm{E}+20$ \\
\hline 5.5 & $8.69 E+20$ & $7.47 E+20$ & $6.88 E+20$ & $6.65 E+20$ & $6.40 E+20$ & $6.58 \mathrm{E}+20$ & $6.92 E+20$ & $7.41 \mathrm{E}+20$ & $8.64 \mathrm{E}+20$ \\
\hline 6.5 & $9.30 \mathrm{E}+20$ & $8.02 \mathrm{E}+20$ & $7.58 \mathrm{E}+20$ & $7.16 \mathrm{E}+20$ & $6.93 E+20$ & $7.19 \mathrm{E}+20$ & $7.56 \mathrm{E}+20$ & $7.99 \mathrm{E}+20$ & $9.34 \mathrm{E}+20$ \\
\hline 7.5 & $9.99 E+20$ & $8.55 E+20$ & $7.89 \mathrm{E}+20$ & $7.61 \mathrm{E}+20$ & $7.27 \mathrm{E}+20$ & $7.62 \mathrm{E}+20$ & $8.03 E+20$ & $8.59 \mathrm{E}+20$ & $9.83 E+20$ \\
\hline 8.5 & $1.03 E+21$ & $8.74 \mathrm{E}+20$ & $8.31 \mathrm{E}+20$ & $7.97 \mathrm{E}+20$ & $7.62 \mathrm{E}+20$ & $8.05 E+20$ & $8.27 E+20$ & $8.96 \mathrm{E}+20$ & $1.05 \mathrm{E}+21$ \\
\hline 9.5 & $1.07 E+21$ & $9.34 \mathrm{E}+20$ & $8.56 \mathrm{E}+20$ & $8.20 \mathrm{E}+20$ & $7.98 \mathrm{E}+20$ & +20 & $8.66 \mathrm{E}+20$ & $9.05 E+20$ & $6 \mathrm{E}+21$ \\
\hline 10.5 & $1.09 \mathrm{E}+21$ & $9.36 \mathrm{E}+20$ & $8.74 \mathrm{E}+20$ & $8.41 \mathrm{E}+20$ & $8.13 E+20$ & $8.43 E+20$ & $8.76 \mathrm{E}+20$ & $9.33 \mathrm{E}+20$ & $1.08 \mathrm{E}+21$ \\
\hline 11.5 & $1.11 \mathrm{E}+21$ & $9.45 \mathrm{E}+20$ & $8.78 E+20$ & $8.51 \mathrm{E}+20$ & $8.23 E+20$ & $8.61 \mathrm{E}+20$ & $8.83 E+20$ & $9.53 \mathrm{E}+20$ & $1.12 \mathrm{E}+21$ \\
\hline 12.5 & $1.13 E+21$ & $9.54 \mathrm{E}+20$ & $8.95 E+20$ & $8.55 E+20$ & $8.24 \mathrm{E}+20$ & $8.43 E+20$ & $8.87 E+20$ & $9.44 \mathrm{E}+20$ & $1.12 \mathrm{E}+21$ \\
\hline 13.5 & $1.11 \mathrm{E}+21$ & $9.38 \mathrm{E}+20$ & $8.75 E+20$ & $8.54 \mathrm{E}+20$ & $8.16 \mathrm{E}+20$ & $8.43 \mathrm{E}+20$ & $8.78 \mathrm{E}+20$ & $9.41 \mathrm{E}+20$ & $1.10 \mathrm{E}+21$ \\
\hline 14.5 & $1.09 \mathrm{E}+21$ & $9.30 \mathrm{E}+20$ & $8.67 E+20$ & $8.37 \mathrm{E}+20$ & $8.08 \mathrm{E}+20$ & $8.31 \mathrm{E}+20$ & $8.57 \mathrm{E}+20$ & $9.26 \mathrm{E}+20$ & $1.09 \mathrm{E}+21$ \\
\hline 15.5 & $1.06 \mathrm{E}+21$ & $8.94 \mathrm{E}+20$ & $8.37 E+20$ & $8.04 \mathrm{E}+20$ & $7.79 \mathrm{E}+20$ & $8.05 E+20$ & $8.35 E+20$ & $9.09 \mathrm{E}+20$ & $1.04 \mathrm{E}+21$ \\
\hline 16.5 & $1.00 \mathrm{E}+21$ & $8.63 E+20$ & $8.12 \mathrm{E}+20$ & $7.78 \mathrm{E}+20$ & $7.50 \mathrm{E}+20$ & $7.72 \mathrm{E}+20$ & $8.06 \mathrm{E}+20$ & $8.53 \mathrm{E}+20$ & $1.00 \mathrm{E}+21$ \\
\hline 17.5 & $9.63 \mathrm{E}+20$ & $8.31 \mathrm{E}+20$ & $7.75 \mathrm{E}+20$ & $7.38 \mathrm{E}+20$ & $7.14 \mathrm{E}+20$ & $7.39 \mathrm{E}+20$ & $7.59 \mathrm{E}+20$ & $8.15 E+20$ & $9.60 \mathrm{E}+20$ \\
\hline 18.5 & $8.93 E+20$ & $7.74 \mathrm{E}+20$ & $7.20 \mathrm{E}+20$ & $7.00 \mathrm{E}+20$ & $6.76 \mathrm{E}+20$ & $6.95 \mathrm{E}+20$ & $7.34 \mathrm{E}+20$ & $7.78 \mathrm{E}+20$ & $8.89 E+20$ \\
\hline 19.5 & $8.35 E+20$ & $7.06 \mathrm{E}+20$ & $6.66 \mathrm{E}+20$ & $6.44 \mathrm{E}+20$ & $6.18 E+20$ & $6.32 \mathrm{E}+20$ & $6.72 E+20$ & $7.18 \mathrm{E}+20$ & $8.27 E+20$ \\
\hline 20.5 & $7.58 \mathrm{E}+20$ & $6.45 \mathrm{E}+20$ & $6.05 E+20$ & $5.79 \mathrm{E}+20$ & $5.61 \mathrm{E}+20$ & $5.79 \mathrm{E}+20$ & $6.08 \mathrm{E}+20$ & $6.44 \mathrm{E}+20$ & $7.49 \mathrm{E}+20$ \\
\hline 21.5 & $6.72 \mathrm{E}+20$ & $5.70 \mathrm{E}+20$ & $5.30 \mathrm{E}+20$ & $5.07 \mathrm{E}+20$ & $4.93 E+20$ & $5.11 \mathrm{E}+20$ & $5.25 \mathrm{E}+20$ & $5.71 \mathrm{E}+20$ & $6.82 \mathrm{E}+20$ \\
\hline 22.5 & $5.97 \mathrm{E}+20$ & $5.11 \mathrm{E}+20$ & $4.55 \mathrm{E}+20$ & $4.41 \mathrm{E}+20$ & $4.17 \mathrm{E}+20$ & $4.39 \mathrm{E}+20$ & $4.63 \mathrm{E}+20$ & $5.00 \mathrm{E}+20$ & $5.91 \mathrm{E}+20$ \\
\hline 23.5 & $7.91 \mathrm{E}+20$ & $6.27 \mathrm{E}+20$ & $5.94 \mathrm{E}+20$ & $5.70 \mathrm{E}+20$ & $5.48 \mathrm{E}+20$ & $5.73 \mathrm{E}+20$ & $5.87 \mathrm{E}+20$ & $6.59 \mathrm{E}+20$ & $7.59 \mathrm{E}+20$ \\
\hline Ax. Avg. & $8.83 E+20$ & $7.51 \mathrm{E}+20$ & $7.01 \mathrm{E}+20$ & $6.74 \mathrm{E}+20$ & $6.51 E+20$ & $6.74 \mathrm{E}+20$ & $7.01 \mathrm{E}+20$ & $7.51 \mathrm{E}+20$ & $8.78 E+20$ \\
\hline
\end{tabular}


Table A-13. Fission Density Data (fissions $/ \mathrm{cm}^{3}$ ) in Fuel Plate 13 in Element Discharged from Reference Mixed Burnup Core: FT loaded with typical samples.

\section{Fuel core width 2.974 inches Fuel core thickness 20 mil}

\begin{tabular}{|c|c|c|c|c|c|c|c|c|c|}
\hline $\begin{array}{c}\text { Axial } \\
\text { position } \\
\text { from top of } \\
\text { fuel (inches) }\end{array}$ & $\begin{array}{c}0.000 \text { to } \\
0.197 \\
\text { inches }\end{array}$ & $\begin{array}{c}0.197 \text { to } \\
0.394 \\
\text { inches }\end{array}$ & $\begin{array}{c}0.394 \text { to } \\
0.591 \\
\text { inches }\end{array}$ & $\begin{array}{c}0.591 \text { to } \\
1.188 \\
\text { inches }\end{array}$ & $\begin{array}{l}1.188 \text { to } \\
1.786 \\
\text { inches }\end{array}$ & $\begin{array}{l}1.786 \text { to } \\
2.384 \\
\text { inches }\end{array}$ & $\begin{array}{c}2.384 \text { to } \\
2.581 \\
\text { inches }\end{array}$ & $\begin{array}{c}2.581 \text { to } \\
2.778 \\
\text { inches }\end{array}$ & $\begin{array}{c}2.778 \text { to } \\
2.974 \\
\text { inches }\end{array}$ \\
\hline 0.5 & $5.92 \mathrm{E}+20$ & $5.04 \mathrm{E}+20$ & $4.78 \mathrm{E}+20$ & $4.52 \mathrm{E}+20$ & $4.40 \mathrm{E}+20$ & $4.52 \mathrm{E}+20$ & $4.65 E+20$ & $4.99 \mathrm{E}+20$ & $5.97 \mathrm{E}+20$ \\
\hline 1.5 & $5.18 \mathrm{E}+20$ & $4.34 \mathrm{E}+20$ & $4.05 E+20$ & $3.83 \mathrm{E}+20$ & $3.70 \mathrm{E}+20$ & $3.85 E+20$ & $3.98 E+20$ & $4.23 E+20$ & $5.05 \mathrm{E}+20$ \\
\hline 2.5 & $6.10 \mathrm{E}+20$ & $5.14 \mathrm{E}+20$ & $4.84 \mathrm{E}+20$ & $4.66 \mathrm{E}+20$ & $4.47 \mathrm{E}+20$ & $4.61 E+20$ & $4.84 \mathrm{E}+20$ & $5.16 \mathrm{E}+20$ & $6.01 E+20$ \\
\hline 3.5 & $7.05 E+20$ & $6.04 \mathrm{E}+20$ & $5.73 E+20$ & $5.41 \mathrm{E}+20$ & $5.25 E+20$ & $5.39 \mathrm{E}+20$ & $5.71 \mathrm{E}+20$ & $6.01 \mathrm{E}+20$ & $7.10 \mathrm{E}+20$ \\
\hline 4.5 & $7.89 \mathrm{E}+20$ & $6.82 \mathrm{E}+20$ & $6.29 E+20$ & $6.11 \mathrm{E}+20$ & $5.89 \mathrm{E}+20$ & $6.14 \mathrm{E}+20$ & $6.39 \mathrm{E}+20$ & $6.74 \mathrm{E}+20$ & $7.96 \mathrm{E}+20$ \\
\hline 5.5 & $8.76 \mathrm{E}+20$ & $7.64 \mathrm{E}+20$ & $6.96 \mathrm{E}+20$ & $6.74 \mathrm{E}+20$ & $6.50 \mathrm{E}+20$ & $6.69 \mathrm{E}+20$ & $7.00 \mathrm{E}+20$ & $7.42 \mathrm{E}+20$ & $8.74 \mathrm{E}+20$ \\
\hline 6.5 & $9.49 \mathrm{E}+20$ & $7.97 \mathrm{E}+20$ & $7.61 \mathrm{E}+20$ & $7.32 \mathrm{E}+20$ & $6.92 \mathrm{E}+20$ & $7.20 \mathrm{E}+20$ & $7.55 \mathrm{E}+20$ & $8.26 \mathrm{E}+20$ & $9.56 \mathrm{E}+20$ \\
\hline 7.5 & $1.01 \mathrm{E}+21$ & $8.71 \mathrm{E}+20$ & $8.00 \mathrm{E}+20$ & $7.68 \mathrm{E}+20$ & $7.41 \mathrm{E}+20$ & $7.72 \mathrm{E}+20$ & $8.05 E+20$ & $8.64 \mathrm{E}+20$ & $1.01 \mathrm{E}+21$ \\
\hline 8.5 & $1.05 \mathrm{E}+21$ & $8.94 \mathrm{E}+20$ & $8.40 \mathrm{E}+20$ & $7.97 \mathrm{E}+20$ & $7.83 \mathrm{E}+20$ & $8.10 \mathrm{E}+20$ & $8.46 \mathrm{E}+20$ & $8.98 \mathrm{E}+20$ & $1.06 \mathrm{E}+21$ \\
\hline 9.5 & $1.08 \mathrm{E}+21$ & $9.33 E+20$ & $8.61 \mathrm{E}+20$ & $8.30 \mathrm{E}+20$ & $8.08 \mathrm{E}+20$ & $8.37 \mathrm{E}+20$ & $8.70 \mathrm{E}+20$ & $9.23 \mathrm{E}+20$ & $1.08 \mathrm{E}+21$ \\
\hline 10.5 & $1.09 \mathrm{E}+21$ & $9.43 E+20$ & $8.87 E+20$ & $8.54 \mathrm{E}+20$ & $8.25 E+20$ & $8.52 E+20$ & $8.84 \mathrm{E}+20$ & $9.67 E+20$ & $1.08 \mathrm{E}+21$ \\
\hline 11.5 & $1.12 \mathrm{E}+21$ & $9.61 \mathrm{E}+20$ & $9.00 \mathrm{E}+20$ & $8.63 E+20$ & $8.36 \mathrm{E}+20$ & $8.65 E+20$ & $9.05 E+20$ & $9.70 \mathrm{E}+20$ & $1.12 \mathrm{E}+21$ \\
\hline 12.5 & $1.13 E+21$ & $9.61 \mathrm{E}+20$ & $9.08 \mathrm{E}+20$ & $8.59 E+20$ & $8.41 E+20$ & $8.64 E+20$ & $9.05 E+20$ & $9.59 \mathrm{E}+20$ & $1.12 \mathrm{E}+21$ \\
\hline 13.5 & $1.12 \mathrm{E}+21$ & $9.59 \mathrm{E}+20$ & $8.92 \mathrm{E}+20$ & $8.55 \mathrm{E}+20$ & $8.18 \mathrm{E}+20$ & $8.52 \mathrm{E}+20$ & $8.94 \mathrm{E}+20$ & $9.53 \mathrm{E}+20$ & $1.12 \mathrm{E}+21$ \\
\hline 14.5 & $1.09 \mathrm{E}+21$ & $9.35 \mathrm{E}+20$ & $8.77 \mathrm{E}+20$ & $8.44 \mathrm{E}+20$ & $8.14 \mathrm{E}+20$ & $8.40 \mathrm{E}+20$ & $8.74 \mathrm{E}+20$ & $9.30 \mathrm{E}+20$ & $1.10 \mathrm{E}+21$ \\
\hline 15.5 & $1.06 \mathrm{E}+21$ & $9.08 \mathrm{E}+20$ & $8.55 E+20$ & $8.16 \mathrm{E}+20$ & $7.89 \mathrm{E}+20$ & $8.15 E+20$ & $8.77 \mathrm{E}+20$ & $9.15 \mathrm{E}+20$ & $1.07 \mathrm{E}+21$ \\
\hline 16.5 & $1.03 E+21$ & $8.73 E+20$ & $8.14 \mathrm{E}+20$ & $7.86 \mathrm{E}+20$ & $7.58 \mathrm{E}+20$ & $7.82 \mathrm{E}+20$ & $8.12 \mathrm{E}+20$ & $8.84 \mathrm{E}+20$ & $1.04 \mathrm{E}+21$ \\
\hline 17.5 & $9.66 \mathrm{E}+20$ & $8.47 \mathrm{E}+20$ & $7.88 \mathrm{E}+20$ & $7.44 \mathrm{E}+20$ & $7.16 \mathrm{E}+20$ & $7.45 \mathrm{E}+20$ & $7.73 E+20$ & $8.26 \mathrm{E}+20$ & $9.67 E+20$ \\
\hline 18.5 & $9.05 E+20$ & $7.78 \mathrm{E}+20$ & $7.30 \mathrm{E}+20$ & $7.09 \mathrm{E}+20$ & $6.74 \mathrm{E}+20$ & $6.92 E+20$ & $7.33 E+20$ & $7.92 \mathrm{E}+20$ & $9.04 \mathrm{E}+20$ \\
\hline 19.5 & $8.48 \mathrm{E}+20$ & $7.34 \mathrm{E}+20$ & $6.71 E+20$ & $6.54 \mathrm{E}+20$ & $6.21 \mathrm{E}+20$ & $6.47 E+20$ & $6.70 \mathrm{E}+20$ & $7.34 \mathrm{E}+20$ & $8.44 \mathrm{E}+20$ \\
\hline 20.5 & $7.72 \mathrm{E}+20$ & $6.59 \mathrm{E}+20$ & $6.02 \mathrm{E}+20$ & $5.79 \mathrm{E}+20$ & $5.69 \mathrm{E}+20$ & $5.88 \mathrm{E}+20$ & $5.99 \mathrm{E}+20$ & $6.57 \mathrm{E}+20$ & $7.74 \mathrm{E}+20$ \\
\hline 21.5 & $6.75 \mathrm{E}+20$ & $5.79 E+20$ & $5.42 \mathrm{E}+20$ & $5.16 \mathrm{E}+20$ & $5.00 \mathrm{E}+20$ & $5.15 \mathrm{E}+20$ & $5.37 \mathrm{E}+20$ & $5.82 \mathrm{E}+20$ & $6.78 \mathrm{E}+20$ \\
\hline 22.5 & $6.08 \mathrm{E}+20$ & $5.09 \mathrm{E}+20$ & $4.60 \mathrm{E}+20$ & $4.50 \mathrm{E}+20$ & $4.27 \mathrm{E}+20$ & $4.47 \mathrm{E}+20$ & $4.69 \mathrm{E}+20$ & $5.15 \mathrm{E}+20$ & $6.08 \mathrm{E}+20$ \\
\hline 23.5 & $7.98 \mathrm{E}+20$ & $6.55 \mathrm{E}+20$ & $5.95 \mathrm{E}+20$ & $5.80 \mathrm{E}+20$ & $5.52 \mathrm{E}+20$ & $5.75 \mathrm{E}+20$ & $5.98 \mathrm{E}+20$ & $6.49 \mathrm{E}+20$ & $7.88 \mathrm{E}+20$ \\
\hline Ax. Avg. & $8.92 \mathrm{E}+20$ & $7.62 \mathrm{E}+20$ & $7.10 \mathrm{E}+20$ & $6.82 \mathrm{E}+20$ & $6.58 \mathrm{E}+20$ & $6.81 E+20$ & $7.11 \mathrm{E}+20$ & $7.62 \mathrm{E}+20$ & $8.92 E+20$ \\
\hline
\end{tabular}


Table A-14. Fission Density Data (fissions $/ \mathrm{cm}^{3}$ ) in Fuel Plate 14 in Element Discharged from Reference Mixed Burnup Core: FT loaded with typical samples.

\section{Fuel core width 3.081 inches Fuel core thickness 20 mil}

\begin{tabular}{|c|c|c|c|c|c|c|c|c|c|}
\hline $\begin{array}{c}\text { Axial } \\
\text { position } \\
\text { from top of } \\
\text { fuel (inches) }\end{array}$ & $\begin{array}{c}0.000 \text { to } \\
0.197 \\
\text { inches }\end{array}$ & $\begin{array}{c}0.197 \text { to } \\
0.394 \\
\text { inches }\end{array}$ & $\begin{array}{c}0.394 \text { to } \\
0.591 \\
\text { inches }\end{array}$ & $\begin{array}{c}0.591 \text { to } \\
1.224 \\
\text { inches }\end{array}$ & $\begin{array}{l}1.224 \text { to } \\
1.857 \\
\text { inches }\end{array}$ & $\begin{array}{l}1.857 \text { to } \\
2.491 \\
\text { inches }\end{array}$ & $\begin{array}{c}2.491 \text { to } \\
2.688 \\
\text { inches }\end{array}$ & $\begin{array}{c}2.688 \text { to } \\
2.884 \\
\text { inches }\end{array}$ & $\begin{array}{c}2.884 \text { to } \\
3.081 \\
\text { inches }\end{array}$ \\
\hline 0.5 & $5.75 \mathrm{E}+20$ & $4.89 \mathrm{E}+20$ & $4.60 \mathrm{E}+20$ & $4.39 \mathrm{E}+20$ & $4.28 \mathrm{E}+20$ & $4.44 \mathrm{E}+20$ & $4.47 \mathrm{E}+20$ & $4.93 \mathrm{E}+20$ & $5.71 \mathrm{E}+20$ \\
\hline 1.5 & $5.11 E+20$ & $4.20 \mathrm{E}+20$ & $3.90 \mathrm{E}+20$ & $3.81 \mathrm{E}+20$ & $3.65 E+20$ & $3.78 \mathrm{E}+20$ & $3.95 E+20$ & $4.31 \mathrm{E}+20$ & $4.98 \mathrm{E}+20$ \\
\hline 2.5 & $6.01 E+20$ & $5.05 E+20$ & $4.84 \mathrm{E}+20$ & $4.64 \mathrm{E}+20$ & $4.45 \mathrm{E}+20$ & $4.57 \mathrm{E}+20$ & $4.75 E+20$ & $5.02 \mathrm{E}+20$ & $6.00 \mathrm{E}+20$ \\
\hline 3.5 & $7.17 E+20$ & $6.01 \mathrm{E}+20$ & $5.61 \mathrm{E}+20$ & $5.40 \mathrm{E}+20$ & $5.21 \mathrm{E}+20$ & $5.41 \mathrm{E}+20$ & $5.66 \mathrm{E}+20$ & $5.93 \mathrm{E}+20$ & $6.97 E+20$ \\
\hline 4.5 & $7.93 E+20$ & $6.67 E+20$ & $6.20 \mathrm{E}+20$ & $6.07 E+20$ & $5.81 E+20$ & $6.09 \mathrm{E}+20$ & $6.35 E+20$ & $6.80 \mathrm{E}+20$ & $7.93 \mathrm{E}+20$ \\
\hline 5.5 & $8.84 E+20$ & $7.51 \mathrm{E}+20$ & $7.08 \mathrm{E}+20$ & $6.74 \mathrm{E}+20$ & $6.49 \mathrm{E}+20$ & $5 E+20$ & $6.94 \mathrm{E}+20$ & $7.54 \mathrm{E}+20$ & $8.79 E+20$ \\
\hline 6.5 & $9.38 \mathrm{E}+20$ & $8.14 \mathrm{E}+20$ & $7.57 \mathrm{E}+20$ & $7.21 \mathrm{E}+20$ & $6.94 \mathrm{E}+20$ & $7.25 \mathrm{E}+20$ & $7.64 \mathrm{E}+20$ & $8.19 \mathrm{E}+20$ & $9.45 \mathrm{E}+20$ \\
\hline 7.5 & $1.00 \mathrm{E}+21$ & $8.68 \mathrm{E}+20$ & $7.86 \mathrm{E}+20$ & $7.67 E+20$ & $7.33 E+20$ & $7.68 \mathrm{E}+20$ & $7.93 E+20$ & $8.61 \mathrm{E}+20$ & $1.02 \mathrm{E}+21$ \\
\hline 8.5 & $1.05 \mathrm{E}+21$ & $9.15 \mathrm{E}+20$ & $8.30 \mathrm{E}+20$ & $7.95 \mathrm{E}+20$ & $7.75 \mathrm{E}+20$ & $7.98 \mathrm{E}+20$ & $8.37 \mathrm{E}+20$ & $8.72 \mathrm{E}+20$ & $1.06 \mathrm{E}+21$ \\
\hline 9.5 & $1.08 \mathrm{E}+21$ & $9.29 E+20$ & $8.51 E+20$ & $8.23 \mathrm{E}+20$ & $8.04 \mathrm{E}+20$ & 8.311 & $8.70 \mathrm{E}+20$ & $E+20$ & $7 E+21$ \\
\hline 10.5 & $1.11 \mathrm{E}+21$ & $9.43 E+20$ & $8.68 \mathrm{E}+20$ & $8.50 \mathrm{E}+20$ & $8.21 E+20$ & $8.50 \mathrm{E}+20$ & $8.92 E+20$ & $9.46 \mathrm{E}+20$ & $1.11 \mathrm{E}+21$ \\
\hline 11.5 & $1.12 \mathrm{E}+21$ & $9.55 \mathrm{E}+20$ & $8.93 E+20$ & $8.63 E+20$ & $8.22 E+20$ & $8.63 E+20$ & $9.01 E+20$ & $9.77 \mathrm{E}+20$ & $1.13 E+21$ \\
\hline 12.5 & $1.13 E+21$ & $9.54 \mathrm{E}+20$ & $9.04 \mathrm{E}+20$ & $8.70 \mathrm{E}+20$ & $8.26 \mathrm{E}+20$ & $8.63 E+20$ & $8.94 \mathrm{E}+20$ & $9.76 \mathrm{E}+20$ & $1.13 \mathrm{E}+21$ \\
\hline 13.5 & $1.12 \mathrm{E}+21$ & $9.55 \mathrm{E}+20$ & $9.03 E+20$ & $8.51 \mathrm{E}+20$ & $8.24 \mathrm{E}+20$ & $8.48 \mathrm{E}+20$ & $8.76 \mathrm{E}+20$ & $9.56 \mathrm{E}+20$ & $1.12 \mathrm{E}+21$ \\
\hline 14.5 & $1.11 \mathrm{E}+21$ & $9.38 \mathrm{E}+20$ & $8.83 E+20$ & $8.39 \mathrm{E}+20$ & $8.10 \mathrm{E}+20$ & $8.34 \mathrm{E}+20$ & $8.67 \mathrm{E}+20$ & $9.33 \mathrm{E}+20$ & $1.11 \mathrm{E}+21$ \\
\hline 15.5 & $1.06 \mathrm{E}+21$ & $9.06 \mathrm{E}+20$ & $8.35 E+20$ & $8.12 \mathrm{E}+20$ & $7.89 \mathrm{E}+20$ & $8.14 \mathrm{E}+20$ & $8.49 \mathrm{E}+20$ & $9.13 \mathrm{E}+20$ & $1.07 \mathrm{E}+21$ \\
\hline 16.5 & $1.03 \mathrm{E}+21$ & $8.75 \mathrm{E}+20$ & $8.09 E+20$ & $7.81 \mathrm{E}+20$ & $7.53 E+20$ & $7.91 \mathrm{E}+20$ & $8.13 E+20$ & $8.71 \mathrm{E}+20$ & $1.04 \mathrm{E}+21$ \\
\hline 17.5 & $9.76 \mathrm{E}+20$ & $8.31 \mathrm{E}+20$ & $7.76 \mathrm{E}+20$ & $7.45 \mathrm{E}+20$ & $7.17 \mathrm{E}+20$ & $7.40 \mathrm{E}+20$ & $7.77 E+20$ & $8.18 \mathrm{E}+20$ & $9.71 E+20$ \\
\hline 18.5 & $9.15 E+20$ & $7.83 \mathrm{E}+20$ & $7.24 \mathrm{E}+20$ & $6.95 \mathrm{E}+20$ & $6.71 E+20$ & $6.99 \mathrm{E}+20$ & $7.24 \mathrm{E}+20$ & $7.73 E+20$ & $9.22 \mathrm{E}+20$ \\
\hline 19.5 & $8.57 E+20$ & $7.25 \mathrm{E}+20$ & $6.67 E+20$ & $6.48 \mathrm{E}+20$ & $6.21 \mathrm{E}+20$ & $6.40 \mathrm{E}+20$ & $6.66 \mathrm{E}+20$ & $7.15 \mathrm{E}+20$ & $8.52 \mathrm{E}+20$ \\
\hline 20.5 & $7.75 E+20$ & $6.49 \mathrm{E}+20$ & $6.02 E+20$ & $5.83 \mathrm{E}+20$ & $5.58 \mathrm{E}+20$ & $5.81 \mathrm{E}+20$ & $5.95 \mathrm{E}+20$ & $6.54 \mathrm{E}+20$ & $7.67 \mathrm{E}+20$ \\
\hline 21.5 & $6.83 E+20$ & $5.73 \mathrm{E}+20$ & $5.37 \mathrm{E}+20$ & $5.10 \mathrm{E}+20$ & $4.93 E+20$ & $5.15 \mathrm{E}+20$ & $5.43 \mathrm{E}+20$ & $5.76 \mathrm{E}+20$ & $6.80 \mathrm{E}+20$ \\
\hline 22.5 & $6.06 \mathrm{E}+20$ & $5.06 \mathrm{E}+20$ & $4.62 \mathrm{E}+20$ & $4.42 \mathrm{E}+20$ & $4.17 \mathrm{E}+20$ & $4.42 \mathrm{E}+20$ & $4.73 E+20$ & $5.09 \mathrm{E}+20$ & $6.03 \mathrm{E}+20$ \\
\hline 23.5 & $7.93 E+20$ & $6.41 \mathrm{E}+20$ & $6.04 \mathrm{E}+20$ & $5.71 \mathrm{E}+20$ & $5.48 \mathrm{E}+20$ & $5.67 \mathrm{E}+20$ & $5.96 \mathrm{E}+20$ & $6.45 \mathrm{E}+20$ & $7.83 \mathrm{E}+20$ \\
\hline Ax. Avg. & $8.93 E+20$ & $7.58 \mathrm{E}+20$ & $7.05 E+20$ & $6.78 \mathrm{E}+20$ & $6.53 E+20$ & $6.78 \mathrm{E}+20$ & $7.06 \mathrm{E}+20$ & $7.58 \mathrm{E}+20$ & $8.92 E+20$ \\
\hline
\end{tabular}


Table A 15. Fission Density Data (fissions $/ \mathrm{cm}^{3}$ ) in Fuel Plate 15 in Element Discharged from Reference Mixed Burnup Core: FT loaded with typical samples.

\section{Fuel core width 3.188 inches Fuel core thickness 20 mil}

\begin{tabular}{|c|c|c|c|c|c|c|c|c|c|}
\hline $\begin{array}{c}\text { Axial } \\
\text { position } \\
\text { from top of } \\
\text { fuel (inches) }\end{array}$ & $\begin{array}{c}0.000 \text { to } \\
0.197 \\
\text { inches }\end{array}$ & $\begin{array}{c}0.197 \text { to } \\
0.394 \\
\text { inches }\end{array}$ & $\begin{array}{c}0.394 \text { to } \\
0.591 \\
\text { inches }\end{array}$ & $\begin{array}{c}0.591 \text { to } \\
1.260 \\
\text { inches }\end{array}$ & $\begin{array}{l}1.260 \text { to } \\
1.929 \\
\text { inches }\end{array}$ & $\begin{array}{l}1.929 \text { to } \\
2.597 \\
\text { inches }\end{array}$ & $\begin{array}{l}2.597 \text { to } \\
2.794 \\
\text { inches }\end{array}$ & $\begin{array}{c}2.794 \text { to } \\
2.991 \\
\text { inches }\end{array}$ & $\begin{array}{c}2.991 \text { to } \\
3.188 \\
\text { inches }\end{array}$ \\
\hline 0.5 & $5.70 \mathrm{E}+20$ & $4.83 \mathrm{E}+20$ & $4.50 \mathrm{E}+20$ & $4.28 \mathrm{E}+20$ & $4.20 \mathrm{E}+20$ & $4.32 \mathrm{E}+20$ & $4.45 \mathrm{E}+20$ & $4.73 \mathrm{E}+20$ & $5.60 \mathrm{E}+20$ \\
\hline 1.5 & $4.95 E+20$ & $4.24 \mathrm{E}+20$ & $3.85 E+20$ & $3.74 \mathrm{E}+20$ & $3.61 \mathrm{E}+20$ & $3.77 \mathrm{E}+20$ & $3.94 \mathrm{E}+20$ & $4.26 \mathrm{E}+20$ & $4.99 \mathrm{E}+20$ \\
\hline 2.5 & $5.98 \mathrm{E}+20$ & $5.15 E+20$ & $4.63 E+20$ & $4.56 \mathrm{E}+20$ & $4.39 \mathrm{E}+20$ & $4.55 \mathrm{E}+20$ & $4.71 \mathrm{E}+20$ & $5.09 \mathrm{E}+20$ & $5.95 \mathrm{E}+20$ \\
\hline 3.5 & $7.02 E+20$ & $6.00 \mathrm{E}+20$ & $5.65 E+20$ & $5.37 E+20$ & $5.13 E+20$ & $5.41 \mathrm{E}+20$ & $5.68 \mathrm{E}+20$ & $5.95 \mathrm{E}+20$ & $7.06 \mathrm{E}+20$ \\
\hline 4.5 & $7.94 \mathrm{E}+20$ & $6.71 \mathrm{E}+20$ & $6.20 \mathrm{E}+20$ & $6.01 \mathrm{E}+20$ & $5.86 \mathrm{E}+20$ & $6.09 \mathrm{E}+20$ & $6.32 E+20$ & $6.74 \mathrm{E}+20$ & $7.94 \mathrm{E}+20$ \\
\hline 5.5 & $8.81 E+20$ & $7.31 \mathrm{E}+20$ & $6.89 E+20$ & $6.66 \mathrm{E}+20$ & $6.38 \mathrm{E}+20$ & $6.68 \mathrm{E}+20$ & $6.85 E+20$ & $7.48 \mathrm{E}+20$ & $8.81 E+20$ \\
\hline 6.5 & $9.51 \mathrm{E}+20$ & $8.00 \mathrm{E}+20$ & $7.48 \mathrm{E}+20$ & $7.11 \mathrm{E}+20$ & $6.97 \mathrm{E}+20$ & $7.16 \mathrm{E}+20$ & $7.45 \mathrm{E}+20$ & $8.10 \mathrm{E}+20$ & $9.43 \mathrm{E}+20$ \\
\hline 7.5 & $1.00 \mathrm{E}+21$ & $8.51 \mathrm{E}+20$ & $7.92 \mathrm{E}+20$ & $7.59 \mathrm{E}+20$ & $7.31 \mathrm{E}+20$ & $7.62 \mathrm{E}+20$ & $7.96 \mathrm{E}+20$ & $8.60 \mathrm{E}+20$ & $1.02 \mathrm{E}+21$ \\
\hline 8.5 & $1.05 \mathrm{E}+21$ & $8.87 \mathrm{E}+20$ & $8.26 \mathrm{E}+20$ & $7.92 \mathrm{E}+20$ & $7.64 \mathrm{E}+20$ & $7.92 \mathrm{E}+20$ & $8.35 E+20$ & $8.82 \mathrm{E}+20$ & $1.07 \mathrm{E}+21$ \\
\hline 9.5 & $1.07 E+21$ & $9.22 \mathrm{E}+20$ & $E+20$ & $8.29 E+20$ & $7.92 \mathrm{E}+20$ & +20 & $8.64 \mathrm{E}+20$ & $9.18 \mathrm{E}+20$ & $1.09 \mathrm{E}+21$ \\
\hline 10.5 & $1.10 \mathrm{E}+21$ & $9.39 E+20$ & $8.71 E+20$ & $8.39 E+20$ & $8.07 E+20$ & $8.41 \mathrm{E}+20$ & $8.66 \mathrm{E}+20$ & $9.45 \mathrm{E}+20$ & $1.12 \mathrm{E}+21$ \\
\hline 11.5 & $1.12 \mathrm{E}+21$ & $9.59 \mathrm{E}+20$ & $8.93 E+20$ & $8.56 \mathrm{E}+20$ & $8.16 \mathrm{E}+20$ & $8.39 E+20$ & $8.90 \mathrm{E}+20$ & $9.45 \mathrm{E}+20$ & $1.13 E+21$ \\
\hline 12.5 & $1.14 \mathrm{E}+21$ & $9.52 \mathrm{E}+20$ & $8.92 \mathrm{E}+20$ & $8.49 E+20$ & $8.23 E+20$ & $8.47 \mathrm{E}+20$ & $8.90 \mathrm{E}+20$ & $9.54 \mathrm{E}+20$ & $1.13 \mathrm{E}+21$ \\
\hline 13.5 & $1.12 \mathrm{E}+21$ & $9.38 \mathrm{E}+20$ & $8.96 \mathrm{E}+20$ & $8.52 \mathrm{E}+20$ & $8.18 \mathrm{E}+20$ & $8.47 \mathrm{E}+20$ & $8.87 \mathrm{E}+20$ & $9.53 \mathrm{E}+20$ & $1.09 \mathrm{E}+21$ \\
\hline 14.5 & $1.11 \mathrm{E}+21$ & $9.34 \mathrm{E}+20$ & $8.80 \mathrm{E}+20$ & $8.38 \mathrm{E}+20$ & $8.01 \mathrm{E}+20$ & $8.25 \mathrm{E}+20$ & $8.69 \mathrm{E}+20$ & $9.25 \mathrm{E}+20$ & $1.10 \mathrm{E}+21$ \\
\hline 15.5 & $1.05 E+21$ & $9.01 \mathrm{E}+20$ & $8.42 \mathrm{E}+20$ & $8.11 \mathrm{E}+20$ & $7.80 \mathrm{E}+20$ & $8.08 \mathrm{E}+20$ & $8.40 \mathrm{E}+20$ & $9.08 \mathrm{E}+20$ & $1.06 \mathrm{E}+21$ \\
\hline 16.5 & $1.03 \mathrm{E}+21$ & $8.63 E+20$ & $8.12 \mathrm{E}+20$ & $7.74 \mathrm{E}+20$ & $7.48 \mathrm{E}+20$ & $7.72 \mathrm{E}+20$ & $8.09 \mathrm{E}+20$ & $8.79 \mathrm{E}+20$ & $1.02 \mathrm{E}+21$ \\
\hline 17.5 & $9.62 E+20$ & $8.38 \mathrm{E}+20$ & $7.73 E+20$ & $7.38 \mathrm{E}+20$ & $7.13 E+20$ & $7.40 \mathrm{E}+20$ & $7.59 \mathrm{E}+20$ & $8.15 E+20$ & $9.71 E+20$ \\
\hline 18.5 & $9.11 \mathrm{E}+20$ & $7.80 \mathrm{E}+20$ & $7.23 E+20$ & $7.06 \mathrm{E}+20$ & $6.69 E+20$ & $6.89 E+20$ & $7.27 E+20$ & $7.74 \mathrm{E}+20$ & $9.12 \mathrm{E}+20$ \\
\hline 19.5 & $8.35 E+20$ & $7.19 \mathrm{E}+20$ & $6.65 E+20$ & $6.41 \mathrm{E}+20$ & $6.11 \mathrm{E}+20$ & $6.34 \mathrm{E}+20$ & $6.60 \mathrm{E}+20$ & $7.22 \mathrm{E}+20$ & $8.40 \mathrm{E}+20$ \\
\hline 20.5 & $7.63 E+20$ & $6.44 \mathrm{E}+20$ & $6.07 E+20$ & $5.79 \mathrm{E}+20$ & $5.60 \mathrm{E}+20$ & $5.77 \mathrm{E}+20$ & $6.17 E+20$ & $6.59 \mathrm{E}+20$ & $7.67 \mathrm{E}+20$ \\
\hline 21.5 & $6.82 \mathrm{E}+20$ & $5.74 \mathrm{E}+20$ & $5.21 \mathrm{E}+20$ & $5.13 \mathrm{E}+20$ & $4.91 E+20$ & $5.10 \mathrm{E}+20$ & $5.36 \mathrm{E}+20$ & $5.76 \mathrm{E}+20$ & $6.77 E+20$ \\
\hline 22.5 & $6.16 \mathrm{E}+20$ & $5.04 \mathrm{E}+20$ & $4.71 \mathrm{E}+20$ & $4.35 \mathrm{E}+20$ & $4.21 \mathrm{E}+20$ & $4.34 \mathrm{E}+20$ & $4.67 \mathrm{E}+20$ & $5.08 \mathrm{E}+20$ & $6.11 \mathrm{E}+20$ \\
\hline 23.5 & $7.90 \mathrm{E}+20$ & $6.39 \mathrm{E}+20$ & $5.91 \mathrm{E}+20$ & $5.64 \mathrm{E}+20$ & $5.41 \mathrm{E}+20$ & $5.72 \mathrm{E}+20$ & $5.94 \mathrm{E}+20$ & $6.34 \mathrm{E}+20$ & $7.89 \mathrm{E}+20$ \\
\hline Ax. Avg. & $8.89 \mathrm{E}+20$ & $7.53 \mathrm{E}+20$ & $7.02 \mathrm{E}+20$ & $6.73 E+20$ & $6.47 E+20$ & $6.71 \mathrm{E}+20$ & $7.02 \mathrm{E}+20$ & $7.54 \mathrm{E}+20$ & $8.91 E+20$ \\
\hline
\end{tabular}


Table A-16. Fission Density Data (fissions $/ \mathrm{cm}^{3}$ ) in Fuel Plate 16 in Element Discharged from Reference Mixed Burnup Core: FT loaded with typical samples.

Fuel core width $\quad 3.295$ inches Fuel core thickness 20 mil

\begin{tabular}{|c|c|c|c|c|c|c|c|c|c|}
\hline $\begin{array}{c}\text { Axial } \\
\text { position } \\
\text { from top of } \\
\text { fuel (inches) }\end{array}$ & $\begin{array}{c}0.000 \text { to } \\
0.197 \\
\text { inches }\end{array}$ & $\begin{array}{c}0.197 \text { to } \\
0.394 \\
\text { inches }\end{array}$ & $\begin{array}{c}0.394 \text { to } \\
0.591 \\
\text { inches }\end{array}$ & $\begin{array}{l}0.591 \text { to } \\
1.295 \\
\text { inches }\end{array}$ & $\begin{array}{l}1.295 \text { to } \\
2.000 \\
\text { inches }\end{array}$ & $\begin{array}{l}2.000 \text { to } \\
2.704 \\
\text { inches }\end{array}$ & $\begin{array}{l}2.704 \text { to } \\
2.901 \\
\text { inches }\end{array}$ & $\begin{array}{c}2.901 \text { to } \\
3.098 \\
\text { inches }\end{array}$ & $\begin{array}{c}3.098 \text { to } \\
3.295 \\
\text { inches }\end{array}$ \\
\hline 0.5 & $5.47 \mathrm{E}+20$ & $4.64 \mathrm{E}+20$ & $4.38 \mathrm{E}+20$ & $4.21 \mathrm{E}+20$ & $4.09 \mathrm{E}+20$ & $4.27 \mathrm{E}+20$ & $4.35 \mathrm{E}+20$ & $4.56 \mathrm{E}+20$ & $5.38 \mathrm{E}+20$ \\
\hline 1.5 & $4.93 E+20$ & $4.18 \mathrm{E}+20$ & $3.97 E+20$ & $3.74 \mathrm{E}+20$ & $3.59 \mathrm{E}+20$ & $3.78 \mathrm{E}+20$ & $3.92 E+20$ & $4.22 \mathrm{E}+20$ & $4.91 \mathrm{E}+20$ \\
\hline 2.5 & $6.09 E+20$ & $5.05 E+20$ & $4.70 \mathrm{E}+20$ & $4.57 \mathrm{E}+20$ & $4.41 \mathrm{E}+20$ & $4.56 \mathrm{E}+20$ & $4.74 \mathrm{E}+20$ & $5.09 \mathrm{E}+20$ & $6.12 \mathrm{E}+20$ \\
\hline 3.5 & $7.03 E+20$ & $5.98 \mathrm{E}+20$ & $5.71 \mathrm{E}+20$ & $5.37 E+20$ & $5.22 \mathrm{E}+20$ & $5.40 \mathrm{E}+20$ & $5.55 E+20$ & $6.02 \mathrm{E}+20$ & $7.27 \mathrm{E}+20$ \\
\hline 4.5 & $8.05 E+20$ & $6.90 \mathrm{E}+20$ & $6.38 \mathrm{E}+20$ & $6.06 \mathrm{E}+20$ & $5.88 \mathrm{E}+20$ & $6.10 \mathrm{E}+20$ & $6.38 \mathrm{E}+20$ & $6.79 \mathrm{E}+20$ & $8.06 \mathrm{E}+20$ \\
\hline 5.5 & $4 E+20$ & $7.54 \mathrm{E}+20$ & $6.97 E+20$ & $6.72 E+20$ & $6.46 E+20$ & $6.73 E+20$ & $7.04 E+20$ & $7.40 \mathrm{E}+20$ & $8.89 E+20$ \\
\hline 6.5 & $9.69 \mathrm{E}+20$ & $8.05 \mathrm{E}+20$ & $7.57 \mathrm{E}+20$ & $7.27 \mathrm{E}+20$ & $6.99 \mathrm{E}+20$ & $7.23 \mathrm{E}+20$ & $7.63 E+20$ & $8.22 \mathrm{E}+20$ & $9.60 \mathrm{E}+20$ \\
\hline 7.5 & $1.02 \mathrm{E}+21$ & $8.50 \mathrm{E}+20$ & $8.07 E+20$ & $7.69 \mathrm{E}+20$ & $7.37 \mathrm{E}+20$ & $7.70 \mathrm{E}+20$ & $7.91 \mathrm{E}+20$ & $8.53 \mathrm{E}+20$ & $1.01 \mathrm{E}+21$ \\
\hline 8.5 & $1.08 \mathrm{E}+21$ & $8.90 \mathrm{E}+20$ & $8.33 \mathrm{E}+20$ & $7.95 \mathrm{E}+20$ & $7.67 \mathrm{E}+20$ & $8.01 \mathrm{E}+20$ & $8.43 E+20$ & $9.03 \mathrm{E}+20$ & $1.07 \mathrm{E}+21$ \\
\hline 9.5 & $1.09 \mathrm{E}+21$ & $9.18 \mathrm{E}+20$ & $8.69 \mathrm{E}+20$ & $8.30 \mathrm{E}+20$ & $8.02 \mathrm{E}+20$ & $8.30 \mathrm{E}+20$ & $8.66 \mathrm{E}+20$ & $9.30 \mathrm{E}+20$ & $1.11 \mathrm{E}+21$ \\
\hline 10.5 & $1.12 \mathrm{E}+21$ & $9.42 \mathrm{E}+20$ & $8.89 E+20$ & $8.47 E+20$ & $8.17 E+20$ & $8.49 \mathrm{E}+20$ & $8.77 E+20$ & $9.52 \mathrm{E}+20$ & $1.14 \mathrm{E}+21$ \\
\hline 11.5 & $1.12 E+21$ & $9.72 E+20$ & $8.99 E+20$ & $8.57 E+20$ & $8.21 E+20$ & $8.52 E+20$ & $9.02 E+20$ & $9.84 \mathrm{E}+20$ & $1.15 E+21$ \\
\hline 12.5 & $1.16 \mathrm{E}+21$ & $9.67 \mathrm{E}+20$ & $9.05 E+20$ & $8.48 \mathrm{E}+20$ & $8.28 E+20$ & $8.61 \mathrm{E}+20$ & $9.07 E+20$ & $9.76 \mathrm{E}+20$ & $1.16 \mathrm{E}+21$ \\
\hline 13.5 & $1.15 E+21$ & $9.62 \mathrm{E}+20$ & $8.93 E+20$ & $8.49 \mathrm{E}+20$ & $8.13 E+20$ & $8.54 \mathrm{E}+20$ & $8.95 E+20$ & $9.62 \mathrm{E}+20$ & $1.13 \mathrm{E}+21$ \\
\hline 14.5 & $1.12 \mathrm{E}+21$ & $9.28 \mathrm{E}+20$ & $8.73 E+20$ & $8.43 \mathrm{E}+20$ & $8.07 \mathrm{E}+20$ & $8.38 \mathrm{E}+20$ & $8.81 \mathrm{E}+20$ & $9.31 \mathrm{E}+20$ & $1.11 \mathrm{E}+21$ \\
\hline 15.5 & $1.08 \mathrm{E}+21$ & $9.10 \mathrm{E}+20$ & $8.47 \mathrm{E}+20$ & $8.14 \mathrm{E}+20$ & $7.86 \mathrm{E}+20$ & $8.04 \mathrm{E}+20$ & $8.47 \mathrm{E}+20$ & $9.13 E+20$ & $1.08 \mathrm{E}+21$ \\
\hline 16.5 & $1.04 \mathrm{E}+21$ & $8.85 E+20$ & $8.22 \mathrm{E}+20$ & $7.83 E+20$ & $7.57 \mathrm{E}+20$ & $7.75 \mathrm{E}+20$ & $8.22 \mathrm{E}+20$ & $8.74 \mathrm{E}+20$ & $1.04 \mathrm{E}+21$ \\
\hline 17.5 & $9.82 \mathrm{E}+20$ & $8.31 \mathrm{E}+20$ & $7.79 \mathrm{E}+20$ & $7.41 \mathrm{E}+20$ & $7.17 \mathrm{E}+20$ & $7.36 \mathrm{E}+20$ & $7.70 \mathrm{E}+20$ & $8.37 \mathrm{E}+20$ & $9.94 \mathrm{E}+20$ \\
\hline 18.5 & $9.46 \mathrm{E}+20$ & $7.82 \mathrm{E}+20$ & $7.38 \mathrm{E}+20$ & $7.08 \mathrm{E}+20$ & $6.72 E+20$ & $7.01 \mathrm{E}+20$ & $7.26 \mathrm{E}+20$ & $7.69 \mathrm{E}+20$ & $9.33 E+20$ \\
\hline 19.5 & $8.66 \mathrm{E}+20$ & $7.22 \mathrm{E}+20$ & $6.85 E+20$ & $6.34 \mathrm{E}+20$ & $6.11 E+20$ & $6.39 \mathrm{E}+20$ & $6.74 \mathrm{E}+20$ & $7.21 \mathrm{E}+20$ & $8.64 \mathrm{E}+20$ \\
\hline 20.5 & $7.72 \mathrm{E}+20$ & $6.55 \mathrm{E}+20$ & $6.08 \mathrm{E}+20$ & $5.83 \mathrm{E}+20$ & $5.65 E+20$ & $5.85 \mathrm{E}+20$ & $6.17 E+20$ & $6.57 \mathrm{E}+20$ & $7.83 E+20$ \\
\hline 21.5 & $6.92 \mathrm{E}+20$ & $5.80 \mathrm{E}+20$ & $5.34 \mathrm{E}+20$ & $5.08 \mathrm{E}+20$ & $4.94 \mathrm{E}+20$ & $5.12 \mathrm{E}+20$ & $5.40 \mathrm{E}+20$ & $5.81 \mathrm{E}+20$ & $6.95 \mathrm{E}+20$ \\
\hline 22.5 & $6.22 \mathrm{E}+20$ & $5.15 \mathrm{E}+20$ & $4.67 \mathrm{E}+20$ & $4.43 \mathrm{E}+20$ & $4.30 \mathrm{E}+20$ & $4.40 \mathrm{E}+20$ & $4.67 \mathrm{E}+20$ & $5.05 \mathrm{E}+20$ & $6.15 \mathrm{E}+20$ \\
\hline 23.5 & $7.98 \mathrm{E}+20$ & $6.44 \mathrm{E}+20$ & $6.10 \mathrm{E}+20$ & $5.70 \mathrm{E}+20$ & $5.57 \mathrm{E}+20$ & $5.62 \mathrm{E}+20$ & $6.06 \mathrm{E}+20$ & $6.52 \mathrm{E}+20$ & $7.70 \mathrm{E}+20$ \\
\hline Ax. Avg. & $9.02 E+20$ & $7.58 \mathrm{E}+20$ & $7.09 E+20$ & $6.76 \mathrm{E}+20$ & $6.52 \mathrm{E}+20$ & $6.76 \mathrm{E}+20$ & $7.08 \mathrm{E}+20$ & $7.60 \mathrm{E}+20$ & $9.03 E+20$ \\
\hline
\end{tabular}


Table A-17. Fission Density Data (fissions $/ \mathrm{cm}^{3}$ ) in Fuel Plate 17 in Element Discharged from Reference Mixed Burnup Core: FT loaded with typical samples.

\section{Fuel core width 3.402 inches Fuel core thickness 20 mil}

\begin{tabular}{|c|c|c|c|c|c|c|c|c|c|}
\hline $\begin{array}{c}\text { Axial } \\
\text { position } \\
\text { from top of } \\
\text { fuel (inches) }\end{array}$ & $\begin{array}{c}0.000 \text { to } \\
0.197 \\
\text { inches }\end{array}$ & $\begin{array}{c}0.197 \text { to } \\
0.394 \\
\text { inches }\end{array}$ & $\begin{array}{c}0.394 \text { to } \\
0.591 \\
\text { inches }\end{array}$ & $\begin{array}{c}0.591 \text { to } \\
1.331 \\
\text { inches }\end{array}$ & $\begin{array}{c}1.331 \text { to } \\
2.071 \\
\text { inches }\end{array}$ & $\begin{array}{c}2.071 \text { to } \\
2.811 \\
\text { inches }\end{array}$ & $\begin{array}{c}2.811 \text { to } \\
3.008 \\
\text { inches }\end{array}$ & $\begin{array}{l}3.008 \text { to } \\
3.205 \\
\text { inches }\end{array}$ & $\begin{array}{c}3.205 \text { to } \\
3.402 \\
\text { inches }\end{array}$ \\
\hline 0.5 & $5.40 \mathrm{E}+20$ & $4.63 \mathrm{E}+20$ & $4.33 \mathrm{E}+20$ & $4.16 \mathrm{E}+20$ & $4.03 E+20$ & $4.13 \mathrm{E}+20$ & $4.33 \mathrm{E}+20$ & $4.50 \mathrm{E}+20$ & $5.45 \mathrm{E}+20$ \\
\hline 1.5 & $5.14 \mathrm{E}+20$ & $4.16 \mathrm{E}+20$ & $3.88 E+20$ & $3.80 \mathrm{E}+20$ & $3.65 E+20$ & $3.76 \mathrm{E}+20$ & $3.91 E+20$ & $4.14 \mathrm{E}+20$ & $5.10 \mathrm{E}+20$ \\
\hline 2.5 & $6.25 E+20$ & $5.27 \mathrm{E}+20$ & $4.94 \mathrm{E}+20$ & $4.66 \mathrm{E}+20$ & $4.51 \mathrm{E}+20$ & $4.72 \mathrm{E}+20$ & $4.92 \mathrm{E}+20$ & $5.26 \mathrm{E}+20$ & $6.29 E+20$ \\
\hline 3.5 & $7.36 \mathrm{E}+20$ & $6.32 \mathrm{E}+20$ & $5.79 E+20$ & $5.59 \mathrm{E}+20$ & $5.35 E+20$ & $5.51 \mathrm{E}+20$ & $5.79 E+20$ & $6.19 \mathrm{E}+20$ & $7.48 \mathrm{E}+20$ \\
\hline 4.5 & $8.25 E+20$ & $7.07 \mathrm{E}+20$ & $6.55 E+20$ & $6.24 \mathrm{E}+20$ & $6.02 E+20$ & $6.19 \mathrm{E}+20$ & $6.52 E+20$ & $6.89 \mathrm{E}+20$ & $8.30 \mathrm{E}+20$ \\
\hline 5.5 & $9.37 E+20$ & $7.70 \mathrm{E}+20$ & $7.25 E+20$ & $6.92 E+20$ & $1 E+20$ & $6.93 E+20$ & $7.22 E+20$ & $7.73 E+20$ & $9.26 \mathrm{E}+20$ \\
\hline 6.5 & $1.00 \mathrm{E}+21$ & $8.38 \mathrm{E}+20$ & $7.87 \mathrm{E}+20$ & $7.49 \mathrm{E}+20$ & $7.20 \mathrm{E}+20$ & $7.40 \mathrm{E}+20$ & $7.71 \mathrm{E}+20$ & $8.52 \mathrm{E}+20$ & $1.00 \mathrm{E}+21$ \\
\hline 7.5 & $1.08 \mathrm{E}+21$ & $8.92 \mathrm{E}+20$ & $8.25 E+20$ & $7.91 \mathrm{E}+20$ & $7.61 \mathrm{E}+20$ & $7.91 \mathrm{E}+20$ & $8.31 \mathrm{E}+20$ & $9.00 \mathrm{E}+20$ & $1.06 \mathrm{E}+21$ \\
\hline 8.5 & $1.11 \mathrm{E}+21$ & $9.47 \mathrm{E}+20$ & $8.71 \mathrm{E}+20$ & $8.25 E+20$ & $7.90 \mathrm{E}+20$ & $8.26 \mathrm{E}+20$ & $8.71 \mathrm{E}+20$ & $9.43 \mathrm{E}+20$ & $1.11 \mathrm{E}+21$ \\
\hline 9.5 & $1.16 \mathrm{E}+21$ & $9.59 \mathrm{E}+20$ & $9.04 \mathrm{E}+20$ & $8.63 E+20$ & $8.29 \mathrm{E}+20$ & $8.50 \mathrm{E}+20$ & $8.97 \mathrm{E}+20$ & $9.68 \mathrm{E}+20$ & $1.15 \mathrm{E}+21$ \\
\hline 10.5 & $1.17 E+21$ & $1.00 \mathrm{E}+21$ & $9.16 \mathrm{E}+20$ & $8.69 \mathrm{E}+20$ & $8.46 \mathrm{E}+20$ & $8.71 \mathrm{E}+20$ & $9.16 \mathrm{E}+20$ & $1.00 \mathrm{E}+21$ & $1.19 \mathrm{E}+21$ \\
\hline 11.5 & $1.20 \mathrm{E}+21$ & $9.91 E+20$ & $9.27 \mathrm{E}+20$ & $8.83 E+20$ & $8.42 E+20$ & $8.87 E+20$ & $9.41 E+20$ & $1.00 \mathrm{E}+21$ & $1.18 \mathrm{E}+21$ \\
\hline 12.5 & $1.20 \mathrm{E}+21$ & $9.91 \mathrm{E}+20$ & $9.33 \mathrm{E}+20$ & $8.85 E+20$ & $8.59 \mathrm{E}+20$ & $8.92 \mathrm{E}+20$ & $9.38 \mathrm{E}+20$ & $1.00 \mathrm{E}+21$ & $1.19 \mathrm{E}+21$ \\
\hline 13.5 & $1.19 \mathrm{E}+21$ & $9.89 \mathrm{E}+20$ & $9.22 \mathrm{E}+20$ & $8.79 \mathrm{E}+20$ & $8.47 E+20$ & $8.71 \mathrm{E}+20$ & $9.11 \mathrm{E}+20$ & $1.00 \mathrm{E}+21$ & $1.17 E+21$ \\
\hline 14.5 & $1.16 \mathrm{E}+21$ & $9.76 \mathrm{E}+20$ & $9.12 \mathrm{E}+20$ & $8.65 E+20$ & $8.26 \mathrm{E}+20$ & $8.62 \mathrm{E}+20$ & $8.98 \mathrm{E}+20$ & $9.61 \mathrm{E}+20$ & $1.15 \mathrm{E}+21$ \\
\hline 15.5 & $1.12 \mathrm{E}+21$ & $9.37 \mathrm{E}+20$ & $8.65 E+20$ & $8.34 \mathrm{E}+20$ & $8.01 E+20$ & $8.29 \mathrm{E}+20$ & $8.69 \mathrm{E}+20$ & $9.46 \mathrm{E}+20$ & $1.15 \mathrm{E}+21$ \\
\hline 16.5 & $1.08 \mathrm{E}+21$ & $9.03 E+20$ & $8.48 \mathrm{E}+20$ & $8.02 E+20$ & $7.82 \mathrm{E}+20$ & $8.06 \mathrm{E}+20$ & $8.44 \mathrm{E}+20$ & $8.97 \mathrm{E}+20$ & $1.08 \mathrm{E}+21$ \\
\hline 17.5 & $1.02 \mathrm{E}+21$ & $8.51 \mathrm{E}+20$ & $7.98 \mathrm{E}+20$ & $7.59 \mathrm{E}+20$ & $7.36 \mathrm{E}+20$ & $7.59 \mathrm{E}+20$ & $7.95 \mathrm{E}+20$ & $8.67 \mathrm{E}+20$ & $1.03 \mathrm{E}+21$ \\
\hline 18.5 & $9.64 \mathrm{E}+20$ & $7.96 \mathrm{E}+20$ & $7.55 \mathrm{E}+20$ & $7.22 \mathrm{E}+20$ & $6.94 \mathrm{E}+20$ & $7.16 \mathrm{E}+20$ & $7.48 \mathrm{E}+20$ & $8.07 \mathrm{E}+20$ & $9.73 E+20$ \\
\hline 19.5 & $8.95 E+20$ & $7.53 \mathrm{E}+20$ & $6.98 \mathrm{E}+20$ & $6.59 E+20$ & $6.40 \mathrm{E}+20$ & $6.61 \mathrm{E}+20$ & $6.81 E+20$ & $7.46 \mathrm{E}+20$ & $8.75 E+20$ \\
\hline 20.5 & $8.07 E+20$ & $6.83 \mathrm{E}+20$ & $6.28 \mathrm{E}+20$ & $6.03 E+20$ & $5.80 \mathrm{E}+20$ & $5.93 E+20$ & $6.22 E+20$ & $6.74 \mathrm{E}+20$ & $8.06 \mathrm{E}+20$ \\
\hline 21.5 & $7.24 \mathrm{E}+20$ & $6.01 \mathrm{E}+20$ & $5.58 \mathrm{E}+20$ & $5.25 \mathrm{E}+20$ & $5.12 \mathrm{E}+20$ & $5.26 \mathrm{E}+20$ & $5.63 \mathrm{E}+20$ & $6.09 \mathrm{E}+20$ & $7.09 \mathrm{E}+20$ \\
\hline 22.5 & $6.46 \mathrm{E}+20$ & $5.22 \mathrm{E}+20$ & $4.80 \mathrm{E}+20$ & $4.58 \mathrm{E}+20$ & $4.41 \mathrm{E}+20$ & $4.57 \mathrm{E}+20$ & $4.82 \mathrm{E}+20$ & $5.17 \mathrm{E}+20$ & $6.40 \mathrm{E}+20$ \\
\hline 23.5 & $8.03 E+20$ & $6.60 \mathrm{E}+20$ & $6.16 \mathrm{E}+20$ & $5.80 \mathrm{E}+20$ & $5.64 \mathrm{E}+20$ & $5.86 \mathrm{E}+20$ & $6.12 \mathrm{E}+20$ & $6.61 \mathrm{E}+20$ & $8.01 \mathrm{E}+20$ \\
\hline Ax. Avg. & $9.38 E+20$ & $7.84 \mathrm{E}+20$ & $7.30 \mathrm{E}+20$ & $6.95 E+20$ & $6.71 E+20$ & $6.94 \mathrm{E}+20$ & $7.28 \mathrm{E}+20$ & $7.85 \mathrm{E}+20$ & $9.36 \mathrm{E}+20$ \\
\hline
\end{tabular}


Table A-18. Fission Density Data (fissions $/ \mathrm{cm}^{3}$ ) in Fuel Plate 18 in Element Discharged from Reference Mixed Burnup Core: FT loaded with typical samples.

\section{Fuel core width 3.508 inches Fuel core thickness 20 mil}

\begin{tabular}{|c|c|c|c|c|c|c|c|c|c|}
\hline $\begin{array}{c}\text { Axial } \\
\text { position } \\
\text { from top of } \\
\text { fuel (inches) }\end{array}$ & $\begin{array}{c}0.000 \text { to } \\
0.197 \\
\text { inches }\end{array}$ & $\begin{array}{c}0.197 \text { to } \\
0.394 \\
\text { inches }\end{array}$ & $\begin{array}{c}0.394 \text { to } \\
0.591 \\
\text { inches }\end{array}$ & $\begin{array}{c}0.591 \text { to } \\
1.366 \\
\text { inches }\end{array}$ & $\begin{array}{l}1.366 \text { to } \\
2.142 \\
\text { inches }\end{array}$ & $\begin{array}{l}2.142 \text { to } \\
2.918 \\
\text { inches }\end{array}$ & $\begin{array}{l}2.918 \text { to } \\
3.115 \\
\text { inches }\end{array}$ & $\begin{array}{c}3.115 \text { to } \\
3.312 \\
\text { inches }\end{array}$ & $\begin{array}{c}3.312 \text { to } \\
3.508 \\
\text { inches }\end{array}$ \\
\hline 0.5 & $5.29 \mathrm{E}+20$ & $4.44 \mathrm{E}+20$ & $4.19 \mathrm{E}+20$ & $4.07 \mathrm{E}+20$ & $3.91 \mathrm{E}+20$ & $4.03 E+20$ & $4.09 \mathrm{E}+20$ & $4.37 \mathrm{E}+20$ & $5.07 \mathrm{E}+20$ \\
\hline 1.5 & $5.03 E+20$ & $4.26 \mathrm{E}+20$ & $3.90 \mathrm{E}+20$ & $3.77 \mathrm{E}+20$ & $3.67 E+20$ & $3.79 E+20$ & $4.00 \mathrm{E}+20$ & $4.16 \mathrm{E}+20$ & $5.10 \mathrm{E}+20$ \\
\hline 2.5 & $6.42 E+20$ & $5.34 \mathrm{E}+20$ & $4.99 E+20$ & $4.75 E+20$ & $4.58 E+20$ & $4.74 E+20$ & $4.94 \mathrm{E}+20$ & $5.40 \mathrm{E}+20$ & $6.49 \mathrm{E}+20$ \\
\hline 3.5 & $7.65 E+20$ & $6.27 \mathrm{E}+20$ & $5.86 \mathrm{E}+20$ & $5.65 E+20$ & $5.41 \mathrm{E}+20$ & $5.62 \mathrm{E}+20$ & $5.93 E+20$ & $6.36 \mathrm{E}+20$ & $7.64 \mathrm{E}+20$ \\
\hline 4.5 & $8.65 E+20$ & $7.32 \mathrm{E}+20$ & $6.71 \mathrm{E}+20$ & $6.33 \mathrm{E}+20$ & $6.12 E+20$ & $6.37 E+20$ & $6.66 \mathrm{E}+20$ & $7.21 \mathrm{E}+20$ & $8.66 \mathrm{E}+20$ \\
\hline 5.5 & $9.47 \mathrm{E}+20$ & $7.78 \mathrm{E}+20$ & $7.42 \mathrm{E}+20$ & $7.03 \mathrm{E}+20$ & $6.84 \mathrm{E}+20$ & $7.04 \mathrm{E}+20$ & $7.36 \mathrm{E}+20$ & $8.02 E+20$ & $9.59 \mathrm{E}+20$ \\
\hline 6.5 & $1.04 \mathrm{E}+21$ & $8.60 \mathrm{E}+20$ & $8.03 E+20$ & $7.62 \mathrm{E}+20$ & $7.33 \mathrm{E}+20$ & $7.61 \mathrm{E}+20$ & $8.00 \mathrm{E}+20$ & $8.63 \mathrm{E}+20$ & $1.04 \mathrm{E}+21$ \\
\hline 7.5 & $1.11 \mathrm{E}+21$ & $9.00 \mathrm{E}+20$ & $8.41 \mathrm{E}+20$ & $8.08 \mathrm{E}+20$ & $7.80 \mathrm{E}+20$ & $8.08 E+20$ & $8.64 \mathrm{E}+20$ & $9.20 \mathrm{E}+20$ & $1.09 \mathrm{E}+21$ \\
\hline 8.5 & $1.16 \mathrm{E}+21$ & $9.60 \mathrm{E}+20$ & $8.91 \mathrm{E}+20$ & $8.41 \mathrm{E}+20$ & $8.12 \mathrm{E}+20$ & $8.42 \mathrm{E}+20$ & $8.78 \mathrm{E}+20$ & $9.67 \mathrm{E}+20$ & $1.16 \mathrm{E}+21$ \\
\hline 9.5 & $1.19 \mathrm{E}+21$ & $9.87 \mathrm{E}+20$ & $9.18 \mathrm{E}+20$ & +20 & $8.47 E+20$ & 8.721 & $9.14 \mathrm{E}+20$ & +20 & $1.18 \mathrm{E}+21$ \\
\hline 10.5 & $1.22 \mathrm{E}+21$ & $1.02 \mathrm{E}+21$ & $9.38 E+20$ & $8.90 \mathrm{E}+20$ & $8.65 E+20$ & $8.90 \mathrm{E}+20$ & $9.48 \mathrm{E}+20$ & $1.00 \mathrm{E}+21$ & $1.22 \mathrm{E}+21$ \\
\hline 11.5 & $1.22 \mathrm{E}+21$ & $1.02 \mathrm{E}+21$ & $9.44 \mathrm{E}+20$ & $9.08 \mathrm{E}+20$ & $8.72 \mathrm{E}+20$ & $9.11 \mathrm{E}+20$ & $9.55 \mathrm{E}+20$ & $1.02 \mathrm{E}+21$ & $1.26 \mathrm{E}+21$ \\
\hline 12.5 & $1.24 \mathrm{E}+21$ & $1.02 \mathrm{E}+21$ & $9.58 \mathrm{E}+20$ & $9.10 \mathrm{E}+20$ & $8.78 \mathrm{E}+20$ & $9.16 \mathrm{E}+20$ & $9.53 E+20$ & $1.04 \mathrm{E}+21$ & $1.24 \mathrm{E}+21$ \\
\hline 13.5 & $1.23 \mathrm{E}+21$ & $1.02 \mathrm{E}+21$ & $9.45 E+20$ & $8.87 \mathrm{E}+20$ & $8.62 E+20$ & $9.00 \mathrm{E}+20$ & $9.46 \mathrm{E}+20$ & $1.01 \mathrm{E}+21$ & $1.23 \mathrm{E}+21$ \\
\hline 14.5 & $1.20 \mathrm{E}+21$ & $9.85 \mathrm{E}+20$ & $9.34 \mathrm{E}+20$ & $8.79 \mathrm{E}+20$ & $8.53 \mathrm{E}+20$ & $8.79 E+20$ & $9.19 \mathrm{E}+20$ & $9.99 \mathrm{E}+20$ & $1.21 \mathrm{E}+21$ \\
\hline 15.5 & $1.16 \mathrm{E}+21$ & $9.67 \mathrm{E}+20$ & $8.96 \mathrm{E}+20$ & $8.48 \mathrm{E}+20$ & $8.24 \mathrm{E}+20$ & $8.52 \mathrm{E}+20$ & $8.82 \mathrm{E}+20$ & $9.54 \mathrm{E}+20$ & $1.16 \mathrm{E}+21$ \\
\hline 16.5 & $1.12 \mathrm{E}+21$ & $9.26 \mathrm{E}+20$ & $8.76 \mathrm{E}+20$ & $8.20 \mathrm{E}+20$ & $7.97 \mathrm{E}+20$ & $8.25 E+20$ & $8.69 \mathrm{E}+20$ & $9.17 E+20$ & $1.12 \mathrm{E}+21$ \\
\hline 17.5 & $1.05 \mathrm{E}+21$ & $8.96 \mathrm{E}+20$ & $8.29 \mathrm{E}+20$ & $7.85 \mathrm{E}+20$ & $7.53 \mathrm{E}+20$ & $7.80 \mathrm{E}+20$ & $8.14 \mathrm{E}+20$ & $8.94 \mathrm{E}+20$ & $1.07 \mathrm{E}+21$ \\
\hline 18.5 & $1.00 \mathrm{E}+21$ & $8.29 E+20$ & $7.66 \mathrm{E}+20$ & $7.39 \mathrm{E}+20$ & $7.14 \mathrm{E}+20$ & $7.33 E+20$ & $7.70 \mathrm{E}+20$ & $8.42 E+20$ & $1.02 \mathrm{E}+21$ \\
\hline 19.5 & $9.29 E+20$ & $7.73 \mathrm{E}+20$ & $7.13 E+20$ & $6.81 \mathrm{E}+20$ & $6.53 E+20$ & $6.75 E+20$ & $7.08 \mathrm{E}+20$ & $7.59 \mathrm{E}+20$ & $9.19 \mathrm{E}+20$ \\
\hline 20.5 & $8.39 \mathrm{E}+20$ & $6.88 \mathrm{E}+20$ & $6.39 \mathrm{E}+20$ & $6.20 \mathrm{E}+20$ & $5.96 \mathrm{E}+20$ & $6.09 E+20$ & $6.41 \mathrm{E}+20$ & $6.93 E+20$ & $8.47 E+20$ \\
\hline 21.5 & $7.53 E+20$ & $6.04 \mathrm{E}+20$ & $5.61 \mathrm{E}+20$ & $5.41 \mathrm{E}+20$ & $5.25 \mathrm{E}+20$ & $5.39 \mathrm{E}+20$ & $5.63 \mathrm{E}+20$ & $6.15 \mathrm{E}+20$ & $7.50 \mathrm{E}+20$ \\
\hline 22.5 & $6.83 E+20$ & $5.46 \mathrm{E}+20$ & $5.06 \mathrm{E}+20$ & $4.61 \mathrm{E}+20$ & $4.50 \mathrm{E}+20$ & $4.69 \mathrm{E}+20$ & $4.98 \mathrm{E}+20$ & $5.34 \mathrm{E}+20$ & $6.84 \mathrm{E}+20$ \\
\hline 23.5 & $8.17 \mathrm{E}+20$ & $6.62 \mathrm{E}+20$ & $6.15 \mathrm{E}+20$ & $5.95 \mathrm{E}+20$ & $5.71 \mathrm{E}+20$ & $5.94 \mathrm{E}+20$ & $6.19 \mathrm{E}+20$ & $6.71 \mathrm{E}+20$ & $8.22 \mathrm{E}+20$ \\
\hline Ax. Avg. & $9.67 \mathrm{E}+20$ & $8.00 \mathrm{E}+20$ & $7.45 \mathrm{E}+20$ & $7.09 \mathrm{E}+20$ & $6.85 E+20$ & $7.09 E+20$ & $7.43 E+20$ & $8.02 E+20$ & $9.70 \mathrm{E}+20$ \\
\hline
\end{tabular}


Table A-19. Fission Density Data (fissions $/ \mathrm{cm}^{3}$ ) in Fuel Plate 19 in Element Discharged from Reference Mixed Burnup Core: FT loaded with typical samples.

Fuel core width $\quad 3.615$ inches Fuel core thickness 20 mil

\begin{tabular}{|c|c|c|c|c|c|c|c|c|c|}
\hline $\begin{array}{c}\text { Axial } \\
\text { position } \\
\text { from top of } \\
\text { fuel (inches) }\end{array}$ & $\begin{array}{c}0.000 \text { to } \\
0.197 \\
\text { inches }\end{array}$ & $\begin{array}{c}0.197 \text { to } \\
0.394 \\
\text { inches }\end{array}$ & $\begin{array}{c}0.394 \text { to } \\
0.591 \\
\text { inches }\end{array}$ & $\begin{array}{c}0.591 \text { to } \\
1.402 \\
\text { inches }\end{array}$ & $\begin{array}{l}1.402 \text { to } \\
2.213 \\
\text { inches }\end{array}$ & $\begin{array}{l}2.213 \text { to } \\
3.025 \\
\text { inches }\end{array}$ & $\begin{array}{c}3.025 \text { to } \\
3.222 \\
\text { inches }\end{array}$ & $\begin{array}{c}3.222 \text { to } \\
3.418 \\
\text { inches }\end{array}$ & $\begin{array}{c}3.418 \text { to } \\
3.615 \\
\text { inches }\end{array}$ \\
\hline 0.5 & $5.29 \mathrm{E}+20$ & $4.48 \mathrm{E}+20$ & $4.24 \mathrm{E}+20$ & $4.10 \mathrm{E}+20$ & $3.96 \mathrm{E}+20$ & $4.07 \mathrm{E}+20$ & $4.20 \mathrm{E}+20$ & $4.43 \mathrm{E}+20$ & $5.19 \mathrm{E}+20$ \\
\hline 1.5 & $5.49 \mathrm{E}+20$ & $4.51 \mathrm{E}+20$ & $4.24 \mathrm{E}+20$ & $4.03 \mathrm{E}+20$ & $3.94 \mathrm{E}+20$ & $4.00 \mathrm{E}+20$ & $4.14 \mathrm{E}+20$ & $4.47 \mathrm{E}+20$ & $5.30 \mathrm{E}+20$ \\
\hline 2.5 & $6.87 E+20$ & $5.75 \mathrm{E}+20$ & $5.39 \mathrm{E}+20$ & $5.14 \mathrm{E}+20$ & $4.96 \mathrm{E}+20$ & $5.11 \mathrm{E}+20$ & $5.31 \mathrm{E}+20$ & $5.79 \mathrm{E}+20$ & $6.94 \mathrm{E}+20$ \\
\hline 3.5 & $8.25 E+20$ & $6.94 \mathrm{E}+20$ & $6.45 E+20$ & $6.15 E+20$ & $5.91 E+20$ & $6.10 \mathrm{E}+20$ & $6.48 \mathrm{E}+20$ & $6.80 \mathrm{E}+20$ & $8.34 \mathrm{E}+20$ \\
\hline 4.5 & $9.51 \mathrm{E}+20$ & $7.79 \mathrm{E}+20$ & $7.33 E+20$ & $6.87 \mathrm{E}+20$ & $6.64 E+20$ & $7.01 \mathrm{E}+20$ & $7.34 \mathrm{E}+20$ & $7.92 \mathrm{E}+20$ & $9.54 \mathrm{E}+20$ \\
\hline 5.5 & $1.05 \mathrm{E}+21$ & $8.65 E+20$ & $8.02 \mathrm{E}+20$ & $7.73 \mathrm{E}+20$ & $7.48 \mathrm{E}+20$ & $7.71 \mathrm{E}+20$ & $8.24 \mathrm{E}+20$ & $8.62 \mathrm{E}+20$ & $1.06 \mathrm{E}+21$ \\
\hline 6.5 & $1.14 \mathrm{E}+21$ & $9.43 \mathrm{E}+20$ & $8.66 \mathrm{E}+20$ & $8.33 \mathrm{E}+20$ & $7.98 \mathrm{E}+20$ & $8.36 \mathrm{E}+20$ & $8.63 E+20$ & $9.52 \mathrm{E}+20$ & $1.14 \mathrm{E}+21$ \\
\hline 7.5 & $1.21 \mathrm{E}+21$ & $9.87 \mathrm{E}+20$ & $9.16 \mathrm{E}+20$ & $8.77 \mathrm{E}+20$ & $8.53 E+20$ & $8.89 \mathrm{E}+20$ & $9.41 \mathrm{E}+20$ & $1.01 \mathrm{E}+21$ & $1.21 \mathrm{E}+21$ \\
\hline 8.5 & $1.27 \mathrm{E}+21$ & $1.07 \mathrm{E}+21$ & $9.78 \mathrm{E}+20$ & $9.17 \mathrm{E}+20$ & $8.88 \mathrm{E}+20$ & $9.24 \mathrm{E}+20$ & $9.71 \mathrm{E}+20$ & $1.05 \mathrm{E}+21$ & $1.26 \mathrm{E}+21$ \\
\hline 9.5 & $1.31 \mathrm{E}+21$ & $1.08 \mathrm{E}+21$ & $9.80 \mathrm{E}+20$ & $9.53 E+20$ & $9.24 \mathrm{E}+20$ & $9.53 \mathrm{E}+20$ & $1.01 \mathrm{E}+21$ & $1.09 \mathrm{E}+21$ & $1.32 \mathrm{E}+21$ \\
\hline 10.5 & $1.33 E+21$ & $1.11 \mathrm{E}+21$ & $1.02 \mathrm{E}+21$ & $9.70 \mathrm{E}+20$ & $9.43 E+20$ & $9.73 E+20$ & $1.02 \mathrm{E}+21$ & $1.09 \mathrm{E}+21$ & $1.33 \mathrm{E}+21$ \\
\hline 11.5 & $1.35 E+21$ & $1.11 \mathrm{E}+21$ & $1.04 \mathrm{E}+21$ & $9.95 \mathrm{E}+20$ & $9.47 E+20$ & $9.94 \mathrm{E}+20$ & $1.04 \mathrm{E}+21$ & $1.12 \mathrm{E}+21$ & $1.35 \mathrm{E}+21$ \\
\hline 12.5 & $1.34 \mathrm{E}+21$ & $1.11 \mathrm{E}+21$ & $1.04 \mathrm{E}+21$ & $9.88 \mathrm{E}+20$ & $9.55 E+20$ & $9.99 \mathrm{E}+20$ & $1.03 E+21$ & $1.14 \mathrm{E}+21$ & $1.35 \mathrm{E}+21$ \\
\hline 13.5 & $1.33 \mathrm{E}+21$ & $1.11 \mathrm{E}+21$ & $1.02 \mathrm{E}+21$ & $9.76 \mathrm{E}+20$ & $9.39 \mathrm{E}+20$ & $9.85 \mathrm{E}+20$ & $1.02 \mathrm{E}+21$ & $1.11 \mathrm{E}+21$ & $1.34 \mathrm{E}+21$ \\
\hline 14.5 & $1.32 \mathrm{E}+21$ & $1.07 \mathrm{E}+21$ & $1.00 \mathrm{E}+21$ & $9.72 \mathrm{E}+20$ & $9.28 \mathrm{E}+20$ & $9.70 \mathrm{E}+20$ & $1.00 \mathrm{E}+21$ & $1.07 \mathrm{E}+21$ & $1.30 \mathrm{E}+21$ \\
\hline 15.5 & $1.27 \mathrm{E}+21$ & $1.05 \mathrm{E}+21$ & $9.90 \mathrm{E}+20$ & $9.30 \mathrm{E}+20$ & $8.95 E+20$ & $9.28 \mathrm{E}+20$ & $9.71 \mathrm{E}+20$ & $1.06 \mathrm{E}+21$ & $1.29 \mathrm{E}+21$ \\
\hline 16.5 & $1.23 \mathrm{E}+21$ & $1.02 \mathrm{E}+21$ & $9.37 E+20$ & $9.04 \mathrm{E}+20$ & $8.74 \mathrm{E}+20$ & $8.97 E+20$ & $9.46 \mathrm{E}+20$ & $1.01 \mathrm{E}+21$ & $1.24 \mathrm{E}+21$ \\
\hline 17.5 & $1.17 \mathrm{E}+21$ & $9.72 \mathrm{E}+20$ & $9.04 \mathrm{E}+20$ & $8.52 \mathrm{E}+20$ & $8.23 E+20$ & $8.51 \mathrm{E}+20$ & $8.94 \mathrm{E}+20$ & $9.55 E+20$ & $1.17 E+21$ \\
\hline 18.5 & $1.11 \mathrm{E}+21$ & $9.08 \mathrm{E}+20$ & $8.32 \mathrm{E}+20$ & $8.09 \mathrm{E}+20$ & $7.76 \mathrm{E}+20$ & $8.07 E+20$ & $8.50 \mathrm{E}+20$ & $9.12 \mathrm{E}+20$ & $1.10 \mathrm{E}+21$ \\
\hline 19.5 & $1.02 \mathrm{E}+21$ & $8.38 \mathrm{E}+20$ & $7.78 \mathrm{E}+20$ & $7.44 \mathrm{E}+20$ & $7.17 \mathrm{E}+20$ & $7.38 \mathrm{E}+20$ & $7.75 \mathrm{E}+20$ & $8.44 \mathrm{E}+20$ & $1.02 \mathrm{E}+21$ \\
\hline 20.5 & $9.15 E+20$ & $7.49 \mathrm{E}+20$ & $7.00 \mathrm{E}+20$ & $6.72 \mathrm{E}+20$ & $6.45 E+20$ & $6.73 E+20$ & $7.03 E+20$ & $7.58 \mathrm{E}+20$ & $9.35 \mathrm{E}+20$ \\
\hline 21.5 & $8.13 E+20$ & $6.60 \mathrm{E}+20$ & $6.07 E+20$ & $5.92 \mathrm{E}+20$ & $5.76 \mathrm{E}+20$ & $5.98 \mathrm{E}+20$ & $6.18 \mathrm{E}+20$ & $6.70 \mathrm{E}+20$ & $8.20 \mathrm{E}+20$ \\
\hline 22.5 & $7.36 \mathrm{E}+20$ & $5.92 \mathrm{E}+20$ & $5.44 \mathrm{E}+20$ & $5.12 \mathrm{E}+20$ & $4.92 \mathrm{E}+20$ & $5.17 \mathrm{E}+20$ & $5.47 \mathrm{E}+20$ & $5.95 \mathrm{E}+20$ & $7.41 \mathrm{E}+20$ \\
\hline 23.5 & $8.79 E+20$ & $7.12 \mathrm{E}+20$ & $6.60 \mathrm{E}+20$ & $6.34 \mathrm{E}+20$ & $6.11 \mathrm{E}+20$ & $6.35 \mathrm{E}+20$ & $6.68 \mathrm{E}+20$ & $7.10 \mathrm{E}+20$ & $8.88 \mathrm{E}+20$ \\
\hline Ax. Avg. & $1.06 \mathrm{E}+21$ & $8.71 \mathrm{E}+20$ & $8.07 E+20$ & $7.72 \mathrm{E}+20$ & $7.45 \mathrm{E}+20$ & $7.74 \mathrm{E}+20$ & $8.10 \mathrm{E}+20$ & $8.73 E+20$ & $1.06 \mathrm{E}+21$ \\
\hline
\end{tabular}


Table A-20. Fission Density Data (fissions $/ \mathrm{cm}^{3}$ ) in Fuel Plate 20 in Element Discharged from Reference Mixed Burnup Core: FT loaded with typical samples.

\section{Fuel core width $\quad 3.723$ inches Fuel core thickness 20 mil}

\begin{tabular}{|c|c|c|c|c|c|c|c|c|c|}
\hline $\begin{array}{c}\text { Axial } \\
\text { position } \\
\text { from top of } \\
\text { fuel (inches) }\end{array}$ & $\begin{array}{c}0.000 \text { to } \\
0.197 \\
\text { inches }\end{array}$ & $\begin{array}{c}0.197 \text { to } \\
0.394 \\
\text { inches }\end{array}$ & $\begin{array}{c}0.394 \text { to } \\
0.591 \\
\text { inches }\end{array}$ & $\begin{array}{c}0.591 \text { to } \\
1.438 \\
\text { inches }\end{array}$ & $\begin{array}{l}1.438 \text { to } \\
2.285 \\
\text { inches }\end{array}$ & $\begin{array}{c}2.285 \text { to } \\
3.132 \\
\text { inches }\end{array}$ & $\begin{array}{c}3.132 \text { to } \\
3.329 \\
\text { inches }\end{array}$ & $\begin{array}{c}3.329 \text { to } \\
3.526 \\
\text { inches }\end{array}$ & $\begin{array}{c}3.526 \text { to } \\
3.723 \\
\text { inches }\end{array}$ \\
\hline 0.5 & $5.17 \mathrm{E}+20$ & $4.48 \mathrm{E}+20$ & $4.19 \mathrm{E}+20$ & $4.10 \mathrm{E}+20$ & $4.02 \mathrm{E}+20$ & $4.14 \mathrm{E}+20$ & $4.15 E+20$ & $4.38 \mathrm{E}+20$ & $5.04 \mathrm{E}+20$ \\
\hline 1.5 & $5.64 \mathrm{E}+20$ & $4.78 \mathrm{E}+20$ & $4.46 \mathrm{E}+20$ & $4.26 \mathrm{E}+20$ & $4.20 \mathrm{E}+20$ & $4.22 \mathrm{E}+20$ & $4.27 E+20$ & $4.68 \mathrm{E}+20$ & $5.57 \mathrm{E}+20$ \\
\hline 2.5 & $7.55 E+20$ & $6.21 \mathrm{E}+20$ & $5.83 \mathrm{E}+20$ & $5.58 \mathrm{E}+20$ & $5.44 \mathrm{E}+20$ & $5.69 E+20$ & $5.95 E+20$ & $6.35 E+20$ & $7.63 E+20$ \\
\hline 3.5 & $9.16 \mathrm{E}+20$ & $7.60 \mathrm{E}+20$ & $6.98 \mathrm{E}+20$ & $6.74 \mathrm{E}+20$ & $6.56 \mathrm{E}+20$ & $6.79 \mathrm{E}+20$ & $7.23 E+20$ & $7.65 \mathrm{E}+20$ & $9.14 \mathrm{E}+20$ \\
\hline 4.5 & $1.04 \mathrm{E}+21$ & $8.68 \mathrm{E}+20$ & $8.03 E+20$ & $7.71 \mathrm{E}+20$ & $7.48 \mathrm{E}+20$ & $7.71 \mathrm{E}+20$ & $8.13 E+20$ & $8.78 \mathrm{E}+20$ & $1.04 \mathrm{E}+21$ \\
\hline 5.5 & $1.15 E+21$ & $9.54 \mathrm{E}+20$ & $9.05 E+20$ & $8.47 E+20$ & $8.26 E+20$ & $8.53 E+20$ & $8.92 E+20$ & $9.61 E+20$ & $1.15 \mathrm{E}+21$ \\
\hline 6.5 & $1.23 E+21$ & $1.03 \mathrm{E}+21$ & $9.71 \mathrm{E}+20$ & $9.17 \mathrm{E}+20$ & $8.83 E+20$ & $9.23 \mathrm{E}+20$ & $9.70 \mathrm{E}+20$ & $1.02 \mathrm{E}+21$ & $1.24 \mathrm{E}+21$ \\
\hline 7.5 & $1.33 E+21$ & $1.08 \mathrm{E}+21$ & $1.00 \mathrm{E}+21$ & $9.84 \mathrm{E}+20$ & $9.43 E+20$ & $9.72 \mathrm{E}+20$ & $1.03 \mathrm{E}+21$ & $1.11 \mathrm{E}+21$ & $1.32 \mathrm{E}+21$ \\
\hline 8.5 & $1.37 \mathrm{E}+21$ & $1.16 \mathrm{E}+21$ & $1.08 \mathrm{E}+21$ & $1.02 \mathrm{E}+21$ & $9.93 \mathrm{E}+20$ & $1.03 \mathrm{E}+21$ & $1.07 \mathrm{E}+21$ & $1.15 \mathrm{E}+21$ & $1.37 \mathrm{E}+21$ \\
\hline 9.5 & $1.42 \mathrm{E}+21$ & $1.18 \mathrm{E}+21$ & $1.11 \mathrm{E}+21$ & $1.06 \mathrm{E}+21$ & $1.03 \mathrm{E}+21$ & $1.06 \mathrm{E}+21$ & $1.10 \mathrm{E}+21$ & $1.20 \mathrm{E}+21$ & $1.43 \mathrm{E}+21$ \\
\hline 10.5 & $1.48 \mathrm{E}+21$ & $1.21 \mathrm{E}+21$ & $1.13 E+21$ & $1.07 \mathrm{E}+21$ & $1.05 E+21$ & $1.09 \mathrm{E}+21$ & $1.12 \mathrm{E}+21$ & $1.22 \mathrm{E}+21$ & $1.47 \mathrm{E}+21$ \\
\hline 11.5 & $1.47 \mathrm{E}+21$ & $1.22 \mathrm{E}+21$ & $1.15 E+21$ & $1.10 \mathrm{E}+21$ & $1.05 E+21$ & $1.10 \mathrm{E}+21$ & $1.14 \mathrm{E}+21$ & $1.22 \mathrm{E}+21$ & $1.48 \mathrm{E}+21$ \\
\hline 12.5 & $1.48 \mathrm{E}+21$ & $1.22 \mathrm{E}+21$ & $1.16 \mathrm{E}+21$ & $1.10 \mathrm{E}+21$ & $1.07 \mathrm{E}+21$ & $1.10 \mathrm{E}+21$ & $1.14 \mathrm{E}+21$ & $1.25 \mathrm{E}+21$ & $1.48 \mathrm{E}+21$ \\
\hline 13.5 & $1.46 \mathrm{E}+21$ & $1.21 \mathrm{E}+21$ & $1.14 \mathrm{E}+21$ & $1.09 \mathrm{E}+21$ & $1.05 E+21$ & $1.09 \mathrm{E}+21$ & $1.15 \mathrm{E}+21$ & $1.20 \mathrm{E}+21$ & $1.48 \mathrm{E}+21$ \\
\hline 14.5 & $1.43 \mathrm{E}+21$ & $1.19 \mathrm{E}+21$ & $1.11 \mathrm{E}+21$ & $1.07 \mathrm{E}+21$ & $1.02 \mathrm{E}+21$ & $1.07 \mathrm{E}+21$ & $1.12 \mathrm{E}+21$ & $1.20 \mathrm{E}+21$ & $1.44 \mathrm{E}+21$ \\
\hline 15.5 & $1.40 \mathrm{E}+21$ & $1.17 \mathrm{E}+21$ & $1.08 \mathrm{E}+21$ & $1.04 \mathrm{E}+21$ & $1.00 \mathrm{E}+21$ & $1.03 \mathrm{E}+21$ & $1.06 \mathrm{E}+21$ & $1.15 \mathrm{E}+21$ & $1.41 \mathrm{E}+21$ \\
\hline 16.5 & $1.36 \mathrm{E}+21$ & $1.13 \mathrm{E}+21$ & $1.06 \mathrm{E}+21$ & $1.00 \mathrm{E}+21$ & $9.65 E+20$ & $9.97 E+20$ & $1.05 \mathrm{E}+21$ & $1.12 \mathrm{E}+21$ & $1.35 \mathrm{E}+21$ \\
\hline 17.5 & $1.28 \mathrm{E}+21$ & $1.08 \mathrm{E}+21$ & $9.96 \mathrm{E}+20$ & $9.45 \mathrm{E}+20$ & $9.12 \mathrm{E}+20$ & $9.49 \mathrm{E}+20$ & $9.91 \mathrm{E}+20$ & $1.05 \mathrm{E}+21$ & $1.27 \mathrm{E}+21$ \\
\hline 18.5 & $1.22 \mathrm{E}+21$ & $1.01 \mathrm{E}+21$ & $9.20 \mathrm{E}+20$ & $8.92 \mathrm{E}+20$ & $8.68 E+20$ & $8.88 \mathrm{E}+20$ & $9.26 \mathrm{E}+20$ & $1.01 \mathrm{E}+21$ & $1.20 \mathrm{E}+21$ \\
\hline 19.5 & $1.12 \mathrm{E}+21$ & $9.19 \mathrm{E}+20$ & $8.54 \mathrm{E}+20$ & $8.24 \mathrm{E}+20$ & $7.94 \mathrm{E}+20$ & $8.20 \mathrm{E}+20$ & $8.65 E+20$ & $9.16 \mathrm{E}+20$ & $1.11 \mathrm{E}+21$ \\
\hline 20.5 & $1.00 \mathrm{E}+21$ & $8.35 \mathrm{E}+20$ & $7.74 \mathrm{E}+20$ & $7.40 \mathrm{E}+20$ & $7.26 \mathrm{E}+20$ & $7.52 \mathrm{E}+20$ & $7.79 \mathrm{E}+20$ & $8.34 \mathrm{E}+20$ & $9.95 \mathrm{E}+20$ \\
\hline 21.5 & $8.99 E+20$ & $7.36 \mathrm{E}+20$ & $6.93 E+20$ & $6.65 E+20$ & $6.34 \mathrm{E}+20$ & $6.63 E+20$ & $6.89 \mathrm{E}+20$ & $7.39 \mathrm{E}+20$ & $8.95 E+20$ \\
\hline 22.5 & $8.20 \mathrm{E}+20$ & $6.54 \mathrm{E}+20$ & $6.05 E+20$ & $5.73 \mathrm{E}+20$ & $5.56 \mathrm{E}+20$ & $5.80 \mathrm{E}+20$ & $6.02 \mathrm{E}+20$ & $6.58 \mathrm{E}+20$ & $8.03 E+20$ \\
\hline 23.5 & $9.41 \mathrm{E}+20$ & $7.68 \mathrm{E}+20$ & $7.24 \mathrm{E}+20$ & $6.87 E+20$ & $6.67 \mathrm{E}+20$ & $6.88 \mathrm{E}+20$ & $7.18 \mathrm{E}+20$ & $7.67 \mathrm{E}+20$ & $9.44 \mathrm{E}+20$ \\
\hline Ax. Avg. & $1.15 E+21$ & $9.55 \mathrm{E}+20$ & $8.93 E+20$ & $8.53 E+20$ & $8.26 \mathrm{E}+20$ & $8.54 \mathrm{E}+20$ & $8.91 E+20$ & $9.57 \mathrm{E}+20$ & $1.15 \mathrm{E}+21$ \\
\hline
\end{tabular}


Table A-21. Fission Density Data (fissions $/ \mathrm{cm}^{3}$ ) in Fuel Plate 21 in Element Discharged from Reference Mixed Burnup Core: FT loaded with typical samples.

\section{Fuel core width $\quad 3.830$ inches Fuel core thickness 20 mil}

\begin{tabular}{|c|c|c|c|c|c|c|c|c|c|}
\hline $\begin{array}{c}\text { Axial } \\
\text { position } \\
\text { from top of } \\
\text { fuel (inches) }\end{array}$ & $\begin{array}{c}0.000 \text { to } \\
0.197 \\
\text { inches }\end{array}$ & $\begin{array}{c}0.197 \text { to } \\
0.394 \\
\text { inches }\end{array}$ & $\begin{array}{c}0.394 \text { to } \\
0.591 \\
\text { inches }\end{array}$ & $\begin{array}{c}0.591 \text { to } \\
1.474 \\
\text { inches }\end{array}$ & $\begin{array}{l}1.474 \text { to } \\
2.357 \\
\text { inches }\end{array}$ & $\begin{array}{c}2.357 \text { to } \\
3.240 \\
\text { inches }\end{array}$ & $\begin{array}{c}3.240 \text { to } \\
3.437 \\
\text { inches }\end{array}$ & $\begin{array}{c}3.437 \text { to } \\
3.634 \\
\text { inches }\end{array}$ & $\begin{array}{c}3.634 \text { to } \\
3.830 \\
\text { inches }\end{array}$ \\
\hline 0.5 & $5.31 \mathrm{E}+20$ & $4.57 \mathrm{E}+20$ & $4.22 \mathrm{E}+20$ & $4.26 \mathrm{E}+20$ & $4.21 \mathrm{E}+20$ & $4.27 \mathrm{E}+20$ & $4.24 \mathrm{E}+20$ & $4.38 \mathrm{E}+20$ & $5.14 \mathrm{E}+20$ \\
\hline 1.5 & $6.09 E+20$ & $5.29 \mathrm{E}+20$ & $4.99 E+20$ & $4.75 \mathrm{E}+20$ & $4.75 E+20$ & $4.80 \mathrm{E}+20$ & $4.93 E+20$ & $5.15 \mathrm{E}+20$ & $6.04 \mathrm{E}+20$ \\
\hline 2.5 & $8.62 E+20$ & $7.24 \mathrm{E}+20$ & $6.83 E+20$ & $6.54 \mathrm{E}+20$ & $6.41 \mathrm{E}+20$ & $6.62 E+20$ & $6.88 \mathrm{E}+20$ & $7.42 \mathrm{E}+20$ & $8.65 E+20$ \\
\hline 3.5 & $1.05 E+21$ & $8.82 \mathrm{E}+20$ & $8.43 E+20$ & $7.97 \mathrm{E}+20$ & $7.78 \mathrm{E}+20$ & $8.07 \mathrm{E}+20$ & $8.36 \mathrm{E}+20$ & $8.91 \mathrm{E}+20$ & $1.06 \mathrm{E}+21$ \\
\hline 4.5 & $1.18 \mathrm{E}+21$ & $1.01 \mathrm{E}+21$ & $9.56 \mathrm{E}+20$ & $9.21 \mathrm{E}+20$ & $8.90 \mathrm{E}+20$ & $9.19 \mathrm{E}+20$ & $9.36 \mathrm{E}+20$ & $1.01 \mathrm{E}+21$ & $1.19 \mathrm{E}+21$ \\
\hline 5.5 & $1.33 E+21$ & $1.11 \mathrm{E}+21$ & $1.06 \mathrm{E}+21$ & $1.01 \mathrm{E}+21$ & $9.78 E+20$ & $1.01 \mathrm{E}+21$ & $1.04 \mathrm{E}+21$ & $1.12 \mathrm{E}+21$ & $1.33 \mathrm{E}+21$ \\
\hline 6.5 & $1.44 \mathrm{E}+21$ & $1.20 \mathrm{E}+21$ & $1.14 \mathrm{E}+21$ & $1.09 \mathrm{E}+21$ & $1.05 \mathrm{E}+21$ & $1.09 \mathrm{E}+21$ & $1.13 E+21$ & $1.20 \mathrm{E}+21$ & $1.42 \mathrm{E}+21$ \\
\hline 7.5 & $1.52 \mathrm{E}+21$ & $1.28 \mathrm{E}+21$ & $1.22 \mathrm{E}+21$ & $1.15 \mathrm{E}+21$ & $1.12 \mathrm{E}+21$ & $1.17 \mathrm{E}+21$ & $1.19 \mathrm{E}+21$ & $1.27 \mathrm{E}+21$ & $1.53 \mathrm{E}+21$ \\
\hline 8.5 & $1.57 \mathrm{E}+21$ & $1.32 \mathrm{E}+21$ & $1.27 \mathrm{E}+21$ & $1.21 \mathrm{E}+21$ & $1.17 \mathrm{E}+21$ & $1.22 \mathrm{E}+21$ & $1.27 \mathrm{E}+21$ & $1.34 \mathrm{E}+21$ & $1.59 \mathrm{E}+21$ \\
\hline 9.5 & $1.64 \mathrm{E}+21$ & $1.39 \mathrm{E}+21$ & $1.31 \mathrm{E}+21$ & $1.27 \mathrm{E}+21$ & $1.22 \mathrm{E}+21$ & $1.26 \mathrm{E}+21$ & $1.30 \mathrm{E}+21$ & $1.39 \mathrm{E}+21$ & $1.63 \mathrm{E}+21$ \\
\hline 10.5 & $1.65 E+21$ & $1.42 \mathrm{E}+21$ & $1.33 E+21$ & $1.27 \mathrm{E}+21$ & $1.25 \mathrm{E}+21$ & $1.29 \mathrm{E}+21$ & $1.30 \mathrm{E}+21$ & $1.40 \mathrm{E}+21$ & $1.66 \mathrm{E}+21$ \\
\hline 11.5 & $1.67 E+21$ & $1.44 \mathrm{E}+21$ & $1.37 \mathrm{E}+21$ & $1.31 \mathrm{E}+21$ & $1.25 E+21$ & $1.31 \mathrm{E}+21$ & $1.35 E+21$ & $1.41 \mathrm{E}+21$ & $1.70 \mathrm{E}+21$ \\
\hline 12.5 & $1.69 \mathrm{E}+21$ & $1.41 \mathrm{E}+21$ & $1.34 \mathrm{E}+21$ & $1.30 \mathrm{E}+21$ & $1.26 \mathrm{E}+21$ & $1.30 \mathrm{E}+21$ & $1.34 \mathrm{E}+21$ & $1.45 \mathrm{E}+21$ & $1.70 \mathrm{E}+21$ \\
\hline 13.5 & $1.68 \mathrm{E}+21$ & $1.43 \mathrm{E}+21$ & $1.34 \mathrm{E}+21$ & $1.28 \mathrm{E}+21$ & $1.24 \mathrm{E}+21$ & $1.29 \mathrm{E}+21$ & $1.32 \mathrm{E}+21$ & $1.41 \mathrm{E}+21$ & $1.69 \mathrm{E}+21$ \\
\hline 14.5 & $1.65 E+21$ & $1.40 \mathrm{E}+21$ & $1.31 \mathrm{E}+21$ & $1.27 \mathrm{E}+21$ & $1.23 \mathrm{E}+21$ & $1.26 \mathrm{E}+21$ & $1.30 \mathrm{E}+21$ & $1.39 \mathrm{E}+21$ & $1.66 \mathrm{E}+21$ \\
\hline 15.5 & $1.61 \mathrm{E}+21$ & $1.37 \mathrm{E}+21$ & $1.27 \mathrm{E}+21$ & $1.23 \mathrm{E}+21$ & $1.19 \mathrm{E}+21$ & $1.23 \mathrm{E}+21$ & $1.27 \mathrm{E}+21$ & $1.34 \mathrm{E}+21$ & $1.59 \mathrm{E}+21$ \\
\hline 16.5 & $1.53 \mathrm{E}+21$ & $1.31 \mathrm{E}+21$ & $1.23 E+21$ & $1.18 \mathrm{E}+21$ & $1.14 \mathrm{E}+21$ & $1.18 \mathrm{E}+21$ & $1.22 \mathrm{E}+21$ & $1.31 \mathrm{E}+21$ & $1.53 \mathrm{E}+21$ \\
\hline 17.5 & $1.45 \mathrm{E}+21$ & $1.23 \mathrm{E}+21$ & $1.16 \mathrm{E}+21$ & $1.13 \mathrm{E}+21$ & $1.09 \mathrm{E}+21$ & $1.11 \mathrm{E}+21$ & $1.16 \mathrm{E}+21$ & $1.23 \mathrm{E}+21$ & $1.45 \mathrm{E}+21$ \\
\hline 18.5 & $1.39 \mathrm{E}+21$ & $1.16 \mathrm{E}+21$ & $1.08 \mathrm{E}+21$ & $1.06 \mathrm{E}+21$ & $1.02 \mathrm{E}+21$ & $1.05 \mathrm{E}+21$ & $1.10 \mathrm{E}+21$ & $1.16 \mathrm{E}+21$ & $1.38 \mathrm{E}+21$ \\
\hline 19.5 & $1.28 \mathrm{E}+21$ & $1.08 \mathrm{E}+21$ & $1.01 \mathrm{E}+21$ & $9.80 \mathrm{E}+20$ & $9.49 E+20$ & $9.73 E+20$ & $1.01 \mathrm{E}+21$ & $1.08 \mathrm{E}+21$ & $1.28 \mathrm{E}+21$ \\
\hline 20.5 & $1.15 E+21$ & $9.65 E+20$ & $9.04 \mathrm{E}+20$ & $8.87 E+20$ & $8.54 \mathrm{E}+20$ & $8.91 \mathrm{E}+20$ & $9.20 \mathrm{E}+20$ & $9.82 \mathrm{E}+20$ & $1.16 \mathrm{E}+21$ \\
\hline 21.5 & $1.03 E+21$ & $8.60 \mathrm{E}+20$ & $8.13 E+20$ & $7.97 \mathrm{E}+20$ & $7.64 \mathrm{E}+20$ & $7.86 \mathrm{E}+20$ & $8.21 \mathrm{E}+20$ & $8.68 \mathrm{E}+20$ & $1.03 \mathrm{E}+21$ \\
\hline 22.5 & $9.30 \mathrm{E}+20$ & $7.73 \mathrm{E}+20$ & $7.22 \mathrm{E}+20$ & $6.95 \mathrm{E}+20$ & $6.68 \mathrm{E}+20$ & $6.89 \mathrm{E}+20$ & $7.00 \mathrm{E}+20$ & $7.63 \mathrm{E}+20$ & $9.41 \mathrm{E}+20$ \\
\hline 23.5 & $1.04 \mathrm{E}+21$ & $8.79 \mathrm{E}+20$ & $8.29 E+20$ & $7.92 \mathrm{E}+20$ & $7.60 \mathrm{E}+20$ & $7.90 \mathrm{E}+20$ & $8.04 \mathrm{E}+20$ & $8.59 \mathrm{E}+20$ & $1.06 \mathrm{E}+21$ \\
\hline Ax. Avg. & $1.31 \mathrm{E}+21$ & $1.11 \mathrm{E}+21$ & $1.05 \mathrm{E}+21$ & $1.01 \mathrm{E}+21$ & $9.75 \mathrm{E}+20$ & $1.01 \mathrm{E}+21$ & $1.04 \mathrm{E}+21$ & $1.11 \mathrm{E}+21$ & $1.32 \mathrm{E}+21$ \\
\hline
\end{tabular}


Table A-22. Fission Density Data (fissions $/ \mathrm{cm}^{3}$ ) in Fuel Plate 22 in Element Discharged from Reference Mixed Burnup Core: FT loaded with typical samples.

\section{Fuel core width 3.938 inches Fuel core thickness 20 mil}

\begin{tabular}{|c|c|c|c|c|c|c|c|c|c|}
\hline $\begin{array}{c}\text { Axial } \\
\text { position } \\
\text { from top of } \\
\text { fuel (inches) }\end{array}$ & $\begin{array}{c}0.000 \text { to } \\
0.197 \\
\text { inches }\end{array}$ & $\begin{array}{c}0.197 \text { to } \\
0.394 \\
\text { inches }\end{array}$ & $\begin{array}{c}0.394 \text { to } \\
0.591 \\
\text { inches }\end{array}$ & $\begin{array}{c}0.591 \text { to } \\
1.510 \\
\text { inches }\end{array}$ & $\begin{array}{l}1.510 \text { to } \\
2.429 \\
\text { inches }\end{array}$ & $\begin{array}{c}2.429 \text { to } \\
3.348 \\
\text { inches }\end{array}$ & $\begin{array}{c}3.348 \text { to } \\
3.544 \\
\text { inches }\end{array}$ & $\begin{array}{c}3.544 \text { to } \\
3.741 \\
\text { inches }\end{array}$ & $\begin{array}{c}3.741 \text { to } \\
3.938 \\
\text { inches }\end{array}$ \\
\hline 0.5 & $5.31 \mathrm{E}+20$ & $4.81 \mathrm{E}+20$ & $4.59 \mathrm{E}+20$ & $4.62 \mathrm{E}+20$ & $4.70 \mathrm{E}+20$ & $4.62 \mathrm{E}+20$ & $4.54 \mathrm{E}+20$ & $4.76 \mathrm{E}+20$ & $5.37 \mathrm{E}+20$ \\
\hline 1.5 & $6.97 E+20$ & $6.23 E+20$ & $6.12 E+20$ & $5.94 \mathrm{E}+20$ & $5.83 E+20$ & $5.90 \mathrm{E}+20$ & $5.88 \mathrm{E}+20$ & $6.11 \mathrm{E}+20$ & $7.08 \mathrm{E}+20$ \\
\hline 2.5 & $1.04 \mathrm{E}+21$ & $9.00 \mathrm{E}+20$ & $8.78 \mathrm{E}+20$ & $8.46 \mathrm{E}+20$ & $8.26 \mathrm{E}+20$ & $8.61 \mathrm{E}+20$ & $8.73 E+20$ & $9.31 \mathrm{E}+20$ & $1.03 E+21$ \\
\hline 3.5 & $1.27 \mathrm{E}+21$ & $1.12 \mathrm{E}+21$ & $1.08 \mathrm{E}+21$ & $1.05 \mathrm{E}+21$ & $1.02 E+21$ & $1.05 \mathrm{E}+21$ & $1.08 \mathrm{E}+21$ & $1.13 E+21$ & $1.28 \mathrm{E}+21$ \\
\hline 4.5 & $1.45 E+21$ & $1.27 \mathrm{E}+21$ & $1.21 \mathrm{E}+21$ & $1.18 \mathrm{E}+21$ & $1.15 \mathrm{E}+21$ & $1.19 \mathrm{E}+21$ & $1.22 \mathrm{E}+21$ & $1.28 \mathrm{E}+21$ & $1.43 \mathrm{E}+21$ \\
\hline 5.5 & $1.63 E+21$ & $1.42 \mathrm{E}+21$ & $1.36 \mathrm{E}+21$ & $1.32 \mathrm{E}+21$ & $1.27 \mathrm{E}+21$ & $1.32 E+21$ & $1.36 \mathrm{E}+21$ & $1.42 \mathrm{E}+21$ & $1.61 \mathrm{E}+21$ \\
\hline 6.5 & $1.74 \mathrm{E}+21$ & $1.52 \mathrm{E}+21$ & $1.45 \mathrm{E}+21$ & $1.41 \mathrm{E}+21$ & $1.37 \mathrm{E}+21$ & $1.42 \mathrm{E}+21$ & $1.48 \mathrm{E}+21$ & $1.52 \mathrm{E}+21$ & $1.76 \mathrm{E}+21$ \\
\hline 7.5 & $1.84 \mathrm{E}+21$ & $1.62 \mathrm{E}+21$ & $1.55 E+21$ & $1.51 \mathrm{E}+21$ & $1.46 \mathrm{E}+21$ & $1.51 \mathrm{E}+21$ & $1.54 \mathrm{E}+21$ & $1.64 \mathrm{E}+21$ & $1.85 \mathrm{E}+21$ \\
\hline 8.5 & $1.91 \mathrm{E}+21$ & $1.71 \mathrm{E}+21$ & $1.63 E+21$ & $1.57 \mathrm{E}+21$ & $1.53 E+21$ & $1.59 \mathrm{E}+21$ & $1.63 E+21$ & $1.71 \mathrm{E}+21$ & $1.91 E+21$ \\
\hline 9.5 & $1.97 \mathrm{E}+21$ & $1.75 \mathrm{E}+21$ & $1.68 \mathrm{E}+21$ & $1.63 \mathrm{E}+21$ & $1.58 \mathrm{E}+21$ & $1.65 \mathrm{E}+21$ & $1.70 \mathrm{E}+21$ & $1.76 \mathrm{E}+21$ & $2.01 \mathrm{E}+21$ \\
\hline 10.5 & $2.02 \mathrm{E}+21$ & $1.80 \mathrm{E}+21$ & $1.73 E+21$ & $1.67 \mathrm{E}+21$ & $1.62 \mathrm{E}+21$ & $1.68 \mathrm{E}+21$ & $1.70 \mathrm{E}+21$ & $1.78 \mathrm{E}+21$ & $2.01 \mathrm{E}+21$ \\
\hline 11.5 & $2.04 \mathrm{E}+21$ & $1.83 E+21$ & $1.75 E+21$ & $1.70 \mathrm{E}+21$ & $1.64 \mathrm{E}+21$ & $1.69 E+21$ & $1.73 E+21$ & $1.80 \mathrm{E}+21$ & $2.07 E+21$ \\
\hline 12.5 & $2.02 \mathrm{E}+21$ & $1.79 \mathrm{E}+21$ & $1.75 E+21$ & $1.69 \mathrm{E}+21$ & $1.64 \mathrm{E}+21$ & $1.69 \mathrm{E}+21$ & $1.74 \mathrm{E}+21$ & $1.80 \mathrm{E}+21$ & $2.04 \mathrm{E}+21$ \\
\hline 13.5 & $2.03 E+21$ & $1.81 \mathrm{E}+21$ & $1.71 \mathrm{E}+21$ & $1.68 \mathrm{E}+21$ & $1.63 E+21$ & $1.68 \mathrm{E}+21$ & $1.72 \mathrm{E}+21$ & $1.79 \mathrm{E}+21$ & $2.04 \mathrm{E}+21$ \\
\hline 14.5 & $2.00 \mathrm{E}+21$ & $1.74 \mathrm{E}+21$ & $1.70 \mathrm{E}+21$ & $1.64 \mathrm{E}+21$ & $1.59 \mathrm{E}+21$ & $1.65 \mathrm{E}+21$ & $1.71 \mathrm{E}+21$ & $1.77 \mathrm{E}+21$ & $2.01 \mathrm{E}+21$ \\
\hline 15.5 & $1.96 \mathrm{E}+21$ & $1.73 E+21$ & $1.61 \mathrm{E}+21$ & $1.61 \mathrm{E}+21$ & $1.55 \mathrm{E}+21$ & $1.58 \mathrm{E}+21$ & $1.63 \mathrm{E}+21$ & $1.72 \mathrm{E}+21$ & $1.94 \mathrm{E}+21$ \\
\hline 16.5 & $1.86 \mathrm{E}+21$ & $1.64 \mathrm{E}+21$ & $1.58 \mathrm{E}+21$ & $1.54 \mathrm{E}+21$ & $1.49 \mathrm{E}+21$ & $1.53 \mathrm{E}+21$ & $1.58 \mathrm{E}+21$ & $1.65 \mathrm{E}+21$ & $1.87 \mathrm{E}+21$ \\
\hline 17.5 & $1.78 \mathrm{E}+21$ & $1.57 \mathrm{E}+21$ & $1.51 \mathrm{E}+21$ & $1.45 \mathrm{E}+21$ & $1.41 \mathrm{E}+21$ & $1.45 \mathrm{E}+21$ & $1.49 \mathrm{E}+21$ & $1.55 \mathrm{E}+21$ & $1.78 \mathrm{E}+21$ \\
\hline 18.5 & $1.69 \mathrm{E}+21$ & $1.46 \mathrm{E}+21$ & $1.41 \mathrm{E}+21$ & $1.38 \mathrm{E}+21$ & $1.34 \mathrm{E}+21$ & $1.36 \mathrm{E}+21$ & $1.41 \mathrm{E}+21$ & $1.47 \mathrm{E}+21$ & $1.67 \mathrm{E}+21$ \\
\hline 19.5 & $1.56 \mathrm{E}+21$ & $1.38 \mathrm{E}+21$ & $1.30 \mathrm{E}+21$ & $1.27 \mathrm{E}+21$ & $1.24 \mathrm{E}+21$ & $1.26 \mathrm{E}+21$ & $1.30 \mathrm{E}+21$ & $1.36 \mathrm{E}+21$ & $1.55 \mathrm{E}+21$ \\
\hline 20.5 & $1.41 \mathrm{E}+21$ & $1.25 \mathrm{E}+21$ & $1.19 \mathrm{E}+21$ & $1.17 \mathrm{E}+21$ & $1.13 E+21$ & $1.16 \mathrm{E}+21$ & $1.18 \mathrm{E}+21$ & $1.25 \mathrm{E}+21$ & $1.41 \mathrm{E}+21$ \\
\hline 21.5 & $1.24 \mathrm{E}+21$ & $1.12 \mathrm{E}+21$ & $1.06 \mathrm{E}+21$ & $1.04 \mathrm{E}+21$ & $1.01 \mathrm{E}+21$ & $1.03 \mathrm{E}+21$ & $1.07 \mathrm{E}+21$ & $1.13 \mathrm{E}+21$ & $1.25 \mathrm{E}+21$ \\
\hline 22.5 & $1.15 E+21$ & $1.00 \mathrm{E}+21$ & $9.57 \mathrm{E}+20$ & $9.24 \mathrm{E}+20$ & $8.90 \mathrm{E}+20$ & $9.20 \mathrm{E}+20$ & $9.43 \mathrm{E}+20$ & $9.73 \mathrm{E}+20$ & $1.15 \mathrm{E}+21$ \\
\hline 23.5 & $1.24 \mathrm{E}+21$ & $1.06 \mathrm{E}+21$ & $1.02 \mathrm{E}+21$ & $9.93 \mathrm{E}+20$ & $9.58 \mathrm{E}+20$ & $1.00 \mathrm{E}+21$ & $1.02 \mathrm{E}+21$ & $1.05 \mathrm{E}+21$ & $1.22 \mathrm{E}+21$ \\
\hline Ax. Avg. & $1.59 \mathrm{E}+21$ & $1.40 \mathrm{E}+21$ & $1.34 \mathrm{E}+21$ & $1.31 \mathrm{E}+21$ & $1.27 \mathrm{E}+21$ & $1.31 \mathrm{E}+21$ & $1.34 \mathrm{E}+21$ & $1.40 \mathrm{E}+21$ & $1.59 \mathrm{E}+21$ \\
\hline
\end{tabular}


Table A-23. Fission Density Data (fissions $/ \mathrm{cm}^{3}$ ) in Fuel Plate 23 in Element Discharged from Reference Mixed Burnup Core: FT loaded with typical samples.

\section{Fuel core width 4.048 inches Fuel core thickness 17 mil}

\begin{tabular}{|c|c|c|c|c|c|c|c|c|c|}
\hline $\begin{array}{c}\text { Axial } \\
\text { position } \\
\text { from top of } \\
\text { fuel (inches) }\end{array}$ & $\begin{array}{c}0.000 \text { to } \\
0.197 \\
\text { inches }\end{array}$ & $\begin{array}{c}0.197 \text { to } \\
0.394 \\
\text { inches }\end{array}$ & $\begin{array}{c}0.394 \text { to } \\
0.591 \\
\text { inches }\end{array}$ & $\begin{array}{c}0.591 \text { to } \\
1.546 \\
\text { inches }\end{array}$ & $\begin{array}{l}1.546 \text { to } \\
2.502 \\
\text { inches }\end{array}$ & $\begin{array}{l}2.502 \text { to } \\
3.457 \\
\text { inches }\end{array}$ & $\begin{array}{c}3.457 \text { to } \\
3.654 \\
\text { inches }\end{array}$ & $\begin{array}{c}3.654 \text { to } \\
3.851 \\
\text { inches }\end{array}$ & $\begin{array}{c}3.851 \text { to } \\
4.048 \\
\text { inches }\end{array}$ \\
\hline 0.5 & $5.82 \mathrm{E}+20$ & $5.34 \mathrm{E}+20$ & $5.28 \mathrm{E}+20$ & $5.42 \mathrm{E}+20$ & $5.58 \mathrm{E}+20$ & $5.38 \mathrm{E}+20$ & $5.20 \mathrm{E}+20$ & $5.33 \mathrm{E}+20$ & $5.81 \mathrm{E}+20$ \\
\hline 1.5 & $8.58 E+20$ & $8.02 \mathrm{E}+20$ & $8.05 E+20$ & $7.93 \mathrm{E}+20$ & $7.90 \mathrm{E}+20$ & $7.93 E+20$ & $7.94 \mathrm{E}+20$ & $7.99 \mathrm{E}+20$ & $8.71 E+20$ \\
\hline 2.5 & $1.31 \mathrm{E}+21$ & $1.22 \mathrm{E}+21$ & $1.20 \mathrm{E}+21$ & $1.18 \mathrm{E}+21$ & $1.16 \mathrm{E}+21$ & $1.19 \mathrm{E}+21$ & $1.19 \mathrm{E}+21$ & $1.24 \mathrm{E}+21$ & $1.32 \mathrm{E}+21$ \\
\hline 3.5 & $1.63 E+21$ & $1.53 \mathrm{E}+21$ & $1.52 \mathrm{E}+21$ & $1.49 \mathrm{E}+21$ & $1.44 \mathrm{E}+21$ & $1.49 \mathrm{E}+21$ & $1.53 E+21$ & $1.55 \mathrm{E}+21$ & $1.66 \mathrm{E}+21$ \\
\hline 4.5 & $1.85 E+21$ & $1.75 \mathrm{E}+21$ & $1.70 \mathrm{E}+21$ & $1.66 \mathrm{E}+21$ & $1.61 \mathrm{E}+21$ & $1.65 E+21$ & $1.67 \mathrm{E}+21$ & $1.74 \mathrm{E}+21$ & $1.86 \mathrm{E}+21$ \\
\hline 5.5 & $2.06 \mathrm{E}+21$ & $1.94 \mathrm{E}+21$ & $1.89 \mathrm{E}+21$ & $1.84 \mathrm{E}+21$ & $1.78 \mathrm{E}+21$ & $1.84 \mathrm{E}+21$ & $1.89 \mathrm{E}+21$ & $1.93 \mathrm{E}+21$ & $2.08 \mathrm{E}+21$ \\
\hline 6.5 & $2.18 \mathrm{E}+21$ & $2.03 \mathrm{E}+21$ & $2.00 \mathrm{E}+21$ & $1.95 \mathrm{E}+21$ & $1.90 \mathrm{E}+21$ & $1.96 \mathrm{E}+21$ & $2.00 \mathrm{E}+21$ & $2.07 \mathrm{E}+21$ & $2.20 \mathrm{E}+21$ \\
\hline 7.5 & $2.33 E+21$ & $2.18 \mathrm{E}+21$ & $2.13 E+21$ & $2.09 \mathrm{E}+21$ & $2.02 \mathrm{E}+21$ & $2.09 \mathrm{E}+21$ & $2.13 \mathrm{E}+21$ & $2.18 \mathrm{E}+21$ & $2.35 \mathrm{E}+21$ \\
\hline 8.5 & $2.43 E+21$ & $2.26 \mathrm{E}+21$ & $2.22 \mathrm{E}+21$ & $2.16 \mathrm{E}+21$ & $2.08 \mathrm{E}+21$ & $2.18 \mathrm{E}+21$ & $2.20 \mathrm{E}+21$ & $2.27 \mathrm{E}+21$ & $2.45 \mathrm{E}+21$ \\
\hline 9.5 & $2.50 \mathrm{E}+21$ & $2.36 \mathrm{E}+21$ & $2.29 \mathrm{E}+21$ & $2.24 \mathrm{E}+21$ & $2.16 \mathrm{E}+21$ & $2.25 \mathrm{E}+21$ & $2.28 \mathrm{E}+21$ & $2.35 \mathrm{E}+21$ & $2.53 \mathrm{E}+21$ \\
\hline 10.5 & $2.58 \mathrm{E}+21$ & $2.42 \mathrm{E}+21$ & $2.32 E+21$ & $2.27 \mathrm{E}+21$ & $2.21 \mathrm{E}+21$ & $2.29 E+21$ & $2.34 \mathrm{E}+21$ & $2.40 \mathrm{E}+21$ & $2.58 \mathrm{E}+21$ \\
\hline 11.5 & $2.59 \mathrm{E}+21$ & $2.42 \mathrm{E}+21$ & $2.35 \mathrm{E}+21$ & $2.31 \mathrm{E}+21$ & $2.25 E+21$ & $2.32 \mathrm{E}+21$ & $2.37 E+21$ & $2.40 \mathrm{E}+21$ & $2.58 \mathrm{E}+21$ \\
\hline 12.5 & $2.56 \mathrm{E}+21$ & $2.39 \mathrm{E}+21$ & $2.34 \mathrm{E}+21$ & $2.30 \mathrm{E}+21$ & $2.25 E+21$ & $2.31 \mathrm{E}+21$ & $2.37 \mathrm{E}+21$ & $2.43 E+21$ & $2.55 \mathrm{E}+21$ \\
\hline 13.5 & $2.56 \mathrm{E}+21$ & $2.37 \mathrm{E}+21$ & $2.33 \mathrm{E}+21$ & $2.28 \mathrm{E}+21$ & $2.22 \mathrm{E}+21$ & $2.29 \mathrm{E}+21$ & $2.32 \mathrm{E}+21$ & $2.38 \mathrm{E}+21$ & $2.55 \mathrm{E}+21$ \\
\hline 14.5 & $2.47 \mathrm{E}+21$ & $2.33 \mathrm{E}+21$ & $2.28 \mathrm{E}+21$ & $2.25 \mathrm{E}+21$ & $2.17 \mathrm{E}+21$ & $2.25 \mathrm{E}+21$ & $2.31 \mathrm{E}+21$ & $2.36 \mathrm{E}+21$ & $2.50 \mathrm{E}+21$ \\
\hline 15.5 & $2.44 \mathrm{E}+21$ & $2.29 E+21$ & $2.21 \mathrm{E}+21$ & $2.17 \mathrm{E}+21$ & $2.11 \mathrm{E}+21$ & $2.17 \mathrm{E}+21$ & $2.23 E+21$ & $2.27 \mathrm{E}+21$ & $2.43 \mathrm{E}+21$ \\
\hline 16.5 & $2.31 \mathrm{E}+21$ & $2.20 \mathrm{E}+21$ & $2.13 \mathrm{E}+21$ & $2.11 \mathrm{E}+21$ & $2.04 \mathrm{E}+21$ & $2.11 \mathrm{E}+21$ & $2.15 \mathrm{E}+21$ & $2.21 \mathrm{E}+21$ & $2.32 \mathrm{E}+21$ \\
\hline 17.5 & $2.25 \mathrm{E}+21$ & $2.09 \mathrm{E}+21$ & $2.03 \mathrm{E}+21$ & $2.00 \mathrm{E}+21$ & $1.95 \mathrm{E}+21$ & $1.99 \mathrm{E}+21$ & $2.05 \mathrm{E}+21$ & $2.10 \mathrm{E}+21$ & $2.21 \mathrm{E}+21$ \\
\hline 18.5 & $2.11 \mathrm{E}+21$ & $1.98 \mathrm{E}+21$ & $1.94 \mathrm{E}+21$ & $1.90 \mathrm{E}+21$ & $1.86 \mathrm{E}+21$ & $1.90 \mathrm{E}+21$ & $1.92 \mathrm{E}+21$ & $2.00 \mathrm{E}+21$ & $2.11 E+21$ \\
\hline 19.5 & $1.94 \mathrm{E}+21$ & $1.83 \mathrm{E}+21$ & $1.79 \mathrm{E}+21$ & $1.76 \mathrm{E}+21$ & $1.70 \mathrm{E}+21$ & $1.76 \mathrm{E}+21$ & $1.78 \mathrm{E}+21$ & $1.83 \mathrm{E}+21$ & $1.95 \mathrm{E}+21$ \\
\hline 20.5 & $1.79 \mathrm{E}+21$ & $1.68 \mathrm{E}+21$ & $1.64 \mathrm{E}+21$ & $1.63 \mathrm{E}+21$ & $1.58 \mathrm{E}+21$ & $1.62 \mathrm{E}+21$ & $1.67 \mathrm{E}+21$ & $1.68 \mathrm{E}+21$ & $1.79 \mathrm{E}+21$ \\
\hline 21.5 & $1.61 \mathrm{E}+21$ & $1.51 \mathrm{E}+21$ & $1.47 \mathrm{E}+21$ & $1.46 \mathrm{E}+21$ & $1.42 \mathrm{E}+21$ & $1.45 \mathrm{E}+21$ & $1.47 \mathrm{E}+21$ & $1.52 \mathrm{E}+21$ & $1.61 \mathrm{E}+21$ \\
\hline 22.5 & $1.50 \mathrm{E}+21$ & $1.37 \mathrm{E}+21$ & $1.34 \mathrm{E}+21$ & $1.33 \mathrm{E}+21$ & $1.28 \mathrm{E}+21$ & $1.31 \mathrm{E}+21$ & $1.34 \mathrm{E}+21$ & $1.38 \mathrm{E}+21$ & $1.48 \mathrm{E}+21$ \\
\hline 23.5 & $1.51 \mathrm{E}+21$ & $1.42 \mathrm{E}+21$ & $1.38 \mathrm{E}+21$ & $1.35 \mathrm{E}+21$ & $1.31 \mathrm{E}+21$ & $1.36 \mathrm{E}+21$ & $1.36 \mathrm{E}+21$ & $1.42 \mathrm{E}+21$ & $1.53 \mathrm{E}+21$ \\
\hline Ax. Avg. & $2.00 \mathrm{E}+21$ & $1.87 \mathrm{E}+21$ & $1.83 E+21$ & $1.80 \mathrm{E}+21$ & $1.74 \mathrm{E}+21$ & $1.80 \mathrm{E}+21$ & $1.83 E+21$ & $1.88 \mathrm{E}+21$ & $2.00 \mathrm{E}+21$ \\
\hline
\end{tabular}




\section{APPENDIX B: Plate Specific Power Density Data in MURR LEU Element}


Table B-1. Power Density Data $\left(\mathrm{kW} / \mathrm{cm}^{3}\right)$ in Fuel Plate 1 in Prototypic Core.

\section{Fuel core width: 1.690 inches / Fuel core thickness: 9 mil}

Plate 1, Core Position X5, 0 MWd Burnup, Xenon-Free Conditions

\begin{tabular}{|c|c|c|c|c|c|c|c|c|c|}
\hline $\begin{array}{c}\text { Axial } \\
\text { position } \\
\text { from } \\
\text { top of } \\
\text { fuel } \\
\text { (inches) }\end{array}$ & $\begin{array}{c}0.000 \\
\text { to } \\
0.197 \\
\text { inches }\end{array}$ & $\begin{array}{c}0.197 \\
\text { to } \\
0.394 \\
\text { inches }\end{array}$ & $\begin{array}{c}0.394 \\
\text { to } \\
0.591 \\
\text { inches }\end{array}$ & $\begin{array}{c}0.591 \\
\text { to } \\
0.760 \\
\text { inches }\end{array}$ & $\begin{array}{c}0.760 \\
\text { to } \\
0.929 \\
\text { inches }\end{array}$ & $\begin{array}{c}0.929 \\
\text { to } \\
1.099 \\
\text { inches }\end{array}$ & $\begin{array}{c}1.099 \\
\text { to } \\
1.296 \\
\text { inches }\end{array}$ & $\begin{array}{c}1.296 \\
\text { to } \\
1.493 \\
\text { inches }\end{array}$ & $\begin{array}{c}1.493 \\
\text { to } \\
\text { inches }\end{array}$ \\
\hline 0.5 & 4.20 & 3.67 & 3.71 & 3.47 & 3.76 & 3.66 & 3.54 & 3.54 & 3.61 \\
\hline 1.5 & 4.03 & 3.78 & 3.80 & 3.73 & 3.53 & 3.80 & 3.54 & 3.75 & 3.74 \\
\hline 2.5 & 4.66 & 4.63 & 4.40 & 4.43 & 3.98 & 4.52 & 4.19 & 4.47 & 4.68 \\
\hline 3.5 & 5.50 & 5.45 & 5.39 & 5.41 & 5.04 & 5.15 & 5.52 & 5.37 & 5.43 \\
\hline 4.5 & 6.67 & 6.10 & 6.36 & 6.53 & 6.07 & 6.42 & 6.39 & 6.30 & 6.55 \\
\hline 5.5 & 7.38 & 7.26 & 7.08 & 7.14 & 6.70 & 7.35 & 7.11 & 7.27 & 7.41 \\
\hline 6.5 & 8.25 & 8.14 & 7.85 & 7.76 & 7.49 & 7.98 & 7.90 & 8.08 & 8.15 \\
\hline 7.5 & 9.17 & 8.59 & 8.92 & 8.66 & 8.12 & 8.71 & 8.33 & 8.65 & 8.99 \\
\hline 8.5 & 10.13 & 9.69 & 9.61 & 9.65 & 9.47 & 9.93 & 9.42 & 9.19 & 9.94 \\
\hline 9.5 & 11.32 & 10.66 & 10.42 & 10.62 & 10.03 & 10.74 & 10.84 & 10.71 & 10.93 \\
\hline 10.5 & 12.18 & 11.97 & 11.54 & 11.73 & 11.00 & 11.71 & 11.79 & 11.86 & 11.98 \\
\hline 11.5 & 12.84 & 12.56 & 12.50 & 12.35 & 11.70 & 13.06 & 12.61 & 12.77 & 13.08 \\
\hline 12.5 & 13.73 & 13.72 & 13.09 & 13.45 & 12.53 & 12.86 & 12.87 & 13.24 & 13.43 \\
\hline 13.5 & 14.32 & 13.64 & 13.22 & 13.27 & 12.92 & 13.28 & 13.83 & 13.62 & 13.94 \\
\hline 14.5 & 14.40 & 13.67 & 13.22 & 13.34 & 13.15 & 13.53 & 13.59 & 13.74 & 13.86 \\
\hline 15.5 & 13.69 & 13.42 & 13.27 & 12.90 & 12.64 & 13.77 & 13.14 & 13.63 & 13.70 \\
\hline 16.5 & 13.33 & 12.68 & 12.50 & 12.87 & 12.33 & 12.65 & 12.60 & 12.96 & 13.04 \\
\hline 17.5 & 12.81 & 12.60 & 12.12 & 12.17 & 11.30 & 11.79 & 11.55 & 11.86 & 12.25 \\
\hline 18.5 & 11.98 & 11.43 & 11.37 & 11.44 & 11.02 & 10.99 & 11.19 & 11.75 & 11.66 \\
\hline 19.5 & 11.13 & 10.68 & 10.73 & 11.05 & 10.43 & 10.23 & 10.35 & 10.46 & 10.81 \\
\hline 20.5 & 10.60 & 10.41 & 10.06 & 10.20 & 9.44 & 9.80 & 9.74 & 10.48 & 10.13 \\
\hline 21.5 & 9.90 & 9.55 & 9.12 & 9.17 & 9.06 & 9.21 & 9.37 & 9.43 & 9.47 \\
\hline 22.5 & 8.99 & 8.67 & 8.84 & 8.47 & 8.05 & 8.14 & 7.96 & 8.37 & 8.58 \\
\hline 23.5 & 9.11 & 8.91 & 8.89 & 8.85 & 8.35 & 8.92 & 8.95 & 8.90 & 9.07 \\
\hline Ax. Avg. & 10.02 & 9.66 & 9.50 & 9.53 & 9.09 & 9.51 & 9.43 & 9.60 & 9.77 \\
\hline & & & & & & & & & \\
\hline
\end{tabular}

POWER DENSITY VALUES NORMALIZED TO A CORE POWER OF 11.57 MW AND ASSUME ALL FISSION ENERGY (EXCLUDING GAMMA-HEATING OF EX-CORE COMPONENTS) IS DEPOSITED IN THE FUEL 
Table B-1. Power Density Data (kW/cc) in Fuel Plate 1 in Prototypic Conditions (continued).

Plate 1, Core Position X5, 3 MWd Burnup, Equilibrium Xenon Conditions

\begin{tabular}{|c|c|c|c|c|c|c|c|c|c|}
\hline $\begin{array}{l}\text { Axial } \\
\text { position } \\
\text { from } \\
\text { top of } \\
\text { fuel } \\
\text { (inches) }\end{array}$ & $\begin{array}{c}0.000 \\
\text { to } \\
0.197 \\
\text { inches } \\
\end{array}$ & $\begin{array}{c}0.197 \\
\text { to } \\
0.394 \\
\text { inches } \\
\end{array}$ & $\begin{array}{c}0.394 \\
\text { to } \\
0.591 \\
\text { inches }\end{array}$ & $\begin{array}{c}0.591 \\
\text { to } \\
0.760 \\
\text { inches }\end{array}$ & $\begin{array}{c}0.760 \\
\text { to } \\
0.929 \\
\text { inches }\end{array}$ & $\begin{array}{c}0.929 \\
\text { to } \\
1.099 \\
\text { inches }\end{array}$ & $\begin{array}{c}1.099 \\
\text { to } \\
1.296 \\
\text { inches }\end{array}$ & $\begin{array}{c}1.296 \\
\text { to } \\
1.493 \\
\text { inches }\end{array}$ & $\begin{array}{c}1.493 \\
\text { to } \\
1.690 \\
\text { inches } \\
\end{array}$ \\
\hline 0.5 & 5.72 & 5.41 & 5.43 & 5.70 & 4.94 & 5.37 & 5.35 & 5.33 & 5.59 \\
\hline 1.5 & 5.78 & 5.49 & 5.48 & 5.46 & 5.19 & 5.57 & 5.32 & 5.52 & 5.52 \\
\hline 2.5 & 6.27 & 6.24 & 6.47 & 6.25 & 6.15 & 6.34 & 6.16 & 6.38 & 6.52 \\
\hline 3.5 & 7.45 & 7.11 & 7.13 & 6.88 & 6.98 & 7.16 & 7.16 & 7.45 & 7.56 \\
\hline 4.5 & 8.53 & 8.16 & 7.86 & 8.05 & 7.75 & 7.96 & 8.34 & 8.35 & 8.86 \\
\hline 5.5 & 9.28 & 9.02 & 9.06 & 8.68 & 8.24 & 8.60 & 8.72 & 9.02 & 9.62 \\
\hline 6.5 & 10.34 & 9.44 & 9.57 & 9.52 & 9.08 & 9.49 & 9.55 & 9.91 & 9.75 \\
\hline 7.5 & 10.62 & 10.23 & 9.99 & 9.98 & 9.48 & 9.89 & 9.97 & 9.98 & 10.09 \\
\hline 8.5 & 11.20 & 10.69 & 10.39 & 10.53 & 9.99 & 10.51 & 10.42 & 10.41 & 10.16 \\
\hline 9.5 & 11.73 & 11.28 & 11.23 & 11.14 & 10.46 & 11.16 & 11.14 & 11.13 & 11.54 \\
\hline 10.5 & 12.19 & 11.32 & 11.31 & 11.54 & 11.08 & 11.40 & 11.27 & 11.77 & 11.79 \\
\hline 11.5 & 12.78 & 12.19 & 11.66 & 11.59 & 11.23 & 11.75 & 11.55 & 11.98 & 12.39 \\
\hline 12.5 & 12.47 & 12.04 & 11.85 & 12.14 & 11.30 & 11.56 & 11.60 & 11.72 & 12.06 \\
\hline 13.5 & 12.47 & 12.04 & 11.61 & 11.67 & 11.17 & 11.93 & 11.86 & 12.12 & 12.25 \\
\hline 14.5 & 12.27 & 11.82 & 11.61 & 11.38 & 10.65 & 11.37 & 11.49 & 11.84 & 12.07 \\
\hline 15.5 & 11.35 & 11.14 & 10.81 & 11.24 & 10.29 & 10.92 & 10.65 & 11.13 & 11.65 \\
\hline 16.5 & 10.59 & 10.28 & 10.22 & 10.10 & 9.87 & 10.13 & 10.00 & 10.35 & 10.94 \\
\hline 17.5 & 10.10 & 9.81 & 9.44 & 9.94 & 9.01 & 9.50 & 9.38 & 9.53 & 9.60 \\
\hline 18.5 & 9.20 & 8.78 & 8.94 & 8.65 & 8.53 & 8.76 & 8.81 & 8.80 & 8.83 \\
\hline 19.5 & 8.79 & 8.52 & 8.38 & 8.27 & 7.51 & 8.32 & 8.14 & 8.12 & 8.31 \\
\hline 20.5 & 7.96 & 8.04 & 7.88 & 7.72 & 7.34 & 7.39 & 7.67 & 7.68 & 7.51 \\
\hline 21.5 & 7.31 & 7.06 & 6.95 & 6.99 & 6.63 & 7.13 & 6.61 & 6.74 & 7.13 \\
\hline 22.5 & 6.86 & 6.43 & 6.23 & 6.64 & 5.84 & 6.24 & 6.42 & 6.67 & 6.58 \\
\hline 23.5 & 7.19 & 6.75 & 6.71 & 7.06 & 6.32 & 6.56 & 6.46 & 6.58 & 7.00 \\
\hline Ax. Avg. & 9.52 & 9.14 & 9.01 & 9.05 & 8.55 & 8.96 & 8.92 & 9.11 & 9.31 \\
\hline
\end{tabular}

POWER DENSITY VALUES NORMALIZED TO A CORE POWER OF 11.57 MW AND ASSUME ALL FISSION ENERGY (EXCLUDING GAMMA-HEATING OF EX-CORE COMPONENTS) IS DEPOSITED IN THE FUEL 
Table B-1. Power Density Data (kW/cc) in Fuel Plate 1 in Prototypic Conditions (continued).

Plate 1, Core Position X3, 80 MWd Burnup, Equilibrium Xenon Conditions

\begin{tabular}{|c|c|c|c|c|c|c|c|c|c|}
\hline $\begin{array}{c}\text { Axial } \\
\text { position } \\
\text { from } \\
\text { top of } \\
\text { fuel } \\
\text { (inches) }\end{array}$ & $\begin{array}{c}0.000 \\
\text { to } \\
0.197 \\
\text { inches }\end{array}$ & $\begin{array}{c}0.197 \\
\text { to } \\
0.394 \\
\text { inches }\end{array}$ & $\begin{array}{c}0.394 \\
\text { to } \\
0.591 \\
\text { inches }\end{array}$ & $\begin{array}{c}0.591 \\
\text { to } \\
0.760 \\
\text { inches }\end{array}$ & $\begin{array}{c}0.760 \\
\text { to } \\
0.929 \\
\text { inches }\end{array}$ & $\begin{array}{c}0.929 \\
\text { to } \\
1.099 \\
\text { inches }\end{array}$ & $\begin{array}{c}1.099 \\
\text { to } \\
1.296 \\
\text { inches }\end{array}$ & $\begin{array}{c}1.296 \\
\text { to } \\
1.493 \\
\text { inches }\end{array}$ & $\begin{array}{c}1.493 \\
\text { to } \\
1.690 \\
\text { inches }\end{array}$ \\
\hline 1.5 & 5.43 & 5.21 & 5.04 & 5.14 & 4.88 & 5.32 & 5.21 & 5.15 & 5.42 \\
\hline 2.5 & 5.91 & 5.82 & 5.73 & 5.74 & 5.54 & 5.76 & 5.52 & 5.61 & 5.97 \\
\hline 3.5 & 6.70 & 6.34 & 6.49 & 6.51 & 6.15 & 6.62 & 6.49 & 6.48 & 6.73 \\
\hline 4.5 & 7.34 & 7.13 & 7.06 & 7.35 & 6.99 & 7.21 & 6.88 & 7.12 & 7.35 \\
\hline 5.5 & 8.13 & 7.88 & 7.90 & 8.04 & 7.42 & 7.94 & 7.73 & 7.90 & 8.02 \\
\hline 6.5 & 8.76 & 8.35 & 8.11 & 8.03 & 7.63 & 8.17 & 8.01 & 8.25 & 8.35 \\
\hline 7.5 & 9.22 & 8.57 & 8.62 & 8.66 & 8.22 & 8.49 & 8.56 & 8.62 & 8.76 \\
\hline 8.5 & 9.35 & 8.89 & 8.97 & 8.71 & 8.56 & 8.78 & 8.67 & 9.10 & 9.02 \\
\hline 9.5 & 9.88 & 9.43 & 9.15 & 9.09 & 8.73 & 9.14 & 9.40 & 9.21 & 9.57 \\
\hline 10.5 & 9.97 & 9.86 & 9.41 & 9.61 & 9.10 & 9.69 & 9.47 & 9.43 & 9.91 \\
\hline 11.5 & 10.34 & 10.08 & 9.71 & 10.17 & 9.25 & 9.81 & 9.79 & 9.93 & 10.04 \\
\hline 12.5 & 10.51 & 10.06 & 10.01 & 10.19 & 9.26 & 10.20 & 9.99 & 9.73 & 10.38 \\
\hline 13.5 & 10.30 & 9.81 & 9.86 & 9.90 & 9.67 & 10.18 & 9.82 & 10.12 & 10.48 \\
\hline 14.5 & 10.25 & 9.88 & 9.75 & 9.55 & 9.34 & 9.81 & 9.80 & 9.94 & 9.93 \\
\hline 15.5 & 9.60 & 9.46 & 9.46 & 9.52 & 8.81 & 9.40 & 9.14 & 9.45 & 9.81 \\
\hline 16.5 & 9.36 & 9.12 & 8.88 & 8.97 & 8.73 & 8.81 & 8.67 & 8.71 & 9.01 \\
\hline 17.5 & 8.70 & 8.50 & 8.44 & 8.49 & 8.11 & 8.30 & 8.50 & 8.35 & 8.65 \\
\hline 18.5 & 8.38 & 8.10 & 7.88 & 7.83 & 7.36 & 7.94 & 7.79 & 7.81 & 8.38 \\
\hline 19.5 & 8.04 & 7.34 & 7.30 & 7.54 & 7.05 & 7.14 & 7.43 & 7.26 & 7.72 \\
\hline 20.5 & 7.20 & 6.98 & 6.66 & 6.98 & 6.64 & 6.60 & 6.78 & 7.00 & 6.94 \\
\hline 21.5 & 6.59 & 6.44 & 6.23 & 6.20 & 5.83 & 6.34 & 6.08 & 6.16 & 6.21 \\
\hline 22.5 & 6.03 & 5.72 & 5.69 & 5.70 & 5.73 & 5.78 & 5.88 & 5.58 & 5.81 \\
\hline 23.5 & 6.22 & 5.97 & 6.03 & 5.99 & 5.70 & 5.89 & 6.02 & 5.87 & 6.16 \\
\hline Ax. Avg. & 8.25 & 7.93 & 7.81 & 7.88 & 7.49 & 7.85 & 7.77 & 7.83 & 8.07 \\
\hline & & & & & & & & & 5.14 \\
\hline
\end{tabular}

POWER DENSITY VALUES NORMALIZED TO A CORE POWER OF 11.57 MW AND ASSUME ALL FISSION ENERGY (EXCLUDING GAMMA-HEATING OF EX-CORE COMPONENTS) IS DEPOSITED IN THE FUEL 
Table B-1. Power Density Data (kW/cc) in Fuel Plate 1 in Prototypic Conditions (continued).

Plate 1, Core Position X2, 99 MWd Burnup, Equilibrium Xenon Conditions

\begin{tabular}{|c|c|c|c|c|c|c|c|c|c|}
\hline $\begin{array}{c}\text { Axial } \\
\text { position } \\
\text { from } \\
\text { top of } \\
\text { fuel } \\
\text { (inches) }\end{array}$ & $\begin{array}{c}0.000 \\
\text { to } \\
0.197 \\
\text { inches }\end{array}$ & $\begin{array}{c}0.197 \\
\text { to } \\
0.394 \\
\text { inches }\end{array}$ & $\begin{array}{c}0.394 \\
\text { to } \\
0.591 \\
\text { inches }\end{array}$ & $\begin{array}{c}0.591 \\
\text { to } \\
0.760 \\
\text { inches }\end{array}$ & $\begin{array}{c}0.760 \\
\text { to } \\
0.929 \\
\text { inches }\end{array}$ & $\begin{array}{c}0.929 \\
\text { to } \\
1.099 \\
\text { inches }\end{array}$ & $\begin{array}{c}1.099 \\
\text { to } \\
1.296 \\
\text { inches }\end{array}$ & $\begin{array}{c}1.296 \\
\text { to } \\
1.493 \\
\text { inches }\end{array}$ & $\begin{array}{c}1.493 \\
\text { to } \\
1.690 \\
\text { inches }\end{array}$ \\
\hline 1.5 & 5.10 & 5.06 & 5.02 & 5.23 & 4.77 & 4.83 & 4.96 & 4.98 & 5.08 \\
\hline 2.5 & 6.11 & 5.69 & 5.62 & 5.74 & 5.67 & 5.76 & 5.47 & 5.49 & 5.89 \\
\hline 3.5 & 6.74 & 6.66 & 6.70 & 6.75 & 6.20 & 6.33 & 6.56 & 6.58 & 6.73 \\
\hline 4.5 & 7.33 & 7.13 & 6.97 & 6.77 & 6.56 & 6.86 & 7.08 & 6.96 & 7.36 \\
\hline 5.5 & 7.83 & 7.51 & 7.62 & 7.72 & 7.56 & 7.58 & 7.69 & 7.79 & 7.91 \\
\hline 6.5 & 8.36 & 8.00 & 7.83 & 7.94 & 7.56 & 8.40 & 8.09 & 8.30 & 8.56 \\
\hline 7.5 & 9.03 & 8.71 & 8.55 & 8.71 & 7.96 & 8.37 & 8.46 & 8.83 & 8.90 \\
\hline 8.5 & 9.01 & 8.85 & 8.99 & 8.87 & 8.53 & 8.85 & 8.61 & 8.78 & 8.86 \\
\hline 9.5 & 9.65 & 9.19 & 9.24 & 9.23 & 9.00 & 9.41 & 9.14 & 9.26 & 9.41 \\
\hline 10.5 & 9.47 & 9.45 & 9.23 & 9.46 & 9.07 & 9.55 & 9.31 & 9.34 & 9.81 \\
\hline 11.5 & 9.77 & 9.69 & 9.53 & 9.45 & 9.16 & 9.18 & 9.47 & 9.57 & 9.73 \\
\hline 12.5 & 10.01 & 9.43 & 9.54 & 9.38 & 9.15 & 9.59 & 9.77 & 9.78 & 10.17 \\
\hline 13.5 & 9.79 & 9.29 & 9.34 & 9.56 & 9.11 & 9.82 & 9.60 & 9.56 & 9.95 \\
\hline 14.5 & 9.56 & 9.48 & 9.49 & 9.53 & 9.07 & 9.52 & 9.45 & 9.72 & 9.72 \\
\hline 15.5 & 9.26 & 9.33 & 9.08 & 8.81 & 8.54 & 9.30 & 9.00 & 9.41 & 9.34 \\
\hline 16.5 & 9.14 & 8.66 & 8.88 & 8.79 & 8.27 & 8.79 & 8.81 & 9.00 & 9.03 \\
\hline 17.5 & 8.53 & 8.29 & 8.14 & 8.20 & 8.03 & 8.36 & 8.25 & 8.28 & 8.42 \\
\hline 18.5 & 8.02 & 8.15 & 7.90 & 8.03 & 7.40 & 7.69 & 7.85 & 7.94 & 8.18 \\
\hline 19.5 & 7.47 & 7.20 & 7.07 & 7.39 & 7.05 & 7.29 & 7.08 & 7.48 & 7.67 \\
\hline 20.5 & 6.88 & 6.84 & 6.39 & 6.76 & 6.35 & 6.84 & 6.67 & 6.88 & 6.98 \\
\hline 21.5 & 6.26 & 6.24 & 6.01 & 6.17 & 5.80 & 6.03 & 5.96 & 6.17 & 6.40 \\
\hline 22.5 & 5.96 & 5.78 & 5.68 & 5.55 & 5.35 & 5.61 & 5.74 & 5.54 & 6.07 \\
\hline 23.5 & 6.01 & 5.86 & 5.64 & 5.75 & 5.38 & 5.70 & 5.76 & 5.91 & 5.98 \\
\hline Ax. Avg. & 7.95 & 7.74 & 7.65 & 7.70 & 7.36 & 7.70 & 7.65 & 7.78 & 7.98 \\
\hline & & & & & & & & & 5.13 \\
\hline
\end{tabular}

POWER DENSITY VALUES NORMALIZED TO A CORE POWER OF 11.57 MW AND ASSUME ALL FISSION ENERGY (EXCLUDING GAMMA-HEATING OF EX-CORE COMPONENTS) IS DEPOSITED IN THE FUEL 
Table B-1. Power Density Data (kW/cc) in Fuel Plate 1 in Prototypic Conditions (continued).

Plate 1, Core Position X4, 173 MWd Burnup, Equilibrium Xenon Conditions

\begin{tabular}{|c|c|c|c|c|c|c|c|c|c|}
\hline $\begin{array}{c}\text { Axial } \\
\text { position } \\
\text { from } \\
\text { top of } \\
\text { fuel } \\
\text { (inches) }\end{array}$ & $\begin{array}{c}0.000 \\
\text { to } \\
0.197 \\
\text { inches }\end{array}$ & $\begin{array}{c}0.197 \\
\text { to } \\
0.394 \\
\text { inches }\end{array}$ & $\begin{array}{c}0.394 \\
\text { to } \\
0.591 \\
\text { inches }\end{array}$ & $\begin{array}{c}0.591 \\
\text { to } \\
0.760 \\
\text { inches }\end{array}$ & $\begin{array}{c}0.760 \\
\text { to } \\
0.929 \\
\text { inches }\end{array}$ & $\begin{array}{c}0.929 \\
\text { to } \\
1.099 \\
\text { inches }\end{array}$ & $\begin{array}{c}1.099 \\
\text { to } \\
1.296 \\
\text { inches }\end{array}$ & $\begin{array}{c}1.296 \\
\text { to } \\
1.493 \\
\text { inches }\end{array}$ & $\begin{array}{c}1.493 \\
\text { to } \\
\text { inches }\end{array}$ \\
\hline 0.5 & 4.65 & 4.38 & 4.52 & 4.43 & 4.28 & 4.78 & 4.32 & 4.50 & 4.78 \\
\hline 1.5 & 4.71 & 4.67 & 4.67 & 4.43 & 4.15 & 4.52 & 4.62 & 4.74 & 4.77 \\
\hline 2.5 & 4.97 & 4.95 & 4.78 & 4.84 & 4.58 & 4.87 & 4.93 & 4.99 & 4.99 \\
\hline 3.5 & 5.84 & 5.39 & 5.58 & 5.61 & 5.18 & 5.50 & 5.60 & 5.91 & 5.97 \\
\hline 4.5 & 6.05 & 5.90 & 6.10 & 5.92 & 5.74 & 6.28 & 6.18 & 6.11 & 6.18 \\
\hline 5.5 & 6.86 & 6.71 & 6.62 & 6.70 & 6.13 & 6.64 & 6.66 & 6.54 & 6.76 \\
\hline 6.5 & 7.01 & 6.66 & 6.77 & 6.84 & 6.50 & 6.94 & 6.75 & 6.64 & 7.02 \\
\hline 7.5 & 7.20 & 6.95 & 6.97 & 7.29 & 6.66 & 7.02 & 6.83 & 7.11 & 7.16 \\
\hline 8.5 & 7.34 & 7.24 & 6.74 & 7.08 & 7.01 & 7.32 & 7.01 & 7.32 & 7.29 \\
\hline 9.5 & 7.81 & 7.71 & 7.61 & 7.45 & 7.10 & 7.54 & 7.37 & 7.69 & 7.65 \\
\hline 10.5 & 7.68 & 7.64 & 7.66 & 7.66 & 7.34 & 7.50 & 7.64 & 7.48 & 7.67 \\
\hline 11.5 & 7.97 & 7.69 & 8.05 & 7.82 & 7.30 & 8.11 & 7.59 & 7.86 & 8.11 \\
\hline 12.5 & 8.06 & 8.33 & 7.83 & 8.11 & 7.67 & 7.99 & 8.09 & 8.04 & 8.03 \\
\hline 13.5 & 8.18 & 7.86 & 7.89 & 7.89 & 7.62 & 7.94 & 8.12 & 8.19 & 8.09 \\
\hline 14.5 & 8.14 & 8.16 & 7.92 & 8.21 & 7.70 & 8.08 & 7.72 & 8.04 & 8.21 \\
\hline 15.5 & 7.51 & 7.62 & 7.25 & 7.54 & 7.25 & 7.75 & 7.39 & 7.51 & 7.61 \\
\hline 16.5 & 7.52 & 7.29 & 7.30 & 7.45 & 7.22 & 7.64 & 7.42 & 7.37 & 7.69 \\
\hline 17.5 & 6.75 & 6.74 & 6.78 & 6.93 & 6.46 & 6.90 & 6.91 & 6.94 & 7.26 \\
\hline 18.5 & 7.12 & 6.62 & 6.66 & 6.49 & 6.35 & 6.90 & 6.67 & 6.84 & 7.04 \\
\hline 19.5 & 6.26 & 6.16 & 5.94 & 5.96 & 5.84 & 6.28 & 6.37 & 6.49 & 6.37 \\
\hline 20.5 & 6.21 & 6.27 & 6.02 & 5.88 & 5.75 & 5.75 & 5.91 & 6.09 & 6.14 \\
\hline 21.5 & 5.42 & 5.43 & 5.48 & 5.32 & 5.16 & 5.29 & 5.40 & 5.43 & 5.67 \\
\hline 22.5 & 5.01 & 4.88 & 4.78 & 5.01 & 4.66 & 4.99 & 5.03 & 5.12 & 5.14 \\
\hline 23.5 & 5.38 & 5.31 & 5.39 & 5.12 & 5.16 & 5.11 & 5.16 & 5.15 & 5.39 \\
\hline Ax. Avg. & 6.65 & 6.52 & 6.48 & 6.50 & 6.20 & 6.57 & 6.49 & 6.59 & 6.70 \\
\hline & & & & & & & & & \\
\hline
\end{tabular}

POWER DENSITY VALUES NORMALIZED TO A CORE POWER OF 11.57 MW AND ASSUME ALL FISSION ENERGY (EXCLUDING GAMMA-HEATING OF EX-CORE COMPONENTS) IS DEPOSITED IN THE FUEL 
Table B-2. Power Density Data $\left(\mathrm{kW} / \mathrm{cm}^{3}\right)$ in Fuel Plate 2 in Prototypic Core.

\section{Fuel core width: 1.797 inches / Fuel core thickness: 12 mil}

Plate 2, Core Position X1/X5, 0 MWd Burnup, Xenon-Free Conditions

\begin{tabular}{|c|c|c|c|c|c|c|c|c|c|}
\hline $\begin{array}{c}\text { Axial } \\
\text { position } \\
\text { from } \\
\text { top of } \\
\text { fuel } \\
\text { (inches) } \\
\end{array}$ & $\begin{array}{c}0.000 \\
\text { to } \\
0.197 \\
\text { inches }\end{array}$ & $\begin{array}{c}0.197 \\
\text { to } \\
0.394 \\
\text { inches } \\
\end{array}$ & $\begin{array}{c}0.394 \\
\text { to } \\
0.591 \\
\text { inches } \\
\end{array}$ & $\begin{array}{c}0.591 \\
\text { to } \\
0.796 \\
\text { inches }\end{array}$ & $\begin{array}{c}0.796 \\
\text { to } \\
1.001 \\
\text { inches }\end{array}$ & $\begin{array}{c}1.001 \\
\text { to } \\
1.207 \\
\text { inches }\end{array}$ & $\begin{array}{c}1.207 \\
\text { to } \\
1.403 \\
\text { inches }\end{array}$ & $\begin{array}{c}1.403 \\
\text { to } \\
1.600 \\
\text { inches }\end{array}$ & $\begin{array}{c}1.600 \\
\text { to } \\
1.797 \\
\text { inches } \\
\end{array}$ \\
\hline 0.5 & 3.10 & 2.70 & 2.57 & 2.65 & 2.49 & 2.66 & 2.71 & 2.73 & 2.96 \\
\hline 1.5 & 2.94 & 2.62 & 2.59 & 2.60 & 2.51 & 2.61 & 2.57 & 2.62 & 2.85 \\
\hline 2.5 & 3.39 & 3.25 & 3.17 & 3.12 & 2.99 & 3.00 & 3.05 & 3.17 & 3.43 \\
\hline 3.5 & 4.21 & 3.81 & 3.65 & 3.88 & 3.41 & 3.56 & 3.60 & 3.71 & 3.98 \\
\hline 4.5 & 4.64 & 4.40 & 4.37 & 4.31 & 4.19 & 4.21 & 4.34 & 4.52 & 4.78 \\
\hline 5.5 & 5.50 & 5.06 & 4.85 & 4.81 & 4.63 & 4.76 & 4.81 & 4.92 & 5.47 \\
\hline 6.5 & 6.12 & 5.59 & 5.60 & 5.43 & 5.23 & 5.54 & 5.53 & 5.75 & 6.11 \\
\hline 7.5 & 6.87 & 6.20 & 6.08 & 6.03 & 5.96 & 6.12 & 6.23 & 6.33 & 6.85 \\
\hline 8.5 & 7.58 & 6.95 & 6.85 & 6.82 & 6.68 & 6.79 & 6.99 & 7.11 & 7.56 \\
\hline 9.5 & 8.37 & 7.76 & 7.60 & 7.56 & 7.32 & 7.42 & 7.66 & 7.74 & 8.57 \\
\hline 10.5 & 9.12 & 8.51 & 8.16 & 8.08 & 7.64 & 8.26 & 8.19 & 8.27 & 8.99 \\
\hline 11.5 & 9.67 & 8.94 & 8.76 & 8.75 & 8.28 & 8.79 & 8.61 & 8.97 & 9.82 \\
\hline 12.5 & 10.35 & 9.57 & 9.19 & 9.21 & 8.68 & 9.17 & 9.10 & 9.36 & 10.11 \\
\hline 13.5 & 10.48 & 9.73 & 9.40 & 9.34 & 8.84 & 9.40 & 9.49 & 9.84 & 10.38 \\
\hline 14.5 & 10.60 & 9.55 & 9.34 & 9.28 & 8.98 & 9.30 & 9.45 & 9.75 & 10.32 \\
\hline 15.5 & 10.61 & 9.52 & 9.14 & 9.11 & 8.73 & 9.30 & 9.24 & 9.48 & 10.31 \\
\hline 16.5 & 10.04 & 9.38 & 8.91 & 8.87 & 8.44 & 9.07 & 8.89 & 9.17 & 9.89 \\
\hline 17.5 & 9.81 & 8.98 & 8.52 & 8.67 & 8.21 & 8.46 & 8.72 & 8.85 & 9.50 \\
\hline 18.5 & 9.15 & 8.32 & 7.89 & 8.09 & 7.72 & 7.97 & 7.99 & 8.53 & 8.97 \\
\hline 19.5 & 8.50 & 7.76 & 7.75 & 7.71 & 7.11 & 7.50 & 7.52 & 7.82 & 8.47 \\
\hline 20.5 & 8.10 & 7.24 & 7.03 & 6.97 & 6.66 & 7.07 & 7.13 & 7.28 & 7.95 \\
\hline 21.5 & 7.20 & 6.72 & 6.69 & 6.56 & 6.19 & 6.45 & 6.45 & 6.60 & 7.16 \\
\hline 22.5 & 6.74 & 6.11 & 5.88 & 5.72 & 5.56 & 5.81 & 5.82 & 6.04 & 6.53 \\
\hline 23.5 & 7.26 & 6.54 & 6.26 & 6.39 & 6.01 & 6.24 & 6.17 & 6.57 & 7.19 \\
\hline Ax. Avg. & 7.52 & 6.89 & 6.68 & 6.66 & 6.35 & 6.64 & 6.68 & 6.88 & 7.42 \\
\hline
\end{tabular}

POWER DENSITY VALUES NORMALIZED TO A CORE POWER OF 11.57 MW AND ASSUME ALL FISSION ENERGY (EXCLUDING GAMMA-HEATING OF EX-CORE COMPONENTS) IS DEPOSITED IN THE FUEL 
Table B-2. Power Density Data (kW/cc) in Fuel Plate 2 in Prototypic Conditions (continued).

Plate 2, Core Position X1/X5, 3 MWd Burnup, Equilibrium Xenon Conditions

\begin{tabular}{|c|c|c|c|c|c|c|c|c|c|}
\hline $\begin{array}{c}\text { Axial } \\
\text { position } \\
\text { from } \\
\text { top of } \\
\text { fuel } \\
\text { (inches) }\end{array}$ & $\begin{array}{c}0.000 \\
\text { to } \\
0.197 \\
\text { inches }\end{array}$ & $\begin{array}{c}0.197 \\
\text { to } \\
0.394 \\
\text { inches }\end{array}$ & $\begin{array}{c}0.394 \\
\text { to } \\
0.591 \\
\text { inches }\end{array}$ & $\begin{array}{c}0.591 \\
\text { to } \\
0.796 \\
\text { inches }\end{array}$ & $\begin{array}{c}0.796 \\
\text { to } \\
1.001 \\
\text { inches }\end{array}$ & $\begin{array}{c}1.001 \\
\text { to } \\
1.207 \\
\text { inches }\end{array}$ & $\begin{array}{c}1.207 \\
\text { to } \\
1.403 \\
\text { inches }\end{array}$ & $\begin{array}{c}1.403 \\
\text { to } \\
1.600 \\
\text { inches }\end{array}$ & $\begin{array}{c}1.600 \\
\text { to } \\
1.797 \\
\text { inches }\end{array}$ \\
\hline 0.5 & 4.45 & 4.02 & 3.90 & 3.90 & 3.76 & 3.99 & 4.10 & 4.09 & 4.41 \\
\hline 1.5 & 4.48 & 3.90 & 3.76 & 3.67 & 3.57 & 3.83 & 3.77 & 4.12 & 4.45 \\
\hline 2.5 & 4.95 & 4.65 & 4.39 & 4.41 & 4.13 & 4.32 & 4.35 & 4.62 & 5.00 \\
\hline 3.5 & 5.76 & 5.34 & 5.12 & 5.04 & 4.88 & 4.96 & 5.03 & 5.25 & 5.78 \\
\hline 4.5 & 6.46 & 5.84 & 5.69 & 5.66 & 5.27 & 5.56 & 5.69 & 5.72 & 6.22 \\
\hline 5.5 & 6.85 & 6.35 & 6.18 & 6.18 & 5.93 & 6.09 & 6.18 & 6.48 & 6.99 \\
\hline 6.5 & 7.52 & 6.92 & 6.86 & 6.77 & 6.38 & 6.73 & 6.58 & 6.89 & 7.73 \\
\hline 7.5 & 8.20 & 7.31 & 7.20 & 7.23 & 6.79 & 7.16 & 7.00 & 7.34 & 7.90 \\
\hline 8.5 & 8.37 & 7.79 & 7.48 & 7.44 & 7.26 & 7.58 & 7.42 & 7.57 & 8.25 \\
\hline 9.5 & 9.13 & 8.23 & 7.78 & 7.81 & 7.26 & 7.79 & 8.05 & 7.98 & 8.62 \\
\hline 10.5 & 9.00 & 8.49 & 8.02 & 7.97 & 7.84 & 8.17 & 8.16 & 8.33 & 9.18 \\
\hline 11.5 & 9.33 & 8.52 & 8.29 & 8.36 & 7.84 & 8.31 & 8.20 & 8.49 & 9.24 \\
\hline 12.5 & 9.39 & 8.43 & 8.25 & 8.21 & 7.84 & 8.09 & 8.09 & 8.53 & 9.23 \\
\hline 13.5 & 9.29 & 8.46 & 8.29 & 8.20 & 7.71 & 8.13 & 8.17 & 8.30 & 9.18 \\
\hline 14.5 & 9.07 & 8.11 & 7.94 & 7.82 & 7.57 & 7.95 & 7.90 & 8.27 & 9.06 \\
\hline 15.5 & 8.32 & 7.82 & 7.66 & 7.58 & 7.37 & 7.69 & 7.67 & 7.95 & 8.63 \\
\hline 16.5 & 8.12 & 7.46 & 7.03 & 7.19 & 7.02 & 7.23 & 7.26 & 7.60 & 8.17 \\
\hline 17.5 & 7.62 & 7.10 & 6.83 & 6.74 & 6.51 & 6.75 & 6.70 & 6.97 & 7.52 \\
\hline 18.5 & 7.12 & 6.51 & 6.19 & 6.41 & 6.03 & 6.31 & 6.25 & 6.37 & 7.05 \\
\hline 19.5 & 6.60 & 6.07 & 5.91 & 5.86 & 5.53 & 5.79 & 5.86 & 6.00 & 6.50 \\
\hline 20.5 & 6.07 & 5.65 & 5.39 & 5.33 & 5.16 & 5.43 & 5.37 & 5.69 & 6.09 \\
\hline 21.5 & 5.63 & 4.95 & 4.82 & 4.87 & 4.64 & 4.71 & 4.77 & 5.05 & 5.52 \\
\hline 22.5 & 4.82 & 4.50 & 4.37 & 4.44 & 4.24 & 4.50 & 4.41 & 4.61 & 5.05 \\
\hline 23.5 & 5.48 & 4.96 & 4.86 & 4.66 & 4.51 & 4.62 & 4.63 & 5.01 & 5.38 \\
\hline Ax. Avg. & 7.17 & 6.56 & 6.34 & 6.32 & 6.04 & 6.32 & 6.32 & 6.55 & 7.13 \\
\hline & & & & & & & & & \\
\hline
\end{tabular}

POWER DENSITY VALUES NORMALIZED TO A CORE POWER OF 11.57 MW AND ASSUME ALL FISSION ENERGY (EXCLUDING GAMMA-HEATING OF EX-CORE COMPONENTS) IS DEPOSITED IN THE FUEL 
Table B-2. Power Density Data (kW/cc) in Fuel Plate 2 in Prototypic Conditions (continued).

Plate 2, Core Position X3/X7, 80 MWd Burnup, Equilibrium Xenon Conditions

\begin{tabular}{|c|c|c|c|c|c|c|c|c|c|}
\hline $\begin{array}{c}\text { Axial } \\
\text { position } \\
\text { from } \\
\text { top of } \\
\text { fuel } \\
\text { (inches) }\end{array}$ & $\begin{array}{c}0.000 \\
\text { to } \\
0.197 \\
\text { inches }\end{array}$ & $\begin{array}{c}0.197 \\
\text { to } \\
0.394 \\
\text { inches }\end{array}$ & $\begin{array}{c}0.394 \\
\text { to } \\
0.591 \\
\text { inches }\end{array}$ & $\begin{array}{c}0.591 \\
\text { to } \\
0.796 \\
\text { inches }\end{array}$ & $\begin{array}{c}0.796 \\
\text { to } \\
1.001 \\
\text { inches }\end{array}$ & $\begin{array}{c}1.001 \\
\text { to } \\
1.207 \\
\text { inches }\end{array}$ & $\begin{array}{c}1.207 \\
\text { to } \\
1.403 \\
\text { inches }\end{array}$ & $\begin{array}{c}1.403 \\
\text { to } \\
1.600 \\
\text { inches }\end{array}$ & $\begin{array}{c}1.600 \\
\text { to } \\
1.797 \\
\text { inches }\end{array}$ \\
\hline 0.5 & 4.15 & 3.69 & 3.72 & 3.67 & 3.51 & 3.70 & 3.76 & 3.83 & 4.19 \\
\hline 1.5 & 3.98 & 3.74 & 3.54 & 3.63 & 3.46 & 3.58 & 3.66 & 3.62 & 4.10 \\
\hline 2.5 & 4.52 & 4.22 & 4.17 & 4.12 & 3.95 & 4.15 & 4.03 & 4.27 & 4.60 \\
\hline 3.5 & 5.37 & 4.94 & 4.76 & 4.82 & 4.72 & 4.75 & 4.87 & 4.91 & 5.21 \\
\hline 4.5 & 5.86 & 5.49 & 5.42 & 5.40 & 5.19 & 5.45 & 5.42 & 5.56 & 5.87 \\
\hline 5.5 & 6.48 & 5.96 & 5.84 & 5.92 & 5.58 & 5.85 & 5.92 & 5.98 & 6.56 \\
\hline 6.5 & 6.82 & 6.39 & 6.06 & 6.11 & 5.85 & 6.14 & 6.19 & 6.23 & 6.80 \\
\hline 7.5 & 7.12 & 6.66 & 6.47 & 6.42 & 6.28 & 6.37 & 6.51 & 6.69 & 7.45 \\
\hline 8.5 & 7.37 & 6.93 & 6.69 & 6.86 & 6.41 & 6.77 & 6.85 & 7.02 & 7.61 \\
\hline 9.5 & 8.09 & 7.37 & 7.14 & 7.03 & 6.79 & 7.15 & 7.13 & 7.43 & 8.07 \\
\hline 10.5 & 8.16 & 7.73 & 7.36 & 7.31 & 7.01 & 7.31 & 7.48 & 7.65 & 8.31 \\
\hline 11.5 & 8.44 & 7.75 & 7.49 & 7.59 & 7.20 & 7.56 & 7.61 & 7.71 & 8.46 \\
\hline 12.5 & 8.35 & 7.85 & 7.68 & 7.62 & 7.41 & 7.74 & 7.70 & 7.85 & 8.60 \\
\hline 13.5 & 8.49 & 7.95 & 7.71 & 7.76 & 7.19 & 7.47 & 7.60 & 7.82 & 8.59 \\
\hline 14.5 & 8.25 & 7.57 & 7.38 & 7.29 & 7.13 & 7.51 & 7.44 & 7.55 & 8.11 \\
\hline 15.5 & 7.93 & 7.37 & 7.09 & 7.30 & 6.57 & 7.05 & 7.08 & 7.25 & 7.84 \\
\hline 16.5 & 7.59 & 7.03 & 6.99 & 6.88 & 6.44 & 6.69 & 6.69 & 6.92 & 7.56 \\
\hline 17.5 & 6.84 & 6.23 & 6.34 & 6.28 & 5.87 & 6.23 & 6.30 & 6.36 & 7.06 \\
\hline 18.5 & 6.51 & 6.06 & 5.87 & 5.82 & 5.50 & 5.86 & 5.94 & 6.00 & 6.58 \\
\hline 19.5 & 5.90 & 5.65 & 5.50 & 5.45 & 5.20 & 5.44 & 5.53 & 5.71 & 5.98 \\
\hline 20.5 & 5.51 & 5.18 & 5.05 & 5.02 & 4.72 & 5.12 & 5.02 & 5.33 & 5.69 \\
\hline 21.5 & 5.04 & 4.62 & 4.48 & 4.50 & 4.30 & 4.58 & 4.72 & 4.57 & 5.05 \\
\hline 22.5 & 4.65 & 4.25 & 4.19 & 4.16 & 3.91 & 4.23 & 4.20 & 4.26 & 4.75 \\
\hline 23.5 & 5.09 & 4.56 & 4.44 & 4.42 & 4.34 & 4.44 & 4.42 & 4.50 & 5.02 \\
\hline Ax. Avg. & 6.52 & 6.05 & 5.89 & 5.89 & 5.61 & 5.88 & 5.92 & 6.04 & 6.59 \\
\hline & & & & & & & & & \\
\hline
\end{tabular}

POWER DENSITY VALUES NORMALIZED TO A CORE POWER OF 11.57 MW AND ASSUME ALL FISSION ENERGY (EXCLUDING GAMMA-HEATING OF EX-CORE COMPONENTS) IS DEPOSITED IN THE FUEL 
Table B-2. Power Density Data (kW/cc) in Fuel Plate 2 in Prototypic Conditions (continued).

Plate 2, Core Position X2/X6, 99 MWd Burnup, Equilibrium Xenon Conditions

\begin{tabular}{|c|c|c|c|c|c|c|c|c|c|}
\hline $\begin{array}{c}\text { Axial } \\
\text { position } \\
\text { from } \\
\text { top of } \\
\text { fuel } \\
\text { (inches) }\end{array}$ & $\begin{array}{c}0.000 \\
\text { to } \\
0.197 \\
\text { inches }\end{array}$ & $\begin{array}{c}0.197 \\
\text { to } \\
0.394 \\
\text { inches }\end{array}$ & $\begin{array}{c}0.394 \\
\text { to } \\
0.591 \\
\text { inches }\end{array}$ & $\begin{array}{c}0.591 \\
\text { to } \\
0.796 \\
\text { inches }\end{array}$ & $\begin{array}{c}0.796 \\
\text { to } \\
1.001 \\
\text { inches }\end{array}$ & $\begin{array}{c}1.001 \\
\text { to } \\
1.207 \\
\text { inches }\end{array}$ & $\begin{array}{c}1.207 \\
\text { to } \\
1.403 \\
\text { inches }\end{array}$ & $\begin{array}{c}1.403 \\
\text { to } \\
1.600 \\
\text { inches }\end{array}$ & $\begin{array}{c}1.600 \\
\text { to } \\
1.797 \\
\text { inches }\end{array}$ \\
\hline 0.5 & 4.13 & 3.72 & 3.53 & 3.74 & 3.52 & 3.61 & 3.57 & 3.77 & 4.07 \\
\hline 1.5 & 3.94 & 3.74 & 3.68 & 3.50 & 3.33 & 3.58 & 3.47 & 3.56 & 3.98 \\
\hline 2.5 & 4.44 & 4.21 & 4.04 & 4.02 & 3.92 & 4.17 & 4.14 & 4.31 & 4.48 \\
\hline 3.5 & 5.24 & 4.98 & 4.90 & 4.87 & 4.62 & 4.74 & 4.87 & 4.95 & 5.25 \\
\hline 4.5 & 5.83 & 5.46 & 5.27 & 5.35 & 5.12 & 5.21 & 5.25 & 5.34 & 5.80 \\
\hline 5.5 & 6.41 & 6.06 & 5.75 & 5.85 & 5.67 & 5.89 & 5.78 & 5.95 & 6.39 \\
\hline 6.5 & 6.66 & 6.14 & 5.94 & 6.08 & 5.85 & 6.19 & 6.20 & 6.20 & 6.78 \\
\hline 7.5 & 7.04 & 6.47 & 6.24 & 6.32 & 5.94 & 6.43 & 6.36 & 6.50 & 6.89 \\
\hline 8.5 & 7.20 & 6.87 & 6.76 & 6.65 & 6.31 & 6.54 & 6.54 & 6.83 & 7.43 \\
\hline 9.5 & 7.79 & 7.24 & 7.10 & 6.91 & 6.85 & 7.01 & 7.10 & 7.13 & 7.61 \\
\hline 10.5 & 7.91 & 7.33 & 7.20 & 7.38 & 6.84 & 7.45 & 7.29 & 7.53 & 8.09 \\
\hline 11.5 & 8.34 & 7.77 & 7.56 & 7.42 & 6.94 & 7.38 & 7.35 & 7.59 & 8.17 \\
\hline 12.5 & 8.09 & 7.57 & 7.39 & 7.36 & 7.29 & 7.61 & 7.60 & 7.81 & 8.22 \\
\hline 13.5 & 7.97 & 7.61 & 7.43 & 7.32 & 7.10 & 7.43 & 7.42 & 7.51 & 8.12 \\
\hline 14.5 & 7.89 & 7.54 & 7.30 & 7.20 & 6.81 & 7.18 & 7.28 & 7.66 & 8.17 \\
\hline 15.5 & 7.64 & 7.18 & 6.92 & 6.84 & 6.64 & 6.92 & 7.02 & 7.14 & 7.54 \\
\hline 16.5 & 7.26 & 6.83 & 6.38 & 6.41 & 6.13 & 6.53 & 6.53 & 6.84 & 7.31 \\
\hline 17.5 & 6.65 & 6.39 & 6.00 & 5.98 & 5.78 & 6.13 & 6.18 & 6.34 & 6.64 \\
\hline 18.5 & 6.47 & 6.02 & 5.81 & 5.86 & 5.35 & 5.71 & 5.67 & 5.86 & 6.48 \\
\hline 19.5 & 5.97 & 5.52 & 5.20 & 5.19 & 5.05 & 5.35 & 5.33 & 5.42 & 5.80 \\
\hline 20.5 & 5.48 & 5.09 & 4.88 & 4.86 & 4.61 & 4.94 & 4.95 & 5.19 & 5.48 \\
\hline 21.5 & 4.93 & 4.56 & 4.54 & 4.47 & 4.21 & 4.60 & 4.54 & 4.70 & 5.04 \\
\hline 22.5 & 4.56 & 4.20 & 4.05 & 3.98 & 3.81 & 4.07 & 4.09 & 4.28 & 4.61 \\
\hline 23.5 & 4.90 & 4.41 & 4.36 & 4.52 & 4.25 & 4.28 & 4.50 & 4.56 & 5.07 \\
\hline Ax. Avg. & 6.36 & 5.95 & 5.76 & 5.75 & 5.50 & 5.79 & 5.79 & 5.96 & 6.39 \\
\hline & & & & & & & & & \\
\hline
\end{tabular}

POWER DENSITY VALUES NORMALIZED TO A CORE POWER OF 11.57 MW AND ASSUME ALL FISSION ENERGY (EXCLUDING GAMMA-HEATING OF EX-CORE COMPONENTS) IS DEPOSITED IN THE FUEL 
Table B-2. Power Density Data (kW/cc) in Fuel Plate 2 in Prototypic Conditions (continued).

Plate 2, Core Position X4/X8, 173 MWd Burnup, Equilibrium Xenon Conditions

\begin{tabular}{|c|c|c|c|c|c|c|c|c|c|}
\hline $\begin{array}{c}\text { Axial } \\
\text { position } \\
\text { from } \\
\text { top of } \\
\text { fuel } \\
\text { (inches) }\end{array}$ & $\begin{array}{c}0.000 \\
\text { to } \\
0.197 \\
\text { inches }\end{array}$ & $\begin{array}{c}0.197 \\
\text { to } \\
0.394 \\
\text { inches }\end{array}$ & $\begin{array}{c}0.394 \\
\text { to } \\
0.591 \\
\text { inches }\end{array}$ & $\begin{array}{c}0.591 \\
\text { to } \\
0.796 \\
\text { inches }\end{array}$ & $\begin{array}{c}0.796 \\
\text { to } \\
1.001 \\
\text { inches }\end{array}$ & $\begin{array}{c}1.001 \\
\text { to } \\
1.207 \\
\text { inches }\end{array}$ & $\begin{array}{c}1.207 \\
\text { to } \\
1.403 \\
\text { inches }\end{array}$ & $\begin{array}{c}1.403 \\
\text { to } \\
1.600 \\
\text { inches }\end{array}$ & $\begin{array}{c}1.600 \\
\text { to } \\
1.797 \\
\text { inches }\end{array}$ \\
\hline 0.5 & 4.03 & 3.57 & 3.54 & 3.54 & 3.46 & 3.66 & 3.62 & 3.64 & 3.94 \\
\hline 1.5 & 3.96 & 3.55 & 3.57 & 3.38 & 3.32 & 3.47 & 3.46 & 3.61 & 3.83 \\
\hline 2.5 & 4.23 & 3.93 & 3.85 & 4.00 & 3.63 & 3.92 & 3.84 & 3.95 & 4.18 \\
\hline 3.5 & 4.90 & 4.59 & 4.52 & 4.44 & 4.31 & 4.62 & 4.63 & 4.64 & 5.02 \\
\hline 4.5 & 5.34 & 5.05 & 4.89 & 4.84 & 4.79 & 4.95 & 4.96 & 5.15 & 5.27 \\
\hline 5.5 & 5.99 & 5.50 & 5.37 & 5.46 & 5.18 & 5.36 & 5.19 & 5.63 & 5.99 \\
\hline 6.5 & 6.25 & 5.70 & 5.63 & 5.80 & 5.36 & 5.71 & 5.68 & 5.90 & 6.08 \\
\hline 7.5 & 6.44 & 6.07 & 5.98 & 5.97 & 5.69 & 5.91 & 5.98 & 6.03 & 6.43 \\
\hline 8.5 & 6.68 & 6.28 & 6.16 & 6.09 & 5.82 & 6.17 & 6.14 & 6.24 & 6.78 \\
\hline 9.5 & 6.97 & 6.64 & 6.42 & 6.55 & 6.16 & 6.44 & 6.49 & 6.49 & 6.96 \\
\hline 10.5 & 6.91 & 6.74 & 6.42 & 6.51 & 6.21 & 6.39 & 6.54 & 6.88 & 7.12 \\
\hline 11.5 & 7.32 & 6.78 & 6.79 & 6.63 & 6.46 & 6.96 & 6.81 & 6.92 & 7.22 \\
\hline 12.5 & 7.48 & 6.99 & 6.75 & 7.01 & 6.36 & 6.77 & 6.86 & 6.88 & 7.18 \\
\hline 13.5 & 7.28 & 6.94 & 6.72 & 6.65 & 6.28 & 6.77 & 6.72 & 6.81 & 7.16 \\
\hline 14.5 & 7.30 & 6.76 & 6.68 & 6.64 & 6.34 & 6.69 & 6.57 & 6.74 & 7.09 \\
\hline 15.5 & 6.82 & 6.52 & 6.34 & 6.41 & 6.05 & 6.30 & 6.26 & 6.46 & 6.72 \\
\hline 16.5 & 6.63 & 6.17 & 5.97 & 6.08 & 5.95 & 6.08 & 6.25 & 6.23 & 6.70 \\
\hline 17.5 & 6.32 & 5.93 & 5.70 & 5.65 & 5.46 & 5.68 & 5.64 & 5.94 & 6.16 \\
\hline 18.5 & 5.99 & 5.68 & 5.57 & 5.40 & 5.16 & 5.47 & 5.47 & 5.67 & 5.93 \\
\hline 19.5 & 5.55 & 5.21 & 5.12 & 5.03 & 4.76 & 5.02 & 5.11 & 5.16 & 5.38 \\
\hline 20.5 & 5.15 & 4.93 & 4.84 & 4.76 & 4.57 & 4.76 & 4.64 & 4.76 & 5.10 \\
\hline 21.5 & 4.68 & 4.48 & 4.23 & 4.35 & 4.11 & 4.29 & 4.34 & 4.49 & 4.77 \\
\hline 22.5 & 4.21 & 3.98 & 3.81 & 3.97 & 3.78 & 3.97 & 4.00 & 4.12 & 4.36 \\
\hline 23.5 & 4.56 & 4.31 & 4.25 & 4.08 & 3.93 & 4.27 & 4.19 & 4.30 & 4.68 \\
\hline Ax. Avg. & 5.87 & 5.51 & 5.38 & 5.39 & 5.13 & 5.40 & 5.39 & 5.53 & 5.83 \\
\hline & & & & & & & & & \\
\hline
\end{tabular}

POWER DENSITY VALUES NORMALIZED TO A CORE POWER OF 11.57 MW AND ASSUME ALL FISSION ENERGY (EXCLUDING GAMMA-HEATING OF EX-CORE COMPONENTS) IS DEPOSITED IN THE FUEL 
Table B- 3. Power Density Data $\left(\mathrm{kW} / \mathrm{cm}^{3}\right)$ in Fuel Plate 3 in Prototypic Core.

\section{Fuel core width: 1.905 inches / Fuel core thickness: 16 mil}

Plate 3, Core Position X1/X5, 0 MWd Burnup, Xenon-Free Conditions

\begin{tabular}{|c|c|c|c|c|c|c|c|c|c|}
\hline $\begin{array}{c}\text { Axial } \\
\text { position } \\
\text { from } \\
\text { top of } \\
\text { fuel } \\
\text { (inches) } \\
\end{array}$ & $\begin{array}{c}0.000 \\
\text { to } \\
0.197 \\
\text { inches }\end{array}$ & $\begin{array}{c}0.197 \\
\text { to } \\
0.394 \\
\text { inches } \\
\end{array}$ & $\begin{array}{c}0.394 \\
\text { to } \\
0.591 \\
\text { inches } \\
\end{array}$ & $\begin{array}{c}0.591 \\
\text { to } \\
0.832 \\
\text { inches }\end{array}$ & $\begin{array}{c}0.832 \\
\text { to } \\
1.073 \\
\text { inches }\end{array}$ & $\begin{array}{c}1.073 \\
\text { to } \\
1.314 \\
\text { inches } \\
\end{array}$ & $\begin{array}{c}1.314 \\
\text { to } \\
1.511 \\
\text { inches }\end{array}$ & $\begin{array}{c}1.511 \\
\text { to } \\
1.708 \\
\text { inches }\end{array}$ & $\begin{array}{c}1.708 \\
\text { to } \\
1.905 \\
\text { inches } \\
\end{array}$ \\
\hline 0.5 & 2.50 & 2.08 & 1.96 & 1.98 & 1.87 & 1.92 & 2.07 & 2.08 & 2.34 \\
\hline 1.5 & 2.25 & 1.95 & 1.84 & 1.82 & 1.73 & 1.86 & 1.82 & 1.93 & 2.18 \\
\hline 2.5 & 2.70 & 2.33 & 2.31 & 2.19 & 2.11 & 2.28 & 2.19 & 2.38 & 2.71 \\
\hline 3.5 & 3.26 & 2.82 & 2.74 & 2.59 & 2.46 & 2.59 & 2.57 & 2.69 & 3.11 \\
\hline 4.5 & 3.71 & 3.17 & 3.11 & 3.05 & 2.94 & 3.07 & 3.06 & 3.15 & 3.74 \\
\hline 5.5 & 4.36 & 3.83 & 3.50 & 3.49 & 3.31 & 3.56 & 3.61 & 3.67 & 4.22 \\
\hline 6.5 & 4.72 & 4.22 & 4.01 & 4.01 & 3.86 & 3.99 & 4.03 & 4.20 & 4.78 \\
\hline 7.5 & 5.46 & 4.66 & 4.45 & 4.41 & 4.24 & 4.43 & 4.52 & 4.65 & 5.32 \\
\hline 8.5 & 5.90 & 5.11 & 4.95 & 5.05 & 4.71 & 4.98 & 5.04 & 5.24 & 5.95 \\
\hline 9.5 & 6.53 & 5.63 & 5.48 & 5.56 & 5.18 & 5.40 & 5.48 & 5.72 & 6.61 \\
\hline 10.5 & 7.11 & 6.08 & 6.00 & 5.91 & 5.60 & 5.90 & 6.02 & 6.21 & 7.08 \\
\hline 11.5 & 7.66 & 6.73 & 6.19 & 6.15 & 6.06 & 6.36 & 6.37 & 6.62 & 7.78 \\
\hline 12.5 & 8.02 & 7.09 & 6.61 & 6.62 & 6.30 & 6.55 & 6.77 & 6.93 & 7.82 \\
\hline 13.5 & 8.20 & 7.16 & 6.78 & 6.65 & 6.32 & 6.74 & 6.77 & 7.03 & 8.08 \\
\hline 14.5 & 8.24 & 7.10 & 6.80 & 6.80 & 6.41 & 6.73 & 6.83 & 7.15 & 8.12 \\
\hline 15.5 & 8.34 & 7.07 & 6.64 & 6.69 & 6.45 & 6.62 & 6.69 & 7.00 & 7.94 \\
\hline 16.5 & 7.98 & 7.08 & 6.52 & 6.44 & 6.34 & 6.58 & 6.66 & 6.88 & 7.70 \\
\hline 17.5 & 7.54 & 6.59 & 6.21 & 6.24 & 5.92 & 6.18 & 6.39 & 6.78 & 7.46 \\
\hline 18.5 & 7.26 & 6.10 & 5.96 & 5.94 & 5.65 & 5.93 & 5.99 & 6.21 & 7.01 \\
\hline 19.5 & 6.59 & 5.80 & 5.64 & 5.61 & 5.23 & 5.61 & 5.61 & 5.80 & 6.49 \\
\hline 20.5 & 6.22 & 5.42 & 5.19 & 5.05 & 4.76 & 5.05 & 5.13 & 5.34 & 6.22 \\
\hline 21.5 & 5.55 & 5.07 & 4.84 & 4.65 & 4.37 & 4.60 & 4.55 & 4.88 & 5.68 \\
\hline 22.5 & 5.15 & 4.43 & 4.17 & 4.13 & 3.91 & 4.10 & 4.33 & 4.40 & 5.16 \\
\hline 23.5 & 5.61 & 4.94 & 4.60 & 4.54 & 4.43 & 4.66 & 4.62 & 4.94 & 5.83 \\
\hline Ax. Avg. & 5.87 & 5.10 & 4.85 & 4.82 & 4.59 & 4.82 & 4.88 & 5.08 & 5.81 \\
\hline
\end{tabular}

POWER DENSITY VALUES NORMALIZED TO A CORE POWER OF 11.57 MW AND ASSUME ALL FISSION ENERGY (EXCLUDING GAMMA-HEATING OF EX-CORE COMPONENTS) IS DEPOSITED IN THE FUEL 
Table B-3. Power Density Data (kW/cc) in Fuel Plate 3 in Prototypic Conditions (continued).

Plate 3, Core Position X1/X5, 3 MWd Burnup, Equilibrium Xenon Conditions

\begin{tabular}{|c|c|c|c|c|c|c|c|c|c|}
\hline $\begin{array}{c}\text { Axial } \\
\text { position } \\
\text { from } \\
\text { top of } \\
\text { fuel } \\
\text { (inches) }\end{array}$ & $\begin{array}{c}0.000 \\
\text { to } \\
0.197 \\
\text { inches }\end{array}$ & $\begin{array}{c}0.197 \\
\text { to } \\
0.394 \\
\text { inches }\end{array}$ & $\begin{array}{c}0.394 \\
\text { to } \\
0.591 \\
\text { inches }\end{array}$ & $\begin{array}{c}0.591 \\
\text { to } \\
0.832 \\
\text { inches }\end{array}$ & $\begin{array}{c}0.832 \\
\text { to } \\
1.073 \\
\text { inches }\end{array}$ & $\begin{array}{c}1.073 \\
\text { to } \\
1.314 \\
\text { inches }\end{array}$ & $\begin{array}{c}1.314 \\
\text { to } \\
1.511 \\
\text { inches }\end{array}$ & $\begin{array}{c}1.511 \\
\text { to } \\
1.708 \\
\text { inches }\end{array}$ & $\begin{array}{c}1.708 \\
\text { to } \\
1.905 \\
\text { inches }\end{array}$ \\
\hline 1.5 & 3.69 & 3.10 & 2.92 & 2.80 & 2.77 & 2.89 & 2.89 & 2.99 & 3.48 \\
\hline 2.5 & 3.82 & 3.39 & 3.11 & 3.11 & 2.95 & 3.01 & 3.13 & 3.33 & 3.76 \\
\hline 3.5 & 4.37 & 3.88 & 3.65 & 3.65 & 3.46 & 3.65 & 3.71 & 3.90 & 4.43 \\
\hline 4.5 & 4.81 & 4.32 & 4.19 & 4.03 & 3.87 & 4.05 & 4.05 & 4.36 & 4.87 \\
\hline 5.5 & 5.45 & 4.70 & 4.49 & 4.57 & 4.28 & 4.42 & 4.53 & 4.82 & 5.41 \\
\hline 6.5 & 5.77 & 5.11 & 4.79 & 4.83 & 4.54 & 4.90 & 5.04 & 5.10 & 5.80 \\
\hline 7.5 & 6.35 & 5.46 & 5.22 & 5.10 & 4.86 & 5.10 & 5.17 & 5.44 & 6.11 \\
\hline 8.5 & 6.58 & 5.70 & 5.51 & 5.46 & 5.10 & 5.43 & 5.31 & 5.67 & 6.31 \\
\hline 9.5 & 6.63 & 5.94 & 5.57 & 5.57 & 5.28 & 5.63 & 5.71 & 5.94 & 6.84 \\
\hline 10.5 & 7.21 & 6.02 & 5.84 & 5.78 & 5.53 & 5.91 & 5.91 & 6.12 & 7.03 \\
\hline 11.5 & 7.26 & 6.25 & 5.92 & 5.99 & 5.59 & 5.83 & 5.97 & 6.24 & 6.95 \\
\hline 12.5 & 7.21 & 6.34 & 5.93 & 5.94 & 5.73 & 5.91 & 5.99 & 6.28 & 7.09 \\
\hline 13.5 & 7.23 & 6.05 & 5.81 & 5.81 & 5.72 & 6.03 & 5.90 & 6.04 & 6.99 \\
\hline 14.5 & 7.07 & 6.00 & 5.65 & 5.70 & 5.42 & 5.84 & 5.71 & 6.01 & 6.88 \\
\hline 15.5 & 6.73 & 5.79 & 5.54 & 5.33 & 5.16 & 5.53 & 5.64 & 5.95 & 6.73 \\
\hline 16.5 & 6.38 & 5.60 & 5.38 & 5.28 & 5.03 & 5.22 & 5.24 & 5.60 & 6.32 \\
\hline 17.5 & 5.96 & 5.15 & 4.94 & 4.94 & 4.68 & 4.91 & 4.92 & 5.15 & 5.89 \\
\hline 18.5 & 5.57 & 4.87 & 4.60 & 4.63 & 4.35 & 4.55 & 4.65 & 4.77 & 5.57 \\
\hline 19.5 & 5.08 & 4.51 & 4.38 & 4.30 & 4.18 & 4.25 & 4.27 & 4.49 & 5.04 \\
\hline 20.5 & 4.66 & 3.99 & 3.95 & 3.81 & 3.71 & 3.90 & 4.05 & 4.12 & 4.75 \\
\hline 21.5 & 4.24 & 3.62 & 3.43 & 3.52 & 3.23 & 3.36 & 3.44 & 3.68 & 4.14 \\
\hline 22.5 & 3.87 & 3.38 & 3.14 & 3.09 & 2.97 & 3.17 & 3.20 & 3.40 & 3.97 \\
\hline 23.5 & 4.55 & 3.69 & 3.55 & 3.47 & 3.22 & 3.52 & 3.50 & 3.74 & 4.36 \\
\hline Ax. Avg. & 5.58 & 4.83 & 4.59 & 4.55 & 4.34 & 4.57 & 4.61 & 4.83 & 5.50 \\
\hline & & & & & & & & 2.65 & \\
\hline
\end{tabular}

POWER DENSITY VALUES NORMALIZED TO A CORE POWER OF 11.57 MW AND ASSUME ALL FISSION ENERGY (EXCLUDING GAMMA-HEATING OF EX-CORE COMPONENTS) IS DEPOSITED IN THE FUEL 
Table B-3. Power Density Data (kW/cc) in Fuel Plate 3 in Prototypic Conditions (continued).

Plate 3, Core Position X3/X7, 80 MWd Burnup, Equilibrium Xenon Conditions

\begin{tabular}{|c|c|c|c|c|c|c|c|c|c|}
\hline $\begin{array}{c}\text { Axial } \\
\text { position } \\
\text { from } \\
\text { top of } \\
\text { fuel } \\
\text { (inches) }\end{array}$ & $\begin{array}{c}0.000 \\
\text { to } \\
0.197 \\
\text { inches }\end{array}$ & $\begin{array}{c}0.197 \\
\text { to } \\
0.394 \\
\text { inches }\end{array}$ & $\begin{array}{c}0.394 \\
\text { to } \\
0.591 \\
\text { inches }\end{array}$ & $\begin{array}{c}0.591 \\
\text { to } \\
0.832 \\
\text { inches }\end{array}$ & $\begin{array}{c}0.832 \\
\text { to } \\
1.073 \\
\text { inches }\end{array}$ & $\begin{array}{c}1.073 \\
\text { to } \\
1.314 \\
\text { inches }\end{array}$ & $\begin{array}{c}1.314 \\
\text { to } \\
1.511 \\
\text { inches }\end{array}$ & $\begin{array}{c}1.511 \\
\text { to } \\
1.708 \\
\text { inches }\end{array}$ & $\begin{array}{c}1.708 \\
\text { to } \\
1.905 \\
\text { inches }\end{array}$ \\
\hline 1.5 & 3.34 & 2.93 & 2.79 & 2.70 & 2.64 & 2.83 & 2.82 & 2.97 & 3.56 \\
\hline 2.5 & 3.64 & 3.17 & 3.06 & 3.06 & 2.94 & 3.03 & 3.11 & 3.19 & 3.78 \\
\hline 3.5 & 4.13 & 3.70 & 3.57 & 3.43 & 3.31 & 3.50 & 3.60 & 3.68 & 4.24 \\
\hline 4.5 & 4.77 & 4.16 & 3.97 & 3.95 & 3.81 & 3.99 & 3.84 & 4.14 & 4.63 \\
\hline 5.5 & 5.05 & 4.50 & 4.40 & 4.31 & 4.22 & 4.43 & 4.40 & 4.64 & 5.22 \\
\hline 6.5 & 5.50 & 4.87 & 4.65 & 4.57 & 4.37 & 4.69 & 4.64 & 4.73 & 5.51 \\
\hline 7.5 & 5.72 & 5.21 & 4.88 & 4.78 & 4.48 & 4.80 & 4.78 & 5.19 & 5.79 \\
\hline 8.5 & 5.94 & 5.33 & 5.22 & 5.01 & 4.73 & 5.05 & 4.94 & 5.32 & 6.10 \\
\hline 9.5 & 6.46 & 5.54 & 5.28 & 5.24 & 5.10 & 5.37 & 5.23 & 5.57 & 6.47 \\
\hline 10.5 & 6.42 & 5.82 & 5.61 & 5.56 & 5.25 & 5.39 & 5.45 & 5.85 & 6.67 \\
\hline 11.5 & 6.51 & 5.73 & 5.59 & 5.60 & 5.28 & 5.61 & 5.59 & 5.96 & 6.58 \\
\hline 12.5 & 6.72 & 5.73 & 5.59 & 5.68 & 5.33 & 5.70 & 5.69 & 5.92 & 6.75 \\
\hline 13.5 & 6.69 & 5.92 & 5.69 & 5.47 & 5.33 & 5.56 & 5.74 & 5.90 & 6.66 \\
\hline 14.5 & 6.53 & 5.72 & 5.49 & 5.42 & 5.24 & 5.42 & 5.58 & 5.64 & 6.69 \\
\hline 15.5 & 6.14 & 5.46 & 5.21 & 5.34 & 5.01 & 5.27 & 5.34 & 5.57 & 6.36 \\
\hline 16.5 & 5.97 & 5.38 & 5.10 & 4.98 & 4.72 & 4.97 & 5.03 & 5.29 & 5.94 \\
\hline 17.5 & 5.45 & 4.97 & 4.80 & 4.72 & 4.27 & 4.66 & 4.74 & 5.03 & 5.47 \\
\hline 18.5 & 5.17 & 4.51 & 4.27 & 4.30 & 4.13 & 4.36 & 4.39 & 4.61 & 5.25 \\
\hline 19.5 & 4.82 & 4.19 & 4.09 & 4.01 & 3.97 & 4.01 & 4.05 & 4.27 & 4.86 \\
\hline 20.5 & 4.38 & 3.78 & 3.83 & 3.72 & 3.59 & 3.68 & 3.76 & 3.89 & 4.43 \\
\hline 21.5 & 3.84 & 3.48 & 3.38 & 3.33 & 3.14 & 3.28 & 3.31 & 3.52 & 4.01 \\
\hline 22.5 & 3.52 & 3.09 & 3.04 & 3.01 & 2.85 & 3.00 & 3.07 & 3.30 & 3.66 \\
\hline 23.5 & 4.04 & 3.50 & 3.45 & 3.48 & 3.13 & 3.38 & 3.32 & 3.47 & 4.20 \\
\hline Ax. Avg. & 5.16 & 4.56 & 4.40 & 4.34 & 4.14 & 4.36 & 4.37 & 4.60 & 5.25 \\
\hline & & & & & & & & 2.53 & 2.79 \\
\hline
\end{tabular}

POWER DENSITY VALUES NORMALIZED TO A CORE POWER OF 11.57 MW AND ASSUME ALL FISSION ENERGY (EXCLUDING GAMMA-HEATING OF EX-CORE COMPONENTS) IS DEPOSITED IN THE FUEL 
Table B-3. Power Density Data (kW/cc) in Fuel Plate 3 in Prototypic Conditions (continued).

Plate 3, Core Position X2/X6, 99 MWd Burnup, Equilibrium Xenon Conditions

\begin{tabular}{|c|c|c|c|c|c|c|c|c|c|}
\hline $\begin{array}{c}\text { Axial } \\
\text { position } \\
\text { from } \\
\text { top of } \\
\text { fuel } \\
\text { (inches) }\end{array}$ & $\begin{array}{c}0.000 \\
\text { to } \\
0.197 \\
\text { inches }\end{array}$ & $\begin{array}{c}0.197 \\
\text { to } \\
0.394 \\
\text { inches }\end{array}$ & $\begin{array}{c}0.394 \\
\text { to } \\
0.591 \\
\text { inches }\end{array}$ & $\begin{array}{c}0.591 \\
\text { to } \\
0.832 \\
\text { inches }\end{array}$ & $\begin{array}{c}0.832 \\
\text { to } \\
1.073 \\
\text { inches }\end{array}$ & $\begin{array}{c}1.073 \\
\text { to } \\
1.314 \\
\text { inches }\end{array}$ & $\begin{array}{c}1.314 \\
\text { to } \\
1.511 \\
\text { inches }\end{array}$ & $\begin{array}{c}1.511 \\
\text { to } \\
1.708 \\
\text { inches }\end{array}$ & $\begin{array}{c}1.708 \\
\text { to } \\
1.905 \\
\text { inches }\end{array}$ \\
\hline 1.5 & 3.30 & 2.92 & 2.74 & 2.73 & 2.57 & 2.75 & 2.82 & 2.77 & 3.36 \\
\hline 2.5 & 3.61 & 3.24 & 3.07 & 3.10 & 2.91 & 3.03 & 3.04 & 3.25 & 3.60 \\
\hline 3.5 & 4.13 & 3.62 & 3.69 & 3.51 & 3.43 & 3.54 & 3.50 & 3.73 & 4.19 \\
\hline 4.5 & 4.67 & 4.11 & 4.01 & 4.02 & 3.75 & 3.88 & 4.04 & 4.19 & 4.70 \\
\hline 5.5 & 5.11 & 4.56 & 4.30 & 4.29 & 4.07 & 4.34 & 4.35 & 4.53 & 5.02 \\
\hline 6.5 & 5.35 & 4.78 & 4.61 & 4.60 & 4.30 & 4.62 & 4.60 & 4.83 & 5.36 \\
\hline 7.5 & 5.67 & 4.87 & 4.90 & 4.73 & 4.56 & 4.83 & 4.84 & 4.91 & 5.62 \\
\hline 8.5 & 5.92 & 5.35 & 5.10 & 5.08 & 4.71 & 4.99 & 5.01 & 5.08 & 5.73 \\
\hline 9.5 & 6.30 & 5.61 & 5.22 & 5.33 & 5.11 & 5.30 & 5.31 & 5.54 & 6.20 \\
\hline 10.5 & 6.33 & 5.78 & 5.47 & 5.55 & 5.22 & 5.50 & 5.46 & 5.60 & 6.49 \\
\hline 11.5 & 6.50 & 5.76 & 5.64 & 5.46 & 5.40 & 5.53 & 5.65 & 5.83 & 6.69 \\
\hline 12.5 & 6.63 & 5.85 & 5.55 & 5.68 & 5.43 & 5.66 & 5.75 & 5.86 & 6.64 \\
\hline 13.5 & 6.47 & 5.80 & 5.51 & 5.61 & 5.29 & 5.49 & 5.49 & 5.87 & 6.51 \\
\hline 14.5 & 6.36 & 5.61 & 5.39 & 5.39 & 5.12 & 5.36 & 5.40 & 5.65 & 6.38 \\
\hline 15.5 & 6.10 & 5.41 & 5.22 & 5.00 & 4.81 & 5.20 & 5.31 & 5.46 & 6.12 \\
\hline 16.5 & 5.87 & 5.15 & 4.95 & 4.93 & 4.72 & 4.96 & 4.95 & 5.16 & 5.84 \\
\hline 17.5 & 5.32 & 4.84 & 4.58 & 4.53 & 4.40 & 4.54 & 4.62 & 4.90 & 5.39 \\
\hline 18.5 & 5.19 & 4.53 & 4.37 & 4.32 & 4.05 & 4.33 & 4.24 & 4.48 & 5.18 \\
\hline 19.5 & 4.84 & 4.24 & 4.06 & 3.95 & 3.78 & 4.06 & 3.96 & 4.27 & 4.59 \\
\hline 20.5 & 4.41 & 3.88 & 3.70 & 3.65 & 3.46 & 3.72 & 3.73 & 3.90 & 4.42 \\
\hline 21.5 & 3.87 & 3.47 & 3.36 & 3.35 & 3.20 & 3.26 & 3.26 & 3.47 & 3.98 \\
\hline 22.5 & 3.55 & 3.07 & 2.99 & 2.91 & 2.81 & 2.92 & 2.96 & 3.19 & 3.57 \\
\hline 23.5 & 4.02 & 3.45 & 3.32 & 3.32 & 3.28 & 3.23 & 3.36 & 3.53 & 4.01 \\
\hline Ax. Avg. & 5.11 & 4.52 & 4.35 & 4.32 & 4.12 & 4.32 & 4.34 & 4.53 & 5.12 \\
\hline & & & & & & & & 2.53 & \\
\hline
\end{tabular}

POWER DENSITY VALUES NORMALIZED TO A CORE POWER OF 11.57 MW AND ASSUME ALL FISSION ENERGY (EXCLUDING GAMMA-HEATING OF EX-CORE COMPONENTS) IS DEPOSITED IN THE FUEL 
Table B-3. Power Density Data (kW/cc) in Fuel Plate 3 in Prototypic Conditions (continued).

Plate 3, Core Position X4/X8, 173 MWd Burnup, Equilibrium Xenon Conditions

\begin{tabular}{|c|c|c|c|c|c|c|c|c|c|}
\hline $\begin{array}{l}\text { Axial } \\
\text { position } \\
\text { from } \\
\text { top of } \\
\text { fuel } \\
\text { (inches) }\end{array}$ & $\begin{array}{c}0.000 \\
\text { to } \\
0.197 \\
\text { inches }\end{array}$ & $\begin{array}{c}0.197 \\
\text { to } \\
0.394 \\
\text { inches }\end{array}$ & $\begin{array}{c}0.394 \\
\text { to } \\
0.591 \\
\text { inches }\end{array}$ & $\begin{array}{c}0.591 \\
\text { to } \\
0.832 \\
\text { inches }\end{array}$ & $\begin{array}{c}0.832 \\
\text { to } \\
1.073 \\
\text { inches }\end{array}$ & $\begin{array}{c}1.073 \\
\text { to } \\
1.314 \\
\text { inches }\end{array}$ & $\begin{array}{c}1.314 \\
\text { to } \\
1.511 \\
\text { inches }\end{array}$ & $\begin{array}{c}1.511 \\
\text { to } \\
1.708 \\
\text { inches }\end{array}$ & $\begin{array}{c}1.708 \\
\text { to } \\
1.905 \\
\text { inches }\end{array}$ \\
\hline 0.5 & 3.20 & 2.76 & 2.69 & 2.77 & 2.65 & 2.75 & 2.75 & 2.86 & 3.32 \\
\hline 1.5 & 3.21 & 2.63 & 2.57 & 2.53 & 2.44 & 2.57 & 2.60 & 2.73 & 3.20 \\
\hline 2.5 & 3.44 & 3.10 & 2.89 & 2.96 & 2.77 & 2.88 & 2.98 & 3.02 & 3.44 \\
\hline 3.5 & 3.98 & 3.52 & 3.48 & 3.39 & 3.20 & 3.38 & 3.41 & 3.56 & 4.00 \\
\hline 4.5 & 4.31 & 3.86 & 3.72 & 3.77 & 3.63 & 3.80 & 3.75 & 4.01 & 4.30 \\
\hline 5.5 & 4.83 & 4.37 & 4.18 & 4.08 & 3.93 & 4.05 & 4.13 & 4.34 & 4.78 \\
\hline 6.5 & 5.03 & 4.60 & 4.41 & 4.38 & 4.21 & 4.43 & 4.40 & 4.56 & 4.99 \\
\hline 7.5 & 5.29 & 4.82 & 4.62 & 4.48 & 4.41 & 4.68 & 4.60 & 4.74 & 5.39 \\
\hline 8.5 & 5.50 & 4.86 & 4.82 & 4.79 & 4.56 & 4.79 & 4.87 & 5.00 & 5.48 \\
\hline 9.5 & 5.79 & 5.16 & 5.03 & 5.06 & 4.73 & 4.93 & 5.06 & 5.16 & 5.90 \\
\hline 10.5 & 6.03 & 5.27 & 5.06 & 5.05 & 4.93 & 4.96 & 5.17 & 5.34 & 5.90 \\
\hline 11.5 & 5.90 & 5.45 & 5.13 & 5.26 & 4.92 & 5.24 & 5.26 & 5.53 & 6.08 \\
\hline 12.5 & 6.17 & 5.43 & 5.38 & 5.35 & 5.04 & 5.28 & 5.30 & 5.60 & 5.97 \\
\hline 13.5 & 6.03 & 5.50 & 5.28 & 5.11 & 4.96 & 5.17 & 5.31 & 5.29 & 6.00 \\
\hline 14.5 & 5.95 & 5.26 & 5.11 & 5.26 & 5.04 & 5.14 & 5.17 & 5.40 & 5.89 \\
\hline 15.5 & 5.71 & 5.20 & 4.89 & 4.99 & 4.71 & 4.97 & 4.91 & 5.09 & 5.63 \\
\hline 16.5 & 5.46 & 4.83 & 4.62 & 4.65 & 4.48 & 4.66 & 4.74 & 4.93 & 5.40 \\
\hline 17.5 & 5.17 & 4.73 & 4.47 & 4.35 & 4.03 & 4.46 & 4.55 & 4.74 & 5.09 \\
\hline 18.5 & 4.89 & 4.25 & 4.29 & 4.17 & 4.03 & 4.19 & 4.26 & 4.47 & 4.87 \\
\hline 19.5 & 4.48 & 4.02 & 3.85 & 3.88 & 3.67 & 3.90 & 3.95 & 4.04 & 4.42 \\
\hline 20.5 & 4.27 & 3.74 & 3.61 & 3.57 & 3.52 & 3.60 & 3.68 & 3.76 & 4.09 \\
\hline 21.5 & 3.79 & 3.38 & 3.23 & 3.30 & 3.11 & 3.25 & 3.23 & 3.43 & 3.87 \\
\hline 22.5 & 3.45 & 3.01 & 2.91 & 2.85 & 2.72 & 2.89 & 2.91 & 2.98 & 3.38 \\
\hline 23.5 & 3.81 & 3.38 & 3.34 & 3.19 & 3.12 & 3.26 & 3.19 & 3.35 & 3.89 \\
\hline Ax. Avg. & 4.82 & 4.30 & 4.15 & 4.13 & 3.95 & 4.14 & 4.17 & 4.33 & 4.80 \\
\hline
\end{tabular}

POWER DENSITY VALUES NORMALIZED TO A CORE POWER OF 11.57 MW AND ASSUME ALL FISSION ENERGY (EXCLUDING GAMMA-HEATING OF EX-CORE COMPONENTS) IS DEPOSITED IN THE FUEL 
Table B- 4. Power Density Data $\left(\mathrm{kW} / \mathrm{cm}^{3}\right)$ in Fuel Plate 4 in Prototypic Core.

\section{Fuel core width: 2.012 inches / Fuel core thickness: 20 mil}

Plate 4, Core Position X1/X5, 0 MWd Burnup, Xenon-Free Conditions

\begin{tabular}{|c|c|c|c|c|c|c|c|c|c|}
\hline $\begin{array}{c}\text { Axial } \\
\text { position } \\
\text { from } \\
\text { top of } \\
\text { fuel } \\
\text { (inches) } \\
\end{array}$ & $\begin{array}{c}0.000 \\
\text { to } \\
0.197 \\
\text { inches }\end{array}$ & $\begin{array}{c}0.197 \\
\text { to } \\
0.394 \\
\text { inches } \\
\end{array}$ & $\begin{array}{c}0.394 \\
\text { to } \\
0.591 \\
\text { inches }\end{array}$ & $\begin{array}{c}0.591 \\
\text { to } \\
0.868 \\
\text { inches }\end{array}$ & $\begin{array}{c}0.868 \\
\text { to } \\
1.145 \\
\text { inches }\end{array}$ & $\begin{array}{c}1.145 \\
\text { to } \\
1.422 \\
\text { inches }\end{array}$ & $\begin{array}{c}1.422 \\
\text { to } \\
1.619 \\
\text { inches }\end{array}$ & $\begin{array}{c}1.619 \\
\text { to } \\
1.815 \\
\text { inches }\end{array}$ & $\begin{array}{c}1.815 \\
\text { to } \\
2.012 \\
\text { inches } \\
\end{array}$ \\
\hline 0.5 & 1.94 & 1.70 & 1.52 & 1.47 & 1.44 & 1.47 & 1.53 & 1.60 & 1.90 \\
\hline 1.5 & 1.86 & 1.50 & 1.42 & 1.44 & 1.31 & 1.38 & 1.40 & 1.50 & 1.77 \\
\hline 2.5 & 2.14 & 1.80 & 1.69 & 1.72 & 1.58 & 1.71 & 1.68 & 1.84 & 2.17 \\
\hline 3.5 & 2.58 & 2.17 & 2.05 & 1.99 & 1.89 & 1.98 & 2.02 & 2.11 & 2.60 \\
\hline 4.5 & 2.99 & 2.46 & 2.37 & 2.28 & 2.25 & 2.30 & 2.31 & 2.49 & 2.84 \\
\hline 5.5 & 3.48 & 2.93 & 2.75 & 2.75 & 2.54 & 2.68 & 2.81 & 2.86 & 3.46 \\
\hline 6.5 & 3.87 & 3.25 & 3.13 & 3.03 & 2.85 & 3.01 & 3.08 & 3.32 & 3.76 \\
\hline 7.5 & 4.38 & 3.71 & 3.49 & 3.34 & 3.21 & 3.41 & 3.50 & 3.62 & 4.34 \\
\hline 8.5 & 4.87 & 4.03 & 3.84 & 3.89 & 3.60 & 3.70 & 3.89 & 4.15 & 4.78 \\
\hline 9.5 & 5.39 & 4.47 & 4.29 & 4.16 & 4.01 & 4.19 & 4.25 & 4.54 & 5.28 \\
\hline 10.5 & 5.64 & 4.89 & 4.58 & 4.51 & 4.34 & 4.49 & 4.55 & 4.94 & 5.73 \\
\hline 11.5 & 6.26 & 5.20 & 4.92 & 4.77 & 4.52 & 4.78 & 4.87 & 5.21 & 6.30 \\
\hline 12.5 & 6.47 & 5.42 & 5.03 & 5.00 & 4.85 & 4.97 & 5.17 & 5.30 & 6.21 \\
\hline 13.5 & 6.55 & 5.51 & 5.14 & 5.15 & 4.90 & 5.11 & 5.16 & 5.45 & 6.63 \\
\hline 14.5 & 6.68 & 5.66 & 5.20 & 5.08 & 4.90 & 5.17 & 5.28 & 5.53 & 6.66 \\
\hline 15.5 & 6.70 & 5.48 & 5.20 & 5.04 & 4.98 & 5.13 & 5.30 & 5.47 & 6.58 \\
\hline 16.5 & 6.43 & 5.44 & 5.21 & 5.09 & 4.77 & 5.00 & 5.19 & 5.35 & 6.42 \\
\hline 17.5 & 6.20 & 5.22 & 4.98 & 4.74 & 4.56 & 4.82 & 4.96 & 5.19 & 6.04 \\
\hline 18.5 & 5.95 & 5.00 & 4.74 & 4.63 & 4.38 & 4.57 & 4.60 & 4.81 & 5.91 \\
\hline 19.5 & 5.35 & 4.59 & 4.46 & 4.37 & 4.21 & 4.26 & 4.32 & 4.51 & 5.46 \\
\hline 20.5 & 5.03 & 4.22 & 3.94 & 4.01 & 3.73 & 3.96 & 3.99 & 4.27 & 4.99 \\
\hline 21.5 & 4.51 & 3.93 & 3.55 & 3.58 & 3.41 & 3.50 & 3.67 & 3.69 & 4.48 \\
\hline 22.5 & 4.20 & 3.45 & 3.21 & 3.22 & 3.01 & 3.09 & 3.20 & 3.39 & 3.97 \\
\hline 23.5 & 4.63 & 3.97 & 3.74 & 3.72 & 3.47 & 3.69 & 3.71 & 3.96 & 4.77 \\
\hline Ax. Avg. & 4.75 & 4.00 & 3.77 & 3.71 & 3.53 & 3.68 & 3.77 & 3.96 & 4.71 \\
\hline
\end{tabular}

POWER DENSITY VALUES NORMALIZED TO A CORE POWER OF 11.57 MW AND ASSUME ALL FISSION ENERGY (EXCLUDING GAMMA-HEATING OF EX-CORE COMPONENTS) IS DEPOSITED IN THE FUEL 
Table B-4. Power Density Data (kW/cc) in Fuel Plate 4 in Prototypic Conditions (continued).

Plate 4, Core Position X1/X5, 3 MWd Burnup, Equilibrium Xenon Conditions

\begin{tabular}{|c|c|c|c|c|c|c|c|c|c|}
\hline $\begin{array}{l}\text { Axial } \\
\text { position } \\
\text { from } \\
\text { top of } \\
\text { fuel } \\
\text { (inches) }\end{array}$ & $\begin{array}{c}0.000 \\
\text { to } \\
0.197 \\
\text { inches }\end{array}$ & $\begin{array}{c}0.197 \\
\text { to } \\
0.394 \\
\text { inches }\end{array}$ & $\begin{array}{c}0.394 \\
\text { to } \\
0.591 \\
\text { inches }\end{array}$ & $\begin{array}{c}0.591 \\
\text { to } \\
0.868 \\
\text { inches }\end{array}$ & $\begin{array}{c}0.868 \\
\text { to } \\
1.145 \\
\text { inches }\end{array}$ & $\begin{array}{c}1.145 \\
\text { to } \\
1.422 \\
\text { inches }\end{array}$ & $\begin{array}{c}1.422 \\
\text { to } \\
1.619 \\
\text { inches }\end{array}$ & $\begin{array}{c}1.619 \\
\text { to } \\
1.815 \\
\text { inches }\end{array}$ & $\begin{array}{c}1.815 \\
\text { to } \\
2.012 \\
\text { inches }\end{array}$ \\
\hline 0.5 & 2.94 & 2.42 & 2.29 & 2.24 & 2.17 & 2.23 & 2.29 & 2.42 & 2.88 \\
\hline 1.5 & 2.62 & 2.22 & 2.08 & 2.03 & 1.94 & 1.99 & 2.07 & 2.23 & 2.71 \\
\hline 2.5 & 3.16 & 2.53 & 2.45 & 2.40 & 2.33 & 2.38 & 2.40 & 2.60 & 3.04 \\
\hline 3.5 & 3.55 & 3.05 & 2.89 & 2.81 & 2.69 & 2.71 & 2.73 & 3.13 & 3.51 \\
\hline 4.5 & 4.02 & 3.37 & 3.06 & 3.17 & 2.92 & 3.13 & 3.18 & 3.33 & 4.01 \\
\hline 5.5 & 4.38 & 3.69 & 3.41 & 3.36 & 3.26 & 3.45 & 3.52 & 3.72 & 4.37 \\
\hline 6.5 & 4.80 & 3.97 & 3.73 & 3.66 & 3.51 & 3.75 & 3.75 & 3.98 & 4.71 \\
\hline 7.5 & 5.10 & 4.28 & 4.08 & 3.85 & 3.66 & 3.89 & 3.97 & 4.12 & 5.04 \\
\hline 8.5 & 5.27 & 4.48 & 4.11 & 4.08 & 3.84 & 4.06 & 4.21 & 4.50 & 5.26 \\
\hline 9.5 & 5.48 & 4.72 & 4.29 & 4.21 & 4.10 & 4.31 & 4.34 & 4.62 & 5.52 \\
\hline 10.5 & 5.87 & 4.83 & 4.47 & 4.27 & 4.19 & 4.49 & 4.44 & 4.78 & 5.63 \\
\hline 11.5 & 5.90 & 4.84 & 4.64 & 4.50 & 4.24 & 4.40 & 4.38 & 4.85 & 5.75 \\
\hline 12.5 & 5.96 & 4.96 & 4.41 & 4.49 & 4.31 & 4.31 & 4.53 & 4.85 & 5.74 \\
\hline 13.5 & 5.95 & 4.95 & 4.43 & 4.46 & 4.23 & 4.44 & 4.53 & 4.86 & 5.70 \\
\hline 14.5 & 5.66 & 4.69 & 4.40 & 4.34 & 4.15 & 4.49 & 4.41 & 4.67 & 5.66 \\
\hline 15.5 & 5.44 & 4.53 & 4.28 & 4.15 & 4.01 & 4.18 & 4.29 & 4.64 & 5.50 \\
\hline 16.5 & 5.11 & 4.40 & 4.07 & 4.08 & 3.87 & 3.97 & 4.03 & 4.23 & 5.10 \\
\hline 17.5 & 4.89 & 4.06 & 3.91 & 3.79 & 3.66 & 3.73 & 3.73 & 4.15 & 4.84 \\
\hline 18.5 & 4.51 & 3.80 & 3.64 & 3.59 & 3.37 & 3.54 & 3.63 & 3.78 & 4.49 \\
\hline 19.5 & 4.01 & 3.50 & 3.40 & 3.25 & 3.19 & 3.26 & 3.29 & 3.46 & 4.07 \\
\hline 20.5 & 3.80 & 3.17 & 3.08 & 2.99 & 2.79 & 2.93 & 3.06 & 3.17 & 3.83 \\
\hline 21.5 & 3.40 & 2.89 & 2.68 & 2.63 & 2.53 & 2.63 & 2.71 & 2.88 & 3.49 \\
\hline 22.5 & 3.06 & 2.55 & 2.33 & 2.31 & 2.24 & 2.29 & 2.36 & 2.57 & 3.17 \\
\hline 23.5 & 3.71 & 2.93 & 2.76 & 2.74 & 2.57 & 2.68 & 2.71 & 2.90 & 3.59 \\
\hline Ax. Avg. & 4.52 & 3.79 & 3.54 & 3.47 & 3.33 & 3.47 & 3.52 & 3.77 & 4.48 \\
\hline
\end{tabular}

POWER DENSITY VALUES NORMALIZED TO A CORE POWER OF 11.57 MW AND ASSUME ALL FISSION ENERGY (EXCLUDING GAMMA-HEATING OF EX-CORE COMPONENTS) IS DEPOSITED IN THE FUEL 
Table B-4. Power Density Data (kW/cc) in Fuel Plate 4 in Prototypic Conditions (continued).

Plate 4, Core Position X3/X7, 80 MWd Burnup, Equilibrium Xenon Conditions

\begin{tabular}{|c|c|c|c|c|c|c|c|c|c|}
\hline $\begin{array}{l}\text { Axial } \\
\text { position } \\
\text { from } \\
\text { top of } \\
\text { fuel } \\
\text { (inches) }\end{array}$ & $\begin{array}{c}0.000 \\
\text { to } \\
0.197 \\
\text { inches }\end{array}$ & $\begin{array}{c}0.197 \\
\text { to } \\
0.394 \\
\text { inches }\end{array}$ & $\begin{array}{c}0.394 \\
\text { to } \\
0.591 \\
\text { inches }\end{array}$ & $\begin{array}{c}0.591 \\
\text { to } \\
0.868 \\
\text { inches }\end{array}$ & $\begin{array}{c}0.868 \\
\text { to } \\
1.145 \\
\text { inches }\end{array}$ & $\begin{array}{c}1.145 \\
\text { to } \\
1.422 \\
\text { inches }\end{array}$ & $\begin{array}{c}1.422 \\
\text { to } \\
1.619 \\
\text { inches }\end{array}$ & $\begin{array}{c}1.619 \\
\text { to } \\
1.815 \\
\text { inches }\end{array}$ & $\begin{array}{c}1.815 \\
\text { to } \\
2.012 \\
\text { inches }\end{array}$ \\
\hline 0.5 & 2.81 & 2.33 & 2.21 & 2.20 & 2.12 & 2.22 & 2.24 & 2.32 & 2.91 \\
\hline 1.5 & 2.60 & 2.18 & 2.00 & 1.98 & 1.89 & 1.98 & 2.05 & 2.13 & 2.61 \\
\hline 2.5 & 2.91 & 2.54 & 2.36 & 2.33 & 2.25 & 2.32 & 2.37 & 2.47 & 3.01 \\
\hline 3.5 & 3.46 & 2.98 & 2.64 & 2.73 & 2.53 & 2.78 & 2.83 & 3.02 & 3.42 \\
\hline 4.5 & 3.95 & 3.22 & 3.11 & 3.05 & 2.89 & 3.08 & 3.07 & 3.24 & 3.85 \\
\hline 5.5 & 4.27 & 3.64 & 3.34 & 3.29 & 3.17 & 3.34 & 3.42 & 3.69 & 4.23 \\
\hline 6.5 & 4.49 & 3.95 & 3.53 & 3.56 & 3.42 & 3.56 & 3.61 & 3.82 & 4.52 \\
\hline 7.5 & 4.72 & 4.12 & 3.91 & 3.83 & 3.52 & 3.79 & 3.84 & 4.00 & 4.77 \\
\hline 8.5 & 4.98 & 4.41 & 3.97 & 4.00 & 3.91 & 3.98 & 4.10 & 4.34 & 5.07 \\
\hline 9.5 & 5.31 & 4.40 & 4.19 & 4.00 & 3.91 & 4.15 & 4.18 & 4.39 & 5.22 \\
\hline 10.5 & 5.37 & 4.56 & 4.37 & 4.31 & 4.05 & 4.12 & 4.43 & 4.64 & 5.54 \\
\hline 11.5 & 5.52 & 4.66 & 4.40 & 4.36 & 4.12 & 4.37 & 4.34 & 4.65 & 5.51 \\
\hline 12.5 & 5.54 & 4.63 & 4.34 & 4.20 & 4.16 & 4.30 & 4.46 & 4.72 & 5.76 \\
\hline 13.5 & 5.67 & 4.84 & 4.48 & 4.34 & 4.12 & 4.26 & 4.40 & 4.64 & 5.48 \\
\hline 14.5 & 5.33 & 4.49 & 4.17 & 4.13 & 3.99 & 4.15 & 4.24 & 4.56 & 5.32 \\
\hline 15.5 & 5.16 & 4.29 & 4.23 & 4.14 & 3.83 & 4.00 & 4.18 & 4.48 & 5.26 \\
\hline 16.5 & 4.93 & 4.16 & 3.98 & 3.98 & 3.80 & 3.84 & 4.00 & 4.27 & 4.93 \\
\hline 17.5 & 4.67 & 3.92 & 3.70 & 3.65 & 3.49 & 3.68 & 3.78 & 3.94 & 4.57 \\
\hline 18.5 & 4.38 & 3.65 & 3.43 & 3.42 & 3.24 & 3.39 & 3.34 & 3.63 & 4.30 \\
\hline 19.5 & 3.98 & 3.43 & 3.21 & 3.15 & 3.14 & 3.20 & 3.18 & 3.36 & 3.87 \\
\hline 20.5 & 3.69 & 3.09 & 2.94 & 2.88 & 2.76 & 2.77 & 2.86 & 2.99 & 3.72 \\
\hline 21.5 & 3.28 & 2.77 & 2.58 & 2.63 & 2.48 & 2.51 & 2.74 & 2.79 & 3.29 \\
\hline 22.5 & 2.92 & 2.42 & 2.32 & 2.27 & 2.14 & 2.20 & 2.37 & 2.61 & 3.01 \\
\hline 23.5 & 3.38 & 2.75 & 2.66 & 2.71 & 2.47 & 2.62 & 2.69 & 2.97 & 3.39 \\
\hline Ax. Avg. & 4.31 & 3.64 & 3.42 & 3.38 & 3.22 & 3.36 & 3.45 & 3.65 & 4.31 \\
\hline
\end{tabular}

POWER DENSITY VALUES NORMALIZED TO A CORE POWER OF 11.57 MW AND ASSUME ALL FISSION ENERGY (EXCLUDING GAMMA-HEATING OF EX-CORE COMPONENTS) IS DEPOSITED IN THE FUEL 
Table B-4. Power Density Data (kW/cc) in Fuel Plate 4 in Prototypic Conditions (continued).

Plate 4, Core Position X2/X6, 99 MWd Burnup, Equilibrium Xenon Conditions

\begin{tabular}{|c|c|c|c|c|c|c|c|c|c|}
\hline $\begin{array}{c}\text { Axial } \\
\text { position } \\
\text { from } \\
\text { top of } \\
\text { fuel } \\
\text { (inches) }\end{array}$ & $\begin{array}{c}0.000 \\
\text { to } \\
0.197 \\
\text { inches }\end{array}$ & $\begin{array}{c}0.197 \\
\text { to } \\
0.394 \\
\text { inches }\end{array}$ & $\begin{array}{c}0.394 \\
\text { to } \\
0.591 \\
\text { inches }\end{array}$ & $\begin{array}{c}0.591 \\
\text { to } \\
0.868 \\
\text { inches }\end{array}$ & $\begin{array}{c}0.868 \\
\text { to } \\
1.145 \\
\text { inches }\end{array}$ & $\begin{array}{c}1.145 \\
\text { to } \\
1.422 \\
\text { inches }\end{array}$ & $\begin{array}{c}1.422 \\
\text { to } \\
1.619 \\
\text { inches }\end{array}$ & $\begin{array}{c}1.619 \\
\text { to } \\
1.815 \\
\text { inches }\end{array}$ & $\begin{array}{c}1.815 \\
\text { to } \\
\text { inches }\end{array}$ \\
\hline 1.5 & 2.75 & 2.32 & 2.20 & 2.20 & 2.06 & 2.15 & 2.23 & 2.34 & 2.79 \\
\hline 2.5 & 2.96 & 2.51 & 2.06 & 2.04 & 1.94 & 2.01 & 1.99 & 2.11 & 2.61 \\
\hline 3.5 & 3.42 & 2.95 & 2.87 & 2.79 & 2.62 & 2.73 & 2.87 & 2.89 & 3.44 \\
\hline 4.5 & 3.80 & 3.27 & 3.21 & 3.04 & 2.94 & 3.03 & 3.15 & 3.23 & 3.89 \\
\hline 5.5 & 4.20 & 3.51 & 3.49 & 3.34 & 3.25 & 3.36 & 3.47 & 3.52 & 4.26 \\
\hline 6.5 & 4.46 & 3.87 & 3.65 & 3.63 & 3.49 & 3.60 & 3.67 & 3.78 & 4.51 \\
\hline 7.5 & 4.70 & 4.11 & 3.86 & 3.74 & 3.61 & 3.66 & 3.78 & 3.95 & 4.76 \\
\hline 8.5 & 5.02 & 4.27 & 4.04 & 3.88 & 3.80 & 3.95 & 3.97 & 4.20 & 4.85 \\
\hline 9.5 & 5.18 & 4.49 & 4.29 & 4.20 & 3.98 & 4.19 & 4.04 & 4.33 & 5.16 \\
\hline 10.5 & 5.40 & 4.54 & 4.36 & 4.21 & 4.04 & 4.16 & 4.21 & 4.63 & 5.35 \\
\hline 11.5 & 5.34 & 4.59 & 4.36 & 4.32 & 4.02 & 4.29 & 4.36 & 4.59 & 5.43 \\
\hline 12.5 & 5.53 & 4.70 & 4.41 & 4.27 & 4.16 & 4.36 & 4.42 & 4.67 & 5.51 \\
\hline 13.5 & 5.28 & 4.56 & 4.39 & 4.25 & 4.13 & 4.31 & 4.46 & 4.67 & 5.46 \\
\hline 14.5 & 5.24 & 4.49 & 4.37 & 4.22 & 4.09 & 4.20 & 4.30 & 4.51 & 5.36 \\
\hline 15.5 & 5.22 & 4.40 & 4.16 & 4.05 & 3.91 & 4.08 & 4.17 & 4.39 & 5.17 \\
\hline 16.5 & 4.88 & 4.21 & 3.92 & 3.82 & 3.71 & 3.85 & 3.93 & 4.15 & 4.77 \\
\hline 17.5 & 4.54 & 3.92 & 3.62 & 3.72 & 3.46 & 3.58 & 3.61 & 3.82 & 4.53 \\
\hline 18.5 & 4.28 & 3.59 & 3.46 & 3.45 & 3.20 & 3.46 & 3.48 & 3.63 & 4.26 \\
\hline 19.5 & 3.89 & 3.28 & 3.24 & 3.15 & 2.99 & 3.08 & 3.08 & 3.37 & 3.76 \\
\hline 20.5 & 3.58 & 3.14 & 2.90 & 2.86 & 2.75 & 2.86 & 2.97 & 3.09 & 3.66 \\
\hline 21.5 & 3.32 & 2.79 & 2.59 & 2.52 & 2.44 & 2.54 & 2.52 & 2.82 & 3.24 \\
\hline 22.5 & 2.99 & 2.46 & 2.35 & 2.23 & 2.20 & 2.22 & 2.37 & 2.43 & 2.87 \\
\hline 23.5 & 3.31 & 2.90 & 2.66 & 2.65 & 2.60 & 2.62 & 2.68 & 2.89 & 3.41 \\
\hline Ax. Avg. & 4.24 & 3.63 & 3.45 & 3.37 & 3.23 & 3.36 & 3.42 & 3.61 & 4.25 \\
\hline & & & & & & & & 2.32 & \\
\hline
\end{tabular}

POWER DENSITY VALUES NORMALIZED TO A CORE POWER OF 11.57 MW AND ASSUME ALL FISSION ENERGY (EXCLUDING GAMMA-HEATING OF EX-CORE COMPONENTS) IS DEPOSITED IN THE FUEL 
Table B-4. Power Density Data (kW/cc) in Fuel Plate 4 in Prototypic Conditions (continued).

Plate 4, Core Position X4/X8, 173 MWd Burnup, Equilibrium Xenon Conditions

\begin{tabular}{|c|c|c|c|c|c|c|c|c|c|}
\hline $\begin{array}{c}\text { Axial } \\
\text { position } \\
\text { from } \\
\text { top of } \\
\text { fuel } \\
\text { (inches) }\end{array}$ & $\begin{array}{c}0.000 \\
\text { to } \\
0.197 \\
\text { inches }\end{array}$ & $\begin{array}{c}0.197 \\
\text { to } \\
0.394 \\
\text { inches }\end{array}$ & $\begin{array}{c}0.394 \\
\text { to } \\
0.591 \\
\text { inches }\end{array}$ & $\begin{array}{c}0.591 \\
\text { to } \\
0.868 \\
\text { inches }\end{array}$ & $\begin{array}{c}0.868 \\
\text { to } \\
\text { inches }\end{array}$ & $\begin{array}{c}1.145 \\
\text { to } \\
1.422 \\
\text { inches }\end{array}$ & $\begin{array}{c}1.422 \\
\text { to } \\
1.619 \\
\text { inches }\end{array}$ & $\begin{array}{c}1.619 \\
\text { to } \\
1.815 \\
\text { inches }\end{array}$ & $\begin{array}{c}1.815 \\
\text { to } \\
\text { inches }\end{array}$ \\
\hline 0.5 & 2.69 & 2.38 & 2.20 & 2.13 & 2.13 & 2.17 & 2.27 & 2.36 & 2.83 \\
\hline 1.5 & 2.55 & 2.12 & 1.96 & 1.88 & 1.84 & 1.92 & 2.03 & 2.24 & 2.67 \\
\hline 2.5 & 2.86 & 2.46 & 2.25 & 2.24 & 2.17 & 2.34 & 2.22 & 2.43 & 2.88 \\
\hline 3.5 & 3.49 & 2.80 & 2.72 & 2.69 & 2.47 & 2.65 & 2.75 & 2.82 & 3.33 \\
\hline 4.5 & 3.62 & 3.14 & 2.94 & 2.95 & 2.82 & 2.98 & 2.98 & 3.14 & 3.69 \\
\hline 5.5 & 4.05 & 3.50 & 3.30 & 3.17 & 3.15 & 3.23 & 3.23 & 3.57 & 4.03 \\
\hline 6.5 & 4.33 & 3.79 & 3.51 & 3.48 & 3.30 & 3.54 & 3.56 & 3.72 & 4.20 \\
\hline 7.5 & 4.64 & 3.98 & 3.78 & 3.68 & 3.46 & 3.70 & 3.65 & 3.98 & 4.59 \\
\hline 8.5 & 4.78 & 4.08 & 3.92 & 3.87 & 3.71 & 3.81 & 3.87 & 4.13 & 4.76 \\
\hline 9.5 & 4.93 & 4.28 & 4.08 & 3.99 & 3.84 & 4.00 & 4.07 & 4.30 & 4.99 \\
\hline 10.5 & 5.05 & 4.27 & 4.17 & 4.07 & 3.95 & 4.09 & 4.13 & 4.34 & 4.89 \\
\hline 11.5 & 5.20 & 4.44 & 4.15 & 4.15 & 4.03 & 4.18 & 4.25 & 4.44 & 5.17 \\
\hline 12.5 & 5.14 & 4.48 & 4.22 & 4.22 & 3.98 & 4.22 & 4.30 & 4.54 & 5.19 \\
\hline 13.5 & 5.08 & 4.43 & 4.16 & 4.10 & 3.99 & 4.15 & 4.26 & 4.49 & 5.18 \\
\hline 14.5 & 5.01 & 4.36 & 4.21 & 4.05 & 3.89 & 4.05 & 4.14 & 4.35 & 5.01 \\
\hline 15.5 & 4.95 & 4.09 & 3.84 & 3.99 & 3.68 & 3.93 & 4.08 & 4.22 & 5.00 \\
\hline 16.5 & 4.67 & 4.05 & 3.80 & 3.84 & 3.66 & 3.74 & 3.74 & 4.01 & 4.59 \\
\hline 17.5 & 4.43 & 3.84 & 3.72 & 3.53 & 3.36 & 3.55 & 3.59 & 3.78 & 4.43 \\
\hline 18.5 & 3.98 & 3.56 & 3.32 & 3.37 & 3.15 & 3.35 & 3.46 & 3.75 & 4.07 \\
\hline 19.5 & 3.84 & 3.24 & 3.17 & 3.07 & 2.97 & 3.13 & 3.13 & 3.30 & 3.77 \\
\hline 20.5 & 3.52 & 3.02 & 2.99 & 2.85 & 2.73 & 2.80 & 2.83 & 3.01 & 3.53 \\
\hline 21.5 & 3.16 & 2.78 & 2.61 & 2.52 & 2.43 & 2.55 & 2.59 & 2.77 & 3.29 \\
\hline 22.5 & 2.95 & 2.33 & 2.32 & 2.29 & 2.18 & 2.28 & 2.32 & 2.53 & 2.89 \\
\hline 23.5 & 3.32 & 2.79 & 2.71 & 2.65 & 2.46 & 2.70 & 2.59 & 2.79 & 3.26 \\
\hline Ax. Avg. & 4.09 & 3.51 & 3.34 & 3.28 & 3.14 & 3.30 & 3.34 & 3.54 & 4.09 \\
\hline & & & & & & & & & \\
\hline
\end{tabular}

POWER DENSITY VALUES NORMALIZED TO A CORE POWER OF 11.57 MW AND ASSUME ALL FISSION ENERGY (EXCLUDING GAMMA-HEATING OF EX-CORE COMPONENTS) IS DEPOSITED IN THE FUEL 
Table B-5. Power Density Data $\left(\mathrm{kW} / \mathrm{cm}^{3}\right)$ in Fuel Plate 5 in Prototypic Core.

\section{Fuel core width: 2.120 inches / Fuel core thickness: 20 mil}

Plate 5, Core Position X1/X5, 0 MWd Burnup, Xenon-Free Conditions

\begin{tabular}{|c|c|c|c|c|c|c|c|c|c|}
\hline $\begin{array}{c}\text { Axial } \\
\text { position } \\
\text { from } \\
\text { top of } \\
\text { fuel } \\
\text { (inches) } \\
\end{array}$ & $\begin{array}{c}0.000 \\
\text { to } \\
0.197 \\
\text { inches }\end{array}$ & $\begin{array}{c}0.197 \\
\text { to } \\
0.394 \\
\text { inches } \\
\end{array}$ & $\begin{array}{c}0.394 \\
\text { to } \\
0.591 \\
\text { inches }\end{array}$ & $\begin{array}{c}0.591 \\
\text { to } \\
0.903 \\
\text { inches }\end{array}$ & $\begin{array}{c}0.903 \\
\text { to } \\
1.216 \\
\text { inches }\end{array}$ & $\begin{array}{c}1.216 \\
\text { to } \\
1.529 \\
\text { inches }\end{array}$ & $\begin{array}{c}1.529 \\
\text { to } \\
1.726 \\
\text { inches }\end{array}$ & $\begin{array}{c}1.726 \\
\text { to } \\
1.923 \\
\text { inches }\end{array}$ & $\begin{array}{c}1.923 \\
\text { to } \\
2.120 \\
\text { inches } \\
\end{array}$ \\
\hline 0.5 & 1.77 & 1.49 & 1.31 & 1.35 & 1.21 & 1.29 & 1.35 & 1.44 & 1.79 \\
\hline 1.5 & 1.64 & 1.28 & 1.12 & 1.14 & 1.13 & 1.16 & 1.19 & 1.26 & 1.63 \\
\hline 2.5 & 1.91 & 1.60 & 1.43 & 1.39 & 1.35 & 1.39 & 1.43 & 1.52 & 1.88 \\
\hline 3.5 & 2.17 & 1.78 & 1.68 & 1.68 & 1.61 & 1.65 & 1.73 & 1.84 & 2.22 \\
\hline 4.5 & 2.54 & 2.16 & 2.04 & 1.92 & 1.88 & 2.01 & 2.00 & 2.09 & 2.57 \\
\hline 5.5 & 2.94 & 2.54 & 2.35 & 2.31 & 2.15 & 2.18 & 2.30 & 2.48 & 3.02 \\
\hline 6.5 & 3.36 & 2.86 & 2.64 & 2.56 & 2.43 & 2.55 & 2.53 & 2.75 & 3.45 \\
\hline 7.5 & 3.85 & 3.14 & 2.96 & 2.84 & 2.70 & 2.86 & 2.97 & 3.12 & 3.73 \\
\hline 8.5 & 4.18 & 3.46 & 3.27 & 3.20 & 3.09 & 3.26 & 3.32 & 3.50 & 4.29 \\
\hline 9.5 & 4.68 & 3.86 & 3.66 & 3.52 & 3.41 & 3.56 & 3.55 & 3.84 & 4.80 \\
\hline 10.5 & 5.06 & 4.17 & 3.93 & 3.82 & 3.58 & 3.70 & 3.77 & 4.03 & 4.93 \\
\hline 11.5 & 5.37 & 4.52 & 4.29 & 4.04 & 3.88 & 4.00 & 4.16 & 4.51 & 5.41 \\
\hline 12.5 & 5.68 & 4.61 & 4.18 & 4.18 & 4.11 & 4.22 & 4.40 & 4.69 & 5.42 \\
\hline 13.5 & 5.66 & 4.73 & 4.34 & 4.33 & 4.16 & 4.36 & 4.48 & 4.73 & 5.67 \\
\hline 14.5 & 5.78 & 4.86 & 4.48 & 4.38 & 4.14 & 4.36 & 4.56 & 4.90 & 5.78 \\
\hline 15.5 & 5.89 & 4.71 & 4.53 & 4.31 & 4.22 & 4.32 & 4.41 & 4.78 & 5.73 \\
\hline 16.5 & 5.64 & 4.64 & 4.40 & 4.28 & 4.12 & 4.25 & 4.37 & 4.67 & 5.75 \\
\hline 17.5 & 5.50 & 4.54 & 4.24 & 4.08 & 3.94 & 4.14 & 4.33 & 4.45 & 5.37 \\
\hline 18.5 & 5.04 & 4.26 & 4.00 & 3.78 & 3.66 & 3.88 & 4.04 & 4.26 & 5.23 \\
\hline 19.5 & 4.78 & 4.04 & 3.68 & 3.62 & 3.48 & 3.65 & 3.68 & 4.07 & 4.84 \\
\hline 20.5 & 4.44 & 3.68 & 3.35 & 3.26 & 3.15 & 3.35 & 3.38 & 3.59 & 4.30 \\
\hline 21.5 & 4.08 & 3.21 & 3.02 & 3.00 & 2.88 & 2.95 & 3.12 & 3.24 & 3.98 \\
\hline 22.5 & 3.66 & 2.93 & 2.66 & 2.67 & 2.51 & 2.59 & 2.73 & 2.91 & 3.64 \\
\hline 23.5 & 4.20 & 3.47 & 3.21 & 3.22 & 3.11 & 3.13 & 3.35 & 3.47 & 4.32 \\
\hline Ax. Avg. & 4.16 & 3.44 & 3.20 & 3.12 & 2.99 & 3.12 & 3.21 & 3.42 & 4.16 \\
\hline
\end{tabular}

POWER DENSITY VALUES NORMALIZED TO A CORE POWER OF 11.57 MW AND ASSUME ALL FISSION ENERGY (EXCLUDING GAMMA-HEATING OF EX-CORE COMPONENTS) IS DEPOSITED IN THE FUEL 
Table B-5. Power Density Data (kW/cc) in Fuel Plate 5 in Prototypic Conditions (continued).

Plate 5, Core Position X1/X5, 3 MWd Burnup, Equilibrium Xenon Conditions

\begin{tabular}{|c|c|c|c|c|c|c|c|c|c|}
\hline $\begin{array}{l}\text { Axial } \\
\text { position } \\
\text { from } \\
\text { top of } \\
\text { fuel } \\
\text { (inches) }\end{array}$ & $\begin{array}{c}0.000 \\
\text { to } \\
0.197 \\
\text { inches }\end{array}$ & $\begin{array}{c}0.197 \\
\text { to } \\
0.394 \\
\text { inches }\end{array}$ & $\begin{array}{c}0.394 \\
\text { to } \\
0.591 \\
\text { inches }\end{array}$ & $\begin{array}{c}0.591 \\
\text { to } \\
0.903 \\
\text { inches }\end{array}$ & $\begin{array}{c}0.903 \\
\text { to } \\
1.216 \\
\text { inches }\end{array}$ & $\begin{array}{c}1.216 \\
\text { to } \\
1.529 \\
\text { inches }\end{array}$ & $\begin{array}{c}1.529 \\
\text { to } \\
1.726 \\
\text { inches }\end{array}$ & $\begin{array}{c}1.726 \\
\text { to } \\
1.923 \\
\text { inches }\end{array}$ & $\begin{array}{c}1.923 \\
\text { to } \\
2.120 \\
\text { inches }\end{array}$ \\
\hline 0.5 & 2.63 & 2.12 & 2.07 & 1.92 & 1.88 & 1.95 & 1.94 & 2.08 & 2.49 \\
\hline 1.5 & 2.35 & 1.88 & 1.74 & 1.65 & 1.61 & 1.72 & 1.76 & 1.87 & 2.34 \\
\hline 2.5 & 2.75 & 2.23 & 2.06 & 1.96 & 1.92 & 2.03 & 2.01 & 2.26 & 2.74 \\
\hline 3.5 & 3.10 & 2.56 & 2.32 & 2.31 & 2.19 & 2.35 & 2.38 & 2.53 & 3.17 \\
\hline 4.5 & 3.56 & 2.96 & 2.67 & 2.61 & 2.52 & 2.59 & 2.74 & 2.87 & 3.48 \\
\hline 5.5 & 3.86 & 3.12 & 2.90 & 2.86 & 2.79 & 2.86 & 2.98 & 3.10 & 3.91 \\
\hline 6.5 & 4.13 & 3.42 & 3.20 & 3.07 & 2.96 & 3.12 & 3.21 & 3.36 & 4.26 \\
\hline 7.5 & 4.49 & 3.66 & 3.42 & 3.36 & 3.14 & 3.26 & 3.33 & 3.58 & 4.44 \\
\hline 8.5 & 4.64 & 3.79 & 3.62 & 3.36 & 3.30 & 3.52 & 3.53 & 3.77 & 4.63 \\
\hline 9.5 & 4.80 & 3.96 & 3.57 & 3.66 & 3.49 & 3.65 & 3.60 & 4.04 & 4.79 \\
\hline 10.5 & 5.13 & 4.09 & 3.84 & 3.68 & 3.54 & 3.66 & 3.79 & 4.03 & 5.01 \\
\hline 11.5 & 5.04 & 4.12 & 3.77 & 3.78 & 3.64 & 3.71 & 3.77 & 4.15 & 5.05 \\
\hline 12.5 & 5.25 & 4.24 & 3.92 & 3.74 & 3.67 & 3.72 & 3.73 & 4.16 & 5.16 \\
\hline 13.5 & 5.13 & 4.17 & 3.75 & 3.80 & 3.58 & 3.74 & 3.89 & 4.17 & 5.06 \\
\hline 14.5 & 4.97 & 3.98 & 3.74 & 3.59 & 3.44 & 3.73 & 3.70 & 4.06 & 5.00 \\
\hline 15.5 & 4.71 & 3.85 & 3.63 & 3.57 & 3.37 & 3.54 & 3.66 & 3.98 & 4.81 \\
\hline 16.5 & 4.61 & 3.76 & 3.55 & 3.39 & 3.24 & 3.42 & 3.47 & 3.74 & 4.49 \\
\hline 17.5 & 4.36 & 3.48 & 3.31 & 3.29 & 3.01 & 3.21 & 3.31 & 3.47 & 4.20 \\
\hline 18.5 & 3.99 & 3.36 & 3.05 & 2.99 & 2.91 & 3.01 & 3.15 & 3.34 & 3.93 \\
\hline 19.5 & 3.65 & 3.08 & 2.86 & 2.80 & 2.72 & 2.81 & 2.82 & 2.97 & 3.56 \\
\hline 20.5 & 3.45 & 2.84 & 2.55 & 2.57 & 2.38 & 2.49 & 2.48 & 2.78 & 3.22 \\
\hline 21.5 & 2.98 & 2.48 & 2.28 & 2.23 & 2.16 & 2.18 & 2.24 & 2.50 & 2.99 \\
\hline 22.5 & 2.62 & 2.18 & 1.97 & 1.91 & 1.86 & 1.95 & 1.97 & 2.19 & 2.74 \\
\hline 23.5 & 3.31 & 2.60 & 2.39 & 2.38 & 2.21 & 2.34 & 2.42 & 2.61 & 3.16 \\
\hline Ax. Avg. & 3.98 & 3.25 & 3.01 & 2.94 & 2.81 & 2.94 & 2.99 & 3.23 & 3.94 \\
\hline
\end{tabular}

POWER DENSITY VALUES NORMALIZED TO A CORE POWER OF 11.57 MW AND ASSUME ALL FISSION ENERGY (EXCLUDING GAMMA-HEATING OF EX-CORE COMPONENTS) IS DEPOSITED IN THE FUEL 
Table B-5. Power Density Data (kW/cc) in Fuel Plate 5 in Prototypic Conditions (continued).

Plate 5, Core Position X3/X7, 80 MWd Burnup, Equilibrium Xenon Conditions

\begin{tabular}{|c|c|c|c|c|c|c|c|c|c|}
\hline $\begin{array}{c}\text { Axial } \\
\text { position } \\
\text { from } \\
\text { top of } \\
\text { fuel } \\
\text { (inches) }\end{array}$ & $\begin{array}{c}0.000 \\
\text { to } \\
0.197 \\
\text { inches }\end{array}$ & $\begin{array}{c}0.197 \\
\text { to } \\
0.394 \\
\text { inches }\end{array}$ & $\begin{array}{c}0.394 \\
\text { to } \\
0.591 \\
\text { inches }\end{array}$ & $\begin{array}{c}0.591 \\
\text { to } \\
0.903 \\
\text { inches }\end{array}$ & $\begin{array}{c}0.903 \\
\text { to } \\
1.216 \\
\text { inches }\end{array}$ & $\begin{array}{c}1.216 \\
\text { to } \\
1.529 \\
\text { inches }\end{array}$ & $\begin{array}{c}1.529 \\
\text { to } \\
1.726 \\
\text { inches }\end{array}$ & $\begin{array}{c}1.726 \\
\text { to } \\
1.923 \\
\text { inches }\end{array}$ & $\begin{array}{c}1.923 \\
\text { to } \\
\text { inches }\end{array}$ \\
\hline 1.5 & 2.50 & 1.99 & 1.98 & 1.92 & 1.83 & 1.88 & 1.88 & 2.13 & 2.54 \\
\hline 2.5 & 2.60 & 2.24 & 1.98 & 1.93 & 1.88 & 1.98 & 2.05 & 2.13 & 2.73 \\
\hline 3.5 & 2.98 & 2.56 & 2.28 & 2.30 & 2.13 & 2.31 & 2.33 & 2.60 & 3.10 \\
\hline 4.5 & 3.41 & 2.83 & 2.67 & 2.57 & 2.44 & 2.60 & 2.70 & 2.81 & 3.40 \\
\hline 5.5 & 3.70 & 3.01 & 2.91 & 2.83 & 2.71 & 2.81 & 2.90 & 3.16 & 3.79 \\
\hline 6.5 & 4.02 & 3.25 & 3.15 & 2.89 & 2.91 & 3.07 & 3.16 & 3.41 & 4.00 \\
\hline 7.5 & 4.28 & 3.55 & 3.33 & 3.21 & 3.05 & 3.27 & 3.37 & 3.56 & 4.26 \\
\hline 8.5 & 4.42 & 3.69 & 3.48 & 3.35 & 3.22 & 3.36 & 3.53 & 3.76 & 4.54 \\
\hline 9.5 & 4.73 & 3.96 & 3.63 & 3.48 & 3.36 & 3.56 & 3.58 & 3.85 & 4.50 \\
\hline 10.5 & 4.79 & 3.99 & 3.76 & 3.61 & 3.37 & 3.61 & 3.66 & 3.92 & 4.92 \\
\hline 11.5 & 4.92 & 4.01 & 3.74 & 3.66 & 3.51 & 3.69 & 3.75 & 3.95 & 4.75 \\
\hline 12.5 & 4.88 & 4.08 & 3.67 & 3.56 & 3.47 & 3.68 & 3.75 & 4.02 & 4.96 \\
\hline 13.5 & 5.04 & 3.98 & 3.63 & 3.71 & 3.51 & 3.62 & 3.72 & 4.08 & 4.80 \\
\hline 14.5 & 4.76 & 3.98 & 3.61 & 3.52 & 3.39 & 3.56 & 3.61 & 3.90 & 4.69 \\
\hline 15.5 & 4.50 & 3.76 & 3.50 & 3.45 & 3.26 & 3.44 & 3.53 & 3.83 & 4.59 \\
\hline 16.5 & 4.26 & 3.65 & 3.36 & 3.32 & 3.18 & 3.36 & 3.40 & 3.60 & 4.36 \\
\hline 17.5 & 4.10 & 3.46 & 3.25 & 3.13 & 2.93 & 3.12 & 3.24 & 3.43 & 4.11 \\
\hline 18.5 & 3.85 & 3.18 & 2.98 & 2.91 & 2.76 & 2.81 & 2.96 & 3.09 & 3.74 \\
\hline 19.5 & 3.47 & 2.91 & 2.81 & 2.69 & 2.60 & 2.75 & 2.79 & 2.90 & 3.52 \\
\hline 20.5 & 3.30 & 2.68 & 2.50 & 2.47 & 2.34 & 2.39 & 2.49 & 2.64 & 3.23 \\
\hline 21.5 & 2.89 & 2.41 & 2.18 & 2.17 & 2.09 & 2.18 & 2.23 & 2.40 & 2.85 \\
\hline 22.5 & 2.54 & 2.03 & 1.98 & 1.94 & 1.80 & 1.87 & 1.94 & 2.15 & 2.56 \\
\hline 23.5 & 3.08 & 2.55 & 2.29 & 2.35 & 2.15 & 2.31 & 2.32 & 2.49 & 3.12 \\
\hline Ax. Avg. & 3.80 & 3.15 & 2.93 & 2.86 & 2.73 & 2.87 & 2.95 & 3.15 & 3.81 \\
\hline & & & & & & & & 1.68 & \\
\hline
\end{tabular}

POWER DENSITY VALUES NORMALIZED TO A CORE POWER OF 11.57 MW AND ASSUME ALL FISSION ENERGY (EXCLUDING GAMMA-HEATING OF EX-CORE COMPONENTS) IS DEPOSITED IN THE FUEL 
Table B-5. Power Density Data (kW/cc) in Fuel Plate 5 in Prototypic Conditions (continued).

Plate 5, Core Position X2/X6, 99 MWd Burnup, Equilibrium Xenon Conditions

\begin{tabular}{|c|c|c|c|c|c|c|c|c|c|}
\hline $\begin{array}{c}\text { Axial } \\
\text { position } \\
\text { from } \\
\text { top of } \\
\text { fuel } \\
\text { (inches) }\end{array}$ & $\begin{array}{c}0.000 \\
\text { to } \\
0.197 \\
\text { inches }\end{array}$ & $\begin{array}{c}0.197 \\
\text { to } \\
0.394 \\
\text { inches }\end{array}$ & $\begin{array}{c}0.394 \\
\text { to } \\
0.591 \\
\text { inches }\end{array}$ & $\begin{array}{c}0.591 \\
\text { to } \\
0.903 \\
\text { inches }\end{array}$ & $\begin{array}{c}0.903 \\
\text { to } \\
1.216 \\
\text { inches }\end{array}$ & $\begin{array}{c}1.216 \\
\text { to } \\
1.529 \\
\text { inches }\end{array}$ & $\begin{array}{c}1.529 \\
\text { to } \\
1.726 \\
\text { inches }\end{array}$ & $\begin{array}{c}1.726 \\
\text { to } \\
1.923 \\
\text { inches }\end{array}$ & $\begin{array}{c}1.923 \\
\text { to } \\
\text { inches }\end{array}$ \\
\hline 1.5 & 2.59 & 2.06 & 1.99 & 1.92 & 1.81 & 1.90 & 1.89 & 2.05 & 2.55 \\
\hline 2.5 & 2.56 & 2.04 & 1.93 & 2.01 & 1.83 & 1.98 & 2.06 & 2.23 & 2.62 \\
\hline 3.5 & 2.94 & 2.59 & 2.33 & 2.36 & 2.17 & 2.31 & 2.29 & 2.51 & 2.99 \\
\hline 4.5 & 3.42 & 2.77 & 2.71 & 2.57 & 2.46 & 2.62 & 2.65 & 2.86 & 3.46 \\
\hline 5.5 & 3.72 & 3.18 & 2.90 & 2.79 & 2.71 & 2.85 & 2.78 & 3.12 & 3.63 \\
\hline 6.5 & 3.88 & 3.42 & 3.11 & 3.03 & 2.82 & 3.06 & 3.13 & 3.27 & 3.86 \\
\hline 7.5 & 4.31 & 3.57 & 3.32 & 3.16 & 3.02 & 3.13 & 3.28 & 3.48 & 4.28 \\
\hline 8.5 & 4.35 & 3.62 & 3.33 & 3.41 & 3.22 & 3.35 & 3.51 & 3.63 & 4.48 \\
\hline 9.5 & 4.62 & 3.90 & 3.63 & 3.57 & 3.37 & 3.57 & 3.48 & 3.80 & 4.63 \\
\hline 10.5 & 4.75 & 3.94 & 3.62 & 3.67 & 3.38 & 3.54 & 3.70 & 4.07 & 4.71 \\
\hline 11.5 & 4.92 & 4.03 & 3.73 & 3.59 & 3.41 & 3.68 & 3.71 & 4.00 & 4.77 \\
\hline 12.5 & 4.88 & 4.03 & 3.73 & 3.68 & 3.46 & 3.60 & 3.83 & 3.99 & 4.85 \\
\hline 13.5 & 4.73 & 3.95 & 3.63 & 3.56 & 3.42 & 3.67 & 3.79 & 4.04 & 4.91 \\
\hline 14.5 & 4.72 & 3.95 & 3.75 & 3.49 & 3.47 & 3.56 & 3.69 & 3.92 & 4.73 \\
\hline 15.5 & 4.53 & 3.81 & 3.58 & 3.41 & 3.28 & 3.48 & 3.52 & 3.66 & 4.45 \\
\hline 16.5 & 4.31 & 3.67 & 3.43 & 3.25 & 3.18 & 3.25 & 3.40 & 3.53 & 4.39 \\
\hline 17.5 & 4.06 & 3.48 & 3.20 & 3.09 & 3.01 & 3.10 & 3.24 & 3.47 & 4.09 \\
\hline 18.5 & 3.77 & 3.22 & 2.97 & 2.96 & 2.80 & 2.91 & 3.04 & 3.20 & 3.78 \\
\hline 19.5 & 3.44 & 2.97 & 2.68 & 2.59 & 2.56 & 2.65 & 2.69 & 2.86 & 3.51 \\
\hline 20.5 & 3.10 & 2.60 & 2.45 & 2.39 & 2.36 & 2.50 & 2.55 & 2.68 & 3.33 \\
\hline 21.5 & 2.83 & 2.37 & 2.22 & 2.15 & 2.07 & 2.10 & 2.17 & 2.36 & 2.82 \\
\hline 22.5 & 2.70 & 2.16 & 2.01 & 1.87 & 1.79 & 1.92 & 1.97 & 2.09 & 2.57 \\
\hline 23.5 & 3.14 & 2.54 & 2.30 & 2.35 & 2.24 & 2.28 & 2.36 & 2.54 & 3.14 \\
\hline Ax. Avg. & 3.77 & 3.15 & 2.93 & 2.86 & 2.73 & 2.86 & 2.94 & 3.13 & 3.78 \\
\hline & & & & & & & & 1.76 & 13 \\
\hline
\end{tabular}

POWER DENSITY VALUES NORMALIZED TO A CORE POWER OF 11.57 MW AND ASSUME ALL FISSION ENERGY (EXCLUDING GAMMA-HEATING OF EX-CORE COMPONENTS) IS DEPOSITED IN THE FUEL 
Table B-5. Power Density Data (kW/cc) in Fuel Plate 5 in Prototypic Conditions (continued).

Plate 5, Core Position X4/X8, 173 MWd Burnup, Equilibrium Xenon Conditions

\begin{tabular}{|c|c|c|c|c|c|c|c|c|c|}
\hline $\begin{array}{c}\text { Axial } \\
\text { position } \\
\text { from } \\
\text { top of } \\
\text { fuel } \\
\text { (inches) }\end{array}$ & $\begin{array}{c}0.000 \\
\text { to } \\
0.197 \\
\text { inches }\end{array}$ & $\begin{array}{c}0.197 \\
\text { to } \\
0.394 \\
\text { inches }\end{array}$ & $\begin{array}{c}0.394 \\
\text { to } \\
0.591 \\
\text { inches }\end{array}$ & $\begin{array}{c}0.591 \\
\text { to } \\
0.903 \\
\text { inches }\end{array}$ & $\begin{array}{c}0.903 \\
\text { to } \\
1.216 \\
\text { inches }\end{array}$ & $\begin{array}{c}1.216 \\
\text { to } \\
1.529 \\
\text { inches }\end{array}$ & $\begin{array}{c}1.529 \\
\text { to } \\
1.726 \\
\text { inches }\end{array}$ & $\begin{array}{c}1.726 \\
\text { to } \\
1.923 \\
\text { inches }\end{array}$ & $\begin{array}{c}1.923 \\
\text { to } \\
\text { inches }\end{array}$ \\
\hline 1.5 & 2.46 & 2.04 & 1.90 & 1.90 & 1.84 & 1.91 & 1.95 & 2.07 & 2.55 \\
\hline 2.5 & 2.46 & 2.11 & 1.97 & 1.90 & 1.85 & 1.95 & 1.98 & 2.14 & 2.52 \\
\hline 3.5 & 2.99 & 2.56 & 2.25 & 2.23 & 2.17 & 2.23 & 2.30 & 2.45 & 2.93 \\
\hline 4.5 & 3.16 & 2.64 & 2.56 & 2.51 & 2.44 & 2.55 & 2.60 & 2.73 & 3.27 \\
\hline 5.5 & 3.58 & 3.02 & 2.93 & 2.85 & 2.62 & 2.78 & 2.95 & 3.01 & 3.61 \\
\hline 6.5 & 3.83 & 3.30 & 3.14 & 3.02 & 2.85 & 2.98 & 3.10 & 3.28 & 3.88 \\
\hline 7.5 & 4.08 & 3.41 & 3.22 & 3.20 & 2.97 & 3.10 & 3.28 & 3.38 & 4.14 \\
\hline 8.5 & 4.24 & 3.61 & 3.39 & 3.32 & 3.14 & 3.36 & 3.31 & 3.67 & 4.18 \\
\hline 9.5 & 4.43 & 3.68 & 3.55 & 3.45 & 3.25 & 3.41 & 3.49 & 3.74 & 4.43 \\
\hline 10.5 & 4.55 & 3.91 & 3.53 & 3.49 & 3.37 & 3.57 & 3.55 & 3.85 & 4.61 \\
\hline 11.5 & 4.65 & 3.98 & 3.67 & 3.56 & 3.44 & 3.49 & 3.72 & 3.75 & 4.48 \\
\hline 12.5 & 4.64 & 3.88 & 3.67 & 3.52 & 3.38 & 3.55 & 3.69 & 3.94 & 4.50 \\
\hline 13.5 & 4.56 & 3.85 & 3.60 & 3.51 & 3.44 & 3.58 & 3.76 & 4.02 & 4.60 \\
\hline 14.5 & 4.56 & 3.73 & 3.60 & 3.47 & 3.29 & 3.41 & 3.61 & 3.76 & 4.56 \\
\hline 15.5 & 4.41 & 3.65 & 3.38 & 3.25 & 3.12 & 3.32 & 3.40 & 3.69 & 4.39 \\
\hline 16.5 & 4.15 & 3.47 & 3.32 & 3.26 & 3.14 & 3.25 & 3.31 & 3.55 & 4.14 \\
\hline 17.5 & 4.03 & 3.36 & 3.15 & 3.05 & 2.89 & 3.07 & 3.14 & 3.39 & 4.00 \\
\hline 18.5 & 3.71 & 3.19 & 2.89 & 2.85 & 2.71 & 2.94 & 2.99 & 3.20 & 3.64 \\
\hline 19.5 & 3.41 & 2.85 & 2.71 & 2.60 & 2.52 & 2.75 & 2.70 & 2.88 & 3.47 \\
\hline 20.5 & 3.06 & 2.54 & 2.48 & 2.38 & 2.32 & 2.33 & 2.46 & 2.62 & 3.12 \\
\hline 21.5 & 2.84 & 2.32 & 2.17 & 2.16 & 2.02 & 2.19 & 2.22 & 2.37 & 2.88 \\
\hline 22.5 & 2.54 & 2.08 & 1.93 & 1.92 & 1.84 & 1.92 & 2.07 & 2.10 & 2.51 \\
\hline 23.5 & 2.95 & 2.36 & 2.41 & 2.29 & 2.17 & 2.29 & 2.37 & 2.48 & 2.92 \\
\hline Ax. Avg. & 3.65 & 3.06 & 2.88 & 2.80 & 2.68 & 2.82 & 2.90 & 3.08 & 3.65 \\
\hline & & & & & & & & 1.66 & \\
\hline
\end{tabular}

POWER DENSITY VALUES NORMALIZED TO A CORE POWER OF 11.57 MW AND ASSUME ALL FISSION ENERGY (EXCLUDING GAMMA-HEATING OF EX-CORE COMPONENTS) IS DEPOSITED IN THE FUEL 
Table B-6. Power Density Data $\left(\mathrm{kW} / \mathrm{cm}^{3}\right)$ in Fuel Plate 6 in Prototypic Core.

\section{Fuel core width: 2.227 inches / Fuel core thickness: 20 mil}

Plate 6, Core Position X1/X5, 0 MWd Burnup, Xenon-Free Conditions

\begin{tabular}{|c|c|c|c|c|c|c|c|c|c|}
\hline $\begin{array}{c}\text { Axial } \\
\text { position } \\
\text { from } \\
\text { top of } \\
\text { fuel } \\
\text { (inches) } \\
\end{array}$ & $\begin{array}{c}0.000 \\
\text { to } \\
0.197 \\
\text { inches }\end{array}$ & $\begin{array}{c}0.197 \\
\text { to } \\
0.394 \\
\text { inches } \\
\end{array}$ & $\begin{array}{c}0.394 \\
\text { to } \\
0.591 \\
\text { inches }\end{array}$ & $\begin{array}{c}0.591 \\
\text { to } \\
0.939 \\
\text { inches }\end{array}$ & $\begin{array}{c}0.939 \\
\text { to } \\
1.288 \\
\text { inches }\end{array}$ & $\begin{array}{c}1.288 \\
\text { to } \\
1.636 \\
\text { inches } \\
\end{array}$ & $\begin{array}{c}1.636 \\
\text { to } \\
1.833 \\
\text { inches }\end{array}$ & $\begin{array}{c}1.833 \\
\text { to } \\
2.030 \\
\text { inches }\end{array}$ & $\begin{array}{c}2.030 \\
\text { to } \\
2.227 \\
\text { inches } \\
\end{array}$ \\
\hline 0.5 & 1.65 & 1.33 & 1.21 & 1.17 & 1.15 & 1.20 & 1.22 & 1.38 & 1.57 \\
\hline 1.5 & 1.49 & 1.18 & 1.07 & 1.02 & 1.02 & 1.03 & 1.06 & 1.15 & 1.46 \\
\hline 2.5 & 1.74 & 1.39 & 1.32 & 1.26 & 1.12 & 1.23 & 1.26 & 1.37 & 1.64 \\
\hline 3.5 & 1.99 & 1.60 & 1.50 & 1.50 & 1.44 & 1.55 & 1.58 & 1.60 & 2.03 \\
\hline 4.5 & 2.28 & 1.88 & 1.79 & 1.73 & 1.68 & 1.77 & 1.77 & 1.95 & 2.37 \\
\hline 5.5 & 2.62 & 2.19 & 2.13 & 2.03 & 1.86 & 1.97 & 2.07 & 2.30 & 2.75 \\
\hline 6.5 & 3.04 & 2.56 & 2.40 & 2.36 & 2.16 & 2.27 & 2.39 & 2.53 & 3.06 \\
\hline 7.5 & 3.44 & 2.86 & 2.64 & 2.58 & 2.42 & 2.59 & 2.59 & 2.92 & 3.47 \\
\hline 8.5 & 3.86 & 3.22 & 3.05 & 2.92 & 2.77 & 2.85 & 2.92 & 3.30 & 3.90 \\
\hline 9.5 & 4.21 & 3.50 & 3.14 & 3.03 & 3.02 & 3.18 & 3.36 & 3.53 & 4.28 \\
\hline 10.5 & 4.60 & 3.72 & 3.54 & 3.34 & 3.26 & 3.46 & 3.47 & 3.74 & 4.52 \\
\hline 11.5 & 4.87 & 4.09 & 3.72 & 3.62 & 3.42 & 3.63 & 3.73 & 4.02 & 4.80 \\
\hline 12.5 & 5.19 & 4.24 & 3.97 & 3.73 & 3.65 & 3.87 & 3.89 & 4.23 & 5.10 \\
\hline 13.5 & 5.08 & 4.22 & 4.02 & 3.90 & 3.68 & 3.91 & 3.94 & 4.27 & 5.19 \\
\hline 14.5 & 5.22 & 4.37 & 4.03 & 3.93 & 3.74 & 3.87 & 4.02 & 4.40 & 5.31 \\
\hline 15.5 & 5.25 & 4.27 & 4.00 & 3.89 & 3.77 & 4.01 & 3.98 & 4.30 & 5.24 \\
\hline 16.5 & 5.05 & 4.16 & 3.91 & 3.86 & 3.67 & 3.85 & 4.00 & 4.20 & 5.12 \\
\hline 17.5 & 5.08 & 4.20 & 3.81 & 3.75 & 3.46 & 3.73 & 3.80 & 4.06 & 4.90 \\
\hline 18.5 & 4.60 & 3.95 & 3.64 & 3.51 & 3.39 & 3.55 & 3.68 & 3.89 & 4.75 \\
\hline 19.5 & 4.48 & 3.70 & 3.35 & 3.26 & 3.11 & 3.28 & 3.32 & 3.57 & 4.42 \\
\hline 20.5 & 4.02 & 3.29 & 3.07 & 3.02 & 2.92 & 2.92 & 3.16 & 3.37 & 4.07 \\
\hline 21.5 & 3.69 & 2.99 & 2.77 & 2.68 & 2.58 & 2.71 & 2.74 & 3.01 & 3.62 \\
\hline 22.5 & 3.31 & 2.60 & 2.46 & 2.38 & 2.23 & 2.37 & 2.43 & 2.62 & 3.31 \\
\hline 23.5 & 4.11 & 3.19 & 3.03 & 2.94 & 2.81 & 2.93 & 3.03 & 3.28 & 3.90 \\
\hline Ax. Avg. & 3.79 & 3.11 & 2.90 & 2.81 & 2.68 & 2.82 & 2.89 & 3.12 & 3.78 \\
\hline
\end{tabular}

POWER DENSITY VALUES NORMALIZED TO A CORE POWER OF 11.57 MW AND ASSUME ALL FISSION ENERGY (EXCLUDING GAMMA-HEATING OF EX-CORE COMPONENTS) IS DEPOSITED IN THE FUEL 
Table B-6. Power Density Data (kW/cc) in Fuel Plate 6 in Prototypic Conditions (continued).

Plate 6, Core Position X1/X5, 3 MWd Burnup, Equilibrium Xenon Conditions

\begin{tabular}{|c|c|c|c|c|c|c|c|c|c|}
\hline $\begin{array}{l}\text { Axial } \\
\text { position } \\
\text { from } \\
\text { top of } \\
\text { fuel } \\
\text { (inches) }\end{array}$ & $\begin{array}{c}0.000 \\
\text { to } \\
0.197 \\
\text { inches }\end{array}$ & $\begin{array}{c}0.197 \\
\text { to } \\
0.394 \\
\text { inches }\end{array}$ & $\begin{array}{c}0.394 \\
\text { to } \\
0.591 \\
\text { inches }\end{array}$ & $\begin{array}{c}0.591 \\
\text { to } \\
0.939 \\
\text { inches }\end{array}$ & $\begin{array}{c}0.939 \\
\text { to } \\
1.288 \\
\text { inches }\end{array}$ & $\begin{array}{c}1.288 \\
\text { to } \\
1.636 \\
\text { inches }\end{array}$ & $\begin{array}{c}1.636 \\
\text { to } \\
1.833 \\
\text { inches }\end{array}$ & $\begin{array}{c}1.833 \\
\text { to } \\
2.030 \\
\text { inches }\end{array}$ & $\begin{array}{c}2.030 \\
\text { to } \\
2.227 \\
\text { inches }\end{array}$ \\
\hline 0.5 & 2.49 & 1.91 & 1.85 & 1.71 & 1.72 & 1.81 & 1.78 & 2.01 & 2.43 \\
\hline 1.5 & 2.20 & 1.73 & 1.59 & 1.52 & 1.42 & 1.55 & 1.61 & 1.73 & 2.16 \\
\hline 2.5 & 2.49 & 2.02 & 1.88 & 1.78 & 1.74 & 1.83 & 1.85 & 1.99 & 2.49 \\
\hline 3.5 & 2.79 & 2.25 & 2.12 & 2.07 & 1.99 & 2.12 & 2.14 & 2.35 & 2.85 \\
\hline 4.5 & 3.22 & 2.64 & 2.43 & 2.37 & 2.22 & 2.42 & 2.37 & 2.59 & 3.19 \\
\hline 5.5 & 3.60 & 2.88 & 2.60 & 2.53 & 2.50 & 2.56 & 2.67 & 2.84 & 3.61 \\
\hline 6.5 & 3.76 & 3.04 & 2.83 & 2.78 & 2.71 & 2.79 & 2.92 & 3.09 & 3.87 \\
\hline 7.5 & 4.01 & 3.33 & 3.12 & 2.98 & 2.86 & 2.99 & 3.07 & 3.36 & 4.05 \\
\hline 8.5 & 4.30 & 3.39 & 3.21 & 3.15 & 3.09 & 3.19 & 3.22 & 3.48 & 4.24 \\
\hline 9.5 & 4.42 & 3.63 & 3.25 & 3.23 & 3.13 & 3.34 & 3.39 & 3.60 & 4.50 \\
\hline 10.5 & 4.62 & 3.65 & 3.42 & 3.37 & 3.12 & 3.34 & 3.40 & 3.70 & 4.57 \\
\hline 11.5 & 4.62 & 3.84 & 3.49 & 3.41 & 3.27 & 3.37 & 3.48 & 3.75 & 4.57 \\
\hline 12.5 & 4.63 & 3.78 & 3.56 & 3.38 & 3.20 & 3.38 & 3.51 & 3.76 & 4.60 \\
\hline 13.5 & 4.62 & 3.67 & 3.44 & 3.43 & 3.23 & 3.33 & 3.47 & 3.77 & 4.58 \\
\hline 14.5 & 4.54 & 3.77 & 3.33 & 3.31 & 3.12 & 3.39 & 3.39 & 3.66 & 4.43 \\
\hline 15.5 & 4.32 & 3.56 & 3.15 & 3.18 & 3.01 & 3.23 & 3.28 & 3.59 & 4.24 \\
\hline 16.5 & 4.27 & 3.45 & 3.25 & 3.08 & 2.90 & 3.04 & 3.18 & 3.37 & 4.12 \\
\hline 17.5 & 3.91 & 3.26 & 2.99 & 2.98 & 2.81 & 2.94 & 3.05 & 3.12 & 3.87 \\
\hline 18.5 & 3.65 & 3.05 & 2.75 & 2.71 & 2.58 & 2.73 & 2.81 & 3.07 & 3.74 \\
\hline 19.5 & 3.34 & 2.85 & 2.55 & 2.55 & 2.45 & 2.45 & 2.55 & 2.73 & 3.35 \\
\hline 20.5 & 3.00 & 2.44 & 2.35 & 2.26 & 2.14 & 2.26 & 2.33 & 2.53 & 2.98 \\
\hline 21.5 & 2.75 & 2.26 & 2.06 & 1.98 & 1.94 & 1.99 & 2.06 & 2.31 & 2.74 \\
\hline 22.5 & 2.41 & 1.96 & 1.79 & 1.75 & 1.70 & 1.72 & 1.79 & 2.03 & 2.51 \\
\hline 23.5 & 3.06 & 2.41 & 2.22 & 2.12 & 2.01 & 2.14 & 2.30 & 2.36 & 3.03 \\
\hline Ax. Avg. & 3.63 & 2.95 & 2.72 & 2.65 & 2.54 & 2.66 & 2.73 & 2.95 & 3.61 \\
\hline
\end{tabular}

POWER DENSITY VALUES NORMALIZED TO A CORE POWER OF 11.57 MW AND ASSUME ALL FISSION ENERGY (EXCLUDING GAMMA-HEATING OF EX-CORE COMPONENTS) IS DEPOSITED IN THE FUEL 
Table B-6. Power Density Data (kW/cc) in Fuel Plate 6 in Prototypic Conditions (continued).

Plate 6, Core Position X3/X7, 80 MWd Burnup, Equilibrium Xenon Conditions

\begin{tabular}{|c|c|c|c|c|c|c|c|c|c|}
\hline $\begin{array}{c}\text { Axial } \\
\text { position } \\
\text { from } \\
\text { top of } \\
\text { fuel } \\
\text { (inches) }\end{array}$ & $\begin{array}{c}0.000 \\
\text { to } \\
0.197 \\
\text { inches }\end{array}$ & $\begin{array}{c}0.197 \\
\text { to } \\
0.394 \\
\text { inches }\end{array}$ & $\begin{array}{c}0.394 \\
\text { to } \\
0.591 \\
\text { inches }\end{array}$ & $\begin{array}{c}0.591 \\
\text { to } \\
0.939 \\
\text { inches }\end{array}$ & $\begin{array}{c}0.939 \\
\text { to } \\
1.288 \\
\text { inches }\end{array}$ & $\begin{array}{c}1.288 \\
\text { to } \\
1.636 \\
\text { inches }\end{array}$ & $\begin{array}{c}1.636 \\
\text { to } \\
1.833 \\
\text { inches }\end{array}$ & $\begin{array}{c}1.833 \\
\text { to } \\
2.030 \\
\text { inches }\end{array}$ & $\begin{array}{c}2.030 \\
\text { to } \\
2.227 \\
\text { inches }\end{array}$ \\
\hline 1.5 & 2.34 & 1.92 & 1.80 & 1.75 & 1.73 & 1.75 & 1.76 & 1.95 & 2.30 \\
\hline 2.5 & 2.45 & 1.95 & 1.81 & 1.79 & 1.69 & 1.73 & 1.80 & 1.88 & 2.37 \\
\hline 3.5 & 2.70 & 2.24 & 2.14 & 2.09 & 1.90 & 2.01 & 2.17 & 2.32 & 2.82 \\
\hline 4.5 & 3.23 & 2.60 & 2.40 & 2.29 & 2.21 & 2.27 & 2.32 & 2.54 & 3.13 \\
\hline 5.5 & 3.40 & 2.80 & 2.62 & 2.57 & 2.44 & 2.55 & 2.63 & 2.84 & 3.53 \\
\hline 6.5 & 3.71 & 2.99 & 2.82 & 2.74 & 2.58 & 2.74 & 2.88 & 3.08 & 3.67 \\
\hline 7.5 & 3.80 & 3.32 & 3.05 & 2.88 & 2.78 & 2.87 & 3.01 & 3.28 & 3.85 \\
\hline 8.5 & 4.07 & 3.41 & 3.10 & 2.99 & 2.89 & 3.00 & 3.22 & 3.40 & 4.09 \\
\hline 9.5 & 4.29 & 3.43 & 3.30 & 3.19 & 2.96 & 3.21 & 3.27 & 3.64 & 4.28 \\
\hline 10.5 & 4.22 & 3.61 & 3.35 & 3.18 & 3.03 & 3.23 & 3.21 & 3.51 & 4.40 \\
\hline 11.5 & 4.38 & 3.59 & 3.34 & 3.28 & 3.17 & 3.22 & 3.36 & 3.58 & 4.41 \\
\hline 12.5 & 4.44 & 3.72 & 3.41 & 3.27 & 3.17 & 3.26 & 3.37 & 3.63 & 4.42 \\
\hline 13.5 & 4.47 & 3.65 & 3.33 & 3.26 & 3.07 & 3.33 & 3.29 & 3.54 & 4.29 \\
\hline 14.5 & 4.25 & 3.59 & 3.33 & 3.15 & 2.99 & 3.21 & 3.25 & 3.42 & 4.25 \\
\hline 15.5 & 4.16 & 3.51 & 3.22 & 3.08 & 2.93 & 3.02 & 3.15 & 3.34 & 4.04 \\
\hline 16.5 & 3.90 & 3.27 & 2.99 & 2.97 & 2.85 & 2.88 & 3.13 & 3.34 & 3.95 \\
\hline 17.5 & 3.76 & 3.03 & 2.88 & 2.81 & 2.73 & 2.75 & 2.87 & 3.13 & 3.67 \\
\hline 18.5 & 3.40 & 2.92 & 2.74 & 2.63 & 2.46 & 2.59 & 2.74 & 2.92 & 3.53 \\
\hline 19.5 & 3.22 & 2.62 & 2.51 & 2.41 & 2.40 & 2.40 & 2.45 & 2.70 & 3.28 \\
\hline 20.5 & 3.02 & 2.46 & 2.28 & 2.16 & 2.08 & 2.18 & 2.27 & 2.47 & 2.91 \\
\hline 21.5 & 2.62 & 2.23 & 1.95 & 1.97 & 1.85 & 1.99 & 2.06 & 2.21 & 2.75 \\
\hline 22.5 & 2.39 & 1.90 & 1.73 & 1.76 & 1.58 & 1.70 & 1.72 & 1.94 & 2.39 \\
\hline 23.5 & 2.91 & 2.36 & 2.16 & 2.13 & 2.03 & 2.07 & 2.18 & 2.37 & 2.91 \\
\hline Ax. Avg. & 3.47 & 2.87 & 2.66 & 2.57 & 2.45 & 2.56 & 2.65 & 2.86 & 3.47 \\
\hline & & & & & & & & & 1.53 \\
\hline
\end{tabular}

POWER DENSITY VALUES NORMALIZED TO A CORE POWER OF 11.57 MW AND ASSUME ALL FISSION ENERGY (EXCLUDING GAMMA-HEATING OF EX-CORE COMPONENTS) IS DEPOSITED IN THE FUEL 
Table B-6. Power Density Data (kW/cc) in Fuel Plate 6 in Prototypic Conditions (continued).

Plate 6, Core Position X2/X6, 99 MWd Burnup, Equilibrium Xenon Conditions

\begin{tabular}{|c|c|c|c|c|c|c|c|c|c|}
\hline $\begin{array}{l}\text { Axial } \\
\text { position } \\
\text { from } \\
\text { top of } \\
\text { fuel } \\
\text { (inches) }\end{array}$ & $\begin{array}{c}0.000 \\
\text { to } \\
0.197 \\
\text { inches }\end{array}$ & $\begin{array}{c}0.197 \\
\text { to } \\
0.394 \\
\text { inches }\end{array}$ & $\begin{array}{c}0.394 \\
\text { to } \\
0.591 \\
\text { inches }\end{array}$ & $\begin{array}{c}0.591 \\
\text { to } \\
0.939 \\
\text { inches }\end{array}$ & $\begin{array}{c}0.939 \\
\text { to } \\
1.288 \\
\text { inches }\end{array}$ & $\begin{array}{c}1.288 \\
\text { to } \\
1.636 \\
\text { inches }\end{array}$ & $\begin{array}{c}1.636 \\
\text { to } \\
1.833 \\
\text { inches }\end{array}$ & $\begin{array}{c}1.833 \\
\text { to } \\
2.030 \\
\text { inches }\end{array}$ & $\begin{array}{c}2.030 \\
\text { to } \\
2.227 \\
\text { inches }\end{array}$ \\
\hline 0.5 & 2.34 & 1.99 & 1.79 & 1.77 & 1.62 & 1.71 & 1.76 & 1.93 & 2.33 \\
\hline 1.5 & 2.14 & 1.64 & 1.55 & 1.51 & 1.40 & 1.45 & 1.54 & 1.68 & 2.09 \\
\hline 2.5 & 2.32 & 1.93 & 1.76 & 1.78 & 1.66 & 1.76 & 1.84 & 1.94 & 2.33 \\
\hline 3.5 & 2.64 & 2.28 & 2.04 & 2.00 & 1.91 & 2.03 & 2.07 & 2.21 & 2.85 \\
\hline 4.5 & 3.09 & 2.51 & 2.38 & 2.32 & 2.15 & 2.27 & 2.39 & 2.62 & 3.15 \\
\hline 5.5 & 3.25 & 2.82 & 2.61 & 2.53 & 2.42 & 2.57 & 2.58 & 2.70 & 3.38 \\
\hline 6.5 & 3.64 & 3.05 & 2.86 & 2.75 & 2.60 & 2.77 & 2.91 & 2.95 & 3.64 \\
\hline 7.5 & 3.89 & 3.28 & 2.98 & 2.91 & 2.82 & 2.92 & 3.04 & 3.19 & 3.83 \\
\hline 8.5 & 4.03 & 3.22 & 3.12 & 3.04 & 2.83 & 2.98 & 3.07 & 3.37 & 4.02 \\
\hline 9.5 & 4.25 & 3.43 & 3.15 & 3.17 & 3.00 & 3.17 & 3.20 & 3.50 & 4.18 \\
\hline 10.5 & 4.26 & 3.58 & 3.24 & 3.22 & 3.08 & 3.25 & 3.36 & 3.65 & 4.35 \\
\hline 11.5 & 4.33 & 3.64 & 3.35 & 3.21 & 3.11 & 3.29 & 3.24 & 3.63 & 4.26 \\
\hline 12.5 & 4.37 & 3.54 & 3.37 & 3.21 & 3.17 & 3.38 & 3.30 & 3.68 & 4.49 \\
\hline 13.5 & 4.30 & 3.61 & 3.32 & 3.23 & 3.13 & 3.27 & 3.38 & 3.69 & 4.35 \\
\hline 14.5 & 4.24 & 3.60 & 3.34 & 3.13 & 3.07 & 3.22 & 3.31 & 3.57 & 4.28 \\
\hline 15.5 & 4.07 & 3.53 & 3.21 & 3.10 & 2.91 & 3.13 & 3.20 & 3.33 & 4.11 \\
\hline 16.5 & 3.92 & 3.35 & 3.08 & 3.07 & 2.85 & 2.94 & 3.13 & 3.38 & 3.93 \\
\hline 17.5 & 3.67 & 3.15 & 2.97 & 2.78 & 2.69 & 2.79 & 2.93 & 3.18 & 3.75 \\
\hline 18.5 & 3.56 & 2.92 & 2.68 & 2.62 & 2.53 & 2.67 & 2.73 & 2.86 & 3.51 \\
\hline 19.5 & 3.12 & 2.62 & 2.48 & 2.38 & 2.30 & 2.44 & 2.44 & 2.59 & 3.28 \\
\hline 20.5 & 2.96 & 2.42 & 2.27 & 2.20 & 2.08 & 2.23 & 2.29 & 2.55 & 2.99 \\
\hline 21.5 & 2.69 & 2.22 & 2.02 & 1.96 & 1.89 & 1.89 & 1.97 & 2.16 & 2.57 \\
\hline 22.5 & 2.46 & 1.93 & 1.75 & 1.71 & 1.62 & 1.70 & 1.75 & 1.88 & 2.28 \\
\hline 23.5 & 2.93 & 2.32 & 2.22 & 2.10 & 2.01 & 2.10 & 2.17 & 2.29 & 2.89 \\
\hline Ax. Avg. & 3.44 & 2.86 & 2.65 & 2.57 & 2.45 & 2.58 & 2.65 & 2.86 & 3.45 \\
\hline
\end{tabular}

POWER DENSITY VALUES NORMALIZED TO A CORE POWER OF 11.57 MW AND ASSUME ALL FISSION ENERGY (EXCLUDING GAMMA-HEATING OF EX-CORE COMPONENTS) IS DEPOSITED IN THE FUEL 
Table B-6. Power Density Data (kW/cc) in Fuel Plate 6 in Prototypic Conditions (continued).

Plate 6, Core Position X4/X8, 173 MWd Burnup, Equilibrium Xenon Conditions

\begin{tabular}{|c|c|c|c|c|c|c|c|c|c|}
\hline $\begin{array}{c}\text { Axial } \\
\text { position } \\
\text { from } \\
\text { top of } \\
\text { fuel } \\
\text { (inches) }\end{array}$ & $\begin{array}{c}0.000 \\
\text { to } \\
0.197 \\
\text { inches }\end{array}$ & $\begin{array}{c}0.197 \\
\text { to } \\
0.394 \\
\text { inches }\end{array}$ & $\begin{array}{c}0.394 \\
\text { to } \\
0.591 \\
\text { inches }\end{array}$ & $\begin{array}{c}0.591 \\
\text { to } \\
0.939 \\
\text { inches }\end{array}$ & $\begin{array}{c}0.939 \\
\text { to } \\
\text { thches } \\
\text { inch }\end{array}$ & $\begin{array}{c}1.288 \\
\text { to } \\
1.636 \\
\text { inches }\end{array}$ & $\begin{array}{c}1.636 \\
\text { to } \\
1.833 \\
\text { inches }\end{array}$ & $\begin{array}{c}1.833 \\
\text { to } \\
2.030 \\
\text { inches }\end{array}$ & $\begin{array}{c}2.030 \\
\text { to } \\
\text { inches }\end{array}$ \\
\hline 1.5 & 2.27 & 1.94 & 1.73 & 1.72 & 1.63 & 1.71 & 1.78 & 1.85 & 2.37 \\
\hline 2.5 & 2.35 & 1.64 & 1.51 & 1.46 & 1.43 & 1.52 & 1.54 & 1.69 & 2.02 \\
\hline 3.5 & 2.80 & 2.33 & 2.08 & 2.02 & 1.93 & 2.07 & 2.04 & 2.27 & 2.67 \\
\hline 4.5 & 3.04 & 2.56 & 2.34 & 2.22 & 2.14 & 2.30 & 2.32 & 2.56 & 2.99 \\
\hline 5.5 & 3.33 & 2.82 & 2.62 & 2.54 & 2.44 & 2.49 & 2.57 & 2.76 & 3.30 \\
\hline 6.5 & 3.57 & 3.04 & 2.72 & 2.68 & 2.54 & 2.65 & 2.83 & 3.02 & 3.47 \\
\hline 7.5 & 3.75 & 3.10 & 2.91 & 2.82 & 2.67 & 2.73 & 2.92 & 3.15 & 3.72 \\
\hline 8.5 & 3.96 & 3.31 & 3.14 & 3.00 & 2.84 & 2.95 & 3.04 & 3.32 & 3.97 \\
\hline 9.5 & 4.08 & 3.32 & 3.21 & 3.05 & 2.91 & 3.01 & 3.18 & 3.39 & 4.00 \\
\hline 10.5 & 4.15 & 3.45 & 3.19 & 3.16 & 3.08 & 3.24 & 3.17 & 3.44 & 4.03 \\
\hline 11.5 & 4.28 & 3.45 & 3.34 & 3.13 & 3.10 & 3.22 & 3.31 & 3.53 & 4.28 \\
\hline 12.5 & 4.22 & 3.53 & 3.30 & 3.20 & 3.03 & 3.22 & 3.31 & 3.63 & 4.16 \\
\hline 13.5 & 4.19 & 3.52 & 3.27 & 3.18 & 3.01 & 3.19 & 3.33 & 3.52 & 4.28 \\
\hline 14.5 & 4.06 & 3.38 & 3.18 & 3.07 & 2.97 & 3.08 & 3.16 & 3.42 & 4.06 \\
\hline 15.5 & 3.99 & 3.36 & 3.03 & 2.93 & 2.87 & 3.00 & 3.05 & 3.35 & 4.11 \\
\hline 16.5 & 3.72 & 3.19 & 2.98 & 2.87 & 2.78 & 2.96 & 2.98 & 3.17 & 3.77 \\
\hline 17.5 & 3.56 & 3.04 & 2.84 & 2.78 & 2.64 & 2.77 & 2.89 & 3.05 & 3.59 \\
\hline 18.5 & 3.38 & 2.83 & 2.72 & 2.55 & 2.46 & 2.59 & 2.65 & 2.89 & 3.45 \\
\hline 19.5 & 3.12 & 2.58 & 2.47 & 2.40 & 2.23 & 2.36 & 2.49 & 2.63 & 3.14 \\
\hline 20.5 & 2.86 & 2.43 & 2.25 & 2.17 & 2.07 & 2.11 & 2.25 & 2.29 & 2.87 \\
\hline 21.5 & 2.56 & 2.13 & 1.94 & 1.92 & 1.81 & 1.91 & 1.93 & 2.21 & 2.54 \\
\hline 22.5 & 2.27 & 1.88 & 1.79 & 1.66 & 1.62 & 1.69 & 1.73 & 1.87 & 2.33 \\
\hline 23.5 & 2.76 & 2.23 & 2.08 & 2.09 & 1.92 & 2.10 & 2.11 & 2.20 & 2.79 \\
\hline Ax. Avg. & 3.35 & 2.79 & 2.60 & 2.52 & 2.41 & 2.52 & 2.60 & 2.80 & 3.34 \\
\hline & & & & & & & & & 2.29 \\
\hline
\end{tabular}

POWER DENSITY VALUES NORMALIZED TO A CORE POWER OF 11.57 MW AND ASSUME ALL FISSION ENERGY (EXCLUDING GAMMA-HEATING OF EX-CORE COMPONENTS) IS DEPOSITED IN THE FUEL 
Table B-7. Power Density Data $\left(\mathrm{kW} / \mathrm{cm}^{3}\right)$ in Fuel Plate 7 in Prototypic Core.

\section{Fuel core width: 2.334 inches / Fuel core thickness: 20 mil}

Plate 7, Core Position X1/X5, 0 MWd Burnup, Xenon-Free Conditions

\begin{tabular}{|c|c|c|c|c|c|c|c|c|c|}
\hline $\begin{array}{c}\text { Axial } \\
\text { position } \\
\text { from } \\
\text { top of } \\
\text { fuel } \\
\text { (inches) } \\
\end{array}$ & $\begin{array}{c}0.000 \\
\text { to } \\
0.197 \\
\text { inches }\end{array}$ & $\begin{array}{c}0.197 \\
\text { to } \\
0.394 \\
\text { inches } \\
\end{array}$ & $\begin{array}{c}0.394 \\
\text { to } \\
0.591 \\
\text { inches }\end{array}$ & $\begin{array}{c}0.591 \\
\text { to } \\
0.975 \\
\text { inches }\end{array}$ & $\begin{array}{c}0.975 \\
\text { to } \\
1.359 \\
\text { inches }\end{array}$ & $\begin{array}{c}1.359 \\
\text { to } \\
1.743 \\
\text { inches }\end{array}$ & $\begin{array}{c}1.743 \\
\text { to } \\
1.940 \\
\text { inches }\end{array}$ & $\begin{array}{c}1.940 \\
\text { to } \\
2.137 \\
\text { inches }\end{array}$ & $\begin{array}{c}2.137 \\
\text { to } \\
2.334 \\
\text { inches } \\
\end{array}$ \\
\hline 0.5 & 1.59 & 1.32 & 1.15 & 1.05 & 1.10 & 1.12 & 1.19 & 1.18 & 1.46 \\
\hline 1.5 & 1.33 & 1.01 & 1.00 & 0.93 & 0.89 & 0.93 & 0.98 & 1.06 & 1.27 \\
\hline 2.5 & 1.62 & 1.30 & 1.23 & 1.14 & 1.10 & 1.12 & 1.20 & 1.30 & 1.58 \\
\hline 3.5 & 1.84 & 1.53 & 1.44 & 1.38 & 1.33 & 1.36 & 1.38 & 1.54 & 1.79 \\
\hline 4.5 & 2.12 & 1.76 & 1.66 & 1.66 & 1.62 & 1.64 & 1.71 & 1.81 & 2.25 \\
\hline 5.5 & 2.47 & 2.08 & 1.98 & 1.88 & 1.77 & 1.86 & 1.88 & 2.00 & 2.50 \\
\hline 6.5 & 2.81 & 2.36 & 2.20 & 2.10 & 1.99 & 2.07 & 2.18 & 2.40 & 2.77 \\
\hline 7.5 & 3.16 & 2.63 & 2.46 & 2.34 & 2.29 & 2.41 & 2.56 & 2.63 & 3.17 \\
\hline 8.5 & 3.60 & 3.01 & 2.86 & 2.79 & 2.58 & 2.65 & 2.79 & 2.93 & 3.56 \\
\hline 9.5 & 3.92 & 3.20 & 3.01 & 2.90 & 2.76 & 2.90 & 3.04 & 3.23 & 3.98 \\
\hline 10.5 & 4.17 & 3.50 & 3.29 & 3.24 & 3.09 & 3.15 & 3.35 & 3.49 & 4.14 \\
\hline 11.5 & 4.55 & 3.70 & 3.41 & 3.32 & 3.20 & 3.40 & 3.42 & 3.82 & 4.53 \\
\hline 12.5 & 4.75 & 3.89 & 3.56 & 3.51 & 3.37 & 3.48 & 3.53 & 3.87 & 4.64 \\
\hline 13.5 & 4.91 & 4.16 & 3.68 & 3.61 & 3.45 & 3.65 & 3.77 & 4.17 & 4.94 \\
\hline 14.5 & 4.87 & 4.14 & 3.77 & 3.72 & 3.57 & 3.68 & 3.76 & 4.11 & 4.95 \\
\hline 15.5 & 4.95 & 4.08 & 3.77 & 3.59 & 3.49 & 3.73 & 3.82 & 4.14 & 4.87 \\
\hline 16.5 & 4.75 & 3.95 & 3.68 & 3.56 & 3.42 & 3.62 & 3.79 & 4.05 & 4.93 \\
\hline 17.5 & 4.52 & 3.88 & 3.53 & 3.52 & 3.33 & 3.40 & 3.57 & 3.78 & 4.62 \\
\hline 18.5 & 4.27 & 3.67 & 3.44 & 3.33 & 3.21 & 3.30 & 3.44 & 3.63 & 4.44 \\
\hline 19.5 & 4.10 & 3.37 & 3.17 & 3.09 & 2.96 & 3.12 & 3.13 & 3.33 & 4.09 \\
\hline 20.5 & 3.74 & 3.14 & 2.97 & 2.92 & 2.76 & 2.82 & 2.95 & 3.17 & 3.84 \\
\hline 21.5 & 3.41 & 2.78 & 2.63 & 2.51 & 2.42 & 2.55 & 2.63 & 2.73 & 3.31 \\
\hline 22.5 & 3.06 & 2.51 & 2.24 & 2.16 & 2.02 & 2.16 & 2.24 & 2.30 & 3.05 \\
\hline 23.5 & 3.84 & 3.13 & 2.79 & 2.76 & 2.66 & 2.73 & 2.83 & 3.03 & 3.76 \\
\hline Ax. Avg. & 3.52 & 2.92 & 2.70 & 2.63 & 2.52 & 2.62 & 2.71 & 2.90 & 3.52 \\
\hline
\end{tabular}

POWER DENSITY VALUES NORMALIZED TO A CORE POWER OF 11.57 MW AND ASSUME ALL FISSION ENERGY (EXCLUDING GAMMA-HEATING OF EX-CORE COMPONENTS) IS DEPOSITED IN THE FUEL 
Table B-7. Power Density Data (kW/cc) in Fuel Plate 7 in Prototypic Conditions (continued).

Plate 7, Core Position X1/X5, 3 MWd Burnup, Equilibrium Xenon Conditions

\begin{tabular}{|c|c|c|c|c|c|c|c|c|c|}
\hline $\begin{array}{c}\text { Axial } \\
\text { position } \\
\text { from } \\
\text { top of } \\
\text { fuel } \\
\text { (inches) }\end{array}$ & $\begin{array}{c}0.000 \\
\text { to } \\
0.197 \\
\text { inches }\end{array}$ & $\begin{array}{c}0.197 \\
\text { to } \\
0.394 \\
\text { inches }\end{array}$ & $\begin{array}{c}0.394 \\
\text { to } \\
0.591 \\
\text { inches }\end{array}$ & $\begin{array}{c}0.591 \\
\text { to } \\
0.975 \\
\text { inches }\end{array}$ & $\begin{array}{c}0.975 \\
\text { to } \\
\text { t.359 } \\
\text { inches }\end{array}$ & $\begin{array}{c}1.359 \\
\text { to } \\
1.743 \\
\text { inches }\end{array}$ & $\begin{array}{c}1.743 \\
\text { to } \\
1.940 \\
\text { inches }\end{array}$ & $\begin{array}{c}1.940 \\
\text { to } \\
2.137 \\
\text { inches }\end{array}$ & $\begin{array}{c}2.137 \\
\text { to } \\
\text { inches }\end{array}$ \\
\hline 1.5 & 2.30 & 1.85 & 1.64 & 1.66 & 1.66 & 1.67 & 1.71 & 1.82 & 2.21 \\
\hline 2.5 & 2.26 & 1.94 & 1.76 & 1.69 & 1.60 & 1.66 & 1.74 & 1.92 & 2.26 \\
\hline 3.5 & 2.57 & 2.14 & 2.01 & 1.97 & 1.81 & 1.92 & 2.10 & 2.24 & 2.62 \\
\hline 4.5 & 2.92 & 2.49 & 2.31 & 2.23 & 2.15 & 2.19 & 2.20 & 2.37 & 3.02 \\
\hline 5.5 & 3.20 & 2.72 & 2.47 & 2.40 & 2.32 & 2.40 & 2.50 & 2.57 & 3.13 \\
\hline 6.5 & 3.48 & 2.94 & 2.73 & 2.67 & 2.54 & 2.61 & 2.61 & 2.89 & 3.48 \\
\hline 7.5 & 3.70 & 3.19 & 2.95 & 2.79 & 2.64 & 2.74 & 2.90 & 3.10 & 3.71 \\
\hline 8.5 & 3.96 & 3.21 & 3.04 & 2.91 & 2.82 & 2.98 & 3.02 & 3.24 & 3.91 \\
\hline 9.5 & 4.08 & 3.38 & 3.16 & 3.07 & 2.85 & 3.04 & 3.15 & 3.41 & 4.15 \\
\hline 10.5 & 4.25 & 3.47 & 3.26 & 3.12 & 2.90 & 3.13 & 3.24 & 3.46 & 4.13 \\
\hline 11.5 & 4.26 & 3.50 & 3.27 & 3.15 & 2.98 & 3.16 & 3.20 & 3.50 & 4.24 \\
\hline 12.5 & 4.34 & 3.50 & 3.20 & 3.18 & 3.03 & 3.14 & 3.27 & 3.51 & 4.28 \\
\hline 13.5 & 4.21 & 3.41 & 3.22 & 3.14 & 2.97 & 3.09 & 3.29 & 3.50 & 4.28 \\
\hline 14.5 & 4.01 & 3.46 & 3.18 & 3.08 & 2.96 & 3.14 & 3.19 & 3.42 & 4.10 \\
\hline 15.5 & 4.01 & 3.41 & 3.04 & 2.96 & 2.86 & 3.01 & 3.16 & 3.30 & 3.95 \\
\hline 16.5 & 3.90 & 3.08 & 2.99 & 2.88 & 2.77 & 2.83 & 3.00 & 3.22 & 3.75 \\
\hline 17.5 & 3.59 & 3.16 & 2.82 & 2.78 & 2.55 & 2.72 & 2.82 & 3.01 & 3.60 \\
\hline 18.5 & 3.33 & 2.80 & 2.60 & 2.55 & 2.44 & 2.59 & 2.70 & 2.85 & 3.38 \\
\hline 19.5 & 3.09 & 2.66 & 2.40 & 2.40 & 2.30 & 2.33 & 2.44 & 2.65 & 3.05 \\
\hline 20.5 & 2.86 & 2.31 & 2.20 & 2.16 & 1.99 & 2.13 & 2.15 & 2.31 & 2.81 \\
\hline 21.5 & 2.56 & 2.05 & 1.97 & 1.86 & 1.80 & 1.82 & 1.99 & 2.09 & 2.49 \\
\hline 22.5 & 2.19 & 1.87 & 1.64 & 1.59 & 1.52 & 1.61 & 1.67 & 1.88 & 2.22 \\
\hline 23.5 & 2.90 & 2.24 & 2.05 & 2.07 & 2.00 & 2.06 & 2.09 & 2.25 & 2.85 \\
\hline Ax. Avg. & 3.33 & 2.77 & 2.56 & 2.49 & 2.37 & 2.48 & 2.57 & 2.75 & 3.32 \\
\hline & & & & & & & & & 1.43 \\
\hline
\end{tabular}

POWER DENSITY VALUES NORMALIZED TO A CORE POWER OF 11.57 MW AND ASSUME ALL FISSION ENERGY (EXCLUDING GAMMA-HEATING OF EX-CORE COMPONENTS) IS DEPOSITED IN THE FUEL 
Table B-7. Power Density Data (kW/cc) in Fuel Plate 7 in Prototypic Conditions (continued).

Plate 7, Core Position X3/X7, 80 MWd Burnup, Equilibrium Xenon Conditions

\begin{tabular}{|c|c|c|c|c|c|c|c|c|c|}
\hline $\begin{array}{l}\text { Axial } \\
\text { position } \\
\text { from } \\
\text { top of } \\
\text { fuel } \\
\text { (inches) }\end{array}$ & $\begin{array}{c}0.000 \\
\text { to } \\
0.197 \\
\text { inches } \\
\end{array}$ & $\begin{array}{c}0.197 \\
\text { to } \\
0.394 \\
\text { inches } \\
\end{array}$ & $\begin{array}{c}0.394 \\
\text { to } \\
0.591 \\
\text { inches }\end{array}$ & $\begin{array}{c}0.591 \\
\text { to } \\
0.975 \\
\text { inches } \\
\end{array}$ & $\begin{array}{c}0.975 \\
\text { to } \\
1.359 \\
\text { inches }\end{array}$ & $\begin{array}{c}1.359 \\
\text { to } \\
1.743 \\
\text { inches }\end{array}$ & $\begin{array}{c}1.743 \\
\text { to } \\
1.940 \\
\text { inches }\end{array}$ & $\begin{array}{c}1.940 \\
\text { to } \\
2.137 \\
\text { inches }\end{array}$ & $\begin{array}{c}2.137 \\
\text { to } \\
2.334 \\
\text { inches } \\
\end{array}$ \\
\hline 0.5 & 2.21 & 1.85 & 1.76 & 1.68 & 1.60 & 1.70 & 1.70 & 1.82 & 2.27 \\
\hline 1.5 & 1.95 & 1.63 & 1.45 & 1.36 & 1.31 & 1.39 & 1.42 & 1.55 & 1.92 \\
\hline 2.5 & 2.20 & 1.77 & 1.67 & 1.58 & 1.54 & 1.63 & 1.67 & 1.84 & 2.21 \\
\hline 3.5 & 2.50 & 2.15 & 1.98 & 1.86 & 1.76 & 1.89 & 2.03 & 2.18 & 2.55 \\
\hline 4.5 & 2.95 & 2.38 & 2.21 & 2.19 & 2.06 & 2.10 & 2.18 & 2.35 & 2.88 \\
\hline 5.5 & 3.30 & 2.76 & 2.51 & 2.38 & 2.32 & 2.37 & 2.41 & 2.71 & 3.27 \\
\hline 6.5 & 3.38 & 2.81 & 2.67 & 2.54 & 2.38 & 2.56 & 2.54 & 2.85 & 3.47 \\
\hline 7.5 & 3.59 & 2.97 & 2.83 & 2.67 & 2.57 & 2.67 & 2.83 & 3.01 & 3.63 \\
\hline 8.5 & 3.85 & 3.22 & 2.84 & 2.77 & 2.74 & 2.84 & 2.92 & 3.12 & 3.81 \\
\hline 9.5 & 3.88 & 3.13 & 3.04 & 2.93 & 2.79 & 2.96 & 3.08 & 3.32 & 3.92 \\
\hline 10.5 & 4.05 & 3.32 & 3.08 & 2.97 & 2.86 & 2.99 & 3.15 & 3.31 & 4.05 \\
\hline 11.5 & 4.20 & 3.40 & 3.14 & 3.04 & 2.88 & 3.07 & 3.11 & 3.37 & 4.05 \\
\hline 12.5 & 4.07 & 3.39 & 3.22 & 3.03 & 2.86 & 3.06 & 3.05 & 3.39 & 4.14 \\
\hline 13.5 & 4.19 & 3.39 & 3.16 & 3.03 & 2.86 & 3.01 & 3.04 & 3.31 & 4.01 \\
\hline 14.5 & 4.07 & 3.26 & 2.98 & 2.92 & 2.77 & 2.93 & 3.03 & 3.23 & 3.93 \\
\hline 15.5 & 3.91 & 3.22 & 2.98 & 2.87 & 2.75 & 2.86 & 2.99 & 3.19 & 3.79 \\
\hline 16.5 & 3.63 & 3.14 & 2.88 & 2.79 & 2.67 & 2.74 & 2.76 & 3.25 & 3.78 \\
\hline 17.5 & 3.59 & 2.91 & 2.67 & 2.64 & 2.47 & 2.62 & 2.66 & 2.89 & 3.51 \\
\hline 18.5 & 3.27 & 2.73 & 2.50 & 2.45 & 2.37 & 2.45 & 2.62 & 2.71 & 3.26 \\
\hline 19.5 & 2.96 & 2.55 & 2.29 & 2.27 & 2.15 & 2.25 & 2.32 & 2.48 & 2.94 \\
\hline 20.5 & 2.73 & 2.26 & 2.15 & 2.03 & 1.98 & 2.06 & 2.10 & 2.31 & 2.73 \\
\hline 21.5 & 2.48 & 2.03 & 1.85 & 1.80 & 1.70 & 1.79 & 1.86 & 2.03 & 2.41 \\
\hline 22.5 & 2.22 & 1.77 & 1.63 & 1.57 & 1.47 & 1.58 & 1.66 & 1.81 & 2.15 \\
\hline 23.5 & 2.79 & 2.22 & 1.90 & 1.99 & 1.90 & 1.97 & 2.07 & 2.32 & 2.75 \\
\hline Ax. Avg. & 3.25 & 2.68 & 2.48 & 2.39 & 2.28 & 2.40 & 2.47 & 2.68 & 3.23 \\
\hline
\end{tabular}

POWER DENSITY VALUES NORMALIZED TO A CORE POWER OF 11.57 MW AND ASSUME ALL FISSION ENERGY (EXCLUDING GAMMA-HEATING OF EX-CORE COMPONENTS) IS DEPOSITED IN THE FUEL 
Table B-7. Power Density Data (kW/cc) in Fuel Plate 7 in Prototypic Conditions (continued).

Plate 7, Core Position X2/X6, 99 MWd Burnup, Equilibrium Xenon Conditions

\begin{tabular}{|c|c|c|c|c|c|c|c|c|c|}
\hline $\begin{array}{c}\text { Axial } \\
\text { position } \\
\text { from } \\
\text { top of } \\
\text { fuel } \\
\text { (inches) }\end{array}$ & $\begin{array}{c}0.000 \\
\text { to } \\
0.197 \\
\text { inches }\end{array}$ & $\begin{array}{c}0.197 \\
\text { to } \\
0.394 \\
\text { inches }\end{array}$ & $\begin{array}{c}0.394 \\
\text { to } \\
0.591 \\
\text { inches }\end{array}$ & $\begin{array}{c}0.591 \\
\text { to } \\
0.975 \\
\text { inches }\end{array}$ & $\begin{array}{c}0.975 \\
\text { to } \\
\text { t..359 } \\
\text { inches }\end{array}$ & $\begin{array}{c}1.359 \\
\text { to } \\
1.743 \\
\text { inches }\end{array}$ & $\begin{array}{c}1.743 \\
\text { to } \\
1.940 \\
\text { inches }\end{array}$ & $\begin{array}{c}1.940 \\
\text { to } \\
2.137 \\
\text { inches }\end{array}$ & $\begin{array}{c}2.137 \\
\text { to } \\
\text { inches }\end{array}$ \\
\hline 1.5 & 2.19 & 1.79 & 1.66 & 1.65 & 1.56 & 1.64 & 1.73 & 1.84 & 2.21 \\
\hline 2.5 & 2.20 & 1.81 & 1.70 & 1.62 & 1.54 & 1.60 & 1.69 & 1.88 & 2.21 \\
\hline 3.5 & 2.56 & 2.14 & 1.98 & 1.87 & 1.82 & 1.90 & 1.92 & 2.11 & 2.62 \\
\hline 4.5 & 2.84 & 2.37 & 2.20 & 2.14 & 2.06 & 2.12 & 2.20 & 2.45 & 2.87 \\
\hline 5.5 & 2.98 & 2.64 & 2.50 & 2.35 & 2.24 & 2.36 & 2.46 & 2.61 & 3.04 \\
\hline 6.5 & 3.32 & 2.82 & 2.65 & 2.56 & 2.39 & 2.54 & 2.64 & 2.81 & 3.41 \\
\hline 7.5 & 3.59 & 3.02 & 2.88 & 2.70 & 2.56 & 2.68 & 2.86 & 3.04 & 3.57 \\
\hline 8.5 & 3.69 & 3.15 & 2.89 & 2.81 & 2.72 & 2.83 & 2.96 & 3.08 & 3.82 \\
\hline 9.5 & 3.92 & 3.18 & 3.02 & 2.88 & 2.83 & 2.94 & 3.03 & 3.23 & 3.87 \\
\hline 10.5 & 4.01 & 3.41 & 3.12 & 3.05 & 2.88 & 3.03 & 3.10 & 3.36 & 4.06 \\
\hline 11.5 & 4.04 & 3.31 & 3.10 & 3.03 & 2.92 & 3.02 & 3.18 & 3.30 & 4.11 \\
\hline 12.5 & 4.00 & 3.47 & 3.19 & 3.12 & 2.96 & 3.00 & 3.14 & 3.45 & 4.28 \\
\hline 13.5 & 4.01 & 3.44 & 3.10 & 2.98 & 2.87 & 3.01 & 3.15 & 3.36 & 4.06 \\
\hline 14.5 & 3.90 & 3.28 & 3.07 & 2.94 & 2.82 & 2.94 & 3.15 & 3.35 & 3.94 \\
\hline 15.5 & 3.87 & 3.24 & 2.95 & 2.86 & 2.80 & 2.84 & 2.94 & 3.06 & 3.83 \\
\hline 16.5 & 3.70 & 3.18 & 2.90 & 2.82 & 2.67 & 2.78 & 2.90 & 3.18 & 3.69 \\
\hline 17.5 & 3.46 & 2.95 & 2.76 & 2.59 & 2.46 & 2.60 & 2.76 & 2.92 & 3.45 \\
\hline 18.5 & 3.25 & 2.73 & 2.59 & 2.45 & 2.37 & 2.48 & 2.54 & 2.70 & 3.14 \\
\hline 19.5 & 2.98 & 2.50 & 2.30 & 2.26 & 2.16 & 2.28 & 2.32 & 2.55 & 3.12 \\
\hline 20.5 & 2.77 & 2.28 & 2.17 & 2.05 & 1.93 & 2.05 & 2.17 & 2.26 & 2.70 \\
\hline 21.5 & 2.43 & 2.00 & 1.90 & 1.78 & 1.69 & 1.76 & 1.86 & 2.06 & 2.51 \\
\hline 22.5 & 2.25 & 1.82 & 1.62 & 1.59 & 1.54 & 1.55 & 1.59 & 1.81 & 2.12 \\
\hline 23.5 & 2.73 & 2.24 & 2.05 & 1.97 & 1.88 & 1.97 & 2.04 & 2.19 & 2.76 \\
\hline Ax. Avg. & 3.19 & 2.68 & 2.49 & 2.39 & 2.29 & 2.39 & 2.49 & 2.67 & 3.22 \\
\hline & & & & & & & & & 1.40 \\
\hline
\end{tabular}

POWER DENSITY VALUES NORMALIZED TO A CORE POWER OF 11.57 MW AND ASSUME ALL FISSION ENERGY (EXCLUDING GAMMA-HEATING OF EX-CORE COMPONENTS) IS DEPOSITED IN THE FUEL 
Table B-7. Power Density Data (kW/cc) in Fuel Plate 7 in Prototypic Conditions (continued).

Plate 7, Core Position X4/X8, 173 MWd Burnup, Equilibrium Xenon Conditions

\begin{tabular}{|c|c|c|c|c|c|c|c|c|c|}
\hline $\begin{array}{l}\text { Axial } \\
\text { position } \\
\text { from } \\
\text { top of } \\
\text { fuel } \\
\text { (inches) }\end{array}$ & $\begin{array}{c}0.000 \\
\text { to } \\
0.197 \\
\text { inches }\end{array}$ & $\begin{array}{c}0.197 \\
\text { to } \\
0.394 \\
\text { inches }\end{array}$ & $\begin{array}{c}0.394 \\
\text { to } \\
0.591 \\
\text { inches }\end{array}$ & $\begin{array}{c}0.591 \\
\text { to } \\
0.975 \\
\text { inches }\end{array}$ & $\begin{array}{c}0.975 \\
\text { to } \\
1.359 \\
\text { inches }\end{array}$ & $\begin{array}{c}1.359 \\
\text { to } \\
1.743 \\
\text { inches }\end{array}$ & $\begin{array}{c}1.743 \\
\text { to } \\
1.940 \\
\text { inches }\end{array}$ & $\begin{array}{c}1.940 \\
\text { to } \\
2.137 \\
\text { inches }\end{array}$ & $\begin{array}{c}2.137 \\
\text { to } \\
2.334 \\
\text { inches }\end{array}$ \\
\hline 0.5 & 2.21 & 1.82 & 1.67 & 1.58 & 1.54 & 1.62 & 1.74 & 1.77 & 2.29 \\
\hline 1.5 & 1.84 & 1.49 & 1.39 & 1.35 & 1.31 & 1.44 & 1.45 & 1.59 & 1.90 \\
\hline 2.5 & 2.16 & 1.82 & 1.68 & 1.58 & 1.55 & 1.60 & 1.68 & 1.87 & 2.16 \\
\hline 3.5 & 2.47 & 2.01 & 1.89 & 1.88 & 1.81 & 1.91 & 1.89 & 2.06 & 2.50 \\
\hline 4.5 & 2.76 & 2.35 & 2.13 & 2.05 & 2.04 & 2.10 & 2.14 & 2.38 & 2.74 \\
\hline 5.5 & 3.07 & 2.63 & 2.54 & 2.28 & 2.20 & 2.32 & 2.35 & 2.59 & 3.14 \\
\hline 6.5 & 3.22 & 2.85 & 2.60 & 2.50 & 2.37 & 2.51 & 2.60 & 2.77 & 3.31 \\
\hline 7.5 & 3.52 & 2.91 & 2.74 & 2.63 & 2.50 & 2.66 & 2.75 & 3.05 & 3.55 \\
\hline 8.5 & 3.68 & 3.06 & 2.86 & 2.75 & 2.58 & 2.77 & 2.91 & 3.07 & 3.70 \\
\hline 9.5 & 3.83 & 3.16 & 2.89 & 2.85 & 2.71 & 2.83 & 3.01 & 3.17 & 3.84 \\
\hline 10.5 & 3.90 & 3.34 & 2.97 & 2.93 & 2.80 & 2.92 & 3.06 & 3.28 & 3.87 \\
\hline 11.5 & 3.88 & 3.35 & 3.06 & 2.96 & 2.87 & 2.99 & 3.12 & 3.33 & 3.92 \\
\hline 12.5 & 3.94 & 3.28 & 3.16 & 2.93 & 2.77 & 2.95 & 3.13 & 3.29 & 3.95 \\
\hline 13.5 & 3.86 & 3.25 & 3.04 & 2.93 & 2.78 & 2.97 & 2.96 & 3.39 & 3.85 \\
\hline 14.5 & 3.87 & 3.24 & 2.92 & 2.83 & 2.77 & 2.84 & 2.91 & 3.22 & 3.80 \\
\hline 15.5 & 3.69 & 3.10 & 2.85 & 2.74 & 2.66 & 2.76 & 2.80 & 3.08 & 3.67 \\
\hline 16.5 & 3.56 & 2.96 & 2.84 & 2.77 & 2.60 & 2.72 & 2.80 & 3.08 & 3.52 \\
\hline 17.5 & 3.39 & 2.85 & 2.71 & 2.61 & 2.42 & 2.61 & 2.67 & 2.92 & 3.31 \\
\hline 18.5 & 3.18 & 2.67 & 2.38 & 2.43 & 2.32 & 2.39 & 2.55 & 2.72 & 3.25 \\
\hline 19.5 & 2.91 & 2.52 & 2.33 & 2.17 & 2.10 & 2.24 & 2.28 & 2.51 & 2.85 \\
\hline 20.5 & 2.72 & 2.31 & 2.08 & 2.05 & 1.92 & 1.99 & 2.07 & 2.21 & 2.62 \\
\hline 21.5 & 2.45 & 2.06 & 1.81 & 1.80 & 1.65 & 1.76 & 1.84 & 1.96 & 2.37 \\
\hline 22.5 & 2.16 & 1.73 & 1.60 & 1.52 & 1.49 & 1.54 & 1.64 & 1.76 & 2.15 \\
\hline 23.5 & 2.61 & 2.11 & 1.99 & 1.97 & 1.86 & 1.94 & 2.04 & 2.13 & 2.60 \\
\hline Ax. Avg. & 3.12 & 2.62 & 2.42 & 2.34 & 2.23 & 2.35 & 2.43 & 2.63 & 3.12 \\
\hline
\end{tabular}

POWER DENSITY VALUES NORMALIZED TO A CORE POWER OF 11.57 MW AND ASSUME ALL FISSION ENERGY (EXCLUDING GAMMA-HEATING OF EX-CORE COMPONENTS) IS DEPOSITED IN THE FUEL 
Table B-8. Power Density Data $\left(\mathrm{kW} / \mathrm{cm}^{3}\right)$ in Fuel Plate 8 in Prototypic Core.

\section{Fuel core width: 2.440 inches / Fuel core thickness: 20 mil}

Plate 8, Core Position X1/X5, 0 MWd Burnup, Xenon-Free Conditions

\begin{tabular}{|c|c|c|c|c|c|c|c|c|c|}
\hline $\begin{array}{c}\text { Axial } \\
\text { position } \\
\text { from } \\
\text { top of } \\
\text { fuel } \\
\text { (inches) }\end{array}$ & $\begin{array}{c}0.000 \\
\text { to } \\
0.197 \\
\text { inches }\end{array}$ & $\begin{array}{c}0.197 \\
\text { to } \\
0.394 \\
\text { inches }\end{array}$ & $\begin{array}{c}0.394 \\
\text { to } \\
0.591 \\
\text { inches }\end{array}$ & $\begin{array}{c}0.591 \\
\text { to } \\
\text { inches }\end{array}$ & $\begin{array}{c}1.010 \\
\text { to } \\
1.430 \\
\text { inches }\end{array}$ & $\begin{array}{c}1.430 \\
\text { to } \\
1.850 \\
\text { inches }\end{array}$ & $\begin{array}{c}\text { 1.850 } \\
\text { to } \\
2.047 \\
\text { inches }\end{array}$ & $\begin{array}{c}2.047 \\
\text { to } \\
2.243 \\
\text { inches }\end{array}$ & $\begin{array}{c}2.243 \\
\text { to } \\
\text { inches }\end{array}$ \\
\hline 1.5 & 1.50 & 1.17 & 1.12 & 1.05 & 1.04 & 1.05 & 1.10 & 1.22 & 1.37 \\
\hline 2.5 & 1.25 & 1.08 & 0.95 & 0.91 & 0.86 & 0.87 & 0.91 & 0.96 & 1.24 \\
\hline 3.5 & 1.68 & 1.45 & 1.36 & 1.32 & 1.24 & 1.34 & 1.28 & 1.37 & 1.71 \\
\hline 4.5 & 1.99 & 1.73 & 1.55 & 1.55 & 1.48 & 1.54 & 1.67 & 1.68 & 1.93 \\
\hline 5.5 & 2.30 & 1.93 & 1.80 & 1.79 & 1.71 & 1.73 & 1.83 & 2.01 & 2.37 \\
\hline 6.5 & 2.64 & 2.20 & 2.01 & 1.99 & 1.92 & 2.01 & 2.03 & 2.30 & 2.71 \\
\hline 7.5 & 2.96 & 2.59 & 2.43 & 2.28 & 2.16 & 2.27 & 2.32 & 2.58 & 2.94 \\
\hline 8.5 & 3.28 & 2.79 & 2.59 & 2.51 & 2.44 & 2.54 & 2.62 & 2.84 & 3.34 \\
\hline 9.5 & 3.62 & 3.12 & 2.84 & 2.83 & 2.67 & 2.75 & 2.89 & 3.03 & 3.72 \\
\hline 10.5 & 3.97 & 3.44 & 3.27 & 2.98 & 2.89 & 2.99 & 3.10 & 3.30 & 3.98 \\
\hline 11.5 & 4.33 & 3.50 & 3.33 & 3.18 & 3.04 & 3.18 & 3.34 & 3.52 & 4.24 \\
\hline 12.5 & 4.45 & 3.76 & 3.30 & 3.29 & 3.14 & 3.30 & 3.44 & 3.72 & 4.35 \\
\hline 13.5 & 4.60 & 3.94 & 3.60 & 3.44 & 3.23 & 3.45 & 3.56 & 3.88 & 4.53 \\
\hline 14.5 & 4.60 & 3.84 & 3.54 & 3.50 & 3.29 & 3.46 & 3.64 & 3.81 & 4.68 \\
\hline 15.5 & 4.63 & 3.84 & 3.59 & 3.54 & 3.36 & 3.47 & 3.54 & 3.87 & 4.75 \\
\hline 16.5 & 4.48 & 3.80 & 3.47 & 3.43 & 3.29 & 3.37 & 3.59 & 3.79 & 4.51 \\
\hline 17.5 & 4.41 & 3.80 & 3.43 & 3.28 & 3.16 & 3.25 & 3.45 & 3.61 & 4.41 \\
\hline 18.5 & 4.10 & 3.52 & 3.25 & 3.22 & 3.03 & 3.18 & 3.30 & 3.56 & 4.17 \\
\hline 19.5 & 3.97 & 3.24 & 3.06 & 2.98 & 2.83 & 2.95 & 3.01 & 3.33 & 3.89 \\
\hline 20.5 & 3.44 & 3.13 & 2.77 & 2.75 & 2.62 & 2.72 & 2.80 & 3.04 & 3.55 \\
\hline 21.5 & 3.21 & 2.71 & 2.49 & 2.37 & 2.24 & 2.34 & 2.48 & 2.64 & 3.11 \\
\hline 22.5 & 2.84 & 2.37 & 2.17 & 2.05 & 1.97 & 2.07 & 2.15 & 2.27 & 2.82 \\
\hline 23.5 & 3.66 & 2.94 & 2.75 & 2.64 & 2.57 & 2.61 & 2.72 & 2.99 & 3.72 \\
\hline Ax. Avg. & 3.31 & 2.79 & 2.58 & 2.50 & 2.38 & 2.48 & 2.58 & 2.77 & 3.31 \\
\hline & & & & & & & & & 1.03 \\
\hline
\end{tabular}

POWER DENSITY VALUES NORMALIZED TO A CORE POWER OF 11.57 MW AND ASSUME ALL FISSION ENERGY (EXCLUDING GAMMA-HEATING OF EX-CORE COMPONENTS) IS DEPOSITED IN THE FUEL 
Table B-8. Power Density Data (kW/cc) in Fuel Plate 8 in Prototypic Conditions (continued).

Plate 8, Core Position X1/X5, 3 MWd Burnup, Equilibrium Xenon Conditions

\begin{tabular}{|c|c|c|c|c|c|c|c|c|c|}
\hline $\begin{array}{l}\text { Axial } \\
\text { position } \\
\text { from } \\
\text { top of } \\
\text { fuel } \\
\text { (inches) }\end{array}$ & $\begin{array}{c}0.000 \\
\text { to } \\
0.197 \\
\text { inches }\end{array}$ & $\begin{array}{c}0.197 \\
\text { to } \\
0.394 \\
\text { inches }\end{array}$ & $\begin{array}{c}0.394 \\
\text { to } \\
0.591 \\
\text { inches }\end{array}$ & $\begin{array}{c}0.591 \\
\text { to } \\
1.010 \\
\text { inches }\end{array}$ & $\begin{array}{c}1.010 \\
\text { to } \\
1.430 \\
\text { inches }\end{array}$ & $\begin{array}{c}1.430 \\
\text { to } \\
1.850 \\
\text { inches }\end{array}$ & $\begin{array}{c}1.850 \\
\text { to } \\
2.047 \\
\text { inches }\end{array}$ & $\begin{array}{c}2.047 \\
\text { to } \\
2.243 \\
\text { inches }\end{array}$ & $\begin{array}{c}2.243 \\
\text { to } \\
2.440 \\
\text { inches }\end{array}$ \\
\hline 0.5 & 2.25 & 1.76 & 1.66 & 1.63 & 1.60 & 1.62 & 1.64 & 1.77 & 2.14 \\
\hline 1.5 & 1.87 & 1.49 & 1.37 & 1.33 & 1.29 & 1.35 & 1.40 & 1.48 & 1.94 \\
\hline 2.5 & 2.12 & 1.78 & 1.63 & 1.57 & 1.52 & 1.60 & 1.63 & 1.77 & 2.08 \\
\hline 3.5 & 2.49 & 2.06 & 1.82 & 1.87 & 1.77 & 1.87 & 1.98 & 2.17 & 2.51 \\
\hline 4.5 & 2.86 & 2.33 & 2.16 & 2.10 & 2.03 & 2.11 & 2.06 & 2.35 & 2.74 \\
\hline 5.5 & 3.10 & 2.54 & 2.37 & 2.32 & 2.21 & 2.30 & 2.45 & 2.55 & 3.10 \\
\hline 6.5 & 3.31 & 2.77 & 2.56 & 2.52 & 2.39 & 2.50 & 2.58 & 2.78 & 3.37 \\
\hline 7.5 & 3.56 & 2.92 & 2.77 & 2.68 & 2.57 & 2.66 & 2.78 & 2.93 & 3.60 \\
\hline 8.5 & 3.75 & 2.99 & 2.83 & 2.84 & 2.63 & 2.85 & 2.90 & 3.12 & 3.73 \\
\hline 9.5 & 3.95 & 3.13 & 2.95 & 2.90 & 2.77 & 2.87 & 2.95 & 3.26 & 3.79 \\
\hline 10.5 & 4.05 & 3.26 & 3.09 & 2.95 & 2.75 & 2.96 & 3.05 & 3.30 & 3.94 \\
\hline 11.5 & 3.98 & 3.40 & 3.07 & 2.98 & 2.84 & 2.99 & 3.10 & 3.31 & 3.93 \\
\hline 12.5 & 4.02 & 3.25 & 3.19 & 3.02 & 2.89 & 3.02 & 3.08 & 3.33 & 4.02 \\
\hline 13.5 & 4.04 & 3.30 & 3.02 & 2.99 & 2.84 & 2.95 & 3.13 & 3.45 & 3.94 \\
\hline 14.5 & 3.92 & 3.22 & 3.03 & 2.96 & 2.92 & 2.96 & 3.00 & 3.32 & 3.94 \\
\hline 15.5 & 3.76 & 3.23 & 2.98 & 2.87 & 2.70 & 2.89 & 3.03 & 3.16 & 3.71 \\
\hline 16.5 & 3.68 & 3.09 & 2.87 & 2.80 & 2.61 & 2.73 & 2.80 & 3.01 & 3.64 \\
\hline 17.5 & 3.35 & 2.98 & 2.66 & 2.63 & 2.49 & 2.57 & 2.72 & 2.86 & 3.35 \\
\hline 18.5 & 3.28 & 2.71 & 2.50 & 2.42 & 2.31 & 2.45 & 2.56 & 2.66 & 3.25 \\
\hline 19.5 & 2.94 & 2.42 & 2.32 & 2.27 & 2.22 & 2.25 & 2.33 & 2.45 & 3.02 \\
\hline 20.5 & 2.65 & 2.25 & 2.07 & 2.03 & 1.89 & 2.04 & 2.13 & 2.25 & 2.68 \\
\hline 21.5 & 2.44 & 2.05 & 1.83 & 1.78 & 1.69 & 1.79 & 1.82 & 2.04 & 2.39 \\
\hline 22.5 & 2.17 & 1.72 & 1.54 & 1.49 & 1.50 & 1.53 & 1.62 & 1.79 & 2.12 \\
\hline 23.5 & 2.73 & 2.23 & 2.06 & 1.97 & 1.89 & 1.96 & 2.01 & 2.15 & 2.75 \\
\hline Ax. Avg. & 3.18 & 2.62 & 2.43 & 2.37 & 2.26 & 2.37 & 2.45 & 2.64 & 3.15 \\
\hline
\end{tabular}

POWER DENSITY VALUES NORMALIZED TO A CORE POWER OF 11.57 MW AND ASSUME ALL FISSION ENERGY (EXCLUDING GAMMA-HEATING OF EX-CORE COMPONENTS) IS DEPOSITED IN THE FUEL 
Table B-8. Power Density Data (kW/cc) in Fuel Plate 8 in Prototypic Conditions (continued).

Plate 8, Core Position X3/X7, 80 MWd Burnup, Equilibrium Xenon Conditions

\begin{tabular}{|c|c|c|c|c|c|c|c|c|c|}
\hline $\begin{array}{l}\text { Axial } \\
\text { position } \\
\text { from } \\
\text { top of } \\
\text { fuel } \\
\text { (inches) }\end{array}$ & $\begin{array}{c}0.000 \\
\text { to } \\
0.197 \\
\text { inches }\end{array}$ & $\begin{array}{c}0.197 \\
\text { to } \\
0.394 \\
\text { inches }\end{array}$ & $\begin{array}{c}0.394 \\
\text { to } \\
0.591 \\
\text { inches }\end{array}$ & $\begin{array}{c}0.591 \\
\text { to } \\
1.010 \\
\text { inches }\end{array}$ & $\begin{array}{c}1.010 \\
\text { to } \\
1.430 \\
\text { inches }\end{array}$ & $\begin{array}{c}1.430 \\
\text { to } \\
1.850 \\
\text { inches }\end{array}$ & $\begin{array}{c}1.850 \\
\text { to } \\
2.047 \\
\text { inches }\end{array}$ & $\begin{array}{c}2.047 \\
\text { to } \\
2.243 \\
\text { inches }\end{array}$ & $\begin{array}{c}2.243 \\
\text { to } \\
2.440 \\
\text { inches }\end{array}$ \\
\hline 0.5 & 2.19 & 1.74 & 1.56 & 1.59 & 1.51 & 1.58 & 1.59 & 1.77 & 2.18 \\
\hline 1.5 & 1.81 & 1.50 & 1.36 & 1.31 & 1.23 & 1.28 & 1.41 & 1.47 & 1.79 \\
\hline 2.5 & 2.15 & 1.73 & 1.61 & 1.56 & 1.50 & 1.58 & 1.62 & 1.82 & 2.08 \\
\hline 3.5 & 2.37 & 2.04 & 1.88 & 1.88 & 1.76 & 1.84 & 1.93 & 2.04 & 2.51 \\
\hline 4.5 & 2.77 & 2.29 & 2.12 & 2.06 & 1.92 & 1.99 & 2.13 & 2.35 & 2.81 \\
\hline 5.5 & 2.94 & 2.55 & 2.37 & 2.28 & 2.16 & 2.24 & 2.34 & 2.42 & 3.04 \\
\hline 6.5 & 3.20 & 2.66 & 2.53 & 2.46 & 2.33 & 2.44 & 2.50 & 2.70 & 3.25 \\
\hline 7.5 & 3.47 & 2.76 & 2.70 & 2.57 & 2.43 & 2.55 & 2.73 & 2.80 & 3.48 \\
\hline 8.5 & 3.58 & 2.94 & 2.73 & 2.66 & 2.64 & 2.70 & 2.71 & 3.01 & 3.64 \\
\hline 9.5 & 3.73 & 3.02 & 2.85 & 2.80 & 2.66 & 2.86 & 2.91 & 3.09 & 3.64 \\
\hline 10.5 & 3.85 & 3.18 & 3.02 & 2.81 & 2.76 & 2.84 & 2.98 & 3.21 & 3.81 \\
\hline 11.5 & 3.86 & 3.35 & 3.03 & 2.95 & 2.70 & 2.95 & 3.02 & 3.18 & 3.72 \\
\hline 12.5 & 3.88 & 3.13 & 2.96 & 2.91 & 2.78 & 2.94 & 3.01 & 3.18 & 3.95 \\
\hline 13.5 & 3.92 & 3.17 & 3.03 & 2.84 & 2.76 & 2.85 & 2.98 & 3.20 & 3.78 \\
\hline 14.5 & 3.70 & 3.26 & 3.04 & 2.77 & 2.70 & 2.83 & 2.95 & 3.12 & 3.79 \\
\hline 15.5 & 3.58 & 3.16 & 2.95 & 2.76 & 2.64 & 2.76 & 2.85 & 3.09 & 3.62 \\
\hline 16.5 & 3.54 & 2.87 & 2.79 & 2.67 & 2.50 & 2.68 & 2.77 & 3.00 & 3.61 \\
\hline 17.5 & 3.31 & 2.79 & 2.57 & 2.51 & 2.37 & 2.51 & 2.59 & 2.77 & 3.35 \\
\hline 18.5 & 3.10 & 2.62 & 2.47 & 2.36 & 2.18 & 2.36 & 2.46 & 2.62 & 3.09 \\
\hline 19.5 & 2.85 & 2.46 & 2.24 & 2.14 & 2.05 & 2.15 & 2.20 & 2.31 & 2.91 \\
\hline 20.5 & 2.60 & 2.20 & 1.98 & 1.90 & 1.86 & 1.96 & 1.96 & 2.12 & 2.56 \\
\hline 21.5 & 2.29 & 1.92 & 1.76 & 1.73 & 1.62 & 1.69 & 1.79 & 2.01 & 2.30 \\
\hline 22.5 & 2.11 & 1.65 & 1.51 & 1.47 & 1.43 & 1.51 & 1.65 & 1.72 & 2.01 \\
\hline 23.5 & 2.67 & 2.08 & 2.04 & 1.87 & 1.83 & 1.95 & 1.98 & 2.18 & 2.72 \\
\hline Ax. Avg. & 3.06 & 2.55 & 2.38 & 2.29 & 2.18 & 2.29 & 2.38 & 2.55 & 3.07 \\
\hline
\end{tabular}

POWER DENSITY VALUES NORMALIZED TO A CORE POWER OF 11.57 MW AND ASSUME ALL FISSION ENERGY (EXCLUDING GAMMA-HEATING OF EX-CORE COMPONENTS) IS DEPOSITED IN THE FUEL 
Table B-8. Power Density Data (kW/cc) in Fuel Plate 8 in Prototypic Conditions (continued).

Plate 8, Core Position X2/X6, 99 MWd Burnup, Equilibrium Xenon Conditions

\begin{tabular}{|c|c|c|c|c|c|c|c|c|c|}
\hline $\begin{array}{l}\text { Axial } \\
\text { position } \\
\text { from } \\
\text { top of } \\
\text { fuel } \\
\text { (inches) }\end{array}$ & $\begin{array}{c}0.000 \\
\text { to } \\
0.197 \\
\text { inches }\end{array}$ & $\begin{array}{c}0.197 \\
\text { to } \\
0.394 \\
\text { inches }\end{array}$ & $\begin{array}{c}0.394 \\
\text { to } \\
0.591 \\
\text { inches }\end{array}$ & $\begin{array}{c}0.591 \\
\text { to } \\
1.010 \\
\text { inches }\end{array}$ & $\begin{array}{c}1.010 \\
\text { to } \\
1.430 \\
\text { inches }\end{array}$ & $\begin{array}{c}1.430 \\
\text { to } \\
1.850 \\
\text { inches }\end{array}$ & $\begin{array}{c}1.850 \\
\text { to } \\
2.047 \\
\text { inches }\end{array}$ & $\begin{array}{c}2.047 \\
\text { to } \\
2.243 \\
\text { inches }\end{array}$ & $\begin{array}{c}2.243 \\
\text { to } \\
2.440 \\
\text { inches }\end{array}$ \\
\hline 0.5 & 2.11 & 1.75 & 1.58 & 1.55 & 1.46 & 1.61 & 1.57 & 1.72 & 2.11 \\
\hline 1.5 & 1.72 & 1.48 & 1.42 & 1.29 & 1.22 & 1.25 & 1.33 & 1.47 & 1.83 \\
\hline 2.5 & 2.04 & 1.83 & 1.66 & 1.56 & 1.49 & 1.53 & 1.61 & 1.79 & 2.08 \\
\hline 3.5 & 2.42 & 2.06 & 1.85 & 1.79 & 1.71 & 1.83 & 1.89 & 2.11 & 2.43 \\
\hline 4.5 & 2.72 & 2.30 & 2.10 & 2.05 & 1.92 & 2.02 & 2.14 & 2.35 & 2.69 \\
\hline 5.5 & 2.99 & 2.46 & 2.35 & 2.25 & 2.15 & 2.25 & 2.33 & 2.51 & 3.04 \\
\hline 6.5 & 3.26 & 2.70 & 2.56 & 2.39 & 2.33 & 2.43 & 2.51 & 2.75 & 3.27 \\
\hline 7.5 & 3.43 & 2.81 & 2.70 & 2.58 & 2.41 & 2.55 & 2.69 & 2.88 & 3.36 \\
\hline 8.5 & 3.54 & 2.97 & 2.76 & 2.74 & 2.54 & 2.71 & 2.87 & 2.89 & 3.61 \\
\hline 9.5 & 3.72 & 3.11 & 2.88 & 2.83 & 2.71 & 2.81 & 2.88 & 3.15 & 3.73 \\
\hline 10.5 & 3.80 & 3.14 & 2.99 & 2.89 & 2.74 & 2.95 & 2.98 & 3.16 & 3.75 \\
\hline 11.5 & 3.90 & 3.15 & 2.96 & 2.88 & 2.78 & 2.91 & 2.98 & 3.15 & 3.85 \\
\hline 12.5 & 3.88 & 3.25 & 2.94 & 2.94 & 2.82 & 2.88 & 3.06 & 3.30 & 3.83 \\
\hline 13.5 & 3.77 & 3.29 & 2.97 & 2.89 & 2.72 & 2.92 & 2.99 & 3.33 & 3.87 \\
\hline 14.5 & 3.77 & 3.13 & 3.00 & 2.83 & 2.72 & 2.83 & 2.90 & 3.11 & 3.75 \\
\hline 15.5 & 3.61 & 3.14 & 2.85 & 2.79 & 2.65 & 2.73 & 2.84 & 3.01 & 3.56 \\
\hline 16.5 & 3.49 & 2.91 & 2.78 & 2.67 & 2.51 & 2.66 & 2.80 & 3.04 & 3.55 \\
\hline 17.5 & 3.25 & 2.77 & 2.63 & 2.57 & 2.40 & 2.53 & 2.61 & 2.81 & 3.24 \\
\hline 18.5 & 3.10 & 2.62 & 2.47 & 2.40 & 2.23 & 2.34 & 2.43 & 2.66 & 3.03 \\
\hline 19.5 & 2.87 & 2.45 & 2.18 & 2.12 & 2.08 & 2.17 & 2.17 & 2.37 & 2.89 \\
\hline 20.5 & 2.67 & 2.21 & 2.04 & 1.96 & 1.82 & 1.96 & 2.00 & 2.19 & 2.59 \\
\hline 21.5 & 2.30 & 1.91 & 1.83 & 1.73 & 1.67 & 1.71 & 1.75 & 1.96 & 2.26 \\
\hline 22.5 & 2.03 & 1.65 & 1.58 & 1.53 & 1.46 & 1.51 & 1.52 & 1.67 & 2.11 \\
\hline 23.5 & 2.66 & 2.06 & 2.00 & 1.92 & 1.91 & 1.90 & 1.90 & 2.09 & 2.76 \\
\hline Ax. Avg. & 3.04 & 2.55 & 2.38 & 2.30 & 2.19 & 2.29 & 2.36 & 2.56 & 3.05 \\
\hline
\end{tabular}

POWER DENSITY VALUES NORMALIZED TO A CORE POWER OF 11.57 MW AND ASSUME ALL FISSION ENERGY (EXCLUDING GAMMA-HEATING OF EX-CORE COMPONENTS) IS DEPOSITED IN THE FUEL 
Table B-8. Power Density Data (kW/cc) in Fuel Plate 8 in Prototypic Conditions (continued).

Plate 8, Core Position X4/X8, 173 MWd Burnup, Equilibrium Xenon Conditions

\begin{tabular}{|c|c|c|c|c|c|c|c|c|c|}
\hline $\begin{array}{c}\text { Axial } \\
\text { position } \\
\text { from } \\
\text { top of } \\
\text { fuel } \\
\text { (inches) }\end{array}$ & $\begin{array}{c}0.000 \\
\text { to } \\
0.197 \\
\text { inches }\end{array}$ & $\begin{array}{c}0.197 \\
\text { to } \\
0.394 \\
\text { inches }\end{array}$ & $\begin{array}{c}0.394 \\
\text { to } \\
0.591 \\
\text { inches }\end{array}$ & $\begin{array}{c}0.591 \\
\text { to } \\
1.010 \\
\text { inches }\end{array}$ & $\begin{array}{c}1.010 \\
\text { to } \\
\text { tnches }\end{array}$ & $\begin{array}{c}1.430 \\
\text { to } \\
1.850 \\
\text { inches }\end{array}$ & $\begin{array}{c}1.850 \\
\text { to } \\
2.047 \\
\text { inches }\end{array}$ & $\begin{array}{c}2.047 \\
\text { to } \\
2.243 \\
\text { inches }\end{array}$ & $\begin{array}{c}2.243 \\
\text { to } \\
\text { inches }\end{array}$ \\
\hline 0.5 & 2.07 & 1.76 & 1.59 & 1.57 & 1.50 & 1.52 & 1.60 & 1.67 & 2.16 \\
\hline 1.5 & 1.78 & 1.44 & 1.30 & 1.28 & 1.24 & 1.27 & 1.42 & 1.47 & 1.77 \\
\hline 2.5 & 2.06 & 1.76 & 1.57 & 1.53 & 1.45 & 1.54 & 1.59 & 1.74 & 1.99 \\
\hline 3.5 & 2.45 & 1.99 & 1.84 & 1.82 & 1.73 & 1.78 & 1.83 & 1.96 & 2.26 \\
\hline 4.5 & 2.64 & 2.22 & 2.03 & 1.93 & 1.89 & 1.99 & 2.01 & 2.14 & 2.62 \\
\hline 5.5 & 2.92 & 2.46 & 2.30 & 2.21 & 2.07 & 2.19 & 2.28 & 2.41 & 2.85 \\
\hline 6.5 & 3.12 & 2.59 & 2.53 & 2.36 & 2.25 & 2.40 & 2.46 & 2.67 & 3.16 \\
\hline 7.5 & 3.36 & 2.76 & 2.66 & 2.48 & 2.39 & 2.48 & 2.66 & 2.83 & 3.32 \\
\hline 8.5 & 3.47 & 2.86 & 2.69 & 2.60 & 2.56 & 2.60 & 2.67 & 2.88 & 3.50 \\
\hline 9.5 & 3.65 & 2.95 & 2.89 & 2.75 & 2.60 & 2.68 & 2.94 & 3.06 & 3.66 \\
\hline 10.5 & 3.73 & 3.03 & 2.94 & 2.82 & 2.66 & 2.76 & 2.85 & 3.06 & 3.76 \\
\hline 11.5 & 3.72 & 3.09 & 2.95 & 2.88 & 2.73 & 2.81 & 2.89 & 3.18 & 3.87 \\
\hline 12.5 & 3.78 & 3.17 & 2.88 & 2.83 & 2.68 & 2.88 & 2.87 & 3.19 & 3.84 \\
\hline 13.5 & 3.72 & 3.10 & 2.85 & 2.78 & 2.63 & 2.81 & 2.93 & 3.18 & 3.68 \\
\hline 14.5 & 3.71 & 3.01 & 2.78 & 2.75 & 2.60 & 2.70 & 2.89 & 3.09 & 3.68 \\
\hline 15.5 & 3.46 & 2.95 & 2.71 & 2.63 & 2.53 & 2.64 & 2.72 & 2.92 & 3.49 \\
\hline 16.5 & 3.38 & 2.82 & 2.62 & 2.57 & 2.42 & 2.59 & 2.70 & 2.87 & 3.42 \\
\hline 17.5 & 3.17 & 2.73 & 2.57 & 2.46 & 2.34 & 2.44 & 2.56 & 2.73 & 3.21 \\
\hline 18.5 & 2.93 & 2.54 & 2.44 & 2.33 & 2.26 & 2.32 & 2.43 & 2.60 & 3.00 \\
\hline 19.5 & 2.79 & 2.35 & 2.20 & 2.12 & 1.95 & 2.10 & 2.17 & 2.38 & 2.86 \\
\hline 20.5 & 2.49 & 2.14 & 2.03 & 1.93 & 1.80 & 1.90 & 1.96 & 2.09 & 2.48 \\
\hline 21.5 & 2.27 & 1.89 & 1.71 & 1.70 & 1.63 & 1.72 & 1.77 & 1.92 & 2.28 \\
\hline 22.5 & 2.06 & 1.67 & 1.52 & 1.46 & 1.38 & 1.48 & 1.49 & 1.71 & 2.03 \\
\hline 23.5 & 2.55 & 2.09 & 1.97 & 1.86 & 1.72 & 1.89 & 1.87 & 2.10 & 2.55 \\
\hline Ax. Avg. & 2.97 & 2.47 & 2.32 & 2.24 & 2.13 & 2.23 & 2.32 & 2.49 & 2.98 \\
\hline & & & & & & & & & \\
\hline
\end{tabular}

POWER DENSITY VALUES NORMALIZED TO A CORE POWER OF 11.57 MW AND ASSUME ALL FISSION ENERGY (EXCLUDING GAMMA-HEATING OF EX-CORE COMPONENTS) IS DEPOSITED IN THE FUEL 
Table B-9. Power Density Data $\left(\mathrm{kW} / \mathrm{cm}^{3}\right)$ in Fuel Plate 9 in Prototypic Core.

\section{Fuel core width: 2.547 inches / Fuel core thickness: 20 mil}

Plate 9, Core Position X1/X5, 0 MWd Burnup, Xenon-Free Conditions

\begin{tabular}{|c|c|c|c|c|c|c|c|c|c|}
\hline $\begin{array}{c}\text { Axial } \\
\text { position } \\
\text { from } \\
\text { top of } \\
\text { fuel } \\
\text { (inches) }\end{array}$ & $\begin{array}{c}0.000 \\
\text { to } \\
0.197 \\
\text { inches }\end{array}$ & $\begin{array}{c}0.197 \\
\text { to } \\
0.394 \\
\text { inches }\end{array}$ & $\begin{array}{c}0.394 \\
\text { to } \\
0.591 \\
\text { inches }\end{array}$ & $\begin{array}{c}0.591 \\
\text { to } \\
\text { inches }\end{array}$ & $\begin{array}{c}1.046 \\
\text { to } \\
1.501 \\
\text { inches }\end{array}$ & $\begin{array}{c}1.501 \\
\text { to } \\
1.957 \\
\text { inches }\end{array}$ & $\begin{array}{c}1.957 \\
\text { to } \\
2.153 \\
\text { inches }\end{array}$ & $\begin{array}{c}2.153 \\
\text { to } \\
2.350 \\
\text { inches }\end{array}$ & $\begin{array}{c}2.350 \\
\text { to } \\
\text { inches }\end{array}$ \\
\hline 0.5 & 1.38 & 1.10 & 1.08 & 1.04 & 0.98 & 1.05 & 1.06 & 1.16 & 1.35 \\
\hline 1.5 & 1.20 & 1.02 & 0.93 & 0.83 & 0.84 & 0.86 & 0.86 & 0.98 & 1.12 \\
\hline 2.5 & 1.35 & 1.17 & 1.07 & 1.04 & 1.01 & 1.04 & 1.10 & 1.21 & 1.44 \\
\hline 3.5 & 1.61 & 1.36 & 1.32 & 1.25 & 1.22 & 1.26 & 1.30 & 1.34 & 1.61 \\
\hline 4.5 & 1.89 & 1.59 & 1.55 & 1.52 & 1.42 & 1.46 & 1.60 & 1.63 & 1.95 \\
\hline 5.5 & 2.16 & 1.86 & 1.71 & 1.69 & 1.56 & 1.65 & 1.76 & 1.84 & 2.21 \\
\hline 6.5 & 2.42 & 2.09 & 1.99 & 1.96 & 1.84 & 1.91 & 2.02 & 2.15 & 2.50 \\
\hline 7.5 & 2.85 & 2.42 & 2.29 & 2.18 & 2.06 & 2.15 & 2.28 & 2.43 & 2.76 \\
\hline 8.5 & 3.19 & 2.78 & 2.58 & 2.48 & 2.35 & 2.46 & 2.51 & 2.74 & 3.15 \\
\hline 9.5 & 3.53 & 2.94 & 2.70 & 2.67 & 2.57 & 2.72 & 2.76 & 2.97 & 3.54 \\
\hline 10.5 & 3.81 & 3.18 & 3.09 & 2.92 & 2.82 & 2.87 & 3.02 & 3.27 & 3.81 \\
\hline 11.5 & 4.10 & 3.38 & 3.23 & 3.10 & 2.94 & 3.07 & 3.19 & 3.56 & 4.12 \\
\hline 12.5 & 4.15 & 3.60 & 3.46 & 3.13 & 3.06 & 3.15 & 3.34 & 3.64 & 4.23 \\
\hline 13.5 & 4.39 & 3.73 & 3.52 & 3.33 & 3.18 & 3.31 & 3.49 & 3.65 & 4.41 \\
\hline 14.5 & 4.31 & 3.73 & 3.53 & 3.37 & 3.20 & 3.39 & 3.57 & 3.78 & 4.37 \\
\hline 15.5 & 4.48 & 3.76 & 3.56 & 3.38 & 3.22 & 3.35 & 3.57 & 3.72 & 4.43 \\
\hline 16.5 & 4.28 & 3.58 & 3.51 & 3.38 & 3.16 & 3.33 & 3.45 & 3.68 & 4.52 \\
\hline 17.5 & 4.14 & 3.63 & 3.37 & 3.25 & 3.10 & 3.18 & 3.33 & 3.59 & 4.21 \\
\hline 18.5 & 3.92 & 3.34 & 3.19 & 3.10 & 2.88 & 3.10 & 3.18 & 3.42 & 3.94 \\
\hline 19.5 & 3.59 & 3.16 & 2.94 & 2.86 & 2.76 & 2.85 & 3.01 & 3.26 & 3.82 \\
\hline 20.5 & 3.38 & 2.76 & 2.76 & 2.53 & 2.55 & 2.62 & 2.77 & 2.94 & 3.39 \\
\hline 21.5 & 3.01 & 2.55 & 2.43 & 2.35 & 2.20 & 2.33 & 2.39 & 2.57 & 3.06 \\
\hline 22.5 & 2.87 & 2.28 & 2.09 & 2.00 & 1.88 & 2.05 & 2.11 & 2.27 & 2.81 \\
\hline 23.5 & 3.54 & 2.86 & 2.71 & 2.62 & 2.54 & 2.54 & 2.66 & 2.89 & 3.63 \\
\hline Ax. Avg. & 3.15 & 2.66 & 2.53 & 2.42 & 2.31 & 2.40 & 2.51 & 2.70 & 3.18 \\
\hline & & & & & & & & & \\
\hline
\end{tabular}

POWER DENSITY VALUES NORMALIZED TO A CORE POWER OF 11.57 MW AND ASSUME ALL FISSION ENERGY (EXCLUDING GAMMA-HEATING OF EX-CORE COMPONENTS) IS DEPOSITED IN THE FUEL 
Table B-9. Power Density Data (kW/cc) in Fuel Plate 9 in Prototypic Conditions (continued).

Plate 9, Core Position X1/X5, 3 MWd Burnup, Equilibrium Xenon Conditions

\begin{tabular}{|c|c|c|c|c|c|c|c|c|c|}
\hline $\begin{array}{c}\text { Axial } \\
\text { position } \\
\text { from } \\
\text { top of } \\
\text { fuel } \\
\text { (inches) }\end{array}$ & $\begin{array}{c}0.000 \\
\text { to } \\
0.197 \\
\text { inches }\end{array}$ & $\begin{array}{c}0.197 \\
\text { to } \\
0.394 \\
\text { inches }\end{array}$ & $\begin{array}{c}0.394 \\
\text { to } \\
0.591 \\
\text { inches }\end{array}$ & $\begin{array}{c}0.591 \\
\text { to } \\
1.046 \\
\text { inches }\end{array}$ & $\begin{array}{c}1.046 \\
\text { to } \\
1.501 \\
\text { inches }\end{array}$ & $\begin{array}{c}1.501 \\
\text { to } \\
1.957 \\
\text { inches }\end{array}$ & $\begin{array}{c}1.957 \\
\text { to } \\
2.153 \\
\text { inches }\end{array}$ & $\begin{array}{c}2.153 \\
\text { to } \\
2.350 \\
\text { inches }\end{array}$ & $\begin{array}{c}2.350 \\
\text { to } \\
\text { inches } \\
\text { inch }\end{array}$ \\
\hline 1.5 & 2.07 & 1.67 & 1.58 & 1.59 & 1.55 & 1.57 & 1.63 & 1.71 & 2.09 \\
\hline 2.5 & 2.09 & 1.44 & 1.33 & 1.33 & 1.26 & 1.31 & 1.28 & 1.45 & 1.75 \\
\hline 3.5 & 2.33 & 1.94 & 1.85 & 1.79 & 1.69 & 1.78 & 1.88 & 1.98 & 2.45 \\
\hline 4.5 & 2.75 & 2.27 & 2.09 & 2.03 & 1.96 & 2.01 & 2.10 & 2.21 & 2.59 \\
\hline 5.5 & 2.93 & 2.46 & 2.33 & 2.21 & 2.14 & 2.22 & 2.31 & 2.49 & 2.97 \\
\hline 6.5 & 3.24 & 2.68 & 2.50 & 2.44 & 2.34 & 2.43 & 2.59 & 2.70 & 3.28 \\
\hline 7.5 & 3.40 & 2.92 & 2.70 & 2.59 & 2.46 & 2.53 & 2.66 & 2.90 & 3.50 \\
\hline 8.5 & 3.58 & 3.02 & 2.79 & 2.69 & 2.64 & 2.76 & 2.88 & 2.95 & 3.55 \\
\hline 9.5 & 3.73 & 3.04 & 2.86 & 2.81 & 2.67 & 2.88 & 2.87 & 3.12 & 3.71 \\
\hline 10.5 & 3.75 & 3.21 & 2.94 & 2.87 & 2.72 & 2.88 & 2.94 & 3.16 & 3.70 \\
\hline 11.5 & 3.93 & 3.19 & 2.97 & 2.89 & 2.83 & 2.94 & 3.09 & 3.28 & 3.87 \\
\hline 12.5 & 3.84 & 3.29 & 3.07 & 2.99 & 2.79 & 2.91 & 3.02 & 3.33 & 3.85 \\
\hline 13.5 & 3.88 & 3.21 & 2.98 & 2.91 & 2.77 & 2.89 & 3.07 & 3.22 & 3.81 \\
\hline 14.5 & 3.71 & 3.08 & 2.91 & 2.87 & 2.77 & 2.87 & 2.90 & 3.09 & 3.78 \\
\hline 15.5 & 3.72 & 3.10 & 2.83 & 2.78 & 2.59 & 2.81 & 2.94 & 3.07 & 3.59 \\
\hline 16.5 & 3.40 & 3.01 & 2.74 & 2.63 & 2.58 & 2.65 & 2.76 & 3.00 & 3.50 \\
\hline 17.5 & 3.27 & 2.79 & 2.56 & 2.52 & 2.40 & 2.50 & 2.59 & 2.75 & 3.21 \\
\hline 18.5 & 3.08 & 2.58 & 2.48 & 2.40 & 2.25 & 2.37 & 2.45 & 2.61 & 3.04 \\
\hline 19.5 & 2.89 & 2.46 & 2.33 & 2.20 & 2.11 & 2.13 & 2.27 & 2.46 & 2.91 \\
\hline 20.5 & 2.63 & 2.16 & 2.00 & 1.99 & 1.86 & 1.97 & 2.01 & 2.13 & 2.59 \\
\hline 21.5 & 2.28 & 1.90 & 1.76 & 1.68 & 1.64 & 1.71 & 1.81 & 1.95 & 2.28 \\
\hline 22.5 & 2.08 & 1.65 & 1.48 & 1.50 & 1.44 & 1.50 & 1.56 & 1.67 & 2.05 \\
\hline 23.5 & 2.65 & 2.15 & 1.91 & 1.86 & 1.86 & 1.88 & 1.94 & 2.03 & 2.66 \\
\hline Ax. Avg. & 3.04 & 2.54 & 2.36 & 2.30 & 2.20 & 2.29 & 2.38 & 2.54 & 3.03 \\
\hline & & & & & & & & & 1.53 \\
\hline
\end{tabular}

POWER DENSITY VALUES NORMALIZED TO A CORE POWER OF 11.57 MW AND ASSUME ALL FISSION ENERGY (EXCLUDING GAMMA-HEATING OF EX-CORE COMPONENTS) IS DEPOSITED IN THE FUEL 
Table B-9. Power Density Data (kW/cc) in Fuel Plate 9 in Prototypic Conditions (continued).

Plate 9, Core Position X3/X7, 80 MWd Burnup, Equilibrium Xenon Conditions

\begin{tabular}{|c|c|c|c|c|c|c|c|c|c|}
\hline $\begin{array}{c}\text { Axial } \\
\text { position } \\
\text { from } \\
\text { top of } \\
\text { fuel } \\
\text { (inches) }\end{array}$ & $\begin{array}{c}0.000 \\
\text { to } \\
0.197 \\
\text { inches }\end{array}$ & $\begin{array}{c}0.197 \\
\text { to } \\
0.394 \\
\text { inches }\end{array}$ & $\begin{array}{c}0.394 \\
\text { to } \\
0.591 \\
\text { inches }\end{array}$ & $\begin{array}{c}0.591 \\
\text { to } \\
1.046 \\
\text { inches }\end{array}$ & $\begin{array}{c}1.046 \\
\text { to } \\
1.501 \\
\text { inches }\end{array}$ & $\begin{array}{c}1.501 \\
\text { to } \\
1.957 \\
\text { inches }\end{array}$ & $\begin{array}{c}1.957 \\
\text { to } \\
2.153 \\
\text { inches }\end{array}$ & $\begin{array}{c}2.153 \\
\text { to } \\
2.350 \\
\text { inches }\end{array}$ & $\begin{array}{c}2.350 \\
\text { to } \\
\text { inches } \\
\text { inch }\end{array}$ \\
\hline 1.5 & 2.06 & 1.72 & 1.60 & 1.52 & 1.46 & 1.48 & 1.53 & 1.65 & 2.05 \\
\hline 2.5 & 1.98 & 1.69 & 1.56 & 1.52 & 1.46 & 1.54 & 1.52 & 1.67 & 2.01 \\
\hline 3.5 & 2.23 & 1.98 & 1.80 & 1.84 & 1.70 & 1.77 & 1.85 & 1.96 & 2.40 \\
\hline 4.5 & 2.63 & 2.21 & 2.03 & 1.97 & 1.88 & 1.95 & 2.01 & 2.19 & 2.59 \\
\hline 5.5 & 2.82 & 2.37 & 2.28 & 2.20 & 2.10 & 2.20 & 2.28 & 2.46 & 2.87 \\
\hline 6.5 & 3.17 & 2.62 & 2.42 & 2.37 & 2.30 & 2.37 & 2.45 & 2.62 & 3.06 \\
\hline 7.5 & 3.20 & 2.75 & 2.57 & 2.48 & 2.38 & 2.47 & 2.58 & 2.80 & 3.34 \\
\hline 8.5 & 3.49 & 2.94 & 2.64 & 2.59 & 2.54 & 2.69 & 2.69 & 2.84 & 3.42 \\
\hline 9.5 & 3.47 & 2.95 & 2.79 & 2.73 & 2.58 & 2.73 & 2.82 & 3.03 & 3.57 \\
\hline 10.5 & 3.66 & 3.09 & 2.86 & 2.75 & 2.64 & 2.80 & 2.84 & 3.01 & 3.71 \\
\hline 11.5 & 3.77 & 3.19 & 3.02 & 2.81 & 2.60 & 2.80 & 2.88 & 3.08 & 3.71 \\
\hline 12.5 & 3.69 & 3.09 & 2.90 & 2.80 & 2.70 & 2.83 & 2.82 & 3.14 & 3.72 \\
\hline 13.5 & 3.66 & 3.11 & 2.91 & 2.77 & 2.62 & 2.77 & 2.80 & 3.14 & 3.63 \\
\hline 14.5 & 3.72 & 3.07 & 2.88 & 2.72 & 2.64 & 2.73 & 2.80 & 3.00 & 3.67 \\
\hline 15.5 & 3.49 & 2.99 & 2.80 & 2.68 & 2.55 & 2.66 & 2.81 & 2.97 & 3.51 \\
\hline 16.5 & 3.39 & 2.84 & 2.64 & 2.56 & 2.49 & 2.55 & 2.61 & 2.86 & 3.36 \\
\hline 17.5 & 3.24 & 2.71 & 2.48 & 2.45 & 2.30 & 2.43 & 2.47 & 2.67 & 3.23 \\
\hline 18.5 & 2.98 & 2.54 & 2.30 & 2.28 & 2.18 & 2.28 & 2.44 & 2.55 & 3.03 \\
\hline 19.5 & 2.69 & 2.38 & 2.19 & 2.08 & 2.00 & 2.08 & 2.20 & 2.31 & 2.72 \\
\hline 20.5 & 2.51 & 2.15 & 1.94 & 1.81 & 1.82 & 1.86 & 1.93 & 2.11 & 2.43 \\
\hline 21.5 & 2.27 & 1.92 & 1.74 & 1.67 & 1.58 & 1.70 & 1.77 & 1.87 & 2.22 \\
\hline 22.5 & 2.00 & 1.58 & 1.43 & 1.45 & 1.44 & 1.45 & 1.52 & 1.67 & 2.01 \\
\hline 23.5 & 2.60 & 2.14 & 1.87 & 1.81 & 1.78 & 1.89 & 1.91 & 2.04 & 2.57 \\
\hline Ax. Avg. & 2.94 & 2.48 & 2.29 & 2.21 & 2.12 & 2.22 & 2.29 & 2.46 & 2.94 \\
\hline & & & & & & & & & 1.34 \\
\hline
\end{tabular}

POWER DENSITY VALUES NORMALIZED TO A CORE POWER OF 11.57 MW AND ASSUME ALL FISSION ENERGY (EXCLUDING GAMMA-HEATING OF EX-CORE COMPONENTS) IS DEPOSITED IN THE FUEL 
Table B-9. Power Density Data (kW/cc) in Fuel Plate 9 in Prototypic Conditions (continued).

Plate 9, Core Position X2/X6, 99 MWd Burnup, Equilibrium Xenon Conditions

\begin{tabular}{|c|c|c|c|c|c|c|c|c|c|}
\hline $\begin{array}{c}\text { Axial } \\
\text { position } \\
\text { from } \\
\text { top of } \\
\text { fuel } \\
\text { (inches) }\end{array}$ & $\begin{array}{c}0.000 \\
\text { to } \\
0.197 \\
\text { inches }\end{array}$ & $\begin{array}{c}0.197 \\
\text { to } \\
0.394 \\
\text { inches }\end{array}$ & $\begin{array}{c}0.394 \\
\text { to } \\
0.591 \\
\text { inches }\end{array}$ & $\begin{array}{c}0.591 \\
\text { to } \\
1.046 \\
\text { inches }\end{array}$ & $\begin{array}{c}1.046 \\
\text { to } \\
1.501 \\
\text { inches }\end{array}$ & $\begin{array}{c}1.501 \\
\text { to } \\
1.957 \\
\text { inches }\end{array}$ & $\begin{array}{c}1.957 \\
\text { to } \\
2.153 \\
\text { inches }\end{array}$ & $\begin{array}{c}2.153 \\
\text { to } \\
2.350 \\
\text { inches }\end{array}$ & $\begin{array}{c}2.350 \\
\text { to } \\
\text { inches } \\
\text { inch }\end{array}$ \\
\hline 1.5 & 2.02 & 1.71 & 1.54 & 1.49 & 1.43 & 1.50 & 1.56 & 1.67 & 2.07 \\
\hline 2.5 & 2.04 & 1.45 & 1.36 & 1.27 & 1.18 & 1.26 & 1.27 & 1.42 & 1.73 \\
\hline 3.5 & 2.36 & 1.91 & 1.82 & 1.74 & 1.65 & 1.77 & 1.92 & 1.99 & 2.34 \\
\hline 4.5 & 2.56 & 2.21 & 2.08 & 2.02 & 1.90 & 1.96 & 1.96 & 2.17 & 2.61 \\
\hline 5.5 & 2.95 & 2.41 & 2.29 & 2.19 & 2.05 & 2.19 & 2.23 & 2.35 & 2.84 \\
\hline 6.5 & 3.16 & 2.55 & 2.49 & 2.36 & 2.23 & 2.36 & 2.44 & 2.55 & 3.01 \\
\hline 7.5 & 3.26 & 2.77 & 2.68 & 2.50 & 2.31 & 2.44 & 2.56 & 2.77 & 3.32 \\
\hline 8.5 & 3.37 & 2.86 & 2.61 & 2.55 & 2.49 & 2.63 & 2.74 & 2.85 & 3.35 \\
\hline 9.5 & 3.61 & 2.98 & 2.76 & 2.67 & 2.59 & 2.70 & 2.80 & 3.03 & 3.49 \\
\hline 10.5 & 3.57 & 3.07 & 2.86 & 2.74 & 2.66 & 2.74 & 2.85 & 3.07 & 3.61 \\
\hline 11.5 & 3.77 & 3.09 & 2.87 & 2.76 & 2.71 & 2.75 & 2.87 & 3.12 & 3.68 \\
\hline 12.5 & 3.69 & 3.11 & 2.88 & 2.84 & 2.67 & 2.79 & 2.93 & 3.12 & 3.71 \\
\hline 13.5 & 3.67 & 3.08 & 2.94 & 2.77 & 2.64 & 2.82 & 2.88 & 3.11 & 3.59 \\
\hline 14.5 & 3.63 & 3.08 & 2.93 & 2.78 & 2.63 & 2.72 & 2.85 & 3.09 & 3.67 \\
\hline 15.5 & 3.50 & 2.95 & 2.78 & 2.70 & 2.58 & 2.65 & 2.71 & 2.92 & 3.57 \\
\hline 16.5 & 3.37 & 2.82 & 2.70 & 2.55 & 2.42 & 2.60 & 2.69 & 2.81 & 3.39 \\
\hline 17.5 & 3.21 & 2.68 & 2.53 & 2.44 & 2.34 & 2.44 & 2.46 & 2.65 & 3.04 \\
\hline 18.5 & 3.01 & 2.54 & 2.35 & 2.28 & 2.18 & 2.23 & 2.35 & 2.54 & 2.99 \\
\hline 19.5 & 2.76 & 2.39 & 2.15 & 2.06 & 2.01 & 2.15 & 2.10 & 2.37 & 2.74 \\
\hline 20.5 & 2.44 & 2.11 & 1.95 & 1.91 & 1.80 & 1.91 & 1.93 & 2.12 & 2.48 \\
\hline 21.5 & 2.25 & 1.87 & 1.79 & 1.68 & 1.59 & 1.64 & 1.66 & 1.84 & 2.22 \\
\hline 22.5 & 1.94 & 1.65 & 1.50 & 1.45 & 1.38 & 1.41 & 1.51 & 1.64 & 2.03 \\
\hline 23.5 & 2.61 & 2.11 & 1.98 & 1.83 & 1.80 & 1.84 & 1.92 & 2.04 & 2.56 \\
\hline Ax. Avg. & 2.93 & 2.46 & 2.31 & 2.21 & 2.11 & 2.21 & 2.28 & 2.46 & 2.92 \\
\hline & & & & & & & & & 1.49 \\
\hline
\end{tabular}

POWER DENSITY VALUES NORMALIZED TO A CORE POWER OF 11.57 MW AND ASSUME ALL FISSION ENERGY (EXCLUDING GAMMA-HEATING OF EX-CORE COMPONENTS) IS DEPOSITED IN THE FUEL 
Table B-9. Power Density Data (kW/cc) in Fuel Plate 9 in Prototypic Conditions (continued).

Plate 9, Core Position X4/X8, 173 MWd Burnup, Equilibrium Xenon Conditions

\begin{tabular}{|c|c|c|c|c|c|c|c|c|c|}
\hline $\begin{array}{l}\text { Axial } \\
\text { position } \\
\text { from } \\
\text { top of } \\
\text { fuel } \\
\text { (inches) }\end{array}$ & $\begin{array}{c}0.000 \\
\text { to } \\
0.197 \\
\text { inches }\end{array}$ & $\begin{array}{c}0.197 \\
\text { to } \\
0.394 \\
\text { inches }\end{array}$ & $\begin{array}{c}0.394 \\
\text { to } \\
0.591 \\
\text { inches }\end{array}$ & $\begin{array}{c}0.591 \\
\text { to } \\
1.046 \\
\text { inches }\end{array}$ & $\begin{array}{c}1.046 \\
\text { to } \\
1.501 \\
\text { inches }\end{array}$ & $\begin{array}{c}1.501 \\
\text { to } \\
1.957 \\
\text { inches }\end{array}$ & $\begin{array}{c}1.957 \\
\text { to } \\
2.153 \\
\text { inches }\end{array}$ & $\begin{array}{c}2.153 \\
\text { to } \\
2.350 \\
\text { inches }\end{array}$ & $\begin{array}{c}2.350 \\
\text { to } \\
2.547 \\
\text { inches }\end{array}$ \\
\hline 0.5 & 2.01 & 1.68 & 1.50 & 1.53 & 1.41 & 1.47 & 1.59 & 1.65 & 2.02 \\
\hline 1.5 & 1.68 & 1.44 & 1.31 & 1.21 & 1.17 & 1.24 & 1.30 & 1.40 & 1.71 \\
\hline 2.5 & 1.94 & 1.66 & 1.53 & 1.45 & 1.43 & 1.45 & 1.55 & 1.64 & 2.03 \\
\hline 3.5 & 2.26 & 1.90 & 1.78 & 1.71 & 1.67 & 1.74 & 1.79 & 1.83 & 2.24 \\
\hline 4.5 & 2.60 & 2.19 & 1.93 & 1.90 & 1.83 & 1.91 & 1.97 & 2.17 & 2.49 \\
\hline 5.5 & 2.80 & 2.37 & 2.19 & 2.12 & 2.03 & 2.18 & 2.21 & 2.36 & 2.80 \\
\hline 6.5 & 2.93 & 2.53 & 2.34 & 2.32 & 2.18 & 2.26 & 2.37 & 2.56 & 3.05 \\
\hline 7.5 & 3.29 & 2.77 & 2.53 & 2.41 & 2.34 & 2.41 & 2.51 & 2.69 & 3.13 \\
\hline 8.5 & 3.33 & 2.75 & 2.62 & 2.56 & 2.45 & 2.57 & 2.57 & 2.87 & 3.28 \\
\hline 9.5 & 3.49 & 2.90 & 2.78 & 2.60 & 2.55 & 2.67 & 2.78 & 2.95 & 3.53 \\
\hline 10.5 & 3.48 & 3.01 & 2.75 & 2.67 & 2.55 & 2.61 & 2.78 & 3.09 & 3.59 \\
\hline 11.5 & 3.55 & 3.05 & 2.75 & 2.74 & 2.54 & 2.74 & 2.89 & 3.04 & 3.68 \\
\hline 12.5 & 3.55 & 3.07 & 2.83 & 2.71 & 2.56 & 2.67 & 2.81 & 3.02 & 3.66 \\
\hline 13.5 & 3.59 & 2.95 & 2.89 & 2.69 & 2.57 & 2.67 & 2.84 & 2.97 & 3.55 \\
\hline 14.5 & 3.39 & 2.97 & 2.75 & 2.66 & 2.53 & 2.66 & 2.79 & 3.00 & 3.52 \\
\hline 15.5 & 3.36 & 2.89 & 2.62 & 2.61 & 2.40 & 2.59 & 2.68 & 2.90 & 3.41 \\
\hline 16.5 & 3.19 & 2.66 & 2.55 & 2.50 & 2.33 & 2.47 & 2.58 & 2.72 & 3.18 \\
\hline 17.5 & 3.13 & 2.67 & 2.46 & 2.40 & 2.31 & 2.39 & 2.47 & 2.65 & 3.13 \\
\hline 18.5 & 2.91 & 2.45 & 2.31 & 2.26 & 2.14 & 2.16 & 2.37 & 2.49 & 2.88 \\
\hline 19.5 & 2.65 & 2.29 & 2.17 & 2.06 & 1.95 & 2.01 & 2.18 & 2.31 & 2.70 \\
\hline 20.5 & 2.42 & 2.03 & 1.93 & 1.81 & 1.74 & 1.84 & 1.97 & 2.01 & 2.49 \\
\hline 21.5 & 2.20 & 1.83 & 1.71 & 1.63 & 1.53 & 1.67 & 1.73 & 1.83 & 2.18 \\
\hline 22.5 & 1.98 & 1.60 & 1.47 & 1.43 & 1.35 & 1.43 & 1.44 & 1.63 & 2.01 \\
\hline 23.5 & 2.43 & 2.00 & 1.87 & 1.80 & 1.71 & 1.80 & 1.94 & 2.03 & 2.47 \\
\hline Ax. Avg. & 2.84 & 2.40 & 2.23 & 2.16 & 2.05 & 2.15 & 2.25 & 2.41 & 2.86 \\
\hline
\end{tabular}

POWER DENSITY VALUES NORMALIZED TO A CORE POWER OF 11.57 MW AND ASSUME ALL FISSION ENERGY (EXCLUDING GAMMA-HEATING OF EX-CORE COMPONENTS) IS DEPOSITED IN THE FUEL 
Table B-10. Power Density Data $\left(\mathrm{kW} / \mathrm{cm}^{3}\right)$ in Fuel Plate 10 in Prototypic Core.

\section{Fuel core width: 2.654 inches / Fuel core thickness: 20 mil}

Plate 10, Core Position X1/X5, 0 MWd Burnup, Xenon-Free Conditions

\begin{tabular}{|c|c|c|c|c|c|c|c|c|c|}
\hline $\begin{array}{c}\text { Axial } \\
\text { position } \\
\text { from } \\
\text { top of } \\
\text { fuel } \\
\text { (inches) }\end{array}$ & $\begin{array}{c}0.000 \\
\text { to } \\
0.197 \\
\text { inches }\end{array}$ & $\begin{array}{c}0.197 \\
\text { to } \\
0.394 \\
\text { inches }\end{array}$ & $\begin{array}{c}0.394 \\
\text { to } \\
0.591 \\
\text { inches }\end{array}$ & $\begin{array}{c}0.591 \\
\text { to } \\
1.082 \\
\text { inches }\end{array}$ & $\begin{array}{c}1.082 \\
\text { to } \\
1.572 \\
\text { inches }\end{array}$ & $\begin{array}{c}1.572 \\
\text { to } \\
2.063 \\
\text { inches }\end{array}$ & $\begin{array}{c}2.063 \\
\text { to } \\
2.260 \\
\text { inches }\end{array}$ & $\begin{array}{c}2.260 \\
\text { to } \\
2.457 \\
\text { inches }\end{array}$ & $\begin{array}{c}2.457 \\
\text { to } \\
\text { inches }\end{array}$ \\
\hline 0.5 & 1.32 & 1.07 & 1.04 & 1.00 & 0.95 & 0.97 & 0.98 & 1.10 & 1.30 \\
\hline 1.5 & 1.10 & 0.93 & 0.85 & 0.83 & 0.83 & 0.82 & 0.87 & 0.92 & 1.11 \\
\hline 2.5 & 1.25 & 1.06 & 1.07 & 0.99 & 1.02 & 1.02 & 1.03 & 1.10 & 1.33 \\
\hline 3.5 & 1.49 & 1.33 & 1.27 & 1.20 & 1.17 & 1.24 & 1.22 & 1.29 & 1.55 \\
\hline 4.5 & 1.76 & 1.54 & 1.50 & 1.43 & 1.43 & 1.39 & 1.42 & 1.57 & 1.80 \\
\hline 5.5 & 2.10 & 1.73 & 1.71 & 1.69 & 1.56 & 1.58 & 1.68 & 1.78 & 2.08 \\
\hline 6.5 & 2.45 & 2.16 & 1.98 & 1.87 & 1.76 & 1.85 & 1.88 & 2.06 & 2.42 \\
\hline 7.5 & 2.70 & 2.35 & 2.20 & 2.12 & 2.04 & 2.10 & 2.29 & 2.36 & 2.65 \\
\hline 8.5 & 3.17 & 2.64 & 2.52 & 2.39 & 2.31 & 2.37 & 2.46 & 2.55 & 3.07 \\
\hline 9.5 & 3.32 & 2.90 & 2.69 & 2.62 & 2.49 & 2.66 & 2.72 & 2.91 & 3.45 \\
\hline 10.5 & 3.70 & 3.13 & 2.94 & 2.84 & 2.68 & 2.87 & 3.02 & 3.10 & 3.72 \\
\hline 11.5 & 3.98 & 3.35 & 3.18 & 3.02 & 2.90 & 3.02 & 3.17 & 3.38 & 3.97 \\
\hline 12.5 & 4.08 & 3.46 & 3.27 & 3.14 & 3.04 & 3.13 & 3.35 & 3.55 & 4.17 \\
\hline 13.5 & 4.30 & 3.60 & 3.39 & 3.24 & 3.09 & 3.20 & 3.42 & 3.76 & 4.30 \\
\hline 14.5 & 4.18 & 3.62 & 3.46 & 3.31 & 3.15 & 3.30 & 3.53 & 3.72 & 4.26 \\
\hline 15.5 & 4.35 & 3.54 & 3.50 & 3.37 & 3.17 & 3.28 & 3.47 & 3.66 & 4.26 \\
\hline 16.5 & 4.18 & 3.51 & 3.31 & 3.25 & 3.05 & 3.23 & 3.32 & 3.69 & 4.20 \\
\hline 17.5 & 4.13 & 3.55 & 3.35 & 3.22 & 3.00 & 3.08 & 3.19 & 3.51 & 4.10 \\
\hline 18.5 & 3.78 & 3.21 & 3.13 & 2.98 & 2.88 & 3.04 & 3.07 & 3.37 & 3.94 \\
\hline 19.5 & 3.56 & 3.08 & 2.89 & 2.76 & 2.67 & 2.80 & 2.94 & 3.20 & 3.65 \\
\hline 20.5 & 3.21 & 2.75 & 2.57 & 2.46 & 2.52 & 2.55 & 2.67 & 2.92 & 3.30 \\
\hline 21.5 & 2.98 & 2.54 & 2.37 & 2.26 & 2.14 & 2.27 & 2.32 & 2.48 & 2.99 \\
\hline 22.5 & 2.79 & 2.23 & 2.03 & 1.96 & 1.85 & 1.97 & 2.10 & 2.27 & 2.78 \\
\hline 23.5 & 3.51 & 2.91 & 2.67 & 2.57 & 2.46 & 2.55 & 2.61 & 2.90 & 3.48 \\
\hline Ax. Avg. & 3.06 & 2.59 & 2.45 & 2.36 & 2.26 & 2.35 & 2.45 & 2.63 & 3.08 \\
\hline & & & & & & & & & \\
\hline
\end{tabular}

POWER DENSITY VALUES NORMALIZED TO A CORE POWER OF 11.57 MW AND ASSUME ALL FISSION ENERGY (EXCLUDING GAMMA-HEATING OF EX-CORE COMPONENTS) IS DEPOSITED IN THE FUEL 
Table B-10. Power Density Data (kW/cc) in Fuel Plate 10 in Prototypic Conditions (continued).

Plate 10, Core Position X1/X5, 3 MWd Burnup, Equilibrium Xenon Conditions

\begin{tabular}{|c|c|c|c|c|c|c|c|c|c|}
\hline $\begin{array}{c}\text { Axial } \\
\text { position } \\
\text { from } \\
\text { top of } \\
\text { fuel } \\
\text { (inches) }\end{array}$ & $\begin{array}{c}0.000 \\
\text { to } \\
0.197 \\
\text { inches }\end{array}$ & $\begin{array}{c}0.197 \\
\text { to } \\
0.394 \\
\text { inches }\end{array}$ & $\begin{array}{c}0.394 \\
\text { to } \\
0.591 \\
\text { inches }\end{array}$ & $\begin{array}{c}0.591 \\
\text { to } \\
1.082 \\
\text { inches }\end{array}$ & $\begin{array}{c}1.082 \\
\text { to } \\
1.572 \\
\text { inches }\end{array}$ & $\begin{array}{c}1.572 \\
\text { to } \\
2.063 \\
\text { inches }\end{array}$ & $\begin{array}{c}\text { 2.063 } \\
\text { to } \\
2.260 \\
\text { inches }\end{array}$ & $\begin{array}{c}2.260 \\
\text { to } \\
2.457 \\
\text { inches }\end{array}$ & $\begin{array}{c}2.457 \\
\text { to } \\
\text { inches }\end{array}$ \\
\hline 1.5 & 2.02 & 1.68 & 1.55 & 1.52 & 1.48 & 1.54 & 1.58 & 1.67 & 2.03 \\
\hline 2.5 & 2.01 & 1.65 & 1.55 & 1.47 & 1.47 & 1.51 & 1.55 & 1.63 & 2.00 \\
\hline 3.5 & 2.26 & 1.96 & 1.86 & 1.77 & 1.67 & 1.73 & 1.89 & 1.92 & 2.33 \\
\hline 4.5 & 2.58 & 2.27 & 2.05 & 1.99 & 1.92 & 2.00 & 2.05 & 2.21 & 2.60 \\
\hline 5.5 & 2.85 & 2.35 & 2.24 & 2.14 & 2.06 & 2.17 & 2.22 & 2.53 & 2.94 \\
\hline 6.5 & 3.14 & 2.69 & 2.48 & 2.39 & 2.32 & 2.34 & 2.48 & 2.60 & 3.21 \\
\hline 7.5 & 3.27 & 2.88 & 2.54 & 2.51 & 2.43 & 2.53 & 2.67 & 2.80 & 3.27 \\
\hline 8.5 & 3.43 & 2.89 & 2.80 & 2.67 & 2.56 & 2.68 & 2.78 & 2.90 & 3.45 \\
\hline 9.5 & 3.62 & 3.07 & 2.84 & 2.69 & 2.69 & 2.77 & 2.77 & 3.03 & 3.69 \\
\hline 10.5 & 3.67 & 3.14 & 2.91 & 2.80 & 2.68 & 2.76 & 2.87 & 3.10 & 3.61 \\
\hline 11.5 & 3.82 & 3.20 & 2.94 & 2.85 & 2.76 & 2.85 & 2.97 & 3.18 & 3.84 \\
\hline 12.5 & 3.77 & 3.15 & 2.94 & 2.82 & 2.78 & 2.89 & 2.93 & 3.20 & 3.84 \\
\hline 13.5 & 3.74 & 3.17 & 2.95 & 2.82 & 2.70 & 2.85 & 3.04 & 3.13 & 3.65 \\
\hline 14.5 & 3.63 & 3.08 & 2.88 & 2.70 & 2.71 & 2.81 & 2.88 & 3.12 & 3.75 \\
\hline 15.5 & 3.57 & 2.99 & 2.75 & 2.70 & 2.62 & 2.67 & 2.81 & 2.95 & 3.57 \\
\hline 16.5 & 3.36 & 2.84 & 2.75 & 2.64 & 2.44 & 2.62 & 2.71 & 2.92 & 3.48 \\
\hline 17.5 & 3.21 & 2.71 & 2.54 & 2.45 & 2.38 & 2.44 & 2.55 & 2.77 & 3.21 \\
\hline 18.5 & 2.96 & 2.54 & 2.33 & 2.31 & 2.21 & 2.32 & 2.39 & 2.57 & 3.02 \\
\hline 19.5 & 2.74 & 2.40 & 2.26 & 2.14 & 2.09 & 2.12 & 2.26 & 2.33 & 2.81 \\
\hline 20.5 & 2.55 & 2.08 & 1.92 & 1.92 & 1.84 & 1.94 & 2.03 & 2.11 & 2.60 \\
\hline 21.5 & 2.26 & 1.97 & 1.68 & 1.72 & 1.65 & 1.70 & 1.75 & 1.91 & 2.22 \\
\hline 22.5 & 2.03 & 1.66 & 1.49 & 1.46 & 1.39 & 1.44 & 1.50 & 1.64 & 2.00 \\
\hline 23.5 & 2.56 & 2.11 & 1.90 & 1.88 & 1.80 & 1.88 & 1.93 & 2.13 & 2.45 \\
\hline Ax. Avg. & 2.95 & 2.49 & 2.31 & 2.23 & 2.16 & 2.24 & 2.33 & 2.49 & 2.97 \\
\hline & & & & & & & & 1.29 & 13 \\
\hline
\end{tabular}

POWER DENSITY VALUES NORMALIZED TO A CORE POWER OF 11.57 MW AND ASSUME ALL FISSION ENERGY (EXCLUDING GAMMA-HEATING OF EX-CORE COMPONENTS) IS DEPOSITED IN THE FUEL 
Table B-10. Power Density Data (kW/cc) in Fuel Plate 10 in Prototypic Conditions (continued).

Plate 10, Core Position X3/X7, 80 MWd Burnup, Equilibrium Xenon Conditions

\begin{tabular}{|c|c|c|c|c|c|c|c|c|c|}
\hline $\begin{array}{l}\text { Axial } \\
\text { position } \\
\text { from } \\
\text { top of } \\
\text { fuel } \\
\text { (inches) }\end{array}$ & $\begin{array}{c}0.000 \\
\text { to } \\
0.197 \\
\text { inches }\end{array}$ & $\begin{array}{c}0.197 \\
\text { to } \\
0.394 \\
\text { inches }\end{array}$ & $\begin{array}{c}0.394 \\
\text { to } \\
0.591 \\
\text { inches }\end{array}$ & $\begin{array}{c}0.591 \\
\text { to } \\
1.082 \\
\text { inches }\end{array}$ & $\begin{array}{c}1.082 \\
\text { to } \\
1.572 \\
\text { inches }\end{array}$ & $\begin{array}{c}1.572 \\
\text { to } \\
2.063 \\
\text { inches }\end{array}$ & $\begin{array}{c}2.063 \\
\text { to } \\
2.260 \\
\text { inches }\end{array}$ & $\begin{array}{c}2.260 \\
\text { to } \\
2.457 \\
\text { inches }\end{array}$ & $\begin{array}{c}2.457 \\
\text { to } \\
2.654 \\
\text { inches }\end{array}$ \\
\hline 0.5 & 1.89 & 1.62 & 1.47 & 1.45 & 1.43 & 1.49 & 1.55 & 1.65 & 1.99 \\
\hline 1.5 & 1.60 & 1.35 & 1.30 & 1.23 & 1.21 & 1.22 & 1.34 & 1.39 & 1.62 \\
\hline 2.5 & 1.97 & 1.57 & 1.56 & 1.51 & 1.41 & 1.45 & 1.50 & 1.63 & 1.95 \\
\hline 3.5 & 2.23 & 1.92 & 1.83 & 1.75 & 1.68 & 1.72 & 1.79 & 1.90 & 2.28 \\
\hline 4.5 & 2.59 & 2.16 & 2.02 & 1.94 & 1.86 & 1.95 & 1.99 & 2.16 & 2.56 \\
\hline 5.5 & 2.73 & 2.28 & 2.26 & 2.14 & 2.07 & 2.13 & 2.17 & 2.39 & 2.80 \\
\hline 6.5 & 2.97 & 2.54 & 2.33 & 2.31 & 2.24 & 2.34 & 2.44 & 2.56 & 3.00 \\
\hline 7.5 & 3.14 & 2.75 & 2.44 & 2.44 & 2.31 & 2.42 & 2.57 & 2.73 & 3.20 \\
\hline 8.5 & 3.27 & 2.87 & 2.65 & 2.54 & 2.50 & 2.59 & 2.64 & 2.82 & 3.39 \\
\hline 9.5 & 3.41 & 2.92 & 2.77 & 2.62 & 2.55 & 2.69 & 2.77 & 2.96 & 3.42 \\
\hline 10.5 & 3.58 & 3.13 & 2.81 & 2.71 & 2.51 & 2.70 & 2.75 & 2.92 & 3.48 \\
\hline 11.5 & 3.53 & 3.06 & 2.87 & 2.76 & 2.61 & 2.71 & 2.83 & 3.03 & 3.57 \\
\hline 12.5 & 3.69 & 3.08 & 2.78 & 2.77 & 2.61 & 2.73 & 2.82 & 3.03 & 3.72 \\
\hline 13.5 & 3.58 & 3.07 & 2.82 & 2.72 & 2.58 & 2.78 & 2.84 & 3.11 & 3.49 \\
\hline 14.5 & 3.52 & 2.95 & 2.89 & 2.71 & 2.53 & 2.66 & 2.71 & 2.97 & 3.44 \\
\hline 15.5 & 3.31 & 2.89 & 2.67 & 2.60 & 2.50 & 2.62 & 2.74 & 2.88 & 3.42 \\
\hline 16.5 & 3.19 & 2.76 & 2.56 & 2.50 & 2.38 & 2.49 & 2.59 & 2.76 & 3.29 \\
\hline 17.5 & 3.11 & 2.66 & 2.43 & 2.37 & 2.26 & 2.36 & 2.39 & 2.63 & 3.09 \\
\hline 18.5 & 2.78 & 2.44 & 2.41 & 2.25 & 2.10 & 2.22 & 2.25 & 2.45 & 2.87 \\
\hline 19.5 & 2.73 & 2.29 & 2.18 & 2.10 & 2.01 & 2.02 & 2.11 & 2.31 & 2.70 \\
\hline 20.5 & 2.47 & 2.06 & 1.90 & 1.85 & 1.76 & 1.84 & 1.93 & 2.06 & 2.44 \\
\hline 21.5 & 2.20 & 1.79 & 1.68 & 1.63 & 1.56 & 1.63 & 1.71 & 1.80 & 2.14 \\
\hline 22.5 & 1.94 & 1.60 & 1.52 & 1.38 & 1.34 & 1.42 & 1.46 & 1.56 & 1.89 \\
\hline 23.5 & 2.54 & 2.05 & 1.87 & 1.80 & 1.75 & 1.81 & 1.88 & 2.01 & 2.50 \\
\hline Ax. Avg. & 2.83 & 2.41 & 2.25 & 2.17 & 2.07 & 2.17 & 2.24 & 2.40 & 2.84 \\
\hline
\end{tabular}

POWER DENSITY VALUES NORMALIZED TO A CORE POWER OF 11.57 MW AND ASSUME ALL FISSION ENERGY (EXCLUDING GAMMA-HEATING OF EX-CORE COMPONENTS) IS DEPOSITED IN THE FUEL 
Table B-10. Power Density Data (kW/cc) in Fuel Plate 10 in Prototypic Conditions (continued).

Plate 10, Core Position X2/X6, 99 MWd Burnup, Equilibrium Xenon Conditions

\begin{tabular}{|c|c|c|c|c|c|c|c|c|c|}
\hline $\begin{array}{l}\text { Axial } \\
\text { position } \\
\text { from } \\
\text { top of } \\
\text { fuel } \\
\text { (inches) }\end{array}$ & $\begin{array}{c}0.000 \\
\text { to } \\
0.197 \\
\text { inches }\end{array}$ & $\begin{array}{c}0.197 \\
\text { to } \\
0.394 \\
\text { inches }\end{array}$ & $\begin{array}{c}0.394 \\
\text { to } \\
0.591 \\
\text { inches }\end{array}$ & $\begin{array}{c}0.591 \\
\text { to } \\
1.082 \\
\text { inches }\end{array}$ & $\begin{array}{c}1.082 \\
\text { to } \\
1.572 \\
\text { inches }\end{array}$ & $\begin{array}{c}1.572 \\
\text { to } \\
2.063 \\
\text { inches }\end{array}$ & $\begin{array}{c}2.063 \\
\text { to } \\
2.260 \\
\text { inches }\end{array}$ & $\begin{array}{c}2.260 \\
\text { to } \\
2.457 \\
\text { inches }\end{array}$ & $\begin{array}{c}2.457 \\
\text { to } \\
2.654 \\
\text { inches }\end{array}$ \\
\hline 0.5 & 2.01 & 1.73 & 1.56 & 1.44 & 1.42 & 1.43 & 1.50 & 1.61 & 2.02 \\
\hline 1.5 & 1.67 & 1.42 & 1.28 & 1.24 & 1.18 & 1.21 & 1.24 & 1.35 & 1.64 \\
\hline 2.5 & 1.88 & 1.63 & 1.54 & 1.46 & 1.46 & 1.43 & 1.56 & 1.63 & 1.93 \\
\hline 3.5 & 2.32 & 1.95 & 1.77 & 1.71 & 1.65 & 1.73 & 1.88 & 1.92 & 2.22 \\
\hline 4.5 & 2.49 & 2.11 & 2.00 & 1.95 & 1.87 & 1.98 & 1.99 & 2.21 & 2.65 \\
\hline 5.5 & 2.78 & 2.40 & 2.14 & 2.12 & 2.08 & 2.12 & 2.25 & 2.33 & 2.78 \\
\hline 6.5 & 2.99 & 2.55 & 2.39 & 2.31 & 2.17 & 2.29 & 2.43 & 2.56 & 2.96 \\
\hline 7.5 & 3.12 & 2.77 & 2.61 & 2.44 & 2.31 & 2.43 & 2.55 & 2.65 & 3.26 \\
\hline 8.5 & 3.27 & 2.82 & 2.62 & 2.55 & 2.43 & 2.61 & 2.74 & 2.84 & 3.35 \\
\hline 9.5 & 3.39 & 2.88 & 2.82 & 2.64 & 2.59 & 2.65 & 2.68 & 2.84 & 3.51 \\
\hline 10.5 & 3.45 & 2.87 & 2.82 & 2.72 & 2.63 & 2.66 & 2.78 & 3.06 & 3.58 \\
\hline 11.5 & 3.50 & 3.09 & 2.93 & 2.78 & 2.64 & 2.71 & 2.75 & 2.97 & 3.52 \\
\hline 12.5 & 3.62 & 3.07 & 2.83 & 2.81 & 2.65 & 2.69 & 2.84 & 3.07 & 3.57 \\
\hline 13.5 & 3.56 & 3.06 & 2.82 & 2.73 & 2.59 & 2.71 & 2.86 & 2.96 & 3.58 \\
\hline 14.5 & 3.48 & 2.94 & 2.77 & 2.69 & 2.57 & 2.72 & 2.76 & 2.97 & 3.51 \\
\hline 15.5 & 3.39 & 2.97 & 2.68 & 2.61 & 2.56 & 2.62 & 2.69 & 2.87 & 3.39 \\
\hline 16.5 & 3.28 & 2.69 & 2.54 & 2.51 & 2.40 & 2.48 & 2.62 & 2.85 & 3.16 \\
\hline 17.5 & 3.11 & 2.65 & 2.45 & 2.40 & 2.30 & 2.38 & 2.39 & 2.56 & 3.04 \\
\hline 18.5 & 2.96 & 2.45 & 2.39 & 2.29 & 2.18 & 2.20 & 2.25 & 2.56 & 2.86 \\
\hline 19.5 & 2.62 & 2.35 & 2.22 & 2.07 & 1.96 & 2.09 & 2.13 & 2.29 & 2.61 \\
\hline 20.5 & 2.38 & 2.08 & 1.92 & 1.84 & 1.78 & 1.87 & 1.83 & 2.12 & 2.48 \\
\hline 21.5 & 2.15 & 1.83 & 1.73 & 1.64 & 1.55 & 1.62 & 1.65 & 1.83 & 2.12 \\
\hline 22.5 & 1.96 & 1.64 & 1.47 & 1.40 & 1.34 & 1.38 & 1.49 & 1.58 & 1.90 \\
\hline 23.5 & 2.46 & 2.09 & 1.93 & 1.85 & 1.73 & 1.84 & 1.94 & 2.07 & 2.45 \\
\hline Ax. Avg. & 2.83 & 2.42 & 2.26 & 2.18 & 2.08 & 2.16 & 2.24 & 2.41 & 2.84 \\
\hline
\end{tabular}

POWER DENSITY VALUES NORMALIZED TO A CORE POWER OF 11.57 MW AND ASSUME ALL FISSION ENERGY (EXCLUDING GAMMA-HEATING OF EX-CORE COMPONENTS) IS DEPOSITED IN THE FUEL 
Table B-10. Power Density Data (kW/cc) in Fuel Plate 10 in Prototypic Conditions (continued).

Plate 10, Core Position X4/X8, 173 MWd Burnup, Equilibrium Xenon Conditions

\begin{tabular}{|c|c|c|c|c|c|c|c|c|c|}
\hline $\begin{array}{l}\text { Axial } \\
\text { position } \\
\text { from } \\
\text { top of } \\
\text { fuel } \\
\text { (inches) }\end{array}$ & $\begin{array}{c}0.000 \\
\text { to } \\
0.197 \\
\text { inches }\end{array}$ & $\begin{array}{c}0.197 \\
\text { to } \\
0.394 \\
\text { inches }\end{array}$ & $\begin{array}{c}0.394 \\
\text { to } \\
0.591 \\
\text { inches }\end{array}$ & $\begin{array}{c}0.591 \\
\text { to } \\
1.082 \\
\text { inches }\end{array}$ & $\begin{array}{c}1.082 \\
\text { to } \\
1.572 \\
\text { inches }\end{array}$ & $\begin{array}{c}1.572 \\
\text { to } \\
2.063 \\
\text { inches }\end{array}$ & $\begin{array}{c}2.063 \\
\text { to } \\
2.260 \\
\text { inches }\end{array}$ & $\begin{array}{c}2.260 \\
\text { to } \\
2.457 \\
\text { inches }\end{array}$ & $\begin{array}{c}2.457 \\
\text { to } \\
2.654 \\
\text { inches }\end{array}$ \\
\hline 0.5 & 1.98 & 1.61 & 1.47 & 1.46 & 1.39 & 1.47 & 1.50 & 1.61 & 1.91 \\
\hline 1.5 & 1.66 & 1.37 & 1.25 & 1.18 & 1.16 & 1.20 & 1.27 & 1.42 & 1.66 \\
\hline 2.5 & 1.90 & 1.65 & 1.49 & 1.44 & 1.38 & 1.43 & 1.49 & 1.59 & 1.85 \\
\hline 3.5 & 2.28 & 1.85 & 1.80 & 1.64 & 1.64 & 1.68 & 1.75 & 1.87 & 2.19 \\
\hline 4.5 & 2.48 & 2.09 & 1.91 & 1.89 & 1.80 & 1.90 & 1.96 & 2.11 & 2.45 \\
\hline 5.5 & 2.72 & 2.34 & 2.19 & 2.09 & 2.03 & 2.11 & 2.17 & 2.33 & 2.76 \\
\hline 6.5 & 2.95 & 2.49 & 2.29 & 2.23 & 2.16 & 2.24 & 2.32 & 2.56 & 2.95 \\
\hline 7.5 & 3.17 & 2.65 & 2.44 & 2.33 & 2.27 & 2.34 & 2.53 & 2.71 & 3.11 \\
\hline 8.5 & 3.20 & 2.70 & 2.51 & 2.50 & 2.35 & 2.48 & 2.60 & 2.77 & 3.22 \\
\hline 9.5 & 3.27 & 2.86 & 2.62 & 2.58 & 2.48 & 2.58 & 2.66 & 2.88 & 3.42 \\
\hline 10.5 & 3.39 & 2.95 & 2.69 & 2.60 & 2.45 & 2.64 & 2.74 & 2.93 & 3.50 \\
\hline 11.5 & 3.42 & 2.90 & 2.69 & 2.69 & 2.53 & 2.60 & 2.81 & 2.87 & 3.47 \\
\hline 12.5 & 3.47 & 2.96 & 2.79 & 2.63 & 2.54 & 2.63 & 2.82 & 2.88 & 3.42 \\
\hline 13.5 & 3.36 & 2.94 & 2.71 & 2.62 & 2.46 & 2.57 & 2.74 & 2.91 & 3.54 \\
\hline 14.5 & 3.42 & 2.88 & 2.73 & 2.55 & 2.47 & 2.63 & 2.68 & 2.87 & 3.34 \\
\hline 15.5 & 3.35 & 2.80 & 2.61 & 2.45 & 2.42 & 2.52 & 2.63 & 2.82 & 3.28 \\
\hline 16.5 & 3.08 & 2.66 & 2.50 & 2.40 & 2.29 & 2.42 & 2.57 & 2.66 & 3.11 \\
\hline 17.5 & 2.97 & 2.54 & 2.37 & 2.32 & 2.24 & 2.27 & 2.31 & 2.51 & 3.10 \\
\hline 18.5 & 2.80 & 2.37 & 2.17 & 2.21 & 2.06 & 2.15 & 2.31 & 2.47 & 2.86 \\
\hline 19.5 & 2.65 & 2.28 & 2.09 & 2.02 & 1.93 & 2.01 & 2.12 & 2.21 & 2.54 \\
\hline 20.5 & 2.30 & 1.97 & 1.98 & 1.83 & 1.73 & 1.82 & 1.87 & 1.99 & 2.40 \\
\hline 21.5 & 2.16 & 1.77 & 1.67 & 1.57 & 1.48 & 1.65 & 1.65 & 1.82 & 2.08 \\
\hline 22.5 & 1.92 & 1.55 & 1.44 & 1.39 & 1.32 & 1.38 & 1.41 & 1.60 & 1.90 \\
\hline 23.5 & 2.57 & 1.98 & 1.88 & 1.77 & 1.67 & 1.74 & 1.86 & 1.99 & 2.51 \\
\hline Ax. Avg. & 2.77 & 2.34 & 2.18 & 2.10 & 2.01 & 2.10 & 2.20 & 2.35 & 2.77 \\
\hline
\end{tabular}

POWER DENSITY VALUES NORMALIZED TO A CORE POWER OF 11.57 MW AND ASSUME ALL FISSION ENERGY (EXCLUDING GAMMA-HEATING OF EX-CORE COMPONENTS) IS DEPOSITED IN THE FUEL 
Table B-11. Power Density Data $\left(\mathrm{kW} / \mathrm{cm}^{3}\right)$ in Fuel Plate 11 in Prototypic Core.

\section{Fuel core width: 2.761 inches / Fuel core thickness: 20 mil}

Plate 11, Core Position X1/X5, 0 MWd Burnup, Xenon-Free Conditions

\begin{tabular}{|c|c|c|c|c|c|c|c|c|c|}
\hline $\begin{array}{c}\text { Axial } \\
\text { position } \\
\text { from } \\
\text { top of } \\
\text { fuel } \\
\text { (inches) }\end{array}$ & $\begin{array}{c}0.000 \\
\text { to } \\
0.197 \\
\text { inches }\end{array}$ & $\begin{array}{c}0.197 \\
\text { to } \\
0.394 \\
\text { inches }\end{array}$ & $\begin{array}{c}0.394 \\
\text { to } \\
0.591 \\
\text { inches }\end{array}$ & $\begin{array}{c}0.591 \\
\text { to } \\
\text { inches }\end{array}$ & $\begin{array}{c}1.117 \\
\text { to } \\
1.644 \\
\text { inches }\end{array}$ & $\begin{array}{c}1.644 \\
\text { to } \\
2.170 \\
\text { inches }\end{array}$ & $\begin{array}{c}2.170 \\
\text { to } \\
2.367 \\
\text { inches }\end{array}$ & $\begin{array}{c}2.367 \\
\text { to } \\
2.564 \\
\text { inches }\end{array}$ & $\begin{array}{c}2.564 \\
\text { to } \\
\text { inches }\end{array}$ \\
\hline 0.5 & 1.29 & 1.07 & 0.99 & 0.95 & 0.93 & 0.99 & 1.01 & 1.09 & 1.22 \\
\hline 1.5 & 1.09 & 0.88 & 0.82 & 0.82 & 0.80 & 0.81 & 0.88 & 0.90 & 1.07 \\
\hline 2.5 & 1.20 & 1.08 & 1.06 & 1.00 & 0.96 & 1.00 & 1.08 & 1.06 & 1.31 \\
\hline 3.5 & 1.52 & 1.29 & 1.22 & 1.19 & 1.16 & 1.17 & 1.20 & 1.27 & 1.48 \\
\hline 4.5 & 1.68 & 1.40 & 1.46 & 1.38 & 1.38 & 1.36 & 1.50 & 1.49 & 1.74 \\
\hline 5.5 & 2.03 & 1.76 & 1.63 & 1.62 & 1.55 & 1.56 & 1.65 & 1.76 & 1.99 \\
\hline 6.5 & 2.37 & 2.04 & 1.90 & 1.80 & 1.79 & 1.88 & 1.91 & 2.05 & 2.25 \\
\hline 7.5 & 2.61 & 2.31 & 2.12 & 2.12 & 2.00 & 2.07 & 2.25 & 2.37 & 2.65 \\
\hline 8.5 & 3.03 & 2.60 & 2.44 & 2.35 & 2.26 & 2.39 & 2.44 & 2.51 & 2.98 \\
\hline 9.5 & 3.38 & 2.81 & 2.66 & 2.58 & 2.44 & 2.56 & 2.70 & 2.82 & 3.28 \\
\hline 10.5 & 3.58 & 3.09 & 2.87 & 2.73 & 2.69 & 2.78 & 2.90 & 3.08 & 3.58 \\
\hline 11.5 & 3.91 & 3.34 & 3.12 & 2.95 & 2.84 & 2.97 & 3.11 & 3.26 & 3.85 \\
\hline 12.5 & 3.99 & 3.41 & 3.23 & 3.08 & 2.93 & 3.09 & 3.21 & 3.58 & 3.94 \\
\hline 13.5 & 4.16 & 3.53 & 3.33 & 3.23 & 3.08 & 3.12 & 3.38 & 3.59 & 4.26 \\
\hline 14.5 & 4.18 & 3.60 & 3.36 & 3.24 & 3.13 & 3.24 & 3.36 & 3.50 & 4.23 \\
\hline 15.5 & 4.22 & 3.53 & 3.39 & 3.24 & 3.12 & 3.22 & 3.37 & 3.55 & 4.19 \\
\hline 16.5 & 4.11 & 3.53 & 3.26 & 3.18 & 3.11 & 3.20 & 3.24 & 3.59 & 4.15 \\
\hline 17.5 & 3.99 & 3.40 & 3.27 & 3.14 & 2.97 & 3.05 & 3.17 & 3.54 & 3.97 \\
\hline 18.5 & 3.76 & 3.16 & 3.01 & 2.91 & 2.81 & 2.96 & 3.15 & 3.21 & 3.75 \\
\hline 19.5 & 3.49 & 3.02 & 2.79 & 2.71 & 2.61 & 2.76 & 2.89 & 3.08 & 3.57 \\
\hline 20.5 & 3.18 & 2.75 & 2.52 & 2.46 & 2.47 & 2.55 & 2.65 & 2.82 & 3.24 \\
\hline 21.5 & 2.90 & 2.47 & 2.32 & 2.21 & 2.08 & 2.16 & 2.27 & 2.55 & 2.97 \\
\hline 22.5 & 2.73 & 2.25 & 2.07 & 1.93 & 1.86 & 1.98 & 2.06 & 2.22 & 2.62 \\
\hline 23.5 & 3.47 & 2.79 & 2.61 & 2.54 & 2.44 & 2.53 & 2.62 & 2.81 & 3.42 \\
\hline Ax. Avg. & 3.00 & 2.55 & 2.39 & 2.31 & 2.22 & 2.31 & 2.42 & 2.57 & 2.99 \\
\hline & & & & & & & & & \\
\hline
\end{tabular}

POWER DENSITY VALUES NORMALIZED TO A CORE POWER OF 11.57 MW AND ASSUME ALL FISSION ENERGY (EXCLUDING GAMMA-HEATING OF EX-CORE COMPONENTS) IS DEPOSITED IN THE FUEL 
Table B-11. Power Density Data (kW/cc) in Fuel Plate 11 in Prototypic Conditions (continued).

Plate 11, Core Position X1/X5, 3 MWd Burnup, Equilibrium Xenon Conditions

\begin{tabular}{|c|c|c|c|c|c|c|c|c|c|}
\hline $\begin{array}{l}\text { Axial } \\
\text { position } \\
\text { from } \\
\text { top of } \\
\text { fuel } \\
\text { (inches) }\end{array}$ & $\begin{array}{c}0.000 \\
\text { to } \\
0.197 \\
\text { inches }\end{array}$ & $\begin{array}{c}0.197 \\
\text { to } \\
0.394 \\
\text { inches }\end{array}$ & $\begin{array}{c}0.394 \\
\text { to } \\
0.591 \\
\text { inches }\end{array}$ & $\begin{array}{c}0.591 \\
\text { to } \\
1.117 \\
\text { inches }\end{array}$ & $\begin{array}{c}1.117 \\
\text { to } \\
1.644 \\
\text { inches }\end{array}$ & $\begin{array}{c}1.644 \\
\text { to } \\
2.170 \\
\text { inches }\end{array}$ & $\begin{array}{c}2.170 \\
\text { to } \\
2.367 \\
\text { inches }\end{array}$ & $\begin{array}{c}2.367 \\
\text { to } \\
2.564 \\
\text { inches }\end{array}$ & $\begin{array}{c}2.564 \\
\text { to } \\
2.761 \\
\text { inches }\end{array}$ \\
\hline 0.5 & 1.94 & 1.64 & 1.50 & 1.50 & 1.43 & 1.48 & 1.51 & 1.58 & 1.95 \\
\hline 1.5 & 1.65 & 1.37 & 1.24 & 1.24 & 1.19 & 1.20 & 1.33 & 1.34 & 1.73 \\
\hline 2.5 & 1.98 & 1.62 & 1.48 & 1.46 & 1.43 & 1.49 & 1.56 & 1.64 & 1.93 \\
\hline 3.5 & 2.26 & 1.92 & 1.75 & 1.72 & 1.62 & 1.71 & 1.82 & 1.93 & 2.24 \\
\hline 4.5 & 2.46 & 2.16 & 1.99 & 1.98 & 1.86 & 1.95 & 2.03 & 2.19 & 2.66 \\
\hline 5.5 & 2.88 & 2.39 & 2.22 & 2.17 & 2.06 & 2.13 & 2.17 & 2.44 & 2.80 \\
\hline 6.5 & 2.97 & 2.64 & 2.43 & 2.31 & 2.24 & 2.27 & 2.40 & 2.66 & 3.12 \\
\hline 7.5 & 3.23 & 2.75 & 2.62 & 2.50 & 2.34 & 2.48 & 2.65 & 2.81 & 3.31 \\
\hline 8.5 & 3.32 & 2.89 & 2.71 & 2.62 & 2.54 & 2.65 & 2.69 & 2.87 & 3.30 \\
\hline 9.5 & 3.45 & 2.93 & 2.80 & 2.65 & 2.63 & 2.77 & 2.82 & 3.04 & 3.50 \\
\hline 10.5 & 3.62 & 3.12 & 2.93 & 2.74 & 2.69 & 2.79 & 2.81 & 3.05 & 3.49 \\
\hline 11.5 & 3.68 & 3.13 & 2.83 & 2.80 & 2.72 & 2.84 & 2.90 & 3.10 & 3.73 \\
\hline 12.5 & 3.71 & 3.17 & 2.93 & 2.78 & 2.68 & 2.80 & 2.94 & 3.09 & 3.73 \\
\hline 13.5 & 3.66 & 3.06 & 2.91 & 2.81 & 2.70 & 2.80 & 2.86 & 3.01 & 3.47 \\
\hline 14.5 & 3.61 & 3.02 & 2.84 & 2.73 & 2.68 & 2.75 & 2.74 & 2.98 & 3.56 \\
\hline 15.5 & 3.48 & 2.98 & 2.76 & 2.66 & 2.55 & 2.66 & 2.74 & 2.97 & 3.46 \\
\hline 16.5 & 3.32 & 2.87 & 2.65 & 2.55 & 2.48 & 2.51 & 2.60 & 2.89 & 3.35 \\
\hline 17.5 & 3.16 & 2.76 & 2.47 & 2.41 & 2.29 & 2.45 & 2.60 & 2.71 & 3.10 \\
\hline 18.5 & 2.90 & 2.50 & 2.38 & 2.26 & 2.20 & 2.30 & 2.42 & 2.49 & 2.92 \\
\hline 19.5 & 2.66 & 2.27 & 2.18 & 2.12 & 2.02 & 2.10 & 2.23 & 2.30 & 2.76 \\
\hline 20.5 & 2.53 & 2.03 & 1.91 & 1.92 & 1.82 & 1.90 & 1.96 & 2.09 & 2.58 \\
\hline 21.5 & 2.20 & 1.88 & 1.76 & 1.66 & 1.60 & 1.63 & 1.71 & 1.87 & 2.15 \\
\hline 22.5 & 1.98 & 1.56 & 1.55 & 1.46 & 1.36 & 1.47 & 1.50 & 1.61 & 1.87 \\
\hline 23.5 & 2.49 & 2.02 & 1.87 & 1.83 & 1.74 & 1.84 & 1.93 & 2.05 & 2.59 \\
\hline Ax. Avg. & 2.88 & 2.44 & 2.28 & 2.20 & 2.12 & 2.21 & 2.29 & 2.45 & 2.89 \\
\hline
\end{tabular}

POWER DENSITY VALUES NORMALIZED TO A CORE POWER OF 11.57 MW AND ASSUME ALL FISSION ENERGY (EXCLUDING GAMMA-HEATING OF EX-CORE COMPONENTS) IS DEPOSITED IN THE FUEL 
Table B-11. Power Density Data (kW/cc) in Fuel Plate 11 in Prototypic Conditions (continued).

Plate 11, Core Position X3/X7, 80 MWd Burnup, Equilibrium Xenon Conditions

\begin{tabular}{|c|c|c|c|c|c|c|c|c|c|}
\hline $\begin{array}{c}\text { Axial } \\
\text { position } \\
\text { from } \\
\text { top of } \\
\text { fuel } \\
\text { (inches) }\end{array}$ & $\begin{array}{c}0.000 \\
\text { to } \\
0.197 \\
\text { inches }\end{array}$ & $\begin{array}{c}0.197 \\
\text { to } \\
0.394 \\
\text { inches }\end{array}$ & $\begin{array}{c}0.394 \\
\text { to } \\
0.591 \\
\text { inches }\end{array}$ & $\begin{array}{c}0.591 \\
\text { to } \\
1.117 \\
\text { inches }\end{array}$ & $\begin{array}{c}1.117 \\
\text { to } \\
\text { inches }\end{array}$ & $\begin{array}{c}1.644 \\
\text { to } \\
\text { inch } \\
\text { inches }\end{array}$ & $\begin{array}{c}\text { 2.170 } \\
\text { to } \\
2.367 \\
\text { inches }\end{array}$ & $\begin{array}{c}2.367 \\
\text { to } \\
2.564 \\
\text { inches }\end{array}$ & $\begin{array}{c}2.564 \\
\text { to } \\
\text { inches }\end{array}$ \\
\hline 1.5 & 1.86 & 1.64 & 1.49 & 1.46 & 1.40 & 1.45 & 1.45 & 1.58 & 1.96 \\
\hline 2.5 & 1.86 & 1.34 & 1.30 & 1.20 & 1.15 & 1.21 & 1.26 & 1.36 & 1.57 \\
\hline 3.5 & 2.19 & 1.83 & 1.81 & 1.72 & 1.65 & 1.68 & 1.78 & 1.90 & 2.22 \\
\hline 4.5 & 2.48 & 2.12 & 1.97 & 1.91 & 1.82 & 1.91 & 1.95 & 2.18 & 2.51 \\
\hline 5.5 & 2.71 & 2.34 & 2.13 & 2.09 & 2.00 & 2.10 & 2.18 & 2.33 & 2.68 \\
\hline 6.5 & 2.89 & 2.51 & 2.32 & 2.28 & 2.20 & 2.30 & 2.40 & 2.50 & 2.91 \\
\hline 7.5 & 3.13 & 2.69 & 2.53 & 2.42 & 2.31 & 2.46 & 2.49 & 2.71 & 3.14 \\
\hline 8.5 & 3.29 & 2.89 & 2.64 & 2.48 & 2.43 & 2.57 & 2.66 & 2.82 & 3.35 \\
\hline 9.5 & 3.41 & 2.89 & 2.78 & 2.60 & 2.50 & 2.64 & 2.73 & 2.92 & 3.44 \\
\hline 10.5 & 3.61 & 2.96 & 2.72 & 2.66 & 2.56 & 2.70 & 2.79 & 2.91 & 3.52 \\
\hline 11.5 & 3.49 & 3.06 & 2.82 & 2.74 & 2.58 & 2.68 & 2.78 & 3.06 & 3.41 \\
\hline 12.5 & 3.51 & 3.08 & 2.82 & 2.68 & 2.61 & 2.66 & 2.78 & 3.03 & 3.51 \\
\hline 13.5 & 3.53 & 2.98 & 2.84 & 2.69 & 2.53 & 2.65 & 2.87 & 3.02 & 3.41 \\
\hline 14.5 & 3.42 & 2.96 & 2.74 & 2.73 & 2.49 & 2.59 & 2.72 & 2.88 & 3.43 \\
\hline 15.5 & 3.29 & 2.83 & 2.56 & 2.49 & 2.47 & 2.53 & 2.55 & 2.82 & 3.32 \\
\hline 16.5 & 3.20 & 2.76 & 2.51 & 2.45 & 2.33 & 2.39 & 2.49 & 2.72 & 3.17 \\
\hline 17.5 & 3.00 & 2.53 & 2.39 & 2.34 & 2.20 & 2.29 & 2.33 & 2.53 & 2.97 \\
\hline 18.5 & 2.85 & 2.52 & 2.32 & 2.25 & 2.17 & 2.19 & 2.29 & 2.39 & 2.83 \\
\hline 19.5 & 2.54 & 2.24 & 2.13 & 2.03 & 1.93 & 1.97 & 2.08 & 2.25 & 2.60 \\
\hline 20.5 & 2.38 & 2.03 & 1.86 & 1.82 & 1.74 & 1.79 & 1.93 & 1.97 & 2.41 \\
\hline 21.5 & 2.21 & 1.77 & 1.61 & 1.57 & 1.53 & 1.57 & 1.68 & 1.80 & 2.05 \\
\hline 22.5 & 1.85 & 1.56 & 1.44 & 1.38 & 1.30 & 1.38 & 1.46 & 1.57 & 1.94 \\
\hline 23.5 & 2.46 & 2.07 & 1.89 & 1.81 & 1.73 & 1.79 & 1.86 & 2.03 & 2.55 \\
\hline Ax. Avg. & 2.78 & 2.38 & 2.22 & 2.14 & 2.04 & 2.12 & 2.21 & 2.37 & 2.79 \\
\hline & & & & & & & & 1.47 & 1.93 \\
\hline
\end{tabular}

POWER DENSITY VALUES NORMALIZED TO A CORE POWER OF 11.57 MW AND ASSUME ALL FISSION ENERGY (EXCLUDING GAMMA-HEATING OF EX-CORE COMPONENTS) IS DEPOSITED IN THE FUEL 
Table B-11. Power Density Data (kW/cc) in Fuel Plate 11 in Prototypic Conditions (continued).

Plate 11, Core Position X2/X6, 99 MWd Burnup, Equilibrium Xenon Conditions

\begin{tabular}{|c|c|c|c|c|c|c|c|c|c|}
\hline $\begin{array}{l}\text { Axial } \\
\text { position } \\
\text { from } \\
\text { top of } \\
\text { fuel } \\
\text { (inches) }\end{array}$ & $\begin{array}{c}0.000 \\
\text { to } \\
0.197 \\
\text { inches }\end{array}$ & $\begin{array}{c}0.197 \\
\text { to } \\
0.394 \\
\text { inches }\end{array}$ & $\begin{array}{c}0.394 \\
\text { to } \\
0.591 \\
\text { inches }\end{array}$ & $\begin{array}{c}0.591 \\
\text { to } \\
1.117 \\
\text { inches }\end{array}$ & $\begin{array}{c}1.117 \\
\text { to } \\
1.644 \\
\text { inches }\end{array}$ & $\begin{array}{c}1.644 \\
\text { to } \\
2.170 \\
\text { inches }\end{array}$ & $\begin{array}{c}2.170 \\
\text { to } \\
2.367 \\
\text { inches }\end{array}$ & $\begin{array}{c}2.367 \\
\text { to } \\
2.564 \\
\text { inches }\end{array}$ & $\begin{array}{c}2.564 \\
\text { to } \\
2.761 \\
\text { inches }\end{array}$ \\
\hline 0.5 & 1.90 & 1.59 & 1.50 & 1.41 & 1.37 & 1.43 & 1.40 & 1.51 & 1.89 \\
\hline 1.5 & 1.66 & 1.42 & 1.30 & 1.20 & 1.13 & 1.20 & 1.19 & 1.26 & 1.63 \\
\hline 2.5 & 1.90 & 1.56 & 1.53 & 1.48 & 1.39 & 1.45 & 1.46 & 1.55 & 1.91 \\
\hline 3.5 & 2.28 & 1.92 & 1.82 & 1.65 & 1.66 & 1.69 & 1.79 & 1.91 & 2.12 \\
\hline 4.5 & 2.41 & 2.12 & 1.91 & 1.86 & 1.80 & 1.89 & 1.88 & 2.12 & 2.55 \\
\hline 5.5 & 2.66 & 2.32 & 2.15 & 2.10 & 1.99 & 2.09 & 2.20 & 2.29 & 2.77 \\
\hline 6.5 & 2.87 & 2.49 & 2.34 & 2.27 & 2.16 & 2.25 & 2.39 & 2.54 & 2.95 \\
\hline 7.5 & 3.09 & 2.62 & 2.46 & 2.43 & 2.24 & 2.39 & 2.44 & 2.69 & 3.04 \\
\hline 8.5 & 3.25 & 2.77 & 2.62 & 2.50 & 2.42 & 2.51 & 2.72 & 2.81 & 3.33 \\
\hline 9.5 & 3.40 & 2.83 & 2.66 & 2.63 & 2.49 & 2.55 & 2.60 & 2.80 & 3.38 \\
\hline 10.5 & 3.31 & 2.87 & 2.73 & 2.65 & 2.53 & 2.66 & 2.75 & 2.88 & 3.51 \\
\hline 11.5 & 3.46 & 3.08 & 2.75 & 2.71 & 2.61 & 2.67 & 2.81 & 2.92 & 3.57 \\
\hline 12.5 & 3.44 & 2.91 & 2.83 & 2.72 & 2.57 & 2.65 & 2.83 & 2.90 & 3.54 \\
\hline 13.5 & 3.44 & 3.04 & 2.75 & 2.70 & 2.58 & 2.75 & 2.73 & 2.96 & 3.49 \\
\hline 14.5 & 3.37 & 2.91 & 2.72 & 2.63 & 2.56 & 2.64 & 2.72 & 2.95 & 3.47 \\
\hline 15.5 & 3.39 & 2.83 & 2.64 & 2.56 & 2.49 & 2.52 & 2.65 & 2.81 & 3.29 \\
\hline 16.5 & 3.14 & 2.77 & 2.57 & 2.44 & 2.37 & 2.43 & 2.52 & 2.68 & 3.21 \\
\hline 17.5 & 3.01 & 2.53 & 2.45 & 2.35 & 2.23 & 2.39 & 2.47 & 2.62 & 3.07 \\
\hline 18.5 & 2.79 & 2.49 & 2.29 & 2.28 & 2.09 & 2.19 & 2.30 & 2.52 & 2.93 \\
\hline 19.5 & 2.64 & 2.22 & 2.12 & 2.00 & 1.91 & 2.07 & 2.04 & 2.19 & 2.59 \\
\hline 20.5 & 2.40 & 2.07 & 1.90 & 1.83 & 1.71 & 1.81 & 1.85 & 2.01 & 2.35 \\
\hline 21.5 & 2.07 & 1.77 & 1.68 & 1.61 & 1.52 & 1.60 & 1.64 & 1.80 & 2.16 \\
\hline 22.5 & 1.92 & 1.58 & 1.45 & 1.37 & 1.29 & 1.39 & 1.46 & 1.60 & 1.81 \\
\hline 23.5 & 2.47 & 2.05 & 1.79 & 1.80 & 1.73 & 1.77 & 1.83 & 1.97 & 2.41 \\
\hline Ax. Avg. & 2.76 & 2.37 & 2.21 & 2.13 & 2.03 & 2.12 & 2.19 & 2.35 & 2.79 \\
\hline
\end{tabular}

POWER DENSITY VALUES NORMALIZED TO A CORE POWER OF 11.57 MW AND ASSUME ALL FISSION ENERGY (EXCLUDING GAMMA-HEATING OF EX-CORE COMPONENTS) IS DEPOSITED IN THE FUEL 
Table B-11. Power Density Data (kW/cc) in Fuel Plate 11 in Prototypic Conditions (continued).

Plate 11, Core Position X4/X8, 173 MWd Burnup, Equilibrium Xenon Conditions

\begin{tabular}{|c|c|c|c|c|c|c|c|c|c|}
\hline $\begin{array}{l}\text { Axial } \\
\text { position } \\
\text { from } \\
\text { top of } \\
\text { fuel } \\
\text { (inches) }\end{array}$ & $\begin{array}{c}0.000 \\
\text { to } \\
0.197 \\
\text { inches }\end{array}$ & $\begin{array}{c}0.197 \\
\text { to } \\
0.394 \\
\text { inches }\end{array}$ & $\begin{array}{c}0.394 \\
\text { to } \\
0.591 \\
\text { inches }\end{array}$ & $\begin{array}{c}0.591 \\
\text { to } \\
1.117 \\
\text { inches }\end{array}$ & $\begin{array}{c}1.117 \\
\text { to } \\
1.644 \\
\text { inches }\end{array}$ & $\begin{array}{c}1.644 \\
\text { to } \\
2.170 \\
\text { inches }\end{array}$ & $\begin{array}{c}2.170 \\
\text { to } \\
2.367 \\
\text { inches }\end{array}$ & $\begin{array}{c}2.367 \\
\text { to } \\
2.564 \\
\text { inches }\end{array}$ & $\begin{array}{c}2.564 \\
\text { to } \\
2.761 \\
\text { inches }\end{array}$ \\
\hline 0.5 & 1.91 & 1.56 & 1.46 & 1.41 & 1.41 & 1.45 & 1.47 & 1.65 & 1.88 \\
\hline 1.5 & 1.60 & 1.34 & 1.27 & 1.13 & 1.14 & 1.22 & 1.21 & 1.33 & 1.59 \\
\hline 2.5 & 1.87 & 1.62 & 1.50 & 1.45 & 1.38 & 1.42 & 1.48 & 1.62 & 1.81 \\
\hline 3.5 & 2.18 & 1.88 & 1.74 & 1.68 & 1.59 & 1.61 & 1.73 & 1.86 & 2.15 \\
\hline 4.5 & 2.41 & 2.10 & 1.91 & 1.82 & 1.81 & 1.85 & 1.90 & 2.11 & 2.42 \\
\hline 5.5 & 2.74 & 2.36 & 2.11 & 2.04 & 1.98 & 2.10 & 2.17 & 2.27 & 2.72 \\
\hline 6.5 & 2.85 & 2.38 & 2.35 & 2.15 & 2.11 & 2.18 & 2.32 & 2.48 & 2.93 \\
\hline 7.5 & 3.06 & 2.68 & 2.43 & 2.32 & 2.21 & 2.34 & 2.43 & 2.67 & 2.96 \\
\hline 8.5 & 3.25 & 2.77 & 2.50 & 2.46 & 2.33 & 2.47 & 2.54 & 2.63 & 3.20 \\
\hline 9.5 & 3.27 & 2.73 & 2.62 & 2.51 & 2.37 & 2.52 & 2.63 & 2.74 & 3.32 \\
\hline 10.5 & 3.33 & 2.85 & 2.59 & 2.58 & 2.43 & 2.61 & 2.60 & 2.83 & 3.35 \\
\hline 11.5 & 3.33 & 2.87 & 2.69 & 2.60 & 2.48 & 2.56 & 2.71 & 2.89 & 3.42 \\
\hline 12.5 & 3.35 & 2.94 & 2.69 & 2.63 & 2.53 & 2.53 & 2.64 & 2.90 & 3.30 \\
\hline 13.5 & 3.34 & 2.88 & 2.67 & 2.56 & 2.46 & 2.55 & 2.69 & 2.89 & 3.32 \\
\hline 14.5 & 3.22 & 2.77 & 2.57 & 2.54 & 2.45 & 2.56 & 2.70 & 2.82 & 3.22 \\
\hline 15.5 & 3.21 & 2.76 & 2.54 & 2.43 & 2.37 & 2.46 & 2.60 & 2.68 & 3.24 \\
\hline 16.5 & 3.10 & 2.57 & 2.43 & 2.38 & 2.29 & 2.39 & 2.46 & 2.69 & 3.04 \\
\hline 17.5 & 2.93 & 2.52 & 2.31 & 2.30 & 2.16 & 2.24 & 2.25 & 2.46 & 2.98 \\
\hline 18.5 & 2.75 & 2.35 & 2.14 & 2.15 & 2.04 & 2.08 & 2.25 & 2.40 & 2.69 \\
\hline 19.5 & 2.52 & 2.19 & 2.04 & 2.00 & 1.86 & 1.95 & 2.06 & 2.22 & 2.55 \\
\hline 20.5 & 2.29 & 1.95 & 1.78 & 1.77 & 1.73 & 1.83 & 1.86 & 1.93 & 2.24 \\
\hline 21.5 & 2.01 & 1.73 & 1.60 & 1.57 & 1.47 & 1.55 & 1.59 & 1.66 & 2.08 \\
\hline 22.5 & 1.88 & 1.57 & 1.44 & 1.37 & 1.28 & 1.36 & 1.39 & 1.57 & 1.86 \\
\hline 23.5 & 2.32 & 1.89 & 1.85 & 1.74 & 1.65 & 1.77 & 1.80 & 1.99 & 2.41 \\
\hline Ax. Avg. & 2.70 & 2.30 & 2.14 & 2.07 & 1.98 & 2.07 & 2.15 & 2.30 & 2.69 \\
\hline
\end{tabular}

POWER DENSITY VALUES NORMALIZED TO A CORE POWER OF 11.57 MW AND ASSUME ALL FISSION ENERGY (EXCLUDING GAMMA-HEATING OF EX-CORE COMPONENTS) IS DEPOSITED IN THE FUEL 
Table B-12. Power Density Data $\left(\mathrm{kW} / \mathrm{cm}^{3}\right)$ in Fuel Plate 12 in Prototypic Core.

\section{Fuel core width: 2.868 inches / Fuel core thickness: 20 mil}

Plate 12, Core Position X1/X5, 0 MWd Burnup, Xenon-Free Conditions

\begin{tabular}{|c|c|c|c|c|c|c|c|c|c|}
\hline $\begin{array}{c}\text { Axial } \\
\text { position } \\
\text { from } \\
\text { top of } \\
\text { fuel } \\
\text { (inches) }\end{array}$ & $\begin{array}{c}0.000 \\
\text { to } \\
0.197 \\
\text { inches }\end{array}$ & $\begin{array}{c}0.197 \\
\text { to } \\
0.394 \\
\text { inches }\end{array}$ & $\begin{array}{c}0.394 \\
\text { to } \\
0.591 \\
\text { inches }\end{array}$ & $\begin{array}{c}0.591 \\
\text { to } \\
\text { inches }\end{array}$ & $\begin{array}{c}1.153 \\
\text { to } \\
1.715 \\
\text { inches }\end{array}$ & $\begin{array}{c}1.715 \\
\text { to } \\
2.277 \\
\text { inches }\end{array}$ & $\begin{array}{c}2.277 \\
\text { to } \\
2.474 \\
\text { inches }\end{array}$ & $\begin{array}{c}2.474 \\
\text { to } \\
2.671 \\
\text { inches }\end{array}$ & $\begin{array}{c}2.671 \\
\text { to } \\
\text { inches }\end{array}$ \\
\hline 0.5 & 1.28 & 1.08 & 0.99 & 0.95 & 0.93 & 0.93 & 0.93 & 1.04 & 1.18 \\
\hline 1.5 & 1.00 & 0.90 & 0.84 & 0.81 & 0.80 & 0.81 & 0.79 & 0.86 & 1.07 \\
\hline 2.5 & 1.17 & 1.07 & 0.97 & 0.99 & 0.94 & 0.98 & 1.03 & 1.02 & 1.18 \\
\hline 3.5 & 1.45 & 1.30 & 1.17 & 1.16 & 1.12 & 1.16 & 1.20 & 1.21 & 1.39 \\
\hline 4.5 & 1.68 & 1.42 & 1.37 & 1.38 & 1.34 & 1.36 & 1.43 & 1.46 & 1.70 \\
\hline 5.5 & 1.99 & 1.81 & 1.63 & 1.59 & 1.59 & 1.58 & 1.65 & 1.77 & 1.95 \\
\hline 6.5 & 2.23 & 1.92 & 1.88 & 1.78 & 1.78 & 1.81 & 1.84 & 1.95 & 2.28 \\
\hline 7.5 & 2.58 & 2.23 & 2.01 & 2.06 & 2.00 & 2.04 & 2.19 & 2.25 & 2.62 \\
\hline 8.5 & 2.88 & 2.50 & 2.34 & 2.30 & 2.17 & 2.31 & 2.35 & 2.54 & 2.93 \\
\hline 9.5 & 3.29 & 2.76 & 2.70 & 2.52 & 2.39 & 2.53 & 2.56 & 2.80 & 3.26 \\
\hline 10.5 & 3.52 & 2.99 & 2.78 & 2.77 & 2.68 & 2.73 & 2.89 & 2.97 & 3.55 \\
\hline 11.5 & 3.77 & 3.30 & 3.05 & 2.91 & 2.82 & 2.91 & 3.13 & 3.25 & 3.72 \\
\hline 12.5 & 3.98 & 3.37 & 3.12 & 3.06 & 2.98 & 3.03 & 3.08 & 3.37 & 4.06 \\
\hline 13.5 & 4.02 & 3.58 & 3.17 & 3.20 & 2.99 & 3.12 & 3.36 & 3.53 & 4.13 \\
\hline 14.5 & 4.16 & 3.56 & 3.30 & 3.17 & 3.06 & 3.20 & 3.28 & 3.50 & 4.03 \\
\hline 15.5 & 4.12 & 3.45 & 3.28 & 3.20 & 3.08 & 3.22 & 3.34 & 3.48 & 4.16 \\
\hline 16.5 & 4.02 & 3.49 & 3.34 & 3.21 & 3.03 & 3.12 & 3.35 & 3.53 & 4.23 \\
\hline 17.5 & 3.90 & 3.41 & 3.30 & 3.03 & 2.91 & 3.04 & 3.17 & 3.47 & 3.86 \\
\hline 18.5 & 3.79 & 3.17 & 2.99 & 2.96 & 2.79 & 2.97 & 3.01 & 3.09 & 3.66 \\
\hline 19.5 & 3.45 & 3.01 & 2.85 & 2.70 & 2.59 & 2.74 & 2.82 & 3.08 & 3.55 \\
\hline 20.5 & 3.21 & 2.76 & 2.60 & 2.49 & 2.42 & 2.50 & 2.56 & 2.82 & 3.27 \\
\hline 21.5 & 2.80 & 2.42 & 2.33 & 2.17 & 2.11 & 2.17 & 2.30 & 2.47 & 3.01 \\
\hline 22.5 & 2.57 & 2.11 & 1.95 & 1.90 & 1.82 & 1.93 & 2.07 & 2.25 & 2.68 \\
\hline 23.5 & 3.36 & 2.78 & 2.62 & 2.48 & 2.38 & 2.47 & 2.54 & 2.81 & 3.44 \\
\hline Ax. Avg. & 2.93 & 2.52 & 2.36 & 2.28 & 2.19 & 2.28 & 2.37 & 2.52 & 2.95 \\
\hline & & & & & & & & & \\
\hline
\end{tabular}

POWER DENSITY VALUES NORMALIZED TO A CORE POWER OF 11.57 MW AND ASSUME ALL FISSION ENERGY (EXCLUDING GAMMA-HEATING OF EX-CORE COMPONENTS) IS DEPOSITED IN THE FUEL 
Table B-12. Power Density Data (kW/cc) in Fuel Plate 12 in Prototypic Conditions (continued).

Plate 12, Core Position X1/X5, 3 MWd Burnup, Equilibrium Xenon Conditions

\begin{tabular}{|c|c|c|c|c|c|c|c|c|c|}
\hline $\begin{array}{c}\text { Axial } \\
\text { position } \\
\text { from } \\
\text { top of } \\
\text { fuel } \\
\text { (inches) }\end{array}$ & $\begin{array}{c}0.000 \\
\text { to } \\
0.197 \\
\text { inches }\end{array}$ & $\begin{array}{c}0.197 \\
\text { to } \\
0.394 \\
\text { inches }\end{array}$ & $\begin{array}{c}0.394 \\
\text { to } \\
0.591 \\
\text { inches }\end{array}$ & $\begin{array}{c}0.591 \\
\text { to } \\
1.153 \\
\text { inches }\end{array}$ & $\begin{array}{c}1.153 \\
\text { to } \\
1.715 \\
\text { inches }\end{array}$ & $\begin{array}{c}1.715 \\
\text { to } \\
2.277 \\
\text { inches }\end{array}$ & $\begin{array}{c}\text { 2.277 } \\
\text { to } \\
2.474 \\
\text { inches }\end{array}$ & $\begin{array}{c}2.474 \\
\text { to } \\
2.671 \\
\text { inches }\end{array}$ & $\begin{array}{c}2.671 \\
\text { to } \\
\text { inches }\end{array}$ \\
\hline 1.5 & 1.92 & 1.60 & 1.49 & 1.48 & 1.40 & 1.44 & 1.44 & 1.58 & 1.85 \\
\hline 2.5 & 1.88 & 1.30 & 1.25 & 1.22 & 1.20 & 1.21 & 1.30 & 1.43 & 1.62 \\
\hline 3.5 & 2.20 & 1.84 & 1.70 & 1.70 & 1.63 & 1.75 & 1.76 & 1.86 & 2.21 \\
\hline 4.5 & 2.56 & 2.16 & 2.06 & 1.97 & 1.86 & 1.90 & 1.98 & 2.18 & 2.47 \\
\hline 5.5 & 2.75 & 2.39 & 2.17 & 2.16 & 2.07 & 2.12 & 2.24 & 2.37 & 2.81 \\
\hline 6.5 & 2.94 & 2.53 & 2.47 & 2.31 & 2.23 & 2.30 & 2.48 & 2.57 & 3.03 \\
\hline 7.5 & 3.15 & 2.73 & 2.56 & 2.46 & 2.40 & 2.47 & 2.60 & 2.74 & 3.04 \\
\hline 8.5 & 3.33 & 2.79 & 2.69 & 2.62 & 2.51 & 2.61 & 2.61 & 2.86 & 3.38 \\
\hline 9.5 & 3.51 & 3.11 & 2.70 & 2.64 & 2.61 & 2.76 & 2.74 & 2.89 & 3.39 \\
\hline 10.5 & 3.58 & 3.07 & 2.86 & 2.73 & 2.62 & 2.71 & 2.80 & 3.06 & 3.42 \\
\hline 11.5 & 3.59 & 2.99 & 2.84 & 2.75 & 2.71 & 2.81 & 2.81 & 3.12 & 3.55 \\
\hline 12.5 & 3.63 & 3.14 & 2.87 & 2.79 & 2.67 & 2.77 & 2.91 & 3.09 & 3.63 \\
\hline 13.5 & 3.61 & 3.10 & 2.79 & 2.74 & 2.70 & 2.74 & 2.84 & 3.05 & 3.53 \\
\hline 14.5 & 3.54 & 2.99 & 2.85 & 2.68 & 2.63 & 2.70 & 2.78 & 3.01 & 3.45 \\
\hline 15.5 & 3.46 & 2.96 & 2.76 & 2.67 & 2.55 & 2.64 & 2.70 & 2.92 & 3.38 \\
\hline 16.5 & 3.27 & 2.82 & 2.58 & 2.52 & 2.38 & 2.50 & 2.62 & 2.83 & 3.13 \\
\hline 17.5 & 3.21 & 2.69 & 2.56 & 2.37 & 2.31 & 2.41 & 2.52 & 2.67 & 3.08 \\
\hline 18.5 & 2.76 & 2.47 & 2.34 & 2.25 & 2.20 & 2.27 & 2.36 & 2.55 & 2.81 \\
\hline 19.5 & 2.74 & 2.26 & 2.20 & 2.09 & 2.00 & 2.03 & 2.21 & 2.37 & 2.62 \\
\hline 20.5 & 2.51 & 2.11 & 1.97 & 1.87 & 1.79 & 1.84 & 1.94 & 2.12 & 2.42 \\
\hline 21.5 & 2.17 & 1.86 & 1.69 & 1.64 & 1.57 & 1.62 & 1.70 & 1.79 & 2.15 \\
\hline 22.5 & 1.86 & 1.64 & 1.43 & 1.43 & 1.38 & 1.42 & 1.46 & 1.57 & 1.84 \\
\hline 23.5 & 2.48 & 2.00 & 1.83 & 1.83 & 1.80 & 1.83 & 1.87 & 2.09 & 2.50 \\
\hline Ax. Avg. & 2.84 & 2.42 & 2.25 & 2.18 & 2.11 & 2.18 & 2.26 & 2.43 & 2.80 \\
\hline & & & & & & & & & 1.48 \\
\hline
\end{tabular}

POWER DENSITY VALUES NORMALIZED TO A CORE POWER OF 11.57 MW AND ASSUME ALL FISSION ENERGY (EXCLUDING GAMMA-HEATING OF EX-CORE COMPONENTS) IS DEPOSITED IN THE FUEL 
Table B-12. Power Density Data (kW/cc) in Fuel Plate 12 in Prototypic Conditions (continued).

Plate 12, Core Position X3/X7, 80 MWd Burnup, Equilibrium Xenon Conditions

\begin{tabular}{|c|c|c|c|c|c|c|c|c|c|}
\hline $\begin{array}{l}\text { Axial } \\
\text { position } \\
\text { from } \\
\text { top of } \\
\text { fuel } \\
\text { (inches) }\end{array}$ & $\begin{array}{c}0.000 \\
\text { to } \\
0.197 \\
\text { inches }\end{array}$ & $\begin{array}{c}0.197 \\
\text { to } \\
0.394 \\
\text { inches }\end{array}$ & $\begin{array}{c}0.394 \\
\text { to } \\
0.591 \\
\text { inches }\end{array}$ & $\begin{array}{c}0.591 \\
\text { to } \\
1.153 \\
\text { inches }\end{array}$ & $\begin{array}{c}1.153 \\
\text { to } \\
1.715 \\
\text { inches }\end{array}$ & $\begin{array}{c}1.715 \\
\text { to } \\
2.277 \\
\text { inches }\end{array}$ & $\begin{array}{c}2.277 \\
\text { to } \\
2.474 \\
\text { inches }\end{array}$ & $\begin{array}{c}2.474 \\
\text { to } \\
2.671 \\
\text { inches }\end{array}$ & $\begin{array}{c}2.671 \\
\text { to } \\
2.868 \\
\text { inches }\end{array}$ \\
\hline 0.5 & 1.80 & 1.58 & 1.45 & 1.42 & 1.37 & 1.42 & 1.42 & 1.59 & 1.84 \\
\hline 1.5 & 1.58 & 1.32 & 1.27 & 1.17 & 1.16 & 1.24 & 1.25 & 1.28 & 1.51 \\
\hline 2.5 & 1.84 & 1.57 & 1.54 & 1.41 & 1.36 & 1.39 & 1.50 & 1.50 & 1.79 \\
\hline 3.5 & 2.21 & 1.85 & 1.75 & 1.67 & 1.63 & 1.68 & 1.67 & 1.81 & 2.08 \\
\hline 4.5 & 2.46 & 2.12 & 1.91 & 1.90 & 1.79 & 1.87 & 1.98 & 2.06 & 2.43 \\
\hline 5.5 & 2.72 & 2.34 & 2.13 & 2.10 & 1.99 & 2.05 & 2.18 & 2.35 & 2.70 \\
\hline 6.5 & 2.90 & 2.51 & 2.36 & 2.23 & 2.15 & 2.22 & 2.29 & 2.52 & 2.98 \\
\hline 7.5 & 3.17 & 2.67 & 2.44 & 2.39 & 2.30 & 2.36 & 2.55 & 2.67 & 3.15 \\
\hline 8.5 & 3.15 & 2.80 & 2.62 & 2.46 & 2.36 & 2.54 & 2.65 & 2.87 & 3.27 \\
\hline 9.5 & 3.35 & 2.89 & 2.70 & 2.57 & 2.48 & 2.57 & 2.74 & 2.91 & 3.31 \\
\hline 10.5 & 3.44 & 2.94 & 2.76 & 2.65 & 2.55 & 2.64 & 2.71 & 2.86 & 3.37 \\
\hline 11.5 & 3.43 & 2.93 & 2.76 & 2.65 & 2.53 & 2.70 & 2.77 & 2.99 & 3.50 \\
\hline 12.5 & 3.47 & 2.99 & 2.82 & 2.66 & 2.53 & 2.55 & 2.71 & 2.89 & 3.59 \\
\hline 13.5 & 3.41 & 2.89 & 2.81 & 2.67 & 2.52 & 2.60 & 2.75 & 2.90 & 3.48 \\
\hline 14.5 & 3.43 & 2.90 & 2.69 & 2.64 & 2.49 & 2.56 & 2.64 & 2.90 & 3.45 \\
\hline 15.5 & 3.29 & 2.81 & 2.68 & 2.50 & 2.41 & 2.48 & 2.59 & 2.84 & 3.18 \\
\hline 16.5 & 3.11 & 2.68 & 2.48 & 2.39 & 2.36 & 2.39 & 2.56 & 2.64 & 3.10 \\
\hline 17.5 & 2.98 & 2.58 & 2.34 & 2.27 & 2.21 & 2.28 & 2.36 & 2.56 & 2.97 \\
\hline 18.5 & 2.83 & 2.40 & 2.28 & 2.24 & 2.09 & 2.14 & 2.25 & 2.41 & 2.78 \\
\hline 19.5 & 2.55 & 2.22 & 2.06 & 2.00 & 1.92 & 1.98 & 2.05 & 2.23 & 2.54 \\
\hline 20.5 & 2.34 & 2.02 & 1.86 & 1.80 & 1.76 & 1.79 & 1.95 & 1.95 & 2.39 \\
\hline 21.5 & 2.08 & 1.80 & 1.65 & 1.59 & 1.49 & 1.58 & 1.62 & 1.77 & 2.17 \\
\hline 22.5 & 1.85 & 1.56 & 1.39 & 1.37 & 1.27 & 1.37 & 1.39 & 1.56 & 1.81 \\
\hline 23.5 & 2.48 & 2.00 & 1.84 & 1.82 & 1.69 & 1.76 & 1.87 & 2.07 & 2.31 \\
\hline Ax. Avg. & 2.74 & 2.35 & 2.19 & 2.11 & 2.02 & 2.09 & 2.19 & 2.34 & 2.74 \\
\hline
\end{tabular}

POWER DENSITY VALUES NORMALIZED TO A CORE POWER OF 11.57 MW AND ASSUME ALL FISSION ENERGY (EXCLUDING GAMMA-HEATING OF EX-CORE COMPONENTS) IS DEPOSITED IN THE FUEL 
Table B-12. Power Density Data (kW/cc) in Fuel Plate 12 in Prototypic Conditions (continued).

Plate 12, Core Position X2/X6, 99 MWd Burnup, Equilibrium Xenon Conditions

\begin{tabular}{|c|c|c|c|c|c|c|c|c|c|}
\hline $\begin{array}{c}\text { Axial } \\
\text { position } \\
\text { from } \\
\text { top of } \\
\text { fuel } \\
\text { (inches) }\end{array}$ & $\begin{array}{c}0.000 \\
\text { to } \\
0.197 \\
\text { inches }\end{array}$ & $\begin{array}{c}0.197 \\
\text { to } \\
0.394 \\
\text { inches }\end{array}$ & $\begin{array}{c}0.394 \\
\text { to } \\
0.591 \\
\text { inches }\end{array}$ & $\begin{array}{c}0.591 \\
\text { to } \\
\text { inches }\end{array}$ & $\begin{array}{c}1.153 \\
\text { to } \\
1.715 \\
\text { inches }\end{array}$ & $\begin{array}{c}1.715 \\
\text { to } \\
2.277 \\
\text { inches }\end{array}$ & $\begin{array}{c}2.277 \\
\text { to } \\
2.474 \\
\text { inches }\end{array}$ & $\begin{array}{c}2.474 \\
\text { to } \\
2.671 \\
\text { inches }\end{array}$ & $\begin{array}{c}2.671 \\
\text { to } \\
\text { inches }\end{array}$ \\
\hline 1.5 & 1.90 & 1.60 & 1.45 & 1.39 & 1.36 & 1.38 & 1.42 & 1.44 & 1.90 \\
\hline 2.5 & 1.92 & 1.34 & 1.21 & 1.17 & 1.13 & 1.20 & 1.21 & 1.31 & 1.56 \\
\hline 3.5 & 2.19 & 1.84 & 1.76 & 1.63 & 1.66 & 1.67 & 1.71 & 1.85 & 2.19 \\
\hline 4.5 & 2.35 & 2.02 & 1.90 & 1.87 & 1.82 & 1.88 & 2.03 & 2.02 & 2.47 \\
\hline 5.5 & 2.71 & 2.33 & 2.17 & 2.03 & 1.99 & 2.05 & 2.11 & 2.33 & 2.70 \\
\hline 6.5 & 2.91 & 2.49 & 2.35 & 2.23 & 2.17 & 2.23 & 2.34 & 2.50 & 2.87 \\
\hline 7.5 & 3.10 & 2.66 & 2.43 & 2.35 & 2.23 & 2.33 & 2.47 & 2.69 & 3.05 \\
\hline 8.5 & 3.19 & 2.72 & 2.57 & 2.47 & 2.39 & 2.50 & 2.59 & 2.80 & 3.32 \\
\hline 9.5 & 3.30 & 2.87 & 2.65 & 2.56 & 2.47 & 2.52 & 2.65 & 2.77 & 3.36 \\
\hline 10.5 & 3.34 & 2.88 & 2.69 & 2.58 & 2.53 & 2.60 & 2.78 & 2.95 & 3.42 \\
\hline 11.5 & 3.43 & 2.98 & 2.73 & 2.68 & 2.56 & 2.69 & 2.79 & 2.82 & 3.48 \\
\hline 12.5 & 3.51 & 2.93 & 2.80 & 2.67 & 2.58 & 2.63 & 2.75 & 3.00 & 3.50 \\
\hline 13.5 & 3.44 & 2.93 & 2.72 & 2.68 & 2.53 & 2.65 & 2.73 & 2.97 & 3.46 \\
\hline 14.5 & 3.34 & 2.95 & 2.71 & 2.63 & 2.50 & 2.57 & 2.70 & 2.89 & 3.45 \\
\hline 15.5 & 3.29 & 2.78 & 2.58 & 2.48 & 2.43 & 2.47 & 2.62 & 2.85 & 3.29 \\
\hline 16.5 & 3.19 & 2.66 & 2.56 & 2.41 & 2.31 & 2.41 & 2.43 & 2.59 & 3.19 \\
\hline 17.5 & 3.01 & 2.58 & 2.44 & 2.30 & 2.26 & 2.32 & 2.33 & 2.50 & 3.01 \\
\hline 18.5 & 2.82 & 2.43 & 2.26 & 2.15 & 2.10 & 2.18 & 2.34 & 2.41 & 2.79 \\
\hline 19.5 & 2.62 & 2.22 & 2.06 & 1.99 & 1.92 & 1.98 & 2.07 & 2.20 & 2.64 \\
\hline 20.5 & 2.36 & 1.98 & 1.90 & 1.80 & 1.75 & 1.82 & 1.89 & 2.01 & 2.27 \\
\hline 21.5 & 2.13 & 1.79 & 1.63 & 1.55 & 1.59 & 1.60 & 1.63 & 1.85 & 2.10 \\
\hline 22.5 & 1.91 & 1.61 & 1.47 & 1.37 & 1.29 & 1.36 & 1.48 & 1.59 & 1.87 \\
\hline 23.5 & 2.40 & 1.94 & 1.90 & 1.74 & 1.71 & 1.78 & 1.78 & 2.07 & 2.31 \\
\hline Ax. Avg. & 2.75 & 2.34 & 2.18 & 2.09 & 2.03 & 2.09 & 2.18 & 2.33 & 2.75 \\
\hline & & & & & & & & & 1.42 \\
\hline
\end{tabular}

POWER DENSITY VALUES NORMALIZED TO A CORE POWER OF 11.57 MW AND ASSUME ALL FISSION ENERGY (EXCLUDING GAMMA-HEATING OF EX-CORE COMPONENTS) IS DEPOSITED IN THE FUEL 
Table B-12. Power Density Data (kW/cc) in Fuel Plate 12 in Prototypic Conditions (continued).

Plate 12, Core Position X4/X8, 173 MWd Burnup, Equilibrium Xenon Conditions

\begin{tabular}{|c|c|c|c|c|c|c|c|c|c|}
\hline $\begin{array}{l}\text { Axial } \\
\text { position } \\
\text { from } \\
\text { top of } \\
\text { fuel } \\
\text { (inches) }\end{array}$ & $\begin{array}{c}0.000 \\
\text { to } \\
0.197 \\
\text { inches }\end{array}$ & $\begin{array}{c}0.197 \\
\text { to } \\
0.394 \\
\text { inches }\end{array}$ & $\begin{array}{c}0.394 \\
\text { to } \\
0.591 \\
\text { inches }\end{array}$ & $\begin{array}{c}0.591 \\
\text { to } \\
1.153 \\
\text { inches }\end{array}$ & $\begin{array}{c}1.153 \\
\text { to } \\
1.715 \\
\text { inches }\end{array}$ & $\begin{array}{c}1.715 \\
\text { to } \\
2.277 \\
\text { inches }\end{array}$ & $\begin{array}{c}2.277 \\
\text { to } \\
2.474 \\
\text { inches }\end{array}$ & $\begin{array}{c}2.474 \\
\text { to } \\
2.671 \\
\text { inches }\end{array}$ & $\begin{array}{c}2.671 \\
\text { to } \\
2.868 \\
\text { inches }\end{array}$ \\
\hline 0.5 & 1.80 & 1.54 & 1.40 & 1.41 & 1.36 & 1.43 & 1.39 & 1.50 & 1.87 \\
\hline 1.5 & 1.57 & 1.37 & 1.23 & 1.16 & 1.14 & 1.16 & 1.27 & 1.34 & 1.55 \\
\hline 2.5 & 1.85 & 1.55 & 1.43 & 1.41 & 1.35 & 1.41 & 1.42 & 1.54 & 1.81 \\
\hline 3.5 & 2.13 & 1.86 & 1.69 & 1.64 & 1.57 & 1.67 & 1.74 & 1.82 & 2.11 \\
\hline 4.5 & 2.45 & 1.98 & 1.95 & 1.83 & 1.78 & 1.79 & 1.92 & 2.07 & 2.37 \\
\hline 5.5 & 2.66 & 2.25 & 2.11 & 2.00 & 1.93 & 1.99 & 2.11 & 2.18 & 2.56 \\
\hline 6.5 & 2.85 & 2.47 & 2.27 & 2.16 & 2.09 & 2.22 & 2.33 & 2.37 & 2.78 \\
\hline 7.5 & 3.03 & 2.61 & 2.41 & 2.29 & 2.14 & 2.35 & 2.40 & 2.61 & 3.01 \\
\hline 8.5 & 3.17 & 2.59 & 2.49 & 2.39 & 2.25 & 2.38 & 2.44 & 2.63 & 3.14 \\
\hline 9.5 & 3.21 & 2.79 & 2.62 & 2.46 & 2.40 & 2.50 & 2.67 & 2.71 & 3.15 \\
\hline 10.5 & 3.28 & 2.79 & 2.59 & 2.54 & 2.44 & 2.58 & 2.62 & 2.77 & 3.20 \\
\hline 11.5 & 3.40 & 2.87 & 2.62 & 2.53 & 2.47 & 2.53 & 2.64 & 2.99 & 3.40 \\
\hline 12.5 & 3.43 & 2.85 & 2.67 & 2.55 & 2.51 & 2.58 & 2.70 & 2.79 & 3.29 \\
\hline 13.5 & 3.38 & 2.77 & 2.59 & 2.55 & 2.44 & 2.52 & 2.62 & 2.81 & 3.28 \\
\hline 14.5 & 3.35 & 2.75 & 2.57 & 2.49 & 2.46 & 2.53 & 2.55 & 2.74 & 3.19 \\
\hline 15.5 & 3.15 & 2.59 & 2.41 & 2.38 & 2.33 & 2.45 & 2.50 & 2.72 & 3.13 \\
\hline 16.5 & 2.94 & 2.61 & 2.50 & 2.39 & 2.31 & 2.33 & 2.45 & 2.60 & 3.05 \\
\hline 17.5 & 2.80 & 2.52 & 2.32 & 2.25 & 2.13 & 2.20 & 2.27 & 2.44 & 2.91 \\
\hline 18.5 & 2.71 & 2.34 & 2.09 & 2.08 & 2.04 & 2.08 & 2.20 & 2.33 & 2.71 \\
\hline 19.5 & 2.51 & 2.11 & 1.99 & 1.95 & 1.87 & 1.89 & 2.07 & 2.17 & 2.51 \\
\hline 20.5 & 2.26 & 1.94 & 1.80 & 1.76 & 1.70 & 1.77 & 1.80 & 1.96 & 2.27 \\
\hline 21.5 & 2.00 & 1.65 & 1.64 & 1.54 & 1.49 & 1.57 & 1.60 & 1.69 & 2.08 \\
\hline 22.5 & 1.82 & 1.57 & 1.36 & 1.33 & 1.26 & 1.32 & 1.44 & 1.51 & 1.84 \\
\hline 23.5 & 2.51 & 1.88 & 1.84 & 1.72 & 1.64 & 1.78 & 1.80 & 1.98 & 2.37 \\
\hline Ax. Avg. & 2.68 & 2.26 & 2.11 & 2.03 & 1.96 & 2.04 & 2.12 & 2.26 & 2.65 \\
\hline
\end{tabular}

POWER DENSITY VALUES NORMALIZED TO A CORE POWER OF 11.57 MW AND ASSUME ALL FISSION ENERGY (EXCLUDING GAMMA-HEATING OF EX-CORE COMPONENTS) IS DEPOSITED IN THE FUEL 
Table B-13. Power Density Data $\left(\mathrm{kW} / \mathrm{cm}^{3}\right)$ in Fuel Plate 13 in Prototypic Core.

\section{Fuel core width: 2.974 inches / Fuel core thickness: 20 mil}

Plate 13, Core Position X1/X5, 0 MWd Burnup, Xenon-Free Conditions

\begin{tabular}{|c|c|c|c|c|c|c|c|c|c|}
\hline $\begin{array}{c}\text { Axial } \\
\text { position } \\
\text { from } \\
\text { top of } \\
\text { fuel } \\
\text { (inches) } \\
\end{array}$ & $\begin{array}{c}0.000 \\
\text { to } \\
0.197 \\
\text { inches }\end{array}$ & $\begin{array}{c}0.197 \\
\text { to } \\
0.394 \\
\text { inches } \\
\end{array}$ & $\begin{array}{c}0.394 \\
\text { to } \\
0.591 \\
\text { inches } \\
\end{array}$ & $\begin{array}{c}0.591 \\
\text { to } \\
1.188 \\
\text { inches }\end{array}$ & $\begin{array}{c}1.188 \\
\text { to } \\
1.786 \\
\text { inches }\end{array}$ & $\begin{array}{c}1.786 \\
\text { to } \\
2.384 \\
\text { inches } \\
\end{array}$ & $\begin{array}{c}2.384 \\
\text { to } \\
2.581 \\
\text { inches } \\
\end{array}$ & $\begin{array}{c}2.581 \\
\text { to } \\
2.778 \\
\text { inches } \\
\end{array}$ & $\begin{array}{c}2.778 \\
\text { to } \\
2.974 \\
\text { inches } \\
\end{array}$ \\
\hline 0.5 & 1.16 & 1.02 & 0.91 & 0.94 & 0.86 & 0.91 & 0.94 & 1.05 & 1.18 \\
\hline 1.5 & 0.91 & 0.85 & 0.85 & 0.81 & 0.77 & 0.77 & 0.82 & 0.87 & 1.04 \\
\hline 2.5 & 1.10 & 1.03 & 0.95 & 0.99 & 0.89 & 0.98 & 1.00 & 1.00 & 1.18 \\
\hline 3.5 & 1.36 & 1.22 & 1.15 & 1.13 & 1.09 & 1.17 & 1.16 & 1.20 & 1.34 \\
\hline 4.5 & 1.59 & 1.38 & 1.36 & 1.35 & 1.29 & 1.34 & 1.41 & 1.43 & 1.68 \\
\hline 5.5 & 1.88 & 1.73 & 1.60 & 1.55 & 1.55 & 1.59 & 1.61 & 1.63 & 1.87 \\
\hline 6.5 & 2.10 & 1.95 & 1.81 & 1.74 & 1.78 & 1.79 & 1.86 & 1.95 & 2.19 \\
\hline 7.5 & 2.52 & 2.13 & 2.02 & 2.03 & 1.97 & 2.01 & 2.15 & 2.22 & 2.48 \\
\hline 8.5 & 2.84 & 2.39 & 2.21 & 2.24 & 2.20 & 2.28 & 2.40 & 2.50 & 2.86 \\
\hline 9.5 & 3.25 & 2.77 & 2.60 & 2.49 & 2.40 & 2.49 & 2.60 & 2.76 & 3.23 \\
\hline 10.5 & 3.46 & 3.00 & 2.75 & 2.72 & 2.69 & 2.80 & 2.76 & 2.97 & 3.43 \\
\hline 11.5 & 3.73 & 3.33 & 2.95 & 2.88 & 2.80 & 2.95 & 2.97 & 3.21 & 3.73 \\
\hline 12.5 & 3.92 & 3.38 & 3.13 & 3.02 & 2.93 & 3.01 & 3.14 & 3.40 & 3.88 \\
\hline 13.5 & 4.04 & 3.56 & 3.31 & 3.12 & 3.03 & 3.16 & 3.33 & 3.60 & 4.08 \\
\hline 14.5 & 4.14 & 3.49 & 3.26 & 3.19 & 3.09 & 3.19 & 3.39 & 3.58 & 4.07 \\
\hline 15.5 & 4.10 & 3.46 & 3.13 & 3.15 & 3.01 & 3.16 & 3.22 & 3.46 & 4.09 \\
\hline 16.5 & 3.97 & 3.55 & 3.30 & 3.15 & 3.00 & 3.15 & 3.27 & 3.50 & 4.08 \\
\hline 17.5 & 4.02 & 3.37 & 3.21 & 3.03 & 2.91 & 3.05 & 3.14 & 3.30 & 3.89 \\
\hline 18.5 & 3.69 & 3.28 & 2.94 & 2.86 & 2.79 & 2.91 & 2.87 & 3.21 & 3.76 \\
\hline 19.5 & 3.47 & 2.96 & 2.78 & 2.69 & 2.59 & 2.69 & 2.78 & 3.05 & 3.45 \\
\hline 20.5 & 3.19 & 2.72 & 2.55 & 2.48 & 2.38 & 2.46 & 2.56 & 2.77 & 3.16 \\
\hline 21.5 & 2.88 & 2.41 & 2.24 & 2.18 & 2.07 & 2.16 & 2.25 & 2.43 & 2.89 \\
\hline 22.5 & 2.66 & 2.18 & 1.94 & 1.85 & 1.82 & 1.90 & 2.00 & 2.20 & 2.50 \\
\hline 23.5 & 3.43 & 2.72 & 2.57 & 2.47 & 2.35 & 2.44 & 2.57 & 2.75 & 3.48 \\
\hline Ax. Avg. & 2.89 & 2.49 & 2.31 & 2.25 & 2.18 & 2.26 & 2.34 & 2.50 & 2.90 \\
\hline
\end{tabular}

POWER DENSITY VALUES NORMALIZED TO A CORE POWER OF 11.57 MW AND ASSUME ALL FISSION ENERGY (EXCLUDING GAMMA-HEATING OF EX-CORE COMPONENTS) IS DEPOSITED IN THE FUEL 
Table B-13. Power Density Data (kW/cc) in Fuel Plate 13 in Prototypic Conditions (continued).

Plate 13, Core Position X1/X5, 3 MWd Burnup, Equilibrium Xenon Conditions

\begin{tabular}{|c|c|c|c|c|c|c|c|c|c|}
\hline $\begin{array}{c}\text { Axial } \\
\text { position } \\
\text { from } \\
\text { top of } \\
\text { fuel } \\
\text { (inches) }\end{array}$ & $\begin{array}{c}0.000 \\
\text { to } \\
0.197 \\
\text { inches }\end{array}$ & $\begin{array}{c}0.197 \\
\text { to } \\
0.394 \\
\text { inches }\end{array}$ & $\begin{array}{c}0.394 \\
\text { to } \\
0.591 \\
\text { inches }\end{array}$ & $\begin{array}{c}0.591 \\
\text { to } \\
1.188 \\
\text { inches }\end{array}$ & $\begin{array}{c}1.188 \\
\text { to } \\
1.786 \\
\text { inches }\end{array}$ & $\begin{array}{c}1.786 \\
\text { to } \\
2.384 \\
\text { inches }\end{array}$ & $\begin{array}{c}2.384 \\
\text { to } \\
2.581 \\
\text { inches }\end{array}$ & $\begin{array}{c}2.581 \\
\text { to } \\
2.778 \\
\text { inches }\end{array}$ & $\begin{array}{c}2.778 \\
\text { to } \\
2.974 \\
\text { inches }\end{array}$ \\
\hline 1.5 & 1.62 & 1.61 & 1.52 & 1.42 & 1.37 & 1.42 & 1.44 & 1.57 & 1.91 \\
\hline 2.5 & 1.91 & 1.66 & 1.53 & 1.18 & 1.18 & 1.23 & 1.28 & 1.40 & 1.54 \\
\hline 3.5 & 2.17 & 1.83 & 1.76 & 1.69 & 1.66 & 1.68 & 1.73 & 1.87 & 2.28 \\
\hline 4.5 & 2.44 & 2.12 & 1.99 & 1.93 & 1.81 & 1.90 & 2.05 & 2.14 & 2.54 \\
\hline 5.5 & 2.65 & 2.39 & 2.13 & 2.12 & 2.07 & 2.09 & 2.19 & 2.37 & 2.75 \\
\hline 6.5 & 2.92 & 2.55 & 2.36 & 2.31 & 2.21 & 2.29 & 2.44 & 2.63 & 3.00 \\
\hline 7.5 & 3.22 & 2.70 & 2.52 & 2.45 & 2.33 & 2.43 & 2.58 & 2.67 & 3.22 \\
\hline 8.5 & 3.40 & 2.81 & 2.62 & 2.54 & 2.47 & 2.58 & 2.63 & 2.79 & 3.29 \\
\hline 9.5 & 3.44 & 3.06 & 2.75 & 2.62 & 2.58 & 2.69 & 2.69 & 2.87 & 3.48 \\
\hline 10.5 & 3.49 & 3.04 & 2.79 & 2.69 & 2.64 & 2.69 & 2.76 & 3.06 & 3.48 \\
\hline 11.5 & 3.59 & 3.07 & 2.82 & 2.75 & 2.67 & 2.75 & 2.87 & 3.11 & 3.51 \\
\hline 12.5 & 3.61 & 2.98 & 2.93 & 2.65 & 2.65 & 2.77 & 2.90 & 3.05 & 3.53 \\
\hline 13.5 & 3.56 & 2.99 & 2.83 & 2.72 & 2.59 & 2.71 & 2.90 & 3.04 & 3.68 \\
\hline 14.5 & 3.45 & 2.96 & 2.77 & 2.70 & 2.59 & 2.69 & 2.78 & 2.85 & 3.40 \\
\hline 15.5 & 3.44 & 2.88 & 2.73 & 2.62 & 2.52 & 2.59 & 2.77 & 2.87 & 3.39 \\
\hline 16.5 & 3.29 & 2.69 & 2.61 & 2.49 & 2.43 & 2.46 & 2.52 & 2.83 & 3.32 \\
\hline 17.5 & 3.06 & 2.68 & 2.53 & 2.36 & 2.25 & 2.38 & 2.48 & 2.63 & 3.07 \\
\hline 18.5 & 2.79 & 2.41 & 2.27 & 2.26 & 2.11 & 2.18 & 2.28 & 2.50 & 2.90 \\
\hline 19.5 & 2.65 & 2.37 & 2.13 & 2.06 & 1.99 & 2.00 & 2.18 & 2.34 & 2.64 \\
\hline 20.5 & 2.53 & 2.10 & 1.88 & 1.82 & 1.85 & 1.81 & 1.91 & 2.08 & 2.42 \\
\hline 21.5 & 2.15 & 1.79 & 1.70 & 1.58 & 1.61 & 1.62 & 1.69 & 1.81 & 2.12 \\
\hline 22.5 & 1.93 & 1.63 & 1.46 & 1.42 & 1.36 & 1.37 & 1.47 & 1.60 & 1.85 \\
\hline 23.5 & 2.51 & 2.00 & 1.84 & 1.80 & 1.72 & 1.79 & 1.86 & 2.02 & 2.49 \\
\hline Ax. Avg. & 2.82 & 2.40 & 2.24 & 2.15 & 2.09 & 2.15 & 2.25 & 2.41 & 2.82 \\
\hline & & & & & & & & & 1.53 \\
\hline
\end{tabular}

POWER DENSITY VALUES NORMALIZED TO A CORE POWER OF 11.57 MW AND ASSUME ALL FISSION ENERGY (EXCLUDING GAMMA-HEATING OF EX-CORE COMPONENTS) IS DEPOSITED IN THE FUEL 
Table B-13. Power Density Data (kW/cc) in Fuel Plate 13 in Prototypic Conditions (continued).

Plate 13, Core Position X3/X7, 80 MWd Burnup, Equilibrium Xenon Conditions

\begin{tabular}{|c|c|c|c|c|c|c|c|c|c|}
\hline $\begin{array}{c}\text { Axial } \\
\text { position } \\
\text { from } \\
\text { top of } \\
\text { fuel } \\
\text { (inches) }\end{array}$ & $\begin{array}{c}0.000 \\
\text { to } \\
0.197 \\
\text { inches }\end{array}$ & $\begin{array}{c}0.197 \\
\text { to } \\
0.394 \\
\text { inches }\end{array}$ & $\begin{array}{c}0.394 \\
\text { to } \\
0.591 \\
\text { inches }\end{array}$ & $\begin{array}{c}0.591 \\
\text { to } \\
\text { inches }\end{array}$ & $\begin{array}{c}1.188 \\
\text { to } \\
1.786 \\
\text { inches }\end{array}$ & $\begin{array}{c}1.786 \\
\text { to } \\
2.384 \\
\text { inches }\end{array}$ & $\begin{array}{c}\text { 2.384 } \\
\text { to } \\
2.581 \\
\text { inches }\end{array}$ & $\begin{array}{c}2.581 \\
\text { to } \\
2.778 \\
\text { inches }\end{array}$ & $\begin{array}{c}2.778 \\
\text { to } \\
\text { inches }\end{array}$ \\
\hline 0.5 & 1.84 & 1.53 & 1.53 & 1.39 & 1.36 & 1.42 & 1.42 & 1.48 & 1.82 \\
\hline 1.5 & 1.66 & 1.37 & 1.27 & 1.18 & 1.15 & 1.18 & 1.23 & 1.29 & 1.53 \\
\hline 2.5 & 1.88 & 1.59 & 1.44 & 1.42 & 1.35 & 1.41 & 1.51 & 1.63 & 1.89 \\
\hline 3.5 & 2.14 & 1.81 & 1.78 & 1.65 & 1.59 & 1.62 & 1.73 & 1.84 & 2.21 \\
\hline 4.5 & 2.40 & 2.11 & 1.95 & 1.86 & 1.80 & 1.88 & 1.93 & 2.01 & 2.48 \\
\hline 5.5 & 2.70 & 2.38 & 2.14 & 2.10 & 1.99 & 2.06 & 2.11 & 2.25 & 2.64 \\
\hline 6.5 & 2.88 & 2.39 & 2.38 & 2.26 & 2.10 & 2.16 & 2.26 & 2.55 & 2.91 \\
\hline 7.5 & 3.05 & 2.68 & 2.40 & 2.31 & 2.28 & 2.31 & 2.45 & 2.69 & 3.09 \\
\hline 8.5 & 3.31 & 2.70 & 2.64 & 2.41 & 2.38 & 2.45 & 2.66 & 2.79 & 3.28 \\
\hline 9.5 & 3.27 & 2.90 & 2.64 & 2.54 & 2.45 & 2.54 & 2.67 & 2.86 & 3.31 \\
\hline 10.5 & 3.35 & 2.88 & 2.81 & 2.64 & 2.53 & 2.62 & 2.66 & 2.98 & 3.31 \\
\hline 11.5 & 3.50 & 2.93 & 2.75 & 2.63 & 2.52 & 2.61 & 2.68 & 3.01 & 3.45 \\
\hline 12.5 & 3.45 & 2.96 & 2.75 & 2.65 & 2.55 & 2.61 & 2.74 & 2.94 & 3.45 \\
\hline 13.5 & 3.45 & 2.86 & 2.74 & 2.59 & 2.54 & 2.61 & 2.69 & 2.89 & 3.41 \\
\hline 14.5 & 3.35 & 2.87 & 2.70 & 2.56 & 2.49 & 2.52 & 2.58 & 2.96 & 3.39 \\
\hline 15.5 & 3.19 & 2.77 & 2.62 & 2.48 & 2.42 & 2.47 & 2.73 & 2.73 & 3.18 \\
\hline 16.5 & 3.13 & 2.72 & 2.44 & 2.39 & 2.30 & 2.40 & 2.45 & 2.63 & 3.05 \\
\hline 17.5 & 3.03 & 2.57 & 2.41 & 2.26 & 2.17 & 2.29 & 2.29 & 2.51 & 2.92 \\
\hline 18.5 & 2.76 & 2.43 & 2.26 & 2.17 & 2.07 & 2.08 & 2.22 & 2.40 & 2.76 \\
\hline 19.5 & 2.60 & 2.25 & 2.01 & 1.99 & 1.91 & 1.97 & 2.03 & 2.23 & 2.63 \\
\hline 20.5 & 2.35 & 2.05 & 1.90 & 1.76 & 1.71 & 1.82 & 1.79 & 2.00 & 2.38 \\
\hline 21.5 & 2.06 & 1.79 & 1.69 & 1.61 & 1.50 & 1.56 & 1.63 & 1.77 & 2.05 \\
\hline 22.5 & 1.81 & 1.56 & 1.42 & 1.35 & 1.29 & 1.38 & 1.40 & 1.53 & 1.90 \\
\hline 23.5 & 2.40 & 1.95 & 1.83 & 1.78 & 1.67 & 1.75 & 1.81 & 1.95 & 2.40 \\
\hline Ax. Avg. & 2.73 & 2.33 & 2.19 & 2.08 & 2.00 & 2.07 & 2.15 & 2.33 & 2.73 \\
\hline & & & & & & & & & \\
\hline
\end{tabular}

POWER DENSITY VALUES NORMALIZED TO A CORE POWER OF 11.57 MW AND ASSUME ALL FISSION ENERGY (EXCLUDING GAMMA-HEATING OF EX-CORE COMPONENTS) IS DEPOSITED IN THE FUEL 
Table B-13. Power Density Data (kW/cc) in Fuel Plate 13 in Prototypic Conditions (continued).

Plate 13, Core Position X2/X6, 99 MWd Burnup, Equilibrium Xenon Conditions

\begin{tabular}{|c|c|c|c|c|c|c|c|c|c|}
\hline $\begin{array}{c}\text { Axial } \\
\text { position } \\
\text { from } \\
\text { top of } \\
\text { fuel } \\
\text { (inches) }\end{array}$ & $\begin{array}{c}0.000 \\
\text { to } \\
0.197 \\
\text { inches }\end{array}$ & $\begin{array}{c}0.197 \\
\text { to } \\
0.394 \\
\text { inches }\end{array}$ & $\begin{array}{c}0.394 \\
\text { to } \\
0.591 \\
\text { inches }\end{array}$ & $\begin{array}{c}0.591 \\
\text { to } \\
\text { inches }\end{array}$ & $\begin{array}{c}1.188 \\
\text { to } \\
1.786 \\
\text { inches }\end{array}$ & $\begin{array}{c}1.786 \\
\text { to } \\
2.384 \\
\text { inches }\end{array}$ & $\begin{array}{c}\text { 2.384 } \\
\text { to } \\
2.581 \\
\text { inches }\end{array}$ & $\begin{array}{c}2.581 \\
\text { to } \\
2.778 \\
\text { inches }\end{array}$ & $\begin{array}{c}2.778 \\
\text { to } \\
\text { inches }\end{array}$ \\
\hline 0.5 & 1.79 & 1.54 & 1.39 & 1.35 & 1.32 & 1.36 & 1.40 & 1.55 & 1.76 \\
\hline 1.5 & 1.54 & 1.31 & 1.26 & 1.18 & 1.10 & 1.14 & 1.19 & 1.25 & 1.52 \\
\hline 2.5 & 1.83 & 1.53 & 1.46 & 1.44 & 1.39 & 1.40 & 1.43 & 1.53 & 1.83 \\
\hline 3.5 & 2.16 & 1.89 & 1.76 & 1.66 & 1.62 & 1.69 & 1.78 & 1.85 & 2.11 \\
\hline 4.5 & 2.41 & 2.04 & 1.85 & 1.87 & 1.84 & 1.89 & 1.95 & 2.10 & 2.39 \\
\hline 5.5 & 2.75 & 2.31 & 2.16 & 2.04 & 1.99 & 2.05 & 2.16 & 2.25 & 2.67 \\
\hline 6.5 & 2.90 & 2.44 & 2.32 & 2.23 & 2.08 & 2.19 & 2.27 & 2.52 & 2.98 \\
\hline 7.5 & 3.06 & 2.67 & 2.46 & 2.38 & 2.24 & 2.40 & 2.45 & 2.62 & 3.05 \\
\hline 8.5 & 3.08 & 2.76 & 2.56 & 2.43 & 2.41 & 2.47 & 2.61 & 2.74 & 3.24 \\
\hline 9.5 & 3.27 & 2.82 & 2.58 & 2.54 & 2.46 & 2.55 & 2.70 & 2.84 & 3.25 \\
\hline 10.5 & 3.29 & 2.84 & 2.69 & 2.60 & 2.54 & 2.61 & 2.75 & 3.01 & 3.33 \\
\hline 11.5 & 3.36 & 2.93 & 2.80 & 2.64 & 2.55 & 2.66 & 2.79 & 2.91 & 3.46 \\
\hline 12.5 & 3.44 & 2.94 & 2.74 & 2.68 & 2.61 & 2.65 & 2.80 & 2.90 & 3.44 \\
\hline 13.5 & 3.41 & 3.01 & 2.75 & 2.63 & 2.48 & 2.59 & 2.74 & 2.93 & 3.47 \\
\hline 14.5 & 3.37 & 2.88 & 2.65 & 2.60 & 2.50 & 2.59 & 2.74 & 2.85 & 3.36 \\
\hline 15.5 & 3.20 & 2.79 & 2.67 & 2.47 & 2.39 & 2.49 & 2.70 & 2.87 & 3.37 \\
\hline 16.5 & 3.23 & 2.67 & 2.53 & 2.42 & 2.29 & 2.39 & 2.52 & 2.69 & 3.23 \\
\hline 17.5 & 2.97 & 2.64 & 2.42 & 2.29 & 2.23 & 2.28 & 2.41 & 2.53 & 2.97 \\
\hline 18.5 & 2.90 & 2.37 & 2.27 & 2.14 & 2.09 & 2.12 & 2.24 & 2.41 & 2.72 \\
\hline 19.5 & 2.55 & 2.27 & 2.06 & 2.02 & 1.87 & 2.02 & 2.05 & 2.24 & 2.52 \\
\hline 20.5 & 2.34 & 2.01 & 1.81 & 1.76 & 1.72 & 1.80 & 1.86 & 2.02 & 2.41 \\
\hline 21.5 & 2.09 & 1.74 & 1.64 & 1.59 & 1.55 & 1.57 & 1.65 & 1.85 & 2.08 \\
\hline 22.5 & 1.84 & 1.58 & 1.42 & 1.39 & 1.30 & 1.37 & 1.44 & 1.63 & 1.86 \\
\hline 23.5 & 2.50 & 2.06 & 1.80 & 1.79 & 1.73 & 1.73 & 1.84 & 2.00 & 2.39 \\
\hline Ax. Avg. & 2.72 & 2.33 & 2.17 & 2.09 & 2.01 & 2.08 & 2.19 & 2.34 & 2.73 \\
\hline & & & & & & & & & \\
\hline
\end{tabular}

POWER DENSITY VALUES NORMALIZED TO A CORE POWER OF 11.57 MW AND ASSUME ALL FISSION ENERGY (EXCLUDING GAMMA-HEATING OF EX-CORE COMPONENTS) IS DEPOSITED IN THE FUEL 
Table B-13. Power Density Data (kW/cc) in Fuel Plate 13 in Prototypic Conditions (continued).

Plate 13, Core Position X4/X8, 173 MWd Burnup, Equilibrium Xenon Conditions

\begin{tabular}{|c|c|c|c|c|c|c|c|c|c|}
\hline $\begin{array}{c}\text { Axial } \\
\text { position } \\
\text { from } \\
\text { top of } \\
\text { fuel } \\
\text { (inches) }\end{array}$ & $\begin{array}{c}0.000 \\
\text { to } \\
0.197 \\
\text { inches }\end{array}$ & $\begin{array}{c}0.197 \\
\text { to } \\
0.394 \\
\text { inches }\end{array}$ & $\begin{array}{c}0.394 \\
\text { to } \\
0.591 \\
\text { inches }\end{array}$ & $\begin{array}{c}0.591 \\
\text { to } \\
1.188 \\
\text { inches }\end{array}$ & $\begin{array}{c}1.188 \\
\text { to } \\
1.786 \\
\text { inches }\end{array}$ & $\begin{array}{c}1.786 \\
\text { to } \\
2.384 \\
\text { inches }\end{array}$ & $\begin{array}{c}\text { 2.384 } \\
\text { to } \\
2.581 \\
\text { inches }\end{array}$ & $\begin{array}{c}2.581 \\
\text { to } \\
2.778 \\
\text { inches }\end{array}$ & $\begin{array}{c}2.778 \\
\text { to } \\
2.974 \\
\text { inches }\end{array}$ \\
\hline 1.5 & 1.78 & 1.49 & 1.41 & 1.37 & 1.34 & 1.33 & 1.43 & 1.51 & 1.83 \\
\hline 2.5 & 1.85 & 1.28 & 1.19 & 1.14 & 1.09 & 1.15 & 1.17 & 1.23 & 1.59 \\
\hline 3.5 & 2.15 & 1.86 & 1.79 & 1.39 & 1.35 & 1.37 & 1.45 & 1.54 & 1.73 \\
\hline 4.5 & 2.40 & 2.07 & 1.92 & 1.81 & 1.74 & 1.84 & 1.89 & 1.99 & 2.34 \\
\hline 5.5 & 2.60 & 2.26 & 2.08 & 1.97 & 1.91 & 1.97 & 2.09 & 2.21 & 2.63 \\
\hline 6.5 & 2.90 & 2.37 & 2.22 & 2.15 & 2.08 & 2.17 & 2.27 & 2.41 & 2.79 \\
\hline 7.5 & 3.02 & 2.60 & 2.42 & 2.26 & 2.21 & 2.29 & 2.37 & 2.59 & 2.98 \\
\hline 8.5 & 3.05 & 2.67 & 2.45 & 2.37 & 2.31 & 2.42 & 2.42 & 2.65 & 3.14 \\
\hline 9.5 & 3.23 & 2.63 & 2.57 & 2.45 & 2.39 & 2.47 & 2.58 & 2.70 & 3.19 \\
\hline 10.5 & 3.21 & 2.79 & 2.54 & 2.51 & 2.37 & 2.50 & 2.64 & 2.77 & 3.12 \\
\hline 11.5 & 3.31 & 2.83 & 2.63 & 2.52 & 2.48 & 2.56 & 2.72 & 2.85 & 3.27 \\
\hline 12.5 & 3.36 & 2.86 & 2.70 & 2.50 & 2.47 & 2.53 & 2.61 & 2.84 & 3.30 \\
\hline 13.5 & 3.27 & 2.87 & 2.57 & 2.51 & 2.39 & 2.51 & 2.62 & 2.79 & 3.19 \\
\hline 14.5 & 3.16 & 2.72 & 2.61 & 2.46 & 2.37 & 2.49 & 2.58 & 2.69 & 3.26 \\
\hline 15.5 & 3.18 & 2.65 & 2.43 & 2.42 & 2.32 & 2.41 & 2.52 & 2.72 & 3.12 \\
\hline 16.5 & 2.96 & 2.58 & 2.37 & 2.32 & 2.27 & 2.31 & 2.45 & 2.67 & 3.06 \\
\hline 17.5 & 2.74 & 2.47 & 2.28 & 2.19 & 2.11 & 2.16 & 2.27 & 2.44 & 2.86 \\
\hline 18.5 & 2.59 & 2.29 & 2.12 & 2.10 & 1.97 & 2.09 & 2.21 & 2.38 & 2.68 \\
\hline 19.5 & 2.58 & 2.08 & 2.00 & 1.92 & 1.84 & 1.90 & 1.94 & 2.17 & 2.54 \\
\hline 20.5 & 2.23 & 1.90 & 1.77 & 1.74 & 1.69 & 1.76 & 1.76 & 1.93 & 2.25 \\
\hline 21.5 & 1.95 & 1.77 & 1.59 & 1.53 & 1.45 & 1.56 & 1.59 & 1.68 & 2.04 \\
\hline 22.5 & 1.86 & 1.43 & 1.32 & 1.34 & 1.27 & 1.34 & 1.43 & 1.55 & 1.82 \\
\hline 23.5 & 2.32 & 2.00 & 1.81 & 1.73 & 1.63 & 1.77 & 1.79 & 1.96 & 2.37 \\
\hline Ax. Avg. & 2.63 & 2.25 & 2.09 & 2.01 & 1.94 & 2.02 & 2.11 & 2.25 & 2.63 \\
\hline & & & & & & & & & \\
\hline
\end{tabular}

POWER DENSITY VALUES NORMALIZED TO A CORE POWER OF 11.57 MW AND ASSUME ALL FISSION ENERGY (EXCLUDING GAMMA-HEATING OF EX-CORE COMPONENTS) IS DEPOSITED IN THE FUEL 
Table B-14. Power Density Data $\left(\mathrm{kW} / \mathrm{cm}^{3}\right)$ in Fuel Plate 14 in Prototypic Core.

\section{Fuel core width: 3.081 inches / Fuel core thickness: 20 mil}

Plate 14, Core Position X1/X5, 0 MWd Burnup, Xenon-Free Conditions

\begin{tabular}{|c|c|c|c|c|c|c|c|c|c|}
\hline $\begin{array}{c}\text { Axial } \\
\text { position } \\
\text { from } \\
\text { top of } \\
\text { fuel } \\
\text { (inches) } \\
\end{array}$ & $\begin{array}{c}0.000 \\
\text { to } \\
0.197 \\
\text { inches }\end{array}$ & $\begin{array}{c}0.197 \\
\text { to } \\
0.394 \\
\text { inches } \\
\end{array}$ & $\begin{array}{c}0.394 \\
\text { to } \\
0.591 \\
\text { inches } \\
\end{array}$ & $\begin{array}{c}0.591 \\
\text { to } \\
1.224 \\
\text { inches }\end{array}$ & $\begin{array}{c}1.224 \\
\text { to } \\
1.857 \\
\text { inches }\end{array}$ & $\begin{array}{c}1.857 \\
\text { to } \\
2.491 \\
\text { inches } \\
\end{array}$ & $\begin{array}{c}2.491 \\
\text { to } \\
2.688 \\
\text { inches }\end{array}$ & $\begin{array}{c}2.688 \\
\text { to } \\
2.884 \\
\text { inches } \\
\end{array}$ & $\begin{array}{c}2.884 \\
\text { to } \\
3.081 \\
\text { inches } \\
\end{array}$ \\
\hline 0.5 & 1.18 & 1.03 & 0.91 & 0.89 & 0.89 & 0.93 & 0.93 & 0.94 & 1.16 \\
\hline 1.5 & 0.95 & 0.88 & 0.82 & 0.77 & 0.77 & 0.76 & 0.81 & 0.86 & 0.97 \\
\hline 2.5 & 1.08 & 1.01 & 0.99 & 0.94 & 0.91 & 0.96 & 0.93 & 1.03 & 1.16 \\
\hline 3.5 & 1.31 & 1.22 & 1.14 & 1.12 & 1.10 & 1.14 & 1.14 & 1.15 & 1.32 \\
\hline 4.5 & 1.46 & 1.39 & 1.36 & 1.34 & 1.26 & 1.34 & 1.38 & 1.51 & 1.57 \\
\hline 5.5 & 1.83 & 1.70 & 1.63 & 1.51 & 1.49 & 1.55 & 1.60 & 1.66 & 1.81 \\
\hline 6.5 & 2.09 & 1.84 & 1.76 & 1.74 & 1.73 & 1.73 & 1.81 & 1.92 & 2.16 \\
\hline 7.5 & 2.47 & 2.21 & 2.03 & 2.00 & 1.95 & 2.03 & 2.09 & 2.15 & 2.50 \\
\hline 8.5 & 2.83 & 2.44 & 2.36 & 2.18 & 2.13 & 2.25 & 2.34 & 2.50 & 2.82 \\
\hline 9.5 & 3.21 & 2.71 & 2.62 & 2.45 & 2.40 & 2.47 & 2.57 & 2.74 & 3.26 \\
\hline 10.5 & 3.45 & 3.03 & 2.82 & 2.73 & 2.59 & 2.72 & 2.88 & 2.97 & 3.56 \\
\hline 11.5 & 3.74 & 3.27 & 2.98 & 2.85 & 2.81 & 2.91 & 2.98 & 3.24 & 3.78 \\
\hline 12.5 & 3.95 & 3.37 & 3.14 & 3.02 & 2.89 & 3.02 & 3.07 & 3.31 & 3.95 \\
\hline 13.5 & 4.02 & 3.43 & 3.30 & 3.11 & 2.99 & 3.11 & 3.20 & 3.46 & 4.01 \\
\hline 14.5 & 4.09 & 3.50 & 3.26 & 3.17 & 3.03 & 3.15 & 3.30 & 3.53 & 4.11 \\
\hline 15.5 & 4.16 & 3.55 & 3.24 & 3.14 & 3.07 & 3.17 & 3.32 & 3.46 & 4.14 \\
\hline 16.5 & 3.94 & 3.39 & 3.28 & 3.09 & 2.92 & 3.08 & 3.30 & 3.53 & 4.08 \\
\hline 17.5 & 3.89 & 3.33 & 3.13 & 3.01 & 2.94 & 3.05 & 3.14 & 3.43 & 3.91 \\
\hline 18.5 & 3.68 & 3.08 & 2.96 & 2.88 & 2.80 & 2.88 & 2.93 & 3.30 & 3.70 \\
\hline 19.5 & 3.37 & 2.90 & 2.80 & 2.64 & 2.59 & 2.66 & 2.82 & 2.96 & 3.48 \\
\hline 20.5 & 3.22 & 2.68 & 2.59 & 2.42 & 2.38 & 2.42 & 2.64 & 2.78 & 3.21 \\
\hline 21.5 & 2.80 & 2.32 & 2.28 & 2.16 & 2.08 & 2.15 & 2.27 & 2.44 & 2.84 \\
\hline 22.5 & 2.53 & 2.09 & 1.99 & 1.89 & 1.82 & 1.95 & 2.07 & 2.11 & 2.54 \\
\hline 23.5 & 3.31 & 2.72 & 2.53 & 2.41 & 2.34 & 2.45 & 2.47 & 2.69 & 3.42 \\
\hline Ax. Avg. & 2.86 & 2.46 & 2.33 & 2.23 & 2.16 & 2.24 & 2.33 & 2.49 & 2.89 \\
\hline
\end{tabular}

POWER DENSITY VALUES NORMALIZED TO A CORE POWER OF 11.57 MW AND ASSUME ALL FISSION ENERGY (EXCLUDING GAMMA-HEATING OF EX-CORE COMPONENTS) IS DEPOSITED IN THE FUEL 
Table B-14. Power Density Data (kW/cc) in Fuel Plate 14 in Prototypic Conditions (continued).

Plate 14, Core Position X1/X5, 3 MWd Burnup, Equilibrium Xenon Conditions

\begin{tabular}{|c|c|c|c|c|c|c|c|c|c|}
\hline $\begin{array}{l}\text { Axial } \\
\text { position } \\
\text { from } \\
\text { top of } \\
\text { fuel } \\
\text { (inches) }\end{array}$ & $\begin{array}{c}0.000 \\
\text { to } \\
0.197 \\
\text { inches } \\
\end{array}$ & $\begin{array}{c}0.197 \\
\text { to } \\
0.394 \\
\text { inches } \\
\end{array}$ & $\begin{array}{c}0.394 \\
\text { to } \\
0.591 \\
\text { inches }\end{array}$ & $\begin{array}{c}0.591 \\
\text { to } \\
1.224 \\
\text { inches }\end{array}$ & $\begin{array}{c}1.224 \\
\text { to } \\
1.857 \\
\text { inches }\end{array}$ & $\begin{array}{c}1.857 \\
\text { to } \\
2.491 \\
\text { inches }\end{array}$ & $\begin{array}{c}2.491 \\
\text { to } \\
2.688 \\
\text { inches } \\
\end{array}$ & $\begin{array}{c}2.688 \\
\text { to } \\
2.884 \\
\text { inches }\end{array}$ & $\begin{array}{c}2.884 \\
\text { to } \\
3.081 \\
\text { inches } \\
\end{array}$ \\
\hline 0.5 & 1.86 & 1.56 & 1.45 & 1.39 & 1.38 & 1.38 & 1.36 & 1.60 & 1.80 \\
\hline 1.5 & 1.53 & 1.28 & 1.20 & 1.20 & 1.15 & 1.19 & 1.26 & 1.35 & 1.56 \\
\hline 2.5 & 1.87 & 1.55 & 1.53 & 1.44 & 1.39 & 1.44 & 1.50 & 1.55 & 1.91 \\
\hline 3.5 & 2.25 & 1.91 & 1.72 & 1.65 & 1.62 & 1.70 & 1.73 & 1.89 & 2.26 \\
\hline 4.5 & 2.48 & 2.13 & 1.93 & 1.90 & 1.84 & 1.93 & 1.98 & 2.11 & 2.49 \\
\hline 5.5 & 2.70 & 2.29 & 2.20 & 2.12 & 2.04 & 2.10 & 2.22 & 2.40 & 2.85 \\
\hline 6.5 & 2.98 & 2.56 & 2.39 & 2.27 & 2.21 & 2.31 & 2.40 & 2.62 & 2.99 \\
\hline 7.5 & 3.12 & 2.78 & 2.54 & 2.43 & 2.30 & 2.46 & 2.46 & 2.73 & 3.23 \\
\hline 8.5 & 3.39 & 2.93 & 2.57 & 2.50 & 2.52 & 2.54 & 2.64 & 2.68 & 3.30 \\
\hline 9.5 & 3.44 & 2.98 & 2.68 & 2.61 & 2.53 & 2.63 & 2.70 & 2.88 & 3.49 \\
\hline 10.5 & 3.50 & 2.99 & 2.78 & 2.71 & 2.59 & 2.69 & 2.72 & 3.01 & 3.52 \\
\hline 11.5 & 3.54 & 3.06 & 2.79 & 2.73 & 2.60 & 2.77 & 2.82 & 3.07 & 3.50 \\
\hline 12.5 & 3.50 & 2.93 & 2.81 & 2.71 & 2.61 & 2.77 & 2.88 & 3.10 & 3.52 \\
\hline 13.5 & 3.54 & 3.12 & 2.85 & 2.66 & 2.67 & 2.69 & 2.80 & 3.14 & 3.52 \\
\hline 14.5 & 3.47 & 2.96 & 2.71 & 2.71 & 2.58 & 2.62 & 2.74 & 2.93 & 3.53 \\
\hline 15.5 & 3.36 & 2.94 & 2.61 & 2.62 & 2.52 & 2.58 & 2.71 & 2.97 & 3.50 \\
\hline 16.5 & 3.24 & 2.81 & 2.55 & 2.48 & 2.40 & 2.48 & 2.61 & 2.74 & 3.27 \\
\hline 17.5 & 3.20 & 2.71 & 2.45 & 2.34 & 2.25 & 2.36 & 2.47 & 2.58 & 3.10 \\
\hline 18.5 & 2.86 & 2.45 & 2.27 & 2.25 & 2.14 & 2.22 & 2.26 & 2.41 & 2.92 \\
\hline 19.5 & 2.73 & 2.33 & 2.12 & 2.06 & 1.97 & 2.04 & 2.14 & 2.25 & 2.68 \\
\hline 20.5 & 2.46 & 2.04 & 1.95 & 1.91 & 1.79 & 1.83 & 1.88 & 2.10 & 2.49 \\
\hline 21.5 & 2.13 & 1.77 & 1.70 & 1.61 & 1.53 & 1.62 & 1.69 & 1.76 & 2.14 \\
\hline 22.5 & 1.92 & 1.60 & 1.48 & 1.40 & 1.34 & 1.39 & 1.51 & 1.62 & 1.89 \\
\hline 23.5 & 2.46 & 1.99 & 1.82 & 1.80 & 1.73 & 1.75 & 1.87 & 2.08 & 2.43 \\
\hline Ax. Avg. & 2.81 & 2.40 & 2.21 & 2.14 & 2.07 & 2.15 & 2.22 & 2.40 & 2.83 \\
\hline
\end{tabular}

POWER DENSITY VALUES NORMALIZED TO A CORE POWER OF 11.57 MW AND ASSUME ALL FISSION ENERGY (EXCLUDING GAMMA-HEATING OF EX-CORE COMPONENTS) IS DEPOSITED IN THE FUEL 
Table B-14. Power Density Data (kW/cc) in Fuel Plate 14 in Prototypic Conditions (continued).

Plate 14, Core Position X3/X7, 80 MWd Burnup, Equilibrium Xenon Conditions

\begin{tabular}{|c|c|c|c|c|c|c|c|c|c|}
\hline $\begin{array}{l}\text { Axial } \\
\text { position } \\
\text { from } \\
\text { top of } \\
\text { fuel } \\
\text { (inches) }\end{array}$ & $\begin{array}{c}0.000 \\
\text { to } \\
0.197 \\
\text { inches }\end{array}$ & $\begin{array}{c}0.197 \\
\text { to } \\
0.394 \\
\text { inches }\end{array}$ & $\begin{array}{c}0.394 \\
\text { to } \\
0.591 \\
\text { inches }\end{array}$ & $\begin{array}{c}0.591 \\
\text { to } \\
1.224 \\
\text { inches }\end{array}$ & $\begin{array}{c}1.224 \\
\text { to } \\
1.857 \\
\text { inches }\end{array}$ & $\begin{array}{c}1.857 \\
\text { to } \\
2.491 \\
\text { inches }\end{array}$ & $\begin{array}{c}2.491 \\
\text { to } \\
2.688 \\
\text { inches }\end{array}$ & $\begin{array}{c}2.688 \\
\text { to } \\
2.884 \\
\text { inches }\end{array}$ & $\begin{array}{c}2.884 \\
\text { to } \\
3.081 \\
\text { inches }\end{array}$ \\
\hline 0.5 & 1.75 & 1.47 & 1.48 & 1.36 & 1.34 & 1.41 & 1.41 & 1.51 & 1.73 \\
\hline 1.5 & 1.59 & 1.30 & 1.21 & 1.19 & 1.12 & 1.16 & 1.25 & 1.36 & 1.50 \\
\hline 2.5 & 1.85 & 1.52 & 1.51 & 1.40 & 1.35 & 1.41 & 1.47 & 1.61 & 1.84 \\
\hline 3.5 & 2.20 & 1.78 & 1.74 & 1.70 & 1.57 & 1.62 & 1.74 & 1.82 & 2.12 \\
\hline 4.5 & 2.47 & 2.05 & 1.89 & 1.85 & 1.77 & 1.84 & 1.89 & 2.10 & 2.49 \\
\hline 5.5 & 2.75 & 2.33 & 2.19 & 2.07 & 1.99 & 2.04 & 2.08 & 2.32 & 2.68 \\
\hline 6.5 & 2.90 & 2.48 & 2.33 & 2.24 & 2.14 & 2.19 & 2.35 & 2.50 & 2.88 \\
\hline 7.5 & 3.11 & 2.65 & 2.41 & 2.36 & 2.26 & 2.31 & 2.50 & 2.68 & 3.14 \\
\hline 8.5 & 3.19 & 2.86 & 2.56 & 2.45 & 2.34 & 2.45 & 2.54 & 2.72 & 3.28 \\
\hline 9.5 & 3.28 & 2.84 & 2.58 & 2.53 & 2.45 & 2.55 & 2.67 & 2.74 & 3.35 \\
\hline 10.5 & 3.42 & 2.89 & 2.67 & 2.63 & 2.53 & 2.59 & 2.71 & 2.86 & 3.40 \\
\hline 11.5 & 3.45 & 2.81 & 2.71 & 2.63 & 2.53 & 2.62 & 2.75 & 2.99 & 3.46 \\
\hline 12.5 & 3.57 & 2.98 & 2.78 & 2.67 & 2.53 & 2.67 & 2.73 & 2.99 & 3.36 \\
\hline 13.5 & 3.46 & 2.92 & 2.69 & 2.62 & 2.48 & 2.62 & 2.68 & 2.91 & 3.41 \\
\hline 14.5 & 3.40 & 2.84 & 2.74 & 2.56 & 2.48 & 2.53 & 2.60 & 2.84 & 3.33 \\
\hline 15.5 & 3.24 & 2.73 & 2.63 & 2.51 & 2.44 & 2.50 & 2.59 & 2.76 & 3.24 \\
\hline 16.5 & 3.21 & 2.70 & 2.50 & 2.39 & 2.31 & 2.43 & 2.43 & 2.65 & 3.17 \\
\hline 17.5 & 2.91 & 2.55 & 2.39 & 2.25 & 2.19 & 2.26 & 2.30 & 2.51 & 2.92 \\
\hline 18.5 & 2.89 & 2.48 & 2.20 & 2.15 & 2.07 & 2.09 & 2.16 & 2.37 & 2.90 \\
\hline 19.5 & 2.64 & 2.23 & 2.01 & 1.97 & 1.90 & 1.94 & 2.07 & 2.13 & 2.59 \\
\hline 20.5 & 2.33 & 2.06 & 1.79 & 1.75 & 1.69 & 1.76 & 1.80 & 2.01 & 2.28 \\
\hline 21.5 & 2.10 & 1.79 & 1.62 & 1.57 & 1.50 & 1.57 & 1.60 & 1.72 & 2.04 \\
\hline 22.5 & 1.91 & 1.61 & 1.41 & 1.37 & 1.26 & 1.36 & 1.41 & 1.50 & 1.82 \\
\hline 23.5 & 2.42 & 1.96 & 1.86 & 1.73 & 1.66 & 1.71 & 1.79 & 1.93 & 2.40 \\
\hline Ax. Avg. & 2.75 & 2.33 & 2.16 & 2.08 & 2.00 & 2.07 & 2.15 & 2.31 & 2.72 \\
\hline
\end{tabular}

POWER DENSITY VALUES NORMALIZED TO A CORE POWER OF 11.57 MW AND ASSUME ALL FISSION ENERGY (EXCLUDING GAMMA-HEATING OF EX-CORE COMPONENTS) IS DEPOSITED IN THE FUEL 
Table B-14. Power Density Data (kW/cc) in Fuel Plate 14 in Prototypic Conditions (continued).

Plate 14, Core Position X2/X6, 99 MWd Burnup, Equilibrium Xenon Conditions

\begin{tabular}{|c|c|c|c|c|c|c|c|c|c|}
\hline $\begin{array}{l}\text { Axial } \\
\text { position } \\
\text { from } \\
\text { top of } \\
\text { fuel } \\
\text { (inches) }\end{array}$ & $\begin{array}{c}0.000 \\
\text { to } \\
0.197 \\
\text { inches }\end{array}$ & $\begin{array}{c}0.197 \\
\text { to } \\
0.394 \\
\text { inches }\end{array}$ & $\begin{array}{c}0.394 \\
\text { to } \\
0.591 \\
\text { inches }\end{array}$ & $\begin{array}{c}0.591 \\
\text { to } \\
1.224 \\
\text { inches }\end{array}$ & $\begin{array}{c}1.224 \\
\text { to } \\
1.857 \\
\text { inches }\end{array}$ & $\begin{array}{c}1.857 \\
\text { to } \\
2.491 \\
\text { inches }\end{array}$ & $\begin{array}{c}2.491 \\
\text { to } \\
2.688 \\
\text { inches }\end{array}$ & $\begin{array}{c}2.688 \\
\text { to } \\
2.884 \\
\text { inches }\end{array}$ & $\begin{array}{c}2.884 \\
\text { to } \\
3.081 \\
\text { inches }\end{array}$ \\
\hline 0.5 & 1.71 & 1.49 & 1.37 & 1.33 & 1.28 & 1.35 & 1.37 & 1.52 & 1.72 \\
\hline 1.5 & 1.61 & 1.32 & 1.22 & 1.15 & 1.10 & 1.14 & 1.14 & 1.31 & 1.53 \\
\hline 2.5 & 1.82 & 1.61 & 1.45 & 1.45 & 1.36 & 1.37 & 1.42 & 1.49 & 1.79 \\
\hline 3.5 & 2.17 & 1.86 & 1.78 & 1.67 & 1.62 & 1.67 & 1.74 & 1.82 & 2.19 \\
\hline 4.5 & 2.38 & 1.98 & 1.91 & 1.87 & 1.82 & 1.86 & 2.01 & 2.15 & 2.40 \\
\hline 5.5 & 2.69 & 2.32 & 2.17 & 2.09 & 2.00 & 2.02 & 2.12 & 2.30 & 2.70 \\
\hline 6.5 & 2.85 & 2.52 & 2.29 & 2.18 & 2.11 & 2.22 & 2.35 & 2.46 & 2.86 \\
\hline 7.5 & 3.09 & 2.69 & 2.37 & 2.34 & 2.23 & 2.38 & 2.38 & 2.59 & 3.18 \\
\hline 8.5 & 3.20 & 2.75 & 2.54 & 2.42 & 2.37 & 2.42 & 2.62 & 2.68 & 3.30 \\
\hline 9.5 & 3.37 & 2.88 & 2.64 & 2.55 & 2.50 & 2.54 & 2.70 & 2.87 & 3.23 \\
\hline 10.5 & 3.36 & 2.86 & 2.65 & 2.56 & 2.52 & 2.64 & 2.77 & 2.85 & 3.44 \\
\hline 11.5 & 3.42 & 2.98 & 2.74 & 2.66 & 2.52 & 2.61 & 2.76 & 3.06 & 3.52 \\
\hline 12.5 & 3.41 & 2.92 & 2.81 & 2.73 & 2.54 & 2.63 & 2.75 & 2.98 & 3.61 \\
\hline 13.5 & 3.49 & 2.85 & 2.82 & 2.62 & 2.52 & 2.57 & 2.66 & 2.89 & 3.44 \\
\hline 14.5 & 3.50 & 2.92 & 2.77 & 2.56 & 2.50 & 2.62 & 2.67 & 2.92 & 3.45 \\
\hline 15.5 & 3.26 & 2.77 & 2.57 & 2.42 & 2.41 & 2.46 & 2.58 & 2.79 & 3.29 \\
\hline 16.5 & 3.18 & 2.65 & 2.47 & 2.39 & 2.30 & 2.47 & 2.46 & 2.64 & 3.19 \\
\hline 17.5 & 3.01 & 2.50 & 2.35 & 2.32 & 2.22 & 2.25 & 2.39 & 2.51 & 2.95 \\
\hline 18.5 & 2.80 & 2.42 & 2.23 & 2.11 & 2.04 & 2.16 & 2.21 & 2.39 & 2.76 \\
\hline 19.5 & 2.55 & 2.19 & 2.09 & 2.02 & 1.91 & 1.95 & 2.02 & 2.25 & 2.63 \\
\hline 20.5 & 2.38 & 1.92 & 1.85 & 1.77 & 1.68 & 1.78 & 1.84 & 1.94 & 2.36 \\
\hline 21.5 & 2.12 & 1.74 & 1.68 & 1.55 & 1.53 & 1.56 & 1.69 & 1.83 & 2.08 \\
\hline 22.5 & 1.84 & 1.51 & 1.41 & 1.34 & 1.28 & 1.36 & 1.44 & 1.61 & 1.85 \\
\hline 23.5 & 2.41 & 1.96 & 1.90 & 1.77 & 1.68 & 1.76 & 1.84 & 1.99 & 2.44 \\
\hline Ax. Avg. & 2.73 & 2.32 & 2.17 & 2.08 & 2.00 & 2.07 & 2.16 & 2.33 & 2.75 \\
\hline
\end{tabular}

POWER DENSITY VALUES NORMALIZED TO A CORE POWER OF 11.57 MW AND ASSUME ALL FISSION ENERGY (EXCLUDING GAMMA-HEATING OF EX-CORE COMPONENTS) IS DEPOSITED IN THE FUEL 
Table B-14. Power Density Data (kW/cc) in Fuel Plate 14 in Prototypic Conditions (continued).

Plate 14, Core Position X4/X8, 173 MWd Burnup, Equilibrium Xenon Conditions

\begin{tabular}{|c|c|c|c|c|c|c|c|c|c|}
\hline $\begin{array}{l}\text { Axial } \\
\text { position } \\
\text { from } \\
\text { top of } \\
\text { fuel } \\
\text { (inches) }\end{array}$ & $\begin{array}{c}0.000 \\
\text { to } \\
0.197 \\
\text { inches }\end{array}$ & $\begin{array}{c}0.197 \\
\text { to } \\
0.394 \\
\text { inches }\end{array}$ & $\begin{array}{c}0.394 \\
\text { to } \\
0.591 \\
\text { inches }\end{array}$ & $\begin{array}{c}0.591 \\
\text { to } \\
1.224 \\
\text { inches }\end{array}$ & $\begin{array}{c}1.224 \\
\text { to } \\
1.857 \\
\text { inches }\end{array}$ & $\begin{array}{c}1.857 \\
\text { to } \\
2.491 \\
\text { inches }\end{array}$ & $\begin{array}{c}2.491 \\
\text { to } \\
2.688 \\
\text { inches }\end{array}$ & $\begin{array}{c}2.688 \\
\text { to } \\
2.884 \\
\text { inches }\end{array}$ & $\begin{array}{c}2.884 \\
\text { to } \\
3.081 \\
\text { inches }\end{array}$ \\
\hline 0.5 & 1.75 & 1.48 & 1.35 & 1.31 & 1.26 & 1.29 & 1.33 & 1.42 & 1.76 \\
\hline 1.5 & 1.51 & 1.24 & 1.15 & 1.14 & 1.11 & 1.15 & 1.21 & 1.26 & 1.51 \\
\hline 2.5 & 1.83 & 1.51 & 1.44 & 1.40 & 1.34 & 1.38 & 1.44 & 1.50 & 1.82 \\
\hline 3.5 & 2.18 & 1.84 & 1.61 & 1.60 & 1.57 & 1.63 & 1.72 & 1.74 & 1.95 \\
\hline 4.5 & 2.40 & 2.04 & 1.87 & 1.81 & 1.70 & 1.85 & 1.90 & 1.95 & 2.34 \\
\hline 5.5 & 2.69 & 2.25 & 2.11 & 1.98 & 1.93 & 2.00 & 2.10 & 2.23 & 2.55 \\
\hline 6.5 & 2.76 & 2.41 & 2.27 & 2.14 & 2.05 & 2.17 & 2.26 & 2.48 & 2.86 \\
\hline 7.5 & 2.97 & 2.52 & 2.33 & 2.28 & 2.19 & 2.27 & 2.39 & 2.57 & 2.93 \\
\hline 8.5 & 3.07 & 2.68 & 2.50 & 2.38 & 2.28 & 2.38 & 2.46 & 2.60 & 3.05 \\
\hline 9.5 & 3.15 & 2.68 & 2.52 & 2.39 & 2.37 & 2.46 & 2.59 & 2.74 & 3.07 \\
\hline 10.5 & 3.27 & 2.82 & 2.53 & 2.52 & 2.43 & 2.49 & 2.72 & 2.89 & 3.29 \\
\hline 11.5 & 3.34 & 2.87 & 2.71 & 2.56 & 2.42 & 2.59 & 2.71 & 2.84 & 3.41 \\
\hline 12.5 & 3.41 & 2.85 & 2.68 & 2.53 & 2.44 & 2.51 & 2.61 & 2.90 & 3.30 \\
\hline 13.5 & 3.29 & 2.83 & 2.71 & 2.53 & 2.44 & 2.52 & 2.61 & 2.78 & 3.33 \\
\hline 14.5 & 3.15 & 2.78 & 2.58 & 2.47 & 2.37 & 2.44 & 2.63 & 2.74 & 3.27 \\
\hline 15.5 & 3.10 & 2.67 & 2.41 & 2.42 & 2.29 & 2.45 & 2.54 & 2.68 & 3.08 \\
\hline 16.5 & 3.03 & 2.57 & 2.39 & 2.32 & 2.23 & 2.29 & 2.48 & 2.64 & 3.05 \\
\hline 17.5 & 2.85 & 2.43 & 2.32 & 2.21 & 2.13 & 2.21 & 2.38 & 2.43 & 2.94 \\
\hline 18.5 & 2.66 & 2.23 & 2.18 & 2.01 & 1.98 & 2.10 & 2.26 & 2.30 & 2.73 \\
\hline 19.5 & 2.60 & 2.14 & 1.95 & 1.90 & 1.83 & 1.93 & 1.94 & 2.14 & 2.55 \\
\hline 20.5 & 2.33 & 1.93 & 1.80 & 1.74 & 1.69 & 1.74 & 1.78 & 1.97 & 2.30 \\
\hline 21.5 & 2.02 & 1.72 & 1.59 & 1.53 & 1.48 & 1.58 & 1.68 & 1.74 & 2.08 \\
\hline 22.5 & 1.76 & 1.49 & 1.36 & 1.32 & 1.24 & 1.32 & 1.44 & 1.51 & 1.83 \\
\hline 23.5 & 2.44 & 1.95 & 1.81 & 1.69 & 1.65 & 1.72 & 1.81 & 1.91 & 2.31 \\
\hline Ax. Avg. & 2.65 & 2.25 & 2.09 & 2.01 & 1.93 & 2.02 & 2.12 & 2.25 & 2.64 \\
\hline
\end{tabular}

POWER DENSITY VALUES NORMALIZED TO A CORE POWER OF 11.57 MW AND ASSUME ALL FISSION ENERGY (EXCLUDING GAMMA-HEATING OF EX-CORE COMPONENTS) IS DEPOSITED IN THE FUEL 
Table B-15. Power Density Data $\left(\mathrm{kW} / \mathrm{cm}^{3}\right)$ in Fuel Plate 15 in Prototypic Core.

\section{Fuel core width: 3.188 inches / Fuel core thickness: 20 mil}

Plate 15, Core Position X1/X5, 0 MWd Burnup, Xenon-Free Conditions

\begin{tabular}{|c|c|c|c|c|c|c|c|c|c|}
\hline $\begin{array}{c}\text { Axial } \\
\text { position } \\
\text { from } \\
\text { top of } \\
\text { fuel } \\
\text { (inches) }\end{array}$ & $\begin{array}{c}0.000 \\
\text { to } \\
0.197 \\
\text { inches }\end{array}$ & $\begin{array}{c}0.197 \\
\text { to } \\
0.394 \\
\text { inches }\end{array}$ & $\begin{array}{c}0.394 \\
\text { to } \\
0.591 \\
\text { inches }\end{array}$ & $\begin{array}{c}0.591 \\
\text { to } \\
\text { inches }\end{array}$ & $\begin{array}{c}1.260 \\
\text { to } \\
1.929 \\
\text { inches }\end{array}$ & $\begin{array}{c}1.929 \\
\text { to } \\
2.597 \\
\text { inches }\end{array}$ & $\begin{array}{c}\text { 2.597 } \\
\text { to } \\
2.794 \\
\text { inches }\end{array}$ & $\begin{array}{c}2.794 \\
\text { to } \\
2.991 \\
\text { inches }\end{array}$ & $\begin{array}{c}2.991 \\
\text { to } \\
\text { inches }\end{array}$ \\
\hline 0.5 & 1.11 & 0.99 & 0.93 & 0.91 & 0.86 & 0.89 & 0.89 & 0.99 & 1.10 \\
\hline 1.5 & 0.94 & 0.85 & 0.80 & 0.75 & 0.75 & 0.75 & 0.78 & 0.82 & 0.99 \\
\hline 2.5 & 1.09 & 0.96 & 0.90 & 0.92 & 0.87 & 0.94 & 0.92 & 1.01 & 1.12 \\
\hline 3.5 & 1.32 & 1.19 & 1.18 & 1.12 & 1.07 & 1.11 & 1.13 & 1.19 & 1.29 \\
\hline 4.5 & 1.51 & 1.38 & 1.28 & 1.31 & 1.26 & 1.29 & 1.31 & 1.45 & 1.57 \\
\hline 5.5 & 1.75 & 1.59 & 1.57 & 1.53 & 1.48 & 1.52 & 1.50 & 1.57 & 1.79 \\
\hline 6.5 & 2.07 & 1.82 & 1.79 & 1.72 & 1.68 & 1.74 & 1.78 & 1.85 & 2.09 \\
\hline 7.5 & 2.45 & 2.12 & 2.05 & 2.00 & 1.94 & 2.00 & 2.03 & 2.10 & 2.38 \\
\hline 8.5 & 2.79 & 2.43 & 2.36 & 2.19 & 2.15 & 2.25 & 2.29 & 2.49 & 2.81 \\
\hline 9.5 & 3.13 & 2.66 & 2.57 & 2.45 & 2.40 & 2.46 & 2.62 & 2.77 & 3.26 \\
\hline 10.5 & 3.56 & 2.94 & 2.78 & 2.68 & 2.61 & 2.74 & 2.79 & 2.97 & 3.48 \\
\hline 11.5 & 3.74 & 3.27 & 3.00 & 2.86 & 2.74 & 2.88 & 2.99 & 3.24 & 3.80 \\
\hline 12.5 & 4.01 & 3.38 & 3.13 & 3.03 & 2.88 & 2.97 & 3.13 & 3.39 & 3.91 \\
\hline 13.5 & 4.18 & 3.45 & 3.24 & 3.11 & 3.01 & 3.12 & 3.26 & 3.53 & 4.06 \\
\hline 14.5 & 4.13 & 3.56 & 3.24 & 3.18 & 3.02 & 3.17 & 3.34 & 3.64 & 4.11 \\
\hline 15.5 & 4.16 & 3.48 & 3.32 & 3.14 & 3.03 & 3.19 & 3.32 & 3.44 & 4.21 \\
\hline 16.5 & 3.97 & 3.45 & 3.24 & 3.14 & 2.98 & 3.08 & 3.33 & 3.52 & 4.07 \\
\hline 17.5 & 3.97 & 3.39 & 3.20 & 3.06 & 2.92 & 3.05 & 3.18 & 3.43 & 3.92 \\
\hline 18.5 & 3.81 & 3.34 & 3.06 & 2.85 & 2.75 & 2.84 & 2.97 & 3.26 & 3.82 \\
\hline 19.5 & 3.45 & 2.91 & 2.81 & 2.64 & 2.56 & 2.72 & 2.76 & 3.06 & 3.46 \\
\hline 20.5 & 3.10 & 2.75 & 2.59 & 2.42 & 2.33 & 2.47 & 2.67 & 2.78 & 3.23 \\
\hline 21.5 & 2.95 & 2.42 & 2.27 & 2.16 & 2.11 & 2.20 & 2.35 & 2.41 & 2.90 \\
\hline 22.5 & 2.57 & 2.12 & 2.01 & 1.87 & 1.81 & 1.92 & 2.03 & 2.18 & 2.63 \\
\hline 23.5 & 3.34 & 2.82 & 2.57 & 2.45 & 2.32 & 2.46 & 2.51 & 2.63 & 3.34 \\
\hline Ax. Avg. & 2.88 & 2.47 & 2.33 & 2.23 & 2.15 & 2.24 & 2.33 & 2.49 & 2.89 \\
\hline & & & & & & & & & \\
\hline
\end{tabular}

POWER DENSITY VALUES NORMALIZED TO A CORE POWER OF 11.57 MW AND ASSUME ALL FISSION ENERGY (EXCLUDING GAMMA-HEATING OF EX-CORE COMPONENTS) IS DEPOSITED IN THE FUEL 
Table B-15. Power Density Data (kW/cc) in Fuel Plate 15 in Prototypic Conditions (continued).

Plate 15, Core Position X1/X5, 3 MWd Burnup, Equilibrium Xenon Conditions

\begin{tabular}{|c|c|c|c|c|c|c|c|c|c|}
\hline $\begin{array}{l}\text { Axial } \\
\text { position } \\
\text { from } \\
\text { top of } \\
\text { fuel } \\
\text { (inches) }\end{array}$ & $\begin{array}{c}0.000 \\
\text { to } \\
0.197 \\
\text { inches }\end{array}$ & $\begin{array}{c}0.197 \\
\text { to } \\
0.394 \\
\text { inches }\end{array}$ & $\begin{array}{c}0.394 \\
\text { to } \\
0.591 \\
\text { inches }\end{array}$ & $\begin{array}{c}0.591 \\
\text { to } \\
1.260 \\
\text { inches }\end{array}$ & $\begin{array}{c}1.260 \\
\text { to } \\
1.929 \\
\text { inches }\end{array}$ & $\begin{array}{c}1.929 \\
\text { to } \\
2.597 \\
\text { inches }\end{array}$ & $\begin{array}{c}2.597 \\
\text { to } \\
2.794 \\
\text { inches }\end{array}$ & $\begin{array}{c}2.794 \\
\text { to } \\
2.991 \\
\text { inches }\end{array}$ & $\begin{array}{c}2.991 \\
\text { to } \\
3.188 \\
\text { inches }\end{array}$ \\
\hline 0.5 & 1.84 & 1.55 & 1.38 & 1.41 & 1.38 & 1.40 & 1.37 & 1.48 & 1.79 \\
\hline 1.5 & 1.60 & 1.38 & 1.21 & 1.17 & 1.17 & 1.19 & 1.27 & 1.33 & 1.56 \\
\hline 2.5 & 1.88 & 1.62 & 1.51 & 1.46 & 1.40 & 1.41 & 1.46 & 1.63 & 1.86 \\
\hline 3.5 & 2.25 & 1.89 & 1.76 & 1.69 & 1.62 & 1.72 & 1.80 & 1.93 & 2.24 \\
\hline 4.5 & 2.50 & 2.07 & 1.89 & 1.89 & 1.87 & 1.93 & 1.99 & 2.13 & 2.57 \\
\hline 5.5 & 2.72 & 2.25 & 2.19 & 2.11 & 2.00 & 2.12 & 2.19 & 2.39 & 2.75 \\
\hline 6.5 & 3.02 & 2.60 & 2.34 & 2.28 & 2.20 & 2.28 & 2.37 & 2.58 & 2.99 \\
\hline 7.5 & 3.24 & 2.66 & 2.45 & 2.41 & 2.32 & 2.43 & 2.50 & 2.69 & 3.24 \\
\hline 8.5 & 3.38 & 2.76 & 2.60 & 2.58 & 2.47 & 2.55 & 2.64 & 2.79 & 3.41 \\
\hline 9.5 & 3.32 & 2.96 & 2.80 & 2.64 & 2.51 & 2.63 & 2.72 & 2.97 & 3.58 \\
\hline 10.5 & 3.49 & 3.04 & 2.79 & 2.67 & 2.56 & 2.68 & 2.78 & 3.00 & 3.53 \\
\hline 11.5 & 3.49 & 3.03 & 2.83 & 2.73 & 2.63 & 2.70 & 2.77 & 3.04 & 3.56 \\
\hline 12.5 & 3.55 & 3.00 & 2.85 & 2.71 & 2.68 & 2.70 & 2.81 & 3.06 & 3.54 \\
\hline 13.5 & 3.61 & 2.98 & 2.91 & 2.71 & 2.61 & 2.71 & 2.78 & 3.07 & 3.52 \\
\hline 14.5 & 3.59 & 3.04 & 2.78 & 2.71 & 2.58 & 2.65 & 2.80 & 3.00 & 3.48 \\
\hline 15.5 & 3.41 & 2.90 & 2.72 & 2.63 & 2.52 & 2.60 & 2.73 & 2.91 & 3.40 \\
\hline 16.5 & 3.29 & 2.73 & 2.59 & 2.48 & 2.42 & 2.45 & 2.61 & 2.83 & 3.21 \\
\hline 17.5 & 3.11 & 2.68 & 2.42 & 2.35 & 2.29 & 2.38 & 2.47 & 2.63 & 3.10 \\
\hline 18.5 & 2.84 & 2.53 & 2.27 & 2.25 & 2.15 & 2.19 & 2.28 & 2.38 & 2.86 \\
\hline 19.5 & 2.63 & 2.27 & 2.03 & 2.05 & 1.98 & 2.03 & 2.12 & 2.26 & 2.74 \\
\hline 20.5 & 2.43 & 2.09 & 1.89 & 1.87 & 1.81 & 1.88 & 1.98 & 2.14 & 2.41 \\
\hline 21.5 & 2.15 & 1.81 & 1.66 & 1.63 & 1.57 & 1.64 & 1.69 & 1.82 & 2.26 \\
\hline 22.5 & 1.92 & 1.61 & 1.45 & 1.37 & 1.32 & 1.37 & 1.51 & 1.58 & 1.97 \\
\hline 23.5 & 2.48 & 1.96 & 1.84 & 1.76 & 1.73 & 1.80 & 1.96 & 2.03 & 2.45 \\
\hline Ax. Avg. & 2.82 & 2.39 & 2.22 & 2.15 & 2.07 & 2.14 & 2.23 & 2.40 & 2.83 \\
\hline
\end{tabular}

POWER DENSITY VALUES NORMALIZED TO A CORE POWER OF 11.57 MW AND ASSUME ALL FISSION ENERGY (EXCLUDING GAMMA-HEATING OF EX-CORE COMPONENTS) IS DEPOSITED IN THE FUEL 
Table B-15. Power Density Data (kW/cc) in Fuel Plate 15 in Prototypic Conditions (continued).

Plate 15, Core Position X3/X7, 80 MWd Burnup, Equilibrium Xenon Conditions

\begin{tabular}{|c|c|c|c|c|c|c|c|c|c|}
\hline $\begin{array}{c}\text { Axial } \\
\text { position } \\
\text { from } \\
\text { top of } \\
\text { fuel } \\
\text { (inches) }\end{array}$ & $\begin{array}{c}0.000 \\
\text { to } \\
0.197 \\
\text { inches }\end{array}$ & $\begin{array}{c}0.197 \\
\text { to } \\
0.394 \\
\text { inches }\end{array}$ & $\begin{array}{c}0.394 \\
\text { to } \\
0.591 \\
\text { inches }\end{array}$ & $\begin{array}{c}0.591 \\
\text { to } \\
1.260 \\
\text { inches }\end{array}$ & $\begin{array}{c}1.260 \\
\text { to } \\
1.929 \\
\text { inches }\end{array}$ & $\begin{array}{c}1.929 \\
\text { to } \\
2.597 \\
\text { inches }\end{array}$ & $\begin{array}{c}\text { 2.597 } \\
\text { to } \\
2.794 \\
\text { inches }\end{array}$ & $\begin{array}{c}2.794 \\
\text { to } \\
2.991 \\
\text { inches }\end{array}$ & $\begin{array}{c}2.991 \\
\text { to } \\
\text { inches }\end{array}$ \\
\hline 0.5 & 1.72 & 1.50 & 1.43 & 1.32 & 1.31 & 1.34 & 1.43 & 1.50 & 1.74 \\
\hline 1.5 & 1.56 & 1.32 & 1.21 & 1.18 & 1.13 & 1.17 & 1.23 & 1.34 & 1.58 \\
\hline 2.5 & 1.84 & 1.66 & 1.44 & 1.40 & 1.37 & 1.41 & 1.49 & 1.59 & 1.80 \\
\hline 3.5 & 2.17 & 1.84 & 1.72 & 1.69 & 1.56 & 1.65 & 1.76 & 1.80 & 2.13 \\
\hline 4.5 & 2.48 & 2.10 & 1.99 & 1.85 & 1.81 & 1.87 & 1.95 & 2.07 & 2.46 \\
\hline 5.5 & 2.81 & 2.28 & 2.12 & 2.05 & 1.99 & 2.06 & 2.15 & 2.29 & 2.79 \\
\hline 6.5 & 2.91 & 2.43 & 2.36 & 2.21 & 2.19 & 2.21 & 2.36 & 2.55 & 2.91 \\
\hline 7.5 & 3.13 & 2.69 & 2.43 & 2.36 & 2.28 & 2.34 & 2.48 & 2.66 & 3.13 \\
\hline 8.5 & 3.25 & 2.75 & 2.60 & 2.45 & 2.36 & 2.44 & 2.61 & 2.81 & 3.33 \\
\hline 9.5 & 3.30 & 2.84 & 2.64 & 2.56 & 2.45 & 2.51 & 2.71 & 2.77 & 3.38 \\
\hline 10.5 & 3.49 & 2.95 & 2.69 & 2.61 & 2.52 & 2.61 & 2.62 & 2.98 & 3.56 \\
\hline 11.5 & 3.46 & 2.97 & 2.75 & 2.66 & 2.54 & 2.59 & 2.73 & 2.97 & 3.49 \\
\hline 12.5 & 3.65 & 2.97 & 2.85 & 2.62 & 2.50 & 2.62 & 2.79 & 2.88 & 3.58 \\
\hline 13.5 & 3.40 & 2.93 & 2.75 & 2.65 & 2.55 & 2.65 & 2.74 & 2.93 & 3.35 \\
\hline 14.5 & 3.36 & 2.81 & 2.69 & 2.60 & 2.47 & 2.52 & 2.68 & 2.86 & 3.40 \\
\hline 15.5 & 3.25 & 2.81 & 2.62 & 2.51 & 2.43 & 2.44 & 2.53 & 2.78 & 3.30 \\
\hline 16.5 & 3.25 & 2.71 & 2.53 & 2.37 & 2.27 & 2.41 & 2.39 & 2.57 & 3.23 \\
\hline 17.5 & 2.95 & 2.53 & 2.43 & 2.25 & 2.20 & 2.25 & 2.36 & 2.46 & 2.98 \\
\hline 18.5 & 2.89 & 2.38 & 2.25 & 2.20 & 2.03 & 2.12 & 2.26 & 2.45 & 2.85 \\
\hline 19.5 & 2.57 & 2.24 & 2.06 & 1.97 & 1.89 & 1.93 & 2.02 & 2.24 & 2.58 \\
\hline 20.5 & 2.42 & 1.94 & 1.84 & 1.81 & 1.71 & 1.73 & 1.88 & 1.97 & 2.37 \\
\hline 21.5 & 2.09 & 1.78 & 1.63 & 1.59 & 1.50 & 1.57 & 1.63 & 1.77 & 2.05 \\
\hline 22.5 & 1.96 & 1.58 & 1.43 & 1.34 & 1.31 & 1.35 & 1.39 & 1.51 & 1.89 \\
\hline 23.5 & 2.51 & 1.97 & 1.85 & 1.77 & 1.67 & 1.72 & 1.83 & 1.94 & 2.42 \\
\hline Ax. Avg. & 2.77 & 2.33 & 2.18 & 2.08 & 2.00 & 2.06 & 2.17 & 2.32 & 2.76 \\
\hline & & & & & & & & & \\
\hline
\end{tabular}

POWER DENSITY VALUES NORMALIZED TO A CORE POWER OF 11.57 MW AND ASSUME ALL FISSION ENERGY (EXCLUDING GAMMA-HEATING OF EX-CORE COMPONENTS) IS DEPOSITED IN THE FUEL 
Table B-15. Power Density Data (kW/cc) in Fuel Plate 15 in Prototypic Conditions (continued).

Plate 15, Core Position X2/X6, 99 MWd Burnup, Equilibrium Xenon Conditions

\begin{tabular}{|c|c|c|c|c|c|c|c|c|c|}
\hline $\begin{array}{l}\text { Axial } \\
\text { position } \\
\text { from } \\
\text { top of } \\
\text { fuel } \\
\text { (inches) }\end{array}$ & $\begin{array}{c}0.000 \\
\text { to } \\
0.197 \\
\text { inches }\end{array}$ & $\begin{array}{c}0.197 \\
\text { to } \\
0.394 \\
\text { inches }\end{array}$ & $\begin{array}{c}0.394 \\
\text { to } \\
0.591 \\
\text { inches }\end{array}$ & $\begin{array}{c}0.591 \\
\text { to } \\
1.260 \\
\text { inches }\end{array}$ & $\begin{array}{c}1.260 \\
\text { to } \\
1.929 \\
\text { inches }\end{array}$ & $\begin{array}{c}1.929 \\
\text { to } \\
2.597 \\
\text { inches }\end{array}$ & $\begin{array}{c}2.597 \\
\text { to } \\
2.794 \\
\text { inches }\end{array}$ & $\begin{array}{c}2.794 \\
\text { to } \\
2.991 \\
\text { inches }\end{array}$ & $\begin{array}{c}2.991 \\
\text { to } \\
3.188 \\
\text { inches }\end{array}$ \\
\hline 0.5 & 1.80 & 1.47 & 1.35 & 1.29 & 1.25 & 1.28 & 1.38 & 1.45 & 1.71 \\
\hline 1.5 & 1.53 & 1.32 & 1.17 & 1.14 & 1.09 & 1.16 & 1.21 & 1.33 & 1.54 \\
\hline 2.5 & 1.84 & 1.58 & 1.42 & 1.39 & 1.35 & 1.42 & 1.46 & 1.56 & 1.87 \\
\hline 3.5 & 2.12 & 1.87 & 1.78 & 1.66 & 1.62 & 1.69 & 1.79 & 1.81 & 2.24 \\
\hline 4.5 & 2.44 & 2.10 & 1.93 & 1.88 & 1.83 & 1.89 & 1.99 & 2.05 & 2.47 \\
\hline 5.5 & 2.71 & 2.26 & 2.16 & 2.05 & 1.96 & 2.08 & 2.12 & 2.39 & 2.72 \\
\hline 6.5 & 2.90 & 2.49 & 2.34 & 2.17 & 2.15 & 2.22 & 2.25 & 2.46 & 2.91 \\
\hline 7.5 & 3.00 & 2.61 & 2.56 & 2.33 & 2.25 & 2.40 & 2.50 & 2.68 & 3.17 \\
\hline 8.5 & 3.22 & 2.79 & 2.58 & 2.38 & 2.34 & 2.43 & 2.58 & 2.71 & 3.29 \\
\hline 9.5 & 3.40 & 2.87 & 2.69 & 2.59 & 2.48 & 2.55 & 2.70 & 2.88 & 3.35 \\
\hline 10.5 & 3.35 & 2.83 & 2.68 & 2.57 & 2.52 & 2.64 & 2.65 & 2.91 & 3.41 \\
\hline 11.5 & 3.44 & 2.94 & 2.73 & 2.65 & 2.53 & 2.55 & 2.84 & 2.90 & 3.58 \\
\hline 12.5 & 3.51 & 2.97 & 2.70 & 2.66 & 2.56 & 2.63 & 2.77 & 2.96 & 3.51 \\
\hline 13.5 & 3.49 & 2.90 & 2.72 & 2.64 & 2.54 & 2.60 & 2.82 & 2.93 & 3.37 \\
\hline 14.5 & 3.49 & 2.90 & 2.82 & 2.60 & 2.51 & 2.57 & 2.72 & 2.84 & 3.39 \\
\hline 15.5 & 3.26 & 2.76 & 2.59 & 2.48 & 2.41 & 2.52 & 2.60 & 2.85 & 3.32 \\
\hline 16.5 & 3.18 & 2.70 & 2.50 & 2.42 & 2.36 & 2.38 & 2.51 & 2.81 & 3.16 \\
\hline 17.5 & 2.98 & 2.63 & 2.37 & 2.32 & 2.22 & 2.30 & 2.33 & 2.53 & 3.03 \\
\hline 18.5 & 2.79 & 2.42 & 2.28 & 2.21 & 2.07 & 2.15 & 2.23 & 2.40 & 2.83 \\
\hline 19.5 & 2.64 & 2.25 & 2.10 & 2.01 & 1.87 & 1.97 & 2.07 & 2.28 & 2.59 \\
\hline 20.5 & 2.34 & 2.02 & 1.92 & 1.75 & 1.72 & 1.76 & 1.92 & 2.03 & 2.37 \\
\hline 21.5 & 2.10 & 1.81 & 1.60 & 1.60 & 1.53 & 1.55 & 1.64 & 1.81 & 2.08 \\
\hline 22.5 & 1.92 & 1.54 & 1.49 & 1.34 & 1.29 & 1.34 & 1.45 & 1.60 & 1.88 \\
\hline 23.5 & 2.41 & 2.02 & 1.84 & 1.78 & 1.65 & 1.79 & 1.83 & 1.96 & 2.51 \\
\hline Ax. Avg. & 2.74 & 2.33 & 2.18 & 2.08 & 2.00 & 2.08 & 2.18 & 2.34 & 2.76 \\
\hline
\end{tabular}

POWER DENSITY VALUES NORMALIZED TO A CORE POWER OF 11.57 MW AND ASSUME ALL FISSION ENERGY (EXCLUDING GAMMA-HEATING OF EX-CORE COMPONENTS) IS DEPOSITED IN THE FUEL 
Table B-15. Power Density Data (kW/cc) in Fuel Plate 15 in Prototypic Conditions (continued).

Plate 15, Core Position X4/X8, 173 MWd Burnup, Equilibrium Xenon Conditions

\begin{tabular}{|c|c|c|c|c|c|c|c|c|c|}
\hline $\begin{array}{l}\text { Axial } \\
\text { position } \\
\text { from } \\
\text { top of } \\
\text { fuel } \\
\text { (inches) }\end{array}$ & $\begin{array}{c}0.000 \\
\text { to } \\
0.197 \\
\text { inches }\end{array}$ & $\begin{array}{c}0.197 \\
\text { to } \\
0.394 \\
\text { inches }\end{array}$ & $\begin{array}{c}0.394 \\
\text { to } \\
0.591 \\
\text { inches }\end{array}$ & $\begin{array}{c}0.591 \\
\text { to } \\
1.260 \\
\text { inches }\end{array}$ & $\begin{array}{c}1.260 \\
\text { to } \\
1.929 \\
\text { inches }\end{array}$ & $\begin{array}{c}1.929 \\
\text { to } \\
2.597 \\
\text { inches }\end{array}$ & $\begin{array}{c}2.597 \\
\text { to } \\
2.794 \\
\text { inches }\end{array}$ & $\begin{array}{c}2.794 \\
\text { to } \\
2.991 \\
\text { inches }\end{array}$ & $\begin{array}{c}2.991 \\
\text { to } \\
3.188 \\
\text { inches }\end{array}$ \\
\hline 0.5 & 1.69 & 1.47 & 1.40 & 1.29 & 1.28 & 1.32 & 1.32 & 1.42 & 1.69 \\
\hline 1.5 & 1.44 & 1.23 & 1.18 & 1.14 & 1.10 & 1.15 & 1.16 & 1.26 & 1.50 \\
\hline 2.5 & 1.85 & 1.51 & 1.37 & 1.40 & 1.30 & 1.39 & 1.42 & 1.53 & 1.84 \\
\hline 3.5 & 2.16 & 1.83 & 1.72 & 1.61 & 1.54 & 1.64 & 1.68 & 1.84 & 2.13 \\
\hline 4.5 & 2.41 & 2.02 & 1.85 & 1.82 & 1.75 & 1.84 & 1.90 & 2.09 & 2.32 \\
\hline 5.5 & 2.66 & 2.26 & 2.06 & 2.03 & 1.95 & 2.01 & 2.01 & 2.18 & 2.63 \\
\hline 6.5 & 2.94 & 2.39 & 2.21 & 2.14 & 2.09 & 2.16 & 2.25 & 2.44 & 2.87 \\
\hline 7.5 & 3.07 & 2.57 & 2.35 & 2.30 & 2.20 & 2.26 & 2.37 & 2.62 & 3.02 \\
\hline 8.5 & 3.09 & 2.67 & 2.44 & 2.41 & 2.29 & 2.39 & 2.50 & 2.59 & 3.18 \\
\hline 9.5 & 3.21 & 2.74 & 2.57 & 2.46 & 2.36 & 2.48 & 2.56 & 2.74 & 3.20 \\
\hline 10.5 & 3.35 & 2.82 & 2.62 & 2.53 & 2.38 & 2.47 & 2.69 & 2.82 & 3.40 \\
\hline 11.5 & 3.50 & 2.93 & 2.76 & 2.56 & 2.41 & 2.56 & 2.67 & 2.78 & 3.34 \\
\hline 12.5 & 3.42 & 2.83 & 2.65 & 2.51 & 2.45 & 2.53 & 2.64 & 2.91 & 3.33 \\
\hline 13.5 & 3.38 & 2.80 & 2.74 & 2.53 & 2.41 & 2.53 & 2.63 & 2.88 & 3.31 \\
\hline 14.5 & 3.27 & 2.82 & 2.58 & 2.46 & 2.35 & 2.48 & 2.55 & 2.76 & 3.31 \\
\hline 15.5 & 3.13 & 2.69 & 2.49 & 2.42 & 2.30 & 2.44 & 2.55 & 2.69 & 3.15 \\
\hline 16.5 & 2.99 & 2.53 & 2.44 & 2.31 & 2.21 & 2.32 & 2.50 & 2.66 & 3.07 \\
\hline 17.5 & 2.88 & 2.53 & 2.34 & 2.22 & 2.12 & 2.24 & 2.24 & 2.48 & 2.91 \\
\hline 18.5 & 2.74 & 2.33 & 2.14 & 2.06 & 2.04 & 2.06 & 2.22 & 2.35 & 2.75 \\
\hline 19.5 & 2.48 & 2.12 & 2.02 & 1.91 & 1.82 & 1.92 & 1.95 & 2.15 & 2.48 \\
\hline 20.5 & 2.25 & 1.92 & 1.85 & 1.74 & 1.69 & 1.78 & 1.86 & 2.04 & 2.34 \\
\hline 21.5 & 2.10 & 1.72 & 1.57 & 1.53 & 1.48 & 1.57 & 1.69 & 1.74 & 1.99 \\
\hline 22.5 & 1.81 & 1.52 & 1.46 & 1.34 & 1.30 & 1.32 & 1.43 & 1.59 & 1.82 \\
\hline 23.5 & 2.38 & 1.94 & 1.78 & 1.67 & 1.65 & 1.76 & 1.74 & 1.92 & 2.37 \\
\hline Ax. Avg. & 2.68 & 2.26 & 2.11 & 2.02 & 1.94 & 2.03 & 2.11 & 2.27 & 2.66 \\
\hline
\end{tabular}

POWER DENSITY VALUES NORMALIZED TO A CORE POWER OF 11.57 MW AND ASSUME ALL FISSION ENERGY (EXCLUDING GAMMA-HEATING OF EX-CORE COMPONENTS) IS DEPOSITED IN THE FUEL 
Table B-16. Power Density Data $\left(\mathrm{kW} / \mathrm{cm}^{3}\right)$ in Fuel Plate 16 in Prototypic Core.

\section{Fuel core width: 3.295 inches / Fuel core thickness: 20 mil}

Plate 16, Core Position X1/X5, 0 MWd Burnup, Xenon-Free Conditions

\begin{tabular}{|c|c|c|c|c|c|c|c|c|c|}
\hline $\begin{array}{c}\text { Axial } \\
\text { position } \\
\text { from } \\
\text { top of } \\
\text { fuel } \\
\text { (inches) } \\
\end{array}$ & $\begin{array}{c}0.000 \\
\text { to } \\
0.197 \\
\text { inches }\end{array}$ & $\begin{array}{c}0.197 \\
\text { to } \\
0.394 \\
\text { inches } \\
\end{array}$ & $\begin{array}{c}0.394 \\
\text { to } \\
0.591 \\
\text { inches } \\
\end{array}$ & $\begin{array}{c}0.591 \\
\text { to } \\
1.295 \\
\text { inches }\end{array}$ & $\begin{array}{c}1.295 \\
\text { to } \\
2.000 \\
\text { inches }\end{array}$ & $\begin{array}{c}2.000 \\
\text { to } \\
2.704 \\
\text { inches } \\
\end{array}$ & $\begin{array}{c}2.704 \\
\text { to } \\
2.901 \\
\text { inches }\end{array}$ & $\begin{array}{c}2.901 \\
\text { to } \\
3.098 \\
\text { inches }\end{array}$ & $\begin{array}{c}3.098 \\
\text { to } \\
3.295 \\
\text { inches } \\
\end{array}$ \\
\hline 0.5 & 1.08 & 0.94 & 0.90 & 0.88 & 0.87 & 0.87 & 0.87 & 0.90 & 1.08 \\
\hline 1.5 & 0.94 & 0.83 & 0.76 & 0.76 & 0.77 & 0.77 & 0.76 & 0.75 & 0.90 \\
\hline 2.5 & 1.09 & 1.01 & 0.93 & 0.94 & 0.91 & 0.92 & 0.97 & 0.96 & 1.09 \\
\hline 3.5 & 1.26 & 1.16 & 1.12 & 1.08 & 1.07 & 1.10 & 1.08 & 1.21 & 1.30 \\
\hline 4.5 & 1.49 & 1.36 & 1.27 & 1.30 & 1.25 & 1.27 & 1.28 & 1.41 & 1.55 \\
\hline 5.5 & 1.77 & 1.62 & 1.62 & 1.50 & 1.45 & 1.50 & 1.46 & 1.64 & 1.79 \\
\hline 6.5 & 1.99 & 1.77 & 1.71 & 1.68 & 1.65 & 1.74 & 1.76 & 1.88 & 2.00 \\
\hline 7.5 & 2.46 & 2.10 & 2.02 & 1.97 & 1.90 & 1.92 & 1.93 & 2.07 & 2.39 \\
\hline 8.5 & 2.82 & 2.48 & 2.31 & 2.23 & 2.16 & 2.22 & 2.30 & 2.51 & 2.89 \\
\hline 9.5 & 3.31 & 2.82 & 2.54 & 2.48 & 2.39 & 2.46 & 2.66 & 2.72 & 3.22 \\
\hline 10.5 & 3.62 & 3.02 & 2.86 & 2.74 & 2.60 & 2.70 & 2.83 & 3.04 & 3.53 \\
\hline 11.5 & 3.80 & 3.34 & 3.07 & 2.90 & 2.77 & 2.85 & 3.05 & 3.25 & 3.84 \\
\hline 12.5 & 3.92 & 3.33 & 3.15 & 2.99 & 2.91 & 3.04 & 3.22 & 3.44 & 3.98 \\
\hline 13.5 & 4.07 & 3.45 & 3.29 & 3.10 & 2.99 & 3.14 & 3.28 & 3.56 & 4.10 \\
\hline 14.5 & 4.27 & 3.59 & 3.41 & 3.16 & 3.06 & 3.19 & 3.45 & 3.55 & 4.20 \\
\hline 15.5 & 4.14 & 3.62 & 3.40 & 3.19 & 3.08 & 3.24 & 3.39 & 3.57 & 4.08 \\
\hline 16.5 & 4.13 & 3.42 & 3.30 & 3.12 & 2.99 & 3.12 & 3.29 & 3.60 & 4.09 \\
\hline 17.5 & 4.04 & 3.47 & 3.24 & 3.07 & 2.90 & 3.06 & 3.16 & 3.37 & 4.03 \\
\hline 18.5 & 3.84 & 3.11 & 3.02 & 2.83 & 2.74 & 2.83 & 3.07 & 3.23 & 3.77 \\
\hline 19.5 & 3.59 & 3.01 & 2.79 & 2.66 & 2.62 & 2.71 & 2.85 & 3.00 & 3.55 \\
\hline 20.5 & 3.21 & 2.82 & 2.58 & 2.48 & 2.33 & 2.48 & 2.65 & 2.76 & 3.37 \\
\hline 21.5 & 3.01 & 2.44 & 2.28 & 2.24 & 2.10 & 2.18 & 2.38 & 2.42 & 3.01 \\
\hline 22.5 & 2.63 & 2.18 & 1.94 & 1.88 & 1.81 & 1.93 & 2.05 & 2.20 & 2.67 \\
\hline 23.5 & 3.33 & 2.67 & 2.56 & 2.52 & 2.35 & 2.43 & 2.48 & 2.78 & 3.44 \\
\hline Ax. Avg. & 2.91 & 2.48 & 2.34 & 2.24 & 2.15 & 2.24 & 2.34 & 2.49 & 2.91 \\
\hline
\end{tabular}

POWER DENSITY VALUES NORMALIZED TO A CORE POWER OF 11.57 MW AND ASSUME ALL FISSION ENERGY (EXCLUDING GAMMA-HEATING OF EX-CORE COMPONENTS) IS DEPOSITED IN THE FUEL 
Table B-16. Power Density Data (kW/cc) in Fuel Plate 16 in Prototypic Conditions (continued).

Plate 16, Core Position X1/X5, 3 MWd Burnup, Equilibrium Xenon Conditions

\begin{tabular}{|c|c|c|c|c|c|c|c|c|c|}
\hline $\begin{array}{l}\text { Axial } \\
\text { position } \\
\text { from } \\
\text { top of } \\
\text { fuel } \\
\text { (inches) }\end{array}$ & $\begin{array}{c}0.000 \\
\text { to } \\
0.197 \\
\text { inches }\end{array}$ & $\begin{array}{c}0.197 \\
\text { to } \\
0.394 \\
\text { inches }\end{array}$ & $\begin{array}{c}0.394 \\
\text { to } \\
0.591 \\
\text { inches }\end{array}$ & $\begin{array}{c}0.591 \\
\text { to } \\
1.295 \\
\text { inches }\end{array}$ & $\begin{array}{c}1.295 \\
\text { to } \\
2.000 \\
\text { inches }\end{array}$ & $\begin{array}{c}2.000 \\
\text { to } \\
2.704 \\
\text { inches }\end{array}$ & $\begin{array}{c}2.704 \\
\text { to } \\
2.901 \\
\text { inches }\end{array}$ & $\begin{array}{c}2.901 \\
\text { to } \\
3.098 \\
\text { inches }\end{array}$ & $\begin{array}{c}3.098 \\
\text { to } \\
3.295 \\
\text { inches }\end{array}$ \\
\hline 0.5 & 1.77 & 1.48 & 1.42 & 1.40 & 1.37 & 1.35 & 1.42 & 1.44 & 1.75 \\
\hline 1.5 & 1.58 & 1.36 & 1.23 & 1.19 & 1.16 & 1.20 & 1.24 & 1.30 & 1.54 \\
\hline 2.5 & 1.93 & 1.60 & 1.45 & 1.44 & 1.42 & 1.42 & 1.51 & 1.56 & 1.93 \\
\hline 3.5 & 2.17 & 1.89 & 1.77 & 1.70 & 1.63 & 1.73 & 1.70 & 1.89 & 2.25 \\
\hline 4.5 & 2.55 & 2.15 & 2.01 & 1.91 & 1.86 & 1.91 & 2.01 & 2.14 & 2.58 \\
\hline 5.5 & 2.74 & 2.33 & 2.28 & 2.14 & 2.04 & 2.13 & 2.28 & 2.38 & 2.79 \\
\hline 6.5 & 2.99 & 2.57 & 2.36 & 2.33 & 2.18 & 2.29 & 2.42 & 2.65 & 3.06 \\
\hline 7.5 & 3.26 & 2.63 & 2.55 & 2.44 & 2.31 & 2.48 & 2.59 & 2.68 & 3.24 \\
\hline 8.5 & 3.45 & 2.76 & 2.66 & 2.54 & 2.46 & 2.56 & 2.69 & 2.93 & 3.32 \\
\hline 9.5 & 3.54 & 2.92 & 2.80 & 2.64 & 2.56 & 2.67 & 2.85 & 3.00 & 3.53 \\
\hline 10.5 & 3.52 & 3.05 & 2.83 & 2.69 & 2.64 & 2.72 & 2.83 & 3.00 & 3.62 \\
\hline 11.5 & 3.54 & 3.04 & 2.80 & 2.71 & 2.62 & 2.71 & 2.86 & 3.09 & 3.67 \\
\hline 12.5 & 3.78 & 3.10 & 2.87 & 2.72 & 2.71 & 2.76 & 2.98 & 3.12 & 3.63 \\
\hline 13.5 & 3.69 & 3.09 & 2.86 & 2.68 & 2.60 & 2.74 & 2.89 & 3.11 & 3.71 \\
\hline 14.5 & 3.58 & 3.04 & 2.76 & 2.68 & 2.57 & 2.68 & 2.82 & 2.98 & 3.51 \\
\hline 15.5 & 3.48 & 2.90 & 2.74 & 2.64 & 2.53 & 2.57 & 2.76 & 2.95 & 3.56 \\
\hline 16.5 & 3.36 & 2.85 & 2.58 & 2.51 & 2.47 & 2.51 & 2.70 & 2.79 & 3.29 \\
\hline 17.5 & 3.19 & 2.62 & 2.52 & 2.39 & 2.30 & 2.35 & 2.55 & 2.66 & 3.16 \\
\hline 18.5 & 2.97 & 2.55 & 2.37 & 2.27 & 2.17 & 2.23 & 2.34 & 2.46 & 2.98 \\
\hline 19.5 & 2.72 & 2.31 & 2.14 & 2.05 & 1.96 & 2.05 & 2.17 & 2.34 & 2.85 \\
\hline 20.5 & 2.47 & 2.09 & 1.94 & 1.88 & 1.82 & 1.88 & 2.01 & 2.14 & 2.54 \\
\hline 21.5 & 2.24 & 1.85 & 1.64 & 1.65 & 1.59 & 1.67 & 1.74 & 1.86 & 2.23 \\
\hline 22.5 & 1.93 & 1.63 & 1.45 & 1.43 & 1.37 & 1.43 & 1.46 & 1.62 & 1.98 \\
\hline 23.5 & 2.50 & 2.06 & 1.90 & 1.79 & 1.72 & 1.81 & 1.93 & 2.11 & 2.51 \\
\hline Ax. Avg. & 2.87 & 2.41 & 2.25 & 2.16 & 2.09 & 2.16 & 2.28 & 2.42 & 2.88 \\
\hline
\end{tabular}

POWER DENSITY VALUES NORMALIZED TO A CORE POWER OF 11.57 MW AND ASSUME ALL FISSION ENERGY (EXCLUDING GAMMA-HEATING OF EX-CORE COMPONENTS) IS DEPOSITED IN THE FUEL 
Table B-16. Power Density Data (kW/cc) in Fuel Plate 16 in Prototypic Conditions (continued).

Plate 16, Core Position X3/X7, 80 MWd Burnup, Equilibrium Xenon Conditions

\begin{tabular}{|c|c|c|c|c|c|c|c|c|c|}
\hline $\begin{array}{c}\text { Axial } \\
\text { position } \\
\text { from } \\
\text { top of } \\
\text { fuel } \\
\text { (inches) }\end{array}$ & $\begin{array}{c}0.000 \\
\text { to } \\
0.197 \\
\text { inches }\end{array}$ & $\begin{array}{c}0.197 \\
\text { to } \\
0.394 \\
\text { inches }\end{array}$ & $\begin{array}{c}0.394 \\
\text { to } \\
0.591 \\
\text { inches }\end{array}$ & $\begin{array}{c}0.591 \\
\text { to } \\
1.295 \\
\text { inches }\end{array}$ & $\begin{array}{c}1.295 \\
\text { to } \\
\text { inches }\end{array}$ & $\begin{array}{c}2.000 \\
\text { to } \\
2.704 \\
\text { inches }\end{array}$ & $\begin{array}{c}\text { 2.704 } \\
\text { to } \\
2.901 \\
\text { inches }\end{array}$ & $\begin{array}{c}2.901 \\
\text { to } \\
3.098 \\
\text { inches }\end{array}$ & $\begin{array}{c}3.098 \\
\text { to } \\
\text { inches }\end{array}$ \\
\hline 0.5 & 1.69 & 1.46 & 1.36 & 1.33 & 1.29 & 1.36 & 1.36 & 1.43 & 1.67 \\
\hline 1.5 & 1.57 & 1.32 & 1.21 & 1.17 & 1.12 & 1.20 & 1.28 & 1.33 & 1.52 \\
\hline 2.5 & 1.92 & 1.58 & 1.48 & 1.41 & 1.35 & 1.41 & 1.48 & 1.65 & 1.93 \\
\hline 3.5 & 2.22 & 1.87 & 1.74 & 1.67 & 1.60 & 1.66 & 1.73 & 1.87 & 2.26 \\
\hline 4.5 & 2.53 & 2.16 & 2.00 & 1.88 & 1.81 & 1.88 & 2.00 & 2.15 & 2.49 \\
\hline 5.5 & 2.78 & 2.40 & 2.13 & 2.08 & 2.02 & 2.06 & 2.13 & 2.27 & 2.85 \\
\hline 6.5 & 2.96 & 2.51 & 2.39 & 2.29 & 2.20 & 2.24 & 2.39 & 2.53 & 2.95 \\
\hline 7.5 & 3.17 & 2.71 & 2.55 & 2.38 & 2.28 & 2.37 & 2.38 & 2.68 & 3.18 \\
\hline 8.5 & 3.41 & 2.75 & 2.59 & 2.47 & 2.36 & 2.49 & 2.56 & 2.82 & 3.34 \\
\hline 9.5 & 3.38 & 2.89 & 2.71 & 2.62 & 2.50 & 2.60 & 2.65 & 2.89 & 3.41 \\
\hline 10.5 & 3.50 & 2.90 & 2.76 & 2.65 & 2.56 & 2.65 & 2.66 & 2.93 & 3.61 \\
\hline 11.5 & 3.49 & 3.01 & 2.79 & 2.66 & 2.54 & 2.63 & 2.80 & 3.11 & 3.55 \\
\hline 12.5 & 3.62 & 3.01 & 2.85 & 2.62 & 2.53 & 2.70 & 2.73 & 2.96 & 3.54 \\
\hline 13.5 & 3.57 & 2.94 & 2.80 & 2.66 & 2.48 & 2.63 & 2.77 & 2.99 & 3.51 \\
\hline 14.5 & 3.48 & 2.77 & 2.68 & 2.63 & 2.49 & 2.56 & 2.71 & 2.85 & 3.45 \\
\hline 15.5 & 3.38 & 2.78 & 2.63 & 2.54 & 2.43 & 2.45 & 2.54 & 2.82 & 3.27 \\
\hline 16.5 & 3.22 & 2.73 & 2.58 & 2.44 & 2.31 & 2.36 & 2.52 & 2.68 & 3.27 \\
\hline 17.5 & 3.10 & 2.60 & 2.44 & 2.29 & 2.22 & 2.25 & 2.31 & 2.56 & 3.05 \\
\hline 18.5 & 2.92 & 2.39 & 2.22 & 2.20 & 2.05 & 2.16 & 2.21 & 2.30 & 2.85 \\
\hline 19.5 & 2.68 & 2.18 & 2.12 & 1.95 & 1.88 & 1.95 & 2.05 & 2.16 & 2.63 \\
\hline 20.5 & 2.46 & 2.02 & 1.87 & 1.80 & 1.73 & 1.79 & 1.82 & 2.02 & 2.44 \\
\hline 21.5 & 2.14 & 1.84 & 1.74 & 1.58 & 1.52 & 1.58 & 1.67 & 1.78 & 2.16 \\
\hline 22.5 & 1.93 & 1.62 & 1.43 & 1.37 & 1.32 & 1.34 & 1.41 & 1.53 & 1.87 \\
\hline 23.5 & 2.53 & 2.08 & 1.93 & 1.78 & 1.73 & 1.73 & 1.84 & 1.92 & 2.38 \\
\hline Ax. Avg. & 2.82 & 2.36 & 2.21 & 2.10 & 2.01 & 2.09 & 2.17 & 2.34 & 2.80 \\
\hline & & & & & & & & & \\
\hline
\end{tabular}

POWER DENSITY VALUES NORMALIZED TO A CORE POWER OF 11.57 MW AND ASSUME ALL FISSION ENERGY (EXCLUDING GAMMA-HEATING OF EX-CORE COMPONENTS) IS DEPOSITED IN THE FUEL 
Table B-16. Power Density Data (kW/cc) in Fuel Plate 16 in Prototypic Conditions (continued).

Plate 16, Core Position X2/X6, 99 MWd Burnup, Equilibrium Xenon Conditions

\begin{tabular}{|c|c|c|c|c|c|c|c|c|c|}
\hline $\begin{array}{c}\text { Axial } \\
\text { position } \\
\text { from } \\
\text { top of } \\
\text { fuel } \\
\text { (inches) }\end{array}$ & $\begin{array}{c}0.000 \\
\text { to } \\
0.197 \\
\text { inches }\end{array}$ & $\begin{array}{c}0.197 \\
\text { to } \\
0.394 \\
\text { inches }\end{array}$ & $\begin{array}{c}0.394 \\
\text { to } \\
0.591 \\
\text { inches }\end{array}$ & $\begin{array}{c}0.591 \\
\text { to } \\
1.295 \\
\text { inches }\end{array}$ & $\begin{array}{c}1.295 \\
\text { to } \\
\text { inches }\end{array}$ & $\begin{array}{c}2.000 \\
\text { to } \\
2.704 \\
\text { inches }\end{array}$ & $\begin{array}{c}\text { 2.704 } \\
\text { to } \\
2.901 \\
\text { inches }\end{array}$ & $\begin{array}{c}2.901 \\
\text { to } \\
3.098 \\
\text { inches }\end{array}$ & $\begin{array}{c}3.098 \\
\text { to } \\
\text { inches }\end{array}$ \\
\hline 0.5 & 1.67 & 1.39 & 1.33 & 1.25 & 1.21 & 1.30 & 1.31 & 1.37 & 1.64 \\
\hline 1.5 & 1.51 & 1.28 & 1.30 & 1.13 & 1.09 & 1.14 & 1.18 & 1.32 & 1.52 \\
\hline 2.5 & 1.83 & 1.53 & 1.49 & 1.41 & 1.36 & 1.44 & 1.48 & 1.57 & 1.88 \\
\hline 3.5 & 2.17 & 1.84 & 1.85 & 1.68 & 1.62 & 1.69 & 1.73 & 1.85 & 2.28 \\
\hline 4.5 & 2.48 & 2.13 & 1.96 & 1.88 & 1.83 & 1.90 & 1.97 & 2.11 & 2.52 \\
\hline 5.5 & 2.67 & 2.31 & 2.19 & 2.09 & 2.00 & 2.08 & 2.20 & 2.30 & 2.73 \\
\hline 6.5 & 3.03 & 2.46 & 2.37 & 2.22 & 2.17 & 2.26 & 2.35 & 2.60 & 2.97 \\
\hline 7.5 & 3.17 & 2.63 & 2.50 & 2.36 & 2.28 & 2.42 & 2.44 & 2.63 & 3.08 \\
\hline 8.5 & 3.24 & 2.82 & 2.56 & 2.46 & 2.40 & 2.50 & 2.69 & 2.72 & 3.37 \\
\hline 9.5 & 3.34 & 2.82 & 2.66 & 2.54 & 2.52 & 2.58 & 2.63 & 2.90 & 3.48 \\
\hline 10.5 & 3.48 & 2.93 & 2.75 & 2.62 & 2.50 & 2.63 & 2.69 & 2.95 & 3.52 \\
\hline 11.5 & 3.43 & 3.04 & 2.81 & 2.68 & 2.55 & 2.64 & 2.82 & 3.04 & 3.60 \\
\hline 12.5 & 3.55 & 2.96 & 2.79 & 2.65 & 2.55 & 2.63 & 2.85 & 3.04 & 3.65 \\
\hline 13.5 & 3.48 & 3.01 & 2.75 & 2.63 & 2.54 & 2.66 & 2.75 & 3.03 & 3.51 \\
\hline 14.5 & 3.51 & 3.00 & 2.74 & 2.63 & 2.53 & 2.61 & 2.69 & 2.84 & 3.42 \\
\hline 15.5 & 3.35 & 2.83 & 2.59 & 2.50 & 2.43 & 2.49 & 2.65 & 2.79 & 3.38 \\
\hline 16.5 & 3.29 & 2.73 & 2.60 & 2.42 & 2.34 & 2.38 & 2.59 & 2.75 & 3.20 \\
\hline 17.5 & 3.05 & 2.57 & 2.40 & 2.30 & 2.22 & 2.29 & 2.35 & 2.60 & 3.08 \\
\hline 18.5 & 2.92 & 2.43 & 2.34 & 2.18 & 2.09 & 2.18 & 2.25 & 2.40 & 2.88 \\
\hline 19.5 & 2.70 & 2.29 & 2.16 & 1.96 & 1.90 & 2.01 & 2.14 & 2.30 & 2.63 \\
\hline 20.5 & 2.37 & 2.05 & 1.92 & 1.78 & 1.74 & 1.82 & 1.95 & 2.01 & 2.35 \\
\hline 21.5 & 2.17 & 1.79 & 1.65 & 1.57 & 1.54 & 1.57 & 1.65 & 1.80 & 2.14 \\
\hline 22.5 & 1.91 & 1.57 & 1.46 & 1.38 & 1.33 & 1.33 & 1.45 & 1.55 & 1.89 \\
\hline 23.5 & 2.51 & 1.95 & 1.89 & 1.78 & 1.76 & 1.73 & 1.92 & 2.04 & 2.34 \\
\hline Ax. Avg. & 2.79 & 2.35 & 2.21 & 2.09 & 2.02 & 2.10 & 2.20 & 2.36 & 2.79 \\
\hline & & & & & & & & & \\
\hline
\end{tabular}

POWER DENSITY VALUES NORMALIZED TO A CORE POWER OF 11.57 MW AND ASSUME ALL FISSION ENERGY (EXCLUDING GAMMA-HEATING OF EX-CORE COMPONENTS) IS DEPOSITED IN THE FUEL 
Table B-16. Power Density Data (kW/cc) in Fuel Plate 16 in Prototypic Conditions (continued).

Plate 16, Core Position X4/X8, 173 MWd Burnup, Equilibrium Xenon Conditions

\begin{tabular}{|c|c|c|c|c|c|c|c|c|c|}
\hline $\begin{array}{c}\text { Axial } \\
\text { position } \\
\text { from } \\
\text { top of } \\
\text { fuel } \\
\text { (inches) }\end{array}$ & $\begin{array}{c}0.000 \\
\text { to } \\
0.197 \\
\text { inches }\end{array}$ & $\begin{array}{c}0.197 \\
\text { to } \\
0.394 \\
\text { inches }\end{array}$ & $\begin{array}{c}0.394 \\
\text { to } \\
0.591 \\
\text { inches }\end{array}$ & $\begin{array}{c}0.591 \\
\text { to } \\
1.295 \\
\text { inches }\end{array}$ & $\begin{array}{c}1.295 \\
\text { to } \\
\text { inches }\end{array}$ & $\begin{array}{c}2.000 \\
\text { to } \\
2.704 \\
\text { inches }\end{array}$ & $\begin{array}{c}\text { 2.704 } \\
\text { to } \\
2.901 \\
\text { inches }\end{array}$ & $\begin{array}{c}2.901 \\
\text { to } \\
3.098 \\
\text { inches }\end{array}$ & $\begin{array}{c}3.098 \\
\text { to } \\
\text { inches }\end{array}$ \\
\hline 0.5 & 1.66 & 1.43 & 1.33 & 1.26 & 1.21 & 1.28 & 1.31 & 1.42 & 1.62 \\
\hline 1.5 & 1.46 & 1.22 & 1.18 & 1.15 & 1.08 & 1.15 & 1.17 & 1.29 & 1.51 \\
\hline 2.5 & 1.88 & 1.56 & 1.39 & 1.42 & 1.35 & 1.37 & 1.41 & 1.52 & 1.85 \\
\hline 3.5 & 2.16 & 1.81 & 1.71 & 1.60 & 1.62 & 1.62 & 1.71 & 1.86 & 2.21 \\
\hline 4.5 & 2.42 & 2.12 & 1.94 & 1.84 & 1.79 & 1.87 & 1.93 & 2.01 & 2.39 \\
\hline 5.5 & 2.66 & 2.30 & 2.05 & 2.02 & 1.95 & 2.07 & 2.13 & 2.21 & 2.64 \\
\hline 6.5 & 3.04 & 2.45 & 2.27 & 2.19 & 2.12 & 2.18 & 2.31 & 2.41 & 2.92 \\
\hline 7.5 & 3.06 & 2.57 & 2.40 & 2.36 & 2.25 & 2.28 & 2.42 & 2.58 & 3.02 \\
\hline 8.5 & 3.26 & 2.69 & 2.52 & 2.39 & 2.29 & 2.39 & 2.51 & 2.74 & 3.23 \\
\hline 9.5 & 3.31 & 2.75 & 2.60 & 2.50 & 2.35 & 2.45 & 2.62 & 2.75 & 3.28 \\
\hline 10.5 & 3.35 & 2.80 & 2.67 & 2.55 & 2.44 & 2.54 & 2.72 & 2.92 & 3.40 \\
\hline 11.5 & 3.41 & 2.94 & 2.75 & 2.57 & 2.47 & 2.58 & 2.70 & 2.95 & 3.47 \\
\hline 12.5 & 3.38 & 2.93 & 2.71 & 2.52 & 2.49 & 2.60 & 2.70 & 2.99 & 3.56 \\
\hline 13.5 & 3.49 & 2.89 & 2.66 & 2.56 & 2.46 & 2.55 & 2.70 & 2.79 & 3.31 \\
\hline 14.5 & 3.27 & 2.68 & 2.64 & 2.52 & 2.41 & 2.54 & 2.71 & 2.88 & 3.35 \\
\hline 15.5 & 3.22 & 2.78 & 2.55 & 2.42 & 2.36 & 2.47 & 2.57 & 2.79 & 3.22 \\
\hline 16.5 & 3.03 & 2.67 & 2.41 & 2.33 & 2.27 & 2.37 & 2.38 & 2.62 & 3.16 \\
\hline 17.5 & 2.84 & 2.51 & 2.31 & 2.22 & 2.15 & 2.24 & 2.35 & 2.57 & 3.05 \\
\hline 18.5 & 2.92 & 2.33 & 2.22 & 2.13 & 2.03 & 2.12 & 2.21 & 2.39 & 2.87 \\
\hline 19.5 & 2.64 & 2.16 & 2.06 & 1.90 & 1.84 & 1.92 & 1.98 & 2.14 & 2.62 \\
\hline 20.5 & 2.28 & 1.95 & 1.80 & 1.78 & 1.73 & 1.76 & 1.89 & 1.99 & 2.40 \\
\hline 21.5 & 2.02 & 1.70 & 1.58 & 1.50 & 1.48 & 1.54 & 1.65 & 1.77 & 2.09 \\
\hline 22.5 & 1.96 & 1.56 & 1.45 & 1.31 & 1.31 & 1.36 & 1.47 & 1.57 & 1.91 \\
\hline 23.5 & 2.33 & 1.90 & 1.84 & 1.71 & 1.70 & 1.71 & 1.83 & 2.03 & 2.33 \\
\hline Ax. Avg. & 2.71 & 2.28 & 2.13 & 2.03 & 1.97 & 2.04 & 2.14 & 2.30 & 2.73 \\
\hline & & & & & & & & & \\
\hline
\end{tabular}

POWER DENSITY VALUES NORMALIZED TO A CORE POWER OF 11.57 MW AND ASSUME ALL FISSION ENERGY (EXCLUDING GAMMA-HEATING OF EX-CORE COMPONENTS) IS DEPOSITED IN THE FUEL 
Table B-17. Power Density Data $\left(\mathrm{kW} / \mathrm{cm}^{3}\right)$ in Fuel Plate 17 in Prototypic Core.

\section{Fuel core width: 3.402 inches / Fuel core thickness: 20 mil}

Plate 17, Core Position X1/X5, 0 MWd Burnup, Xenon-Free Conditions

\begin{tabular}{|c|c|c|c|c|c|c|c|c|c|}
\hline $\begin{array}{c}\text { Axial } \\
\text { position } \\
\text { from } \\
\text { top of } \\
\text { fuel } \\
\text { (inches) } \\
\end{array}$ & $\begin{array}{c}0.000 \\
\text { to } \\
0.197 \\
\text { inches }\end{array}$ & $\begin{array}{c}0.197 \\
\text { to } \\
0.394 \\
\text { inches } \\
\end{array}$ & $\begin{array}{c}0.394 \\
\text { to } \\
0.591 \\
\text { inches } \\
\end{array}$ & $\begin{array}{c}0.591 \\
\text { to } \\
1.331 \\
\text { inches }\end{array}$ & $\begin{array}{c}1.331 \\
\text { to } \\
2.071 \\
\text { inches }\end{array}$ & $\begin{array}{c}2.071 \\
\text { to } \\
2.811 \\
\text { inches } \\
\end{array}$ & $\begin{array}{c}2.811 \\
\text { to } \\
3.008 \\
\text { inches }\end{array}$ & $\begin{array}{c}3.008 \\
\text { to } \\
3.205 \\
\text { inches }\end{array}$ & $\begin{array}{c}3.205 \\
\text { to } \\
3.402 \\
\text { inches } \\
\end{array}$ \\
\hline 0.5 & 1.04 & 0.86 & 0.82 & 0.88 & 0.82 & 0.86 & 0.91 & 0.91 & 1.04 \\
\hline 1.5 & 0.85 & 0.80 & 0.73 & 0.77 & 0.75 & 0.75 & 0.78 & 0.79 & 0.88 \\
\hline 2.5 & 1.06 & 0.98 & 0.92 & 0.90 & 0.90 & 0.90 & 0.87 & 0.96 & 1.05 \\
\hline 3.5 & 1.29 & 1.12 & 1.03 & 1.07 & 1.04 & 1.11 & 1.05 & 1.21 & 1.27 \\
\hline 4.5 & 1.46 & 1.29 & 1.22 & 1.29 & 1.30 & 1.27 & 1.28 & 1.37 & 1.49 \\
\hline 5.5 & 1.73 & 1.55 & 1.51 & 1.46 & 1.47 & 1.50 & 1.44 & 1.59 & 1.79 \\
\hline 6.5 & 1.99 & 1.75 & 1.70 & 1.67 & 1.67 & 1.73 & 1.75 & 1.77 & 1.97 \\
\hline 7.5 & 2.35 & 2.06 & 1.99 & 1.94 & 1.91 & 2.00 & 1.91 & 2.00 & 2.38 \\
\hline 8.5 & 2.71 & 2.46 & 2.28 & 2.26 & 2.17 & 2.23 & 2.37 & 2.51 & 2.76 \\
\hline 9.5 & 3.29 & 2.75 & 2.62 & 2.51 & 2.43 & 2.52 & 2.63 & 2.85 & 3.25 \\
\hline 10.5 & 3.59 & 3.08 & 2.83 & 2.78 & 2.66 & 2.75 & 2.84 & 3.12 & 3.56 \\
\hline 11.5 & 3.90 & 3.34 & 3.14 & 2.95 & 2.83 & 2.93 & 3.07 & 3.33 & 3.85 \\
\hline 12.5 & 4.12 & 3.43 & 3.18 & 3.05 & 2.93 & 3.06 & 3.23 & 3.60 & 4.11 \\
\hline 13.5 & 4.22 & 3.57 & 3.39 & 3.17 & 3.03 & 3.15 & 3.24 & 3.60 & 4.30 \\
\hline 14.5 & 4.25 & 3.63 & 3.40 & 3.21 & 3.06 & 3.25 & 3.52 & 3.71 & 4.25 \\
\hline 15.5 & 4.27 & 3.58 & 3.38 & 3.20 & 3.13 & 3.21 & 3.48 & 3.67 & 4.27 \\
\hline 16.5 & 4.27 & 3.61 & 3.34 & 3.16 & 3.06 & 3.23 & 3.37 & 3.58 & 4.26 \\
\hline 17.5 & 4.07 & 3.53 & 3.27 & 3.08 & 2.99 & 3.09 & 3.33 & 3.54 & 4.17 \\
\hline 18.5 & 3.90 & 3.23 & 3.09 & 2.92 & 2.79 & 2.97 & 3.14 & 3.35 & 4.08 \\
\hline 19.5 & 3.63 & 3.11 & 2.85 & 2.74 & 2.71 & 2.73 & 2.86 & 3.20 & 3.70 \\
\hline 20.5 & 3.26 & 2.83 & 2.67 & 2.50 & 2.40 & 2.48 & 2.65 & 2.83 & 3.34 \\
\hline 21.5 & 2.97 & 2.50 & 2.37 & 2.23 & 2.17 & 2.19 & 2.36 & 2.50 & 3.00 \\
\hline 22.5 & 2.79 & 2.21 & 2.01 & 1.94 & 1.86 & 1.92 & 2.10 & 2.26 & 2.71 \\
\hline 23.5 & 3.50 & 2.84 & 2.68 & 2.51 & 2.41 & 2.45 & 2.54 & 2.79 & 3.49 \\
\hline Ax. Avg. & 2.94 & 2.50 & 2.35 & 2.26 & 2.19 & 2.26 & 2.36 & 2.54 & 2.96 \\
\hline
\end{tabular}

POWER DENSITY VALUES NORMALIZED TO A CORE POWER OF 11.57 MW AND ASSUME ALL FISSION ENERGY (EXCLUDING GAMMA-HEATING OF EX-CORE COMPONENTS) IS DEPOSITED IN THE FUEL 
Table B-17. Power Density Data (kW/cc) in Fuel Plate 17 in Prototypic Conditions (continued).

Plate 17, Core Position X1/X5, 3 MWd Burnup, Equilibrium Xenon Conditions

\begin{tabular}{|c|c|c|c|c|c|c|c|c|c|}
\hline $\begin{array}{l}\text { Axial } \\
\text { position } \\
\text { from } \\
\text { top of } \\
\text { fuel } \\
\text { (inches) }\end{array}$ & $\begin{array}{c}0.000 \\
\text { to } \\
0.197 \\
\text { inches }\end{array}$ & $\begin{array}{c}0.197 \\
\text { to } \\
0.394 \\
\text { inches }\end{array}$ & $\begin{array}{c}0.394 \\
\text { to } \\
0.591 \\
\text { inches }\end{array}$ & $\begin{array}{c}0.591 \\
\text { to } \\
1.331 \\
\text { inches }\end{array}$ & $\begin{array}{c}1.331 \\
\text { to } \\
2.071 \\
\text { inches }\end{array}$ & $\begin{array}{c}2.071 \\
\text { to } \\
2.811 \\
\text { inches } \\
\end{array}$ & $\begin{array}{c}2.811 \\
\text { to } \\
3.008 \\
\text { inches }\end{array}$ & $\begin{array}{c}3.008 \\
\text { to } \\
3.205 \\
\text { inches }\end{array}$ & $\begin{array}{c}3.205 \\
\text { to } \\
3.402 \\
\text { inches }\end{array}$ \\
\hline 0.5 & 1.76 & 1.53 & 1.40 & 1.38 & 1.33 & 1.34 & 1.34 & 1.41 & 1.68 \\
\hline 1.5 & 1.62 & 1.34 & 1.27 & 1.21 & 1.19 & 1.19 & 1.22 & 1.26 & 1.61 \\
\hline 2.5 & 1.90 & 1.64 & 1.59 & 1.46 & 1.36 & 1.48 & 1.56 & 1.63 & 1.96 \\
\hline 3.5 & 2.29 & 1.97 & 1.79 & 1.73 & 1.67 & 1.74 & 1.75 & 1.91 & 2.35 \\
\hline 4.5 & 2.58 & 2.16 & 2.04 & 1.91 & 1.88 & 1.95 & 2.02 & 2.17 & 2.59 \\
\hline 5.5 & 2.94 & 2.44 & 2.27 & 2.16 & 2.07 & 2.18 & 2.28 & 2.43 & 2.89 \\
\hline 6.5 & 3.19 & 2.68 & 2.45 & 2.36 & 2.25 & 2.31 & 2.39 & 2.72 & 3.14 \\
\hline 7.5 & 3.35 & 2.67 & 2.59 & 2.48 & 2.38 & 2.47 & 2.66 & 2.87 & 3.32 \\
\hline 8.5 & 3.47 & 2.94 & 2.74 & 2.58 & 2.51 & 2.61 & 2.71 & 2.91 & 3.56 \\
\hline 9.5 & 3.64 & 3.01 & 2.89 & 2.71 & 2.63 & 2.70 & 2.83 & 3.04 & 3.65 \\
\hline 10.5 & 3.75 & 3.14 & 2.89 & 2.73 & 2.66 & 2.74 & 2.95 & 3.22 & 3.73 \\
\hline 11.5 & 3.74 & 2.97 & 2.88 & 2.76 & 2.67 & 2.78 & 2.98 & 3.14 & 3.75 \\
\hline 12.5 & 3.76 & 3.09 & 2.95 & 2.77 & 2.72 & 2.84 & 2.94 & 3.18 & 3.79 \\
\hline 13.5 & 3.77 & 3.11 & 2.97 & 2.79 & 2.71 & 2.79 & 2.94 & 3.19 & 3.77 \\
\hline 14.5 & 3.72 & 3.10 & 2.89 & 2.75 & 2.63 & 2.69 & 2.78 & 3.08 & 3.66 \\
\hline 15.5 & 3.54 & 2.99 & 2.73 & 2.63 & 2.58 & 2.67 & 2.77 & 3.00 & 3.65 \\
\hline 16.5 & 3.42 & 2.87 & 2.67 & 2.52 & 2.49 & 2.55 & 2.65 & 2.81 & 3.44 \\
\hline 17.5 & 3.27 & 2.72 & 2.56 & 2.46 & 2.34 & 2.44 & 2.47 & 2.75 & 3.23 \\
\hline 18.5 & 3.03 & 2.46 & 2.38 & 2.29 & 2.22 & 2.24 & 2.33 & 2.55 & 3.05 \\
\hline 19.5 & 2.86 & 2.33 & 2.18 & 2.10 & 2.03 & 2.14 & 2.16 & 2.35 & 2.80 \\
\hline 20.5 & 2.53 & 2.11 & 1.97 & 1.89 & 1.85 & 1.91 & 1.97 & 2.18 & 2.55 \\
\hline 21.5 & 2.33 & 1.91 & 1.75 & 1.68 & 1.60 & 1.68 & 1.79 & 1.95 & 2.23 \\
\hline 22.5 & 2.02 & 1.67 & 1.52 & 1.44 & 1.41 & 1.45 & 1.47 & 1.64 & 1.98 \\
\hline 23.5 & 2.52 & 2.08 & 1.94 & 1.81 & 1.75 & 1.84 & 1.85 & 2.08 & 2.50 \\
\hline Ax. Avg. & 2.96 & 2.46 & 2.30 & 2.19 & 2.12 & 2.20 & 2.28 & 2.48 & 2.95 \\
\hline
\end{tabular}

POWER DENSITY VALUES NORMALIZED TO A CORE POWER OF 11.57 MW AND ASSUME ALL FISSION ENERGY (EXCLUDING GAMMA-HEATING OF EX-CORE COMPONENTS) IS DEPOSITED IN THE FUEL 
Table B-17. Power Density Data (kW/cc) in Fuel Plate 17 in Prototypic Conditions (continued).

Plate 17, Core Position X3/X7, 80 MWd Burnup, Equilibrium Xenon Conditions

\begin{tabular}{|c|c|c|c|c|c|c|c|c|c|}
\hline $\begin{array}{l}\text { Axial } \\
\text { position } \\
\text { from } \\
\text { top of } \\
\text { fuel } \\
\text { (inches) }\end{array}$ & $\begin{array}{c}0.000 \\
\text { to } \\
0.197 \\
\text { inches }\end{array}$ & $\begin{array}{c}0.197 \\
\text { to } \\
0.394 \\
\text { inches }\end{array}$ & $\begin{array}{c}0.394 \\
\text { to } \\
0.591 \\
\text { inches }\end{array}$ & $\begin{array}{c}0.591 \\
\text { to } \\
1.331 \\
\text { inches }\end{array}$ & $\begin{array}{c}1.331 \\
\text { to } \\
2.071 \\
\text { inches }\end{array}$ & $\begin{array}{c}2.071 \\
\text { to } \\
2.811 \\
\text { inches }\end{array}$ & $\begin{array}{c}2.811 \\
\text { to } \\
3.008 \\
\text { inches }\end{array}$ & $\begin{array}{c}3.008 \\
\text { to } \\
3.205 \\
\text { inches }\end{array}$ & $\begin{array}{c}3.205 \\
\text { to } \\
3.402 \\
\text { inches }\end{array}$ \\
\hline 0.5 & 1.68 & 1.43 & 1.32 & 1.28 & 1.24 & 1.28 & 1.38 & 1.34 & 1.62 \\
\hline 1.5 & 1.59 & 1.30 & 1.16 & 1.19 & 1.14 & 1.20 & 1.23 & 1.32 & 1.55 \\
\hline 2.5 & 1.98 & 1.64 & 1.50 & 1.42 & 1.39 & 1.45 & 1.50 & 1.67 & 1.96 \\
\hline 3.5 & 2.28 & 1.94 & 1.87 & 1.74 & 1.64 & 1.67 & 1.82 & 1.91 & 2.28 \\
\hline 4.5 & 2.53 & 2.18 & 2.06 & 1.97 & 1.82 & 1.89 & 2.01 & 2.17 & 2.53 \\
\hline 5.5 & 2.92 & 2.35 & 2.17 & 2.16 & 2.07 & 2.09 & 2.17 & 2.40 & 2.85 \\
\hline 6.5 & 3.12 & 2.57 & 2.44 & 2.35 & 2.24 & 2.25 & 2.31 & 2.59 & 3.11 \\
\hline 7.5 & 3.44 & 2.92 & 2.59 & 2.46 & 2.30 & 2.41 & 2.59 & 2.73 & 3.29 \\
\hline 8.5 & 3.52 & 2.93 & 2.65 & 2.56 & 2.45 & 2.55 & 2.70 & 2.89 & 3.47 \\
\hline 9.5 & 3.64 & 2.91 & 2.73 & 2.71 & 2.54 & 2.63 & 2.77 & 3.02 & 3.58 \\
\hline 10.5 & 3.62 & 3.11 & 2.86 & 2.67 & 2.61 & 2.65 & 2.76 & 3.03 & 3.74 \\
\hline 11.5 & 3.79 & 3.10 & 2.84 & 2.73 & 2.58 & 2.73 & 2.81 & 2.99 & 3.65 \\
\hline 12.5 & 3.72 & 3.06 & 2.83 & 2.71 & 2.64 & 2.71 & 2.89 & 3.04 & 3.62 \\
\hline 13.5 & 3.68 & 3.01 & 2.86 & 2.71 & 2.60 & 2.63 & 2.82 & 3.00 & 3.59 \\
\hline 14.5 & 3.54 & 2.93 & 2.82 & 2.67 & 2.52 & 2.63 & 2.73 & 2.99 & 3.51 \\
\hline 15.5 & 3.42 & 2.87 & 2.60 & 2.53 & 2.40 & 2.50 & 2.60 & 2.85 & 3.48 \\
\hline 16.5 & 3.36 & 2.78 & 2.69 & 2.47 & 2.39 & 2.43 & 2.52 & 2.67 & 3.31 \\
\hline 17.5 & 3.15 & 2.64 & 2.35 & 2.32 & 2.26 & 2.28 & 2.41 & 2.62 & 3.19 \\
\hline 18.5 & 3.07 & 2.48 & 2.30 & 2.19 & 2.12 & 2.15 & 2.25 & 2.41 & 2.97 \\
\hline 19.5 & 2.71 & 2.32 & 2.09 & 1.98 & 1.95 & 1.99 & 2.09 & 2.20 & 2.69 \\
\hline 20.5 & 2.42 & 2.08 & 1.91 & 1.86 & 1.75 & 1.76 & 1.89 & 2.01 & 2.45 \\
\hline 21.5 & 2.18 & 1.79 & 1.75 & 1.59 & 1.55 & 1.56 & 1.74 & 1.86 & 2.18 \\
\hline 22.5 & 2.00 & 1.58 & 1.45 & 1.39 & 1.33 & 1.40 & 1.45 & 1.59 & 1.98 \\
\hline 23.5 & 2.42 & 2.03 & 1.91 & 1.79 & 1.71 & 1.76 & 1.86 & 1.98 & 2.47 \\
\hline Ax. Avg. & 2.91 & 2.41 & 2.24 & 2.14 & 2.05 & 2.11 & 2.22 & 2.39 & 2.88 \\
\hline
\end{tabular}

POWER DENSITY VALUES NORMALIZED TO A CORE POWER OF 11.57 MW AND ASSUME ALL FISSION ENERGY (EXCLUDING GAMMA-HEATING OF EX-CORE COMPONENTS) IS DEPOSITED IN THE FUEL 
Table B-17. Power Density Data (kW/cc) in Fuel Plate 17 in Prototypic Conditions (continued).

Plate 17, Core Position X2/X6, 99 MWd Burnup, Equilibrium Xenon Conditions

\begin{tabular}{|c|c|c|c|c|c|c|c|c|c|}
\hline $\begin{array}{l}\text { Axial } \\
\text { position } \\
\text { from } \\
\text { top of } \\
\text { fuel } \\
\text { (inches) }\end{array}$ & $\begin{array}{c}0.000 \\
\text { to } \\
0.197 \\
\text { inches }\end{array}$ & $\begin{array}{c}0.197 \\
\text { to } \\
0.394 \\
\text { inches }\end{array}$ & $\begin{array}{c}0.394 \\
\text { to } \\
0.591 \\
\text { inches }\end{array}$ & $\begin{array}{c}0.591 \\
\text { to } \\
1.331 \\
\text { inches }\end{array}$ & $\begin{array}{c}1.331 \\
\text { to } \\
2.071 \\
\text { inches }\end{array}$ & $\begin{array}{c}2.071 \\
\text { to } \\
2.811 \\
\text { inches } \\
\end{array}$ & $\begin{array}{c}2.811 \\
\text { to } \\
3.008 \\
\text { inches }\end{array}$ & $\begin{array}{c}3.008 \\
\text { to } \\
3.205 \\
\text { inches }\end{array}$ & $\begin{array}{c}3.205 \\
\text { to } \\
3.402 \\
\text { inches }\end{array}$ \\
\hline 0.5 & 1.62 & 1.36 & 1.27 & 1.26 & 1.19 & 1.25 & 1.30 & 1.37 & 1.68 \\
\hline 1.5 & 1.57 & 1.28 & 1.18 & 1.15 & 1.07 & 1.12 & 1.19 & 1.28 & 1.58 \\
\hline 2.5 & 1.87 & 1.61 & 1.50 & 1.41 & 1.39 & 1.46 & 1.49 & 1.63 & 1.96 \\
\hline 3.5 & 2.24 & 1.92 & 1.75 & 1.73 & 1.65 & 1.71 & 1.78 & 1.90 & 2.32 \\
\hline 4.5 & 2.51 & 2.18 & 1.96 & 1.89 & 1.85 & 1.89 & 2.05 & 2.07 & 2.58 \\
\hline 5.5 & 2.80 & 2.33 & 2.23 & 2.11 & 2.08 & 2.15 & 2.26 & 2.37 & 2.86 \\
\hline 6.5 & 3.00 & 2.57 & 2.42 & 2.28 & 2.21 & 2.29 & 2.41 & 2.67 & 3.12 \\
\hline 7.5 & 3.26 & 2.74 & 2.51 & 2.42 & 2.37 & 2.47 & 2.50 & 2.85 & 3.25 \\
\hline 8.5 & 3.35 & 2.91 & 2.63 & 2.54 & 2.41 & 2.55 & 2.67 & 2.95 & 3.38 \\
\hline 9.5 & 3.48 & 2.92 & 2.78 & 2.61 & 2.56 & 2.61 & 2.73 & 2.95 & 3.58 \\
\hline 10.5 & 3.50 & 3.06 & 2.77 & 2.65 & 2.60 & 2.66 & 2.81 & 3.08 & 3.59 \\
\hline 11.5 & 3.62 & 3.06 & 2.93 & 2.72 & 2.58 & 2.72 & 2.94 & 3.12 & 3.61 \\
\hline 12.5 & 3.76 & 3.07 & 2.88 & 2.76 & 2.64 & 2.74 & 2.87 & 3.06 & 3.73 \\
\hline 13.5 & 3.66 & 3.11 & 2.78 & 2.69 & 2.59 & 2.68 & 2.77 & 3.10 & 3.61 \\
\hline 14.5 & 3.63 & 3.07 & 2.79 & 2.63 & 2.55 & 2.67 & 2.78 & 2.88 & 3.54 \\
\hline 15.5 & 3.48 & 2.87 & 2.69 & 2.59 & 2.46 & 2.55 & 2.68 & 2.94 & 3.54 \\
\hline 16.5 & 3.31 & 2.78 & 2.59 & 2.45 & 2.42 & 2.48 & 2.65 & 2.83 & 3.29 \\
\hline 17.5 & 3.06 & 2.57 & 2.53 & 2.31 & 2.25 & 2.33 & 2.48 & 2.64 & 3.21 \\
\hline 18.5 & 2.90 & 2.42 & 2.34 & 2.26 & 2.11 & 2.24 & 2.31 & 2.49 & 2.99 \\
\hline 19.5 & 2.73 & 2.33 & 2.19 & 2.03 & 1.99 & 2.07 & 2.10 & 2.38 & 2.67 \\
\hline 20.5 & 2.50 & 2.16 & 1.95 & 1.86 & 1.80 & 1.83 & 1.92 & 2.10 & 2.47 \\
\hline 21.5 & 2.26 & 1.85 & 1.71 & 1.62 & 1.59 & 1.62 & 1.69 & 1.83 & 2.16 \\
\hline 22.5 & 1.95 & 1.59 & 1.50 & 1.45 & 1.37 & 1.39 & 1.53 & 1.61 & 1.98 \\
\hline 23.5 & 2.50 & 2.00 & 1.90 & 1.80 & 1.76 & 1.80 & 1.97 & 2.05 & 2.45 \\
\hline Ax. Avg. & 2.86 & 2.41 & 2.24 & 2.13 & 2.06 & 2.14 & 2.25 & 2.42 & 2.88 \\
\hline
\end{tabular}

POWER DENSITY VALUES NORMALIZED TO A CORE POWER OF 11.57 MW AND ASSUME ALL FISSION ENERGY (EXCLUDING GAMMA-HEATING OF EX-CORE COMPONENTS) IS DEPOSITED IN THE FUEL 
Table B-17. Power Density Data (kW/cc) in Fuel Plate 17 in Prototypic Conditions (continued).

Plate 17, Core Position X4/X8, 173 MWd Burnup, Equilibrium Xenon Conditions

\begin{tabular}{|c|c|c|c|c|c|c|c|c|c|}
\hline $\begin{array}{l}\text { Axial } \\
\text { position } \\
\text { from } \\
\text { top of } \\
\text { fuel } \\
\text { (inches) }\end{array}$ & $\begin{array}{c}0.000 \\
\text { to } \\
0.197 \\
\text { inches }\end{array}$ & $\begin{array}{c}0.197 \\
\text { to } \\
0.394 \\
\text { inches }\end{array}$ & $\begin{array}{c}0.394 \\
\text { to } \\
0.591 \\
\text { inches }\end{array}$ & $\begin{array}{c}0.591 \\
\text { to } \\
1.331 \\
\text { inches }\end{array}$ & $\begin{array}{c}1.331 \\
\text { to } \\
2.071 \\
\text { inches }\end{array}$ & $\begin{array}{c}2.071 \\
\text { to } \\
2.811 \\
\text { inches }\end{array}$ & $\begin{array}{c}2.811 \\
\text { to } \\
3.008 \\
\text { inches }\end{array}$ & $\begin{array}{c}3.008 \\
\text { to } \\
3.205 \\
\text { inches }\end{array}$ & $\begin{array}{c}3.205 \\
\text { to } \\
3.402 \\
\text { inches }\end{array}$ \\
\hline 0.5 & 1.58 & 1.39 & 1.35 & 1.20 & 1.20 & 1.21 & 1.30 & 1.41 & 1.72 \\
\hline 1.5 & 1.53 & 1.20 & 1.17 & 1.12 & 1.09 & 1.11 & 1.17 & 1.23 & 1.51 \\
\hline 2.5 & 1.94 & 1.59 & 1.48 & 1.44 & 1.39 & 1.41 & 1.50 & 1.52 & 1.84 \\
\hline 3.5 & 2.24 & 1.94 & 1.69 & 1.67 & 1.62 & 1.66 & 1.76 & 1.88 & 2.25 \\
\hline 4.5 & 2.52 & 2.16 & 1.99 & 1.89 & 1.83 & 1.88 & 1.93 & 2.05 & 2.50 \\
\hline 5.5 & 2.86 & 2.36 & 2.24 & 2.07 & 2.01 & 2.10 & 2.16 & 2.29 & 2.78 \\
\hline 6.5 & 2.99 & 2.49 & 2.36 & 2.22 & 2.16 & 2.23 & 2.36 & 2.49 & 2.90 \\
\hline 7.5 & 3.25 & 2.61 & 2.45 & 2.36 & 2.30 & 2.36 & 2.46 & 2.60 & 3.21 \\
\hline 8.5 & 3.31 & 2.85 & 2.70 & 2.45 & 2.34 & 2.45 & 2.61 & 2.83 & 3.24 \\
\hline 9.5 & 3.47 & 2.95 & 2.72 & 2.57 & 2.46 & 2.51 & 2.70 & 2.88 & 3.36 \\
\hline 10.5 & 3.58 & 3.00 & 2.75 & 2.62 & 2.52 & 2.66 & 2.76 & 2.99 & 3.51 \\
\hline 11.5 & 3.56 & 3.03 & 2.72 & 2.63 & 2.51 & 2.66 & 2.84 & 3.08 & 3.53 \\
\hline 12.5 & 3.46 & 2.96 & 2.81 & 2.62 & 2.56 & 2.68 & 2.83 & 3.03 & 3.52 \\
\hline 13.5 & 3.49 & 2.91 & 2.73 & 2.61 & 2.51 & 2.60 & 2.67 & 3.00 & 3.45 \\
\hline 14.5 & 3.35 & 2.89 & 2.71 & 2.57 & 2.46 & 2.60 & 2.74 & 2.87 & 3.37 \\
\hline 15.5 & 3.36 & 2.79 & 2.60 & 2.50 & 2.42 & 2.48 & 2.64 & 2.84 & 3.45 \\
\hline 16.5 & 3.20 & 2.66 & 2.46 & 2.41 & 2.31 & 2.45 & 2.55 & 2.71 & 3.17 \\
\hline 17.5 & 3.09 & 2.54 & 2.36 & 2.25 & 2.19 & 2.29 & 2.42 & 2.65 & 3.06 \\
\hline 18.5 & 2.86 & 2.42 & 2.26 & 2.13 & 2.09 & 2.15 & 2.30 & 2.47 & 2.96 \\
\hline 19.5 & 2.71 & 2.26 & 2.11 & 1.98 & 1.90 & 1.92 & 2.01 & 2.24 & 2.59 \\
\hline 20.5 & 2.47 & 2.03 & 1.89 & 1.80 & 1.73 & 1.80 & 1.87 & 1.99 & 2.44 \\
\hline 21.5 & 2.13 & 1.84 & 1.66 & 1.56 & 1.56 & 1.62 & 1.70 & 1.85 & 2.15 \\
\hline 22.5 & 1.96 & 1.58 & 1.43 & 1.34 & 1.31 & 1.39 & 1.47 & 1.51 & 1.93 \\
\hline 23.5 & 2.44 & 2.00 & 1.82 & 1.72 & 1.72 & 1.80 & 1.83 & 2.02 & 2.42 \\
\hline Ax. Avg. & 2.81 & 2.35 & 2.19 & 2.07 & 2.01 & 2.08 & 2.19 & 2.35 & 2.79 \\
\hline
\end{tabular}

POWER DENSITY VALUES NORMALIZED TO A CORE POWER OF 11.57 MW AND ASSUME ALL FISSION ENERGY (EXCLUDING GAMMA-HEATING OF EX-CORE COMPONENTS) IS DEPOSITED IN THE FUEL 
Table B-18. Power Density Data $\left(\mathrm{kW} / \mathrm{cm}^{3}\right)$ in Fuel Plate 18 in Prototypic Core.

\section{Fuel core width: 3.508 inches / Fuel core thickness: 20 mil}

Plate 18, Core Position X1/X5, 0 MWd Burnup, Xenon-Free Conditions

\begin{tabular}{|c|c|c|c|c|c|c|c|c|c|}
\hline $\begin{array}{c}\text { Axial } \\
\text { position } \\
\text { from } \\
\text { top of } \\
\text { fuel } \\
\text { (inches) }\end{array}$ & $\begin{array}{c}0.000 \\
\text { to } \\
0.197 \\
\text { inches }\end{array}$ & $\begin{array}{c}0.197 \\
\text { to } \\
0.394 \\
\text { inches }\end{array}$ & $\begin{array}{c}0.394 \\
\text { to } \\
0.591 \\
\text { inches }\end{array}$ & $\begin{array}{c}0.591 \\
\text { to } \\
\text { inches }\end{array}$ & $\begin{array}{c}1.366 \\
\text { to } \\
2.142 \\
\text { inches }\end{array}$ & $\begin{array}{c}2.142 \\
\text { to } \\
2.918 \\
\text { inches }\end{array}$ & $\begin{array}{c}2.918 \\
\text { to } \\
3.115 \\
\text { inches }\end{array}$ & $\begin{array}{c}3.115 \\
\text { to } \\
3.312 \\
\text { inches }\end{array}$ & $\begin{array}{c}3.312 \\
\text { to } \\
\text { inches }\end{array}$ \\
\hline 1.5 & 1.03 & 0.88 & 0.84 & 0.84 & 0.85 & 0.86 & 0.89 & 0.91 & 1.07 \\
\hline 2.5 & 0.85 & 0.77 & 0.73 & 0.76 & 0.74 & 0.76 & 0.79 & 0.78 & 0.87 \\
\hline 3.5 & 1.02 & 1.00 & 0.94 & 0.89 & 0.91 & 0.89 & 0.89 & 0.93 & 1.06 \\
\hline 4.5 & 1.48 & 1.25 & 1.26 & 1.25 & 1.28 & 1.29 & 1.30 & 1.26 & 1.46 \\
\hline 5.5 & 1.74 & 1.49 & 1.50 & 1.49 & 1.48 & 1.51 & 1.50 & 1.48 & 1.66 \\
\hline 6.5 & 1.98 & 1.70 & 1.69 & 1.71 & 1.69 & 1.73 & 1.72 & 1.79 & 1.88 \\
\hline 7.5 & 2.32 & 2.09 & 1.98 & 1.91 & 1.95 & 1.96 & 2.05 & 2.07 & 2.42 \\
\hline 8.5 & 2.96 & 2.42 & 2.30 & 2.28 & 2.16 & 2.31 & 2.44 & 2.52 & 2.90 \\
\hline 9.5 & 3.43 & 2.84 & 2.74 & 2.56 & 2.48 & 2.56 & 2.69 & 2.87 & 3.52 \\
\hline 10.5 & 3.85 & 3.16 & 2.93 & 2.80 & 2.68 & 2.81 & 2.95 & 3.19 & 3.76 \\
\hline 11.5 & 4.11 & 3.50 & 3.15 & 3.01 & 2.89 & 3.00 & 3.22 & 3.47 & 4.19 \\
\hline 12.5 & 4.33 & 3.62 & 3.30 & 3.12 & 2.98 & 3.16 & 3.41 & 3.56 & 4.39 \\
\hline 13.5 & 4.49 & 3.66 & 3.42 & 3.25 & 3.10 & 3.29 & 3.40 & 3.71 & 4.42 \\
\hline 14.5 & 4.48 & 3.78 & 3.50 & 3.31 & 3.15 & 3.35 & 3.46 & 3.73 & 4.50 \\
\hline 15.5 & 4.54 & 3.75 & 3.40 & 3.32 & 3.21 & 3.37 & 3.52 & 3.67 & 4.53 \\
\hline 16.5 & 4.44 & 3.63 & 3.40 & 3.24 & 3.13 & 3.25 & 3.44 & 3.74 & 4.51 \\
\hline 17.5 & 4.33 & 3.71 & 3.32 & 3.16 & 3.08 & 3.21 & 3.33 & 3.60 & 4.46 \\
\hline 18.5 & 4.01 & 3.33 & 3.14 & 2.98 & 2.92 & 3.03 & 3.19 & 3.58 & 4.20 \\
\hline 19.5 & 3.85 & 3.25 & 2.95 & 2.83 & 2.78 & 2.83 & 3.07 & 3.25 & 3.76 \\
\hline 20.5 & 3.52 & 2.91 & 2.75 & 2.60 & 2.47 & 2.60 & 2.77 & 2.93 & 3.45 \\
\hline 21.5 & 3.19 & 2.61 & 2.41 & 2.29 & 2.22 & 2.29 & 2.47 & 2.58 & 3.11 \\
\hline 22.5 & 2.82 & 2.24 & 2.11 & 2.02 & 1.94 & 2.00 & 2.11 & 2.33 & 2.90 \\
\hline 23.5 & 3.53 & 2.92 & 2.69 & 2.55 & 2.46 & 2.51 & 2.65 & 2.86 & 3.69 \\
\hline Ax. Avg. & 3.06 & 2.57 & 2.40 & 2.30 & 2.23 & 2.32 & 2.43 & 2.58 & 3.08 \\
\hline & & & & & & & & & 1.07 \\
\hline
\end{tabular}

POWER DENSITY VALUES NORMALIZED TO A CORE POWER OF 11.57 MW AND ASSUME ALL FISSION ENERGY (EXCLUDING GAMMA-HEATING OF EX-CORE COMPONENTS) IS DEPOSITED IN THE FUEL 
Table B-18. Power Density Data (kW/cc) in Fuel Plate 18 in Prototypic Conditions (continued).

Plate 18, Core Position X1/X5, 3 MWd Burnup, Equilibrium Xenon Conditions

\begin{tabular}{|c|c|c|c|c|c|c|c|c|c|}
\hline $\begin{array}{l}\text { Axial } \\
\text { position } \\
\text { from } \\
\text { top of } \\
\text { fuel } \\
\text { (inches) }\end{array}$ & $\begin{array}{c}0.000 \\
\text { to } \\
0.197 \\
\text { inches }\end{array}$ & $\begin{array}{c}0.197 \\
\text { to } \\
0.394 \\
\text { inches }\end{array}$ & $\begin{array}{c}0.394 \\
\text { to } \\
0.591 \\
\text { inches }\end{array}$ & $\begin{array}{c}0.591 \\
\text { to } \\
1.366 \\
\text { inches }\end{array}$ & $\begin{array}{c}1.366 \\
\text { to } \\
2.142 \\
\text { inches }\end{array}$ & $\begin{array}{c}2.142 \\
\text { to } \\
2.918 \\
\text { inches }\end{array}$ & $\begin{array}{c}2.918 \\
\text { to } \\
3.115 \\
\text { inches }\end{array}$ & $\begin{array}{c}3.115 \\
\text { to } \\
3.312 \\
\text { inches }\end{array}$ & $\begin{array}{c}3.312 \\
\text { to } \\
3.508 \\
\text { inches }\end{array}$ \\
\hline 0.5 & 1.70 & 1.44 & 1.37 & 1.38 & 1.32 & 1.34 & 1.30 & 1.44 & 1.61 \\
\hline 1.5 & 1.58 & 1.33 & 1.26 & 1.20 & 1.20 & 1.19 & 1.27 & 1.32 & 1.64 \\
\hline 2.5 & 2.01 & 1.74 & 1.59 & 1.49 & 1.42 & 1.50 & 1.60 & 1.74 & 2.11 \\
\hline 3.5 & 2.44 & 1.97 & 1.85 & 1.74 & 1.71 & 1.76 & 1.88 & 2.01 & 2.35 \\
\hline 4.5 & 2.66 & 2.29 & 2.10 & 1.99 & 1.92 & 2.02 & 2.13 & 2.23 & 2.72 \\
\hline 5.5 & 2.93 & 2.46 & 2.33 & 2.19 & 2.13 & 2.24 & 2.40 & 2.51 & 2.98 \\
\hline 6.5 & 3.23 & 2.75 & 2.59 & 2.38 & 2.29 & 2.37 & 2.55 & 2.77 & 3.23 \\
\hline 7.5 & 3.52 & 2.95 & 2.66 & 2.53 & 2.44 & 2.56 & 2.77 & 2.92 & 3.46 \\
\hline 8.5 & 3.72 & 3.07 & 2.84 & 2.63 & 2.58 & 2.67 & 2.71 & 3.05 & 3.67 \\
\hline 9.5 & 3.75 & 3.15 & 2.97 & 2.79 & 2.68 & 2.75 & 2.91 & 3.16 & 3.77 \\
\hline 10.5 & 3.89 & 3.20 & 2.93 & 2.80 & 2.73 & 2.83 & 2.98 & 3.21 & 3.91 \\
\hline 11.5 & 3.79 & 3.22 & 3.02 & 2.83 & 2.74 & 2.93 & 3.07 & 3.30 & 4.07 \\
\hline 12.5 & 3.92 & 3.20 & 2.98 & 2.89 & 2.80 & 2.94 & 3.00 & 3.36 & 3.88 \\
\hline 13.5 & 3.99 & 3.26 & 3.07 & 2.82 & 2.76 & 2.89 & 3.01 & 3.26 & 4.00 \\
\hline 14.5 & 3.81 & 3.09 & 3.07 & 2.84 & 2.74 & 2.80 & 2.99 & 3.17 & 3.94 \\
\hline 15.5 & 3.80 & 3.11 & 2.89 & 2.66 & 2.64 & 2.78 & 2.90 & 3.02 & 3.67 \\
\hline 16.5 & 3.57 & 3.00 & 2.79 & 2.64 & 2.54 & 2.60 & 2.79 & 2.89 & 3.54 \\
\hline 17.5 & 3.35 & 2.89 & 2.78 & 2.52 & 2.44 & 2.49 & 2.60 & 2.88 & 3.41 \\
\hline 18.5 & 3.19 & 2.59 & 2.42 & 2.35 & 2.31 & 2.32 & 2.49 & 2.73 & 3.26 \\
\hline 19.5 & 3.00 & 2.43 & 2.31 & 2.14 & 2.08 & 2.22 & 2.26 & 2.44 & 2.95 \\
\hline 20.5 & 2.64 & 2.16 & 2.06 & 1.96 & 1.90 & 1.95 & 2.05 & 2.18 & 2.75 \\
\hline 21.5 & 2.50 & 1.90 & 1.79 & 1.74 & 1.67 & 1.73 & 1.80 & 2.01 & 2.36 \\
\hline 22.5 & 2.19 & 1.79 & 1.61 & 1.47 & 1.43 & 1.49 & 1.59 & 1.65 & 2.18 \\
\hline 23.5 & 2.63 & 2.07 & 1.97 & 1.86 & 1.81 & 1.88 & 1.96 & 2.10 & 2.57 \\
\hline Ax. Avg. & 3.08 & 2.54 & 2.38 & 2.24 & 2.18 & 2.26 & 2.37 & 2.56 & 3.08 \\
\hline
\end{tabular}

POWER DENSITY VALUES NORMALIZED TO A CORE POWER OF 11.57 MW AND ASSUME ALL FISSION ENERGY (EXCLUDING GAMMA-HEATING OF EX-CORE COMPONENTS) IS DEPOSITED IN THE FUEL 
Table B-18. Power Density Data (kW/cc) in Fuel Plate 18 in Prototypic Conditions (continued).

Plate 18, Core Position X3/X7, 80 MWd Burnup, Equilibrium Xenon Conditions

\begin{tabular}{|c|c|c|c|c|c|c|c|c|c|}
\hline $\begin{array}{l}\text { Axial } \\
\text { position } \\
\text { from } \\
\text { top of } \\
\text { fuel } \\
\text { (inches) }\end{array}$ & $\begin{array}{c}0.000 \\
\text { to } \\
0.197 \\
\text { inches }\end{array}$ & $\begin{array}{c}0.197 \\
\text { to } \\
0.394 \\
\text { inches }\end{array}$ & $\begin{array}{c}0.394 \\
\text { to } \\
0.591 \\
\text { inches }\end{array}$ & $\begin{array}{c}0.591 \\
\text { to } \\
1.366 \\
\text { inches }\end{array}$ & $\begin{array}{c}1.366 \\
\text { to } \\
2.142 \\
\text { inches }\end{array}$ & $\begin{array}{c}2.142 \\
\text { to } \\
2.918 \\
\text { inches }\end{array}$ & $\begin{array}{c}2.918 \\
\text { to } \\
3.115 \\
\text { inches }\end{array}$ & $\begin{array}{c}3.115 \\
\text { to } \\
3.312 \\
\text { inches }\end{array}$ & $\begin{array}{c}3.312 \\
\text { to } \\
3.508 \\
\text { inches }\end{array}$ \\
\hline 0.5 & 1.66 & 1.37 & 1.27 & 1.29 & 1.27 & 1.29 & 1.28 & 1.37 & 1.59 \\
\hline 1.5 & 1.58 & 1.35 & 1.29 & 1.20 & 1.15 & 1.21 & 1.24 & 1.29 & 1.61 \\
\hline 2.5 & 2.08 & 1.67 & 1.57 & 1.47 & 1.44 & 1.48 & 1.54 & 1.65 & 2.00 \\
\hline 3.5 & 2.40 & 1.97 & 1.79 & 1.79 & 1.70 & 1.74 & 1.86 & 1.97 & 2.36 \\
\hline 4.5 & 2.72 & 2.30 & 2.10 & 1.99 & 1.90 & 1.95 & 2.02 & 2.26 & 2.73 \\
\hline 5.5 & 3.06 & 2.42 & 2.31 & 2.22 & 2.12 & 2.14 & 2.23 & 2.46 & 3.06 \\
\hline 6.5 & 3.27 & 2.73 & 2.54 & 2.42 & 2.31 & 2.34 & 2.45 & 2.69 & 3.27 \\
\hline 7.5 & 3.43 & 2.76 & 2.65 & 2.52 & 2.42 & 2.51 & 2.65 & 2.82 & 3.46 \\
\hline 8.5 & 3.65 & 2.97 & 2.78 & 2.64 & 2.53 & 2.64 & 2.76 & 3.02 & 3.65 \\
\hline 9.5 & 3.72 & 3.00 & 2.81 & 2.75 & 2.64 & 2.73 & 2.77 & 3.12 & 3.75 \\
\hline 10.5 & 3.81 & 3.18 & 2.96 & 2.79 & 2.68 & 2.77 & 2.94 & 3.10 & 3.88 \\
\hline 11.5 & 3.79 & 3.19 & 2.97 & 2.83 & 2.70 & 2.83 & 2.94 & 3.12 & 3.90 \\
\hline 12.5 & 3.99 & 3.17 & 2.97 & 2.81 & 2.73 & 2.79 & 2.95 & 3.15 & 3.86 \\
\hline 13.5 & 3.84 & 3.24 & 2.95 & 2.74 & 2.68 & 2.79 & 2.89 & 3.10 & 3.83 \\
\hline 14.5 & 3.75 & 3.09 & 2.85 & 2.70 & 2.65 & 2.73 & 2.85 & 3.07 & 3.65 \\
\hline 15.5 & 3.62 & 3.05 & 2.72 & 2.63 & 2.50 & 2.58 & 2.71 & 2.94 & 3.54 \\
\hline 16.5 & 3.45 & 2.86 & 2.72 & 2.52 & 2.47 & 2.51 & 2.64 & 2.77 & 3.47 \\
\hline 17.5 & 3.29 & 2.81 & 2.58 & 2.42 & 2.29 & 2.39 & 2.48 & 2.81 & 3.33 \\
\hline 18.5 & 3.13 & 2.57 & 2.37 & 2.25 & 2.18 & 2.24 & 2.33 & 2.57 & 3.20 \\
\hline 19.5 & 2.93 & 2.40 & 2.20 & 2.10 & 1.98 & 2.04 & 2.19 & 2.36 & 2.86 \\
\hline 20.5 & 2.62 & 2.11 & 1.92 & 1.91 & 1.81 & 1.86 & 1.96 & 2.08 & 2.56 \\
\hline 21.5 & 2.26 & 1.83 & 1.69 & 1.65 & 1.59 & 1.62 & 1.71 & 1.88 & 2.30 \\
\hline 22.5 & 2.12 & 1.63 & 1.54 & 1.39 & 1.38 & 1.43 & 1.47 & 1.64 & 2.10 \\
\hline 23.5 & 2.55 & 2.05 & 1.90 & 1.85 & 1.74 & 1.82 & 1.94 & 2.05 & 2.62 \\
\hline Ax. Avg. & 3.03 & 2.49 & 2.31 & 2.20 & 2.12 & 2.18 & 2.28 & 2.47 & 3.02 \\
\hline
\end{tabular}

POWER DENSITY VALUES NORMALIZED TO A CORE POWER OF 11.57 MW AND ASSUME ALL FISSION ENERGY (EXCLUDING GAMMA-HEATING OF EX-CORE COMPONENTS) IS DEPOSITED IN THE FUEL 
Table B-18. Power Density Data (kW/cc) in Fuel Plate 18 in Prototypic Conditions (continued).

Plate 18, Core Position X2/X6, 99 MWd Burnup, Equilibrium Xenon Conditions

\begin{tabular}{|c|c|c|c|c|c|c|c|c|c|}
\hline $\begin{array}{l}\text { Axial } \\
\text { position } \\
\text { from } \\
\text { top of } \\
\text { fuel } \\
\text { (inches) }\end{array}$ & $\begin{array}{c}0.000 \\
\text { to } \\
0.197 \\
\text { inches }\end{array}$ & $\begin{array}{c}0.197 \\
\text { to } \\
0.394 \\
\text { inches }\end{array}$ & $\begin{array}{c}0.394 \\
\text { to } \\
0.591 \\
\text { inches }\end{array}$ & $\begin{array}{c}0.591 \\
\text { to } \\
1.366 \\
\text { inches }\end{array}$ & $\begin{array}{c}1.366 \\
\text { to } \\
2.142 \\
\text { inches }\end{array}$ & $\begin{array}{c}2.142 \\
\text { to } \\
2.918 \\
\text { inches }\end{array}$ & $\begin{array}{c}2.918 \\
\text { to } \\
3.115 \\
\text { inches }\end{array}$ & $\begin{array}{c}3.115 \\
\text { to } \\
3.312 \\
\text { inches }\end{array}$ & $\begin{array}{c}3.312 \\
\text { to } \\
3.508 \\
\text { inches }\end{array}$ \\
\hline 0.5 & 1.64 & 1.39 & 1.33 & 1.21 & 1.14 & 1.19 & 1.21 & 1.30 & 1.56 \\
\hline 1.5 & 1.57 & 1.34 & 1.17 & 1.16 & 1.11 & 1.17 & 1.27 & 1.31 & 1.59 \\
\hline 2.5 & 1.95 & 1.65 & 1.50 & 1.48 & 1.44 & 1.46 & 1.49 & 1.70 & 2.06 \\
\hline 3.5 & 2.39 & 1.90 & 1.81 & 1.78 & 1.69 & 1.75 & 1.82 & 1.99 & 2.41 \\
\hline 4.5 & 2.70 & 2.28 & 2.12 & 1.95 & 1.94 & 2.01 & 2.09 & 2.22 & 2.74 \\
\hline 5.5 & 2.87 & 2.40 & 2.26 & 2.18 & 2.14 & 2.23 & 2.27 & 2.50 & 3.04 \\
\hline 6.5 & 3.22 & 2.64 & 2.45 & 2.35 & 2.30 & 2.39 & 2.53 & 2.69 & 3.29 \\
\hline 7.5 & 3.38 & 2.80 & 2.66 & 2.52 & 2.45 & 2.56 & 2.71 & 2.86 & 3.42 \\
\hline 8.5 & 3.56 & 2.99 & 2.71 & 2.62 & 2.55 & 2.63 & 2.78 & 3.05 & 3.65 \\
\hline 9.5 & 3.69 & 3.04 & 2.79 & 2.76 & 2.66 & 2.71 & 2.97 & 3.14 & 3.70 \\
\hline 10.5 & 3.78 & 3.18 & 2.92 & 2.76 & 2.72 & 2.75 & 2.97 & 3.08 & 3.81 \\
\hline 11.5 & 3.81 & 3.19 & 2.91 & 2.81 & 2.74 & 2.81 & 2.93 & 3.21 & 3.88 \\
\hline 12.5 & 3.78 & 3.20 & 3.02 & 2.85 & 2.74 & 2.90 & 2.93 & 3.30 & 3.90 \\
\hline 13.5 & 3.79 & 3.15 & 2.98 & 2.78 & 2.69 & 2.78 & 3.00 & 3.24 & 3.86 \\
\hline 14.5 & 3.79 & 3.06 & 2.91 & 2.74 & 2.64 & 2.73 & 2.80 & 3.14 & 3.74 \\
\hline 15.5 & 3.57 & 2.99 & 2.82 & 2.68 & 2.60 & 2.65 & 2.69 & 2.98 & 3.72 \\
\hline 16.5 & 3.59 & 2.88 & 2.82 & 2.56 & 2.51 & 2.61 & 2.70 & 2.92 & 3.49 \\
\hline 17.5 & 3.30 & 2.74 & 2.50 & 2.44 & 2.38 & 2.44 & 2.57 & 2.80 & 3.26 \\
\hline 18.5 & 3.08 & 2.60 & 2.37 & 2.33 & 2.24 & 2.30 & 2.39 & 2.58 & 3.11 \\
\hline 19.5 & 2.87 & 2.44 & 2.24 & 2.14 & 2.06 & 2.13 & 2.20 & 2.36 & 2.88 \\
\hline 20.5 & 2.62 & 2.17 & 2.01 & 1.95 & 1.86 & 1.91 & 1.97 & 2.23 & 2.68 \\
\hline 21.5 & 2.36 & 1.92 & 1.77 & 1.69 & 1.66 & 1.67 & 1.73 & 1.93 & 2.35 \\
\hline 22.5 & 2.08 & 1.72 & 1.59 & 1.46 & 1.42 & 1.47 & 1.61 & 1.73 & 2.18 \\
\hline 23.5 & 2.50 & 2.02 & 1.89 & 1.85 & 1.78 & 1.87 & 1.89 & 2.13 & 2.58 \\
\hline Ax. Avg. & 3.00 & 2.49 & 2.31 & 2.21 & 2.14 & 2.21 & 2.31 & 2.52 & 3.04 \\
\hline
\end{tabular}

POWER DENSITY VALUES NORMALIZED TO A CORE POWER OF 11.57 MW AND ASSUME ALL FISSION ENERGY (EXCLUDING GAMMA-HEATING OF EX-CORE COMPONENTS) IS DEPOSITED IN THE FUEL 
Table B-18. Power Density Data (kW/cc) in Fuel Plate 18 in Prototypic Conditions (continued).

Plate 18, Core Position X4/X8, 173 MWd Burnup, Equilibrium Xenon Conditions

\begin{tabular}{|c|c|c|c|c|c|c|c|c|c|}
\hline $\begin{array}{l}\text { Axial } \\
\text { position } \\
\text { from } \\
\text { top of } \\
\text { fuel } \\
\text { (inches) }\end{array}$ & $\begin{array}{c}0.000 \\
\text { to } \\
0.197 \\
\text { inches }\end{array}$ & $\begin{array}{c}0.197 \\
\text { to } \\
0.394 \\
\text { inches }\end{array}$ & $\begin{array}{c}0.394 \\
\text { to } \\
0.591 \\
\text { inches }\end{array}$ & $\begin{array}{c}0.591 \\
\text { to } \\
1.366 \\
\text { inches }\end{array}$ & $\begin{array}{c}1.366 \\
\text { to } \\
2.142 \\
\text { inches }\end{array}$ & $\begin{array}{c}2.142 \\
\text { to } \\
2.918 \\
\text { inches }\end{array}$ & $\begin{array}{c}2.918 \\
\text { to } \\
3.115 \\
\text { inches }\end{array}$ & $\begin{array}{c}3.115 \\
\text { to } \\
3.312 \\
\text { inches }\end{array}$ & $\begin{array}{c}3.312 \\
\text { to } \\
3.508 \\
\text { inches }\end{array}$ \\
\hline 0.5 & 1.58 & 1.32 & 1.25 & 1.19 & 1.14 & 1.21 & 1.30 & 1.34 & 1.56 \\
\hline 1.5 & 1.52 & 1.28 & 1.14 & 1.13 & 1.11 & 1.15 & 1.19 & 1.26 & 1.50 \\
\hline 2.5 & 1.94 & 1.59 & 1.55 & 1.46 & 1.39 & 1.45 & 1.53 & 1.63 & 1.90 \\
\hline 3.5 & 2.29 & 1.97 & 1.84 & 1.71 & 1.62 & 1.75 & 1.83 & 1.94 & 2.38 \\
\hline 4.5 & 2.67 & 2.23 & 2.02 & 1.95 & 1.85 & 1.95 & 2.04 & 2.25 & 2.57 \\
\hline 5.5 & 2.90 & 2.41 & 2.34 & 2.16 & 2.11 & 2.15 & 2.27 & 2.51 & 2.82 \\
\hline 6.5 & 3.15 & 2.58 & 2.42 & 2.33 & 2.21 & 2.36 & 2.41 & 2.60 & 3.08 \\
\hline 7.5 & 3.45 & 2.70 & 2.47 & 2.48 & 2.39 & 2.42 & 2.62 & 2.84 & 3.26 \\
\hline 8.5 & 3.54 & 2.92 & 2.77 & 2.57 & 2.44 & 2.53 & 2.66 & 2.90 & 3.46 \\
\hline 9.5 & 3.64 & 3.10 & 2.88 & 2.66 & 2.55 & 2.66 & 2.71 & 2.95 & 3.45 \\
\hline 10.5 & 3.65 & 3.08 & 2.85 & 2.72 & 2.64 & 2.73 & 2.91 & 3.07 & 3.64 \\
\hline 11.5 & 3.83 & 3.10 & 2.85 & 2.83 & 2.65 & 2.78 & 2.96 & 3.07 & 3.77 \\
\hline 12.5 & 3.76 & 3.04 & 2.94 & 2.77 & 2.65 & 2.76 & 2.99 & 3.17 & 3.75 \\
\hline 13.5 & 3.63 & 3.04 & 2.75 & 2.68 & 2.59 & 2.74 & 2.86 & 3.00 & 3.57 \\
\hline 14.5 & 3.49 & 3.01 & 2.79 & 2.66 & 2.58 & 2.68 & 2.81 & 3.06 & 3.67 \\
\hline 15.5 & 3.46 & 2.89 & 2.72 & 2.58 & 2.51 & 2.61 & 2.69 & 2.93 & 3.48 \\
\hline 16.5 & 3.28 & 2.78 & 2.56 & 2.48 & 2.39 & 2.54 & 2.68 & 2.82 & 3.43 \\
\hline 17.5 & 3.10 & 2.70 & 2.46 & 2.40 & 2.26 & 2.39 & 2.46 & 2.62 & 3.32 \\
\hline 18.5 & 3.08 & 2.54 & 2.38 & 2.26 & 2.17 & 2.27 & 2.37 & 2.61 & 3.11 \\
\hline 19.5 & 2.75 & 2.33 & 2.13 & 2.09 & 2.00 & 2.01 & 2.17 & 2.28 & 2.74 \\
\hline 20.5 & 2.56 & 2.11 & 1.97 & 1.89 & 1.85 & 1.85 & 2.01 & 2.13 & 2.54 \\
\hline 21.5 & 2.25 & 1.87 & 1.73 & 1.65 & 1.62 & 1.69 & 1.77 & 1.83 & 2.31 \\
\hline 22.5 & 2.12 & 1.65 & 1.56 & 1.41 & 1.37 & 1.44 & 1.52 & 1.60 & 2.04 \\
\hline 23.5 & 2.49 & 2.11 & 1.91 & 1.84 & 1.77 & 1.82 & 1.91 & 2.06 & 2.44 \\
\hline Ax. Avg. & 2.92 & 2.43 & 2.26 & 2.16 & 2.08 & 2.16 & 2.28 & 2.44 & 2.91 \\
\hline
\end{tabular}

POWER DENSITY VALUES NORMALIZED TO A CORE POWER OF 11.57 MW AND ASSUME ALL FISSION ENERGY (EXCLUDING GAMMA-HEATING OF EX-CORE COMPONENTS) IS DEPOSITED IN THE FUEL 
Table B-19. Power Density Data $\left(\mathrm{kW} / \mathrm{cm}^{3}\right)$ in Fuel Plate 19 in Prototypic Core.

\section{Fuel core width: 3.615 inches / Fuel core thickness: 20 mil}

Plate 19, Core Position X1/X5, 0 MWd Burnup, Xenon-Free Conditions

\begin{tabular}{|c|c|c|c|c|c|c|c|c|c|}
\hline $\begin{array}{c}\text { Axial } \\
\text { position } \\
\text { from } \\
\text { top of } \\
\text { fuel } \\
\text { (inches) } \\
\end{array}$ & $\begin{array}{c}0.000 \\
\text { to } \\
0.197 \\
\text { inches }\end{array}$ & $\begin{array}{c}0.197 \\
\text { to } \\
0.394 \\
\text { inches } \\
\end{array}$ & $\begin{array}{c}0.394 \\
\text { to } \\
0.591 \\
\text { inches } \\
\end{array}$ & $\begin{array}{c}0.591 \\
\text { to } \\
1.402 \\
\text { inches }\end{array}$ & $\begin{array}{c}1.402 \\
\text { to } \\
2.213 \\
\text { inches }\end{array}$ & $\begin{array}{c}2.213 \\
\text { to } \\
3.025 \\
\text { inches } \\
\end{array}$ & $\begin{array}{c}3.025 \\
\text { to } \\
3.222 \\
\text { inches }\end{array}$ & $\begin{array}{c}3.222 \\
\text { to } \\
3.418 \\
\text { inches }\end{array}$ & $\begin{array}{c}3.418 \\
\text { to } \\
3.615 \\
\text { inches } \\
\end{array}$ \\
\hline 0.5 & 0.98 & 0.81 & 0.79 & 0.86 & 0.83 & 0.88 & 0.85 & 0.87 & 0.98 \\
\hline 1.5 & 0.85 & 0.83 & 0.76 & 0.76 & 0.77 & 0.76 & 0.81 & 0.80 & 0.87 \\
\hline 2.5 & 1.03 & 0.91 & 0.91 & 0.91 & 0.94 & 0.92 & 0.86 & 0.89 & 0.99 \\
\hline 3.5 & 1.26 & 1.09 & 1.08 & 1.10 & 1.07 & 1.11 & 1.07 & 1.09 & 1.17 \\
\hline 4.5 & 1.46 & 1.37 & 1.26 & 1.27 & 1.28 & 1.31 & 1.27 & 1.33 & 1.40 \\
\hline 5.5 & 1.65 & 1.58 & 1.50 & 1.46 & 1.49 & 1.52 & 1.48 & 1.55 & 1.69 \\
\hline 6.5 & 1.95 & 1.79 & 1.75 & 1.74 & 1.71 & 1.73 & 1.75 & 1.75 & 1.88 \\
\hline 7.5 & 2.29 & 2.09 & 1.99 & 1.99 & 2.03 & 1.99 & 2.05 & 2.07 & 2.37 \\
\hline 8.5 & 3.01 & 2.61 & 2.40 & 2.39 & 2.25 & 2.42 & 2.46 & 2.66 & 3.03 \\
\hline 9.5 & 3.49 & 3.02 & 2.77 & 2.68 & 2.61 & 2.67 & 2.87 & 3.07 & 3.58 \\
\hline 10.5 & 3.97 & 3.33 & 3.05 & 2.95 & 2.78 & 2.96 & 3.20 & 3.39 & 4.13 \\
\hline 11.5 & 4.42 & 3.69 & 3.30 & 3.16 & 3.05 & 3.16 & 3.37 & 3.64 & 4.32 \\
\hline 12.5 & 4.68 & 3.87 & 3.49 & 3.30 & 3.15 & 3.42 & 3.57 & 3.84 & 4.59 \\
\hline 13.5 & 4.82 & 3.92 & 3.67 & 3.44 & 3.25 & 3.42 & 3.72 & 3.85 & 4.75 \\
\hline 14.5 & 4.87 & 4.05 & 3.69 & 3.52 & 3.37 & 3.52 & 3.57 & 3.89 & 4.79 \\
\hline 15.5 & 4.70 & 3.86 & 3.67 & 3.46 & 3.43 & 3.46 & 3.71 & 4.10 & 4.80 \\
\hline 16.5 & 4.67 & 3.87 & 3.65 & 3.47 & 3.35 & 3.43 & 3.62 & 3.88 & 4.65 \\
\hline 17.5 & 4.62 & 3.85 & 3.61 & 3.37 & 3.26 & 3.32 & 3.51 & 3.78 & 4.65 \\
\hline 18.5 & 4.46 & 3.56 & 3.29 & 3.22 & 3.06 & 3.22 & 3.30 & 3.63 & 4.41 \\
\hline 19.5 & 4.02 & 3.38 & 3.19 & 2.98 & 2.94 & 3.02 & 3.21 & 3.40 & 4.06 \\
\hline 20.5 & 3.86 & 3.08 & 2.93 & 2.75 & 2.63 & 2.77 & 2.89 & 3.01 & 3.84 \\
\hline 21.5 & 3.28 & 2.69 & 2.48 & 2.41 & 2.34 & 2.46 & 2.55 & 2.76 & 3.41 \\
\hline 22.5 & 2.97 & 2.38 & 2.23 & 2.14 & 2.08 & 2.14 & 2.20 & 2.45 & 3.02 \\
\hline 23.5 & 3.62 & 2.96 & 2.82 & 2.63 & 2.57 & 2.63 & 2.74 & 3.00 & 3.72 \\
\hline Ax. Avg. & 3.21 & 2.69 & 2.51 & 2.42 & 2.34 & 2.43 & 2.53 & 2.70 & 3.21 \\
\hline
\end{tabular}

POWER DENSITY VALUES NORMALIZED TO A CORE POWER OF 11.57 MW AND ASSUME ALL FISSION ENERGY (EXCLUDING GAMMA-HEATING OF EX-CORE COMPONENTS) IS DEPOSITED IN THE FUEL 
Table B-19. Power Density Data (kW/cc) in Fuel Plate 19 in Prototypic Conditions (continued).

Plate 19, Core Position X1/X5, 3 MWd Burnup, Equilibrium Xenon Conditions

\begin{tabular}{|c|c|c|c|c|c|c|c|c|c|}
\hline $\begin{array}{l}\text { Axial } \\
\text { position } \\
\text { from } \\
\text { top of } \\
\text { fuel } \\
\text { (inches) }\end{array}$ & $\begin{array}{c}0.000 \\
\text { to } \\
0.197 \\
\text { inches }\end{array}$ & $\begin{array}{c}0.197 \\
\text { to } \\
0.394 \\
\text { inches }\end{array}$ & $\begin{array}{c}0.394 \\
\text { to } \\
0.591 \\
\text { inches }\end{array}$ & $\begin{array}{c}0.591 \\
\text { to } \\
1.402 \\
\text { inches }\end{array}$ & $\begin{array}{c}1.402 \\
\text { to } \\
2.213 \\
\text { inches }\end{array}$ & $\begin{array}{c}2.213 \\
\text { to } \\
3.025 \\
\text { inches }\end{array}$ & $\begin{array}{c}3.025 \\
\text { to } \\
3.222 \\
\text { inches }\end{array}$ & $\begin{array}{c}3.222 \\
\text { to } \\
3.418 \\
\text { inches }\end{array}$ & $\begin{array}{c}3.418 \\
\text { to } \\
3.615 \\
\text { inches }\end{array}$ \\
\hline 0.5 & 1.63 & 1.47 & 1.49 & 1.35 & 1.31 & 1.36 & 1.35 & 1.42 & 1.61 \\
\hline 1.5 & 1.70 & 1.38 & 1.31 & 1.28 & 1.24 & 1.25 & 1.33 & 1.41 & 1.62 \\
\hline 2.5 & 2.13 & 1.74 & 1.66 & 1.60 & 1.49 & 1.57 & 1.62 & 1.78 & 2.07 \\
\hline 3.5 & 2.51 & 2.03 & 1.90 & 1.86 & 1.78 & 1.86 & 1.96 & 2.05 & 2.45 \\
\hline 4.5 & 2.83 & 2.44 & 2.23 & 2.06 & 2.00 & 2.13 & 2.26 & 2.38 & 2.91 \\
\hline 5.5 & 3.11 & 2.56 & 2.33 & 2.34 & 2.25 & 2.32 & 2.54 & 2.69 & 3.23 \\
\hline 6.5 & 3.51 & 2.96 & 2.66 & 2.51 & 2.41 & 2.55 & 2.69 & 2.89 & 3.49 \\
\hline 7.5 & 3.73 & 3.03 & 2.78 & 2.66 & 2.56 & 2.68 & 2.90 & 3.10 & 3.70 \\
\hline 8.5 & 3.98 & 3.30 & 3.07 & 2.81 & 2.70 & 2.81 & 2.95 & 3.18 & 3.94 \\
\hline 9.5 & 3.96 & 3.32 & 3.01 & 2.88 & 2.78 & 2.88 & 3.09 & 3.45 & 4.11 \\
\hline 10.5 & 4.15 & 3.41 & 3.13 & 2.96 & 2.88 & 2.98 & 3.12 & 3.38 & 4.09 \\
\hline 11.5 & 4.15 & 3.39 & 3.16 & 3.03 & 2.90 & 3.06 & 3.21 & 3.44 & 4.26 \\
\hline 12.5 & 4.17 & 3.44 & 3.24 & 3.07 & 2.94 & 3.05 & 3.17 & 3.51 & 4.21 \\
\hline 13.5 & 4.18 & 3.54 & 3.10 & 2.99 & 2.93 & 3.04 & 3.16 & 3.38 & 4.20 \\
\hline 14.5 & 4.03 & 3.38 & 3.08 & 2.99 & 2.87 & 2.98 & 3.03 & 3.29 & 4.07 \\
\hline 15.5 & 3.98 & 3.30 & 3.07 & 2.89 & 2.81 & 2.94 & 3.05 & 3.31 & 4.05 \\
\hline 16.5 & 3.91 & 3.15 & 2.88 & 2.78 & 2.69 & 2.77 & 2.97 & 3.07 & 3.88 \\
\hline 17.5 & 3.66 & 3.05 & 2.86 & 2.64 & 2.59 & 2.64 & 2.80 & 3.02 & 3.76 \\
\hline 18.5 & 3.48 & 2.79 & 2.51 & 2.50 & 2.42 & 2.55 & 2.59 & 2.79 & 3.37 \\
\hline 19.5 & 3.22 & 2.69 & 2.41 & 2.29 & 2.25 & 2.30 & 2.41 & 2.67 & 3.20 \\
\hline 20.5 & 2.86 & 2.32 & 2.13 & 2.08 & 2.02 & 2.09 & 2.18 & 2.41 & 2.89 \\
\hline 21.5 & 2.52 & 2.04 & 1.89 & 1.84 & 1.79 & 1.84 & 1.95 & 2.15 & 2.56 \\
\hline 22.5 & 2.28 & 1.83 & 1.72 & 1.57 & 1.51 & 1.57 & 1.72 & 1.83 & 2.31 \\
\hline 23.5 & 2.78 & 2.19 & 1.98 & 1.94 & 1.89 & 1.95 & 2.03 & 2.25 & 2.78 \\
\hline Ax. Avg. & 3.27 & 2.70 & 2.48 & 2.37 & 2.29 & 2.38 & 2.50 & 2.70 & 3.28 \\
\hline
\end{tabular}

POWER DENSITY VALUES NORMALIZED TO A CORE POWER OF 11.57 MW AND ASSUME ALL FISSION ENERGY (EXCLUDING GAMMA-HEATING OF EX-CORE COMPONENTS) IS DEPOSITED IN THE FUEL 
Table B-19. Power Density Data (kW/cc) in Fuel Plate 19 in Prototypic Conditions (continued).

Plate 19, Core Position X3/X7, 80 MWd Burnup, Equilibrium Xenon Conditions

\begin{tabular}{|c|c|c|c|c|c|c|c|c|c|}
\hline $\begin{array}{l}\text { Axial } \\
\text { position } \\
\text { from } \\
\text { top of } \\
\text { fuel } \\
\text { (inches) }\end{array}$ & $\begin{array}{c}0.000 \\
\text { to } \\
0.197 \\
\text { inches }\end{array}$ & $\begin{array}{c}0.197 \\
\text { to } \\
0.394 \\
\text { inches }\end{array}$ & $\begin{array}{c}0.394 \\
\text { to } \\
0.591 \\
\text { inches }\end{array}$ & $\begin{array}{c}0.591 \\
\text { to } \\
1.402 \\
\text { inches }\end{array}$ & $\begin{array}{c}1.402 \\
\text { to } \\
2.213 \\
\text { inches }\end{array}$ & $\begin{array}{c}2.213 \\
\text { to } \\
3.025 \\
\text { inches }\end{array}$ & $\begin{array}{c}3.025 \\
\text { to } \\
3.222 \\
\text { inches }\end{array}$ & $\begin{array}{c}3.222 \\
\text { to } \\
3.418 \\
\text { inches }\end{array}$ & $\begin{array}{c}3.418 \\
\text { to } \\
3.615 \\
\text { inches }\end{array}$ \\
\hline 0.5 & 1.59 & 1.34 & 1.23 & 1.28 & 1.25 & 1.27 & 1.34 & 1.35 & 1.59 \\
\hline 1.5 & 1.62 & 1.40 & 1.31 & 1.25 & 1.24 & 1.23 & 1.24 & 1.35 & 1.65 \\
\hline 2.5 & 2.09 & 1.73 & 1.67 & 1.55 & 1.52 & 1.56 & 1.60 & 1.75 & 2.10 \\
\hline 3.5 & 2.60 & 2.12 & 1.97 & 1.90 & 1.83 & 1.85 & 1.95 & 2.10 & 2.58 \\
\hline 4.5 & 2.93 & 2.38 & 2.30 & 2.09 & 2.01 & 2.10 & 2.17 & 2.37 & 2.88 \\
\hline 5.5 & 3.28 & 2.66 & 2.54 & 2.37 & 2.28 & 2.30 & 2.52 & 2.65 & 3.21 \\
\hline 6.5 & 3.52 & 2.89 & 2.65 & 2.60 & 2.45 & 2.53 & 2.62 & 2.97 & 3.57 \\
\hline 7.5 & 3.78 & 3.06 & 2.82 & 2.67 & 2.63 & 2.72 & 2.83 & 3.09 & 3.68 \\
\hline 8.5 & 3.82 & 3.27 & 3.00 & 2.81 & 2.68 & 2.82 & 2.95 & 3.27 & 3.87 \\
\hline 9.5 & 3.97 & 3.33 & 3.04 & 2.88 & 2.82 & 2.90 & 2.99 & 3.34 & 4.06 \\
\hline 10.5 & 4.11 & 3.46 & 3.13 & 2.95 & 2.84 & 2.93 & 3.03 & 3.33 & 4.12 \\
\hline 11.5 & 4.20 & 3.44 & 3.19 & 3.03 & 2.84 & 2.99 & 3.18 & 3.36 & 4.20 \\
\hline 12.5 & 4.13 & 3.41 & 3.12 & 2.94 & 2.86 & 3.01 & 3.07 & 3.47 & 4.05 \\
\hline 13.5 & 4.08 & 3.35 & 3.17 & 2.93 & 2.84 & 2.97 & 3.05 & 3.36 & 4.12 \\
\hline 14.5 & 3.95 & 3.22 & 3.02 & 2.94 & 2.79 & 2.92 & 3.05 & 3.25 & 3.89 \\
\hline 15.5 & 3.97 & 3.22 & 2.94 & 2.75 & 2.63 & 2.74 & 2.88 & 3.15 & 3.90 \\
\hline 16.5 & 3.74 & 3.04 & 2.90 & 2.70 & 2.59 & 2.63 & 2.80 & 3.02 & 3.76 \\
\hline 17.5 & 3.50 & 2.86 & 2.67 & 2.56 & 2.44 & 2.54 & 2.63 & 2.83 & 3.43 \\
\hline 18.5 & 3.29 & 2.71 & 2.47 & 2.44 & 2.30 & 2.38 & 2.51 & 2.72 & 3.33 \\
\hline 19.5 & 3.00 & 2.54 & 2.40 & 2.22 & 2.11 & 2.17 & 2.32 & 2.55 & 3.08 \\
\hline 20.5 & 2.77 & 2.31 & 2.17 & 2.01 & 1.88 & 1.96 & 2.09 & 2.23 & 2.80 \\
\hline 21.5 & 2.47 & 1.94 & 1.79 & 1.78 & 1.70 & 1.79 & 1.79 & 1.99 & 2.44 \\
\hline 22.5 & 2.27 & 1.78 & 1.61 & 1.54 & 1.47 & 1.56 & 1.60 & 1.77 & 2.16 \\
\hline 23.5 & 2.65 & 2.22 & 2.06 & 1.92 & 1.82 & 1.91 & 1.99 & 2.11 & 2.65 \\
\hline Ax. Avg. & 3.22 & 2.65 & 2.47 & 2.34 & 2.24 & 2.32 & 2.43 & 2.64 & 3.21 \\
\hline
\end{tabular}

POWER DENSITY VALUES NORMALIZED TO A CORE POWER OF 11.57 MW AND ASSUME ALL FISSION ENERGY (EXCLUDING GAMMA-HEATING OF EX-CORE COMPONENTS) IS DEPOSITED IN THE FUEL 
Table B-19. Power Density Data (kW/cc) in Fuel Plate 19 in Prototypic Conditions (continued).

Plate 19, Core Position X2/X6, 99 MWd Burnup, Equilibrium Xenon Conditions

\begin{tabular}{|c|c|c|c|c|c|c|c|c|c|}
\hline $\begin{array}{l}\text { Axial } \\
\text { position } \\
\text { from } \\
\text { top of } \\
\text { fuel } \\
\text { (inches) }\end{array}$ & $\begin{array}{c}0.000 \\
\text { to } \\
0.197 \\
\text { inches }\end{array}$ & $\begin{array}{c}0.197 \\
\text { to } \\
0.394 \\
\text { inches }\end{array}$ & $\begin{array}{c}0.394 \\
\text { to } \\
0.591 \\
\text { inches }\end{array}$ & $\begin{array}{c}0.591 \\
\text { to } \\
1.402 \\
\text { inches }\end{array}$ & $\begin{array}{c}1.402 \\
\text { to } \\
2.213 \\
\text { inches }\end{array}$ & $\begin{array}{c}2.213 \\
\text { to } \\
3.025 \\
\text { inches }\end{array}$ & $\begin{array}{c}3.025 \\
\text { to } \\
3.222 \\
\text { inches }\end{array}$ & $\begin{array}{c}3.222 \\
\text { to } \\
3.418 \\
\text { inches }\end{array}$ & $\begin{array}{c}3.418 \\
\text { to } \\
3.615 \\
\text { inches }\end{array}$ \\
\hline 0.5 & 1.63 & 1.36 & 1.23 & 1.19 & 1.11 & 1.14 & 1.16 & 1.27 & 1.52 \\
\hline 1.5 & 1.67 & 1.36 & 1.29 & 1.18 & 1.16 & 1.19 & 1.26 & 1.36 & 1.62 \\
\hline 2.5 & 2.08 & 1.76 & 1.59 & 1.54 & 1.51 & 1.55 & 1.62 & 1.77 & 2.12 \\
\hline 3.5 & 2.45 & 2.13 & 1.96 & 1.87 & 1.77 & 1.85 & 1.96 & 2.08 & 2.62 \\
\hline 4.5 & 2.89 & 2.31 & 2.17 & 2.08 & 2.04 & 2.16 & 2.27 & 2.41 & 2.96 \\
\hline 5.5 & 3.11 & 2.53 & 2.45 & 2.34 & 2.28 & 2.39 & 2.50 & 2.66 & 3.30 \\
\hline 6.5 & 3.41 & 2.83 & 2.59 & 2.51 & 2.44 & 2.54 & 2.59 & 2.94 & 3.51 \\
\hline 7.5 & 3.55 & 2.89 & 2.77 & 2.70 & 2.60 & 2.72 & 2.89 & 3.01 & 3.72 \\
\hline 8.5 & 3.74 & 3.19 & 2.93 & 2.78 & 2.73 & 2.83 & 2.95 & 3.23 & 3.81 \\
\hline 9.5 & 3.89 & 3.22 & 2.92 & 2.92 & 2.85 & 2.91 & 3.14 & 3.32 & 4.02 \\
\hline 10.5 & 4.02 & 3.31 & 3.09 & 2.96 & 2.88 & 2.97 & 3.16 & 3.30 & 4.03 \\
\hline 11.5 & 4.08 & 3.36 & 3.15 & 3.01 & 2.91 & 3.04 & 3.14 & 3.43 & 3.97 \\
\hline 12.5 & 3.99 & 3.34 & 3.14 & 3.03 & 2.94 & 3.11 & 3.16 & 3.49 & 4.17 \\
\hline 13.5 & 3.99 & 3.35 & 3.10 & 3.00 & 2.87 & 3.04 & 3.17 & 3.42 & 4.12 \\
\hline 14.5 & 4.07 & 3.26 & 3.00 & 2.98 & 2.83 & 2.97 & 3.07 & 3.25 & 3.97 \\
\hline 15.5 & 3.80 & 3.20 & 3.11 & 2.87 & 2.71 & 2.82 & 2.93 & 3.21 & 3.89 \\
\hline 16.5 & 3.67 & 3.07 & 2.86 & 2.76 & 2.69 & 2.75 & 2.87 & 3.14 & 3.83 \\
\hline 17.5 & 3.57 & 2.97 & 2.79 & 2.59 & 2.49 & 2.57 & 2.73 & 2.88 & 3.55 \\
\hline 18.5 & 3.40 & 2.79 & 2.63 & 2.48 & 2.38 & 2.46 & 2.67 & 2.85 & 3.37 \\
\hline 19.5 & 3.12 & 2.53 & 2.35 & 2.28 & 2.20 & 2.30 & 2.34 & 2.57 & 3.06 \\
\hline 20.5 & 2.76 & 2.27 & 2.10 & 2.07 & 1.97 & 2.07 & 2.14 & 2.27 & 2.87 \\
\hline 21.5 & 2.47 & 2.08 & 1.86 & 1.80 & 1.77 & 1.80 & 1.91 & 2.02 & 2.49 \\
\hline 22.5 & 2.17 & 1.81 & 1.65 & 1.57 & 1.50 & 1.58 & 1.67 & 1.81 & 2.31 \\
\hline 23.5 & 2.61 & 2.16 & 1.96 & 1.90 & 1.88 & 1.92 & 2.04 & 2.18 & 2.69 \\
\hline Ax. Avg. & 3.17 & 2.63 & 2.44 & 2.35 & 2.27 & 2.36 & 2.47 & 2.66 & 3.23 \\
\hline
\end{tabular}

POWER DENSITY VALUES NORMALIZED TO A CORE POWER OF 11.57 MW AND ASSUME ALL FISSION ENERGY (EXCLUDING GAMMA-HEATING OF EX-CORE COMPONENTS) IS DEPOSITED IN THE FUEL 
Table B-19. Power Density Data (kW/cc) in Fuel Plate 19 in Prototypic Conditions (continued).

Plate 19, Core Position X4/X8, 173 MWd Burnup, Equilibrium Xenon Conditions

\begin{tabular}{|c|c|c|c|c|c|c|c|c|c|}
\hline $\begin{array}{l}\text { Axial } \\
\text { position } \\
\text { from } \\
\text { top of } \\
\text { fuel } \\
\text { (inches) }\end{array}$ & $\begin{array}{c}0.000 \\
\text { to } \\
0.197 \\
\text { inches }\end{array}$ & $\begin{array}{c}0.197 \\
\text { to } \\
0.394 \\
\text { inches }\end{array}$ & $\begin{array}{c}0.394 \\
\text { to } \\
0.591 \\
\text { inches }\end{array}$ & $\begin{array}{c}0.591 \\
\text { to } \\
1.402 \\
\text { inches }\end{array}$ & $\begin{array}{c}1.402 \\
\text { to } \\
2.213 \\
\text { inches }\end{array}$ & $\begin{array}{c}2.213 \\
\text { to } \\
3.025 \\
\text { inches }\end{array}$ & $\begin{array}{c}3.025 \\
\text { to } \\
3.222 \\
\text { inches }\end{array}$ & $\begin{array}{c}3.222 \\
\text { to } \\
3.418 \\
\text { inches }\end{array}$ & $\begin{array}{c}3.418 \\
\text { to } \\
3.615 \\
\text { inches }\end{array}$ \\
\hline 0.5 & 1.56 & 1.27 & 1.21 & 1.16 & 1.14 & 1.17 & 1.24 & 1.35 & 1.57 \\
\hline 1.5 & 1.66 & 1.31 & 1.22 & 1.19 & 1.14 & 1.19 & 1.18 & 1.29 & 1.53 \\
\hline 2.5 & 2.03 & 1.73 & 1.63 & 1.56 & 1.49 & 1.52 & 1.59 & 1.72 & 2.12 \\
\hline 3.5 & 2.43 & 2.12 & 1.99 & 1.82 & 1.78 & 1.82 & 1.99 & 2.00 & 2.44 \\
\hline 4.5 & 2.87 & 2.33 & 2.18 & 2.09 & 2.00 & 2.10 & 2.19 & 2.44 & 2.79 \\
\hline 5.5 & 3.21 & 2.73 & 2.37 & 2.32 & 2.25 & 2.33 & 2.42 & 2.44 & 3.07 \\
\hline 6.5 & 3.35 & 2.76 & 2.61 & 2.47 & 2.36 & 2.50 & 2.57 & 2.71 & 3.23 \\
\hline 7.5 & 3.62 & 3.00 & 2.72 & 2.60 & 2.54 & 2.65 & 2.78 & 2.99 & 3.54 \\
\hline 8.5 & 3.84 & 3.17 & 2.85 & 2.72 & 2.65 & 2.72 & 2.92 & 3.05 & 3.67 \\
\hline 9.5 & 4.02 & 3.22 & 2.91 & 2.87 & 2.74 & 2.86 & 2.97 & 3.10 & 3.76 \\
\hline 10.5 & 3.90 & 3.31 & 3.02 & 2.88 & 2.83 & 2.90 & 3.01 & 3.26 & 3.87 \\
\hline 11.5 & 3.90 & 3.27 & 3.12 & 2.99 & 2.82 & 2.95 & 3.11 & 3.34 & 3.99 \\
\hline 12.5 & 3.97 & 3.27 & 3.09 & 2.93 & 2.83 & 2.94 & 3.14 & 3.38 & 3.89 \\
\hline 13.5 & 3.91 & 3.26 & 2.96 & 2.91 & 2.75 & 2.89 & 3.03 & 3.23 & 3.77 \\
\hline 14.5 & 3.88 & 3.15 & 3.03 & 2.87 & 2.76 & 2.88 & 2.97 & 3.20 & 3.89 \\
\hline 15.5 & 3.64 & 3.01 & 2.87 & 2.77 & 2.71 & 2.76 & 2.93 & 3.14 & 3.74 \\
\hline 16.5 & 3.60 & 3.07 & 2.71 & 2.72 & 2.61 & 2.73 & 2.84 & 2.94 & 3.58 \\
\hline 17.5 & 3.46 & 2.91 & 2.64 & 2.53 & 2.46 & 2.58 & 2.68 & 2.86 & 3.41 \\
\hline 18.5 & 3.28 & 2.71 & 2.45 & 2.38 & 2.32 & 2.39 & 2.52 & 2.68 & 3.28 \\
\hline 19.5 & 2.99 & 2.40 & 2.28 & 2.23 & 2.14 & 2.18 & 2.33 & 2.45 & 3.06 \\
\hline 20.5 & 2.71 & 2.18 & 2.09 & 1.98 & 1.95 & 2.05 & 2.12 & 2.29 & 2.77 \\
\hline 21.5 & 2.40 & 1.93 & 1.82 & 1.76 & 1.72 & 1.82 & 1.85 & 1.97 & 2.46 \\
\hline 22.5 & 2.21 & 1.76 & 1.62 & 1.53 & 1.49 & 1.56 & 1.66 & 1.82 & 2.20 \\
\hline 23.5 & 2.64 & 2.05 & 2.00 & 1.92 & 1.82 & 1.92 & 2.03 & 2.07 & 2.65 \\
\hline Ax. Avg. & 3.13 & 2.58 & 2.39 & 2.30 & 2.22 & 2.31 & 2.42 & 2.57 & 3.09 \\
\hline
\end{tabular}

POWER DENSITY VALUES NORMALIZED TO A CORE POWER OF 11.57 MW AND ASSUME ALL FISSION ENERGY (EXCLUDING GAMMA-HEATING OF EX-CORE COMPONENTS) IS DEPOSITED IN THE FUEL 
Table B-20. Power Density Data $\left(\mathrm{kW} / \mathrm{cm}^{3}\right)$ in Fuel Plate 20 in Prototypic Core.

\section{Fuel core width: 3.723 inches / Fuel core thickness: 20 mil}

Plate 20, Core Position X1/X5, 0 MWd Burnup, Xenon-Free Conditions

\begin{tabular}{|c|c|c|c|c|c|c|c|c|c|}
\hline $\begin{array}{c}\text { Axial } \\
\text { position } \\
\text { from } \\
\text { top of } \\
\text { fuel } \\
\text { (inches) } \\
\end{array}$ & $\begin{array}{c}0.000 \\
\text { to } \\
0.197 \\
\text { inches }\end{array}$ & $\begin{array}{c}0.197 \\
\text { to } \\
0.394 \\
\text { inches } \\
\end{array}$ & $\begin{array}{c}0.394 \\
\text { to } \\
0.591 \\
\text { inches } \\
\end{array}$ & $\begin{array}{c}0.591 \\
\text { to } \\
1.438 \\
\text { inches } \\
\end{array}$ & $\begin{array}{c}1.438 \\
\text { to } \\
2.285 \\
\text { inches }\end{array}$ & $\begin{array}{c}2.285 \\
\text { to } \\
3.132 \\
\text { inches } \\
\end{array}$ & $\begin{array}{c}3.132 \\
\text { to } \\
3.329 \\
\text { inches }\end{array}$ & $\begin{array}{c}3.329 \\
\text { to } \\
3.526 \\
\text { inches }\end{array}$ & $\begin{array}{c}3.526 \\
\text { to } \\
3.723 \\
\text { inches } \\
\end{array}$ \\
\hline 0.5 & 0.93 & 0.82 & 0.78 & 0.88 & 0.86 & 0.88 & 0.86 & 0.88 & 0.96 \\
\hline 1.5 & 0.89 & 0.75 & 0.81 & 0.79 & 0.79 & 0.78 & 0.75 & 0.72 & 0.91 \\
\hline 2.5 & 1.05 & 1.00 & 0.95 & 0.94 & 0.95 & 0.97 & 0.88 & 0.89 & 0.98 \\
\hline 3.5 & 1.18 & 1.12 & 1.15 & 1.13 & 1.16 & 1.13 & 1.09 & 1.09 & 1.19 \\
\hline 4.5 & 1.43 & 1.30 & 1.26 & 1.32 & 1.36 & 1.31 & 1.25 & 1.32 & 1.50 \\
\hline 5.5 & 1.63 & 1.58 & 1.50 & 1.52 & 1.54 & 1.54 & 1.44 & 1.53 & 1.72 \\
\hline 6.5 & 1.99 & 1.74 & 1.79 & 1.77 & 1.77 & 1.80 & 1.72 & 1.74 & 1.90 \\
\hline 7.5 & 2.36 & 2.12 & 2.09 & 2.06 & 2.10 & 2.09 & 2.08 & 2.15 & 2.46 \\
\hline 8.5 & 3.15 & 2.76 & 2.52 & 2.51 & 2.43 & 2.53 & 2.66 & 2.73 & 3.16 \\
\hline 9.5 & 3.77 & 3.18 & 3.07 & 2.90 & 2.78 & 2.86 & 3.09 & 3.24 & 3.92 \\
\hline 10.5 & 4.45 & 3.68 & 3.36 & 3.16 & 3.04 & 3.23 & 3.39 & 3.59 & 4.41 \\
\hline 11.5 & 4.83 & 3.95 & 3.57 & 3.39 & 3.29 & 3.45 & 3.73 & 3.94 & 4.87 \\
\hline 12.5 & 4.91 & 4.17 & 3.82 & 3.60 & 3.41 & 3.62 & 3.77 & 4.27 & 4.95 \\
\hline 13.5 & 5.08 & 4.28 & 3.88 & 3.70 & 3.56 & 3.72 & 3.86 & 4.10 & 5.12 \\
\hline 14.5 & 5.05 & 4.35 & 3.98 & 3.72 & 3.57 & 3.79 & 3.98 & 4.33 & 5.21 \\
\hline 15.5 & 5.15 & 4.31 & 3.99 & 3.81 & 3.64 & 3.76 & 4.02 & 4.26 & 5.25 \\
\hline 16.5 & 5.16 & 4.26 & 3.99 & 3.82 & 3.65 & 3.78 & 3.92 & 4.28 & 5.08 \\
\hline 17.5 & 5.06 & 4.31 & 3.96 & 3.66 & 3.59 & 3.64 & 3.80 & 4.20 & 4.98 \\
\hline 18.5 & 4.81 & 4.03 & 3.60 & 3.48 & 3.36 & 3.49 & 3.75 & 3.98 & 4.81 \\
\hline 19.5 & 4.41 & 3.68 & 3.53 & 3.26 & 3.21 & 3.30 & 3.42 & 3.62 & 4.41 \\
\hline 20.5 & 4.11 & 3.39 & 3.06 & 3.03 & 2.90 & 3.04 & 3.17 & 3.41 & 4.20 \\
\hline 21.5 & 3.64 & 3.05 & 2.79 & 2.68 & 2.60 & 2.70 & 2.86 & 3.01 & 3.71 \\
\hline 22.5 & 3.27 & 2.68 & 2.49 & 2.33 & 2.27 & 2.35 & 2.44 & 2.68 & 3.37 \\
\hline 23.5 & 3.90 & 3.20 & 2.89 & 2.86 & 2.73 & 2.79 & 2.97 & 3.21 & 3.94 \\
\hline Ax. Avg. & 3.42 & 2.90 & 2.70 & 2.60 & 2.52 & 2.61 & 2.70 & 2.88 & 3.46 \\
\hline
\end{tabular}

POWER DENSITY VALUES NORMALIZED TO A CORE POWER OF 11.57 MW AND ASSUME ALL FISSION ENERGY (EXCLUDING GAMMA-HEATING OF EX-CORE COMPONENTS) IS DEPOSITED IN THE FUEL 
Table B-20. Power Density Data (kW/cc) in Fuel Plate 20 in Prototypic Conditions (continued).

Plate 20, Core Position X1/X5, 3 MWd Burnup, Equilibrium Xenon Conditions

\begin{tabular}{|c|c|c|c|c|c|c|c|c|c|}
\hline $\begin{array}{l}\text { Axial } \\
\text { position } \\
\text { from } \\
\text { top of } \\
\text { fuel } \\
\text { (inches) }\end{array}$ & $\begin{array}{c}0.000 \\
\text { to } \\
0.197 \\
\text { inches }\end{array}$ & $\begin{array}{c}0.197 \\
\text { to } \\
0.394 \\
\text { inches }\end{array}$ & $\begin{array}{c}0.394 \\
\text { to } \\
0.591 \\
\text { inches }\end{array}$ & $\begin{array}{c}0.591 \\
\text { to } \\
1.438 \\
\text { inches }\end{array}$ & $\begin{array}{c}1.438 \\
\text { to } \\
2.285 \\
\text { inches }\end{array}$ & $\begin{array}{c}2.285 \\
\text { to } \\
3.132 \\
\text { inches }\end{array}$ & $\begin{array}{c}3.132 \\
\text { to } \\
3.329 \\
\text { inches }\end{array}$ & $\begin{array}{c}3.329 \\
\text { to } \\
3.526 \\
\text { inches }\end{array}$ & $\begin{array}{c}3.526 \\
\text { to } \\
3.723 \\
\text { inches }\end{array}$ \\
\hline 0.5 & 1.61 & 1.48 & 1.37 & 1.40 & 1.40 & 1.40 & 1.36 & 1.40 & 1.55 \\
\hline 1.5 & 1.74 & 1.51 & 1.38 & 1.33 & 1.32 & 1.33 & 1.34 & 1.43 & 1.69 \\
\hline 2.5 & 2.23 & 1.86 & 1.75 & 1.68 & 1.62 & 1.72 & 1.80 & 1.97 & 2.32 \\
\hline 3.5 & 2.78 & 2.29 & 2.13 & 2.00 & 1.93 & 2.03 & 2.17 & 2.27 & 2.74 \\
\hline 4.5 & 3.15 & 2.54 & 2.43 & 2.25 & 2.18 & 2.25 & 2.43 & 2.58 & 3.21 \\
\hline 5.5 & 3.41 & 2.88 & 2.68 & 2.51 & 2.44 & 2.54 & 2.71 & 2.89 & 3.48 \\
\hline 6.5 & 3.78 & 3.16 & 2.91 & 2.69 & 2.57 & 2.74 & 2.91 & 3.09 & 3.78 \\
\hline 7.5 & 4.06 & 3.39 & 3.00 & 2.93 & 2.77 & 2.91 & 3.09 & 3.41 & 4.08 \\
\hline 8.5 & 4.19 & 3.56 & 3.29 & 3.04 & 2.98 & 3.05 & 3.19 & 3.48 & 4.29 \\
\hline 9.5 & 4.38 & 3.57 & 3.45 & 3.20 & 3.03 & 3.14 & 3.35 & 3.64 & 4.44 \\
\hline 10.5 & 4.62 & 3.74 & 3.43 & 3.27 & 3.13 & 3.29 & 3.36 & 3.77 & 4.49 \\
\hline 11.5 & 4.65 & 3.61 & 3.49 & 3.34 & 3.19 & 3.36 & 3.51 & 3.76 & 4.67 \\
\hline 12.5 & 4.74 & 3.78 & 3.53 & 3.30 & 3.25 & 3.32 & 3.46 & 3.85 & 4.51 \\
\hline 13.5 & 4.67 & 3.79 & 3.45 & 3.30 & 3.24 & 3.35 & 3.53 & 3.80 & 4.55 \\
\hline 14.5 & 4.46 & 3.62 & 3.43 & 3.27 & 3.13 & 3.26 & 3.45 & 3.69 & 4.43 \\
\hline 15.5 & 4.38 & 3.63 & 3.34 & 3.18 & 3.18 & 3.22 & 3.28 & 3.59 & 4.28 \\
\hline 16.5 & 4.29 & 3.48 & 3.12 & 3.07 & 3.03 & 3.04 & 3.25 & 3.49 & 4.21 \\
\hline 17.5 & 4.00 & 3.30 & 3.08 & 2.91 & 2.81 & 2.92 & 3.06 & 3.35 & 4.09 \\
\hline 18.5 & 3.88 & 3.15 & 2.87 & 2.73 & 2.64 & 2.74 & 2.88 & 3.18 & 3.74 \\
\hline 19.5 & 3.49 & 2.85 & 2.61 & 2.54 & 2.45 & 2.52 & 2.71 & 2.85 & 3.55 \\
\hline 20.5 & 3.12 & 2.55 & 2.29 & 2.23 & 2.24 & 2.30 & 2.45 & 2.58 & 3.05 \\
\hline 21.5 & 2.94 & 2.29 & 2.16 & 2.01 & 1.91 & 2.06 & 2.10 & 2.23 & 2.75 \\
\hline 22.5 & 2.57 & 2.01 & 1.86 & 1.78 & 1.66 & 1.73 & 1.84 & 2.00 & 2.50 \\
\hline 23.5 & 2.94 & 2.38 & 2.28 & 2.08 & 2.00 & 2.10 & 2.23 & 2.36 & 2.91 \\
\hline Ax. Avg. & 3.59 & 2.93 & 2.72 & 2.58 & 2.50 & 2.60 & 2.73 & 2.94 & 3.56 \\
\hline
\end{tabular}

POWER DENSITY VALUES NORMALIZED TO A CORE POWER OF 11.57 MW AND ASSUME ALL FISSION ENERGY (EXCLUDING GAMMA-HEATING OF EX-CORE COMPONENTS) IS DEPOSITED IN THE FUEL 
Table B-20. Power Density Data (kW/cc) in Fuel Plate 20 in Prototypic Conditions (continued).

Plate 20, Core Position X3/X7, 80 MWd Burnup, Equilibrium Xenon Conditions

\begin{tabular}{|c|c|c|c|c|c|c|c|c|c|}
\hline $\begin{array}{l}\text { Axial } \\
\text { position } \\
\text { from } \\
\text { top of } \\
\text { fuel } \\
\text { (inches) }\end{array}$ & $\begin{array}{c}0.000 \\
\text { to } \\
0.197 \\
\text { inches }\end{array}$ & $\begin{array}{c}0.197 \\
\text { to } \\
0.394 \\
\text { inches }\end{array}$ & $\begin{array}{c}0.394 \\
\text { to } \\
0.591 \\
\text { inches }\end{array}$ & $\begin{array}{c}0.591 \\
\text { to } \\
1.438 \\
\text { inches }\end{array}$ & $\begin{array}{c}1.438 \\
\text { to } \\
2.285 \\
\text { inches }\end{array}$ & $\begin{array}{c}2.285 \\
\text { to } \\
3.132 \\
\text { inches }\end{array}$ & $\begin{array}{c}3.132 \\
\text { to } \\
3.329 \\
\text { inches }\end{array}$ & $\begin{array}{c}3.329 \\
\text { to } \\
3.526 \\
\text { inches }\end{array}$ & $\begin{array}{c}3.526 \\
\text { to } \\
3.723 \\
\text { inches }\end{array}$ \\
\hline 0.5 & 1.54 & 1.34 & 1.31 & 1.29 & 1.26 & 1.30 & 1.30 & 1.31 & 1.51 \\
\hline 1.5 & 1.73 & 1.48 & 1.37 & 1.33 & 1.32 & 1.30 & 1.31 & 1.43 & 1.67 \\
\hline 2.5 & 2.31 & 1.90 & 1.77 & 1.69 & 1.68 & 1.74 & 1.76 & 1.85 & 2.38 \\
\hline 3.5 & 2.87 & 2.31 & 2.09 & 2.04 & 2.00 & 2.04 & 2.14 & 2.38 & 2.91 \\
\hline 4.5 & 3.26 & 2.75 & 2.52 & 2.38 & 2.26 & 2.33 & 2.46 & 2.68 & 3.17 \\
\hline 5.5 & 3.61 & 2.93 & 2.81 & 2.60 & 2.51 & 2.58 & 2.67 & 2.88 & 3.57 \\
\hline 6.5 & 3.87 & 3.20 & 2.96 & 2.86 & 2.69 & 2.84 & 2.97 & 3.17 & 3.89 \\
\hline 7.5 & 4.01 & 3.22 & 3.08 & 3.04 & 2.86 & 2.95 & 3.04 & 3.38 & 4.11 \\
\hline 8.5 & 4.22 & 3.56 & 3.28 & 3.09 & 2.96 & 3.14 & 3.34 & 3.56 & 4.16 \\
\hline 9.5 & 4.32 & 3.61 & 3.41 & 3.23 & 3.11 & 3.23 & 3.33 & 3.67 & 4.34 \\
\hline 10.5 & 4.47 & 3.66 & 3.46 & 3.25 & 3.18 & 3.30 & 3.41 & 3.69 & 4.48 \\
\hline 11.5 & 4.35 & 3.75 & 3.48 & 3.31 & 3.18 & 3.32 & 3.38 & 3.70 & 4.48 \\
\hline 12.5 & 4.40 & 3.72 & 3.53 & 3.28 & 3.18 & 3.29 & 3.38 & 3.70 & 4.52 \\
\hline 13.5 & 4.46 & 3.72 & 3.45 & 3.25 & 3.16 & 3.26 & 3.45 & 3.54 & 4.66 \\
\hline 14.5 & 4.37 & 3.67 & 3.39 & 3.22 & 3.10 & 3.21 & 3.32 & 3.56 & 4.34 \\
\hline 15.5 & 4.24 & 3.45 & 3.15 & 3.12 & 2.90 & 3.07 & 3.15 & 3.46 & 4.31 \\
\hline 16.5 & 4.08 & 3.37 & 3.19 & 2.98 & 2.80 & 2.93 & 3.15 & 3.31 & 4.03 \\
\hline 17.5 & 3.87 & 3.22 & 3.01 & 2.83 & 2.66 & 2.81 & 2.98 & 3.13 & 3.79 \\
\hline 18.5 & 3.69 & 3.04 & 2.70 & 2.65 & 2.56 & 2.61 & 2.74 & 3.03 & 3.60 \\
\hline 19.5 & 3.35 & 2.70 & 2.58 & 2.44 & 2.30 & 2.40 & 2.55 & 2.70 & 3.40 \\
\hline 20.5 & 3.01 & 2.47 & 2.36 & 2.21 & 2.11 & 2.18 & 2.29 & 2.49 & 3.05 \\
\hline 21.5 & 2.68 & 2.22 & 2.01 & 1.98 & 1.88 & 1.91 & 2.02 & 2.27 & 2.73 \\
\hline 22.5 & 2.57 & 1.99 & 1.76 & 1.70 & 1.67 & 1.72 & 1.75 & 1.97 & 2.36 \\
\hline 23.5 & 2.82 & 2.31 & 2.16 & 2.07 & 2.00 & 2.07 & 2.12 & 2.29 & 2.90 \\
\hline Ax. Avg. & 3.50 & 2.90 & 2.70 & 2.57 & 2.47 & 2.56 & 2.67 & 2.88 & 3.52 \\
\hline
\end{tabular}

POWER DENSITY VALUES NORMALIZED TO A CORE POWER OF 11.57 MW AND ASSUME ALL FISSION ENERGY (EXCLUDING GAMMA-HEATING OF EX-CORE COMPONENTS) IS DEPOSITED IN THE FUEL 
Table B-20. Power Density Data (kW/cc) in Fuel Plate 20 in Prototypic Conditions (continued).

Plate 20, Core Position X2/X6, 99 MWd Burnup, Equilibrium Xenon Conditions

\begin{tabular}{|c|c|c|c|c|c|c|c|c|c|}
\hline $\begin{array}{l}\text { Axial } \\
\text { position } \\
\text { from } \\
\text { top of } \\
\text { fuel } \\
\text { (inches) }\end{array}$ & $\begin{array}{c}0.000 \\
\text { to } \\
0.197 \\
\text { inches }\end{array}$ & $\begin{array}{c}0.197 \\
\text { to } \\
0.394 \\
\text { inches }\end{array}$ & $\begin{array}{c}0.394 \\
\text { to } \\
0.591 \\
\text { inches }\end{array}$ & $\begin{array}{c}0.591 \\
\text { to } \\
1.438 \\
\text { inches }\end{array}$ & $\begin{array}{c}1.438 \\
\text { to } \\
2.285 \\
\text { inches }\end{array}$ & $\begin{array}{c}2.285 \\
\text { to } \\
3.132 \\
\text { inches }\end{array}$ & $\begin{array}{c}3.132 \\
\text { to } \\
3.329 \\
\text { inches }\end{array}$ & $\begin{array}{c}3.329 \\
\text { to } \\
3.526 \\
\text { inches }\end{array}$ & $\begin{array}{c}3.526 \\
\text { to } \\
3.723 \\
\text { inches }\end{array}$ \\
\hline 0.5 & 1.60 & 1.31 & 1.25 & 1.15 & 1.11 & 1.18 & 1.15 & 1.27 & 1.48 \\
\hline 1.5 & 1.65 & 1.39 & 1.34 & 1.26 & 1.22 & 1.25 & 1.27 & 1.44 & 1.70 \\
\hline 2.5 & 2.28 & 1.85 & 1.79 & 1.71 & 1.65 & 1.72 & 1.79 & 1.92 & 2.30 \\
\hline 3.5 & 2.66 & 2.24 & 2.10 & 2.06 & 2.01 & 2.09 & 2.20 & 2.34 & 2.71 \\
\hline 4.5 & 3.08 & 2.61 & 2.40 & 2.35 & 2.30 & 2.39 & 2.52 & 2.68 & 3.19 \\
\hline 5.5 & 3.44 & 2.81 & 2.73 & 2.58 & 2.55 & 2.63 & 2.74 & 3.08 & 3.54 \\
\hline 6.5 & 3.58 & 3.09 & 2.96 & 2.79 & 2.76 & 2.81 & 2.97 & 3.14 & 3.77 \\
\hline 7.5 & 3.95 & 3.21 & 3.04 & 2.98 & 2.90 & 2.99 & 3.20 & 3.40 & 3.94 \\
\hline 8.5 & 4.08 & 3.44 & 3.27 & 3.12 & 3.06 & 3.12 & 3.21 & 3.51 & 4.08 \\
\hline 9.5 & 4.29 & 3.54 & 3.28 & 3.24 & 3.20 & 3.23 & 3.36 & 3.63 & 4.34 \\
\hline 10.5 & 4.38 & 3.65 & 3.39 & 3.22 & 3.25 & 3.32 & 3.45 & 3.67 & 4.51 \\
\hline 11.5 & 4.53 & 3.72 & 3.53 & 3.34 & 3.19 & 3.32 & 3.52 & 3.72 & 4.47 \\
\hline 12.5 & 4.44 & 3.67 & 3.53 & 3.38 & 3.27 & 3.41 & 3.52 & 3.83 & 4.51 \\
\hline 13.5 & 4.35 & 3.64 & 3.51 & 3.34 & 3.22 & 3.36 & 3.52 & 3.67 & 4.57 \\
\hline 14.5 & 4.25 & 3.55 & 3.36 & 3.27 & 3.13 & 3.23 & 3.41 & 3.66 & 4.42 \\
\hline 15.5 & 4.24 & 3.62 & 3.32 & 3.19 & 3.06 & 3.12 & 3.19 & 3.55 & 4.32 \\
\hline 16.5 & 4.09 & 3.44 & 3.34 & 3.07 & 2.96 & 3.03 & 3.23 & 3.43 & 4.15 \\
\hline 17.5 & 3.84 & 3.31 & 3.06 & 2.86 & 2.84 & 2.90 & 2.99 & 3.12 & 3.84 \\
\hline 18.5 & 3.64 & 3.05 & 2.83 & 2.73 & 2.68 & 2.72 & 2.80 & 3.05 & 3.60 \\
\hline 19.5 & 3.46 & 2.85 & 2.57 & 2.51 & 2.46 & 2.54 & 2.66 & 2.79 & 3.27 \\
\hline 20.5 & 3.05 & 2.51 & 2.36 & 2.27 & 2.22 & 2.35 & 2.36 & 2.51 & 2.95 \\
\hline 21.5 & 2.68 & 2.24 & 2.20 & 2.05 & 1.93 & 2.05 & 2.15 & 2.23 & 2.71 \\
\hline 22.5 & 2.38 & 1.98 & 1.87 & 1.75 & 1.71 & 1.78 & 1.87 & 2.01 & 2.49 \\
\hline 23.5 & 2.82 & 2.32 & 2.21 & 2.11 & 2.02 & 2.08 & 2.22 & 2.38 & 2.81 \\
\hline Ax. Avg. & 3.45 & 2.88 & 2.72 & 2.60 & 2.53 & 2.61 & 2.72 & 2.92 & 3.49 \\
\hline
\end{tabular}

POWER DENSITY VALUES NORMALIZED TO A CORE POWER OF 11.57 MW AND ASSUME ALL FISSION ENERGY (EXCLUDING GAMMA-HEATING OF EX-CORE COMPONENTS) IS DEPOSITED IN THE FUEL 
Table B-20. Power Density Data (kW/cc) in Fuel Plate 20 in Prototypic Conditions (continued).

Plate 20, Core Position X4/X8, 173 MWd Burnup, Equilibrium Xenon Conditions

\begin{tabular}{|c|c|c|c|c|c|c|c|c|c|}
\hline $\begin{array}{l}\text { Axial } \\
\text { position } \\
\text { from } \\
\text { top of } \\
\text { fuel } \\
\text { (inches) }\end{array}$ & $\begin{array}{c}0.000 \\
\text { to } \\
0.197 \\
\text { inches }\end{array}$ & $\begin{array}{c}0.197 \\
\text { to } \\
0.394 \\
\text { inches }\end{array}$ & $\begin{array}{c}0.394 \\
\text { to } \\
0.591 \\
\text { inches }\end{array}$ & $\begin{array}{c}0.591 \\
\text { to } \\
1.438 \\
\text { inches }\end{array}$ & $\begin{array}{c}1.438 \\
\text { to } \\
2.285 \\
\text { inches }\end{array}$ & $\begin{array}{c}2.285 \\
\text { to } \\
3.132 \\
\text { inches }\end{array}$ & $\begin{array}{c}3.132 \\
\text { to } \\
3.329 \\
\text { inches }\end{array}$ & $\begin{array}{c}3.329 \\
\text { to } \\
3.526 \\
\text { inches }\end{array}$ & $\begin{array}{c}3.526 \\
\text { to } \\
3.723 \\
\text { inches }\end{array}$ \\
\hline 0.5 & 1.49 & 1.31 & 1.13 & 1.13 & 1.11 & 1.13 & 1.22 & 1.33 & 1.55 \\
\hline 1.5 & 1.71 & 1.41 & 1.31 & 1.24 & 1.22 & 1.22 & 1.26 & 1.34 & 1.67 \\
\hline 2.5 & 2.30 & 1.89 & 1.72 & 1.67 & 1.62 & 1.69 & 1.86 & 1.94 & 2.21 \\
\hline 3.5 & 2.77 & 2.36 & 2.12 & 2.05 & 1.99 & 2.04 & 2.24 & 2.26 & 2.68 \\
\hline 4.5 & 3.05 & 2.59 & 2.36 & 2.33 & 2.30 & 2.33 & 2.41 & 2.66 & 3.02 \\
\hline 5.5 & 3.42 & 2.92 & 2.71 & 2.54 & 2.48 & 2.54 & 2.65 & 2.73 & 3.28 \\
\hline 6.5 & 3.69 & 3.02 & 2.90 & 2.74 & 2.63 & 2.76 & 2.86 & 2.95 & 3.51 \\
\hline 7.5 & 4.07 & 3.30 & 3.03 & 2.93 & 2.86 & 2.89 & 3.05 & 3.27 & 3.80 \\
\hline 8.5 & 4.07 & 3.44 & 3.22 & 3.04 & 3.00 & 3.07 & 3.18 & 3.31 & 4.05 \\
\hline 9.5 & 4.22 & 3.55 & 3.34 & 3.18 & 3.09 & 3.16 & 3.26 & 3.60 & 4.19 \\
\hline 10.5 & 4.38 & 3.55 & 3.41 & 3.24 & 3.17 & 3.26 & 3.36 & 3.60 & 4.22 \\
\hline 11.5 & 4.29 & 3.63 & 3.45 & 3.33 & 3.19 & 3.30 & 3.41 & 3.61 & 4.27 \\
\hline 12.5 & 4.34 & 3.60 & 3.49 & 3.27 & 3.18 & 3.30 & 3.42 & 3.68 & 4.31 \\
\hline 13.5 & 4.20 & 3.52 & 3.41 & 3.23 & 3.10 & 3.24 & 3.43 & 3.51 & 4.11 \\
\hline 14.5 & 4.23 & 3.50 & 3.30 & 3.19 & 3.02 & 3.20 & 3.40 & 3.58 & 4.25 \\
\hline 15.5 & 4.03 & 3.38 & 3.20 & 3.11 & 2.96 & 3.06 & 3.15 & 3.34 & 4.10 \\
\hline 16.5 & 3.95 & 3.36 & 3.09 & 2.97 & 2.89 & 3.06 & 3.06 & 3.31 & 3.91 \\
\hline 17.5 & 3.75 & 3.16 & 2.87 & 2.83 & 2.71 & 2.84 & 2.96 & 3.14 & 3.70 \\
\hline 18.5 & 3.55 & 3.01 & 2.72 & 2.67 & 2.60 & 2.65 & 2.80 & 2.98 & 3.57 \\
\hline 19.5 & 3.20 & 2.69 & 2.58 & 2.49 & 2.39 & 2.45 & 2.53 & 2.73 & 3.22 \\
\hline 20.5 & 2.91 & 2.57 & 2.34 & 2.23 & 2.20 & 2.26 & 2.33 & 2.52 & 2.99 \\
\hline 21.5 & 2.59 & 2.15 & 2.00 & 1.99 & 1.94 & 2.01 & 2.06 & 2.19 & 2.63 \\
\hline 22.5 & 2.39 & 1.93 & 1.83 & 1.70 & 1.68 & 1.77 & 1.82 & 1.97 & 2.35 \\
\hline 23.5 & 2.81 & 2.28 & 2.11 & 2.05 & 2.05 & 2.07 & 2.11 & 2.23 & 2.79 \\
\hline Ax. Avg. & 3.39 & 2.84 & 2.65 & 2.55 & 2.47 & 2.56 & 2.66 & 2.82 & 3.35 \\
\hline
\end{tabular}

POWER DENSITY VALUES NORMALIZED TO A CORE POWER OF 11.57 MW AND ASSUME ALL FISSION ENERGY (EXCLUDING GAMMA-HEATING OF EX-CORE COMPONENTS) IS DEPOSITED IN THE FUEL 
Table B-21. Power Density Data $\left(\mathrm{kW} / \mathrm{cm}^{3}\right)$ in Fuel Plate 21 in Prototypic Core.

\section{Fuel core width: 3.830 inches / Fuel core thickness: 20 mil}

Plate 21, Core Position X1/X5, 0 MWd Burnup, Xenon-Free Conditions

\begin{tabular}{|c|c|c|c|c|c|c|c|c|c|}
\hline $\begin{array}{c}\text { Axial } \\
\text { position } \\
\text { from } \\
\text { top of } \\
\text { fuel } \\
\text { (inches) } \\
\end{array}$ & $\begin{array}{c}0.000 \\
\text { to } \\
0.197 \\
\text { inches }\end{array}$ & $\begin{array}{c}0.197 \\
\text { to } \\
0.394 \\
\text { inches } \\
\end{array}$ & $\begin{array}{c}0.394 \\
\text { to } \\
0.591 \\
\text { inches } \\
\end{array}$ & $\begin{array}{c}0.591 \\
\text { to } \\
1.474 \\
\text { inches }\end{array}$ & $\begin{array}{c}1.474 \\
\text { to } \\
2.357 \\
\text { inches }\end{array}$ & $\begin{array}{c}2.357 \\
\text { to } \\
3.240 \\
\text { inches }\end{array}$ & $\begin{array}{c}3.240 \\
\text { to } \\
3.437 \\
\text { inches }\end{array}$ & $\begin{array}{c}3.437 \\
\text { to } \\
3.634 \\
\text { inches } \\
\end{array}$ & $\begin{array}{c}3.634 \\
\text { to } \\
3.830 \\
\text { inches } \\
\end{array}$ \\
\hline 0.5 & 0.90 & 0.83 & 0.79 & 0.88 & 0.92 & 0.90 & 0.85 & 0.88 & 0.88 \\
\hline 1.5 & 0.86 & 0.77 & 0.77 & 0.84 & 0.84 & 0.83 & 0.74 & 0.79 & 0.87 \\
\hline 2.5 & 1.02 & 0.93 & 0.96 & 1.01 & 0.99 & 1.00 & 0.90 & 0.96 & 1.00 \\
\hline 3.5 & 1.18 & 1.16 & 1.12 & 1.19 & 1.24 & 1.18 & 1.12 & 1.16 & 1.22 \\
\hline 4.5 & 1.46 & 1.34 & 1.28 & 1.40 & 1.44 & 1.39 & 1.33 & 1.35 & 1.52 \\
\hline 5.5 & 1.66 & 1.58 & 1.55 & 1.58 & 1.67 & 1.59 & 1.55 & 1.53 & 1.68 \\
\hline 6.5 & 1.95 & 1.78 & 1.78 & 1.87 & 1.95 & 1.90 & 1.79 & 1.85 & 1.96 \\
\hline 7.5 & 2.43 & 2.28 & 2.20 & 2.27 & 2.29 & 2.23 & 2.18 & 2.25 & 2.49 \\
\hline 8.5 & 3.50 & 2.99 & 2.82 & 2.74 & 2.71 & 2.79 & 2.84 & 2.97 & 3.36 \\
\hline 9.5 & 4.35 & 3.66 & 3.37 & 3.29 & 3.14 & 3.32 & 3.45 & 3.62 & 4.40 \\
\hline 10.5 & 4.83 & 4.07 & 3.80 & 3.59 & 3.48 & 3.68 & 3.81 & 4.10 & 4.84 \\
\hline 11.5 & 5.23 & 4.47 & 4.08 & 3.88 & 3.74 & 3.98 & 4.30 & 4.53 & 5.30 \\
\hline 12.5 & 5.55 & 4.66 & 4.28 & 4.08 & 3.93 & 4.12 & 4.42 & 4.67 & 5.55 \\
\hline 13.5 & 5.84 & 4.87 & 4.47 & 4.23 & 4.06 & 4.23 & 4.51 & 4.73 & 5.69 \\
\hline 14.5 & 5.76 & 4.95 & 4.40 & 4.33 & 4.13 & 4.29 & 4.62 & 4.89 & 6.01 \\
\hline 15.5 & 5.87 & 4.84 & 4.66 & 4.43 & 4.24 & 4.42 & 4.75 & 4.93 & 5.88 \\
\hline 16.5 & 5.77 & 4.94 & 4.59 & 4.47 & 4.20 & 4.37 & 4.59 & 4.80 & 5.89 \\
\hline 17.5 & 5.51 & 4.79 & 4.43 & 4.25 & 4.14 & 4.25 & 4.45 & 4.88 & 5.69 \\
\hline 18.5 & 5.42 & 4.48 & 4.17 & 4.02 & 3.88 & 4.01 & 4.21 & 4.50 & 5.32 \\
\hline 19.5 & 4.91 & 4.19 & 4.09 & 3.79 & 3.67 & 3.88 & 4.06 & 4.10 & 5.01 \\
\hline 20.5 & 4.63 & 3.76 & 3.70 & 3.48 & 3.39 & 3.50 & 3.64 & 3.94 & 4.53 \\
\hline 21.5 & 4.20 & 3.58 & 3.16 & 3.19 & 3.12 & 3.15 & 3.41 & 3.51 & 4.19 \\
\hline 22.5 & 3.78 & 3.17 & 2.97 & 2.81 & 2.74 & 2.70 & 2.97 & 3.01 & 3.80 \\
\hline 23.5 & 4.32 & 3.59 & 3.28 & 3.19 & 3.09 & 3.22 & 3.45 & 3.57 & 4.38 \\
\hline Ax. Avg. & 3.79 & 3.24 & 3.03 & 2.95 & 2.88 & 2.95 & 3.08 & 3.23 & 3.81 \\
\hline
\end{tabular}

POWER DENSITY VALUES NORMALIZED TO A CORE POWER OF 11.57 MW AND ASSUME ALL FISSION ENERGY (EXCLUDING GAMMA-HEATING OF EX-CORE COMPONENTS) IS DEPOSITED IN THE FUEL 
Table B-21. Power Density Data (kW/cc) in Fuel Plate 21 in Prototypic Conditions (continued).

Plate 21, Core Position X1/X5, 3 MWd Burnup, Equilibrium Xenon Conditions

\begin{tabular}{|c|c|c|c|c|c|c|c|c|c|}
\hline $\begin{array}{l}\text { Axial } \\
\text { position } \\
\text { from } \\
\text { top of } \\
\text { fuel } \\
\text { (inches) }\end{array}$ & $\begin{array}{c}0.000 \\
\text { to } \\
0.197 \\
\text { inches }\end{array}$ & $\begin{array}{c}0.197 \\
\text { to } \\
0.394 \\
\text { inches }\end{array}$ & $\begin{array}{c}0.394 \\
\text { to } \\
0.591 \\
\text { inches }\end{array}$ & $\begin{array}{c}0.591 \\
\text { to } \\
1.474 \\
\text { inches }\end{array}$ & $\begin{array}{c}1.474 \\
\text { to } \\
2.357 \\
\text { inches }\end{array}$ & $\begin{array}{c}2.357 \\
\text { to } \\
3.240 \\
\text { inches }\end{array}$ & $\begin{array}{c}3.240 \\
\text { to } \\
3.437 \\
\text { inches }\end{array}$ & $\begin{array}{c}3.437 \\
\text { to } \\
3.634 \\
\text { inches }\end{array}$ & $\begin{array}{c}3.634 \\
\text { to } \\
3.830 \\
\text { inches }\end{array}$ \\
\hline 0.5 & 1.70 & 1.46 & 1.34 & 1.46 & 1.53 & 1.49 & 1.41 & 1.41 & 1.67 \\
\hline 1.5 & 1.83 & 1.62 & 1.54 & 1.46 & 1.50 & 1.52 & 1.54 & 1.58 & 1.85 \\
\hline 2.5 & 2.59 & 2.10 & 2.03 & 1.89 & 1.85 & 1.97 & 2.03 & 2.24 & 2.61 \\
\hline 3.5 & 3.05 & 2.59 & 2.47 & 2.26 & 2.22 & 2.34 & 2.37 & 2.55 & 3.18 \\
\hline 4.5 & 3.44 & 2.91 & 2.82 & 2.68 & 2.49 & 2.66 & 2.70 & 2.97 & 3.55 \\
\hline 5.5 & 3.96 & 3.18 & 3.08 & 2.87 & 2.76 & 2.92 & 3.09 & 3.25 & 3.90 \\
\hline 6.5 & 4.23 & 3.58 & 3.31 & 3.13 & 2.99 & 3.12 & 3.26 & 3.52 & 4.30 \\
\hline 7.5 & 4.53 & 3.87 & 3.51 & 3.33 & 3.17 & 3.36 & 3.52 & 3.79 & 4.57 \\
\hline 8.5 & 4.78 & 3.91 & 3.68 & 3.49 & 3.32 & 3.50 & 3.70 & 3.95 & 4.76 \\
\hline 9.5 & 5.01 & 4.17 & 3.90 & 3.69 & 3.51 & 3.64 & 3.89 & 4.22 & 4.96 \\
\hline 10.5 & 5.13 & 4.23 & 4.16 & 3.78 & 3.65 & 3.80 & 3.93 & 4.21 & 5.11 \\
\hline 11.5 & 5.15 & 4.35 & 4.06 & 3.89 & 3.66 & 3.84 & 4.05 & 4.25 & 5.24 \\
\hline 12.5 & 5.27 & 4.24 & 4.05 & 3.90 & 3.76 & 3.89 & 3.97 & 4.36 & 5.11 \\
\hline 13.5 & 5.18 & 4.40 & 4.09 & 3.87 & 3.76 & 3.90 & 4.00 & 4.31 & 5.27 \\
\hline 14.5 & 4.98 & 4.17 & 3.97 & 3.86 & 3.66 & 3.76 & 4.00 & 4.21 & 5.17 \\
\hline 15.5 & 4.97 & 4.17 & 3.93 & 3.70 & 3.62 & 3.72 & 3.86 & 4.09 & 4.93 \\
\hline 16.5 & 4.64 & 3.92 & 3.69 & 3.60 & 3.46 & 3.51 & 3.69 & 3.96 & 4.63 \\
\hline 17.5 & 4.41 & 3.68 & 3.59 & 3.40 & 3.34 & 3.30 & 3.56 & 3.78 & 4.52 \\
\hline 18.5 & 4.29 & 3.53 & 3.27 & 3.20 & 3.07 & 3.21 & 3.36 & 3.57 & 4.26 \\
\hline 19.5 & 3.96 & 3.27 & 3.08 & 2.92 & 2.91 & 2.96 & 3.08 & 3.30 & 3.97 \\
\hline 20.5 & 3.49 & 2.91 & 2.63 & 2.66 & 2.55 & 2.70 & 2.79 & 3.02 & 3.57 \\
\hline 21.5 & 3.26 & 2.63 & 2.42 & 2.39 & 2.27 & 2.39 & 2.50 & 2.62 & 3.24 \\
\hline 22.5 & 2.87 & 2.38 & 2.18 & 2.07 & 2.02 & 2.06 & 2.13 & 2.39 & 2.86 \\
\hline 23.5 & 3.17 & 2.69 & 2.42 & 2.34 & 2.25 & 2.37 & 2.43 & 2.69 & 3.22 \\
\hline Ax. Avg. & 4.00 & 3.33 & 3.13 & 2.99 & 2.89 & 3.00 & 3.12 & 3.34 & 4.02 \\
\hline
\end{tabular}

POWER DENSITY VALUES NORMALIZED TO A CORE POWER OF 11.57 MW AND ASSUME ALL FISSION ENERGY (EXCLUDING GAMMA-HEATING OF EX-CORE COMPONENTS) IS DEPOSITED IN THE FUEL 
Table B-21. Power Density Data (kW/cc) in Fuel Plate 21 in Prototypic Conditions (continued).

Plate 21, Core Position X3/X7, 80 MWd Burnup, Equilibrium Xenon Conditions

\begin{tabular}{|c|c|c|c|c|c|c|c|c|c|}
\hline $\begin{array}{l}\text { Axial } \\
\text { position } \\
\text { from } \\
\text { top of } \\
\text { fuel } \\
\text { (inches) }\end{array}$ & $\begin{array}{c}0.000 \\
\text { to } \\
0.197 \\
\text { inches }\end{array}$ & $\begin{array}{c}0.197 \\
\text { to } \\
0.394 \\
\text { inches }\end{array}$ & $\begin{array}{c}0.394 \\
\text { to } \\
0.591 \\
\text { inches }\end{array}$ & $\begin{array}{c}0.591 \\
\text { to } \\
1.474 \\
\text { inches }\end{array}$ & $\begin{array}{c}1.474 \\
\text { to } \\
2.357 \\
\text { inches }\end{array}$ & $\begin{array}{c}2.357 \\
\text { to } \\
3.240 \\
\text { inches }\end{array}$ & $\begin{array}{c}3.240 \\
\text { to } \\
3.437 \\
\text { inches }\end{array}$ & $\begin{array}{c}3.437 \\
\text { to } \\
3.634 \\
\text { inches }\end{array}$ & $\begin{array}{c}3.634 \\
\text { to } \\
3.830 \\
\text { inches }\end{array}$ \\
\hline 0.5 & 1.58 & 1.34 & 1.34 & 1.31 & 1.32 & 1.33 & 1.30 & 1.30 & 1.50 \\
\hline 1.5 & 1.83 & 1.63 & 1.49 & 1.45 & 1.49 & 1.46 & 1.49 & 1.52 & 1.85 \\
\hline 2.5 & 2.57 & 2.20 & 2.06 & 1.98 & 1.98 & 2.00 & 2.03 & 2.17 & 2.56 \\
\hline 3.5 & 3.21 & 2.69 & 2.58 & 2.43 & 2.37 & 2.44 & 2.54 & 2.76 & 3.32 \\
\hline 4.5 & 3.67 & 3.13 & 3.00 & 2.78 & 2.67 & 2.77 & 2.82 & 3.08 & 3.59 \\
\hline 5.5 & 4.05 & 3.46 & 3.22 & 3.08 & 2.92 & 3.00 & 3.07 & 3.35 & 4.00 \\
\hline 6.5 & 4.46 & 3.67 & 3.45 & 3.35 & 3.16 & 3.26 & 3.47 & 3.64 & 4.30 \\
\hline 7.5 & 4.66 & 3.82 & 3.71 & 3.48 & 3.34 & 3.48 & 3.55 & 3.82 & 4.77 \\
\hline 8.5 & 4.82 & 3.97 & 3.94 & 3.68 & 3.50 & 3.69 & 3.85 & 4.11 & 4.86 \\
\hline 9.5 & 4.91 & 4.21 & 3.94 & 3.81 & 3.67 & 3.79 & 3.88 & 4.20 & 4.99 \\
\hline 10.5 & 5.01 & 4.28 & 3.91 & 3.78 & 3.67 & 3.83 & 3.80 & 4.17 & 5.05 \\
\hline 11.5 & 5.04 & 4.33 & 4.08 & 3.89 & 3.73 & 3.89 & 4.08 & 4.30 & 5.17 \\
\hline 12.5 & 5.14 & 4.30 & 3.97 & 3.84 & 3.73 & 3.82 & 4.02 & 4.40 & 5.09 \\
\hline 13.5 & 5.09 & 4.27 & 4.01 & 3.82 & 3.63 & 3.77 & 3.94 & 4.15 & 4.95 \\
\hline 14.5 & 5.15 & 4.24 & 3.88 & 3.75 & 3.62 & 3.69 & 3.75 & 4.12 & 4.96 \\
\hline 15.5 & 4.95 & 4.09 & 3.78 & 3.58 & 3.46 & 3.61 & 3.66 & 3.91 & 4.72 \\
\hline 16.5 & 4.58 & 3.90 & 3.61 & 3.44 & 3.28 & 3.41 & 3.57 & 3.85 & 4.51 \\
\hline 17.5 & 4.35 & 3.65 & 3.45 & 3.23 & 3.10 & 3.24 & 3.41 & 3.57 & 4.27 \\
\hline 18.5 & 4.19 & 3.39 & 3.18 & 3.09 & 2.94 & 3.06 & 3.21 & 3.40 & 4.15 \\
\hline 19.5 & 3.86 & 3.22 & 2.91 & 2.88 & 2.70 & 2.81 & 3.02 & 3.22 & 3.83 \\
\hline 20.5 & 3.46 & 2.90 & 2.74 & 2.59 & 2.49 & 2.59 & 2.71 & 2.89 & 3.48 \\
\hline 21.5 & 3.11 & 2.55 & 2.40 & 2.34 & 2.23 & 2.25 & 2.44 & 2.51 & 3.00 \\
\hline 22.5 & 2.72 & 2.22 & 2.11 & 2.05 & 1.95 & 1.98 & 2.04 & 2.18 & 2.78 \\
\hline 23.5 & 3.14 & 2.61 & 2.45 & 2.34 & 2.26 & 2.34 & 2.44 & 2.56 & 3.29 \\
\hline Ax. Avg. & 3.98 & 3.34 & 3.13 & 3.00 & 2.88 & 2.98 & 3.09 & 3.30 & 3.96 \\
\hline
\end{tabular}

POWER DENSITY VALUES NORMALIZED TO A CORE POWER OF 11.57 MW AND ASSUME ALL FISSION ENERGY (EXCLUDING GAMMA-HEATING OF EX-CORE COMPONENTS) IS DEPOSITED IN THE FUEL 
Table B-21. Power Density Data (kW/cc) in Fuel Plate 21 in Prototypic Conditions (continued).

Plate 21, Core Position X2/X6, 99 MWd Burnup, Equilibrium Xenon Conditions

\begin{tabular}{|c|c|c|c|c|c|c|c|c|c|}
\hline $\begin{array}{l}\text { Axial } \\
\text { position } \\
\text { from } \\
\text { top of } \\
\text { fuel } \\
\text { (inches) }\end{array}$ & $\begin{array}{c}0.000 \\
\text { to } \\
0.197 \\
\text { inches }\end{array}$ & $\begin{array}{c}0.197 \\
\text { to } \\
0.394 \\
\text { inches }\end{array}$ & $\begin{array}{c}0.394 \\
\text { to } \\
0.591 \\
\text { inches }\end{array}$ & $\begin{array}{c}0.591 \\
\text { to } \\
1.474 \\
\text { inches }\end{array}$ & $\begin{array}{c}1.474 \\
\text { to } \\
2.357 \\
\text { inches }\end{array}$ & $\begin{array}{c}2.357 \\
\text { to } \\
3.240 \\
\text { inches }\end{array}$ & $\begin{array}{c}3.240 \\
\text { to } \\
3.437 \\
\text { inches }\end{array}$ & $\begin{array}{c}3.437 \\
\text { to } \\
3.634 \\
\text { inches }\end{array}$ & $\begin{array}{c}3.634 \\
\text { to } \\
3.830 \\
\text { inches }\end{array}$ \\
\hline 0.5 & 1.53 & 1.39 & 1.16 & 1.16 & 1.11 & 1.15 & 1.17 & 1.22 & 1.46 \\
\hline 1.5 & 1.85 & 1.51 & 1.51 & 1.39 & 1.34 & 1.40 & 1.41 & 1.50 & 1.70 \\
\hline 2.5 & 2.57 & 2.14 & 2.04 & 1.99 & 1.92 & 1.96 & 2.10 & 2.27 & 2.64 \\
\hline 3.5 & 3.07 & 2.56 & 2.47 & 2.39 & 2.34 & 2.43 & 2.57 & 2.68 & 3.10 \\
\hline 4.5 & 3.45 & 2.99 & 2.78 & 2.78 & 2.74 & 2.78 & 2.88 & 3.05 & 3.64 \\
\hline 5.5 & 3.83 & 3.25 & 3.08 & 3.06 & 3.03 & 3.09 & 3.24 & 3.40 & 4.05 \\
\hline 6.5 & 4.23 & 3.56 & 3.40 & 3.29 & 3.25 & 3.32 & 3.49 & 3.64 & 4.33 \\
\hline 7.5 & 4.43 & 3.78 & 3.71 & 3.48 & 3.45 & 3.60 & 3.68 & 3.80 & 4.56 \\
\hline 8.5 & 4.51 & 3.95 & 3.75 & 3.67 & 3.60 & 3.70 & 3.84 & 4.05 & 4.83 \\
\hline 9.5 & 4.87 & 4.09 & 3.85 & 3.83 & 3.74 & 3.83 & 3.93 & 4.12 & 4.93 \\
\hline 10.5 & 4.84 & 4.21 & 3.89 & 3.82 & 3.79 & 3.93 & 3.92 & 4.25 & 4.97 \\
\hline 11.5 & 4.91 & 4.27 & 4.19 & 3.95 & 3.80 & 3.96 & 4.04 & 4.23 & 5.08 \\
\hline 12.5 & 4.94 & 4.25 & 4.07 & 3.93 & 3.82 & 3.97 & 4.07 & 4.31 & 5.21 \\
\hline 13.5 & 4.94 & 4.25 & 4.00 & 3.87 & 3.77 & 3.97 & 4.03 & 4.26 & 5.11 \\
\hline 14.5 & 4.80 & 4.17 & 3.96 & 3.82 & 3.75 & 3.82 & 3.97 & 4.18 & 4.89 \\
\hline 15.5 & 4.73 & 3.99 & 3.76 & 3.77 & 3.59 & 3.68 & 3.87 & 4.06 & 4.78 \\
\hline 16.5 & 4.59 & 3.94 & 3.73 & 3.58 & 3.45 & 3.59 & 3.76 & 3.93 & 4.72 \\
\hline 17.5 & 4.34 & 3.74 & 3.45 & 3.47 & 3.35 & 3.37 & 3.45 & 3.75 & 4.31 \\
\hline 18.5 & 4.02 & 3.59 & 3.30 & 3.19 & 3.13 & 3.19 & 3.31 & 3.45 & 4.09 \\
\hline 19.5 & 3.74 & 3.26 & 3.09 & 2.98 & 2.87 & 2.94 & 3.00 & 3.21 & 3.79 \\
\hline 20.5 & 3.45 & 2.77 & 2.70 & 2.70 & 2.58 & 2.67 & 2.74 & 2.94 & 3.43 \\
\hline 21.5 & 2.97 & 2.54 & 2.47 & 2.42 & 2.32 & 2.40 & 2.45 & 2.62 & 3.11 \\
\hline 22.5 & 2.78 & 2.35 & 2.19 & 2.10 & 2.02 & 2.11 & 2.11 & 2.34 & 2.82 \\
\hline 23.5 & 3.03 & 2.60 & 2.50 & 2.41 & 2.29 & 2.36 & 2.39 & 2.52 & 3.10 \\
\hline Ax. Avg. & 3.85 & 3.30 & 3.13 & 3.05 & 2.96 & 3.05 & 3.14 & 3.32 & 3.94 \\
\hline
\end{tabular}

POWER DENSITY VALUES NORMALIZED TO A CORE POWER OF 11.57 MW AND ASSUME ALL FISSION ENERGY (EXCLUDING GAMMA-HEATING OF EX-CORE COMPONENTS) IS DEPOSITED IN THE FUEL 
Table B-21. Power Density Data (kW/cc) in Fuel Plate 21 in Prototypic Conditions (continued).

Plate 21, Core Position X4/X8, 173 MWd Burnup, Equilibrium Xenon Conditions

\begin{tabular}{|c|c|c|c|c|c|c|c|c|c|}
\hline $\begin{array}{l}\text { Axial } \\
\text { position } \\
\text { from } \\
\text { top of } \\
\text { fuel } \\
\text { (inches) }\end{array}$ & $\begin{array}{c}0.000 \\
\text { to } \\
0.197 \\
\text { inches }\end{array}$ & $\begin{array}{c}0.197 \\
\text { to } \\
0.394 \\
\text { inches }\end{array}$ & $\begin{array}{c}0.394 \\
\text { to } \\
0.591 \\
\text { inches }\end{array}$ & $\begin{array}{c}0.591 \\
\text { to } \\
1.474 \\
\text { inches }\end{array}$ & $\begin{array}{c}1.474 \\
\text { to } \\
2.357 \\
\text { inches }\end{array}$ & $\begin{array}{c}2.357 \\
\text { to } \\
3.240 \\
\text { inches }\end{array}$ & $\begin{array}{c}3.240 \\
\text { to } \\
3.437 \\
\text { inches }\end{array}$ & $\begin{array}{c}3.437 \\
\text { to } \\
3.634 \\
\text { inches }\end{array}$ & $\begin{array}{c}3.634 \\
\text { to } \\
3.830 \\
\text { inches }\end{array}$ \\
\hline 0.5 & 1.52 & 1.25 & 1.20 & 1.17 & 1.09 & 1.14 & 1.18 & 1.31 & 1.52 \\
\hline 1.5 & 1.72 & 1.55 & 1.40 & 1.35 & 1.34 & 1.35 & 1.44 & 1.54 & 1.80 \\
\hline 2.5 & 2.53 & 2.18 & 2.01 & 1.92 & 1.87 & 1.95 & 2.02 & 2.15 & 2.48 \\
\hline 3.5 & 3.14 & 2.67 & 2.53 & 2.41 & 2.32 & 2.39 & 2.46 & 2.60 & 3.07 \\
\hline 4.5 & 3.43 & 2.94 & 2.78 & 2.71 & 2.68 & 2.72 & 2.73 & 2.96 & 3.37 \\
\hline 5.5 & 3.97 & 3.36 & 3.19 & 3.01 & 2.91 & 3.00 & 2.98 & 3.27 & 3.83 \\
\hline 6.5 & 4.27 & 3.51 & 3.37 & 3.19 & 3.05 & 3.20 & 3.20 & 3.43 & 3.99 \\
\hline 7.5 & 4.54 & 3.72 & 3.53 & 3.44 & 3.36 & 3.44 & 3.39 & 3.65 & 4.28 \\
\hline 8.5 & 4.55 & 3.90 & 3.71 & 3.56 & 3.54 & 3.58 & 3.67 & 3.85 & 4.46 \\
\hline 9.5 & 4.76 & 4.05 & 3.94 & 3.73 & 3.59 & 3.70 & 3.72 & 3.95 & 4.52 \\
\hline 10.5 & 4.73 & 4.21 & 3.87 & 3.79 & 3.73 & 3.80 & 3.86 & 3.99 & 4.56 \\
\hline 11.5 & 4.77 & 4.22 & 3.93 & 3.86 & 3.71 & 3.84 & 3.95 & 4.00 & 4.73 \\
\hline 12.5 & 4.84 & 4.02 & 3.92 & 3.81 & 3.72 & 3.83 & 3.87 & 4.23 & 4.81 \\
\hline 13.5 & 4.81 & 4.06 & 3.85 & 3.74 & 3.65 & 3.75 & 3.76 & 4.02 & 4.77 \\
\hline 14.5 & 4.74 & 4.04 & 3.77 & 3.72 & 3.57 & 3.75 & 3.82 & 4.07 & 4.73 \\
\hline 15.5 & 4.50 & 4.07 & 3.72 & 3.58 & 3.48 & 3.62 & 3.72 & 3.83 & 4.53 \\
\hline 16.5 & 4.38 & 3.77 & 3.58 & 3.48 & 3.38 & 3.54 & 3.53 & 3.85 & 4.36 \\
\hline 17.5 & 4.22 & 3.57 & 3.34 & 3.30 & 3.19 & 3.32 & 3.43 & 3.59 & 4.21 \\
\hline 18.5 & 4.05 & 3.30 & 3.15 & 3.15 & 3.04 & 3.10 & 3.22 & 3.46 & 3.92 \\
\hline 19.5 & 3.68 & 3.16 & 2.97 & 2.90 & 2.82 & 2.89 & 2.97 & 3.15 & 3.65 \\
\hline 20.5 & 3.24 & 2.93 & 2.68 & 2.61 & 2.56 & 2.66 & 2.72 & 2.85 & 3.32 \\
\hline 21.5 & 2.95 & 2.54 & 2.40 & 2.34 & 2.28 & 2.33 & 2.38 & 2.59 & 2.95 \\
\hline 22.5 & 2.71 & 2.26 & 2.12 & 2.05 & 1.97 & 2.05 & 2.06 & 2.18 & 2.75 \\
\hline 23.5 & 3.00 & 2.57 & 2.50 & 2.32 & 2.25 & 2.34 & 2.33 & 2.47 & 3.07 \\
\hline Ax. Avg. & 3.79 & 3.24 & 3.06 & 2.97 & 2.88 & 2.97 & 3.02 & 3.21 & 3.74 \\
\hline
\end{tabular}

POWER DENSITY VALUES NORMALIZED TO A CORE POWER OF 11.57 MW AND ASSUME ALL FISSION ENERGY (EXCLUDING GAMMA-HEATING OF EX-CORE COMPONENTS) IS DEPOSITED IN THE FUEL 
Table B-22. Power Density Data $\left(\mathrm{kW} / \mathrm{cm}^{3}\right)$ in Fuel Plate 22 in Prototypic Core.

\section{Fuel core width: 3.938 inches / Fuel core thickness: 20 mil}

Plate 22, Core Position X1, 0 MWd Burnup, Xenon-Free Conditions

\begin{tabular}{|c|c|c|c|c|c|c|c|c|c|}
\hline $\begin{array}{c}\text { Axial } \\
\text { position } \\
\text { from } \\
\text { top of } \\
\text { fuel } \\
\text { (inches) } \\
\end{array}$ & $\begin{array}{c}0.000 \\
\text { to } \\
0.197 \\
\text { inches }\end{array}$ & $\begin{array}{c}0.197 \\
\text { to } \\
0.394 \\
\text { inches } \\
\end{array}$ & $\begin{array}{c}0.394 \\
\text { to } \\
0.591 \\
\text { inches }\end{array}$ & $\begin{array}{c}0.591 \\
\text { to } \\
1.510 \\
\text { inches }\end{array}$ & $\begin{array}{c}1.510 \\
\text { to } \\
2.429 \\
\text { inches }\end{array}$ & $\begin{array}{c}2.429 \\
\text { to } \\
3.348 \\
\text { inches } \\
\end{array}$ & $\begin{array}{c}3.348 \\
\text { to } \\
3.544 \\
\text { inches } \\
\end{array}$ & $\begin{array}{c}3.544 \\
\text { to } \\
3.741 \\
\text { inches }\end{array}$ & $\begin{array}{c}3.741 \\
\text { to } \\
3.938 \\
\text { inches } \\
\end{array}$ \\
\hline 0.5 & 0.78 & 0.75 & 0.66 & 0.75 & 0.90 & 0.78 & 0.66 & 0.73 & 0.81 \\
\hline 1.5 & 0.77 & 0.65 & 0.68 & 0.75 & 0.89 & 0.74 & 0.72 & 0.70 & 0.75 \\
\hline 2.5 & 0.89 & 0.91 & 0.86 & 0.94 & 1.03 & 0.89 & 0.80 & 0.78 & 0.89 \\
\hline 3.5 & 1.09 & 1.02 & 1.01 & 1.06 & 1.20 & 1.05 & 0.95 & 0.96 & 1.04 \\
\hline 4.5 & 1.29 & 1.11 & 1.16 & 1.24 & 1.41 & 1.23 & 1.18 & 1.08 & 1.24 \\
\hline 5.5 & 1.42 & 1.39 & 1.37 & 1.42 & 1.72 & 1.44 & 1.36 & 1.36 & 1.46 \\
\hline 6.5 & 1.71 & 1.56 & 1.55 & 1.79 & 2.03 & 1.75 & 1.66 & 1.67 & 1.91 \\
\hline 7.5 & 2.27 & 2.14 & 2.13 & 2.26 & 2.56 & 2.23 & 2.14 & 2.15 & 2.25 \\
\hline 8.5 & 3.83 & 3.44 & 3.47 & 3.28 & 3.21 & 3.23 & 3.24 & 3.38 & 3.86 \\
\hline 9.5 & 5.35 & 4.70 & 4.31 & 4.17 & 3.93 & 4.17 & 4.48 & 4.56 & 5.21 \\
\hline 10.5 & 6.02 & 5.17 & 5.03 & 4.70 & 4.47 & 4.81 & 5.00 & 5.19 & 5.84 \\
\hline 11.5 & 6.64 & 5.64 & 5.48 & 5.08 & 4.92 & 5.14 & 5.64 & 5.73 & 6.58 \\
\hline 12.5 & 6.73 & 6.02 & 5.74 & 5.51 & 5.08 & 5.45 & 5.74 & 5.98 & 6.74 \\
\hline 13.5 & 7.32 & 6.29 & 6.18 & 5.66 & 5.39 & 5.59 & 5.83 & 5.97 & 7.13 \\
\hline 14.5 & 7.32 & 6.44 & 6.00 & 5.76 & 5.48 & 5.67 & 6.03 & 6.38 & 7.40 \\
\hline 15.5 & 7.23 & 6.23 & 5.87 & 5.73 & 5.49 & 5.75 & 6.09 & 5.95 & 7.18 \\
\hline 16.5 & 7.23 & 6.21 & 6.00 & 5.73 & 5.39 & 5.55 & 5.68 & 6.17 & 7.02 \\
\hline 17.5 & 7.05 & 6.06 & 5.80 & 5.46 & 5.25 & 5.56 & 5.59 & 6.01 & 6.83 \\
\hline 18.5 & 6.59 & 5.78 & 5.58 & 5.24 & 4.94 & 5.22 & 5.36 & 5.86 & 6.51 \\
\hline 19.5 & 6.13 & 5.28 & 5.22 & 4.94 & 4.79 & 4.95 & 5.33 & 5.27 & 6.28 \\
\hline 20.5 & 5.83 & 4.95 & 4.90 & 4.51 & 4.48 & 4.58 & 4.71 & 4.95 & 5.81 \\
\hline 21.5 & 5.27 & 4.50 & 4.29 & 4.20 & 3.98 & 4.14 & 4.22 & 4.39 & 5.07 \\
\hline 22.5 & 4.63 & 3.99 & 4.00 & 3.72 & 3.57 & 3.65 & 3.92 & 4.06 & 4.69 \\
\hline 23.5 & 5.54 & 4.52 & 4.30 & 4.01 & 3.92 & 3.94 & 4.12 & 4.36 & 4.95 \\
\hline Ax. Avg. & 4.54 & 3.95 & 3.82 & 3.66 & 3.58 & 3.65 & 3.77 & 3.90 & 4.48 \\
\hline
\end{tabular}

POWER DENSITY VALUES NORMALIZED TO A CORE POWER OF 11.57 MW AND ASSUME ALL FISSION ENERGY (EXCLUDING GAMMA-HEATING OF EX-CORE COMPONENTS) IS DEPOSITED IN THE FUEL 
Table B-22. Power Density Data (kW/cc) in Fuel Plate 22 in Prototypic Conditions (continued).

Plate 22, Core Position X1, 3 MWd Burnup, Equilibrium Xenon Conditions

\begin{tabular}{|c|c|c|c|c|c|c|c|c|c|}
\hline $\begin{array}{l}\text { Axial } \\
\text { position } \\
\text { from } \\
\text { top of } \\
\text { fuel } \\
\text { (inches) }\end{array}$ & $\begin{array}{c}0.000 \\
\text { to } \\
0.197 \\
\text { inches }\end{array}$ & $\begin{array}{c}0.197 \\
\text { to } \\
0.394 \\
\text { inches }\end{array}$ & $\begin{array}{c}0.394 \\
\text { to } \\
0.591 \\
\text { inches }\end{array}$ & $\begin{array}{c}0.591 \\
\text { to } \\
1.510 \\
\text { inches }\end{array}$ & $\begin{array}{c}1.510 \\
\text { to } \\
2.429 \\
\text { inches }\end{array}$ & $\begin{array}{c}2.429 \\
\text { to } \\
3.348 \\
\text { inches }\end{array}$ & $\begin{array}{c}3.348 \\
\text { to } \\
3.544 \\
\text { inches }\end{array}$ & $\begin{array}{c}3.544 \\
\text { to } \\
3.741 \\
\text { inches }\end{array}$ & $\begin{array}{c}3.741 \\
\text { to } \\
3.938 \\
\text { inches }\end{array}$ \\
\hline 0.5 & 1.66 & 1.40 & 1.34 & 1.42 & 1.68 & 1.41 & 1.22 & 1.39 & 1.55 \\
\hline 1.5 & 2.08 & 1.79 & 1.74 & 1.77 & 1.88 & 1.79 & 1.64 & 1.74 & 2.13 \\
\hline 2.5 & 3.12 & 2.82 & 2.58 & 2.51 & 2.51 & 2.53 & 2.65 & 2.73 & 3.07 \\
\hline 3.5 & 3.93 & 3.33 & 3.15 & 3.06 & 2.98 & 3.12 & 3.15 & 3.42 & 3.78 \\
\hline 4.5 & 4.42 & 3.87 & 3.75 & 3.54 & 3.39 & 3.49 & 3.60 & 3.89 & 4.27 \\
\hline 5.5 & 5.09 & 4.19 & 4.01 & 3.92 & 3.75 & 3.89 & 4.15 & 4.20 & 4.77 \\
\hline 6.5 & 5.62 & 4.67 & 4.38 & 4.21 & 4.08 & 4.19 & 4.49 & 4.54 & 5.53 \\
\hline 7.5 & 5.89 & 4.90 & 4.73 & 4.56 & 4.32 & 4.53 & 4.73 & 5.01 & 5.71 \\
\hline 8.5 & 6.06 & 5.32 & 5.04 & 4.71 & 4.51 & 4.80 & 4.84 & 5.23 & 5.95 \\
\hline 9.5 & 6.20 & 5.55 & 5.12 & 4.89 & 4.67 & 4.98 & 5.20 & 5.26 & 6.10 \\
\hline 10.5 & 6.32 & 5.60 & 5.48 & 4.98 & 4.77 & 5.08 & 5.23 & 5.73 & 6.33 \\
\hline 11.5 & 6.65 & 5.76 & 5.42 & 5.10 & 4.84 & 5.03 & 5.30 & 5.59 & 6.39 \\
\hline 12.5 & 6.36 & 5.51 & 5.41 & 5.04 & 4.79 & 5.05 & 5.15 & 5.62 & 6.35 \\
\hline 13.5 & 6.42 & 5.52 & 5.36 & 5.11 & 4.90 & 5.11 & 5.17 & 5.44 & 6.36 \\
\hline 14.5 & 6.21 & 5.36 & 5.32 & 4.95 & 4.74 & 4.91 & 5.19 & 5.37 & 6.17 \\
\hline 15.5 & 6.19 & 5.23 & 4.89 & 4.83 & 4.64 & 4.78 & 4.87 & 5.35 & 5.88 \\
\hline 16.5 & 6.03 & 5.13 & 4.93 & 4.66 & 4.45 & 4.55 & 4.76 & 5.06 & 5.82 \\
\hline 17.5 & 5.44 & 5.01 & 4.66 & 4.45 & 4.21 & 4.37 & 4.53 & 4.76 & 5.38 \\
\hline 18.5 & 5.34 & 4.60 & 4.32 & 4.20 & 3.93 & 4.12 & 4.33 & 4.56 & 5.31 \\
\hline 19.5 & 4.93 & 4.30 & 4.08 & 3.94 & 3.78 & 3.89 & 4.02 & 4.32 & 4.92 \\
\hline 20.5 & 4.43 & 3.77 & 3.59 & 3.51 & 3.37 & 3.47 & 3.71 & 4.05 & 4.30 \\
\hline 21.5 & 4.13 & 3.50 & 3.39 & 3.21 & 3.03 & 3.16 & 3.22 & 3.55 & 3.97 \\
\hline 22.5 & 3.63 & 3.13 & 2.99 & 2.87 & 2.70 & 2.72 & 2.97 & 3.08 & 3.66 \\
\hline 23.5 & 3.89 & 3.35 & 3.24 & 3.02 & 2.87 & 3.07 & 3.28 & 3.44 & 3.91 \\
\hline Ax. Avg. & 5.00 & 4.32 & 4.12 & 3.94 & 3.78 & 3.92 & 4.06 & 4.31 & 4.90 \\
\hline
\end{tabular}

POWER DENSITY VALUES NORMALIZED TO A CORE POWER OF 11.57 MW AND ASSUME ALL FISSION ENERGY (EXCLUDING GAMMA-HEATING OF EX-CORE COMPONENTS) IS DEPOSITED IN THE FUEL 
Table B-22. Power Density Data (kW/cc) in Fuel Plate 22 in Prototypic Conditions (continued).

Plate 22, Core Position X7, 80 MWd Burnup, Equilibrium Xenon Conditions

\begin{tabular}{|c|c|c|c|c|c|c|c|c|c|}
\hline $\begin{array}{l}\text { Axial } \\
\text { position } \\
\text { from } \\
\text { top of } \\
\text { fuel } \\
\text { (inches) }\end{array}$ & $\begin{array}{c}0.000 \\
\text { to } \\
0.197 \\
\text { inches }\end{array}$ & $\begin{array}{c}0.197 \\
\text { to } \\
0.394 \\
\text { inches }\end{array}$ & $\begin{array}{c}0.394 \\
\text { to } \\
0.591 \\
\text { inches }\end{array}$ & $\begin{array}{c}0.591 \\
\text { to } \\
1.510 \\
\text { inches }\end{array}$ & $\begin{array}{c}1.510 \\
\text { to } \\
2.429 \\
\text { inches }\end{array}$ & $\begin{array}{c}2.429 \\
\text { to } \\
3.348 \\
\text { inches }\end{array}$ & $\begin{array}{c}3.348 \\
\text { to } \\
3.544 \\
\text { inches }\end{array}$ & $\begin{array}{c}3.544 \\
\text { to } \\
3.741 \\
\text { inches }\end{array}$ & $\begin{array}{c}3.741 \\
\text { to } \\
3.938 \\
\text { inches }\end{array}$ \\
\hline 0.5 & 1.39 & 1.34 & 1.21 & 1.28 & 1.47 & 1.65 & 1.49 & 1.46 & 1.56 \\
\hline 1.5 & 1.97 & 1.72 & 1.85 & 1.72 & 1.80 & 1.95 & 1.83 & 1.81 & 2.11 \\
\hline 2.5 & 3.02 & 2.69 & 2.66 & 2.54 & 2.48 & 2.58 & 2.61 & 2.70 & 3.13 \\
\hline 3.5 & 3.81 & 3.48 & 3.23 & 3.16 & 3.10 & 3.13 & 3.16 & 3.36 & 3.77 \\
\hline 4.5 & 4.31 & 3.94 & 3.53 & 3.56 & 3.41 & 3.55 & 3.39 & 3.76 & 4.20 \\
\hline 5.5 & 4.90 & 4.36 & 4.23 & 4.04 & 3.82 & 3.87 & 4.03 & 4.18 & 4.77 \\
\hline 6.5 & 5.36 & 4.61 & 4.21 & 4.31 & 4.03 & 4.12 & 4.28 & 4.50 & 5.22 \\
\hline 7.5 & 5.65 & 5.05 & 4.66 & 4.55 & 4.29 & 4.39 & 4.46 & 4.59 & 5.59 \\
\hline 8.5 & 5.71 & 5.08 & 4.97 & 4.74 & 4.54 & 4.68 & 4.71 & 5.04 & 5.71 \\
\hline 9.5 & 5.98 & 5.17 & 5.10 & 4.93 & 4.68 & 4.86 & 4.88 & 5.18 & 5.93 \\
\hline 10.5 & 6.12 & 5.63 & 5.21 & 5.06 & 4.82 & 4.83 & 4.91 & 5.24 & 5.90 \\
\hline 11.5 & 6.25 & 5.59 & 5.44 & 5.16 & 4.85 & 4.86 & 5.00 & 5.19 & 6.08 \\
\hline 12.5 & 6.15 & 5.37 & 5.39 & 5.10 & 4.76 & 4.77 & 5.14 & 5.29 & 5.93 \\
\hline 13.5 & 6.13 & 5.68 & 5.18 & 5.02 & 4.66 & 4.74 & 5.04 & 5.16 & 6.09 \\
\hline 14.5 & 6.13 & 5.26 & 5.18 & 4.89 & 4.56 & 4.67 & 4.91 & 5.19 & 5.97 \\
\hline 15.5 & 5.98 & 5.19 & 4.72 & 4.70 & 4.40 & 4.39 & 4.60 & 4.87 & 5.60 \\
\hline 16.5 & 5.64 & 4.88 & 4.55 & 4.53 & 4.21 & 4.30 & 4.56 & 4.74 & 5.24 \\
\hline 17.5 & 5.30 & 4.70 & 4.46 & 4.21 & 3.88 & 4.05 & 4.21 & 4.36 & 5.21 \\
\hline 18.5 & 5.11 & 4.28 & 4.15 & 3.99 & 3.75 & 3.79 & 4.02 & 4.29 & 4.81 \\
\hline 19.5 & 4.43 & 4.16 & 3.91 & 3.66 & 3.47 & 3.54 & 3.71 & 3.96 & 4.59 \\
\hline 20.5 & 4.13 & 3.60 & 3.67 & 3.44 & 3.17 & 3.21 & 3.41 & 3.62 & 4.14 \\
\hline 21.5 & 3.56 & 3.30 & 3.07 & 2.99 & 2.85 & 2.98 & 3.02 & 3.23 & 3.72 \\
\hline 22.5 & 3.28 & 2.98 & 2.94 & 2.64 & 2.58 & 2.63 & 2.78 & 2.83 & 3.46 \\
\hline 23.5 & 3.68 & 3.11 & 3.05 & 2.93 & 2.82 & 2.88 & 2.99 & 3.12 & 3.59 \\
\hline Ax. Avg. & 4.75 & 4.22 & 4.02 & 3.88 & 3.68 & 3.77 & 3.88 & 4.07 & 4.68 \\
\hline
\end{tabular}

POWER DENSITY VALUES NORMALIZED TO A CORE POWER OF 11.57 MW AND ASSUME ALL FISSION ENERGY (EXCLUDING GAMMA-HEATING OF EX-CORE COMPONENTS) IS DEPOSITED IN THE FUEL 
Table B-22. Power Density Data (kW/cc) in Fuel Plate 22 in Prototypic Conditions (continued).

Plate 22, Core Position X6, 99 MWd Burnup, Equilibrium Xenon Conditions

\begin{tabular}{|c|c|c|c|c|c|c|c|c|c|}
\hline $\begin{array}{l}\text { Axial } \\
\text { position } \\
\text { from } \\
\text { top of } \\
\text { fuel } \\
\text { (inches) }\end{array}$ & $\begin{array}{c}0.000 \\
\text { to } \\
0.197 \\
\text { inches }\end{array}$ & $\begin{array}{c}0.197 \\
\text { to } \\
0.394 \\
\text { inches }\end{array}$ & $\begin{array}{c}0.394 \\
\text { to } \\
0.591 \\
\text { inches }\end{array}$ & $\begin{array}{c}0.591 \\
\text { to } \\
1.510 \\
\text { inches }\end{array}$ & $\begin{array}{c}1.510 \\
\text { to } \\
2.429 \\
\text { inches }\end{array}$ & $\begin{array}{c}2.429 \\
\text { to } \\
3.348 \\
\text { inches }\end{array}$ & $\begin{array}{c}3.348 \\
\text { to } \\
3.544 \\
\text { inches }\end{array}$ & $\begin{array}{c}3.544 \\
\text { to } \\
3.741 \\
\text { inches }\end{array}$ & $\begin{array}{c}3.741 \\
\text { to } \\
3.938 \\
\text { inches }\end{array}$ \\
\hline 0.5 & 1.57 & 1.35 & 1.24 & 1.18 & 1.12 & 1.17 & 1.29 & 1.27 & 1.49 \\
\hline 1.5 & 1.96 & 1.89 & 1.80 & 1.61 & 1.55 & 1.62 & 1.61 & 1.74 & 1.95 \\
\hline 2.5 & 3.07 & 2.44 & 2.55 & 2.44 & 2.38 & 2.48 & 2.60 & 2.67 & 3.07 \\
\hline 3.5 & 3.53 & 3.11 & 3.09 & 3.01 & 3.00 & 3.12 & 3.26 & 3.37 & 3.75 \\
\hline 4.5 & 4.03 & 3.71 & 3.36 & 3.47 & 3.43 & 3.54 & 3.63 & 3.81 & 4.20 \\
\hline 5.5 & 4.38 & 3.90 & 3.89 & 3.80 & 3.75 & 3.88 & 4.02 & 4.39 & 4.99 \\
\hline 6.5 & 4.80 & 4.37 & 4.28 & 4.08 & 4.18 & 4.26 & 4.40 & 4.57 & 5.32 \\
\hline 7.5 & 5.01 & 4.56 & 4.39 & 4.44 & 4.42 & 4.48 & 4.61 & 4.91 & 5.51 \\
\hline 8.5 & 5.33 & 4.88 & 4.65 & 4.65 & 4.52 & 4.84 & 4.94 & 5.00 & 5.76 \\
\hline 9.5 & 5.31 & 4.90 & 4.89 & 4.79 & 4.81 & 4.99 & 4.95 & 5.33 & 5.97 \\
\hline 10.5 & 5.35 & 4.92 & 4.78 & 4.83 & 4.81 & 4.94 & 5.32 & 5.15 & 5.88 \\
\hline 11.5 & 5.73 & 5.16 & 4.99 & 4.99 & 4.82 & 5.07 & 5.11 & 5.47 & 6.15 \\
\hline 12.5 & 5.69 & 5.14 & 4.99 & 4.96 & 4.93 & 5.10 & 5.41 & 5.32 & 6.17 \\
\hline 13.5 & 5.79 & 5.11 & 4.86 & 4.88 & 4.89 & 4.96 & 5.15 & 5.43 & 6.09 \\
\hline 14.5 & 5.61 & 5.05 & 4.82 & 4.91 & 4.80 & 4.96 & 5.19 & 5.44 & 6.21 \\
\hline 15.5 & 5.49 & 5.07 & 4.56 & 4.77 & 4.57 & 4.79 & 4.98 & 5.06 & 5.86 \\
\hline 16.5 & 5.18 & 4.82 & 4.60 & 4.52 & 4.53 & 4.67 & 4.69 & 5.00 & 5.70 \\
\hline 17.5 & 5.06 & 4.50 & 4.44 & 4.33 & 4.27 & 4.30 & 4.39 & 4.51 & 5.24 \\
\hline 18.5 & 4.75 & 4.19 & 4.13 & 4.07 & 4.00 & 4.13 & 4.07 & 4.25 & 4.84 \\
\hline 19.5 & 4.33 & 3.94 & 3.70 & 3.74 & 3.77 & 3.69 & 3.79 & 3.94 & 4.54 \\
\hline 20.5 & 4.05 & 3.59 & 3.47 & 3.43 & 3.31 & 3.42 & 3.37 & 3.70 & 4.05 \\
\hline 21.5 & 3.55 & 3.09 & 3.07 & 3.01 & 2.94 & 3.07 & 3.05 & 3.35 & 3.65 \\
\hline 22.5 & 3.18 & 2.82 & 2.75 & 2.71 & 2.63 & 2.73 & 2.71 & 2.84 & 3.48 \\
\hline 23.5 & 3.57 & 2.98 & 2.87 & 2.87 & 2.81 & 2.86 & 2.87 & 3.07 & 3.64 \\
\hline Ax. Avg. & 4.43 & 3.98 & 3.84 & 3.81 & 3.76 & 3.88 & 3.97 & 4.15 & 4.73 \\
\hline
\end{tabular}

POWER DENSITY VALUES NORMALIZED TO A CORE POWER OF 11.57 MW AND ASSUME ALL FISSION ENERGY (EXCLUDING GAMMA-HEATING OF EX-CORE COMPONENTS) IS DEPOSITED IN THE FUEL 
Table B-22. Power Density Data (kW/cc) in Fuel Plate 22 in Prototypic Conditions (continued).

Plate 22, Core Position X8, 173 MWd Burnup, Equilibrium Xenon Conditions

\begin{tabular}{|c|c|c|c|c|c|c|c|c|c|}
\hline $\begin{array}{l}\text { Axial } \\
\text { position } \\
\text { from } \\
\text { top of } \\
\text { fuel } \\
\text { (inches) }\end{array}$ & $\begin{array}{c}0.000 \\
\text { to } \\
0.197 \\
\text { inches }\end{array}$ & $\begin{array}{c}0.197 \\
\text { to } \\
0.394 \\
\text { inches }\end{array}$ & $\begin{array}{c}0.394 \\
\text { to } \\
0.591 \\
\text { inches }\end{array}$ & $\begin{array}{c}0.591 \\
\text { to } \\
1.510 \\
\text { inches }\end{array}$ & $\begin{array}{c}1.510 \\
\text { to } \\
2.429 \\
\text { inches }\end{array}$ & $\begin{array}{c}2.429 \\
\text { to } \\
3.348 \\
\text { inches }\end{array}$ & $\begin{array}{c}3.348 \\
\text { to } \\
3.544 \\
\text { inches }\end{array}$ & $\begin{array}{c}3.544 \\
\text { to } \\
3.741 \\
\text { inches }\end{array}$ & $\begin{array}{c}3.741 \\
\text { to } \\
3.938 \\
\text { inches }\end{array}$ \\
\hline 0.5 & 1.51 & 1.32 & 1.25 & 1.19 & 1.13 & 1.17 & 1.24 & 1.30 & 1.41 \\
\hline 1.5 & 2.05 & 1.70 & 1.78 & 1.71 & 1.61 & 1.60 & 1.70 & 1.70 & 2.04 \\
\hline 2.5 & 2.99 & 2.67 & 2.57 & 2.53 & 2.44 & 2.55 & 2.63 & 2.81 & 3.06 \\
\hline 3.5 & 3.80 & 3.30 & 3.20 & 3.12 & 3.07 & 3.13 & 3.17 & 3.37 & 3.76 \\
\hline 4.5 & 4.13 & 3.67 & 3.65 & 3.47 & 3.40 & 3.49 & 3.59 & 3.69 & 4.27 \\
\hline 5.5 & 4.73 & 4.10 & 3.96 & 3.92 & 3.86 & 3.92 & 3.91 & 4.22 & 4.67 \\
\hline 6.5 & 4.80 & 4.39 & 4.12 & 4.18 & 4.03 & 4.14 & 4.36 & 4.44 & 4.87 \\
\hline 7.5 & 5.38 & 4.68 & 4.52 & 4.35 & 4.33 & 4.45 & 4.38 & 4.69 & 5.18 \\
\hline 8.5 & 5.35 & 4.79 & 4.59 & 4.50 & 4.52 & 4.50 & 4.78 & 5.10 & 5.51 \\
\hline 9.5 & 5.59 & 4.95 & 4.86 & 4.68 & 4.71 & 4.75 & 4.87 & 5.10 & 5.81 \\
\hline 10.5 & 5.66 & 5.11 & 4.89 & 4.87 & 4.70 & 4.84 & 4.72 & 5.03 & 5.61 \\
\hline 11.5 & 5.50 & 5.06 & 4.81 & 4.81 & 4.72 & 4.93 & 5.00 & 5.14 & 5.83 \\
\hline 12.5 & 5.63 & 4.90 & 4.81 & 4.80 & 4.74 & 4.85 & 5.02 & 5.06 & 5.65 \\
\hline 13.5 & 5.43 & 4.92 & 4.65 & 4.69 & 4.64 & 4.83 & 4.91 & 5.08 & 5.71 \\
\hline 14.5 & 5.32 & 4.71 & 4.56 & 4.60 & 4.49 & 4.83 & 4.99 & 5.17 & 5.68 \\
\hline 15.5 & 5.28 & 4.66 & 4.46 & 4.60 & 4.47 & 4.62 & 4.72 & 4.90 & 5.39 \\
\hline 16.5 & 5.13 & 4.46 & 4.46 & 4.41 & 4.31 & 4.54 & 4.46 & 4.74 & 5.32 \\
\hline 17.5 & 5.00 & 4.40 & 4.24 & 4.12 & 4.15 & 4.29 & 4.47 & 4.48 & 5.06 \\
\hline 18.5 & 4.63 & 4.23 & 4.09 & 4.02 & 3.98 & 3.94 & 3.96 & 4.36 & 4.84 \\
\hline 19.5 & 4.39 & 3.94 & 3.77 & 3.63 & 3.62 & 3.66 & 3.79 & 3.94 & 4.41 \\
\hline 20.5 & 3.76 & 3.70 & 3.44 & 3.41 & 3.33 & 3.46 & 3.40 & 3.54 & 3.98 \\
\hline 21.5 & 3.57 & 3.22 & 3.12 & 2.99 & 3.00 & 3.04 & 3.23 & 3.24 & 3.49 \\
\hline 22.5 & 3.26 & 2.93 & 2.67 & 2.67 & 2.68 & 2.79 & 2.80 & 2.86 & 3.32 \\
\hline 23.5 & 3.65 & 3.18 & 3.04 & 2.90 & 2.79 & 2.97 & 3.02 & 3.04 & 3.56 \\
\hline Ax. Avg. & 4.44 & 3.96 & 3.81 & 3.76 & 3.70 & 3.80 & 3.88 & 4.04 & 4.52 \\
\hline
\end{tabular}

POWER DENSITY VALUES NORMALIZED TO A CORE POWER OF 11.57 MW AND ASSUME ALL FISSION ENERGY (EXCLUDING GAMMA-HEATING OF EX-CORE COMPONENTS) IS DEPOSITED IN THE FUEL 
Table B-23. Power Density Data $\left(\mathrm{kW} / \mathrm{cm}^{3}\right)$ in Fuel Plate 23 in Prototypic Core.

\section{Fuel core width: 4.048 inches / Fuel core thickness: 17 mil}

Plate 23, Core Position X1, 0 MWd Burnup, Xenon-Free Conditions

\begin{tabular}{|c|c|c|c|c|c|c|c|c|c|}
\hline $\begin{array}{c}\text { Axial } \\
\text { position } \\
\text { from } \\
\text { top of } \\
\text { fuel } \\
\text { (inches) } \\
\end{array}$ & $\begin{array}{c}0.000 \\
\text { to } \\
0.197 \\
\text { inches }\end{array}$ & $\begin{array}{c}0.197 \\
\text { to } \\
0.394 \\
\text { inches } \\
\end{array}$ & $\begin{array}{c}0.394 \\
\text { to } \\
0.591 \\
\text { inches } \\
\end{array}$ & $\begin{array}{c}0.591 \\
\text { to } \\
1.546 \\
\text { inches }\end{array}$ & $\begin{array}{c}1.546 \\
\text { to } \\
2.502 \\
\text { inches }\end{array}$ & $\begin{array}{c}2.502 \\
\text { to } \\
3.457 \\
\text { inches } \\
\end{array}$ & $\begin{array}{c}3.457 \\
\text { to } \\
3.654 \\
\text { inches }\end{array}$ & $\begin{array}{c}3.654 \\
\text { to } \\
3.851 \\
\text { inches }\end{array}$ & $\begin{array}{c}3.851 \\
\text { to } \\
4.048 \\
\text { inches } \\
\end{array}$ \\
\hline 0.5 & 0.82 & 0.69 & 0.67 & 0.87 & 1.10 & 0.89 & 0.76 & 0.84 & 0.80 \\
\hline 1.5 & 0.75 & 0.65 & 0.73 & 0.88 & 1.15 & 0.85 & 0.73 & 0.75 & 0.75 \\
\hline 2.5 & 0.95 & 0.85 & 0.88 & 1.03 & 1.30 & 1.01 & 0.81 & 0.85 & 0.89 \\
\hline 3.5 & 1.12 & 1.02 & 1.06 & 1.23 & 1.59 & 1.17 & 0.98 & 1.07 & 1.14 \\
\hline 4.5 & 1.25 & 1.19 & 1.20 & 1.43 & 1.81 & 1.51 & 1.18 & 1.17 & 1.24 \\
\hline 5.5 & 1.62 & 1.47 & 1.47 & 1.61 & 2.23 & 1.69 & 1.40 & 1.35 & 1.48 \\
\hline 6.5 & 1.84 & 1.72 & 1.75 & 2.11 & 2.63 & 2.05 & 1.82 & 1.79 & 1.83 \\
\hline 7.5 & 2.66 & 2.48 & 2.56 & 2.80 & 3.32 & 2.67 & 2.56 & 2.47 & 2.52 \\
\hline 8.5 & 4.79 & 4.56 & 4.43 & 4.50 & 4.56 & 4.40 & 4.25 & 4.42 & 4.70 \\
\hline 9.5 & 6.76 & 6.42 & 6.12 & 5.96 & 5.66 & 5.86 & 6.12 & 6.21 & 6.58 \\
\hline 10.5 & 8.07 & 7.53 & 7.04 & 6.88 & 6.48 & 6.80 & 7.14 & 7.30 & 7.77 \\
\hline 11.5 & 8.57 & 8.08 & 8.06 & 7.43 & 7.00 & 7.35 & 7.87 & 8.14 & 8.48 \\
\hline 12.5 & 9.06 & 8.48 & 8.31 & 7.93 & 7.44 & 7.87 & 8.31 & 8.37 & 9.03 \\
\hline 13.5 & 9.43 & 8.98 & 8.52 & 8.27 & 7.74 & 8.08 & 8.55 & 8.56 & 9.12 \\
\hline 14.5 & 9.32 & 8.97 & 8.68 & 8.31 & 7.93 & 8.18 & 8.62 & 8.62 & 9.45 \\
\hline 15.5 & 9.69 & 8.78 & 8.73 & 8.32 & 8.02 & 8.40 & 8.67 & 8.77 & 9.57 \\
\hline 16.5 & 9.62 & 8.95 & 8.74 & 8.19 & 7.93 & 8.17 & 8.55 & 9.06 & 9.17 \\
\hline 17.5 & 9.25 & 8.66 & 8.16 & 7.98 & 7.49 & 8.01 & 8.33 & 8.54 & 9.13 \\
\hline 18.5 & 8.61 & 8.39 & 8.00 & 7.57 & 7.20 & 7.48 & 8.00 & 8.47 & 8.79 \\
\hline 19.5 & 8.12 & 7.56 & 7.39 & 7.21 & 6.79 & 7.25 & 7.55 & 7.52 & 8.29 \\
\hline 20.5 & 7.77 & 7.13 & 6.76 & 6.74 & 6.50 & 6.72 & 6.94 & 7.35 & 7.65 \\
\hline 21.5 & 7.03 & 6.56 & 6.35 & 6.13 & 5.87 & 6.13 & 6.38 & 6.26 & 6.80 \\
\hline 22.5 & 6.25 & 5.76 & 5.91 & 5.56 & 5.31 & 5.55 & 5.51 & 5.86 & 6.11 \\
\hline 23.5 & 6.69 & 6.08 & 5.99 & 5.73 & 5.53 & 5.59 & 5.68 & 6.15 & 6.63 \\
\hline Ax. Avg. & 5.83 & 5.46 & 5.31 & 5.19 & 5.11 & 5.16 & 5.28 & 5.41 & 5.74 \\
\hline
\end{tabular}

POWER DENSITY VALUES NORMALIZED TO A CORE POWER OF 11.57 MW AND ASSUME ALL FISSION ENERGY (EXCLUDING GAMMA-HEATING OF EX-CORE COMPONENTS) IS DEPOSITED IN THE FUEL 
Table B-23. Power Density Data (kW/cc) in Fuel Plate 23 in Prototypic Conditions (continued).

Plate 23, Core Position X1, 3 MWd Burnup, Equilibrium Xenon Conditions

\begin{tabular}{|c|c|c|c|c|c|c|c|c|c|}
\hline $\begin{array}{c}\text { Axial } \\
\text { position } \\
\text { from } \\
\text { top of } \\
\text { fuel } \\
\text { (inches) }\end{array}$ & $\begin{array}{c}0.000 \\
\text { to } \\
0.197 \\
\text { inches }\end{array}$ & $\begin{array}{c}0.197 \\
\text { to } \\
0.394 \\
\text { inches }\end{array}$ & $\begin{array}{c}0.394 \\
\text { to } \\
0.591 \\
\text { inches }\end{array}$ & $\begin{array}{c}0.591 \\
\text { to } \\
1.546 \\
\text { inches }\end{array}$ & $\begin{array}{c}1.546 \\
\text { to } \\
2.502 \\
\text { inches }\end{array}$ & $\begin{array}{c}2.502 \\
\text { to } \\
3.457 \\
\text { inches }\end{array}$ & $\begin{array}{c}\text { 3.457 } \\
\text { to } \\
3.654 \\
\text { inches }\end{array}$ & $\begin{array}{c}3.654 \\
\text { to } \\
3.851 \\
\text { inches }\end{array}$ & $\begin{array}{c}3.851 \\
\text { to } \\
4.048 \\
\text { inches }\end{array}$ \\
\hline 1.5 & 1.77 & 1.60 & 1.55 & 1.70 & 2.11 & 1.69 & 1.41 & 1.50 & 1.75 \\
\hline 2.5 & 4.23 & 3.88 & 3.65 & 3.69 & 3.61 & 3.63 & 3.72 & 3.84 & 4.14 \\
\hline 3.5 & 5.21 & 4.71 & 4.87 & 4.50 & 4.44 & 4.59 & 4.69 & 4.78 & 5.23 \\
\hline 4.5 & 6.12 & 5.66 & 5.44 & 5.22 & 4.91 & 5.14 & 5.29 & 5.36 & 5.99 \\
\hline 5.5 & 6.53 & 6.21 & 5.92 & 5.80 & 5.49 & 5.75 & 5.72 & 5.99 & 6.72 \\
\hline 6.5 & 7.26 & 6.65 & 6.66 & 6.24 & 5.99 & 6.18 & 6.49 & 6.79 & 7.27 \\
\hline 7.5 & 7.82 & 7.20 & 7.19 & 6.69 & 6.44 & 6.77 & 6.95 & 7.18 & 7.63 \\
\hline 8.5 & 8.29 & 7.54 & 7.30 & 7.00 & 6.60 & 7.04 & 7.23 & 7.44 & 8.02 \\
\hline 9.5 & 8.55 & 7.97 & 7.61 & 7.24 & 6.87 & 7.27 & 7.57 & 7.66 & 8.46 \\
\hline 10.5 & 8.45 & 8.00 & 7.84 & 7.30 & 7.05 & 7.41 & 7.47 & 8.05 & 8.76 \\
\hline 11.5 & 8.80 & 8.00 & 7.56 & 7.43 & 7.14 & 7.48 & 7.83 & 8.05 & 8.55 \\
\hline 12.5 & 8.66 & 7.85 & 7.82 & 7.42 & 7.15 & 7.39 & 7.74 & 8.13 & 8.41 \\
\hline 13.5 & 8.88 & 7.97 & 7.77 & 7.37 & 7.20 & 7.46 & 7.61 & 7.77 & 8.40 \\
\hline 14.5 & 8.39 & 7.85 & 7.46 & 7.29 & 6.97 & 7.31 & 7.71 & 7.69 & 8.43 \\
\hline 15.5 & 8.09 & 7.60 & 7.18 & 7.03 & 6.83 & 7.03 & 7.30 & 7.64 & 8.40 \\
\hline 16.5 & 7.55 & 7.29 & 6.90 & 6.85 & 6.59 & 6.71 & 7.18 & 7.28 & 7.86 \\
\hline 17.5 & 7.54 & 7.00 & 6.60 & 6.52 & 6.32 & 6.42 & 6.70 & 6.90 & 7.23 \\
\hline 18.5 & 6.94 & 6.66 & 6.37 & 6.17 & 5.80 & 5.98 & 6.44 & 6.72 & 7.21 \\
\hline 19.5 & 6.55 & 6.09 & 5.93 & 5.78 & 5.47 & 5.61 & 5.89 & 6.14 & 6.36 \\
\hline 20.5 & 6.18 & 5.69 & 5.25 & 5.27 & 5.01 & 5.13 & 5.43 & 5.52 & 5.88 \\
\hline 21.5 & 5.35 & 5.08 & 4.83 & 4.75 & 4.55 & 4.73 & 4.88 & 4.99 & 5.47 \\
\hline 22.5 & 4.97 & 4.38 & 4.52 & 4.31 & 4.12 & 4.26 & 4.40 & 4.44 & 5.01 \\
\hline 23.5 & 4.96 & 4.72 & 4.30 & 4.43 & 4.16 & 4.35 & 4.55 & 4.71 & 4.95 \\
\hline Ax. Avg. & 6.65 & 6.16 & 5.95 & 5.77 & 5.56 & 5.74 & 5.94 & 6.12 & 6.61 \\
\hline & & & & & & & & 2.49 & \\
\hline
\end{tabular}

POWER DENSITY VALUES NORMALIZED TO A CORE POWER OF 11.57 MW AND ASSUME ALL FISSION ENERGY (EXCLUDING GAMMA-HEATING OF EX-CORE COMPONENTS) IS DEPOSITED IN THE FUEL 
Table B-23. Power Density Data (kW/cc) in Fuel Plate 23 in Prototypic Conditions (continued).

Plate 23, Core Position X7, 80 MWd Burnup, Equilibrium Xenon Conditions

\begin{tabular}{|c|c|c|c|c|c|c|c|c|c|}
\hline $\begin{array}{l}\text { Axial } \\
\text { position } \\
\text { from } \\
\text { top of } \\
\text { fuel } \\
\text { (inches) }\end{array}$ & $\begin{array}{c}0.000 \\
\text { to } \\
0.197 \\
\text { inches }\end{array}$ & $\begin{array}{c}0.197 \\
\text { to } \\
0.394 \\
\text { inches }\end{array}$ & $\begin{array}{c}0.394 \\
\text { to } \\
0.591 \\
\text { inches }\end{array}$ & $\begin{array}{c}0.591 \\
\text { to } \\
1.546 \\
\text { inches }\end{array}$ & $\begin{array}{c}1.546 \\
\text { to } \\
2.502 \\
\text { inches }\end{array}$ & $\begin{array}{c}2.502 \\
\text { to } \\
3.457 \\
\text { inches }\end{array}$ & $\begin{array}{c}3.457 \\
\text { to } \\
3.654 \\
\text { inches }\end{array}$ & $\begin{array}{c}3.654 \\
\text { to } \\
3.851 \\
\text { inches }\end{array}$ & $\begin{array}{c}3.851 \\
\text { to } \\
4.048 \\
\text { inches }\end{array}$ \\
\hline 0.5 & 1.55 & 1.44 & 1.39 & 1.43 & 1.91 & 2.15 & 1.83 & 1.68 & 1.72 \\
\hline 1.5 & 2.42 & 2.30 & 2.40 & 2.32 & 2.61 & 2.79 & 2.53 & 2.54 & 2.81 \\
\hline 2.5 & 4.09 & 3.67 & 3.58 & 3.63 & 3.62 & 3.69 & 3.67 & 3.88 & 4.28 \\
\hline 3.5 & 4.97 & 4.63 & 4.61 & 4.55 & 4.38 & 4.55 & 4.54 & 4.71 & 5.01 \\
\hline 4.5 & 5.67 & 5.49 & 5.18 & 5.18 & 4.94 & 5.07 & 5.00 & 5.38 & 5.58 \\
\hline 5.5 & 6.53 & 6.09 & 5.96 & 5.76 & 5.44 & 5.52 & 5.81 & 5.82 & 6.33 \\
\hline 6.5 & 7.00 & 6.38 & 6.34 & 6.09 & 5.78 & 5.95 & 6.03 & 6.46 & 6.72 \\
\hline 7.5 & 7.21 & 6.83 & 6.58 & 6.60 & 6.18 & 6.33 & 6.22 & 6.61 & 7.28 \\
\hline 8.5 & 7.55 & 7.08 & 6.92 & 6.74 & 6.38 & 6.63 & 6.68 & 6.79 & 7.48 \\
\hline 9.5 & 7.65 & 7.23 & 7.13 & 7.00 & 6.62 & 6.80 & 6.71 & 6.99 & 7.57 \\
\hline 10.5 & 7.95 & 7.62 & 7.21 & 7.06 & 6.73 & 6.79 & 7.11 & 7.34 & 7.85 \\
\hline 11.5 & 7.96 & 7.60 & 7.46 & 7.08 & 6.68 & 6.85 & 7.01 & 7.12 & 7.61 \\
\hline 12.5 & 7.96 & 7.44 & 7.24 & 7.11 & 6.54 & 6.77 & 6.98 & 7.11 & 7.55 \\
\hline 13.5 & 8.13 & 7.55 & 7.28 & 6.95 & 6.51 & 6.63 & 6.75 & 7.32 & 7.65 \\
\hline 14.5 & 7.63 & 7.20 & 6.88 & 6.82 & 6.31 & 6.48 & 6.60 & 6.82 & 7.37 \\
\hline 15.5 & 7.50 & 7.03 & 6.54 & 6.44 & 6.15 & 6.11 & 6.51 & 6.73 & 6.98 \\
\hline 16.5 & 7.15 & 6.69 & 6.46 & 6.37 & 5.78 & 6.01 & 6.34 & 6.60 & 6.87 \\
\hline 17.5 & 6.99 & 6.50 & 5.99 & 5.99 & 5.48 & 5.60 & 5.88 & 6.14 & 6.46 \\
\hline 18.5 & 6.35 & 5.89 & 5.86 & 5.57 & 5.26 & 5.31 & 5.33 & 5.86 & 6.15 \\
\hline 19.5 & 5.89 & 5.57 & 5.46 & 5.15 & 4.85 & 4.99 & 5.11 & 5.44 & 5.95 \\
\hline 20.5 & 5.34 & 5.02 & 4.88 & 4.73 & 4.53 & 4.63 & 4.87 & 5.04 & 5.21 \\
\hline 21.5 & 4.86 & 4.46 & 4.46 & 4.28 & 4.12 & 4.23 & 4.27 & 4.52 & 4.92 \\
\hline 22.5 & 4.51 & 4.16 & 3.96 & 3.93 & 3.75 & 3.83 & 4.03 & 4.14 & 4.35 \\
\hline 23.5 & 4.55 & 4.29 & 4.25 & 4.04 & 3.95 & 3.94 & 4.10 & 4.22 & 4.59 \\
\hline Ax. Avg. & 6.14 & 5.76 & 5.58 & 5.45 & 5.19 & 5.32 & 5.41 & 5.64 & 6.01 \\
\hline
\end{tabular}

POWER DENSITY VALUES NORMALIZED TO A CORE POWER OF 11.57 MW AND ASSUME ALL FISSION ENERGY (EXCLUDING GAMMA-HEATING OF EX-CORE COMPONENTS) IS DEPOSITED IN THE FUEL 
Table B-23. Power Density Data (kW/cc) in Fuel Plate 23 in Prototypic Conditions (continued).

Plate 23, Core Position X2, 99 MWd Burnup, Equilibrium Xenon Conditions

\begin{tabular}{|c|c|c|c|c|c|c|c|c|c|}
\hline $\begin{array}{c}\text { Axial } \\
\text { position } \\
\text { from } \\
\text { top of } \\
\text { fuel } \\
\text { (inches) }\end{array}$ & $\begin{array}{c}0.000 \\
\text { to } \\
0.197 \\
\text { inches }\end{array}$ & $\begin{array}{c}0.197 \\
\text { to } \\
0.394 \\
\text { inches }\end{array}$ & $\begin{array}{c}0.394 \\
\text { to } \\
0.591 \\
\text { inches }\end{array}$ & $\begin{array}{c}0.591 \\
\text { to } \\
1.546 \\
\text { inches }\end{array}$ & $\begin{array}{c}1.546 \\
\text { to } \\
2.502 \\
\text { inches }\end{array}$ & $\begin{array}{c}2.502 \\
\text { to } \\
3.457 \\
\text { inches }\end{array}$ & $\begin{array}{c}\text { 3.457 } \\
\text { to } \\
3.654 \\
\text { inches }\end{array}$ & $\begin{array}{c}3.654 \\
\text { to } \\
3.851 \\
\text { inches }\end{array}$ & $\begin{array}{c}3.851 \\
\text { to } \\
4.048 \\
\text { inches }\end{array}$ \\
\hline 1.5 & 1.63 & 1.35 & 1.33 & 1.30 & 1.21 & 1.34 & 1.27 & 1.51 & 1.78 \\
\hline 2.5 & 3.69 & 3.56 & 2.27 & 2.20 & 2.17 & 2.24 & 2.26 & 2.37 & 2.70 \\
\hline 3.5 & 4.83 & 4.66 & 4.60 & 4.56 & 4.51 & 4.59 & 4.58 & 4.66 & 5.10 \\
\hline 4.5 & 5.58 & 5.37 & 5.14 & 5.07 & 5.08 & 5.14 & 5.14 & 5.37 & 5.80 \\
\hline 5.5 & 6.08 & 5.88 & 5.59 & 5.64 & 5.52 & 5.74 & 5.98 & 5.94 & 6.47 \\
\hline 6.5 & 6.19 & 6.13 & 5.99 & 6.05 & 5.90 & 6.11 & 5.87 & 6.08 & 6.56 \\
\hline 7.5 & 6.94 & 6.50 & 6.32 & 6.44 & 6.28 & 6.54 & 6.72 & 6.48 & 6.92 \\
\hline 8.5 & 7.18 & 6.86 & 6.65 & 6.56 & 6.51 & 6.72 & 6.59 & 6.76 & 7.31 \\
\hline 9.5 & 7.43 & 7.07 & 6.86 & 6.77 & 6.64 & 6.82 & 7.07 & 7.10 & 7.41 \\
\hline 10.5 & 7.84 & 7.12 & 7.01 & 6.78 & 6.62 & 6.87 & 7.12 & 7.05 & 7.76 \\
\hline 11.5 & 7.74 & 7.30 & 7.02 & 6.90 & 6.77 & 6.98 & 6.88 & 6.93 & 7.49 \\
\hline 12.5 & 7.52 & 7.05 & 6.96 & 6.89 & 6.78 & 6.93 & 7.04 & 7.29 & 7.49 \\
\hline 13.5 & 7.32 & 7.03 & 7.00 & 6.77 & 6.65 & 6.83 & 6.78 & 7.02 & 7.31 \\
\hline 14.5 & 7.36 & 6.88 & 6.79 & 6.80 & 6.57 & 6.60 & 6.98 & 6.91 & 7.28 \\
\hline 15.5 & 7.38 & 7.00 & 6.67 & 6.61 & 6.34 & 6.52 & 6.52 & 6.58 & 7.14 \\
\hline 16.5 & 6.84 & 6.67 & 6.64 & 6.35 & 6.03 & 6.27 & 6.44 & 6.51 & 6.66 \\
\hline 17.5 & 6.58 & 6.33 & 6.13 & 6.08 & 5.96 & 5.91 & 6.11 & 6.29 & 6.60 \\
\hline 18.5 & 6.34 & 5.87 & 5.95 & 5.79 & 5.62 & 5.85 & 5.66 & 5.84 & 6.20 \\
\hline 19.5 & 5.84 & 5.49 & 5.51 & 5.43 & 5.16 & 5.37 & 5.34 & 5.63 & 5.63 \\
\hline 20.5 & 5.38 & 5.11 & 5.04 & 5.02 & 4.84 & 4.91 & 4.93 & 4.99 & 5.24 \\
\hline 21.5 & 4.70 & 4.50 & 4.48 & 4.50 & 4.41 & 4.38 & 4.38 & 4.56 & 4.74 \\
\hline 22.5 & 4.64 & 4.17 & 4.29 & 4.05 & 3.95 & 4.00 & 4.04 & 4.17 & 4.44 \\
\hline 23.5 & 4.53 & 4.20 & 4.23 & 4.11 & 4.09 & 4.21 & 4.10 & 4.26 & 4.64 \\
\hline Ax. Avg. & 5.92 & 5.59 & 5.50 & 5.43 & 5.30 & 5.44 & 5.47 & 5.59 & 5.93 \\
\hline & & & & & & & & 3.59 & \\
\hline
\end{tabular}

POWER DENSITY VALUES NORMALIZED TO A CORE POWER OF 11.57 MW AND ASSUME ALL FISSION ENERGY (EXCLUDING GAMMA-HEATING OF EX-CORE COMPONENTS) IS DEPOSITED IN THE FUEL 
Table B-23. Power Density Data (kW/cc) in Fuel Plate 23 in Prototypic Conditions (continued).

Plate 23, Core Position X8, 173 MWd Burnup, Equilibrium Xenon Conditions

\begin{tabular}{|c|c|c|c|c|c|c|c|c|c|}
\hline $\begin{array}{c}\text { Axial } \\
\text { position } \\
\text { from } \\
\text { top of } \\
\text { fuel } \\
\text { (inches) }\end{array}$ & $\begin{array}{c}0.000 \\
\text { to } \\
0.197 \\
\text { inches }\end{array}$ & $\begin{array}{c}0.197 \\
\text { to } \\
0.394 \\
\text { inches }\end{array}$ & $\begin{array}{c}0.394 \\
\text { to } \\
0.591 \\
\text { inches }\end{array}$ & $\begin{array}{c}0.591 \\
\text { to } \\
1.546 \\
\text { inches }\end{array}$ & $\begin{array}{c}1.546 \\
\text { to } \\
2.502 \\
\text { inches }\end{array}$ & $\begin{array}{c}2.502 \\
\text { to } \\
3.457 \\
\text { inches }\end{array}$ & $\begin{array}{c}\text { 3.457 } \\
\text { to } \\
3.654 \\
\text { inches }\end{array}$ & $\begin{array}{c}3.654 \\
\text { to } \\
3.851 \\
\text { inches }\end{array}$ & $\begin{array}{c}3.851 \\
\text { to } \\
4.048 \\
\text { inches }\end{array}$ \\
\hline 1.5 & 1.59 & 1.44 & 1.30 & 1.31 & 1.28 & 1.30 & 1.36 & 1.47 & 1.52 \\
\hline 2.5 & 3.84 & 2.44 & 2.31 & 2.24 & 2.09 & 2.16 & 2.14 & 2.20 & 2.46 \\
\hline 3.5 & 4.65 & 4.62 & 4.55 & 4.46 & 4.30 & 4.39 & 4.33 & 4.58 & 4.67 \\
\hline 4.5 & 5.38 & 5.06 & 4.97 & 4.73 & 4.78 & 4.72 & 4.68 & 4.84 & 5.25 \\
\hline 5.5 & 5.84 & 5.72 & 5.49 & 5.38 & 5.31 & 5.34 & 5.36 & 5.56 & 5.82 \\
\hline 6.5 & 5.84 & 5.53 & 5.42 & 5.51 & 5.38 & 5.46 & 5.51 & 5.77 & 6.08 \\
\hline 7.5 & 6.51 & 6.12 & 5.99 & 5.81 & 5.82 & 5.93 & 5.96 & 6.07 & 6.44 \\
\hline 8.5 & 6.52 & 6.23 & 6.23 & 6.07 & 5.93 & 6.00 & 6.07 & 6.29 & 6.34 \\
\hline 9.5 & 6.69 & 6.57 & 6.27 & 6.33 & 6.06 & 6.21 & 6.04 & 6.30 & 6.86 \\
\hline 10.5 & 6.90 & 6.33 & 6.40 & 6.21 & 6.08 & 6.29 & 6.23 & 6.38 & 6.70 \\
\hline 11.5 & 6.92 & 6.36 & 6.27 & 6.34 & 6.15 & 6.36 & 6.28 & 6.32 & 6.80 \\
\hline 12.5 & 6.40 & 6.30 & 6.19 & 6.09 & 6.16 & 6.27 & 6.52 & 6.52 & 6.81 \\
\hline 13.5 & 6.74 & 6.26 & 6.05 & 6.12 & 6.00 & 6.31 & 6.48 & 6.22 & 6.77 \\
\hline 14.5 & 6.51 & 6.10 & 6.09 & 6.10 & 5.90 & 6.23 & 6.39 & 6.38 & 6.70 \\
\hline 15.5 & 6.35 & 5.91 & 5.80 & 5.81 & 5.78 & 5.96 & 5.99 & 6.14 & 6.38 \\
\hline 16.5 & 6.11 & 5.87 & 5.84 & 5.77 & 5.71 & 5.87 & 6.05 & 6.14 & 6.28 \\
\hline 17.5 & 5.75 & 5.40 & 5.49 & 5.39 & 5.46 & 5.52 & 5.60 & 5.57 & 5.89 \\
\hline 18.5 & 5.65 & 5.38 & 5.27 & 5.30 & 5.32 & 5.34 & 5.50 & 5.50 & 5.71 \\
\hline 19.5 & 5.27 & 4.91 & 5.21 & 4.89 & 4.80 & 4.97 & 5.09 & 5.22 & 5.40 \\
\hline 20.5 & 4.91 & 4.56 & 4.76 & 4.61 & 4.52 & 4.77 & 4.73 & 4.75 & 5.05 \\
\hline 21.5 & 4.34 & 4.28 & 4.07 & 4.17 & 4.09 & 4.12 & 4.25 & 4.22 & 4.44 \\
\hline 22.5 & 4.22 & 3.92 & 3.77 & 3.77 & 3.70 & 3.75 & 3.89 & 3.93 & 4.11 \\
\hline 23.5 & 4.30 & 3.99 & 3.90 & 3.89 & 3.75 & 3.89 & 3.94 & 4.07 & 4.36 \\
\hline Ax. Avg. & 5.41 & 5.12 & 5.04 & 4.99 & 4.91 & 5.03 & 5.07 & 5.16 & 5.45 \\
\hline & & & & & & & & 3.49 & \\
\hline
\end{tabular}

POWER DENSITY VALUES NORMALIZED TO A CORE POWER OF 11.57 MW AND ASSUME ALL FISSION ENERGY (EXCLUDING GAMMA-HEATING OF EX-CORE COMPONENTS) IS DEPOSITED IN THE FUEL 
(This page left intentionally blank) 


\section{Argonne}

Nuclear Science \& Engineering Division

Argonne National Laboratory

9700 South Cass Avenue, Bldg. 208

Argonne, IL 60439

www.anl.gov 